\title{
Wind Environment Study of High-rise Residential Building by using Multiple Computational Tools
}

BY

Yi He

MSc., MArch., BE., BSc..

\begin{abstract}
A thesis
submitted to the Victoria University of Wellington in fulfilment of the requirements for the degree of

Doctor of Philosophy

in

Architecture
\end{abstract}

Supervisors:

Dr. Marc Aurel Schnabel, Professor

Dr. Yi Mei, Senior Lecturer

VICTORIA UNIVERSITY OF WELLINGTON 


\section{Acknowledgements}

Foremost, I would like to express my deepest gratitude and appreciation to my supervisors, Prof. Marc Aurel Schnabel and Dr. Yi Mei, for their considerable support through my $\mathrm{PhD}$ program and valuable encouragement at various stages of this thesis. It is a rewarding and enjoyable experience to work with them. I could never complete this thesis without them.

First, I would like to express my special thanks to my primary supervisor Prof. Marc Aurel Schnabel, who has given me endless encouragement and the opportunity of this $\mathrm{PhD}$ program. I and Marc Aurel have known each other since my master program in Hong Kong. After my graduation, I worked as an architect and engineer in a large national design institute for two years before Marc Aurel invited me to join his new research project in Victoria University of Wellington. I hesitated for a time and it was his encouragement and sincerity that moved me to make the decision. Marc is a respectable leader and an excellent scholar, who always motivates me to focus on my research by his kind advice and boundless energy. I appreciate all his contribution to guide my $\mathrm{PhD}$ program.

Second, I am blessed to be supervised by my secondary supervisor Dr. Yi Mei, who has given me technical suggestion and helped in developing logic thinking for my thesis. I came to know Dr. Mei in the second year of my PhD program, when I was in a difficult time. Dr. Mei is a responsible teacher and a resourceful researcher, who provides me great confidence to expound my research capability in the thesis. I appreciate his patience for listening my research ideas all the time. He has set up a benchmark as a college teacher for me to learn from.

Third, there are teachers and colleague friends I am grateful for their kindness and support. So many thanks for the warm help from the Student Advocate of University, the Educational Branch of Chinese Embassy, and the teachers who cared. I also appreciate the professional viewpoints and friendship from my $\mathrm{PhD}$ colleagues.

In the end, I would like to thank my family for their endless love, support and sacrifice. I will never have the courage to give up my previous work and start my $\mathrm{PhD}$ journey without them. They have tolerated a hard time and shared all burdens with me. They always incent me striving forward and taught me never give up. All my conviction and willpower are from them. 


\section{Table of contents}

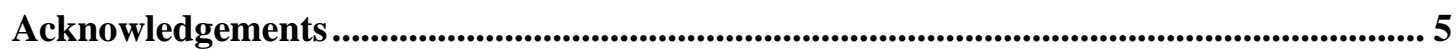

List of figures .............................................................................................................................. 15

List of tables .............................................................................................................................................. 26

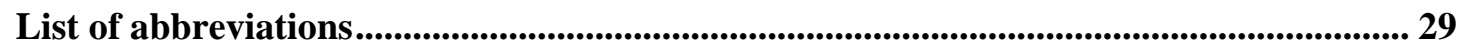

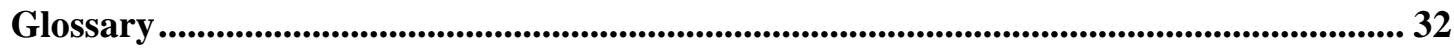

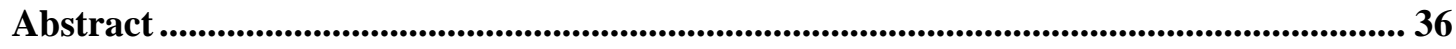

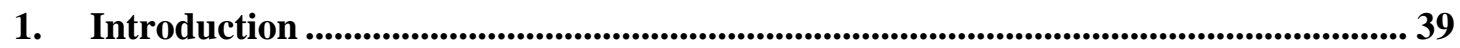

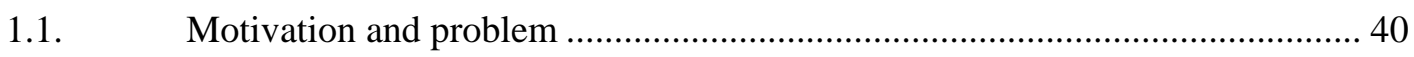

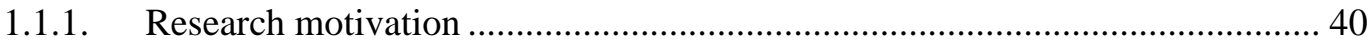

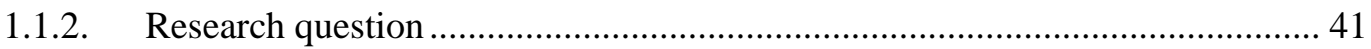

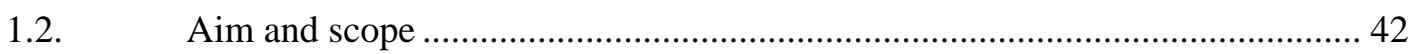

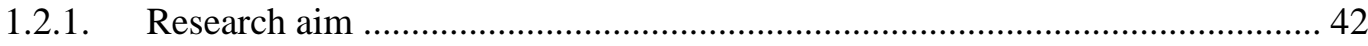

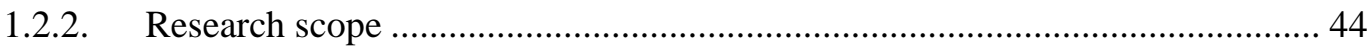

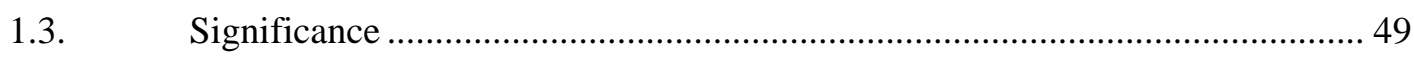

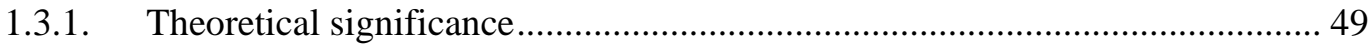

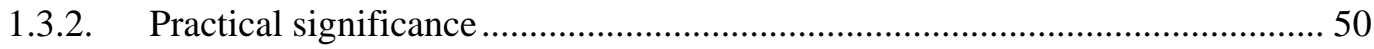

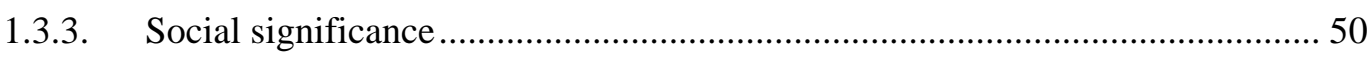

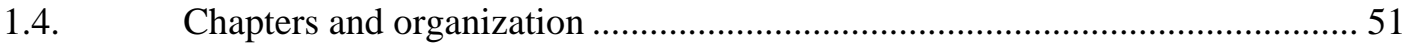

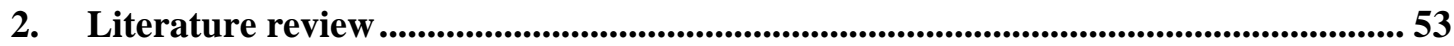

2.1. Development of wind environment studies ..................................................... 53

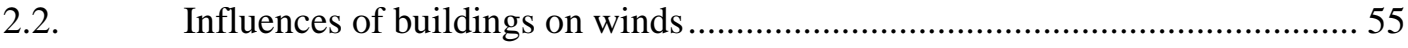

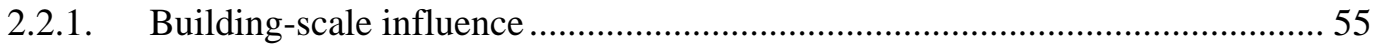

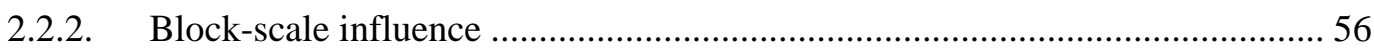

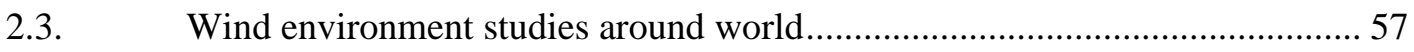

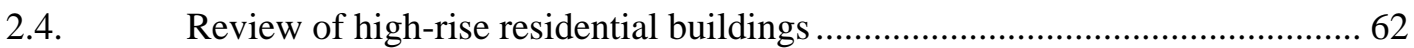

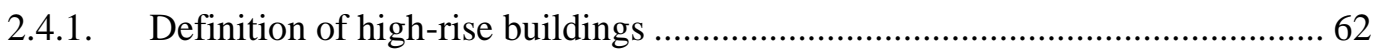

2.4.2. Development of high-rise residential buildings.............................................. 63

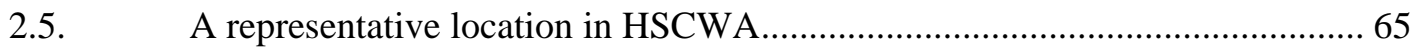

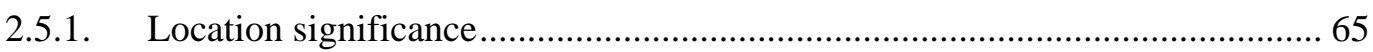

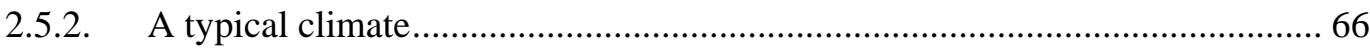




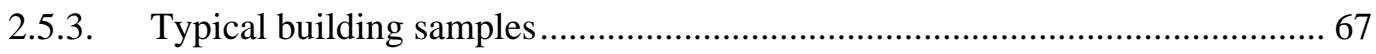

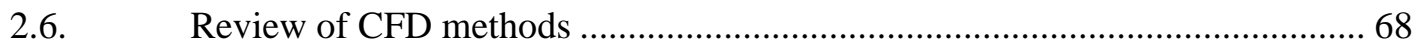

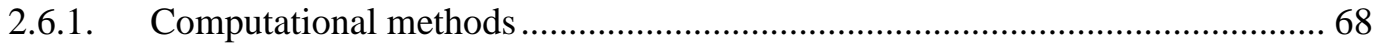

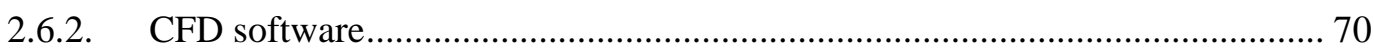

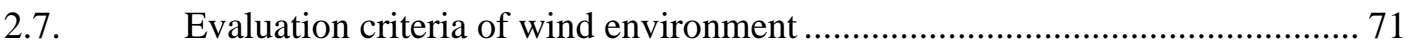

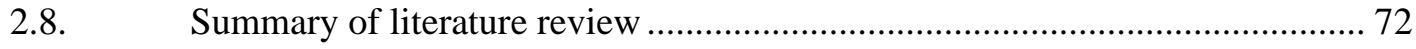

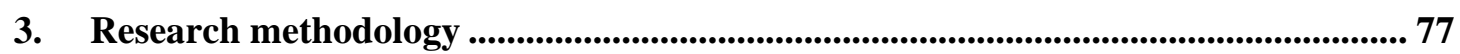

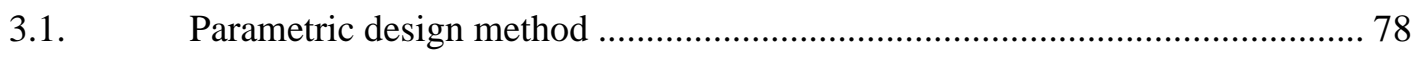

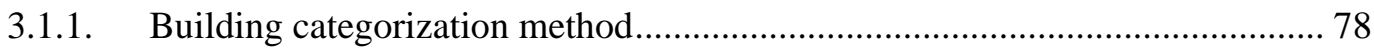

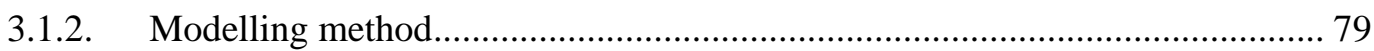

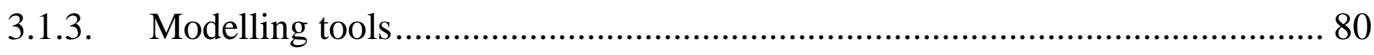

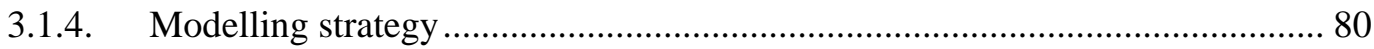

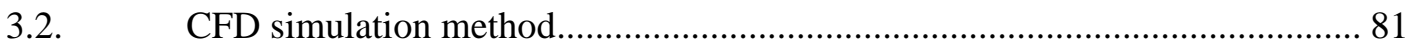

3.2.1. Computational domain and boundary conditions .......................................... 82

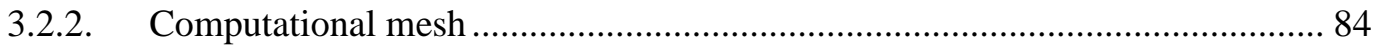

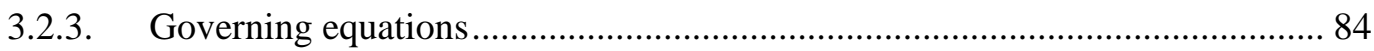

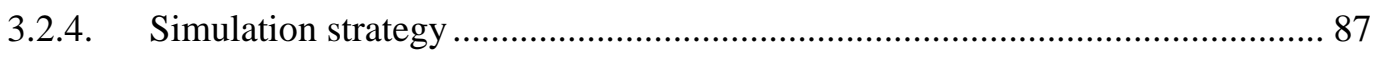

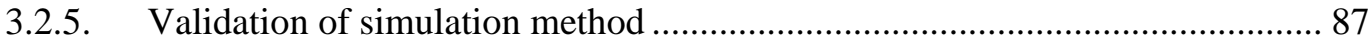

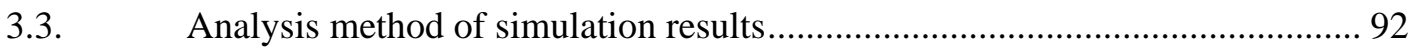

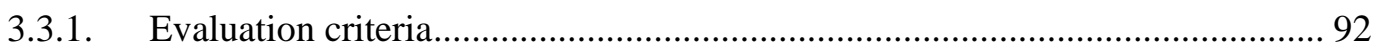

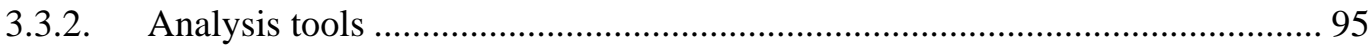

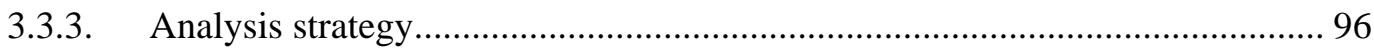

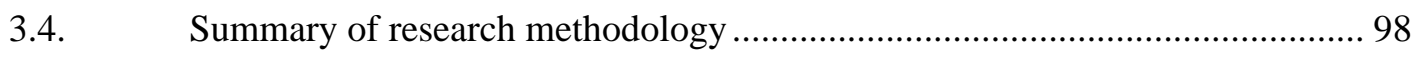

4. Parametric design of the buildings ......................................................................................... 101

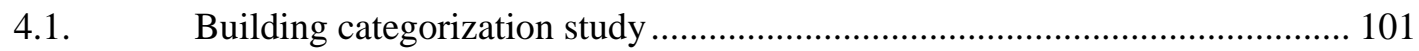

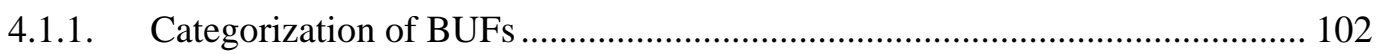

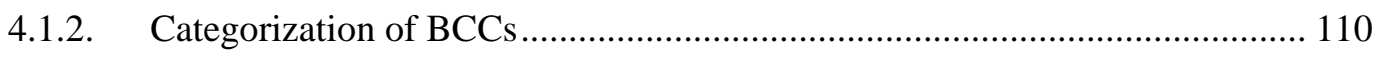

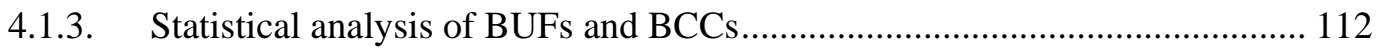

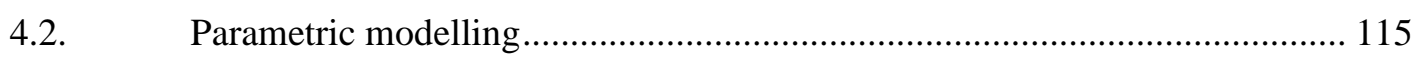

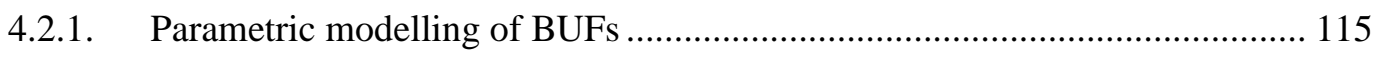

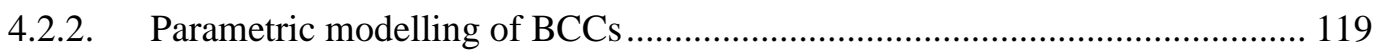

4.2.3. Comparison of parametric modelling and manual modelling ........................ 124 


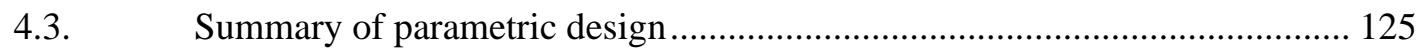

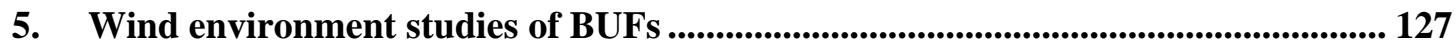

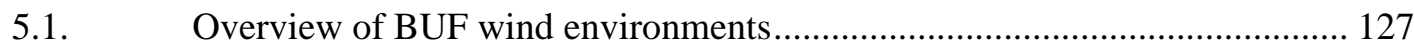

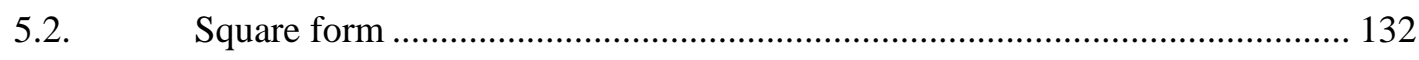

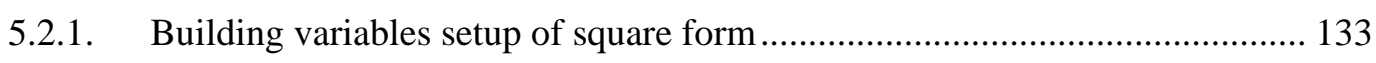

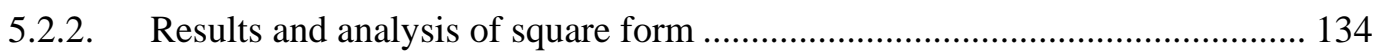

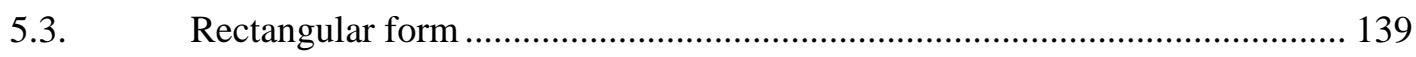

5.3.1. Building variables setup of rectangular form …......................................... 140

5.3.2. Results and analysis of rectangular form ...................................................... 144

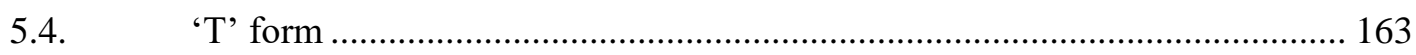

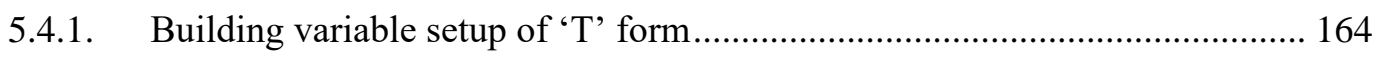

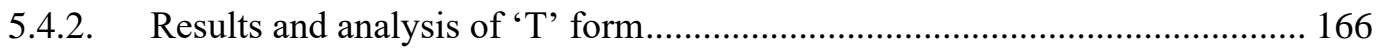

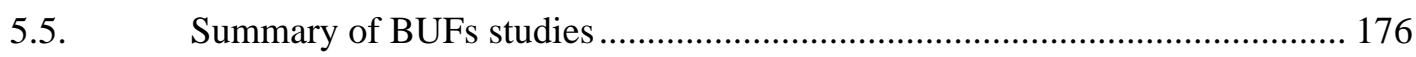

6. Wind environment studies of BCCs................................................................... 179

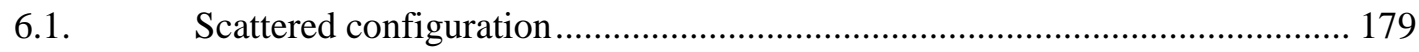

6.1.1. Building variable setup of scattered configuration ...................................... 180

6.1.2. Results and analysis of scattered configuration........................................... 186

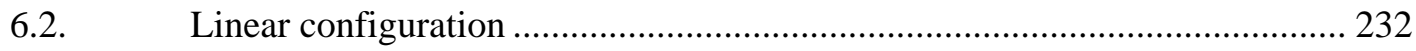

6.2.1. Building variable setup of linear configuration ............................................ 233

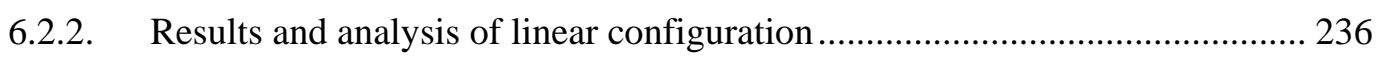

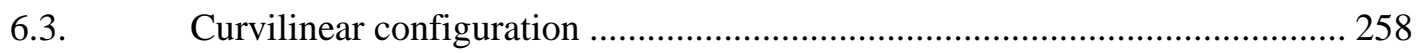

6.3.1. Building-variable setup of curvilinear configuration .................................. 259

6.3.2. Results and analysis of curvilinear configuration........................................ 260

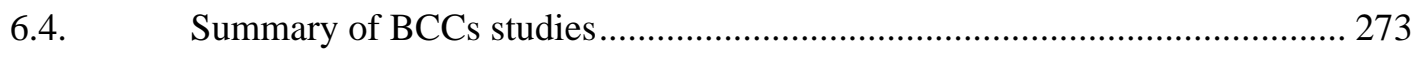

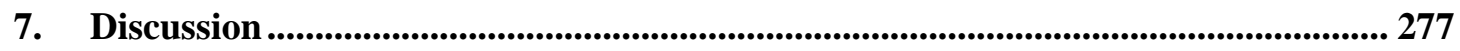

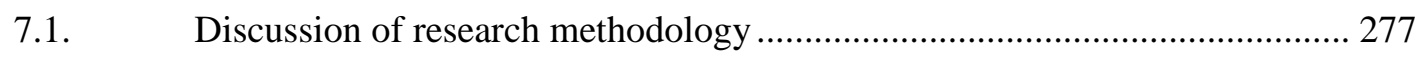

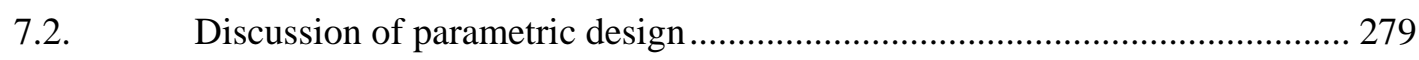

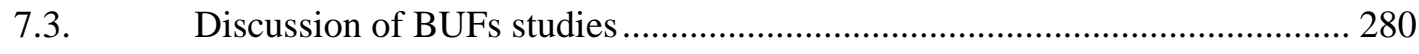

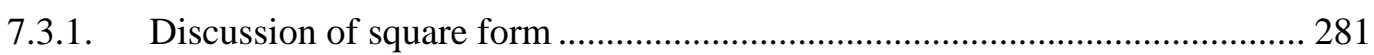

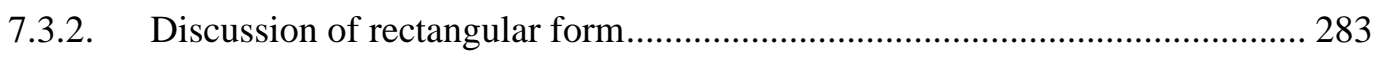

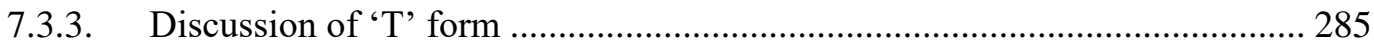

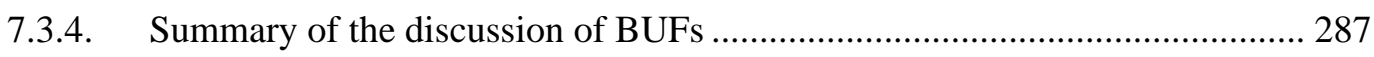




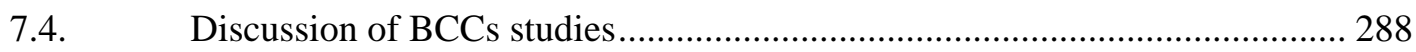

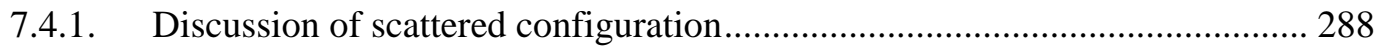

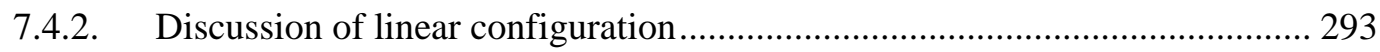

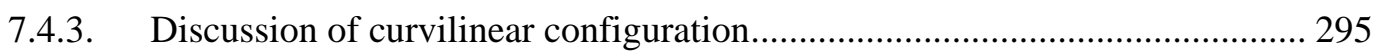

7.4.4. Summary of the discussion of BCCs ............................................................ 297

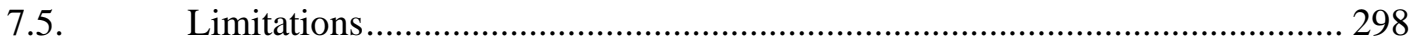

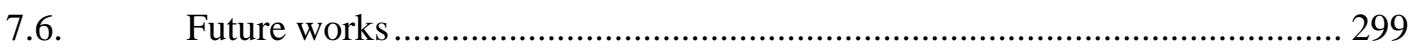

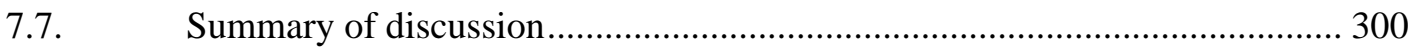

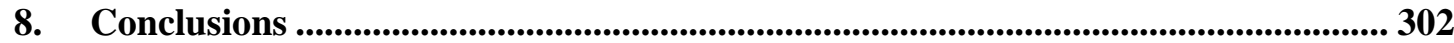

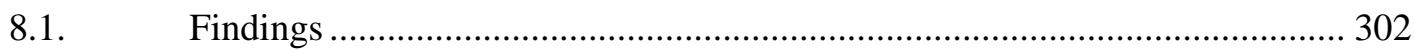

8.1.1. A novel research methodology ……………............................................... 302

8.1.2. A parametric design system ........................................................................... 303

8.1.3. Relationships between the influences and variables of the BUFs ................. 304

8.1.4. Relationships between the influences and variables of the BCCs ................. 308

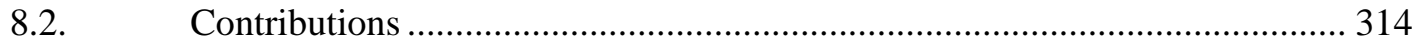

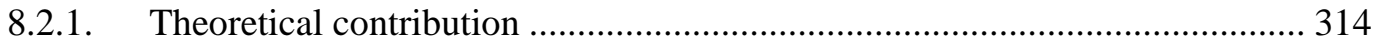

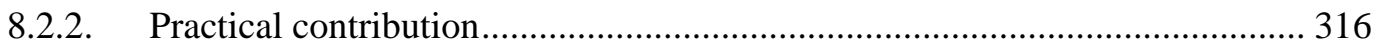

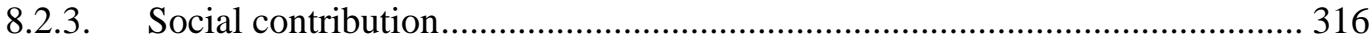

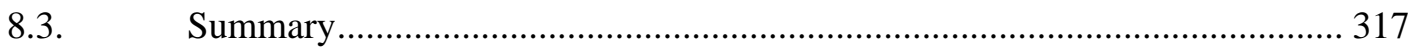

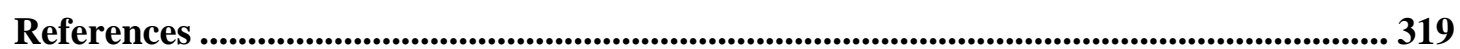

Publications ............................................................................................................................................ 331

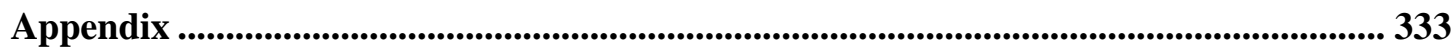




\section{List of figures}

Figure 1. The HSCWA in China (Wang, Wu, Ding, Feng \& Wang, 2014)..................... 45

Figure 2. The location and map of Wuhan City (captured in Google map, 2019).......... 46

Figure 3. The figure on the left shows "the typical air flow features around a building"

(Aynsley, 1999). The figure on the right shows the interaction of air flow and an ordinary rectilinear floor plan building. It explains "the mechanism of downwash and high-speed wind areas may be expected at the pedestrian-level corners" (Cochran, 2004) 56

Figure 4. The wind speed profile of podium layer, urban canopy layer (UCL), roughness sublayer (RSL) and urban boundary layer (UBL) (Oke, 2006; Ng et al., 2011). In the figure, $\mathrm{Vp}, \mathrm{Vc}, \mathrm{Vs}$ are the wind speeds of these layers. 57

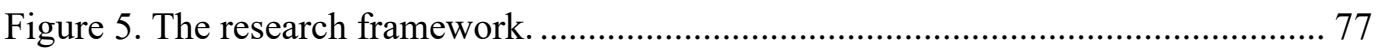

Figure 6. The figure shows the data of monthly average wind velocities of Wuhan City.

The data is collected and calculated from the source of the Chinese Standard Weather Data (CSWD). 83

Figure 7. The figure on the left shows the structured Cartesian immersed-body mesh. The right one shows the refined mesh (Sobachkin \& Dumnoy, 2014). 84

Figure 8 . The figure above shows wind direction, building array and the 12 positions of vertical profiles measured in the simulation and experiment. The figure on the bottom shows the comparison of the horizontal velocity (V) and turbulent kinetic energy (TKE) profiles between CFD simulation (blue lines) and wind tunnel experiment (orange lines). 90

Figure 9. The figure above shows comparisons of wind velocities and TKE of 3 grid sets

(coarse, medium and fine grids) at $\mathrm{x}=-0.075 \mathrm{~m}$ 91

Figure 10. The calculation process of influenced area................................................ 96

Figure 11. An example of simplified floor plan of square form. ….............................. 103

Figure 12. An example of simplified floor plan of rectangular form.......................... 104

Figure 13. An example of simplified floor plan of ' $\mathrm{T}$ ' form........................................ 105

Figure 14. An example of simplified floor plan of frog form...................................... 106

Figure 15. An example of simplified floor plan of hash form. .................................... 106

Figure 16. An example of simplified floor plan of cross form. ................................... 107

Figure 17. An example of simplified floor plan of ' $Y$ ' form. ....................................... 108

Figure 18. An example of simplified floor plan of ' $\mathrm{H}$ ' form. ....................................... 108

Figure 19. Two examples of simplified floor plans of ' $V$ ' form (butterfly form).......... 109

Figure 20. An example of simplified floor plan of circular form. ….............................110

Figure 21. The comparison of numbers of 11 BUFs (codes: F01-F11) in the Optics Valley

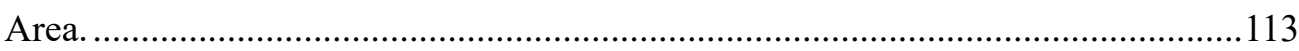

Figure 22. The comparison of numbers of 4 BCCs (codes: C01-C04) in the Optics Valley

Area. 114

Figure 23. The parameters set in the parametric modelling of square and rectangular form.

Figure 24. The parametric model of rectangular form. 
Figure 25. The parameters set in the parametric modelling of ' $\mathrm{T}$ ' form. 119

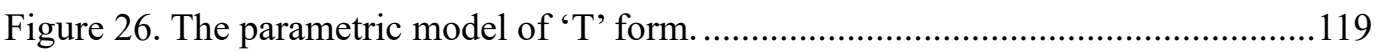

Figure 27. The parameters set in the parametric modelling of scattered configuration. 121

Figure 28. The parametric model of scattered configuration..................................... 121

Figure 29. The parametric model of linear configuration........................................ 122

Figure 30. The parameters set in the parametric modelling of curvilinear configuration.

Figure 31. The parametric model of curvilinear configuration.................................. 124

Figure 32. The comparison of times of creating each single parametric and manual models.

Figure 33. The comparison of total times of parametric modelling and manual modelling.

Figure 34 . An example of the wind velocity magnitudes on horizontal $(1.5 \mathrm{~m}$ pedestrian level) and vertical planes of the square-form building with the size of $36 \mathrm{~m} * 20 \mathrm{~m} * 20 \mathrm{~m}$.

Figure 35 . An example of the wind velocity magnitudes on horizontal $(1.5 \mathrm{~m}$ pedestrian level) and vertical planes of the rectangular-form building with the size of $36 \mathrm{~m} * 15 \mathrm{~m} * 30 \mathrm{~m}$. 128

Figure 36. An example of the wind velocity magnitudes on horizontal $(1.5 \mathrm{~m}$ pedestrian level) and vertical planes of the ' $\mathrm{T}$ '-form building with the major part of $12 \mathrm{~m} * 39 \mathrm{~m} * 72 \mathrm{~m}$ and bulge part of $12 \mathrm{~m} * 18 \mathrm{~m}$.

Figure 37. An example of the air-pressure magnitudes of the square-form building with the size of $36 \mathrm{~m} * 20 \mathrm{~m} * 20 \mathrm{~m}$. 130

Figure 38. An example of the air-pressure magnitudes of the rectangular-form building with the size of $36 \mathrm{~m} * 15 \mathrm{~m} * 30 \mathrm{~m}$. 130

Figure 39. An example of the air-pressure magnitudes of the ' $T$ '-form building with the major part of $12 \mathrm{~m} * 39 \mathrm{~m} * 72 \mathrm{~m}$ and bulge part of $12 \mathrm{~m} * 18 \mathrm{~m}$. 130

Figure 40. An example of the wind-flow streamlines of the square-form buildings with the size of $36 \mathrm{~m} * 20 \mathrm{~m} * 20 \mathrm{~m}$. 131

Figure 41. An example of the wind-flow streamlines of the rectangular-form building with the size of $36 \mathrm{~m} * 15 \mathrm{~m} * 30 \mathrm{~m}$.

Figure 42. An example of the wind-flow streamlines of the ' $T$ '-form building with the major part of $12 \mathrm{~m} * 39 \mathrm{~m} * 72 \mathrm{~m}$ and bulge part of $12 \mathrm{~m} * 18 \mathrm{~m}$. 132

Figure 43. The building variables of square form. 132

Figure 44. The comparison of wind-velocity magnitudes on horizontal and vertical planes of the square-form buildings with the sizes of $36 \mathrm{~m} *(10 \mathrm{~m} * 10 \mathrm{~m}, 15 \mathrm{~m} * 15 \mathrm{~m}, 20 \mathrm{~m} * 20 \mathrm{~m})$.

Figure 45. The influenced areas with different velocities in Group 1 of square form... 135 Figure 46. The figure shows the comparisons of streamlines and air pressure magnitudes on horizontal, vertical planes and building surfaces of the square-form buildings with the sizes of $36 \mathrm{~m} *(10 \mathrm{~m} * 10 \mathrm{~m}, 15 \mathrm{~m} * 15 \mathrm{~m}, 20 \mathrm{~m} * 20 \mathrm{~m})$. 136

Figure 47 . The comparison of wind velocity magnitudes on horizontal and vertical planes of the square-form buildings with different rotation angles of $0,15,30$ and 45 degrees. 
Figure 48. The influenced areas with different velocities in Group 2 of square form... 138

Figure 49. The air pressure magnitudes (right) and streamlines (left) of the square-form buildings with rotation angles of $0,15,30$ and 45 degrees.

Figure 50. The building variables of rectangular form.

Figure 51. The comparison of wind velocity magnitudes on horizontal and vertical planes of the rectangular-form buildings with the sizes of $36 \mathrm{~m} * 10 \mathrm{~m} *(10 \mathrm{~m}, 20 \mathrm{~m}, 30 \mathrm{~m}, 40 \mathrm{~m})$ in Group 1.

Figure 52. The influenced areas with different velocities in Group 1 of rectangular form.

Figure 53. The comparison of low-velocity areas $(0-0.533 \mathrm{~m} / \mathrm{s})$ of the wind velocity magnitude on horizontal plane of the rectangular-form buildings in Group 1....... 146

Figure 54 . The trend of the lengths of low-velocity areas $(0-0.533 \mathrm{~m} / \mathrm{s})$ of the wind velocity magnitude on horizontal plane of the rectangular-form buildings in Group 1....... 146

Figure 55. The comparison of wind velocity magnitudes on horizontal and vertical planes of the rectangular-form buildings in Group 2.

Figure 56. The influenced areas with different velocities in Group 2 of rectangular form.

Figure 57. The comparison of wind velocity magnitudes on horizontal and vertical planes of the rectangular-form buildings in Group 3.

Figure 58. The influenced areas with different velocities in Group 3 of rectangular form.

Figure 59. The comparisons of streamlines (right) and air-pressure magnitudes (left) on horizontal, vertical planes and building surfaces of the rectangular-form buildings in Group 1 ......

Figure 60. The comparisons of streamlines (right) and air-pressure magnitudes (left) on horizontal, vertical planes and building surfaces of the rectangular-form buildings in Group 2.

Figure 61. The comparisons of streamlines (right) and air-pressure magnitudes (left) on horizontal, vertical planes and building surfaces of the rectangular-form buildings in Group 3.

Figure 62. The comparison of wind velocity magnitudes on horizontal and vertical planes of the rectangular-form buildings with different heights in Group 4. 150

Figure 63. The influenced areas with different velocities in Group 4 of rectangular form.

Figure 64. The comparisons of streamlines (right) and air-pressure magnitudes (left) on horizontal, vertical planes and building surfaces of the rectangular-form buildings in Group 4

Figure 65 . The comparison of wind velocity magnitudes on horizontal and vertical planes of the rectangular-form buildings with different heights in Group 5.

Figure 66. The influenced areas with different velocities in Group 5 of rectangular form. 154

Figure 67. The comparison of wind velocity magnitudes on horizontal and vertical planes of the rectangular-form buildings with different heights in Group 6 . 154

Figure 68. The influenced areas with different velocities in Group 6 of rectangular form. 
Figure 69. The comparison of wind velocity magnitudes on horizontal and vertical planes of the rectangular-form buildings with different heights in Group 7.

Figure 70. The influenced areas with different velocities in Group 7 of rectangular form.

Figure 71. The comparisons of streamlines (right) and air-pressure magnitudes (left) on horizontal, vertical planes and building surfaces of the rectangular-form buildings in Group 5 .

Figure 72. The comparisons of streamlines (right) and air-pressure magnitudes (left) on horizontal, vertical planes and building surfaces of the rectangular-form buildings in Group 6.

Figure 73. The comparisons of streamlines (right) and air-pressure magnitudes (left) on horizontal, vertical planes and building surfaces of the rectangular-form buildings in Group 7 .

Figure 74. The comparison of wind velocity magnitudes on horizontal and vertical planes of the rectangular-form buildings with different heights in Group 8.

Figure 75. The influenced areas with different velocities in Group 8 of rectangular form.

Figure 76. The comparison of wind velocity magnitudes on horizontal and vertical planes of the rectangular-form buildings with different heights in Group 9.

Figure 77. The influenced areas with different velocities in Group 9 of rectangular form. 160

Figure 78. The comparison of wind velocity magnitudes on horizontal and vertical planes of the rectangular-form buildings with different heights in Group 10. 160

Figure 79. The influenced areas with different velocities in Group 10 of rectangular form.

Figure 80. The comparisons of streamlines (right) and air-pressure magnitudes (left) on horizontal, vertical planes and building surfaces of the rectangular-form buildings in Group 8 . 162

Figure 81. The comparisons of streamlines (right) and air-pressure magnitudes (left) on horizontal, vertical planes and building surfaces of the rectangular-form buildings in Group 9. 162

Figure 82. The comparisons of streamlines (right) and air-pressure magnitudes (left) on horizontal, vertical planes and building surfaces of the rectangular-form buildings in Group 10 . 163

Figure 83. The building variables of ' $\mathrm{T}$ ' form. 164

Figure 84 . The comparison of wind velocity magnitudes on horizontal and vertical planes of the ' $\mathrm{T}$ '-form buildings with different heights in Group 1. 168

Figure 85. The influenced areas with different velocities in Group 1 of ' $\mathrm{T}$ ' form......... 169

Figure 86. The comparisons of streamlines (right) and air-pressure magnitudes (left) on horizontal, vertical planes and building surfaces of the ' $\mathrm{T}$ '-form buildings in Group 1.

Figure 87 . The comparison of wind velocity magnitudes on horizontal and vertical planes of the ' $\mathrm{T}$ '-form buildings with different heights in Group 2. 
Figure 88. The influenced areas with different velocities in Group 2 of ' $T$ ' form. 172

Figure 89 . The comparison of wind velocity magnitudes on horizontal and vertical planes of the ' $\mathrm{T}$ '-form buildings with different heights in Group 3.

Figure 90. The influenced areas with different velocities in Group 3 of ' $T$ ' form......... 173

Figure 91. The comparison of wind velocity magnitudes on horizontal and vertical planes of the ' $\mathrm{T}$ '-form buildings with different heights in Group 4.

Figure 92. The influenced areas with different velocities in Group 4 of ' $T$ ' form......... 174

Figure 93. The comparisons of wind-flow streamlines (right) and air-pressure magnitudes (left) on horizontal, vertical planes and building surfaces of the ' $\mathrm{T}$ '-form buildings in Group 2 . 175

Figure 94. The comparisons of wind-flow streamlines (right) and air-pressure magnitudes (left) on horizontal, vertical planes and building surfaces of the ' $\mathrm{T}$ '-form buildings in Group 3. 175

Figure 95. The comparisons of wind-flow streamlines (right) and air-pressure magnitudes (left) on horizontal, vertical planes and building surfaces of the ' $\mathrm{T}$ '-form buildings in Group 4 176

Figure 96. The building variables of staggered configuration. 180

Figure 97. The comparison of wind velocity magnitudes on horizontal and vertical planes of the building clusters in Group 1 of scattered configuration. 188

Figure 98. The influenced areas with different velocities in Group 1 of scattered configuration

Figure 99. The comparison of air-pressure magnitudes on the horizontal and vertical planes of the building clusters in Group 1 of scattered configuration

Figure 100. The comparison of wind-flow streamlines (right) and air-pressure magnitudes (left) on the horizontal plane and building surfaces in Group 1 of scattered configuration

Figure 101. The comparison of wind-velocity magnitudes on horizontal and vertical planes of the building clusters in Group 2 of scattered configuration.

Figure 102. The influenced areas with different velocities in Group 2 of scattered configuration.

Figure 103. The comparison of air-pressure magnitudes on the horizontal and vertical planes of the building clusters in Group 2 of scattered configuration. 193

Figure 104. The comparison of wind-flow streamlines (right) and air-pressure magnitudes (left) on the horizontal plane and building surfaces in Group 2 of scattered configuration.

Figure 105. The comparison of wind-velocity magnitudes on horizontal and vertical planes of the building clusters in Group 3 of scattered configuration. 195

Figure 106. The influenced areas with different velocities in Group 3 of scattered configuration.

Figure 107. The comparison of wind-flow streamlines (right) and air-pressure magnitudes (left) on the horizontal plane and building surfaces in Group 3 of scattered configuration.

Figure 108. The comparison of wind-velocity magnitudes on horizontal and vertical planes of the building clusters in Group 4 of scattered configuration. 198 
Figure 109. The influenced areas with different velocities in Group 4 of scattered configuration.

Figure 110. The comparison of wind-flow streamlines (right) and air-pressure magnitudes (left) on the horizontal plane and building surfaces in Group 4 of scattered configuration 200

Figure 111. The comparison of wind-velocity magnitudes on horizontal and vertical planes of the building clusters in Group 5 of scattered configuration.

Figure 112. The influenced areas with different velocities in Group 5 of scattered configuration. 202

Figure 113. The comparison of wind-velocity magnitudes on horizontal and vertical planes of the building clusters in Group 6 of scattered configuration 204

Figure 114. The influenced areas with different velocities in Group 6 of scattered configuration 204

Figure 115. The comparison of wind-velocity magnitudes on horizontal and vertical planes of the building clusters in Group 7 of scattered configuration.

Figure 116. The influenced areas with different velocities in Group 7 of scattered configuration. 205

Figure 117. The comparison of air-pressure magnitudes on the horizontal and vertical planes of the building clusters in Group 5 of scattered configuration.

Figure 118. The comparison of wind-flow streamlines (right) and air-pressure magnitudes (left) on the horizontal plane and building surfaces in Group 5 of scattered configuration. 207

Figure 119. The comparison of air-pressure magnitudes on the horizontal and vertical planes of the building clusters in Group 6 of scattered configuration. 208

Figure 120. The comparison of wind-flow streamlines (right) and air-pressure magnitudes (left) on the horizontal plane and building surfaces in Group 6 of scattered configuration. 208

Figure 121. The comparison of air-pressure magnitudes on the horizontal and vertical planes of the building clusters in Group 7 of scattered configuration. 209

Figure 122. The comparison of wind-flow streamlines (right) and air-pressure magnitudes (left) on the horizontal plane and building surfaces in Group 7 of scattered configuration 209

Figure 123. The comparison of wind-velocity magnitudes on horizontal and vertical planes of the building clusters in Group 8 of scattered configuration.

Figure 124. The influenced areas with different velocities in Group 8 of scattered configuration.

Figure 125. The comparison of wind-velocity magnitudes on horizontal and vertical planes of the building clusters in Group 9 of scattered configuration. 213

Figure 126. The influenced areas with different velocities in Group 9 of scattered configuration 213

Figure 127. The comparison of wind-flow streamlines (right) and air-pressure magnitudes (left) on the horizontal plane and building surfaces in Group 8 of scattered configuration

Figure 128. The comparison of wind-flow streamlines (right) and air-pressure magnitudes 
(left) on the horizontal plane and building surfaces in Group 9 of scattered configuration

Figure 129. The comparison of wind-velocity magnitudes on horizontal and vertical planes of the building clusters in Group 10 of scattered configuration.

Figure 130. The influenced areas with different velocities in Group 10 of scattered configuration.

Figure 131. The comparison of wind-velocity magnitudes on horizontal and vertical planes of the building clusters in Group 11 of scattered configuration.

Figure 132. The influenced areas with different velocities in Group 11 of scattered configuration.

Figure 133. The comparison of wind-flow streamlines (right) and air-pressure magnitudes (left) on the horizontal plane and building surfaces in Group 10 of scattered configuration

Figure 134. The comparison of wind-flow streamlines (right) and air-pressure magnitudes (left) on the horizontal plane and building surfaces in Group 11 of scattered configuration.

Figure 135. The comparison of wind-velocity magnitudes on horizontal and vertical planes of the building clusters in Group 12 of scattered configuration.

Figure 136. The influenced areas with different velocities in Group 12 of scattered configuration.

Figure 137. The comparison of air-pressure magnitudes on the horizontal and vertical planes of the building clusters in Group 12 of scattered configuration 224

Figure 138. The comparison of wind-flow streamlines (right) and air-pressure magnitudes (left) on the horizontal plane and building surfaces in Group 12 of scattered configuration. 225

Figure 139. The comparison of wind-velocity magnitudes on horizontal and vertical planes of the building clusters in Group 13 of scattered configuration. 226

Figure 140. The influenced areas with different velocities in Group 13 of scattered configuration 226

Figure 141. The comparison of air-pressure magnitudes on the horizontal and vertical planes of the building clusters in Group 13 of scattered configuration.....

Figure 142. The comparison of wind-flow streamlines (right) and air-pressure magnitudes (left) on the horizontal plane and building surfaces in Group 13 of scattered configuration.

Figure 143. The comparison of wind-velocity magnitudes on horizontal and vertical planes of the building clusters in Group 14 of scattered configuration.

Figure 144. The influenced areas with different velocities in Group 14 of scattered configuration.

Figure 145. The comparison of air-pressure magnitudes on the horizontal and vertical planes of the building clusters in Group 14 of scattered configuration

Figure 146. The comparison of wind-flow streamlines (right) and air-pressure magnitudes (left) on the horizontal plane and building surfaces in Group 14 of scattered configuration

Figure 147 . The building variables of linear configuration. 233 
Figure 148. The comparison of wind-velocity magnitudes on horizontal and vertical planes of the building clusters in Group 1 of linear configuration.

Figure 149. The influenced areas with different velocities in Group 1 of linear configuration.

Figure 150. The comparison of air-pressure magnitudes on the horizontal and vertical planes of the building clusters in Group 1 of linear configuration. 239

Figure 151. The comparison of wind-flow streamlines (right) and air-pressure magnitudes (left) on the horizontal plane and building surfaces in Group 1 of linear configuration.

Figure 152. The comparison of wind-velocity magnitudes on horizontal and vertical planes of the building clusters in Group 2 of linear configuration.

Figure 153. The influenced areas with different velocities in Group 2 of linear configuration

Figure 154. The comparison of air-pressure magnitudes on the horizontal and vertical planes of the building clusters in Group 1 of linear configuration

Figure 155. The comparison of wind-flow streamlines (right) and air-pressure magnitudes (left) on the horizontal plane and building surfaces in Group 2 of linear configuration.

Figure 156. The comparison of wind-velocity magnitudes on horizontal and vertical planes of the building clusters in Group 3 of linear configuration.

Figure 157. The influenced areas with different velocities in Group 3 of linear configuration. 245

Figure 158. The comparison of air-pressure magnitudes on the horizontal and vertical planes of the building clusters in Group 3 of linear configuration. 246

Figure 159. The comparison of wind-flow streamlines (right) and air-pressure magnitudes (left) on the horizontal plane and building surfaces in Group 3 of linear configuration.

Figure 160. The comparison of wind-velocity magnitudes on horizontal and vertical planes of the building clusters in Group 4 of linear configuration

Figure 161. The influenced areas with different velocities in Group 4 of linear configuration.

Figure 162. The comparison of air-pressure magnitudes on the horizontal and vertical planes of the building clusters in Group 4 of linear configuration..... 250

Figure 163. The comparison of wind-flow streamlines (right) and air-pressure magnitudes (left) on the horizontal plane and building surfaces in Group 4 of linear configuration. 250

Figure 164. The comparison of wind-velocity magnitudes on horizontal and vertical planes of the building clusters in Group 5 of linear configuration. 252

Figure 165. The influenced areas with different velocities in Group 5 of linear configuration 252

Figure 166. The comparison of air-pressure magnitudes on the horizontal and vertical planes of the building clusters in Group 5 of linear configuration 253

Figure 167. The comparison of wind-flow streamlines (right) and air-pressure magnitudes (left) on the horizontal plane and building surfaces in Group 5 of linear configuration. 
Figure 168. The comparison of wind-velocity magnitudes on horizontal and vertical planes of the building clusters in Group 6 of linear configuration. 256

Figure 169. The influenced areas with different velocities in Group 6 of linear configuration 256

Figure 170. The comparison of air-pressure magnitudes on the horizontal and vertical planes of the building clusters in Group 6 of linear configuration

Figure 171. The comparison of wind-flow streamlines (right) and air-pressure magnitudes (left) on the horizontal plane and building surfaces in Group 6 of linear configuration.

Figure 172. The building variables of curvilinear configuration. 259

Figure 173. The comparison of wind-velocity magnitudes on horizontal and vertical planes of the building clusters in Group 1 of curvilinear configuration. 262

Figure 174. The influenced areas with different velocities in Group 1 of curvilinear configuration. 262

Figure 175. The influenced areas with the velocity range of $0.000-0.533 \mathrm{~m} / \mathrm{s}$ in Group 1 of curvilinear configuration. 263

Figure 176. The influenced areas with the velocity range of $0.000-0.178 \mathrm{~m} / \mathrm{s}$ in Group 1 of curvilinear configuration 263

Figure 177. The influenced areas with the velocity range of $0.178-0.356 \mathrm{~m} / \mathrm{s}$ in Group 1 of curvilinear configuration. 264

Figure 178. The influenced areas with the velocity range of $0.356-0.533 \mathrm{~m} / \mathrm{s}$ in Group 1 of curvilinear configuration

Figure 179. The influenced areas with the velocity range of $1.422-1.600 \mathrm{~m} / \mathrm{s}$ in Group 1 of curvilinear configuration. 265

Figure 180. The comparison of air-pressure magnitudes on the horizontal and vertical planes of the building clusters in Group 1 of curvilinear configuration 266

Figure 181. The comparison of wind-flow streamlines (top) and air-pressure magnitudes (bottom) on the horizontal plane and building surfaces in Group 1 of curvilinear configuration.

Figure 182. The comparison of wind-velocity magnitudes on horizontal and vertical planes of the building clusters in Group 2 of curvilinear configuration. 268

Figure 183. The influenced areas with different velocities in Group 2 of curvilinear configuration

Figure 184. The influenced areas with the velocity range of $0.000-0.533 \mathrm{~m} / \mathrm{s}$ in Group 2 of curvilinear configuration.

Figure 185. The influenced areas with the velocity range of $0.000-0.178 \mathrm{~m} / \mathrm{s}$ in Group 2 of curvilinear configuration

Figure 186. The influenced areas with the velocity range of $0.178-0.356 \mathrm{~m} / \mathrm{s}$ in Group 2 of curvilinear configuration.

Figure 187. The influenced areas with the velocity range of $0.356-0.533 \mathrm{~m} / \mathrm{s}$ in Group 2 of curvilinear configuration.

Figure 188. The influenced areas with the velocity range of $1.422-1.600 \mathrm{~m} / \mathrm{s}$ in Group 2 of curvilinear configuration 
Figure 189. The comparison of air-pressure magnitudes on the horizontal and vertical planes of the building clusters in Group 2 of curvilinear configuration.

Figure 190. The comparison of wind-flow streamlines (top) and air-pressure magnitudes (bottom) on the horizontal plane and building surfaces in Group 2 of curvilinear configuration

Figure 191. The wind environment influenced by the variables of square form. .......... 282

Figure 192. The wind environment influenced by the variables of the rectangular form.

Figure 193. The wind environment influenced by the variables of ' $\mathrm{T}$ ' form. 287

Figure 194. The wind environment influenced by the variables of scattered configuration.

Figure 195. The wind environment influenced by the variables of linear configuration.

Figure 196. The wind environment influenced by the variables of curvilinear configuration.

Figure 197. The influences on wind environments are relevant to the windward projective areas. 306

Figure 198. The wind environment influenced by the rectangular-form building (the surface with the width on the windward side). 307

Figure 199. The comparison of the square-form buildings with the surfaces which are vertical and not vertical from the wind direction 307

Figure 200. The wind environment influenced by the ' $\mathrm{T}$ '-form building. 308

Figure 201. The windward projective area of the entire building cluster. 312

Figure 202. The comparison of the influences of the increases of the longitudinal and transverse distances of the scattered-configuration building cluster. 312

Figure 203. The comparison of the influences of the normal scattered configuration and the staggered configuration. 313

Figure 204. The comparison of the influences of the curvilinear building rows with the convex and concave surfaces on the windward side. 


\section{List of tables}

Table 1. Comparison of several common CFD tools.

Table 2. The relationship of human comfort and wind velocity provided by Wise and Pendwarden (Penwarden \& Wise, 1975).

Table 3. The table shows the calculation results of erms and GCI of wind velocity (V) and turbulent kinetic energy (TKE) at $\mathrm{x}=-0.075 \mathrm{~m}$

Table 4. The table shows the calculation results of the statistical discrepancies of wind velocity (V) and turbulent kinetic energy (TKE) in the experiment and simulation.91

Table 5. Categorization of 11 BUFs. 102

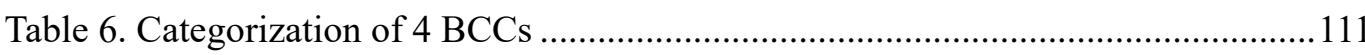

Table 7. The three cases in Group 1 of square form. ................................................ 133

Table 8. The four cases in Group 2 of square form................................................... 133

Table 9. The four cases in Group 1 of rectangular form............................................. 140

Table 10. The four cases in Group 2 of rectangular form.......................................... 141

Table 11. The three cases in Group 3 of rectangular form........................................... 141

Table 12. The three cases in Group 4 of rectangular form.......................................... 141

Table 13. The four cases in Group 5 of rectangular form. ........................................... 142

Table 14. The four cases in Group 6 of rectangular form............................................ 142

Table 15. The three cases in Group 7 of rectangular form........................................... 142

Table 16. The four cases in Group 8 of rectangular form. .......................................... 143

Table 17. The four cases in Group 9 of rectangular form......................................... 143

Table 18. The four cases in Group 10 of rectangular form........................................... 143

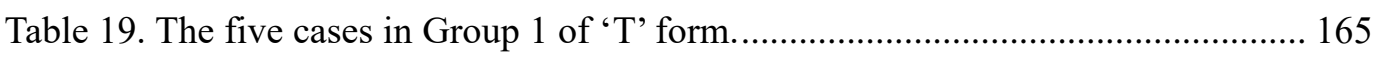

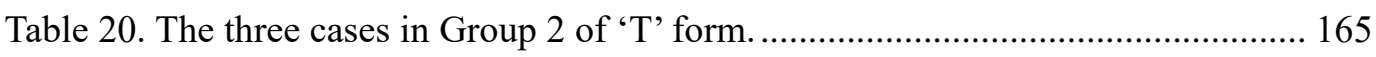

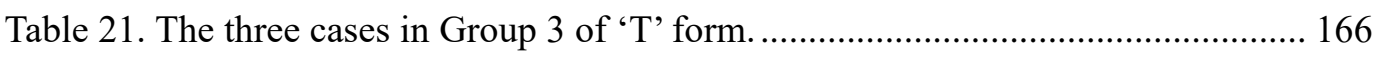

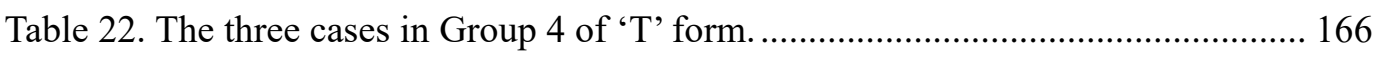

Table 23. The three cases in Group 1 of scattered configuration.................................. 181

Table 24. The three cases in Group 2 of scattered configuration................................. 181

Table 25. The three cases in Group 3 of scattered configuration................................. 181

Table 26. The three cases in Group 4 of scattered configuration.................................. 182

Table 27. The three cases in Group 5 of scattered configuration.................................. 182

Table 28. The three cases in Group 6 of scattered configuration................................. 183

Table 29. The three cases in Group 7 of scattered configuration.................................. 183

Table 30. The three cases in Group 8 of scattered configuration................................. 183

Table 31. The three cases in Group 9 of scattered configuration.................................. 184

Table 32. The three cases in Group 10 of scattered configuration............................... 184

Table 33. The three cases in Group 11 of scattered configuration................................. 184

Table 34. The three cases in Group 12 of scattered configuration................................. 185

Table 35. The three cases in Group 13 of scattered configuration................................. 185

Table 36. The three cases in Group 14 of scattered configuration................................. 186

Table 37. The three cases in Group 1 of linear configuration...................................... 234

Table 38. The three cases in Group 2 of linear configuration...................................... 234 
Table 39. The three cases in Group 3 of linear configuration.

Table 40. The three cases in Group 4 of linear configuration.

Table 41. The three cases in Group 5 of linear configuration

Table 42. The three cases in Group 6 of linear configuration

Table 43. The cases in Group 1 and Group 2 of curvilinear configuration.

Table 44. Relationships between the influences and building variables of square form 282

Table 45. Relationships between the influences and building variables of rectangular form

Table 46. Relationships between the influences and building variables of ' $\mathrm{T}$ ' form ..... 287

Table 47. Relationships between the influences and variables of scattered configuration 292

Table 48. Relationships between the influences and variables of linear configuration . 295 Table 49. Relationships between the influences and variables of curvilinear configuration

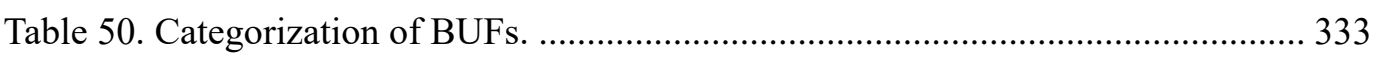

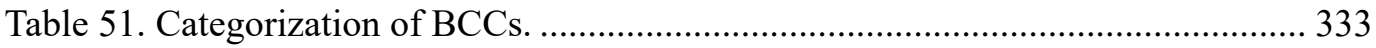

Table 52. Categorization record of BUFs and BCCs for high-rise residential buildings in the Optics Valley area, Wuhan, China. 


\section{List of abbreviations}

ASHRAE

BCC

BUF

BEAM

BREEAM

CAAD

CFD

CSWD

CTBUH

CTYW

DES

DNS

GDP

GH

HSCWA

IWEC

LES

LEED

MHURDC

$\mathrm{NZBCH}$

RANS

RSL

SRANS

UBL

UCL
American Society of Heating, Refrigerating and Air-Conditioning

Engineers

Building Cluster Configuration

Building Unit Form

Building Environmental Assessment Method, Hong Kong

Building Research Establishment Environment Assessment Method,

United Kingdom

Computer Aided Architecture Design

Computational Fluid Dynamics

Chinese Standard Weather Data

The Council on Tall Buildings and Urban Habitat

Chinese Typical Year Weather

Detached Eddy Simulation

Direct Numerical Simulation

Gross Domestic Product

Grasshopper for Rhino

Hot Summer and Cold Winter Area

International Weather for Energy Calculation

Large Eddies Simulation

Leadership in Energy and Environment Design, United States

Ministry of Housing and Urban-Rural Development of China

New Zealand Building Code Handbook

Reynolds Average Navier-Stokes

Roughness Sublayer

Steady Reynolds Averaged Navier-Stokes

Urban Boundary Layer

Urban Canopy Layer 
UHI

UN

WB
Urban Heat Island Effect

United Nations

World Bank 


\section{Glossary}

\begin{tabular}{|c|c|}
\hline $\begin{array}{l}\text { Building Cluster Configuration } \\
\text { (BCC) }\end{array}$ & $\begin{array}{l}\text { The arrangement (layout) of a group of several building } \\
\text { units. }\end{array}$ \\
\hline Building unit & $\begin{array}{l}\text { A single building with at least an independent entrance } \\
\text { and an independent vertical traffic system (the } \\
\text { definition is explained in details in both Literature } \\
\text { Review Chapter and Parametric Design Chapter). }\end{array}$ \\
\hline Building Unit Form (BUF) & $\begin{array}{l}\text { Building-Unit Form (BUF) is the shape (geometry) of } \\
\text { a single building unit (the definition is explained in } \\
\text { details in the Parametric Design Chapter). }\end{array}$ \\
\hline Building variables & $\begin{array}{l}\text { The variables that describe geometric attributes of } \\
\text { BUFs and BCCs, such as length, height and transverse } \\
\text { distance etc.. }\end{array}$ \\
\hline Circular form & $\begin{array}{l}\text { A BUF with an architectural plane which is similar to } \\
\text { the shape of a circle. }\end{array}$ \\
\hline $\begin{array}{l}\text { Cross form (' } \mathrm{X} \text { ' form, Windmill } \\
\text { form) }\end{array}$ & $\begin{array}{l}\text { A BUF with an architectural plane which is similar to } \\
\text { the shape of a cross. }\end{array}$ \\
\hline Compositive configuration & A BCC with several kinds of configurations. \\
\hline Curvilinear configuration & $\begin{array}{l}\text { A BCC with several building units connected one by } \\
\text { one to form one curvilinear building row or several } \\
\text { curvilinear building rows. }\end{array}$ \\
\hline Frog form & $\begin{array}{l}\text { A BUF with an architectural plane which is similar to } \\
\text { the shape of a frog. }\end{array}$ \\
\hline ' $\mathrm{H}$ ' form & $\begin{array}{l}\text { A BUF with an architectural plane which is similar to } \\
\text { the shape of a capital letter ' } \mathrm{H} \text { '. }\end{array}$ \\
\hline Hash form ('\#’ form) & $\begin{array}{l}\text { A BUF with an architectural plane which is similar to } \\
\text { the shape of a hash. }\end{array}$ \\
\hline High-velocity areas & The areas with relatively high wind velocities on the \\
\hline
\end{tabular}




\begin{tabular}{|c|c|}
\hline & $\begin{array}{l}\text { pedestrian level around the buildings. Usually, there } \\
\text { are high-velocity areas on the lateral sides of a building } \\
\text { (the front sides are windward). }\end{array}$ \\
\hline $\begin{array}{l}\text { Hot Summer and Cold Winter } \\
\text { Area (HSCWA) }\end{array}$ & $\begin{array}{l}\text { The 'Hot-Summer and Cold-Winter Area' refers to the } \\
\text { (urban) area with the climate which is hot in summer } \\
\left(25-30^{\circ} \mathrm{C} \text { ) and cold in winter }\left(0-10^{\circ} \mathrm{C}\right) \text {. This research }\right. \\
\text { focuses on the contemporary high-rise residential } \\
\text { buildings in the Optic Valley Area of Wuhan due to its } \\
\text { representativeness of developing regions. }\end{array}$ \\
\hline Influences & $\begin{array}{l}\text { The influences on wind environments of buildings. The } \\
\text { influences are evaluated by the criteria. This research } \\
\text { focuses on the analysis of wind velocities on the } \\
\text { pedestrian level. Air-pressure magnitudes and wind- } \\
\text { flow streamlines help in explaining the mechanisms. }\end{array}$ \\
\hline Irregular form & $\begin{array}{l}\text { A BUF with an architectural plane which is in irregular } \\
\text { shape. }\end{array}$ \\
\hline Linear configuration & $\begin{array}{l}\text { A BCC with several building units connected one by } \\
\text { one to form one linear building row or several linear } \\
\text { building rows. }\end{array}$ \\
\hline Low-velocity areas & $\begin{array}{l}\text { The areas with relatively low wind velocities on the } \\
\text { pedestrian level around the buildings. Usually there are } \\
\text { relatively large low-velocity areas on the leeward sides } \\
\text { of buildings. }\end{array}$ \\
\hline Outdoor ventilation & $\begin{array}{l}\text { The act of supplying fresh air in the outdoor spaces } \\
\text { around the buildings. The outdoor ventilation is } \\
\text { directly relevant to the wind velocities. }\end{array}$ \\
\hline Pixels & $\begin{array}{l}\text { The magnitude maps (of wind velocities and air } \\
\text { pressures) are shown in pictures after CFD simulations. } \\
\text { A pixel is the smallest area on a picture which can be }\end{array}$ \\
\hline
\end{tabular}




\begin{tabular}{|l|l|}
\hline & given a separate color according to the value. \\
\hline Rectangular form & $\begin{array}{l}\text { A BUF with an architectural plane of rectangular } \\
\text { shape. }\end{array}$ \\
\hline Scattered configuration & $\begin{array}{l}\text { A BCC with several scattered building units which are } \\
\text { independent from each other. }\end{array}$ \\
\hline Square form & A BUF with an architectural plane of square shape. \\
\hline Multiple computational tools & $\begin{array}{l}\text { The computational tools used in the wind environment } \\
\text { studies of this research, including the modelling tool, } \\
\text { simulation tool and analysis tool. }\end{array}$ \\
\hline ' $\mathrm{T}$ ' form & $\begin{array}{l}\text { A BUF with an architectural plane which is similar to } \\
\text { the shape of a capital letter ' } \mathrm{T} \text { '. }\end{array}$ \\
\hline 'V' form (Butterfly form) & $\begin{array}{l}\text { A BUF with an architectural plane which is similar to } \\
\text { the shape of a capital letter ' } \mathrm{V} \text { '. }\end{array}$ \\
\hline ' $\mathrm{Y}$ ' form & A BUF with an architectural plane which is similar to \\
\end{tabular}




\section{Abstract}

Wind environment is a key element of sustainable design of architecture. Concerning major trends of climate changes and urbanizations, this research aims to study the relationships between the influences on wind environments and variables related to forms and configurations of contemporary high-rise residential buildings. A novel methodology consisting of parametric design, CFD simulation, and analysis is developed by integrating multiple computational tools, and the evaluation criteria. The integration provides abundant functions and an efficient modelling-simulation-analysis solution for iterative comparison studies. By using a parametric modelling method, building models can be created automatically to help in mesh generation for CFD simulations; the actual influenced areas with different wind velocity ranges can be calculated and compared quantitatively through the calculations of wind-velocity magnitudes from simulation results, at each pixel location on a rendered section. Based on the architectural morphology of Building-Unit Forms (BUFs) and Building-Cluster Configurations (BCCs) in an area classified in China as a Hot-Summer and Cold-Winter Area (HSCWA), the parametric design sets up a bridge between building variables and CFD simulations. A series of representative BUFs and BCCs of high-rise residential buildings are designed for CFD simulations by establishing parametric design system based on the building categorization study. In the wind environment studies, influences of buildings are evaluated based on the windvelocity magnitudes according to the criteria. The trends of influences can be studied through iterative analysis of several cases with different variables. The mechanisms are illustrated through the air-pressure magnitudes and the wind-flow streamlines. In the wind environment studies of BUFs, relationships between influences on wind environments and building variables of three representative BUFs are studied, including square form, rectangular form, and ' $\mathrm{T}$ ' form. The results of the BUFs studies can be summarized: (1) the influences on wind environments increase as the height and windward length are increased, because more winds are obstructed by the increasing windward surface; (2) the influences on wind environments decrease as the ratio of length and width is increased before the ratio reaches a particular value, because influenced air-pressure area is decreased; (3) the influences on the wind environments decrease 
as the bulge-part sizes of the ' $T$ '-form buildings increase, because the increases of bulge-part sizes help to divide winds and lead them to flow around the buildings; (4) the outdoor ventilation is improved as the rotation angle increases, because the non-vertical windward surface promotes the wind flow. In the wind environment studies of BCCs, relationships between influences on wind environments and building variables of three representative BCCs are studied, including scattered configuration, linear configuration, and curvilinear configuration. Results of the BCCs studies can be summarized: (1) the outdoor ventilation of scattered configuration is the best, because it is relatively easy for winds to flow around the scattered building units; (2) the outdoor ventilation can be improved as the longitudinal distance and staggered distance are increased, because the larger building interval promotes winds to flow through the building cluster; (3) for curvilinear configuration, the convex surface on the windward side can promote the wind flow, and the concave surface on the windward side can obstruct the wind flow. The results of the BUFs studies and the BCCs studies all show that the increases of the windward projective areas of buildings can increase the influences on wind environments, because more winds are obstructed. Therefore, the relationships between the influences on wind environments and the building variables of the BUFs and BCCs can be discovered, which can give information to the optimization of wind environments. In summary, the thesis presents a challenging and significant research that contributes original knowledge for wind environment studies in the urban micro climate. And the knowledge is universal and applicable to the practical design projects and also beneficial to the sustainability.

Keywords: Wind environment, Sustainability, Parametric method, CFD, Influences on winds, Building variables 


\section{Introduction}

'Urban settlements are formed by replacing natural surroundings by urban environments and the latter create their own, unique microclimates' (Toparlar et al., 2017). Research of wind environment is directly associated with urban microclimates. In particular, it focuses on studying the wind flow influenced by surrounding objects such as buildings in urban areas. As a key element in sustainable design, wind environment study is becoming popular due to the continuous increase of importance of urban sustainability in the $21^{\text {st }}$ century. Appropriate wind environments can contribute to the improvements of thermal comfort, prevention of safety issue, reduction of air pollution, building performance for saving energy of cooling and heating. The accumulation of changes of microclimates could potentially influence the climates of large urban areas. Take Urban Heat Island (UHI) effect for example. In short, the UHI effect is a phenomenon that the temperature of an urban area is significantly higher than its surrounding rural area (Oke, 2006). The UHI effect is the most apparent when winds are weak (Surhone, Timpledon, \& Marseken, 2010). The UHI intensity decreases when the wind speed increases (Ackerman, 1985; Park, 1986; Travis et al., 1987; Kidder \& Essenwanger, 1995; Eliasson 1996; Ripley, 1996; Figuerola \& Mazzoe, 1998; Magee et al., 1999; Morris et al., 2001; Unger et al., 2001). Changes of wind environments in large areas could potentially influence the effects of UHI or even global warming.

Research of wind environment started more than fifty years ago. People found that winds can generate loads on buildings. At the same time, buildings can also influence winds (Blocken \& Carmeliet, 2004). The influence largely depends on the building shapes (forms) and layouts (configurations) of building clusters. Wind flow is either obstructed or accelerated because of the influences from buildings. Low wind velocities reduce the potential for cooling in hot summers and effective dilution of air pollutants. Further, this may lead to UHI effect or air pollution. On the other hand, high wind velocities can affect human activities in outdoor space. High wind velocities reduce the potential for heat preservation in cold winters. Extremely high wind velocities will even increase the danger for pedestrians to walk outdoors.

This thesis presents a research of wind environments of contemporary high-rise residential 
buildings in the hot-summer and cold winter area by making multiple computational tools work in coordination. In this chapter, the motivation and problem of the research are explained first. The research aim is given to respond to the research question. The study objects including buildings and winds are defined in the research scope. After the research scope, the research significance is explained. In the end, the chapters and organization of the thesis are presented.

\subsection{Motivation and problem}

The research is motivated by the trend towards urbanizations and the challenge of sustainable habitats. Studies of urban microclimate and related topics will continue to gain popularity in the $21^{\text {st }}$ century (Toparlar et al., 2017). Based on the motivation, the research question is formulated from the research gaps.

\subsubsection{Research motivation}

According to the reports of the United Nations (UN) and the World Bank (WB), there will be great increases of the numbers of cities, the migration from rural areas to urban areas and the urbanization of rural areas in the $21^{\text {st }}$ century. They both anticipated a rapid increase of population living in urban areas around the world (Angel et al., 2005; United Nations, 2014). 'Making cities and human settlements climate resilient and sustainable' is marked as one of the sustainable development goals by the UN (United Nations, 2011). Therefore, the research of sustainable habitats and related topics such as wind environments are gaining importance. And the situation will be continuous in the coming years (United Nations, 2015).

Architecture is an essential component in cities that largely influences environments. It is necessary to consider the sustainability on the architectural design stage. Architects and architectural researchers are responsible to seek sustainable building designs not only to fulfill the requirement of architectural functions, but also be friendly to environments. Especially for high-rise residential buildings, they are increased rapidly in urban areas because of the limitation of land resource and the increase of populations in cities. With larger sizes, high-rise 
buildings exert larger influences on the wind flow than previous relatively low buildings.

Wind-environment studies can give information for the optimization of microclimates around buildings, which contributes to the better everyday life and the sustainability for both human being and the planet. The research can benefit people in different fields, including: (1) architects, designers, urban planners; (2) city administrators, related organizations who define the design guidelines (for green buildings); (3) researchers who study the sustainable design (for wind environments); (4) ordinary city dwellers.

\subsubsection{Research question}

According to the literature review, most previous studies of wind environments are undertaken in the urban areas of Europe, Americas and East Asia. The studies usually consider the promotion of natural outdoor ventilation. This is suitable for cities with hot and humid climates in summers. Especially for the cities like Tokyo, Singapore, and Hong Kong, their densities are extremely high. There is an urgent need to improve wind environments in urban areas by encouraging outdoor ventilation.

The researches that consider newly-built residential buildings are needed continuously due to the rapid urbanization processes. Though researches of urban microclimates are gaining popularity, the investigated urban locations do not have a large variety. The CFD urban microclimate knowledge needs to be expanded to the developing regions of the World (Toparlar et al., 2017). Especially for the Hot-Summer and Cold-Winter Climate Area (HSCWA) (the specific definition of HSCWA is given in the section 1.2.2. Research scope as follows), a lot of residential buildings are constructed with novel shapes, geometries(forms), and arrangements, plans (configurations) of building clusters.

In summary, the research question is generated from the research gaps found in the literature review (the gap is explained in details in the Literature Review Chapter, Page 74). In short, the research gap can be summarized as follows: 'Wind-environment studies of novel contemporary high-rise residential buildings are needed continuously, especially for developing regions such as the HSCWA. Previous studies lack of systematic categorization 
of the buildings. The relationships between the influences and building variables related $\underline{\text { to forms and configurations are not clear enough. Using existing wind-environment-study }}$ methods are usually complex and time-consuming'. The buildings in the area are newly built with novel forms and configurations of clusters. And residential buildings usually rely much on the natural ventilation (including outdoor and indoor spaces). Therefore, there is a need to explore the influences on wind environments of the high-rise residential buildings. Based on the research gaps, the research question is formulated: 'How are wind environments influenced by contemporary high-rise residential buildings?' In addition, nowadays windenvironment researches often require a large amount of comparisons of simulations to study the influences of different factors related to building shapes and arrangements. However, the iterative analysis usually needs to repeat the process of modelling, simulation, and analysis, which is time-consuming. Therefore, an appropriate efficient and accurate methodology is needed.

\subsection{Aim and scope}

This section is consisted of the research aim and research scope. The research aim is given first to respond to the research question in the last section. Challenges and an overview of research are presented after the research aim. In the second subsection, the research scope is explained in two aspects, including winds and buildings.

\subsubsection{Research aim}

The research aim is to: study the relationships between the influences on wind environments and the variables related to forms and configurations of contemporary high-rise residential buildings.

To achieve the research aim, there are three major challenges in studying the influences on wind environments: (1) How to evaluate the influences on the wind flow? It is difficult to study the wind flow because winds are erratic and invisible. This signifies that a reliable and 
accessible method to reveal the wind flow influenced by buildings are needed. (2) How to find out the trend of the influences? This signifies that iterative tests for multiple variables and distinguishing the differences of results in a limited time are necessary. (3) How to apply the study outcome to general architectural practices? There is a need to 'design' the representative buildings as study objects. These lead to the development of the methodology which makes the multiple computational tools work in coordination.

In this research, the methodology is developed by integrating the parametric modelling tool, CFD simulation tool, and image processing tool. It is convenient to use multiple computational tools because the research requires a lot of comparison studies for building designs with different variables. Computational tools are used in several aspects: (1) The parametric tool helps in collecting building information and the statistical calculation. The parametric modelling can automatically create building models that improves mesh generation efficiency for CFD simulations. (2) The CFD tool can simulate the outdoor wind environments of high-rise residential buildings. In the CFD simulation, the mesh generation technology is developed from the refinement of Cartesian-based mesh. The Reynold-Averaged Navier-Stokes (RANS) method is used in the CFD simulation as it has been widely used in most previous studies due to its acceptable accuracy and efficiency. A modified k-epsilon model is used as the turbulence model for the simulation. The simulation method is validated by the wind tunnel experimental data. (3) The image processing tool can accurately distinguish the differences among the CFD simulation results by calculating the pixel numbers of wind-velocitymagnitude maps and converting them into actual influenced areas. Then the influences on wind environments of buildings with different variables can be compared quantitively.

The parametric design is an essential step before CFD simulations for high-rise residential buildings. It is based on the building morphology as the wind flow is directly influenced by building forms and configurations. Building information is collected from 5,669 building samples in 383 residential districts in a selected developing urban area. The buildings in the area are categorized based on the definitions of forms and configurations. The most common forms and configurations are summarized from the statistical calculation of building number. Most typical sizes and intervals of buildings are discovered. Parametric modelling scripts are developed to generate the building models, which largely improves the efficiency of mesh 
generation for CFD simulations. The parametric design based on the investigation in building morphology sets up a bridge between building variables and CFD simulations to study building forms and configurations which are representative for generic high-rise residential buildings in the area.

The influences of different single building units on wind environments are studied first. In this research, three Building-Unit Forms (BUF) are studied, including square form, rectangular form, and ' $\mathrm{T}$ ' form. In particular, CFD simulation results with different variables of lengths, widths, heights, rotation angles, and bulge-part sizes (of ' $\mathrm{T}$ ' form) are analyzed. The influenced areas with different wind velocities on pedestrian level are calculated and compared. The trends of the influences of BUFs are analyzed based on the wind-velocity magnitudes, air-pressure magnitudes, and wind-flow streamlines. The relationships between the BUF variables and influences on wind environments can be discovered.

The influences of different building clusters with multiple building units on wind environments are studied based on the studies of BUFs. In this research, three Building-Cluster Configurations (BCCs) are studied, including scattered configuration, linear configuration, and curvilinear configuration. In particular, CFD simulation results with different variables of building-unit numbers, building intervals, and configurations are analyzed. Similar to studies of BUFs, the influenced areas with different wind velocities on pedestrian level are calculated and compared. The trends of the influences of BCC are analyzed based on the wind-velocity magnitudes, air-pressure magnitudes, and wind-flow streamlines. The relationships between the $\mathrm{BCC}$ variables and the influences on wind environments can be discovered.

\subsubsection{Research scope}

Based on the research aim and overview presented above, the research scope is explained in two major aspects of buildings and winds in this subsection.

\section{(1) Buildings}

This research focuses on contemporary high-rise residential buildings in urban areas in the 
HSCWA. In this research, the 'Hot-Summer and Cold-Winter Area' (HSCWA) refers to the (urban) areas with the climates which are hot in summers $\left(25-30^{\circ} \mathrm{C}\right)$ and cold in winters $(0-$ $10^{\circ} \mathrm{C}$ ) (Figure 1). The terminology 'hot-summer and cold-winter area' comes originally from a definition in the 'Code for Design of Civil Buildings' of China (code number: GB 50352-2005). Because China is a large country with a vast territory, it has been divided into seven major climate zones with different requirements of building designs in the code. The HSCWA covers Shanghai City, Zhejiang Province, Jiangxi Province, Hubei Province, Hunan Province, major parts of Jiangsu Province, Anhui Province, Sichuan Province, southern parts of Shanxi Province, Henan Province, Gansu Province, northern parts of Fujian Province, Guangdong Province, Guangxi Province and the eastern part of Guizhou. With a population of more than 500 million people, the HSCWA is the most populous and economically-developed area, contributing to more than a half of the entire Gross Domestic Product (GDP) of the country. Though the research starts initially from the area, the outcome applies universally.

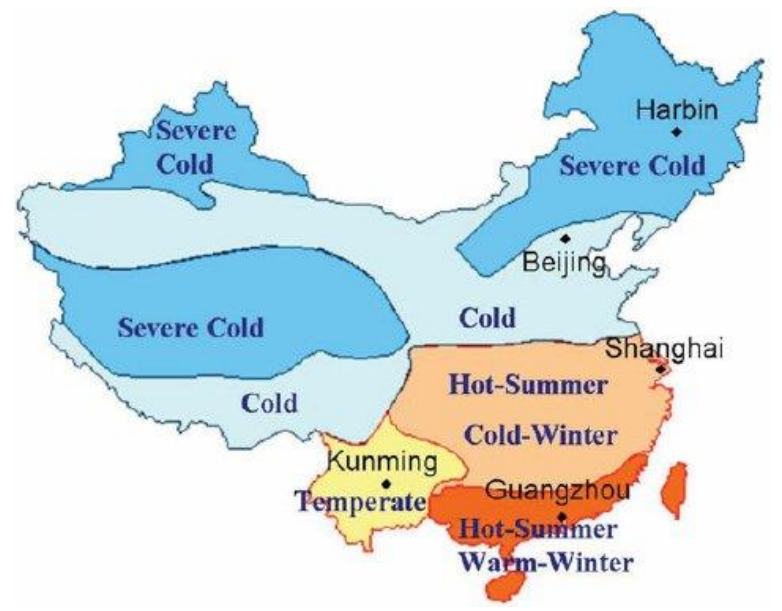

Figure 1. The HSCWA in China (Wang, Wu, Ding, Feng \& Wang, 2014).

There are two major reasons for the rapid increase of newly-built high-rise residential buildings in the HSCWA: one reason is the great demands of residential buildings due to the rapid increase of population in cities; another reason is the higher requirements for residential buildings due to the modifications of relevant architectural standards and the needs for better living conditions by residents. Moreover, temperatures in most urban areas are relatively high in summers and relatively low in winters. Extremely high wind velocities are not suitable for 
cold climates. The promotion of wind flow might not always be good in some situations.

Most newly-built residential buildings are high-rise buildings according to previous studies, architectural practices and site investigations. These existing high-rise residential buildings are usually between 9-30 stories and 27-100 meters in height according to the definition of high-rise buildings in relevant architectural standards. Most of them are built between the years of 2000 and 2019, which represent the newest generation and the development trend of residential building in modern cities.

In the categorization study of the Parametric Design Chapter, the building information is collected from 5,699 buildings in 383 residential districts in the Optic Valley Area of Wuhan City. The location is selected because it is representative for high-rise residential buildings in the HSCWA (Figure 2). The reason is explained in details in the Literature Review Chapter.

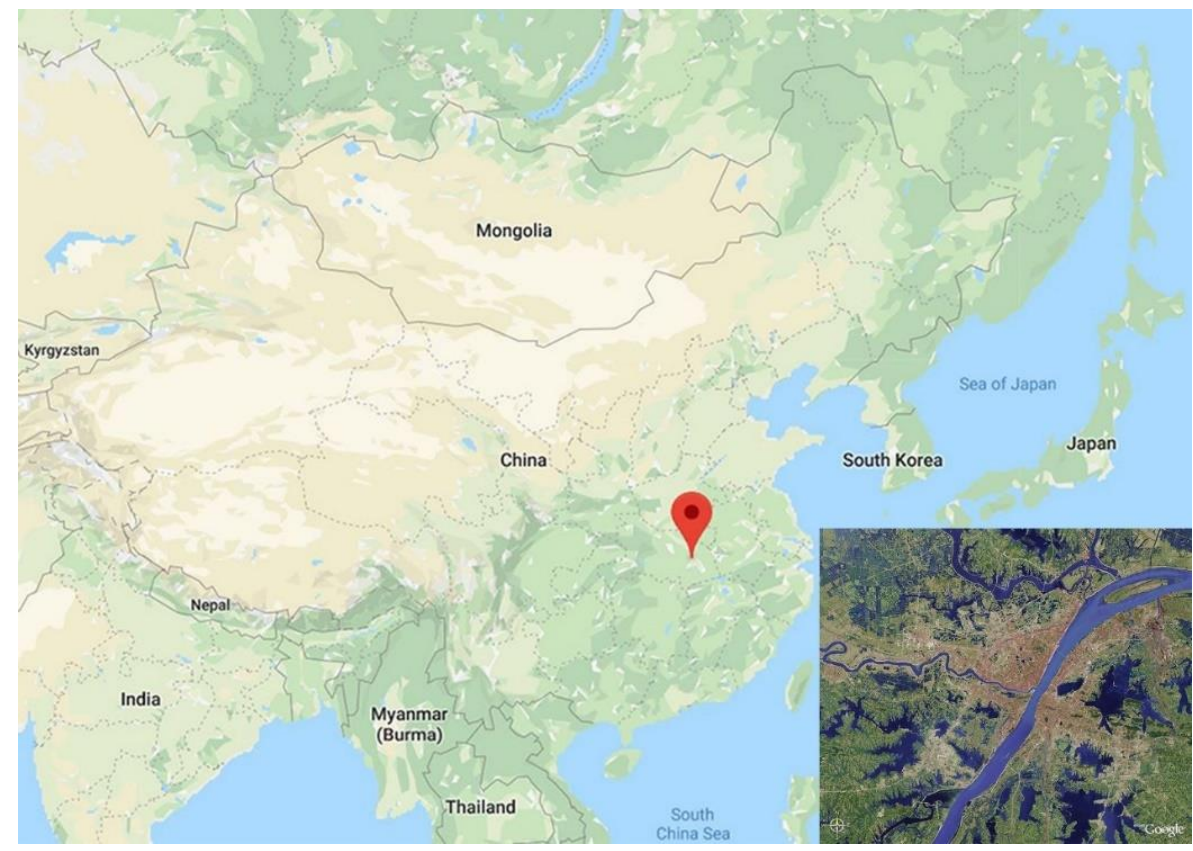

Figure 2. The location and map of Wuhan City (captured in Google map, 2019).

In this research, Building-Unit Form (BUF) is defined as the shape (geometry) of a single building unit. A building unit is defined as a single building with at least an independent entrance and an independent vertical traffic system (the definition is explained in details in both Literature Review Chapter and Parametric Design Chapter). Building-Cluster Configuration (BCC) is defined as the arrangement (layout) of a group of several building units. (The specific 
definitions are explained in details in both Literature Review Chapter and Parametric Design Chapter).

In this research, definitions of eleven BUFs and four BCCs are given for the categorization of all kinds of high-rise residential buildings in the area (in the Parametric Design Chapter). The three BUFs and three BCCs are selected because of their most numbers according to statistical calculations of all building forms and configurations in the area. They are representative for the most common and typical high-rise residential buildings in the area. As they are presented in the Parametric Design Chapter: the three BUFs are square form, rectangular form, and ' $\mathrm{T}$ ' form; the three $\mathrm{BCC}$ s are scattered configuration, linear configuration, and curvilinear configuration.

The influences of BUFs are studied first for a single building unit (in the Fifth Chapter). Based on this, influences of BCCs are studied afterwards for a cluster with several building units (in the Sixth Chapter). In the studies of BUFs, influences of the variables related to the building forms are studied, including: length, height, and orientation (rotation angle) of square form; length, width, height, and orientation (rotation angle) of rectangular form; sizes of bulge parts of ' $T$ ' form. In the studies of BCCs, influences of the variables related to the building configurations are studied, including: building unit number, building intervals (longitudinal and transverse distances), and staggered distance of scattered configuration; building unit number, building interval (longitudinal distance), and staggered distance of linear configuration; curvature (central angle) of curvilinear configuration. In the BCCs studies, all the building units in the clusters are the same in a typical rectangular form.

The parametric method is used to create numerous models of BUFs and BCCs automatically for the CFD mesh generations. Building models are simplified appropriately to only retain major geometrical attributes such as sizes of forms, intervals of configurations. Building details such as decorations and small components are eliminated. This is because each specific building is different from each other in details. And this research aims to generate generally applicable outcomes, which are not for specific buildings. Simplified models can be representative for more generic buildings. Moreover, the influences of building details on wind environments are relatively small. In addition, the simplification can save the computational resource for running the parametric modelling scripts and CFD simulations. So, the influences 
of some building details will not be studied in this research.

\section{(2) Winds}

In the realistic urban environments, winds are dynamic as their velocities and directions are changing all the time. In the time dimension, urban macroscopic wind velocities and directions usually have obvious variation trends in a season or an entire year. In the space dimension, urban wind environments vary significantly from the horizontal and vertical coordinates.

In this research, wind attributes on the time dimension and space dimension are simplified according to the local wind condition record in Wuhan City. The major wind direction and average wind velocity are used to generalize the initial wind flow in a time period. The winds can be simplified effectively by transferring actual dynamic winds to ideal steady winds.

In this research, the 'influence' is the overall influenced areas of wind environments. The wind velocities of the areas are different from the initial velocity because of the influences of buildings. This research focuses on the influenced wind velocities of the horizontal plane on $1.5 \mathrm{~m}$ height (the pedestrian level). Moreover, the air-pressure magnitudes on the building surfaces and horizontal plane and airflow streamlines around buildings are considered for analyzing the mechanisms. The evaluations of the influences are based on the criteria established in the methodology. To generate the initial winds in CFD simulations, the annual average wind velocity calculated from the monthly average velocities measured on sites in the city is used as the reference velocity on $10 \mathrm{~m}$ height. In this research, the wind direction has not been defined specifically because different orientations (rotation angles) of building forms are studied. The influences of different wind directions are studied for square form and rectangular form. For the other situations, the default wind direction is defined as coming from south to north in the summers and transitional seasons; the direction is opposite in winter. Because the northern part and southern part of most building forms and configurations are symmetrical, only one wind direction is considered in CFD simulations in this research.

In this research, the wind environments are studied through simulations using the RANS method in the CFD software. A modified k-epsilon turbulence model is used for the simulations according to the Reynolds Number. The simulation method is validated by a well-recognized wind tunnel experiment (Brown, Lawson, Decroix \& Lee, 2001). The simulations are done 
following a well-recognized guideline 'COST Action 732' (Franke et al., 2007). The simulation results of wind environments are evaluated by the criteria established in the Research Methodology Chapter. Following elements are considered for the evaluations in this research: (1) Wind velocity magnitudes. Wind velocities define the wind-flow intensities and present how strong or how weak the winds are. The research focuses on the influenced areas on the horizontal plane instead of specific points. The velocity-magnitude maps are measured in pixels and converted into actual influenced areas for quantitative comparisons by using the image processing tool. (2) Air-pressure magnitudes. The pressure differences are the reason for wind flow. (3) Wind-flow streamlines. The streamlines illustrate how winds flow around the buildings. (The three elements are explained in details in the Evaluation Criteria Section of Research Methodology Chapter.)

\subsection{Significance}

This research intends to study the wind environments of newly-built high-rise residential buildings (2000-2019) by developing an integrated methodology by making multiple computational tools work in coordination. In the parametric design, the categorization study and parametric modelling scripts will be done based on the architectural morphology for representative buildings in the area. Then, the relationships between the influences on wind environments and variables related to building forms and configurations will be discovered. The significance of the above anticipated outcomes can be summarized in the theoretical, practical and social aspects.

\subsubsection{Theoretical significance}

The research will contribute to the wind environment studies of newly-built high-rise residential buildings in response to the major trend of urbanizations around world. Influences on wind environments of novel building forms and configurations will be studied. Relationships between the influences and building variables will be discovered. 
In particular, for single building units, the relationships between the influences on wind environments and the variables of square form, rectangular form, and ' $\mathrm{T}$ ' form will be explored. For building clusters with multiple units, the relationships between the influences on wind environments and the variables of scattered configuration, linear configuration, and curvilinear configuration will be explored. The research can give information to the optimization of wind environments of high-rise residential communities and improvements of microclimates in urban areas.

The development of the methodology will show that making multiple computational tools work in coordination can provide efficient iterative analysis and implement quantitative comparisons for studying influences on wind environments. There will be great potentials for integrations of multiple computational tools for sustainable and environmental researches.

\subsubsection{Practical significance}

Architects and urban planners will be able to use the knowledge to improve their designs on early stages. City administrators and local authorities will be able to use the knowledge to help in making urban development strategies and defining design guidelines. The knowledge can be helpful for researchers who study sustainable habitats and related topics.

The methodology developed in this research will be used for new studies of wind environments. Especially for applications in architectural practices, it will provide the immediate analysis of wind environments on the early stage, which allows just-in-time design decisions. It will be easy to change any functional modular or even the tools if there are better alternatives. Because more advanced modelling scripts or CFD software will be developed in future works. The parametric modelling will seamlessly be employed to analyze other factors of sustainable design on computational platforms.

\subsubsection{Social significance}

By studying how buildings influence the wind flow, the research will give information to 
building designs and modifications for creating appropriate wind environments in urban areas ultimately, especially for the increasing high-rise residential communities in the HSCWA. Appropriate wind environments will contribute to the improvements of thermal comfort, prevention of safety issue, reduction of air pollution, building performance for saving energy of cooling and heating. The accumulation of changes of microclimates will potentially influence the climates of large urban areas.

\subsection{Chapters and organization}

The rest of the thesis is organized with following chapters: '2. Literature review', '3. Research methodology', '4. Parametric design of the buildings', '5. Wind environment studies of BUFs', '6. Wind environment studies of BCCs', '7. Discussion' and '8. Conclusion'.

In the Second Chapter Literature Review, it presents the development of wind environment studies, influences of buildings on winds, wind environment studies around world, review of high-rise residential buildings, a representative location in the HSCWA, review of CFD methods, and evaluation criteria of wind environments. A summary is made at the end of the literature review to summarize the research gaps. In the Third Chapter, the research methodology is explained, including the parametric design method, CFD simulation method, and analysis method. In the Fourth Chapter Parametric Design of the Buildings, the building categorization study and parametric modelling of the buildings are presented. The Fifth Chapter presents the studies of wind environment studies of BUFs in 49 pages, including an overview of BUF wind environments, square form, rectangular form, and ' $\mathrm{T}$ ' form. The Sixth Chapter presents wind environment studies of BCCs in 95 pages, including the scattered configuration, linear configuration, and curvilinear configuration. In the Seventh Chapter Discussion, the research outcomes are discussed, including the research methodology, parametric design, BUFs studies and BCCs studies. Then the limitations of the research and future works are discussed. In the end, the Eighth Chapter Conclusion presents the findings and contributions of the research. 


\section{Literature review}

In the literature review, the development history of wind environment studies is introduced first. Influences on wind environments of buildings are presented in the building scale and block scale. Wind environment studies around world are reviewed, including studies in Europe, America, Oceania, and Asia. The definitions and development of high-rise residential buildings are presented. A representative location of HSCWA selected for this study is explained. Then the CFD methods and the evaluation criteria of wind environments are reviewed. Based on these, research gaps can be summarized in the end (Section 2.8.).

\subsection{Development of wind environment studies}

People started to study winds in the early years in the $20^{\text {th }}$ century because it could generate loads on high-rise buildings. It is difficult to study winds because the air flow is hard to predict for its invisibility. The new subject of building aerodynamics was established in 1960's, when accurate simulations could be implemented using improved facilities such as wind tunnel (Blocken \& Carmeliet, 2004). At first, wind environment studies were primitive. At that time, a vague standard stated that if the distance between two adjacent buildings was more than about six to eight times the average building width, their mutual influences could be ignored (Simiu, 1985). But the truths are that the urban land resources are becoming limited and the urban densities are becoming even higher nowadays, which make such large distances between adjacent buildings is basically impossible. Since the importance of urban microclimates has been widely recognized, many institutes started to study pedestrian wind environments as required by urban authorities, especially for large construction projects.

Four major methods were developed for the wind environment studies, including the theoretical analysis, site measurement, wind tunnel test and numerical simulation. The theoretical analysis is lack of accuracy as it is usually qualitative based on empirical theories. The site measurement and the wind tunnel test are experimental methods which need experimental facilities. The experimental setting up and data measurement are usually time- 
consuming for them. The numerical simulation method which is computational has the advantage of high efficiency and accessibility. After several decades' development, its accuracy has been improved and verified by researchers in different studies. Though a lot of studies were done by using the site measurement and the wind tunnel test in the past, the computational fluid dynamics (CFD) method has become available as an essential tool which has been widely used in many practices in the last four decades (Blocken \& Carmeliet, 2004).

In the middle of 1980 s, the CFD method was introduced to study architectural wind environments. Turbulence model is usually used to study the air flow around buildings by solving Navier-Stokes equations. Models such as the Reynolds Equation (Dowson, 1962) and the Smagorinsky Model (Gromke, Buccolieri, Sabatino \& Ruck, 2008) are often used. The k- $\varepsilon$ turbulence model is one of the most used models for urban microclimates (Toparlar et al., 2017). Meanwhile, mesh generation technologies such as the Cartesian Grid were developed for CFD simulations.

In 1990s, more relevant models were established and some previous models were improved, especially for the modification of turbulence models. These made simulations of wind environments closer to actual situations. Some studies were done by using CFD methods and wind tunnel tests together. This is a reliable approach that combines simulations and experiments. Most researchers started to focusing on architectural practices. CFD simulations were used to study the influences of rectangular building shapes on the wind flow. In 1996, researcher Mingde Su and his colleagues did the research for velocities and pressures of winds around cuboid high-rise buildings under different wind directions. The simulation was done and compared with experimental data $(\mathrm{Su}, 1996)$. In the mid-90s, Launder and Kato proposed a modified turbulence model (Launder and Kato Model) to improve the turbulence energy equation. The model was then further amended (Launder and Kato, 1993; Selvam, 1996). Murakami and other researchers set up a CFD simulation using the Large Eddies Simulation (LES) method (Kondo, Murakami \& Mochida, 1997). It was found that the results of using the LES method were closer to the data of wind tunnel tests than using the Reynolds Equation Method (Murakami, 1998).

In the 2000s, researchers kept on improving simulation methods. Some novel models came out as the technology was developing. In 2000, a new model for CFD simulations was suggested 
by Kawamoto (Kawamoto, 2000). Besides DNS (Direct Numerical Simulation) method and LES method, RANS (Reynolds Average Navier-Stokes) method has been widely used in CFD simulations for practices because of its high efficiency. In 2007, researchers used a dynamic pressure analysis model to study the peak wind pressure on the surfaces of high-rise buildings (Wu \& Chen, 2007). Nowadays, studies for large architectural projects are often requested in the urban development. The importance of architectural wind environments has been widely recognized because it is a key element of sustainability for buildings and cities. The reliability of the CFD method has been widely admitted as countless researches and practices have proven it is an efficient and stable approach to simulate wind environments.

\subsection{Influences of buildings on winds}

In a relatively large scale, a previous study shows the main reason of UHI effect is the modifications of terrain surfaces in urban areas. This indicates influences of buildings that occupy the land are the most important factor (Solecki et al., 2005). In a relatively small scale, researchers found influences on winds of buildings depended on the shape, size, orientation of a single building and arrangement of a group of several buildings (Blocken \& Carmeliet, 2004). In summary, the influences on wind environments are closely related to building forms and configurations.

\subsubsection{Building-scale influence}

Winds can cause pressure toward building surfaces. As winds flow around a building, they exert varying degrees of air pressure on the building surfaces (Aynsley, 1999). There are positive pressures that indicate the pressures acting toward building surfaces, while there are also the negative pressures that indicate the pressures acting away from building surfaces or suctions (Aynsley, 1999). Forces on buildings generated by winds are closely related to wind pressures on building surfaces. According to different formation mechanisms, there are the lift force and drag force (Aynsley, 1999). The lift force is caused by the differences of air pressures that are 
usually perpendicular to the wind direction between the building surfaces on the opposite sides. The drag force is the sum of the friction force and pressure force of building surfaces resulted from the differences of air pressures that are usually parallel to the wind direction between the building surfaces on the windward and leeward sides (Aynsley, 1999).

Most buildings with flat surfaces and sharp corners are called "bluff bodies" (Aynsley, 1999). The buildings are usually referred to cuboid-shape blocks. When winds meet an obstacle such like a building, coming winds are separated at the windward surfaces and then flow around at the corners. Winds flow around the building and rejoin somewhere behind them (Figure 3). Usually, there is a low-velocity area between the building and juncture point, which is also called the wind-shadow area. The areas can usually be shown on the wind-velocity-magnitude maps generated from CFD simulation results. In the wind-shadow area, the wind velocity usually decreases to half of the original one. Its length is about 15 times of the building height (Krishan \& Arvind, 2001). On the other hand, fast high-elevation winds can be induced to lower levels by a relatively tall building if a significant part of it is exposed to the direct wind flow, such as the phenomenon of 'downwash mechanism' (Cochran, 2004) (Figure 3).

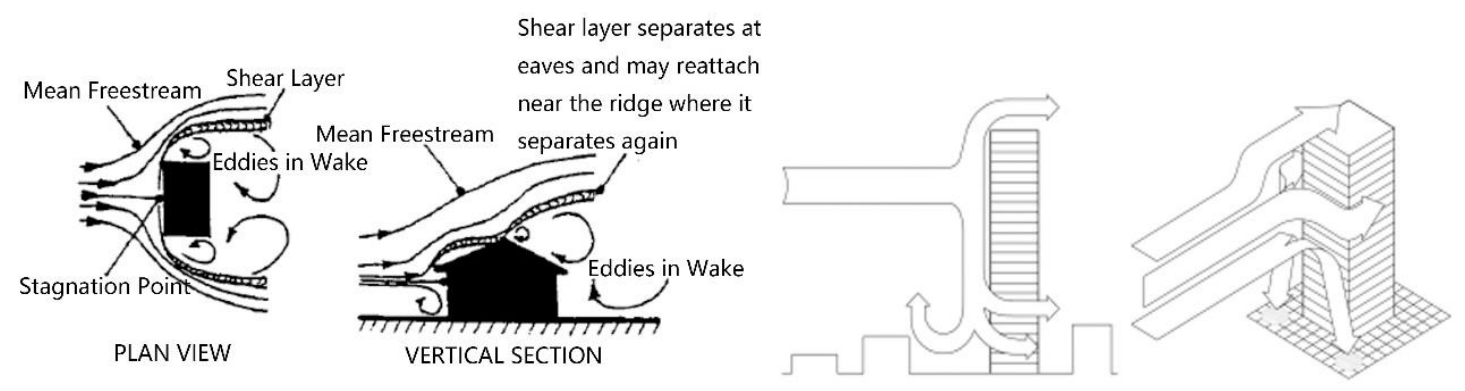

Figure 3. The figure on the left shows "the typical air flow features around a building" (Aynsley, 1999). The figure on the right shows the interaction of air flow and an ordinary rectilinear floor plan building. It explains "the mechanism of downwash and high-speed wind areas may be expected at the pedestrian-level corners" (Cochran, 2004).

\subsubsection{Block-scale influence}

In the block scale, the roughness properties of urban surfaces are introduced to study influences 
on winds (Figure 4), including the velocities and flow paths of winds, the scale and intensity of turbulence (Landsberg, 1981). The total drag force on a roughness surface is the sum of a pressure drag force on the roughness elements, a skin drag force on the underlying surface, and a skin drag force on the ground surface (Raupach, 1992; Shao and Yang, 2005). Because the skin drag forces are relatively small and are not a factor controlled by urban scale, so only the pressure drag force is usually considered in most studies $(\mathrm{Ng}, 2011)$. A logarithmic function is developed to set up a semi-empirical relationship by taking the "two aerodynamic characteristics of roughness length" and the "zero-plane displacement height" into consideration (Oke, 1987). Based on this, it is reliable for depicting the "aerodynamic characteristics of urban areas" and predicting the "urban wind behaviors" (Grimmond and Oke, 1999). Nowadays, there are mainly three classes of methods for estimating the surface roughness: Davenport roughness classification method (Davenport et al., 2000), morphometric method and micrometeorological method (Grimmond and Oke, 1999).

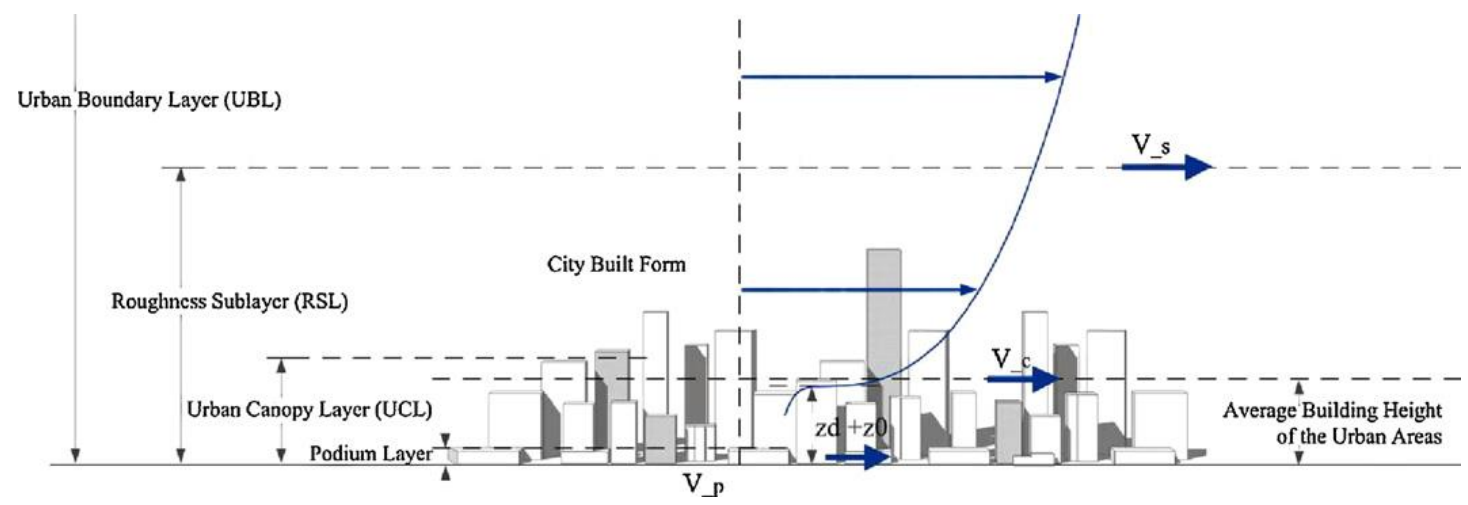

Figure 4. The wind speed profile of podium layer, urban canopy layer (UCL), roughness sublayer (RSL) and urban boundary layer (UBL) (Oke, 2006; Ng et al., 2011). In the figure, Vp, $\mathrm{Vc}, \mathrm{Vs}$ are the wind speeds of these layers.

\subsection{Wind environment studies around world}

Wind environment studies started originally in Europe and America. In the urbanization process, topics related to urban microclimates were gaining importance and caught people's attention, which led to the increases of wind environment studies. 
In 1989, researchers studied a statistical procedure for comparing the performances of two or more than two air quality simulation models by using a performance index (Cox et al., 1989). Researchers kept on improving the numerical simulation method in every aspect to make it become one of most widely-used study methods around the world. Some software packages were developed for wind environment analysis, such as the well-known ANSYS Fluent, CHAM PHOENICS, ENVI-met and so on. Through intersections of different methods, the research accuracy and efficiency were improved. In 2008, researchers compared the CFD data with experimental data in the evaluation of wind environments (Gromke et al., 2008). In another example, researchers used both the wind tunnel experiment and the CFD simulation to study the pedestrian-level wind speed in the downtown area of Auckland, New Zealand (Richards et al., 2002). As technologies developed, not only new study methods came out, but also research boundaries were broadened in different objects from block scale to building scale. For example, researchers studied the relationship of influences of wind, sunlight and temperature on the human comfort in urban spaces (Walton et al., 2007). In Germany, urban climatic maps were produced to factor wind-environment characteristics for guiding future developments and planning decisions (Katzschner, 2000). Relevant principles were established to improve the indoor ventilation in the indoor environment quality (IEQ) section of many standards in western countries, such as BREEAM, LEED and so on (Dimitroulopoulou, 2012). However, principles of outdoor wind environments were indistinct or even primitive, though relevant studies were increased as the urban sustainability is becoming important. For outdoor wind environments, researchers found that the urban form and design had impacts on microclimates. Researchers discussed and assessed the impacts of geometries of street designs on urban microclimates for the semi-arid climate by utilizing a series of site measurements. (Bourbia \& Boucheriba, 2010). In a study in Phoenix local climate zones, it was found that the cooling was dependent on the form and spatial arrangement of urban features (Middel et al, 2014). Not only building shapes and arrangements influence wind environments, but also other factors have impacts on wind environments. Researchers found the airflow field around buildings was changed significantly as the surfaces of buildings and ground were heated by solar radiations and thereby induced the air buoyancy. Then they validated CFD simulations by comparing using a 2D steady RANS method for buoyant flows in urban street canyons with wind tunnel experiments (Allegrini, 
Dorer \& Carmeliet, 2014). In 2015, influences from buildings to even avenue trees on the wind flow were studied (Gromke \& Blocken, 2015). In 2016, researchers did a study for the validation and optimization for the turbulence model (Yu, 2016). A comparison was made between the software packages ENVI-met and Autal2000 to evaluate their modelling performances (Paas \& Schneider, 2016). Researchers did a monitoring study in the street canyon in Thessaloniki. They calibrated and evaluated microclimate simulation models with the ENVI-met software for a comparative analysis of the effects of geometric characteristics on the microclimate and pedestrian comfort. (Chatzidimitriou \& Axarli, 2017).

Besides building shapes (forms), influences on winds are also related to building arrangements (configurations). Linear configuration is common and popular in the arrangement of building plan. As heights of newly-built buildings increase, scattered configuration is becoming popular in high-rise residential communities. Because building units are independent from each other and their arrangements are more flexible. An appropriate configuration can encourage winds to go through intervals of the building cluster and improve the outdoor ventilation.

According to previous studies, when angles between wind directions and windward surfaces of building rows are in the range of 30-60 degree, it is easier for winds to pass through interior spaces in summer seasons (Givoni, 1994). Different building arrangements have different influences on the winds in airflow directions and interior ventilation performances. Vortices are created after winds flow around obstacles, which is similar to the situation that water meets rocks in a river and swirls are generated behind rocks. Besides the wind velocity and direction, the sizes of vortices are related to the size, shape, surface roughness and orientation of the obstacle. Researchers found it was easier for winds to go around buildings with a cylinder shape and the wind-shadow area is relatively small (Watson \& Labs, 1983), which suggests curvilinear configurations may have the similar performance. Theoretically, the curvilinear configuration makes the airflow moves smoothly on the concave windward surfaces of the building rows. According to previous researches, cold winds can take away heat and make city dwellers consume more energy for heating in cold winters. Even keeping the lowest air exchange for the required minimum times (0.5-1 times per hour) in cold winters will cost 1/4-1/3 of the total heating load (Givoni, 1994). 
In Asia, relevant researches started early in Japan. From 1980s, researchers improved computational models based on previous studies, including Launder and Kato Model (Launder \& Kato, 1993), LES method for CFD simulations (Kondo, Murakami \& Mochida, 1997), and so on. Research subjects covered from building scale to block scale in Japanese cities. The pedestrian wind environments in the residential neighborhoods in major cities were studied (Kubota, Miura, Tominaga \& Mochida, 2008). CFD methods had been widely used in studying subjects such as urban climates (Murakami, Ooka, Mochida, Yoshida \& Kim, 1999) and the urban air pollution (Kondo, Asahi, Tomizuka \& Suzuki, 2006). In Singapore, researches mainly focused on the outdoor ventilation of high-rise residential buildings in the tropics (Lee, Jusuf, \& Wong, 2015). In Hong Kong, wind environments had been recognized as an important study, especially the high city density and skyscrapers imped the natural ventilation. Pioneer researchers applied CFD methods to improve the ventilation performance of green features in buildings (Mak, Cheng, \& Niu, 2005) (Niu, Tang \& Mak, 2005).

As CFD methods developed, researchers sought proper turbulence models to simulate wind environments around isolated high-rise buildings, including Steady Reynolds Averaged Navier-Stokes (SRANS), Large Eddy Simulation (LES) and Detached Eddy Simulation (DES) models. They compared the performances of those models in simulating wind environments around the buildings and found that the Delayed DES model could provide the flow field and the instantaneous characteristics of the wind flow (Liu \& Niu, 2016). Influences from detailed architectural components such as balconies, windcatchers were considered (Liu, Mak \& Niu, 2011). Researchers studied certain kinds of buildings such as lift-up type buildings (Liu, Niu, Mak, Xia, Liu \& Niu, 2017). And microclimate issues such as the wind comfort in a long street canyon were studied (Du, Mak, Huang \& Niu, 2017). The researches of building and urban ventilations contributed systematically to the field from advanced numerical techniques to different creative design strategies. Researchers studied the influences of the urban morphology and the surface roughness for improving the wind environments in high-density cities $(\mathrm{Ng}$, Yuan, Chen, Ren \& Fung, 2011). Researchers from Taiwan studied the influence of building length on ventilation in 2014. They used LES method and a wind tunnel for their research. Their results showed that the outdoor ventilation rate was declined as the building length increased. This suggested that the wind environments can be influenced by variables related to building 
shapes such as sizes (Chu, Ren, \& Chiang, 2014).

In mainland China, there were multifarious researches of wind environments in different cities. Because of the formation of local residential districts, studies are usually for practical projects such as a specific residential community. In 2001, CFD simulations were used to study a residential district with different arrangements of outdoor-ventilation openings under different wind velocities. The wind velocity magnitudes were illustrated as a qualitative basis for the planning and design (Tang \& Meng, 2001). In 2002, the CFD method and a wind tunnel were used to study a financial high-rise building in Beijing. The results of the CFD simulation and wind tunnel experiment were compared for dynamic analysis (Chow \& Gao, 2002). In 2003, researchers from Tsinghua set up 2D and 3D models to analyze residential building clusters in Beijing. Impacts on wind environments were studied through numerical simulations (Chen, 2007). In 2008, a study of two adjacent buildings was done by taking into account of the spacing ratio, building height and other conditions. The results revealed the areas of wind loads on building surfaces which provided a theoretical basis for design (Zhang \& Gu, 2008). Researchers from Tongji University explored the climatic adaptability of high-rise buildings in wind environment studies (Chen, 2008). The strategy of climate-adapted designs such as the planning of grand urban windways was suggested, especially for the HSCWA. (Hong, Yu \& Li, 2011) (Gan, Peng, Li, \& Chen, 2013). Wind tunnels were usually used for studying large-scale objects like buildings. Climate chambers were used for studying micro-scale objects, such as the influences of natural wind flow on humans. In 2013, researchers from Tsinghua University compared the influences on human comforts and the performances of constant mechanical winds and simulated natural winds. They evaluated the performances and comforts by using simulated tasks and questionnaires. The results showed that simulated natural winds were better for human comforts at the relatively higher temperature and constant mechanical winds were better at the relatively lower temperature. But results were nearly the same in the performance. In summary, differences between the influences of constant mechanical winds and simulated natural winds were relatively small. (Cui, Cao, Ouyang, \& Zhu, 2013). Researchers usually evaluated outdoor wind environments by using performance indexes, such as the Air Change Rate per hour $(\mathrm{ACH})$. Researchers found that the $\mathrm{ACH}$ might reach a theoretically minimum value depending on the local roof ventilation if the urban size is infinite. The overall urban 
canopy layer (UCL) ventilation capacity with a square urban form was better for parallel approaching winds than oblique winds, and it exceeded that of a staggered urban form under all wind directions, but was less than that of a rectangular urban form under most wind directions. (Lin, Hang, Li, Luo, \& Sandberg, 2014). Researchers used the RANS k-epsilon model and found that the air flow patterns and pollutant dispersion were greatly influenced by the diurnal variation of surface temperatures of buildings and ground. (Tan, Dong, Xiao, \& Tu, 2015)

\subsection{Review of high-rise residential buildings}

This section is consisted of the definition and development of high-rise residential buildings. The definition of high-rise building is reviewed and discussed first. The history and attributes of high-rise residential building are reviewed afterwards.

\subsubsection{Definition of high-rise buildings}

There is no common definition of high-rise buildings as the definition varies in different contexts (Ilgin, 2006). For example, a 14-story building may be considered as a high-rise building in a regional European city. But it may not be considered as a high-rise building in the megacities such as Hong Kong, because it is not high compared to surrounding buildings in the city. Some researchers defined a high-rise building by the need for extra operations and technical measures due to its actual height, instead of its overall height or number of stories (Beedle, 1971). Some researchers defined a high-rise building as a multi-story building that was constructed by a structural frame, installed with high-speed elevators, and combined extraordinary height with ordinary room spaces (Ali \& Armstrong, 1995). From the structural perspective, a high-rise building was defined as its structural analysis and design were in some way affected by lateral loads (Taranath, 1998). From the architectural perspective, a high-rise building was defined as its height became a concern that affects planning, aesthetics, and surrounding environments (Ilgin, 2006). The Council on Tall Buildings and Urban Habitat 
proposed three subjective categories for defining high-rise buildings (tall buildings). In the first category, a high-rise building was usually significantly higher than its surrounding buildings. In the second category, a high-rise building was a building with a tall and slender shape. In the third category, a high-rise building was a building that embraced relevant specific technologies such as vertical transport systems and structural systems.

The definition of existing high-rise contemporary residential building of China was clearly described in relevant architectural standards. The multi-story residential buildings with 9-30 stories and heights of 27-100 meters were called high-rise residential buildings (Zhu, Wei, \& Long, 2011). The common volume ratio of the residential districts was in the range of 1.5-6.0. The regular cuboid shapes such as square form, rectangular form and ' $\mathrm{T}$ ' form were the common forms for high-rise residential buildings. The regular arrangements such as scattered configuration, linear configuration and curvilinear configuration were the common configurations for high-rise residential buildings. These buildings referred to the high-rise residential buildings that are studied in this research. The building forms and configurations are defined and analyzed in the fourth chapter of parametric design.

\subsubsection{Development of high-rise residential buildings}

With the development of cities, spaces for residential buildings became limited. As city densities kept on increasing, high-rise residential buildings came out first in developed metropolises to solve the issue, such as cities in United States, Britain, Germany, France and Japan. The increases of high-rise buildings were due to the growths in populations, land costs and aesthetic features (Ayoub, 2012). In the last decades, advanced construction technologies and novel architectural materials had provided suitable conditions for high-rise residential buildings. Besides huge cities, high-rise residential buildings were even built in some towns to meet the needs of local developments. From this, there seemed to be an inevitable trend to build high-rise residential buildings in the contemporary urban development (except for few cities with special cases such as earthquake), for their significant advantages in saving lands and improving urban landscapes. 
In China, high-rise residential buildings appeared early in big cities like Shanghai in 30s in the $20^{\text {th }}$ century. The expansion of high-rise residential buildings was suspended not until $70 \mathrm{~s}$ because of the Second World War and the economic recovery. After that, residential districts had been developed vigorously in many cities since 1980s. As the economy grew up and lots of buildings were built in cities, urban lands became limited in China. Meanwhile the populations kept on increasing rapidly. More and more people immigrated to cities. Therefore, these led to massive needs of high-rise residential buildings in mega cities such as Beijing, Chongqing, Shanghai, Guangzhou, Shenyang and so on. More high-rise residential buildings had been built since $80 \mathrm{~s}$ in the last century.

It is said that the numbers and heights of high-rise residential buildings represent the modernism of a city. But this may be actually a misunderstanding of the essence and function of high-rise residential buildings. Though high-rise residential buildings can significantly save lands, some issues come out at the same time. Therefore, it needs to be cautious to design and construct high-rise residential buildings as they should be adaptive to local situations. In particular, following five aspects need to be considered for the buildings. (1) A safe and highefficient public transportation system. As heights of high-rise residential buildings increase, elevators become the major transportation tools instead of the conventional stairs. The transportation systems need to be economic, safe and convenient for people to use. Therefore, the floor layouts of high-rise buildings must be specially designed to fulfill the requirements. (2) Designs that adapted to the structure systems of high-rise residential buildings. As the building heights go up, vertical and horizontal loads increase at the same time. The buildings require stronger civil structures (such as the frame structure), which are different from relatively lower multi-floor buildings with the masonry-concrete structure. For instance, the core-tube structures are usually required in most cases. It is uncompromised to design the floor layouts adapted to the structure systems. At the same time, the designs have to meet requirements of client usages to ensure both functions and stabilities. (3) Fulfill requirements of the water supply, electric supply, evacuation and fire safety. As the building height increases, new requirements come out in the water supply, electric supply, evacuation and fire safety. The designs of highrise residential buildings should take those into consideration. (4) Consider the influences of larger building volumes on the environments, residential psychologies and urban forms. High- 
rise residential buildings are not only higher but also larger than conventional residential buildings. Their influences on the surrounding environments, psychologies of residents, landscapes and urban forms become significantly larger and cannot be ignored. (5) Higher investments in construction processes and maintenances. High-rise residential buildings are usually more expensive than conventional residential buildings. High-rise residential buildings cost more materials to build because of their higher height and larger building volume. Moreover, structure systems and supporting facilities are usually more complicated and expensive. (Zhu, Wei, \& Long, 2011).

\subsection{A representative location in HSCWA}

As mentioned in the introduction, the 'Hot-Summer and Cold-Winter Area' (HSCWA) refers to the urban areas with the climates which are hot in summer $\left(25-30^{\circ} \mathrm{C}\right)$ and cold in winter $(0-$ $10^{\circ} \mathrm{C}$ ). It comes from the definition in architectural standards. The HSCWA covers the most populous and economically-developed areas of the country. The rapid increase of newly-built high-rise residential buildings in the HSCWA is due to the rapid increase of population in cities, the modifications of relevant architectural standards and the needs for better living conditions by residents. In this research, the residential building samples are collected from the Optic Valley area in the City of Wuhan, China. The local wind condition is used for simulations. The location is selected because of its representativeness for high-rise residential buildings in the HSCWA.

\subsubsection{Location significance}

Located in the center of China, Wuhan city is the capital city of Hubei province. It is one of the most important cities of the country all the time even from 700 B. C., because of its geographical location, economical position and cultural heritage. Geographically, distances from Wuhan city to Shanghai in the east, Guangzhou in the south, Chengdu in the west, Beijing in the north are all about 1,000 kilometers. Two great rivers of Yangtze River and Han River 
meet in Wuhan and divide the city into three parts of Wuchang Area, Hankou Area and Han yang Area, which is a unique urban form. Because of its position on both water and land routes, Wuhan city has become a traffic center since ancient time. Now as the mid-hub of the national high-speed express system, Wuhan city connects major cities from all directions. Starting from the ports in Wuhan, cargo ships can go up to reach Chongqing, or go down to meet the Pacific Ocean through Shanghai. These conditions have promoted the economic development of Wuhan. In addition, the city has lots of enterprises from high-tech to heavy industries, higher education institutes from top universities to research centers. Most of them are in the Optic Valley Area.

The Optic Valley Area is the most significant new developing area in Wuhan, which is officially called 'The East Lake High-Tech Development Zone'. It was established in 1988 and approved as the one of the first national-level high-tech zones in 1991. Because it is famous for new technology developments especially for optoelectronics technologies, it is labeled as the 'Optic Valley'. With the area of $518.06 \mathrm{~km}^{2}$, it includes the administrative divisions of Wuchang, Hongshan, Jiangxia and Qingshan. There are eight industry parks, more than five commercial centers, more than 90 high-level universities and research institutes in the Optic Valley Area. As the area develops, a huge number of residential buildings are constructed since then. Therefore, the high-rise residential buildings in the area can well represent the new generation of residential buildings, which are epitomes for the development of residential buildings in the HSCWA.

\subsubsection{A typical climate}

In general, the hot-summer and cold-winter climate is one of the most common climates for many cities all around the world. In China, the HSCWA is a transient climate zone between the cold region and hot region. In this region, the outdoor average temperature is in the range of $25-30^{\circ} \mathrm{C}$ in summers. The highest temperature can go above $40^{\circ} \mathrm{C}$ occasionally. The outdoor average temperature is in the range of $0-10^{\circ} \mathrm{C}$ in winters. The lowest temperature can go under

$0^{\circ} \mathrm{C}$ sometimes. The official definition of HSCWA comes from three architectural standards 
established by the Ministry of Housing and Urban-Rural Development of China, including 'Design standard for energy efficiency of residential buildings in hot-summer and cold-winter zone' (standard number: JGJ 75), 'Standard for thermal design of civil buildings' (standard number: GB 50176) and 'Standard for energy efficiency design of public buildings' (standard number: GB 50189). The region covers an area of $1,800,000 \mathrm{~km}^{2}$ including five entire provinces of Hubei, Hunan, Jiangxi, Anhui, Zhejiang, the northern part of Fujian, Guangdong, Guangxi provinces, the southern part of Henan, Jiangsu, Shanxi and Gansu provinces, the eastern part of Sichuan and Guizhou provinces, and two municipalities of Shanghai and Chongqing. With a population of more than 500 million people, the region is the most populous and economically-developed area, contributing to more than a half of the entire gross domestic product (GDP) of China.

The City of Wuhan is such a typical example in the HSCWA. It is hot in summer and cold in winter, which is a typical climate for most metropolises in the temperate climate zone in the middle east of China, like Shanghai, Nanjing, Hefei, Changsha, Zhengzhou, Nanchang, Chongqing, Chengdu and so on. Its average try bulb temperature is in the range of $4-12^{\circ} \mathrm{C}$ in winters and in the range of $22-29^{\circ} \mathrm{C}$ in summers. Compared with other cities, the climate of Wuhan is more extreme in summers and winters. In this research, the local climate data which comes from the Chinese Standard Weather Data (CSWD) will be used. The major wind direction and the average wind velocity are calculated statistically based on the CSWD data to generalize the characteristics of wind conditions in this research.

\subsubsection{Typical building samples}

As Wuhan is the most populous city in the center of China, a large number of contemporary residential buildings are built continuously since 1990. Especially in the newly-developed Optic Valley Area, there is a great demand of residential districts with high-rise residential buildings for city dwellers. Because city dwellers are increasing as the city is developing and expanding. Also, there is a need to renew some previous residential districts which are too old and not suitable to live in any more. In this research, more than 5,000 high-rise residential buildings in 
more than 380 residential districts are investigated. From the investigation, the usual volume ratio of the residential districts is in the range of 1.5-6.0 according to the common urban density of the city. Based on the statistical analysis in building categorization study of 11 BUFs and 4 BCCs, the most common typical BUFs and BCCs are studied in this research.

\subsection{Review of CFD methods}

Numerical methods are essential tools for engineers, architects, urban planners and policy makers to compare urban and architectural design alternatives and to manifest guidelines. As mentioned in the development of wind environment studies, they have the advantages of high efficiency and accessibility for urban microclimate analysis, compared to theoretical analysis, site measurement, wind tunnel test ( $\mathrm{Li}$ et al., 2011). CFD is one of these numerical tools, which has been frequently used in the wind environment studies at various spatial scales (Kubota, Miura, Tominaga, \& Mochida, 2008). Urban microclimates such as wind environment have been intensively studied by using CFD approach due to its flexibility and highly efficient prediction of the complex wind flow in building environment (Xie et al, 2005; Memon et al., 2010). CFD studies on the meteorological microscale, where typical spatial distances are less than $2 \mathrm{~km}$, are gaining popularity due to their advantages such as the explicit modeling of urban and building geometry and resolving the flow field with high spatial resolution (Toparlar et al., 2017).

\subsubsection{Computational methods}

CFD refers to Computational Fluid Dynamics. CFD simulation is based on computational technology of solving the Navier-Stokes equations (N-S equations). Development of computational technology makes CFD simulation a reliable and accessible method for wind environment studies. By utilizing computational graphic technology, simulation results can be illustrated visually. The method has been widely used in architectural design and engineering practices, especially in the study of urban planning issues such as influences of winds on 
structure, heating and ventilation and building fire prevention and so on (Kaijima et al., 2013). There are laminar flow and turbulence flow of fluids movements. Wind environments of buildings are usually described using turbulence flow according to the calculation of Reynolds Number. Turbulence is a nonlinear and irregular fluid movement with complicated dynamics (Yu, 2016). Generally, there are three common methods: (1) Direct Numerical Simulation (DNS) method; (2) Reynolds Average Naviers-Stokes (RANS) method; (3) Large Eddy Simulation (LES) method. In 2016, researchers compared the performances of those models in simulating wind environments around buildings (Liu \& Niu, 2016).

The DNS method calculates every transient dynamic following the change of time in the three-dimension fluid field, by solving the N-S equation group directly. It has the following advantages. (1) Artificial assumption and using empirical constants can be avoided to improve the accuracy. (2) Theoretically, there will be no problem for closing the equation system, as to solve the N-S equations directly. (3) DNS method can provide results of every transient fluid dynamics according to every time and space, including some data that cannot be measured through experiments. (4) Supported by computational graphic technology, the structure of turbulence can be presented clearly and vividly from both large-scale and small-scale fluid movement using massive simulating grid and high-precision calculation methods. The major shortcoming of DNS method is its requirement of huge computational internal memories and time-consuming calculation in central processing units.

Turbulence is made up by many eddies with different sizes. Only the large eddies influence the major fluid movement most. The strategy of LES method is to filter the small-scale eddies and achieve the equations for calculating the large-scale ones (Chu, Ren, \& Chiang, 2014). Researchers found that the Delayed DES model could provide the flow field and the instantaneous characteristics of wind flow (Liu \& Niu, 2016). LES method can solve smallscale turbulence movement problems by consuming much less calculation time compared to DNS method. Compared to RANS, LES method is able to calculate more large-scale turbulence movement, which is more universal (Germano, Pomelli, Moin, \& Cabot, 1991). Shortcomings of LES method include requirements of large computational resources, because of high density of grids, big data processing and solving partial differential equations.

The RANS method uses turbulent statistical theories to solve N-S equations and make the 
average Reynolds equation system closed. It has the following advantages. (1) RANS method does not rely much on high computational performance. (2) The calculation results are practical enough to cover most engineering applications in the Reynolds range. In summary, RANS method is widely used in research and industrial applications nowadays for its high efficiency, especially for steady RANS (SRANS) method. (Franke, Hellsten, Schlünzen, \& Carissimo, 2007). The k- $\varepsilon$ turbulence model is one of the most used models for urban microclimates (Toparlar et al., 2017).

\subsubsection{CFD software}

CFD software generally can provide a variety of computational models. Users can select the models according to different situations, in order to speed up calculations, achieve optimum stability and accuracy. Usually, there is an appropriate model for a specific physical problem according to the flow features. There are many CFD software such as Fluent, Phoenics, OpenFOAM, CFX, STAR-CD, FIFIP, Flow Design, CFD Rhino and other commercial software for scientific and engineering applications.

"ANSYS Fluent software contains broad physical modeling capabilities needed to model flow, turbulence, heat transfer, and reactions for industrial applications" (ANSYS Fluent Website, 2016. Retrieved from: http://www.ansys.com/Products/Fluids/ANSYS-Fluent). Built for multi-physics, it has a flexible workflow. It can solve complex models for wind environment studies. Moreover, a simulated design can be optimized efficiently by linking it to the design tool of CATIA V5.

Phoenics is the first commercial software for fluid dynamics and heat transfer simulation. "It is a reliable, cost-effective CFD program with proven track record simulating scenarios" (CHAM Website, 2016. Retrieved from: http://www.cham.co.uk/phoenics.php). It facilitates the input of problem-defining data and formulae without recompiling. Phoenics possesses an auto-meshing feature that generates a default grid based on the geometry and domain size. Grid regions are created around each object automatically. This removes the need for the timeconsuming and arduous task of creating complex meshes. The software is designed to ensure 
solutions to converge, which relieves the need of setting convergence-promoting parameters.

Fluent and Phoenics are widely-used CFD tools, but they are both time-consuming in mesh generation and simulation. Whereas, they are very expensive. OpenFOAM is a free software, which is relatively easy in application using Rhino and Grasshopper. One of its great superiorities is its convenience in mesh generation due to the powerful modelling capacity of Rhino and Grasshopper. Models with common formats can be either easily switched or imported for the software (Sobachkin et al., 2014). In Fluent, a user usually has to draw a model line-by-line in Gambit which is complex. Besides the mentioned three popular software, there are some other software, including some relatively new software such as CFD Rhino developed in the end of 2016. Most of the software use the common computational models. A brief summary is made to compare several common CFD tools in the table (Table 1.).

Table 1. Comparison of several common CFD tools.

\begin{tabular}{|l|l|l|l|l|}
\hline CFD software & Modeling Time & Calculation Time & Accuracy & Costs \\
\hline Fluent (Ansys) & Long & Long & High & Expensive \\
\hline Phoenics & Long & Long & High & Expensive \\
\hline Open FOAM & Medium & Medium & High & Open source \\
\hline Flow Simulation & Short & Medium & Medium & Medium \\
\hline ENVI-MET & Long & Long & Medium & Free \\
\hline Vasari & Short & Short & Low & Discontinued \\
\hline
\end{tabular}

\subsection{Evaluation criteria of wind environment}

The assessments for wind environment generally include the ratio of wind velocity assessment, the relevant comfort assessment and the probability and statistic of wind velocity assessment. There are several green building evaluation criteria such as LEED (Leadership in Energy and Environment Design, United States), BREEAM (Building Research Establishment Environment Assessment Method, United Kingdom), BEAM (Building Environmental Assessment Method, Hong Kong), NZBCH (New Zealand Building Code Handbook, New 
Zealand) and the 'Green Building Evaluation Standard' (China, standard number: GB/ T503782014). In these evaluation criteria, the standards from various manuals are different. Some are indistinct or even primitive for outdoor wind environment among buildings. In the 'Green Building Evaluation Standard' of China, there are some specific requirements for wind environment by defining the suitable velocity and pressure. For winters, the wind velocities are suggested to be less than $5 \mathrm{~m} / \mathrm{s}$, and the pressure differences between the windward and leeward surfaces are suggested to be less than 5Pa. For summers and transitional seasons, vortexes and areas without airflows are both suggested to be prevented; window areas with the pressure differences over $0.5 \mathrm{~Pa}$ between indoor and outdoor spaces are suggested to be more than $50 \%$ of the total areas.

According to the themes of evaluation criteria of wind speed probability and statistics, the balance of wind velocity is proportional to human sense of comfort (Table 2.). The evaluation criteria of outdoor wind environment can be established based on the relevant requirements of the above standards, by considering the wind velocity, influenced areas with various velocities, air pressure and streamlines of wind flow.

Table 2. The relationship of human comfort and wind velocity provided by Wise and Pendwarden (Penwarden \& Wise, 1975).

\begin{tabular}{|l|l|}
\hline Velocity of wind & Comfort \\
\hline $1 \mathrm{~m} / \mathrm{s}<\mathrm{V}<5 \mathrm{~m} / \mathrm{s}$ & Comfortable \\
\hline $5 \mathrm{~m} / \mathrm{s}<\mathrm{V}<10 \mathrm{~m} / \mathrm{s}$ & Uncomfortable \\
\hline $10 \mathrm{~m} / \mathrm{s}<\mathrm{V}<15 \mathrm{~m} / \mathrm{s}$ & Very uncomfortable \\
\hline $15 \mathrm{~m} / \mathrm{s}<\mathrm{V}<20 \mathrm{~m} / \mathrm{s}$ & Intolerable \\
\hline $20 \mathrm{~m} / \mathrm{s}<\mathrm{V}$ & Dangerous \\
\hline
\end{tabular}

\subsection{Summary of literature review}

In summary, it can be found that wind environment studies of newly-built contemporary highrise residential buildings are needed continuously according to the rapid urbanization process around the world. There is a need to expand the urban climate knowledge to the developing 
regions (Toparlar et al., 2017). Especially for the developing regions in the temperate zone, a large amount of newly-built residential buildings are constructed in novel forms and configurations, which are mostly more or less different from previous buildings. Previous studies lack of systematic categorizations of building forms and configurations. It is known that influences on winds are closely related to the forms and configurations. But the relationships between the influences and some building variables are not clear enough. The existing windenvironment-study methods are usually complex and time-consuming. It is challenging to improve the efficiency of the entire analysis process. Using computational tools (such as the parametric modelling and CFD simulation) should be the most appropriate method for this research.

From the development of wind environment studies, the architectural wind environment study is a crucial topic related to sustainable habitats. There are mainly four methods of wind environment studies. The numerical simulation would be the most appropriate method in this research for its efficiency and accessibility. However, the simulation is still time-consuming and complex, especially for comparisons of different designs which requires iterative analysis.

By reviewing the influences of buildings on winds, previous studies show that buildings can obstruct the wind flow and winds can exert forces on buildings. Influences on urban microclimates are related to the modifications of terrain surfaces in urban areas. Influences of buildings are closely related to the shape, size, orientation of a single building and arrangement of a building cluster with several building units. The air-pressure difference is the reason for wind flow. However, previous studies do not discuss much about the relationships between the wind-velocity magnitudes and air-pressure magnitudes very often. In this research, the airpressure magnitudes and wind-flow streamlines will be used to explain the mechanisms together.

By reviewing wind environment studies around the world, studies of building influences on winds are not systematic enough, especially for forms and configurations of buildings in the HSCWA. For the buildings in previous wind environment studies, the urban locations investigated do not have a large variety. Especially for the cities located in the developing regions, very few studies can be identified (Toparlar et al., 2017). There is a need to expand CFD urban microclimate knowledge to developing regions of the world, such as the HSCWA. 
Though there are some previous researches of HSCWA, most of them are for block scales or for specific buildings, which give limited information to common building designs. In the previous studies, influences of some forms such as the cuboid shape are studied. But the studies are not systematic enough. Especially, the studies of influences of building variables of the forms and configurations are not many. The relationships between the influences and building variables are not clear enough. Therefore, there is a need to explore how influences on wind environments are changed as the building variables are changed. On the other hand, studies that present and analyze overall building influences on surrounding environments are few. In this research, the influenced areas of wind-velocity magnitudes on the horizontal and vertical planes will be both presented and analyzed. The study will give intuitive results that present the overall influences on wind environments. The influences are described by the influenced areas with different wind-velocity ranges (which are changed from the initial wind velocity). Therefore, the research helps architects to understand the influences of buildings on wind environments.

By reviewing the high-rise residential buildings, previous studies of novel high-rise residential buildings in the HSCWA are few, especially for representative building forms and configurations. Previous studies lack systematic categorizations of forms and configurations of buildings, especially for newly-built high-rise residential buildings. Studies of morphological attributes of high-rise residential buildings are needed. Because influences of buildings on wind environments are closely related to forms and configurations. This research will study the newly-built (built in 2000-2018) high-rise residential buildings with novel forms and configurations in the area. The buildings are the focus of this research, instead of the climate. Wind environment studies of the novel building forms and configurations will contribute to the original knowledge of urban microclimates.

For the methods used in the previous wind environment studies, the site measurements, wind tunnel tests and CFD simulations have been used in those researches. Experiments such as wind tunnel tests are often used for validations of the CFD methods. It is usually complex to use those methods. For CFD simulations, there are many procedures such as mesh generation, setting up boundary conditions, using an appropriate computational model and validations. The simulation time is relatively long and the evaluation methods are also complex. Therefore, the entire processes are usually time-consuming. Nowadays, many computational tools have been 
widely used in the architectural researches and designs. For example, parametric modelling tools are usually used for the generation of architectural appearance such as facades. The tools have great advantages in creating mass duplicate building models with complex shapes (forms) and layouts (configurations) by using algorithms. But the emerging tools have been rarely used in wind environment studies. In this research, multiple computational tools will be used in three major aspects, including parametric design (that helps in the mesh generation for CFD simulations), CFD simulations and result analysis. 


\section{Research methodology}

The research methodology will be explained in this chapter, including parametric design, simulation, and analysis. The structure of the research methodology is shown in the figure (Figure 5). In order to study the relationships between the influences on wind environment and the different building variables of building-unit forms (BUFs) and building-cluster configurations (BCCs), this research requires a large amount of comparison studies. The methodology is developed by making multiple computational tools work in coordination. The parametric modelling tool can assist the mesh generations for CFD simulations. Image processing tool can be used to analyze the results quantitatively. The integration of multiple tools can improve the efficiency of comparison studies for building designs with different variables.

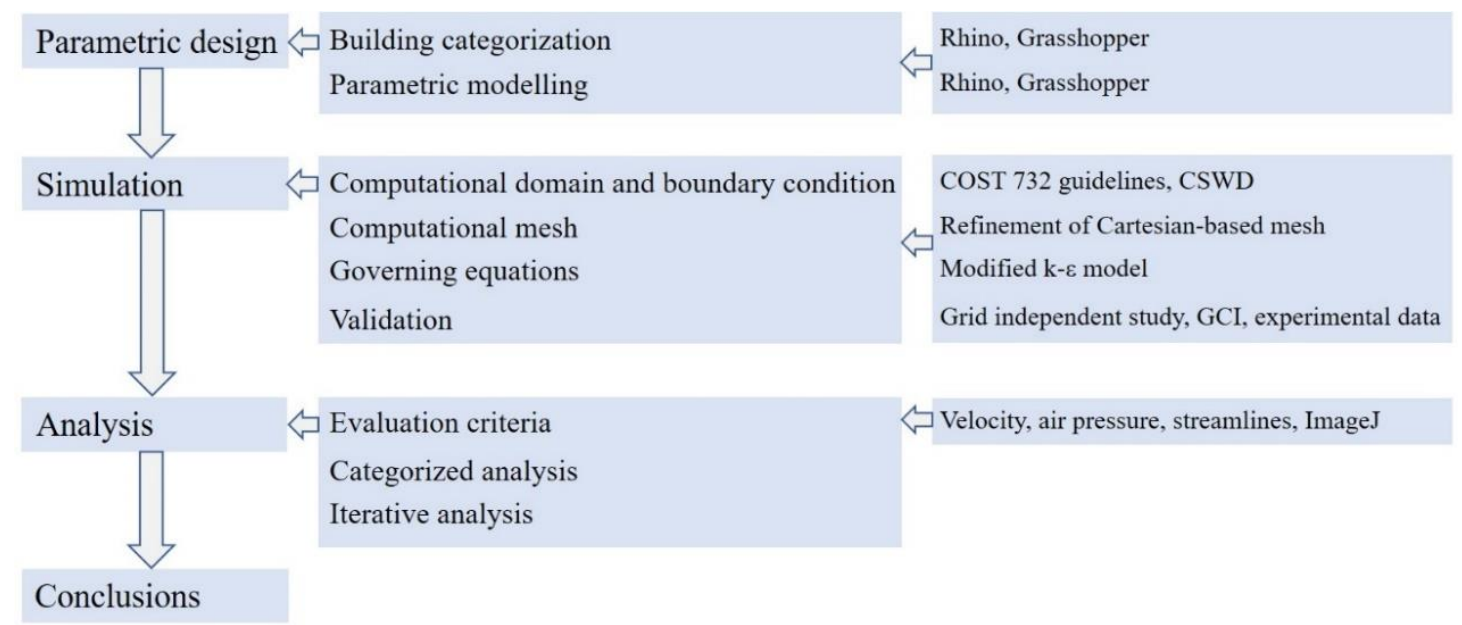

Figure 5. The research framework.

Before simulations, the parametric tool are used in the parametric design, including collecting building information and statistical calculation, automatic creation of parametric building models for mesh generation of CFD simulations. Then, the CFD tool is used to simulate the wind environment around the buildings. The mesh generation technology is based on the refinement of Cartesian-based mesh. The Reynold-averaged Navier-Stokes (RANS) method is used in the CFD simulations and a modified k-epsilon model is used as the turbulence 
model. The computational method has been widely recognized due to its acceptable accuracy and efficiency. The simulation method has been validated by grid-independence studies and wind tunnel experiments. After simulations, the image processing tool is used to accurately evaluate influences on wind environment by calculating the pixel numbers of wind velocity magnitude maps and converting them into actual influenced areas.

This research intends to study the influences of BUFs on wind environment first (as shown in the fifth chapter). And the influences of BCCs are studied based on the studies of BUF as building clusters are composed by multiple building units (as shown in the sixth chapter). Three BUFs and three BCCs are studied respectively. The influenced areas of wind velocity magnitudes on pedestrian level are calculated and compared. Air pressure magnitudes and wind flow streamlines are used to analyze the mechanisms. The relationships between the building variables and the influences on wind environment can be discovered.

\subsection{Parametric design method}

As an essential step before CFD simulations, the parametric design is consisted of categorization study and parametric modelling of buildings. Based on investigation in building morphology, it sets up a bridge between building variables and CFD simulations to study building forms and configurations which are representative for generic high-rise residential buildings in the area.

\subsubsection{Building categorization method}

In general, residential buildings vary from free standing houses to multi-story buildings and high-rise buildings. Each specific building is different from the others. Influences on wind environment are closely related to building shapes and layouts. However, time is limited to simulate thousands of specific buildings in the area in this research. And the research outcomes are expected to be widely applied to general high-rise residential buildings in the HSCWA. Therefore, it is necessary to collect the information of all buildings in the area, to categorize 
them according to definitions and to find out representative forms and configurations. Then parametric modelling scripts are developed based on the attributes of building forms and configurations for mesh generations in CFD simulations.

In this research, information of more than five thousand buildings in the Optic Valley Area of Wuhan City is collected. The rapidly-developing urban area is a typical example in the HSCWA. The definitions of forms and configurations are made from building morphology consisted of previous studies, architectural practices and site investigations. In the definitions, the geometrical attributes of the buildings are summarized according to common situations. Then, buildings in the area are categorized based on the definitions. For building units, they can be categorized into regular forms, such as square form, rectangular form, or irregular forms such as the relatively novel ' $\mathrm{T}$ ' form. For building clusters, they can be categorized into regular configurations such as linear configuration, or irregular configurations such as the relatively novel curvilinear configuration (Zhang, 2012). The regular and irregular forms and configurations are representative for most high-rise residential buildings in the area. This research focuses on the most representative building forms and configurations. The forms and configurations are found through quantitative comparison in the statistical calculation of building number. In this research, the calculation was done based on the investigation of 5,669 residential building samples from 383 residential districts. At the same time, most typical sizes and intervals of the buildings are summarized. The parametric tools Rhino and Grasshopper are used to help marking building locations on the map.

\subsubsection{Modelling method}

After finding the most representative building forms and configurations in the categorization study, parametric design systems are established to link building variables and simulation. The building models are used to help mesh generation for CFD simulations. Parametric modelling is used due to its high efficiency in modelling. Unlike conventional modelling, there is no need to draw lines or surfaces one by one to create a model in parametric modelling. Instead, one or several parameters are used to define one model or several models. Scripts are created to link 
every parameter logically to generate building models. Though development of algorithms is time-consuming, parametric modelling is fast and accurate on the generation stage. Moreover, it is convenient in modelling adjustment, which is suitable for iterative analysis to compare simulation results for different parameters (building variables).

\subsubsection{Modelling tools}

Software tools of Rhino and its plugin Grasshopper are again applied on the modelling stage. There are other approaches such as using Autodesk Dynamo together with Revit and Flow Design. However, they lack in previous studies and practical tests as they are relatively new. In addition, it is difficult to use other plugins in their environment as they are not open-source software. According to the real situation, Rhino and Grasshopper are more suitable on early design stage in practical projects (He \& Schnabel, 2018). Besides, there are more open-resource programs to support the analysis on the platform. It has the potential of bridging different analysis software, such as Flow Simulation and Fluent. In addition, these have already been proved reliable and stable by relevant studies in this filed.

\subsubsection{Modelling strategy}

In the modelling process, modelling strategy is the foundation for creating modelling algorithm. The variables of buildings and attributes of forms and configurations are considered in the strategy. Most building models are divided into several sub models created by modelling script modules. Different algorithms are developed by using abundant components in Grasshopper. For example, an arrangement of points can be created by an algorithm for positions of several objects. Then, the objects can be located by attaching them to the points using another algorithm. For mass-duplication modelling, the strategy is to generate a single architectural component first, and then several components are assembled together to create a complex building model using one or several algorithms. The architectural components can be adjusted anytime by changing the initial parameters. In this research, building models are simplified appropriately, 
because major geometrical information of the models is the key element concerned. Some details and small architectural components such as windows, shading boards and decorations will not be modelled due to their relatively small influences on wind environment. Building models are checked and edited before simulations to reduce computational resource and fit the CFD simulation environment.

Parametric modelling systems are established for each form and configuration. Parametric modelling improves modelling efficiency for following mesh generation for CFD simulations in iterative studies. Moreover, there are great potentials to use parametric modelling to create complex models for analysis on multiple platforms if new algorithm modules or new software tools are developed in the future.

\subsection{CFD simulation method}

In this research, CFD (Computational Fluid Dynamics) tool is used to simulate wind environment of buildings. The numerical method is suitable for requirements and conditions of the research. The CFD simulation aims to study the wind environment of different building forms and configurations comprehensively. The CFD simulations are done following the Best Practice Guideline COST 732 (European Cooperation in Science and Technology) (Frank, Hellsten, Schlünzen, \& Carissimo, 2007), which is well recognized. For CFD simulation, the wind fluids parameters, the mesh generation of simulation and the mathematical model of fluid regions are closely related to accuracy and efficiency of the simulation. Therefore, using a proper setup for the simulation is crucial for the study.

At this stage, the software of Flow Simulation is used for the CFD simulation. In this section, the source of local wind data and the setup of wind fluids parameters are explained first. Then, simulation mesh generation and mathematical model of fluid region are described. And simulation strategy is explained in quick simulation and comprehensive simulation. The simulation method is validated in the end. 


\subsubsection{Computational domain and boundary conditions}

Wind conditions of simulations are set according to local climate data. In this study, the Chinese Standard Weather Data (CSWD) will be used to define wind directions, velocities and other parameters for CFD simulations.

There are some other data resources such as International Weather for Energy Calculation (IWEC), Chinese Typical Year Weather (CTYW) and Solar and Wind Energy Resource Assessment (SWERA). The IWEC data comes from the research project of American Society of Heating, Refrigerating and Air-Conditioning Engineers (ASHRAE). It is archived originally for a period of 1982-1999 at the United States National Climatic Data Center. Part of the data is estimated based on the geometric relationship between sun and earth (ASHRAE, 2001). The CTYW data is achieved by using the same method as the IWEC. It comes from the United States National Climatic Data Center based on a period of 1982-1997 (Zhang \& Huang, 2004). The SWERA data is from the project funded by United Nations Environment Program. The data is measured and recorded from special satellites.

The CSWD Data is selected for this study because it is obtained by measuring actual cases. The data also covers longer period up to the year of 2005. Its hourly weather files are more than the others. The CSWD is developed by researchers from the government institute China Meteorological Bureau, the Climate Information Center and the Department of Building Science and Technology, Tsinghua University (published by China Meteorological Bureau, Climate Information Center, Climate Data Office and Tsinghua University, Department of Building Science and Technology in 2005). The CSWD are recorded through accumulations of measurements of actual data on sites in different cities. It aims to provide various climate data, including daylight, solar radiation, humility, enthalpy, temperature and wind. There are annual data, typical year data and extreme year data. The CSWD has been widely used in studies of building energy and environment.

According to the CSWD (Figure 6), the local monthly average wind velocities fluctuate in a small range of $1.0-1.5 \mathrm{~m} / \mathrm{s}$, which are relatively stable. The average high wind velocities of every month are around $2.0-3.0 \mathrm{~m} / \mathrm{s}$. The average low wind velocities of every month are between $0-1.0 \mathrm{~m} / \mathrm{s}$. The average wind velocities, average high wind velocities and average low 
wind velocities are relatively close. Based on this, the average wind velocity $(1.33 \mathrm{~m} / \mathrm{s})$ is calculated by using the local monthly average wind velocities, which is in the middle of the velocity range. Therefore, to simplify the setting for easy comparisons, the average wind velocity $1.33 \mathrm{~m} / \mathrm{s}$ will be used as the reference velocity in all simulations.

According to the CSWD, wind direction is from the south or south east in most time of summer and transitional seasons. In winter, wind usually comes from the north. Therefore, wind direction is simplified as 'from south to north' in summer and transitional seasons; and it is opposite in winter. In this research, most simulations are done using one wind direction because the north part and south part of most buildings are symmetrical. In some simulations such as square form, the wind direction is not fixed, because building models are rotated in several angles to test the influence on wind environment under different wind directions in this study.

The computational domains are created in cuboid shapes in the CFD software. The sizes of computational domains are decided by following the guidelines COST 732 (Frank et al., 2007). ' $\mathrm{H}$ ' is defined as the height of a building or the height of the tallest building in multiple buildings. The vertical extension of the domain is set as $6 \mathrm{H}$. The lateral extension of the domain is set as $5 \mathrm{H}$ on each side. For extension of the domain in flow direction, the distance between the inflow boundary and the building(s) is set as $5 \mathrm{H}$; the distance between the outflow boundary and the building(s) is set as $15 \mathrm{H}$. The analysis sizes of CFD simulation results (such as the velocity magnitude map, air pressure magnitude map and airflow streamlines) will be smaller than the boundary sizes.

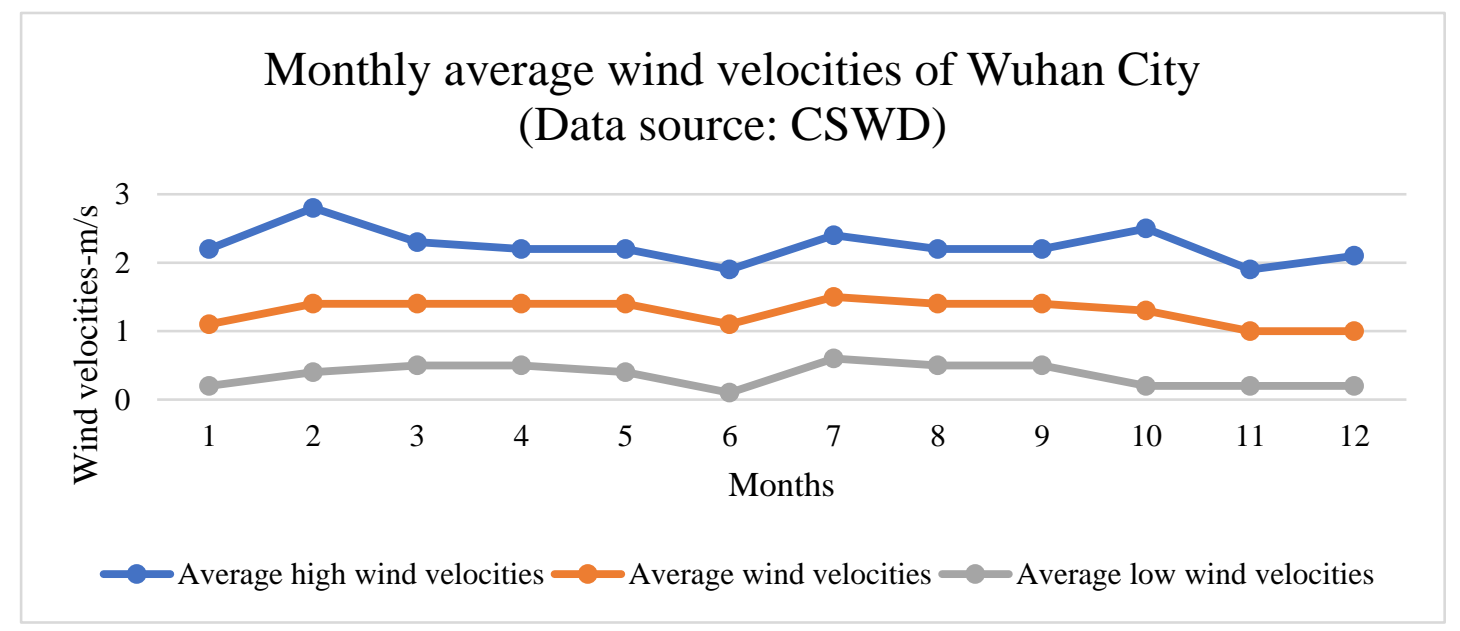

Figure 6. The figure shows the data of monthly average wind velocities of Wuhan City. The 
data is collected and calculated from the source of the Chinese Standard Weather Data (CSWD).

\subsubsection{Computational mesh}

The flow space of CFD is usually defined by mesh from subtraction of solid model based on body-fitted algorithms. In this research, the mesh creation technology is based upon the use of Cartesian-based meshes. The generation process is started from the division of the rectangular computational domain. The domain is divided into 'a set of cuboid cells by intersecting planes parallel to the axes of coordinate system' (Sobachkin \& Dumnoy, 2014). Then, the refinement of the mesh is done through 'splitting each cuboid into eight geometrically-similar cuboids using various adaptation criteria defined for each solid body automatically' (Sobachkin \& Dumnoy, 2014) (Figure 7).

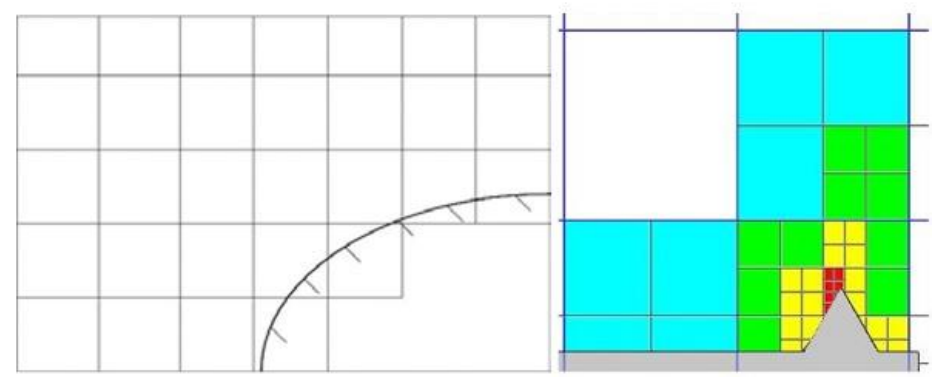

Figure 7. The figure on the left shows the structured Cartesian immersed-body mesh. The right one shows the refined mesh (Sobachkin \& Dumnoy, 2014).

\subsubsection{Governing equations}

The CFD software Flow Simulation solves the Navier-Stokes equations which are presented in Part 1 below. It is consisted of mass, momentum and energy conservation laws. It is supplemented by fluid state equations defining the nature of the fluid and empirical dependencies (Sobachkin \& Dumnoy, 2014). Both laminar and turbulent flows can be considered. Laminar flows are usually existed when Reynolds number is in low values. The Reynolds-Averaged Navier-Stoke (RANS) equations are used for the prediction of turbulence 
flow. To close the system of equations, Flow Simulation employs transport equations for the turbulent kinetic energy and its dissipation rate, using a modified k- $\varepsilon$ model with damping functions (Aynsley, 1985). The modified k- $\varepsilon$ turbulence model can describe laminar, turbulent and transitional flows. The equations of the model that consisted of turbulence conservation laws are presented in Part 2 below.

Part 1: the Navier-Stokes equations

$$
\begin{gathered}
\frac{\partial \rho}{\partial t}+\frac{\partial\left(\rho u_{i}\right)}{\partial x_{i}}=0 \\
\frac{\partial\left(\rho u_{i}\right)}{\partial t}+\frac{\partial}{\partial x_{j}}\left(\rho u_{i} u_{j}\right)+\frac{\partial P}{\partial x_{i}}=\frac{\partial}{\partial x_{j}}\left(\tau_{i j}+\tau_{i j}^{R}\right)+S_{i} \\
\frac{\partial \rho H}{\partial t}+\frac{\partial \rho u_{i} H}{\partial x_{i}}=\frac{\partial}{\partial x_{i}}\left(u_{j}\left(\tau_{i j}+\tau_{i j}^{R}\right)+q_{i}\right)+\frac{\partial P}{\partial t}-\tau_{i j}^{R} \frac{\partial u_{i}}{\partial x_{j}}+\rho \varepsilon+S_{i} u_{i}+Q_{H} \\
H=h+\frac{u^{2}}{2}
\end{gathered}
$$

Part 2: the equations of modified k- $\varepsilon$ model with damping functions

$$
\begin{gathered}
\frac{\partial \rho k}{\partial t}+\frac{\partial \rho k u_{i}}{\partial x_{i}}=\frac{\partial}{\partial x_{i}}\left(\left(\mu+\frac{u_{i}}{\sigma_{k}}\right) \frac{\partial k}{\partial x_{i}}\right)+\tau_{i j}^{R} \frac{\partial u_{i}}{\partial x_{j}}-\rho \varepsilon+u_{i} P_{B} \\
\frac{\partial \rho \varepsilon}{\partial t}+\frac{\partial \rho k u_{i}}{\partial x_{i}}=\frac{\partial}{\partial x_{i}}\left(\left(\mu+\frac{u_{i}}{\sigma_{\varepsilon}}\right) \frac{\partial \varepsilon}{\partial x_{i}}\right)+C_{\varepsilon 1} \frac{\varepsilon}{k}\left(f_{1} \tau_{i j}^{R}+C_{B} \mu_{i} P_{B}\right)-f_{2} C_{\varepsilon 2} \frac{\rho \varepsilon^{2}}{k} \\
\tau_{i j}=\mu s_{i j} \\
\tau_{i j}^{R}=\mu_{i} s_{i j}-\frac{2}{3} \rho \mathrm{k} \delta_{i j}
\end{gathered}
$$




$$
\begin{gathered}
s_{i j}=\frac{\partial u_{i}}{\partial x_{j}}+\frac{\partial u_{i}}{\partial x_{i}}-\frac{2}{3} \delta_{i j} \frac{\partial u_{k}}{\partial x_{k}} \\
P_{B}=-\frac{g_{i}}{\sigma_{B}} \frac{1}{\rho} \frac{\partial \rho}{\partial x_{i}}
\end{gathered}
$$

In the above equations, equation (1) is based on the mass conservation law, equation (2) is based on the momentum conservation law, equation (3) is based on the energy conservation law, $x_{i}$ is the coordinate of the unit volume, $u_{i}$ is the velocity of the unit volume $x_{i}, u_{j}$ is the second-order velocity of the unit volume $x_{j}, P$ is the pressure, $H$ is the total energy of the volume, $h$ is the thermodynamic energy of the volume, $u$ is the velocity of the volume, $Q_{H}$ is the heat release or absorption rate per unit volume, $\rho$ is the fluid density, $S_{i}$ is the momentum of the unit volume, $q_{i}$ is the heat flux, $k$ is the turbulence Prandtl number, $\varepsilon$ is another turbulence Prandtl number, $\mu$ is the dynamic viscosity, $C_{\mu}=0.09, C_{\varepsilon 1}=1.44$, $C_{\varepsilon 2}=1.92, \sigma_{k}=1, \sigma_{\varepsilon}=1.3, \sigma_{B}=0.9, C_{B}=1$, if $P_{B}>0, C_{B}=0$, if $P_{B}<0$, the turbulence viscosity is determined by the following equation.

$$
\begin{gathered}
P_{B}=-\frac{g_{i}}{\sigma_{B}} \frac{1}{\rho} \frac{\partial \rho}{\partial x_{i}} \\
f_{\mu}=\left(1-e^{-0.025 R_{y}}\right)^{2}\left(1+\frac{20.5}{R_{t}}\right) \\
R_{y}=\frac{\rho \sqrt{k} y}{\mu} \\
R_{i}=\frac{\rho k^{2}}{\mu \varepsilon} \\
f_{1}=1+\left(\frac{0.05}{f_{\mu}}\right) \\
f_{2}=1-e^{R_{t}^{2}}
\end{gathered}
$$


In the above equations, $e$ is the natural logarithm, $R_{y}$ is the Reynolds number based on average velocity pf fluctuations and distance from the wall; $y$ is the distance from point to the wall; $f_{\mu}, f_{1}$ and $f_{2}$ are Lam and Bremhorst's damping functions. When the Reynolds number $R_{y}$ becomes too small, the functions $f_{\mu}, f_{1}$ and $f_{2}$ decrease the turbulence viscosity and turbulence energy, and increase turbulence dissipation rate. The approach reverts back to the original k- $\varepsilon$ model if the functions $f_{\mu}=1, f_{1}=1$ and $f_{2}=1$. (Sobachkin \& Dumnoy, 2014).

\subsubsection{Simulation strategy}

For simulation strategy, there are quick simulation and comprehensive simulation for different purposes. Quick simulation aims to get an overview of the wind environment of buildings in a relatively short time. Comprehensive simulation aims to get a relatively accurate simulation result and multiple factors (velocity, air pressure and streamlines) to analyze the wind environment of buildings comprehensively.

In quick simulation, the CFD simulation is done by using a rough geometry and coarse girds. The quick simulation can present the result in a relatively short time, which is close to a real-time simulation. Simulation results of building models with different parameters can be achieved almost immediately. In this research, the quick simulations are usually done at the beginning for testing. In comprehensive simulation, the CFD simulation is done by using a detailed geometry and fine grid. Though the comprehensive simulation takes more time, the simulation result is more accurate. In this research, comprehensive simulations are usually done after several rounds of quick simulations to ensure the accuracy. The simulation results presented in following chapters are from comprehensive simulations.

\subsubsection{Validation of simulation method}

The simulation method was validated by a well-recognized wind tunnel experiment in the US Environmental Protection Agency to prove the accuracy of reliability (Brown, Lawson, Decroix $\&$ Lee, 2001). There are totally 77 cubes with an edge length of $0.15 \mathrm{~m}$ and an interval of $0.15 \mathrm{~m}$ 
placed in 11 rows and 7 columns in the experiment.

First, a grid-independent analysis was performed by constructing three sets of grids described in previous studies, including coarse gird, medium grid and fine grid (Hang \& Li, 2011). The coarse grid had approximate cells. It confirms that numerical results using the medium and fine grids are similarly good and much better than those using the coarse grid. The figure shows that the velocity profile at the position was predicted quite well (Figure 9). The gird convergence index (GCI) was calculated to ensure grid accuracy (Richardson, 1911; Roache, 1994). Though GCI has no fixed value, various researchers suggested it should be less than 5\% to ensure grid accuracy (Vinchurkar \& Longest, 2008). According to the calculation, the GCI is less than 5\% in sets of grids as shown in the table (Table 4.). Based on the analysis, the medium grid was selected for following simulations.

In the validation, velocity (V) and turbulence kinetic energy (TKE) of air fluid on 12 profiles in the center of the cube array are compared (Figure 8). The Predicted Root Mean Square Division (PRMSD) methodology (Wilks, 2006), Normalized Mean Square Error (NMSE), Correlation Coefficient (CC) and Fractional Bias (FB) (Chang \& Hanna, 2004) are considered to evaluate the statistical discrepancies between simulation and experimental results. The following equations present how the four parameters are calculated.

$$
\begin{gathered}
G C I=F_{s} \frac{\varepsilon_{r m s}}{r^{p}-1} \\
\varepsilon_{r m s}=\left(\frac{\sum_{i=1}^{i=n} \varepsilon_{i}^{2}}{n}\right)^{\frac{1}{2}} \\
P R M S D=\frac{1}{E_{m}} \times \sqrt{\sum_{i=1}^{n} \frac{\left(E_{i}-S_{i}\right)^{2}}{n}} \\
N M S E=\frac{\sum_{i=1}^{n}\left(E_{i}-S_{i}\right)^{2}}{\sum_{i=1}^{n}\left(E_{i} \times S_{i}\right)}
\end{gathered}
$$




$$
\begin{gathered}
F B=\frac{E_{m}-S_{m}}{0.5 \times\left(E_{m}+S_{m}\right)} \\
C C=\frac{\sum_{i=1}^{n}\left[\left(E_{i}-E_{m}\right) \times\left(S_{i}-S_{m}\right)\right]}{\sqrt{\sum_{i=1}^{n}\left(E_{i}-E_{m}\right)^{2}} \times \sqrt{\sum_{i=1}^{n}\left(S_{i}-S_{m}\right)^{2}}}
\end{gathered}
$$

In the equations, $F s$ is the safety factor $(F s=1.25), \varepsilon_{r m s}$ is the root mean square relative error, $r$ is the grid refinement ratio $(r=2), p$ is based on the second-order discretization of all terms $(p=2), n$ is the number of measuring points. $E m$ is the mean measurement value of all profiles in the experiment. $E i$ is the measurement value of a profile in the experiment. $\mathrm{Sm}$ is the mean value of all profiles in the simulation. $S i$ is the value of a profile in the simulation. An ideal situation could have the PRMSD of 0, NMSE of $0, \mathrm{CC}$ of 1 and FB of 0 . According to the criteria of COST Action 732 (Frank et al., 2007), the evaluation result is suggested to meet the standards of NMSE $<1.5$ and $-0.3<\mathrm{FB}<0.3$.

As shown in the table (Table 2.), both the calculation results of wind velocity and turbulent kinetic energy have demonstrated the accuracy of our CFD simulation method is acceptable. Especially for the results of velocity (V), the values of PRMSD, NMSE and FB are all very low, which is close to 0 . The correction coefficient is very high, which is close to 1 . 


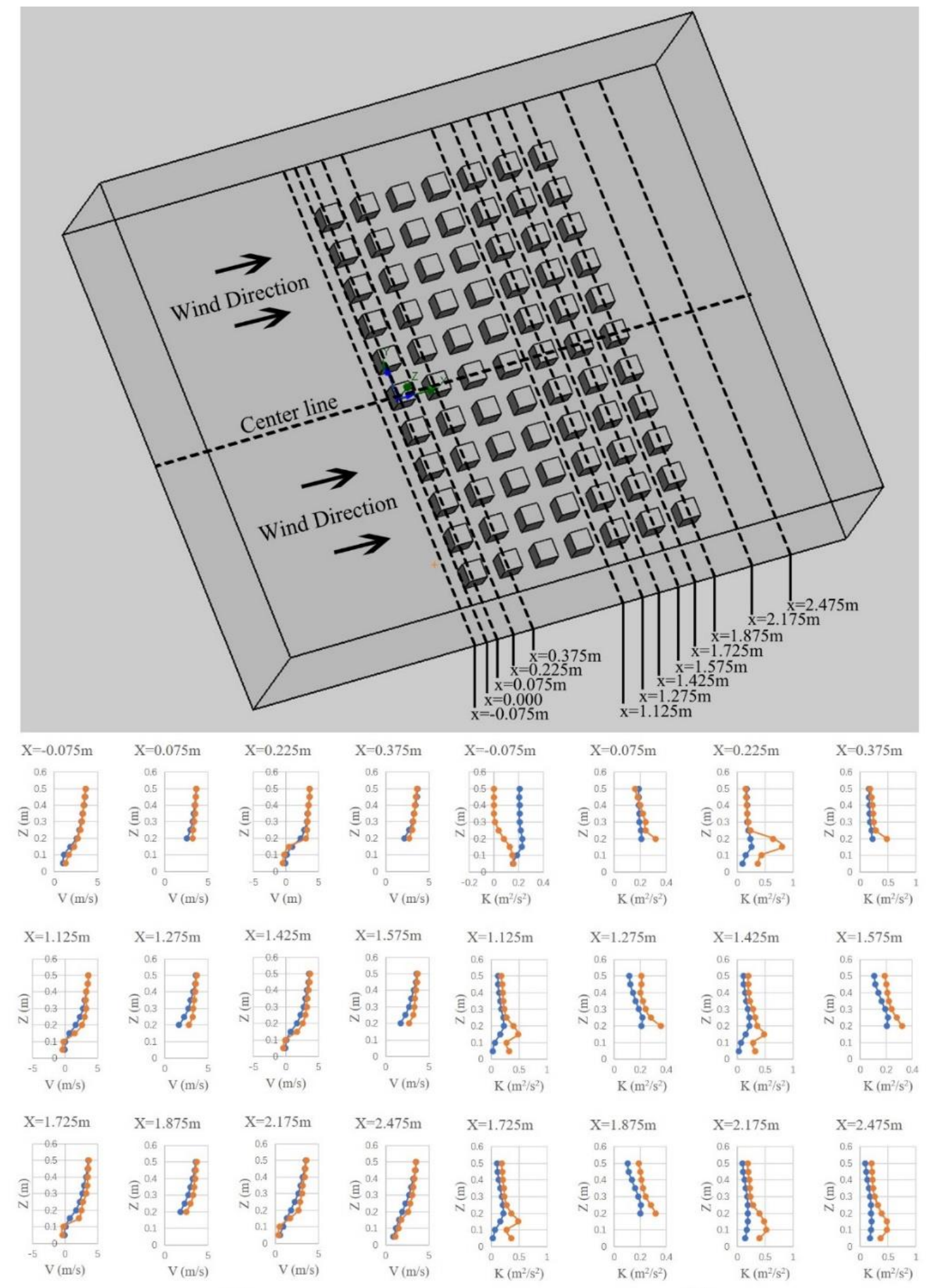

(a)

(b)

Figure 8 . The figure above shows wind direction, building array and the 12 positions of vertical profiles measured in the simulation and experiment. The figure on the bottom shows the comparison of the horizontal velocity (V) and turbulent kinetic energy (TKE) profiles between CFD simulation (blue lines) and wind tunnel experiment (orange lines). 

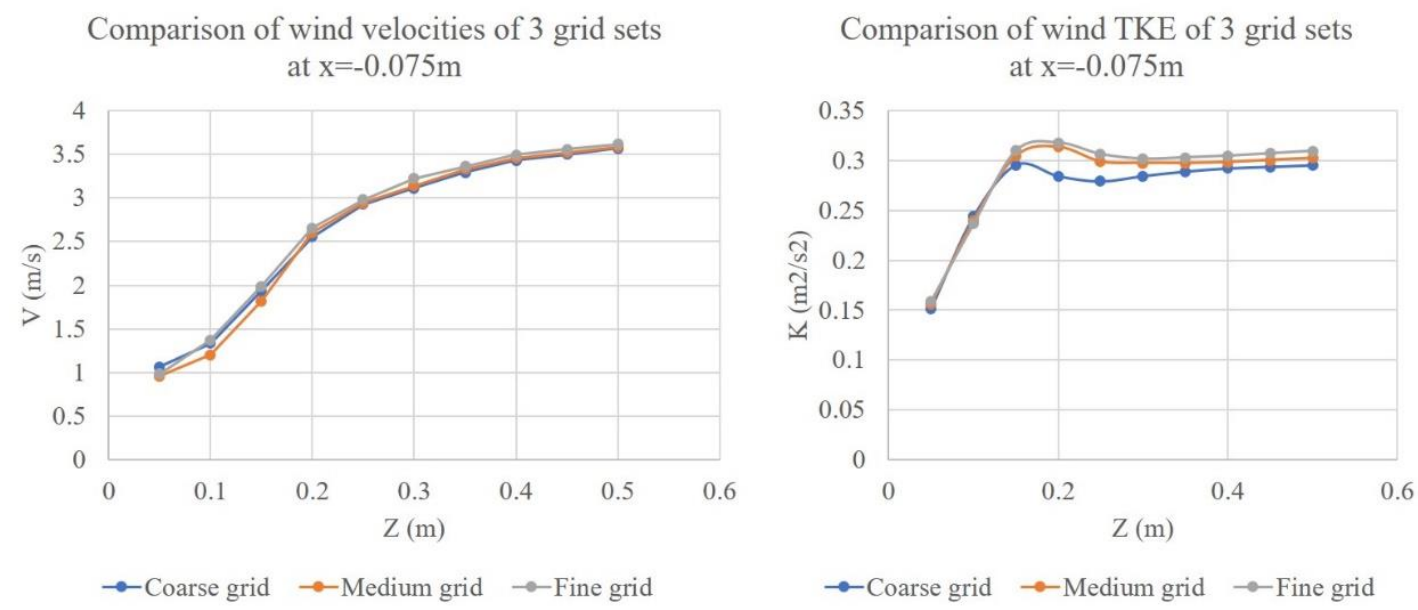

Figure 9. The figure above shows comparisons of wind velocities and TKE of 3 grid sets (coarse, medium and fine grids) at $\mathrm{x}=-0.075 \mathrm{~m}$.

Table 3. The table shows the calculation results of $\varepsilon_{r m s}$ and GCI of wind velocity (V) and turbulent kinetic energy (TKE) at $\mathrm{x}=-0.075 \mathrm{~m}$.

\begin{tabular}{|c|c|c|c|c|}
\hline & Grids & Grid 1-2 & Grid 2-3 & Grid 1-3 \\
\hline \multirow{2}{*}{ V } & $\boldsymbol{\varepsilon}_{\boldsymbol{r m} \boldsymbol{s}}(\mathbf{\%})$ & 6.931 & 8.534 & 6.890 \\
\cline { 2 - 5 } & GCI (\%) & 2.888 & 3.556 & 2.871 \\
\hline \multirow{2}{*}{ TKE } & $\boldsymbol{\varepsilon}_{\boldsymbol{r m s} \boldsymbol{s}}(\mathbf{\%})$ & 1.312 & 0.564 & 1.795 \\
\cline { 2 - 5 } & GCI (\%) & 0.547 & 0.235 & 0.748 \\
\hline
\end{tabular}

Note: $\varepsilon_{r m s}$ - The Root Mean Square Relative Error; GCI- Grid Convergence Index.

Table 4. The table shows the calculation results of the statistical discrepancies of wind velocity (V) and turbulent kinetic energy (TKE) in the experiment and simulation.

\begin{tabular}{|c|c|c|c|c|}
\hline & PRMSD & NMSE & FB & CC \\
\hline V & 0.158991 & 0.026475 & 0.104121 & 0.972412 \\
\hline TKE & 0.562225 & 0.502987 & 0.4033 & 0.523149 \\
\hline
\end{tabular}

Note: PRMSD-Predicted Mean Root Squared Division; NMSE-Normalized Mean Square Error; FB-Fractional Bias (FB); CC-Correction Coefficient. 


\subsection{Analysis method of simulation results}

After simulation, the results need to be judged whether they are negative or positive for the optimization of wind environment. In some situations, quantitative analysis is needed for comparison of different designs. Therefore, it is necessary to establish reasonable criteria to evaluate the wind environment of the buildings.

\subsubsection{Evaluation criteria}

The evaluation criteria will be developed in three aspects based on previous theory and existing standards. The three aspects are listed as follows: (1) wind velocity magnitude (as the major element); (2) air pressure magnitude (on building surfaces and around buildings); (3) wind flow streamlines (around buildings). In this research, the criteria mainly consider wind velocity as the key element for evaluating wind environment. Influences on wind environments are compared quantitatively by measuring the areas with different wind velocities which is accurate and easy for ordinary people to understand and use. Air pressure and wind flow streamlines are also considered to help in analyzing the mechanism of the influence on wind environment.

\section{Element 1: Wind velocity}

Wind velocity is the most important element for wind environment evaluation, as it influences human comfort, safety issue, exchange of heat and renewal of fresh air. Previous research suggests wind velocity is the most important in determining user satisfaction (Walton et al., 2007). For wind velocity, the evaluation criteria are developed based on existing standards, previous studies and practical requirements, including the criteria of wind environment in the 'Green Building Evaluation Standard of China' and the relationship of human comfort and wind velocity in the probability and statistic of wind velocity assessment method (Table 2.). Two aspects are considered for wind velocity: an appropriate range of wind velocity; the distribution of influenced areas with different velocities.

In order to create a favorable wind environment, an appropriate range of wind velocity is 
necessary. Slow wind flow reduces dissipation of heat and dilution of air pollutants, which increases potentials of urban heat island (UHI) phenomenon and air pollution. In hot summers, this can cause higher temperature in cities and cost more electricity for air conditioning. Fast wind flow increases dissipation of heat and may affect human activities in outdoor space. Extremely strong wind can even cause safety issues as people might be blown down. In cold winters, high-speed wind flow will make city dwellers feel cold and consume more energy for heating. Therefore, wind velocity should not be too high nor too low.

In a realistic situation, wind velocities vary in different areas in the space when wind flow is influenced by obstacles such like terrains and buildings. As this research focuses on the wind environment influenced by buildings, influences from other factors such like terrains are eliminated. Different wind velocities in the space can be found in the results of CFD simulations. According to the results, the planar distribution of wind velocity looks like a colorful map to present different velocities in different areas. The distribution of wind velocity is concerned because it is closely related to human comfort and safety. If the wind velocities are changed greatly in adjacent areas, it is uncomfortable for people to adapt to the suddenly changed environments. Take a common high-rise building in cuboid shape for example, high-velocity areas are usually generated on the lateral left and right sides of the building; a relatively small low-velocity area is generated on the windward side in front of the building, a relatively large low-velocity area is generated on the leeward side behind the building; most of the rest areas are ordinary-velocity areas with the velocity closed to initial velocity. The differences among the ordinary-velocity area, the high-velocity area and the low-velocity area are usually large. When people move from an ordinary-velocity area into the high-velocity area or the lowvelocity area, they may feel uncomfortable because the wind velocity is changed violently. Especially for the high-velocity area, the sudden increase of wind velocity can even cause safety issues as people might be blown down by extremely strong wind.

Therefore, based on previous studies, existing standards and practical requirements, the criteria of wind velocity are defined as follows. In all seasons, a favorable wind velocity should be in the range of $0<v<5 \mathrm{~m} / \mathrm{s}$ (' $\mathrm{v}$ ' stands for wind velocity). The distribution of influenced areas with different velocities is encouraged to be as even as possible. For an ideal distribution, wind velocity should be equal everywhere. Both low-velocity areas and high-velocity areas are 
suggested to be reduced. Vortexes and areas without natural air flow should be prevented.

In this research, areas with different wind velocities on pedestrian level (1.5m height) will be measured for quantitative comparisons. Computational tools are used in the measurement, which is explained in the next section of analysis method.

\section{Element 2: Air pressure}

Air pressure is an important element for evaluation of wind environment. Because air pressure difference is the reason for air flow which creates wind. Winds always flow from high-airpressure areas to low-air-pressure areas. When difference of air pressure increases, wind flow will be strengthened. The phenomenon usually happens near the lateral right and left sides of the windward surface of a building, because the air pressure varies remarkably between windward side and leeward side. For interior space, natural ventilation is caused by the air pressure difference between two or more openings on the building. A window on the windward side usually has higher air pressure than a window on the leeward side. If the windows are opened at the same time in a connected indoor space, winds usually can find paths to go through the space in the building. This is the natural ventilation for interior space in a building. For cold winter, air pressure difference between the windward and leeward sides is encouraged to be less than $5 \mathrm{~Pa}$. For hot summer and transitional seasons, the air pressure difference of more than $50 \%$ openings of a building is encouraged to be more than $0.5 \mathrm{~Pa}$. In summary, air pressure is closely related to wind velocity. Air pressure is considered to help in analyzing wind velocity in this research.

\section{Element 3: Streamlines of wind flow}

After CFD simulations, streamlines of wind flow can be presented in the results. The streamlines can clearly show how winds go around buildings and how winds are accelerated or decelerated because of the influences of buildings. These can explain the phenomena such as vortexes, high-velocity area and low-velocity area. As mentioned in the element of velocity above, vortexes, high-velocity area and low-velocity area are all suggested to be reduced. And they can be presented in the streamlines. In this research, streamlines of wind flow are used to help in analyzing wind velocity. 


\subsubsection{Analysis tools}

The simulation results can be visualized through images by using the software of Flow Simulation, including wind velocity magnitude, air pressure magnitude and streamlines of wind flow. For wind velocity, the magnitude and influenced areas can be presented in different colors on planes, including horizontal and vertical planes. Similarly, air pressure magnitude can be presented in different colors on horizontal and vertical planes and surfaces of building models. After setting one surface or several surfaces as study objects, the streamlines of wind flow can be presented in colorful pipes and arrows.

The images are further processed in the free software of ImageJ, which is another computational tool that aims to distinguish slight differences of different CFD simulation results of wind environments. Of course, other software such as Photoshop can also be used on this stage, but ImageJ is more suitable for the research because it is a professional software developed for academic analysis in image preprocessing of life science. It has multiple functions for different image formats, including displaying, editing, analyzing, processing, saving and printing. Moreover, it is accurate and efficient for pixel value statistics and calculations of distances and areas. Therefore, it is used for analyzing the images of simulation results of wind environment, such as calculating the areas with different wind velocities, or measuring the lengths of low-velocity areas and high-velocity areas. In this way, the areas and lengths are compared quantitatively for the evaluation of wind environment.

For wind velocity, the magnitude and influenced areas can be presented in different colors on the horizontal planes. A pixel is the smallest area on an image which can be given a separate color according to the value. To calculate the actual influenced area, the area of each pixel and the total pixel number of the influenced area are both needed. The calculation process is presented in the equations in Part 4 below. In the calculation of influenced areas, $L_{1}$ is the set length (the length of the analysis boundary is used in this study), $X_{1}$ is the number of pixels on the length, $L_{\mathrm{p}}$ is the length of each pixel, $A_{\mathrm{p}}$ is the area of each pixel, $X_{2}$ is the number of pixels on the calculation area, $A_{1}$ is the calculation area. First, the scale can be set in the 
image processing program: a length $L_{1}$ equals a pixel number $X_{1}$; then the length $L_{\mathrm{p}}$ and area $A_{\mathrm{p}}$ of each pixel can be calculated; the scale can be used in the further calculation of the influenced area $A_{1}$ that equals the multiplication of $A_{\mathrm{p}}$ and pixel number $X_{2}$. The calculation process will be done for each simulation case. In the study of a series of buildings, the windvelocity magnitude maps are usually generated in the same sizes and resolutions. The actual areas and lengths in the images with different resolutions can be calculated, because the scale has been set for each case as mentioned in the calculation. In this way, the areas with different velocity ranges can be compared quantitatively (Figure 10).

$$
\begin{gathered}
L_{p}=\frac{L_{1}}{X_{1}} \\
A_{p}=\left(L_{p}\right)^{2} \\
A_{1}=A_{p} \times X_{2}
\end{gathered}
$$

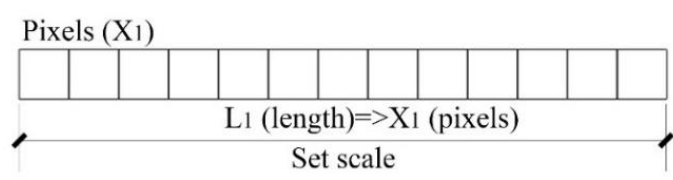

Pixels $\left(\mathrm{X}_{2}\right)$

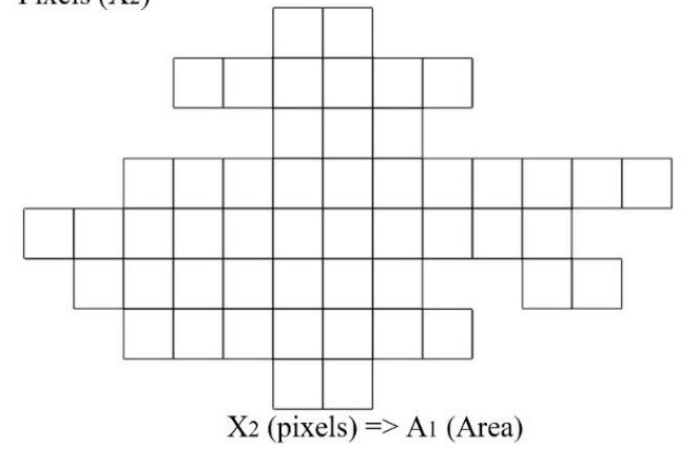

Figure 10. The calculation process of influenced area.

\subsubsection{Analysis strategy}

In this subsection, two analysis strategies used in this research will be explained, including 
categorized analysis and iterative analysis.

\section{(1) Categorized analysis}

The analysis will be implemented respectively according to different conditions of winds, forms and configurations of buildings.

For winds, two situations are mainly considered in the categorized analysis for different seasons: (1) hot summer and transition seasons; (2) cold winter. In the two situations, strengthen of wind is suggested to be reduced in cold wind (obstruction of wind flow); and outdoor ventilation is encouraged to be enhanced in hot summer (promotion of wind flow). As wind direction and building orientation vary in different situations, different angles between wind direction and windward surface of building are considered in some simulations in the following chapters.

For high-rise residential buildings, the categorized analysis is based on the building categorization that aims to find the most common types and layouts. Single building types are analyzed first respectively, as presented in the fifth chapter. Then, building layouts are analyzed for a group of buildings, as presented in the sixth chapter. For building types, three building types of square type, rectangular type and ' $T$ ' type are analyzed one by one. For building layouts, three building layouts of dotted layout, linear layout, and curved layout are analyzed one by one.

\section{(2) Iterative analysis}

Iterative analysis aims to explore the trends and mechanisms of influences of different building variables on wind environments. In order to do so, simulation results of building models with serial parameters are compared to find the tendency of influence on wind environment. After CFD simulations, results will be evaluated according to the criteria. Then parametric models of buildings are modified by changing the initial parameters in a certain degree step by step. Continuous CFD simulations and evaluations are done for every step to see how these modifications of building models influence the wind environment. Through the comparisons, the trends and mechanisms of phenomena can be discovered. For building forms, iterative analysis focuses on different parameters of shape, such as sizes (lengths, widths and heights) 
and orientations (rotation angles). For building configurations, iterative analysis focuses on different parameters of arrangement, such as building unit numbers and building intervals (transverse distances, longitudinal distances and staggered distances).

\subsection{Summary of research methodology}

The research methodology has been explained in this chapter, including parametric design method, CFD simulation method, and analysis method of simulation results.

Based on investigation in building morphology, parametric design sets up a bridge between building variables and CFD simulations to study building forms and configurations which are representative for generic high-rise residential buildings in the area. After finding the most representative building forms and configurations in the categorization study, parametric design systems are established to link building variables and simulations. The building models are used to help mesh generation for CFD simulations. Parametric modelling is used due to its high efficiency in modelling. Software tools of Rhino and its plugin Grasshopper are applied on the modelling stage. Building variables and attributes of building forms and configurations are considered in the modelling strategy. Most models can be divided into several sub models created by modelling script modules. Different algorithms are developed by using abundant components of the software tools.

The CFD simulation method has been explained for wind environment studies of building forms and configurations comprehensively. Wind conditions of the simulations are set according to the local climate data (CSWD). The computational domains are created in cuboid shapes by following the guidelines (COST 732) in the CFD software. The mesh creation technology is based upon the use of Cartesian-based meshes. The RANS method is used in simulations. A modified k- $\varepsilon$ model with damping functions has been explained in the governing equations. Simulation strategies are explained for different purposes. The quick simulation uses a rough geometry and coarse girds and the comprehensive simulation uses a detailed geometry and fine grid. The simulation method was validated by the wind tunnel experiment. The gridindependent analysis was performed by constructing coarse gird, medium grid and fine grid. 
The GCI was calculated to ensure grid accuracy. The PRMSD methodology, NMSE, CC and FB are considered to evaluate the statistical discrepancies between simulation and experimental results. The calculation results of wind velocity and turbulent kinetic energy have demonstrated the accuracy of the method is acceptable.

In the analysis of simulation results, reasonable criteria are established to evaluate the wind environment of the buildings, including three aspects of wind-velocity magnitude, air-pressure magnitude, wind-flow streamlines. By using the analysis tools, the simulation results can be visualized through images of the three aspects; the images are processed to distinguish slight differences of different CFD simulation results of wind environments. Two analysis strategies are used in this research, including categorized analysis and iterative analysis. 


\section{Parametric design of the buildings}

The overall goal of this chapter is to design a series of representative forms and configurations of high-rise residential buildings in the HSCWA for CFD simulations by establishing a parametric design system based on the building categorization study. Because influences on wind environments are closely related to building forms and configurations as mentioned in the literature review, there is a need to study the forms and configurations through categorizations. In the building categorization study, definitions of building-unit forms (BUFs) and buildingcluster configurations (BCCs) will be explained. And numbers of BUFs and BCCs in the area will be calculated statistically. In the parametric modelling, scripts of the representative BUFs and BCCs are developed based on their geometric attributes. The scripts can generate the building models automatically, which largely improves the efficiency of mesh generations for CFD simulations. The parametric models can be adjusted efficiently as the parameters are changed, which enables iterative analysis to study the influences of different building variables. The summary in the end (Section 4.3) summarizes the studies of parametric design briefly.

\subsection{Building categorization study}

In the categorization study, definitions of eleven BUFs and four BCCs will be given first according to the previous studies (mentioned in the literature review), site investigations and their geometric attributes. As mentioned, Optic Valley Area is selected for study due to its representativeness of the HSCWA. Its area is more than 200,000,000 $\mathrm{m}^{2}$. The definitions can cover almost all the forms and configurations of high-rise residential buildings in the area. Information of 5,699 high-rise residential buildings in 383 residential districts were collected and analyzed (detailed building information is presented in the appendix). These tremendous number of samples can show the development trend of high-rise residential buildings. Numbers of eleven BUFs and four BCCs in the area are calculated and compared. Most representative BUFs and BCCs can be found in the analysis. In this research, three BUFs and three BCCs are selected for wind environment studies due to their representativeness. Then, parametric models 
will be developed for CFD simulations afterwards.

\subsubsection{Categorization of BUFs}

BUF (Building Unit Form) is defined as the shape (geometry) of the standard floor plan of a single building unit in this research. A building unit is defined as a single building with at least an independent entrance and an independent vertical traffic system. Specific definitions of eleven BUFs are explained in this subsection, which can cover most situations of high-rise residential buildings in the HSCWA.

The concept of BUF is from the development of contemporary high-rise residential buildings in urban areas, which is clearer and more accurate than the vague definitions of plate form and tower form. Though two or more units can be joined together as one, one single building unit can provide complete architectural functions. The definition of building unit comes from the essential features of nowadays high-rise residential buildings, including independent entrances (for evacuations), vertical transportations and civil structures (frame structure). Usually, there are one or more sets of elevator shafts and evacuation stairs in a vertical transportation system, which is usually built in the tube-structure core of the building. Considering influences of winds (such as hurricanes) and earthquakes, frame structure made by reinforced concrete is usually applied in contemporary high-rise residential buildings. Highrise residential building units can be categorized according to different forms of floor plans (Table 5.). BUFs are largely depended on the arrangements of the vertical transportation system and apartments. Definitions of eleven BUFs are explained in the following subsection.

Table 5. Categorization of 11 BUFs.

\begin{tabular}{|c|c|}
\hline Codes & Names \\
\hline F01 & Square form \\
\hline F02 & Rectangular form \\
\hline F03 & 'T' form \\
\hline F04 & Frog form \\
\hline
\end{tabular}




\begin{tabular}{|c|c|}
\hline F05 & Hash form ('\#' form) \\
\hline F06 & Cross form (' $\mathrm{X}$ ' form, Windmill form) \\
\hline F07 & ' $\mathrm{Y}$ ' form \\
\hline F08 & 'H' form \\
\hline F09 & 'V' form (Butterfly form) \\
\hline F10 & Circular form \\
\hline F11 & Irregular form \\
\hline
\end{tabular}

Note: the ' $F$ ' in the codes stands for 'form' of BUF.

\section{(1) Square form (F01)}

A BUF with an architectural plane of square shape is defined as square form (Figure 11). There are four sides with a same edge length in the square form. The vertical transportation system and core-tube structure are usually in the center of a square-form building. Aisles are designed to surround the core and connect all the apartments that distribute along the 4 sides of the square plane. There are usually more than 4 apartments on every floor in square-form residential buildings. Therefore, the edge length of square form is relatively long, around 30 meters or more. The advantage of square form is its symmetric shape makes the structure very stable. But there are two disadvantages: (1) aisles occupy a lot of areas on floors; (2) some apartments do not have favorable orientations. Therefore, square form has become less popular than before, as clients require better residential conditions.

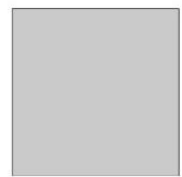

Figure 11. An example of simplified floor plan of square form.

\section{(2) Rectangular form (F02)}

A BUF with an architectural plane of rectangular shape is defined as rectangular form (Figure 12). It has been one of the most common forms since early ages. It is so popular that it also appears in the multi-floor residential buildings with relatively low heights and public buildings 
such like office buildings, commercial buildings and educational buildings. The major differences among them are the building sizes. Therefore, wind environment studies of rectangular form of high-rise residential building can be used as references for other buildings with rectangular floor planes. The size of the rectangular floor plane of a high-rise residential building largely depends on the number of apartments on each floor and building functions. The core-tube structure with a transportation system is usually in the center or in the middle on one side of the building. In the first situation, the apartments are arranged to surround the transportation system, which is similar to square form. In the second situation, the transportation system is usually on the north side and the apartments are usually on the south side for better orientations. And the aisle is shorter which saves more public area; smoke can be exhausted to outside through the vertical transportation system without mechanic ventilation system in the case of fire. Therefore, the rectangular form in the second situation becomes popular nowadays. The rectangular form is especially suitable for relatively large apartments (usually around 4 apartments on each floor). Because many other forms are developed from the modifications of the rectangular form.

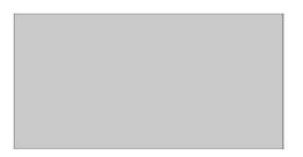

Figure 12. An example of simplified floor plan of rectangular form.

\section{(3) ' $T$ ' form (F03)}

A BUF with an architectural plane which is similar to the shape of a capital letter ' $\mathrm{T}$ ' is defined as ' $\mathrm{T}$ ' form (Figure 13). It can be derived from the rectangular form by bulging in its middle on one side. Usually, the bulge part can be balconies. This is common in residential buildings, especially for high-rise residential buildings with 10-12 floors. The bulge part becomes bigger due to the demand for larger apartments and the rise of building height. For the ' $\mathrm{T}$ '-form buildings with more than 18 floors, the bulge part is usually big enough for two apartments. It is a typical floor plane with four apartments on each floor in the ' $\mathrm{T}$ '-form buildings. A proper number of apartments can save more aisle area. Because the bulge part with apartments is usually in the south for better orientation, the transportation core is set in the center on the north 
side of the building. An aisle is usually in the middle to separate the elevator shaft and the staircase. In the case of fire, smoke can be exhausted to outside through the staircase without mechanic ventilation system. The main advantages of ' $\mathrm{T}$ ' form are its capacity of large apartments and keeping a good balance of saving lands at the same time. Nearly all the apartments have sensible orientations. ' $\mathrm{T}$ '-form buildings can meet most requirements of both developers and clients by adjusting the sizes. Therefore, ' $\mathrm{T}$ ' form is popular in urban area nowadays. But the disadvantage is that seasonal winds cannot go through the apartments of the bulge part in the south-north direction. Because there are not any window openings on the north of the apartments due to the aisle and transportation core on the north. Another disadvantage is that the bulge parts can shade the sunlight for the other parts of ' $\mathrm{T}$ '-form buildings sometimes, especially for the bulge parts with large sizes. In summary, the ' $\mathrm{T}$ ' form is a reasonable and popular form in the HSCWA. Moreover, some other forms are modified from the ' $\mathrm{T}$ ' form, especially for the irregular forms.

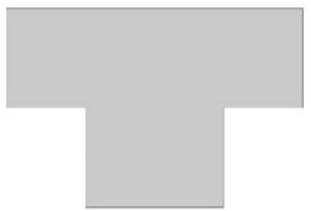

Figure 13. An example of simplified floor plan of ' $T$ ' form.

\section{(4) Frog form (F04)}

A BUF with an architectural plane which is similar to the shape of a frog is defined as frog form (Figure 14). On the floor plane, there are usually four rectangular parts distributed in the four corners like a frog stretching its four limbs. Generally, the four rectangular parts are actually four apartments, which are arranged to surround the aisle and core area of the building. The core-tube structure and the transportation core are usually in the center of the building. The aisle is designed to surround the elevator shaft and the stair case to link the four apartments and transportation core. Compared to ' $\mathrm{T}$ ' form, frog form increases the residential density and reduces the building length. However, the two apartments on the south always block daylight and winds from the other two apartments behind them. Though the two apartments on the south are usually smaller than the other two apartments on the north, it cannot solve the problem 
thoroughly. Therefore, the issue is the major drawback of frog form. Because of this, frog form is not as popular as ' $\mathrm{T}$ ' form nowadays.

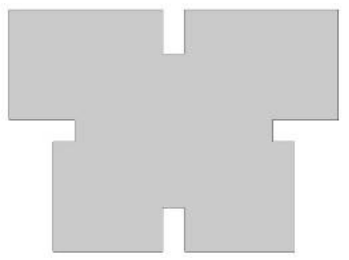

Figure 14. An example of simplified floor plan of frog form.

\section{(5) Hash form ('\#' form) (F05)}

A BUF with an architectural plane which is similar to the shape of a hash is defined as hash form (Figure 15). It is like a large square form with two extending parts on each corner. It aims to provide more apartments by arranging them on the 4 sides and 8 extending parts on each floor of a hash-form building. Therefore, a hash-form building usually has a relatively large size (more than 30 meters on each side). There are usually 8 or more apartments on each floor. The tube-core structure and transportation core are usually in the center of the building. On the floor plane, the elevator shaft and staircase are surrounded by aisle and apartments. The residential density is relatively high due to a large number of apartments on each floor. Therefore, hash form is very suitable in high-density urban areas, such as cities like Hong Kong, Shanghai and so on. However, its disadvantages are also obvious. First, its apartments are usually smaller than rectangular form and ' $\mathrm{T}$ ' form. Second, there are unavoidable negative influences on orientations of apartments, especially for the apartments on the north side. Therefore, hash form is becoming less popular nowadays. It is more suitable in metropolises with extremely high densities.

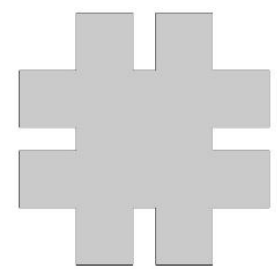

Figure 15. An example of simplified floor plan of hash form. 


\section{(6) Cross form ('X' form, Windmill form) (F06)}

A BUF with an architectural plane which is similar to the shape of a cross is defined as cross form (Figure 16). Cross form can also be called ' $\mathrm{X}$ ' form or windmill form due to its similar shape to the capital letter ' $\mathrm{X}$ ' and windmill. In a cross-form building, there are four extending parts. Each extending part is orthogonal from its two adjacent extending parts. The core-tube structure and transportation core are in the center. The aisle is usually around the elevator shaft and stair case. There are usually two apartments on each extending part. A standard floor plane with 8 apartments is a common example. There are three main disadvantages of cross form. Almost half of its apartments have unfavorable orientations. And usually seasonal winds cannot go through the apartments. Its thermal isolation performance should be affected due to its larger façade areas which promote the heat exchange. In summary, it is difficult for cross form to meet requirements nowadays. It is more suitable for high-density cities where land is very limited.

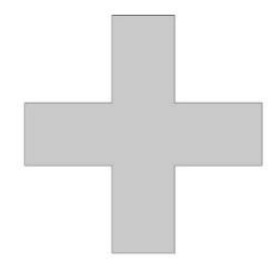

Figure 16. An example of simplified floor plan of cross form.

\section{(7) 'Y' form (F07)}

A BUF with an architectural plane which is similar to the shape of a capital letter ' $\mathrm{Y}$ ' is defined as ' $\mathrm{Y}$ ' form (Figure 17). There are three extending parts of the floor plan. A standard floor plane with 6 apartments is a common example for ' $\mathrm{Y}$ ' form. In the example, there are usually two apartments on each extending part. The angles between two adjacent parts are 60 degree on the floor plane. The core-tube structure and the transportation core are usually in the center of the building. Aisle is designed to surround the elevator shaft and the stair case. Apartments are arranged on the three extending parts that surround the aisle and the core area. The major advantage of ' $\mathrm{Y}$ ' form is that it can provide relatively large capacity for big apartments and increase residential density at the same time. The apartments in ' $\mathrm{Y}$ '-form buildings are usually larger than hash form and square form. However, the three main disadvantages of ' $\mathrm{Y}$ ' form are 
similar to cross form. First, not all the apartments can have good orientations. If one extending part is on the south, the rest two extending parts will be influenced in sunlight. If two extending parts is on the south, the rest one extending part will be influenced. Second, seasonal winds usually cannot go through its apartments due to its high residential density and center position of transportation core. Third, the facade area of ' $\mathrm{Y}$ ' form is larger than common forms such as square form and rectangular form, which may influence thermal-isolation performance of the building. Therefore, ' $\mathrm{Y}$ ' form is more suitable in metropolises with very high density.

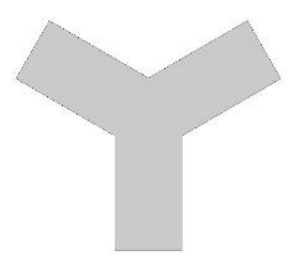

Figure 17. An example of simplified floor plan of ' $Y$ ' form.

\section{(8) 'H' form (F08)}

A BUF with an architectural plane which is similar to the shape of a capital letter ' $\mathrm{H}$ ' is defined as ' $\mathrm{H}$ ' form (Figure 18). There are four extending parts in the ' $\mathrm{H}$ ' form. The core-tube structure and transportation core are usually in the center of a ' $\mathrm{H}$ '-form building. The aisle goes through the middle of the building to link the apartments on two sides of it. The arrangement of apartments, aisle and vertical transportation core depends on the number of apartments on each floor. ' $\mathrm{H}$ ' form has similar advantages and disadvantages like ' $\mathrm{Y}$ ' form and cross form. Because there are usually more than four apartments on each floor, the residential density is relatively high, which is close to cross form. However, ' $\mathrm{H}$ ' form has the similar problems in orientations, interior ventilation and thermal isolation performance. Therefore, ' $\mathrm{H}$ ' form becomes less popular nowadays.

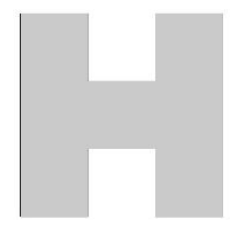

Figure 18. An example of simplified floor plan of ' $\mathrm{H}$ ' form. 


\section{(9) 'V' form (butterfly form) (F09)}

A BUF with an architectural plane which is similar to the shape of a capital letter ' $V$ ' is defined as ' $\mathrm{V}$ ' form (butterfly form) (Figure 19). The form can be thought as a modified version of rectangular form by bending its rectangular floor plane in the middle into a ' $\mathrm{V}$ '-letter shape. The aisle, the core-tube structure and the transportation core with the elevator shaft and the staircase are usually in the middle on each floor. Apartments in a ' $\mathrm{V}$ '-form building can have a relatively wide view. Because of this, it is popular in high-rise residential buildings beside water shore. Sometimes, areas of apartments and facades can be further increased by attaching bulge parts, which is similar to the shape of a butterfly. Though apartments in one extending part can be influenced by the other one in sunlight, the influences are limited. ' $V$ ' form does not have the similar orientation problem with ' $\mathrm{Y}$ ' form, 'H' form and cross form.
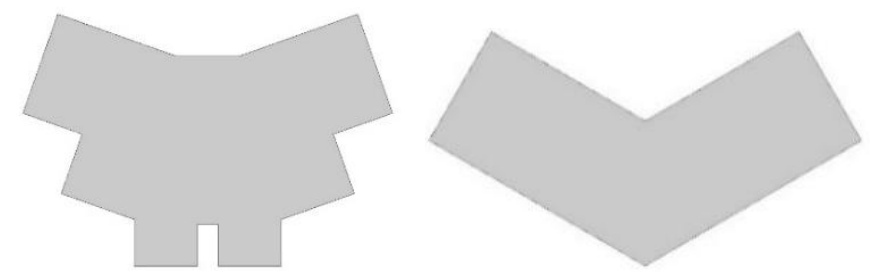

Figure 19. Two examples of simplified floor plans of ' $V$ ' form (butterfly form).

\section{(10) Circular form (F10)}

A BUF with an architectural plane which is similar to the shape of a circle is defined as circular form (Figure 20). In most situations, the main reason of creation of circular form is the need of the specific appearance. The core-tube structure and the vertical transportation system are usually in the center or in the middle on the north side of the building. This depends on the arrangement of apartments, aisle and vertical transportation system. The size of the floor plane is decided by the number of apartments. Due to its unique outlooking, a circular-form building has the advantage of minimum façade area, which saves construction materials. In addition, there is a potential of promoting thermal isolation performance. Because a relatively small surface area can reduce heat exchange between interior and outdoor spaces of the building. However, the practical usage of apartment areas is affected due to the sacrifice of the circular floor-plane shape. Moreover, the complexity of circular form makes its construction process 
becomes relatively difficult.

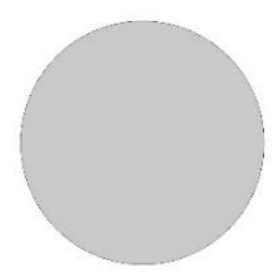

Figure 20. An example of simplified floor plan of circular form.

\section{(11) Irregular form (F11)}

A BUF with an architectural plane which is in irregular shape is defined as irregular form. According to the definition, irregular form is different from any BUF defined above. However, almost all the irregular-form buildings are developed from the other relatively regular BUFs. No matter how complex is its appearance, it can be evolved from the fundamental shapes mentioned above.

\subsubsection{Categorization of BCCs}

BCC (Building-Cluster Configuration) is defined as the arrangement (layout) of a group of several building units in this research. Specific definitions of 4 BCCs are explained in this subsection, which can cover most situations of high-rise residential buildings in the HSCWA.

The concept of $\mathrm{BCC}$ is from the development of contemporary high-rise residential communities in urban areas. Contemporary high-rise residential communities in the HSCWA are developed by local authorities and developers. In a community, several building units are usually built closely to form a building cluster. The outdoor spaces among building units can be used for landscapes and public-activity grounds. In the categorization study of BCCs, different relationships of building units in building clusters are clarified. Based on previous studies (mentioned in literature review) and site investigations, definitions of four typical BCCs are explained in this section, including scattered configuration, linear configuration, curvilinear configuration and composite configuration (Table 6.). 
Table 6. Categorization of 4 BCCs

\begin{tabular}{|c|c|}
\hline Codes & Names \\
\hline $\mathrm{C} 01$ & Scattered configuration \\
\hline $\mathrm{C} 02$ & Linear configuration \\
\hline $\mathrm{C} 03$ & Curvilinear configuration \\
\hline $\mathrm{C} 04$ & Compositive configuration \\
\hline Note: the ' $\mathrm{C}$ ' in the codes stands for 'configuration' of BCC. \\
\hline
\end{tabular}

\section{(1) Scattered configuration (C01)}

A BCC with several scattered building units which are independent from each other is defined as scattered configuration. Scattered configuration is one of the most common configurations nowadays. As sizes of high-rise residential buildings are increased, scattered configuration becomes popular. It is very flexible in building arrangement. The building units can be lined up or arranged to form a specific pattern. The flexibility of scattered configuration promotes the creation of better outdoor environments in residential communities. Spaces among building units can be used as greening, public-activity grounds or landscapes that service the residents. Because of the gaps among building units, usually it is relatively easy for winds to go through the building cluster, which is beneficial for the outdoor ventilation of the residential community.

\section{(2) Linear configuration (C02)}

A BCC with several building units connected one by one to form one linear building row or several linear building rows is defined as linear configuration. Linear configuration is one of the most popular configurations for contemporary buildings. The major advantage of linear configuration is the increase of residential density. As sizes of high-rise residential building units are increased nowadays, building units in a linear building row are usually less than three. This aims to prevent the 'wall effect' by reducing the size of the linear building row, especially for the length. Winds and sunshine can be blocked by several high-rise buildings lined up like a wall (He \& Schnabel, 2016). Wall effect is a typical phenomenon in high-density cities, such as Hong Kong. As soon as people realized the issue, the size of linear building row has been 
limited in new-built buildings.

\section{(3) Curvilinear configuration (C03)}

A BCC with several building units connected one by one to form one curvilinear building row or several curvilinear building rows is defined as curvilinear configuration. Curvilinear configuration can be created by modifying linear configuration. The curvilinear building row can be created by bending the linear building row. Curvilinear configuration becomes popular in contemporary high-rise residential communities due to its advantages of elegant outlooking and flexibility in arrangement. Its innate aesthetic function has enriched the diversity of residential district planning. The relatively large spaces among curvilinear building rows can be used for greening, public-activity grounds and landscapes (He \& Schnabel, 2016). Therefore, a well-designed curvilinear configuration can benefit in improving the living condition of a residential community, which is the reason for its popularity. Similar to linear configuration, the size of curvilinear building row is usually limited, which aims to prevent wall effect.

\section{(4) Composite configuration (C04)}

A BCC with several kinds of configurations is defined as composite configuration. Usually, the composite configuration is the combination of two or three kinds of BCCs defined above.

\subsubsection{Statistical analysis of BUFs and BCCs}

BUFs and BCCs in Optic Valley Area are analyzed statistically in this subsection. A summary is made after the analysis. Three BUFs (square form, rectangular form and ' $\mathrm{T}$ ' form) and three BCCs (scattered configuration, linear configuration and curvilinear configuration) are selected for study in this research.

According to the statistical analysis of BUFs (Figure 21), ' $\mathrm{T}$ ' form (F03) and rectangular form (F02) are the most of all, which are much more than the other forms in the area. 'T'-form buildings are more than rectangular-form buildings. Number of buildings with irregular form comes third (F11), which is much less than ' $\mathrm{T}$ '-form and rectangular-form buildings. Square 
form comes fourth (F01), flog form comes fifth (F04) and ' $\mathrm{V}$ ' form comes sixth (F09). Numbers of the four BUFs (irregular form, square form, flog form and ' $\mathrm{V}$ ' form) are relatively close. ' $\mathrm{Y}$ ' form comes seventh (F07) and cross form comes eighth (F06), which are much less than the former four BUFs. The ninth and the tenth are circular form (F10) and ' $\mathrm{H}$ ' form (F08) respectively. There are very few circular-form and ' $\mathrm{H}$ '-form buildings. Hash form comes eleventh (F05). There are the least number of hash-form buildings in the area.

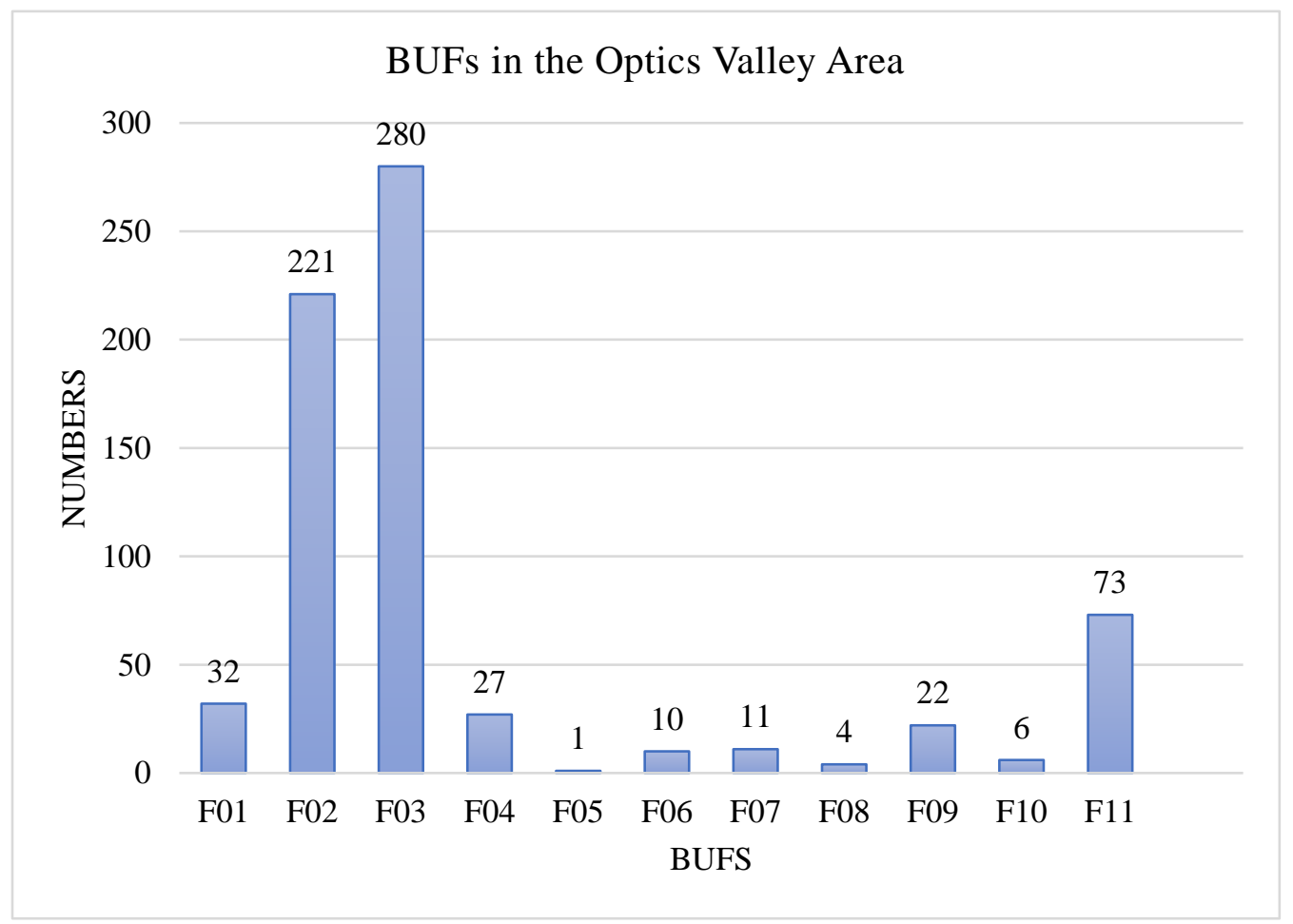

Figure 21. The comparison of numbers of 11 BUFs (codes: F01-F11) in the Optics Valley Area.

According to the statistical analysis of BCCs, differences among numbers of the four BCCs are much less than the BUFs (Figure 22). There is the greatest number of linear configuration (C02) in the area. Scattered configuration (C01) comes second and its number is $7.5 \%$ less than linear configuration. Composite configuration (C04) comes third, which is $14.5 \%$ less than scattered configuration. Though the number of curvilinear configuration is the least (C03) in the area, it is nearly $30 \%$ of the number of linear configuration. There are still a lot of curvilinear configurations in the area, especially in the new-built residential communities. 


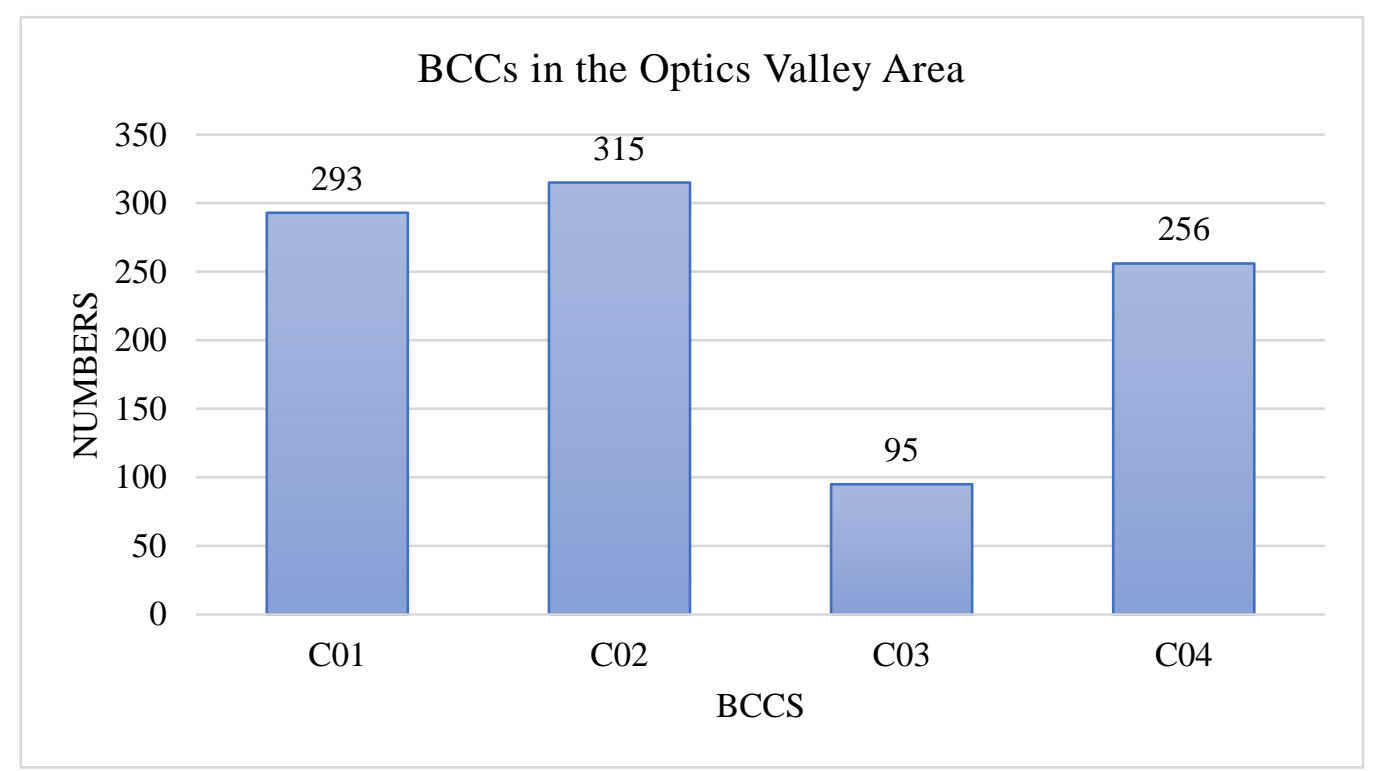

Figure 22. The comparison of numbers of 4 BCCs (codes: C01-C04) in the Optics Valley Area.

Therefore, representative BUFs and BCCs in the area are selected for study in this research. For BUFs, square form, rectangular form and ' $\mathrm{T}$ ' form will be studied. ' $\mathrm{T}$ ' form and rectangular form are the most representative BUFs in the area, as their numbers are the most. Square form can be thought as a special rectangular form. For BCCs, scattered configuration, linear configuration and curvilinear configuration will be studied. The differences among the 4 BCCs are relatively small. Though curvilinear configuration comes fourth, it is popular in new-built residential communities. According to site investigations, around $90 \%$ buildings with curvilinear configuration are built after year 2000. Composite configuration will not be studied in this research as it is a combination of the other BCCs.

The representative ranges of common building variables of the three BUFs are summarized according to previous studies and collected building information. For square form, the edge length is in the range of $10-30 \mathrm{~m}$. For rectangular form, the length is in the range of 10$40 \mathrm{~m}$; the width is in the range of $10-20 \mathrm{~m}$. For ' $\mathrm{T}$ ' form, the ranges of length and width are close to rectangular form; the length of bulge part is in the range of $6-10 \mathrm{~m}$ for balconies and $15-21 \mathrm{~m}$ for apartments; the width of bulge part is in the range of 6-18m for apartments. For all BUFs, the height is in the range of $36-72 \mathrm{~m}$, which is a typical height range for high-rise residential buildings with 12-36 floors.

The representative ranges of common building variables of the three BCCs are also 
summarized according to previous studies and collected building information. For scattered configuration, the number of building unit in a building row is in the range of 1-3; the number of building unit in a building column is in the range of 1-3; the transverse distance between the two adjacent building units is in the range of $10-20 \mathrm{~m}$. For linear configuration, the number of building unit in a building row is in the range of 2-4; and the number of building row is the same as scattered configuration. For curvilinear configuration, the arc angle of a curvilinear building row is in the range of 10-90 degree. For all BCCs, the longitudinal distance between adjacent building rows (units) is in the range of $0.7 \mathrm{H}-1.1 \mathrm{H}$, which is required by architectural standards. 'H' stands for the building height.

\section{2. $\quad$ Parametric modelling}

This section aims to develop the parametric models based on the building categorization study. In parametric modelling, scripts and parameters are used to create building models. Unlike conventional modelling, it is not necessary to draw lines or surfaces one by one to create a model. Instead, scripts are created based on different algorithms to link every parameter logically for modelling generation. Parametric models can be modified easily following the adjustment of parameters. The crucial advantage can highly improve modelling efficiency, and enable iterative analysis. In this section, modelling strategies are discussed and modelling logic are explained. A parametric design system is established to connect building objects and simulations.

\subsubsection{Parametric modelling of BUFs}

This subsection focuses on explaining parametric modelling of BUFs. Modelling scripts are developed in the environment of 'Grasshopper for Rhino' (GH), which is a parametric

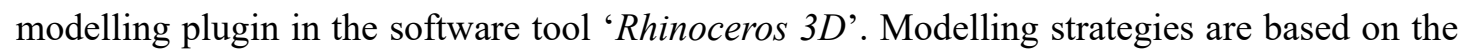
architectural essence, inheritance of conventional drawing expression and actual construction process. 


\section{(1) Modelling of square and rectangular form}

Buildings with square form and rectangular form are similar to the shapes of cuboids. A cuboid can be defined by using three parameters, which are length, width and height. For square form, the length and width of the cuboid are the same, which is a special case of rectangular form. There are many methods to create a cuboid in parametric modelling. The simplest way is to use a series of components related to 'Box' in the surface panel in GH. In this research, a different approach that considers architectural essence is implemented. The building models are created from their planes and facades. A floor-plane surface is created based on several control points first and then the height is defined to generate the entire building. The method is efficient in adjustment of the building model by changing the parameters. And there are great potentials to further modify the modelling script for the other BUFs.

Four points are constructed to define the rectangular surface of floor plane. Each point needs three parameters of $\{x, y, z\}$ coordinates for definition. In this study, the width and length of the rectangular plane are used as the original parameters to develop the coordinates based on scripts with mathematical logic behind it. The height is used as the original parameter to decide the height of the building. The value of $\{x\}$ coordinates is calculated as half of the width; the value of $\{y\}$ coordinates is calculated as half of the length. In this way, the model is created at the original point in the special coordinate system (Figure 23) and the surface edges are created by connecting the points. Then the rectangular floor plane is formed using 'Boundary Surfaces' component based on boundary edge collections. In the end, the 'Extrude' component is used to create the building with the parameter of height. Because the building is defined by the original parameters of width, length and height, it is convenient to adjust the model according to simulation requirements. Moreover, it is able to adjust the details of the model in a relatively large extent, as the building plane is created by constructing points. After parametric modelling of the building, the simulation boundaries are created by using the box component in GH. Three parameters are used to control the size of box in $\{x, y, z\}$ directions. The size of the simulation boundaries is decided according to the Best Practice Guideline COST 732 (Frank, Hellsten, Schlünzen, \& Carissimo, 2007). Then the script with moving function is used to move the boundaries to a suitable position. In the modelling process, scripts with specific functions are 
collected as different functional modules. These functional modules can be used in the other parametric modelling processes.

In summary, there are mainly six original parameters in the parametric modelling of square form and rectangular form, including two groups of widths, lengths and heights. The building model is defined by one group of width, length and height. The simulation boundaries are defined by another group. For the remaining parameters, there are six auxiliary parameters for defining position points of the building model and the boundaries. They only need minor adjustment in different situations after the setup. (Figure 24)

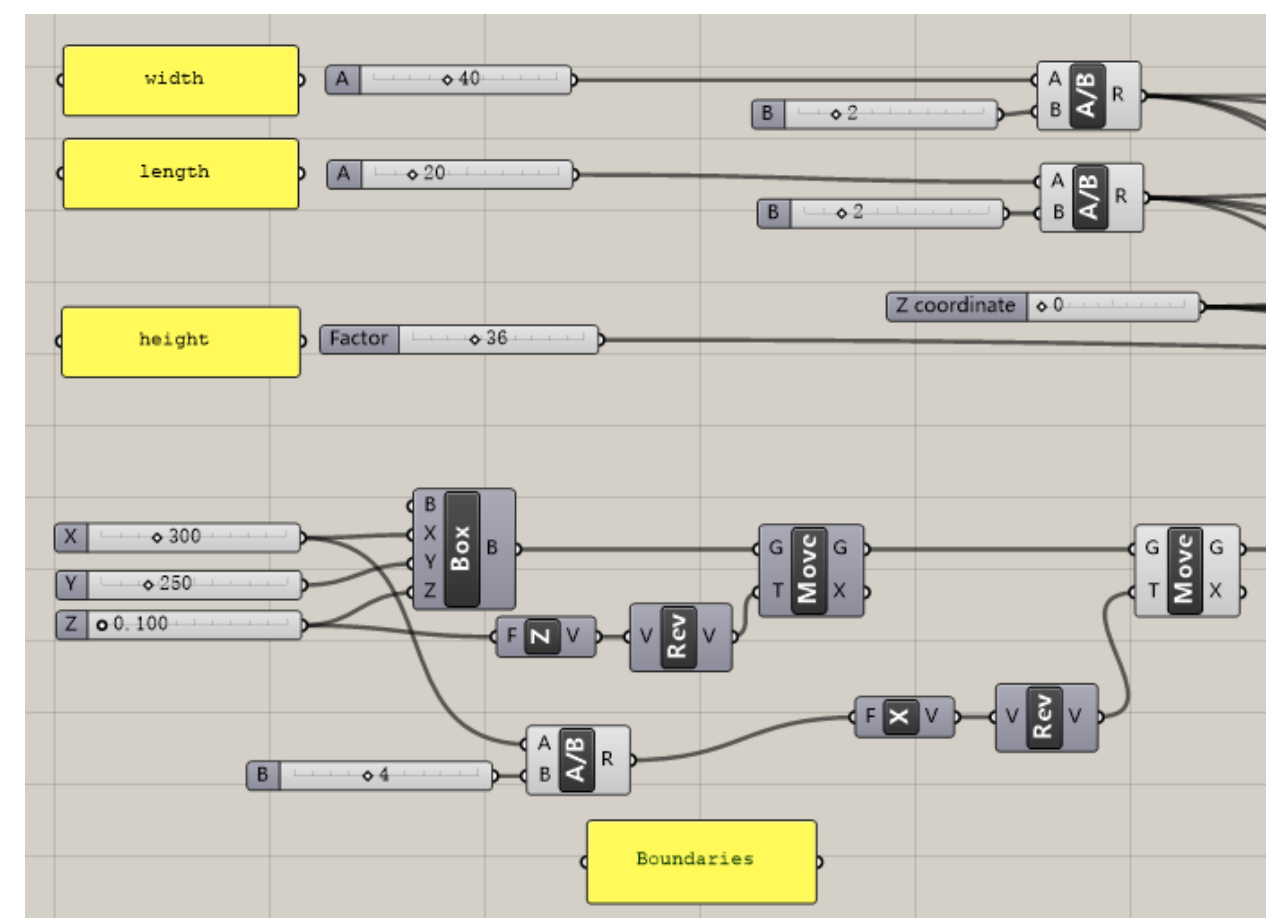

Figure 23. The parameters set in the parametric modelling of square and rectangular form.

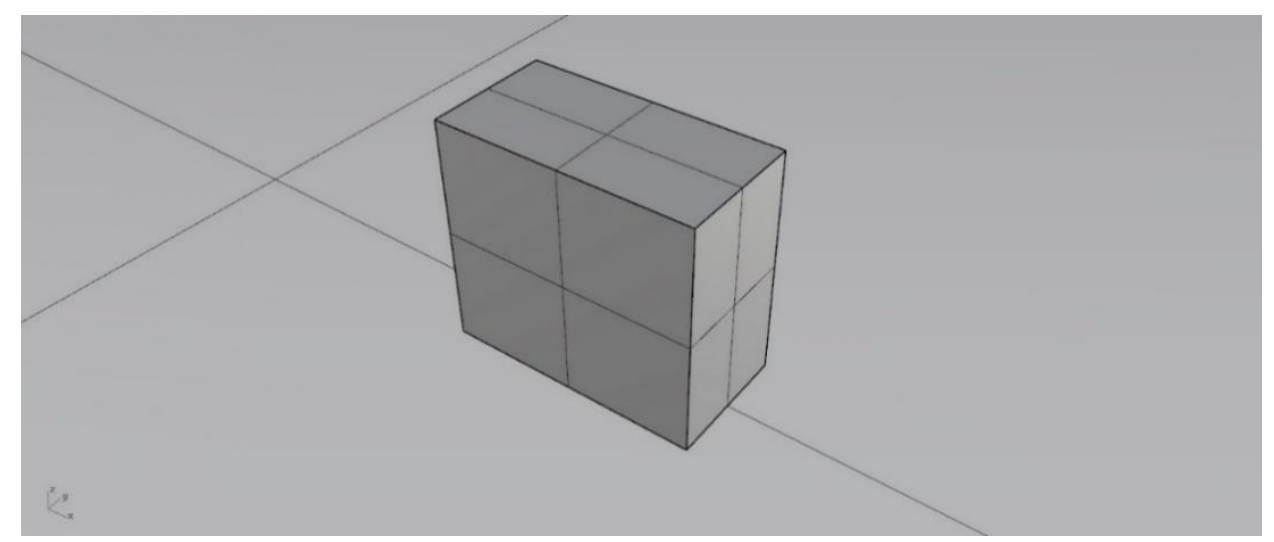

Figure 24. The parametric model of rectangular form. 


\section{(2) Modelling of ' $T$ ' form}

A ' $\mathrm{T}$ '-form building can be thought as a combination of one major part with a relatively large cuboid shape and one bulge part with a relatively small cuboid shape. There are several modelling methods to create ' $\mathrm{T}$ '-form buildings. As mentioned above, a cuboid can be defined by using three parameters of length, width and height. Then a ' $T$ '-form building model can be created by putting two cuboid volumes together. Another easy approach is to use the modelling modules of square and rectangular form. However, the two cuboid volumes are separate as their contact surfaces are not joint. This may cause problems in both modelling and simulations, such as errors in the generation of computational meshes.

Therefore, a modelling strategy which is similar to the square and rectangular form is used. The creation of building model starts from the floor-plane surface and facades. The floor-plane surface is created based on several points first and then the height is defined to generate the entire building. Totally eight points are constructed to define the floor-plane surface of ' $\mathrm{T}$ ' form. First, four points are constructed to define a rectangular surface as the major part. Second, another four points are constructed to define a smaller rectangular surface as the bulge part. In the process, each point needs three parameters of $\{\mathrm{x}, \mathrm{y}, \mathrm{z}\}$ coordinates for definition. There are two groups of widths and lengths for defining the major part and bulge part respectively. They are the original parameters for developing coordinates by using scripts with mathematical logics (Figure 25). Positions of the two parts are also considered in the modelling. In the end, the 'Extrude' component is used to create the entire building model with the parameter of height. After parametric modelling of the building, the creation of simulation boundaries is the same as the parametric modelling of square and rectangular form.

There are five original parameters in the modelling of ' $T$ ' form, including length-a, width$a$, length-b, width-b and height. The major part of ' $T$ ' form is defined using the length-a, width$\mathrm{a}$ and height. The bulge part is defined using the length-b, width-b and height. The simulation boundaries are defined by using the other width, length and height. Similar to the parametric modelling of square and rectangular form, there are the other six auxiliary parameters. (Figure 26) 


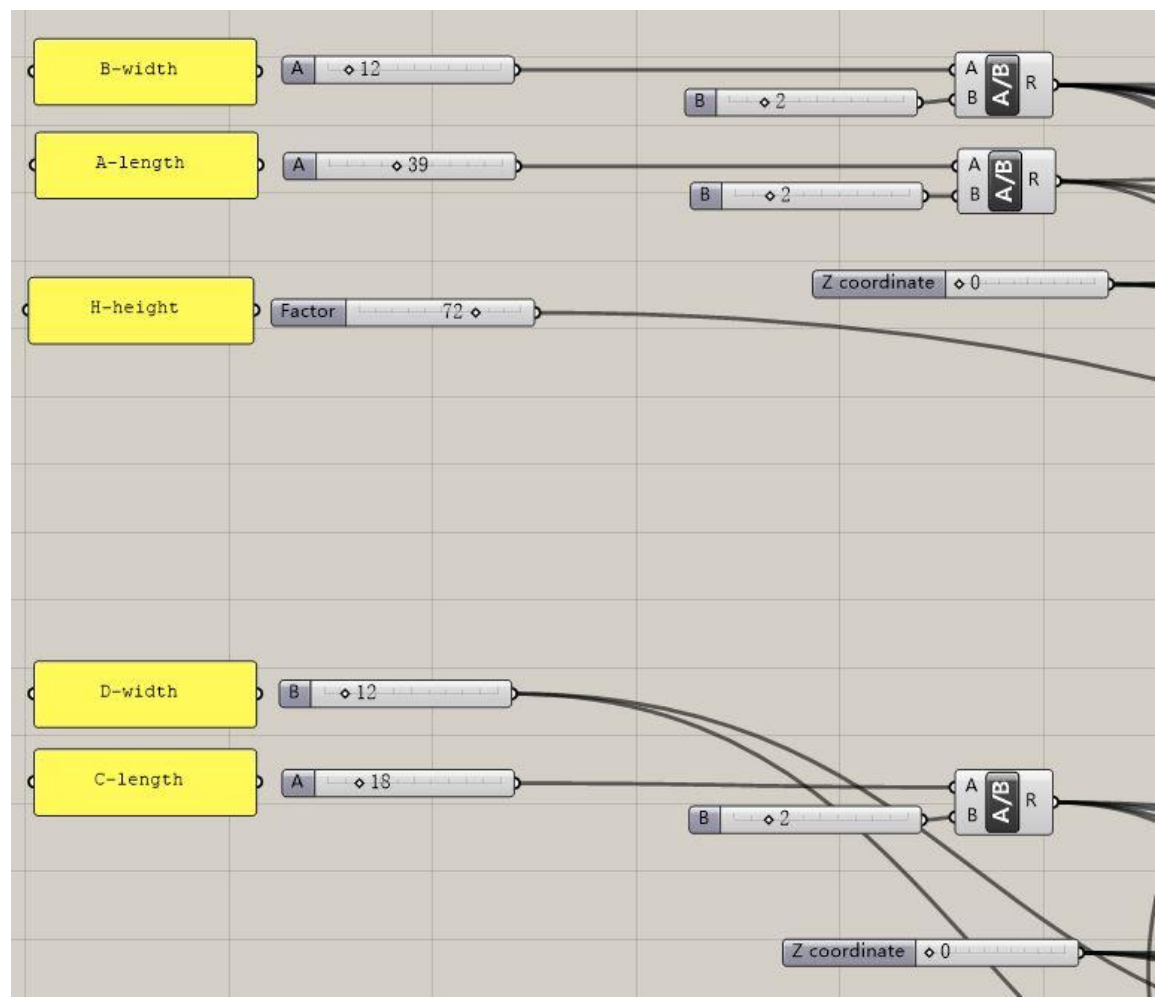

Figure 25. The parameters set in the parametric modelling of ' $T$ ' form.

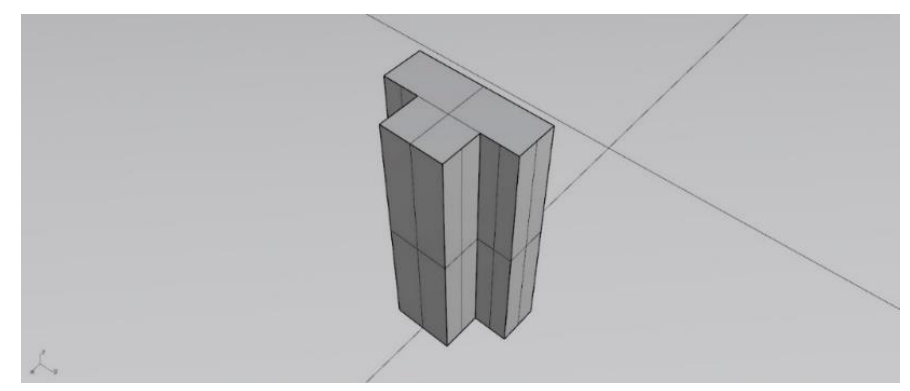

Figure 26. The parametric model of ' $T$ ' form.

\subsubsection{Parametric modelling of BCCs}

Parametric modelling of BCCs is explained in this subsection, including scattered configuration, linear configuration and curvilinear configuration. The modelling scripts of BCCs are also developed in the environment of GH. The modelling strategy is the same as BUFs.

\section{(1) Modelling of scattered configuration}

In the parametric modelling of scattered configuration, building-unit models are created first. 
The parametric modelling script of square and rectangular form is used on this stage. The modelling modules of building unit are created first. The modelling script of scattered configuration is created with the modelling modules of building unit. The relationship and positions of building units are arranged by the script.

There are totally five parameters in the parametric modelling script of scattered configuration, including transverse distance, longitudinal distance, number of columns, number of rows and staggered distance (Figure 27). In this research, transverse distance is used to define the interval between two adjacent building units in the transverse direction, which is usually perpendicular to the windward direction; longitudinal distance is used to define the distance between two adjacent building units in the longitudinal direction, which is usually parallel with the windward direction. In the parametric modelling script, the longitudinal distance is relevant to the height of the building unit with a mathematical multiplication relation. Number of columns is used to define the number of building units in a building row in the transverse direction. Number of rows is used to define the number of building units in a building column in the longitudinal direction. Staggered distance is used to define the transverse distance between two adjacent building rows in longitudinal direction for staggered scattered configuration. In the modelling script, the number slider components are used to set up values of these parameters.

In the modelling scripts, there are two linear-array components. The first linear-array component is used to create an array with several building units in the transverse direction to form a building row. Then the modelling module of building unit is linked to the linear array component as the 'base geometry'. The direction and gap of the array are defined by a vector which is parallel to $\{y\}$ axis. The value of the vector is the sum of building length and transverse distance. The number of elements in the array is defined by the number of columns. The second linear array component is used to create an array of several building rows in the longitudinal direction to form the scatter-configuration cluster. The building row created by the first linear array component is linked to the second linear array component as the 'base geometry'. Direction and gap of the linear array are defined by a vector created based on the sum of two vector products from two multiplications. The first product comes from the multiplication of the vector unit which is parallel to $\{\mathrm{y}\}$ axis and the staggered distance. The second product 
comes from the multiplication of the vector unit which is paralleled to $\{\mathrm{x}\}$ axis and the sum of building width and longitudinal distance. The number of elements in the array is defined by the number of rows. (Figure 28)

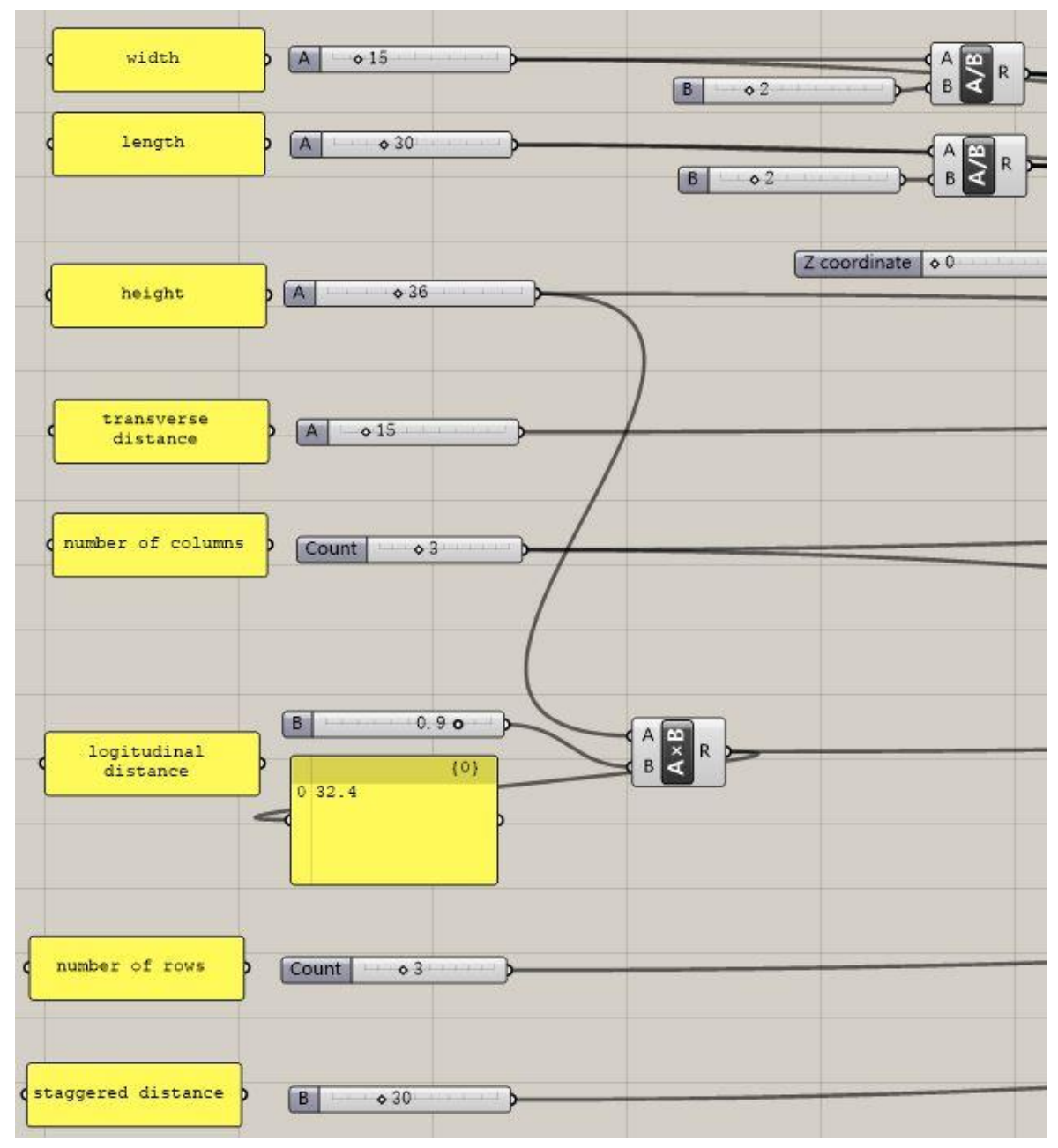

Figure 27. The parameters set in the parametric modelling of scattered configuration.

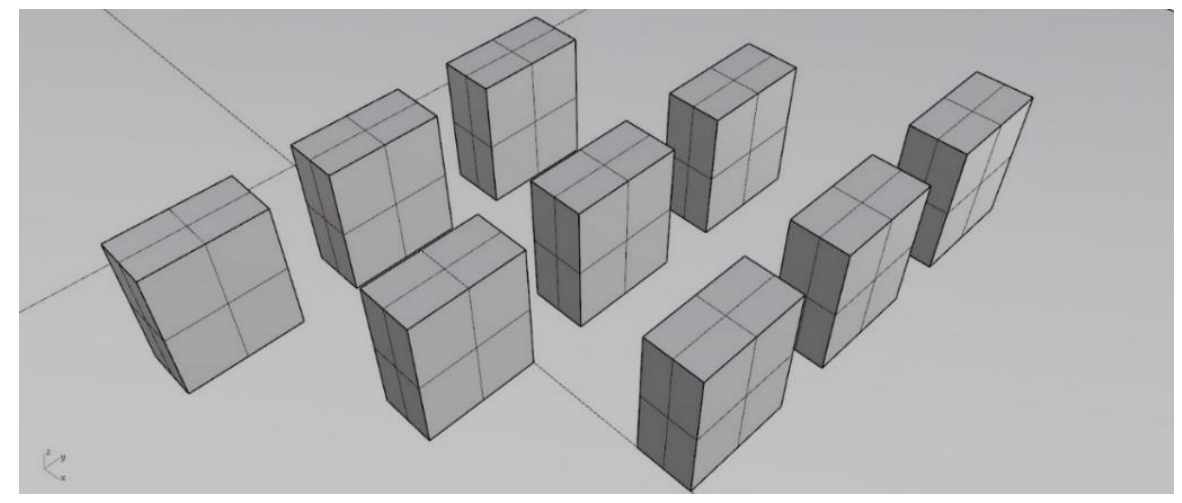

Figure 28. The parametric model of scattered configuration. 
The creation of simulation boundaries is the same as the parametric modelling of BUFs. In the modelling process, scripts with specific functions are collected as different functional modules. These functional modules can be used in the further parametric modelling process.

\section{(2) Modelling of linear configuration}

The parametric modelling script of linear configuration can be done by modifying the scripts of scattered configuration. Similar to scattered configuration, building-unit models are created first. Then the relationship and positions of building units are arranged by the script (Figure 29). In linear configuration, building units are connected one by one to form a linear building row. The linear configuration can be thought as a special case of scattered configuration with a transverse distance of $0 \mathrm{~m}$. Therefore, the parametric model of linear configuration can be created by modifying the parametric modelling script of scattered configuration with the transverse distance of $0 \mathrm{~m}$. Only minor modifications of the script and parameters are needed. The previous functional script modules are used in the parametric modelling. The modelling efficiency is promoted because of the modification. The creation of simulation boundaries is the same as the parametric modelling of scattered configuration.

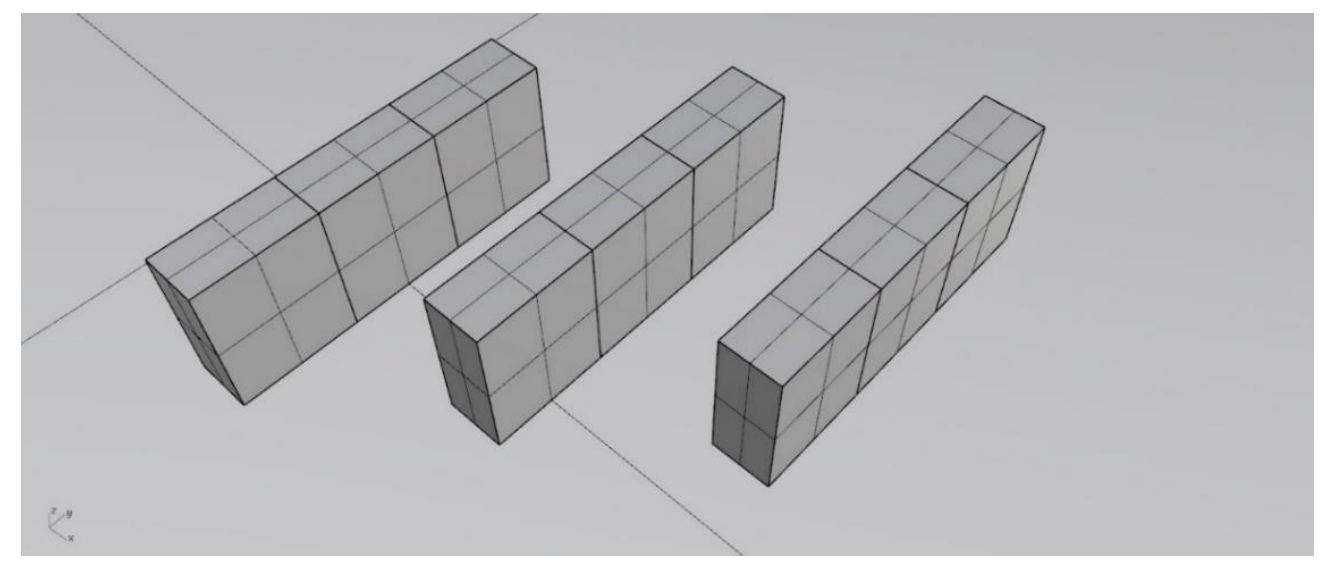

Figure 29. The parametric model of linear configuration.

\section{(3) Modelling of curvilinear configuration}

In curvilinear configuration, building units are connected one by one to follow a curve. In the parametric modelling of curvilinear configuration, the connected building units are simplified 
as a unity. There are totally four initial parameters of a curvilinear building row, including the height, width, length and angle. The length is the arc length of the curvilinear building row. And the central angle defines the curvature of the curvilinear building row (Figure 30). The arc of the curvilinear configuration is created first by using the arc component in GH. In order to study influences on wind environment of different central angles (curvatures) of the curvilinear building row, the arc length is fixed to a setting length no matter how the central angle is changed. There are three input parameters of the arc component, including the base plane, radius of arc and angle domain in radians. The base plane of the arc is set to be the XY plane. The radius of arc is set to be the division result of the arc length and radian. The angle domain is set to be the central angle, which is the product of multiplication of Pi and the division result of the angle and 180 degree. After that, an offset component is used to create another edge of the curvilinear building row with the parameter of building width. Then the floor-plane surface is generated by using an edge surface component. The floor-plane surface is extruded to create the model of the curvilinear building row. In the end, the building model is moved and rotated based on the reference point (Figure 31). The creation of simulation boundaries is the same as the parametric modelling of scattered configuration and linear configuration.

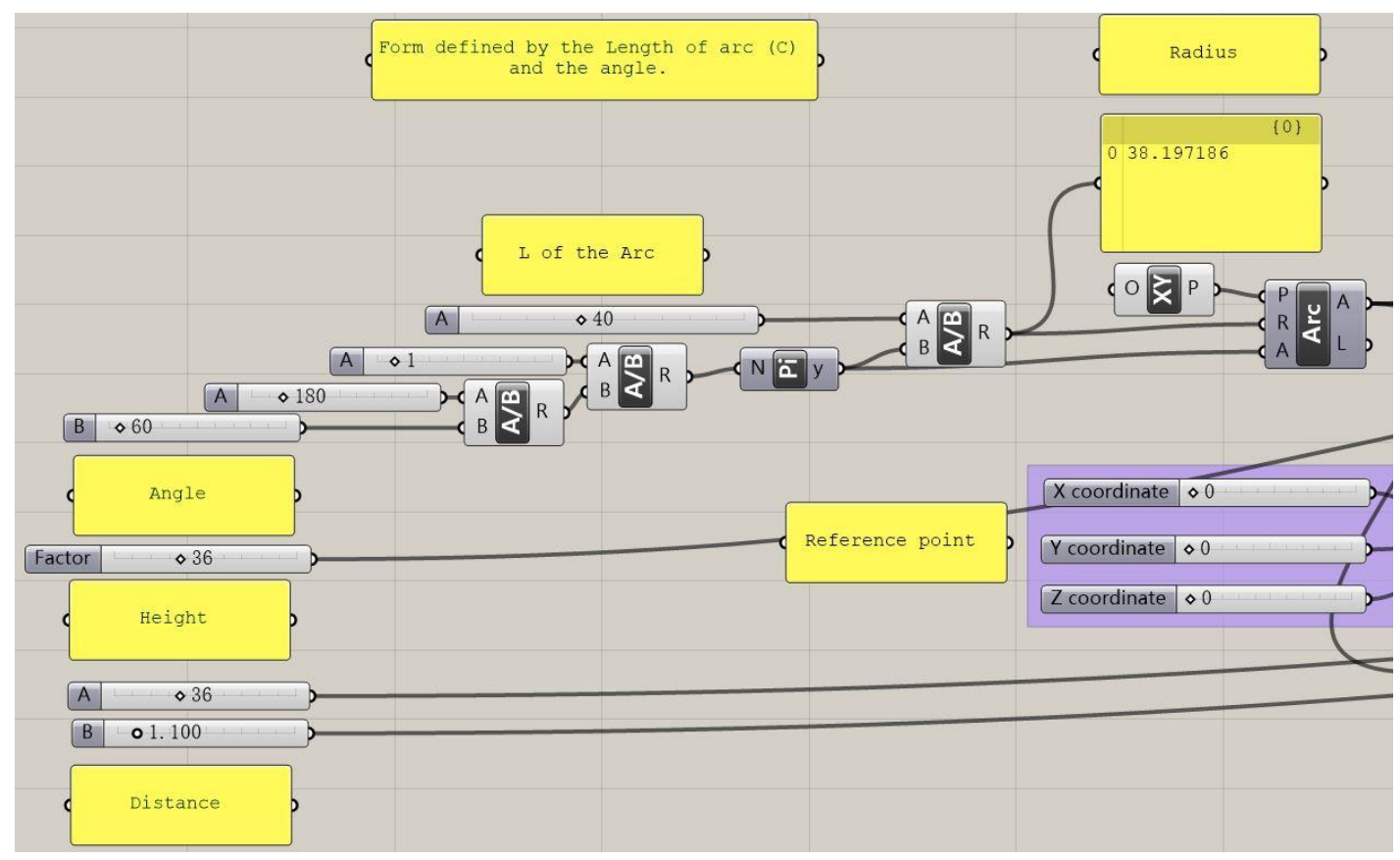

Figure 30. The parameters set in the parametric modelling of curvilinear configuration. 


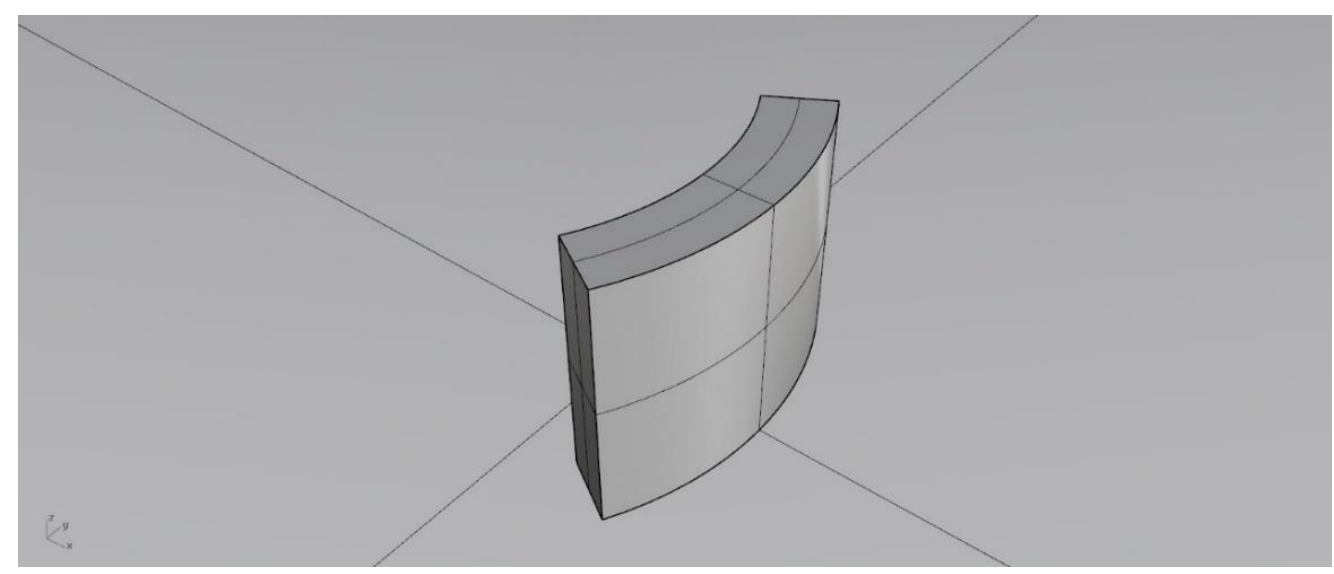

Figure 31. The parametric model of curvilinear configuration.

\subsubsection{Comparison of parametric modelling and manual modelling}

The parametric modelling helps in high-efficient iterative analysis as there are huge number of cases. Assume the time of creating each parametric model (Tp) as 30 minutes; assume the times of creating each manual model $(\mathrm{Tm})$ as 5 minutes. The figure below shows the comparison of time of parametric modelling and time of manual modelling for each case (Figure 32). In this research, there are at least 24 cases in studies of BUFs and 51 cases in studies of BCCs; there are approximately 75 cases in total. Then, the total times of parametric and manual modelling for the cases can be calculated and compared, as shown in the figure below (Figure 33). The parametric method saves approximately half in time than manual modelling. If there are more cases, the advantage could be more significant.

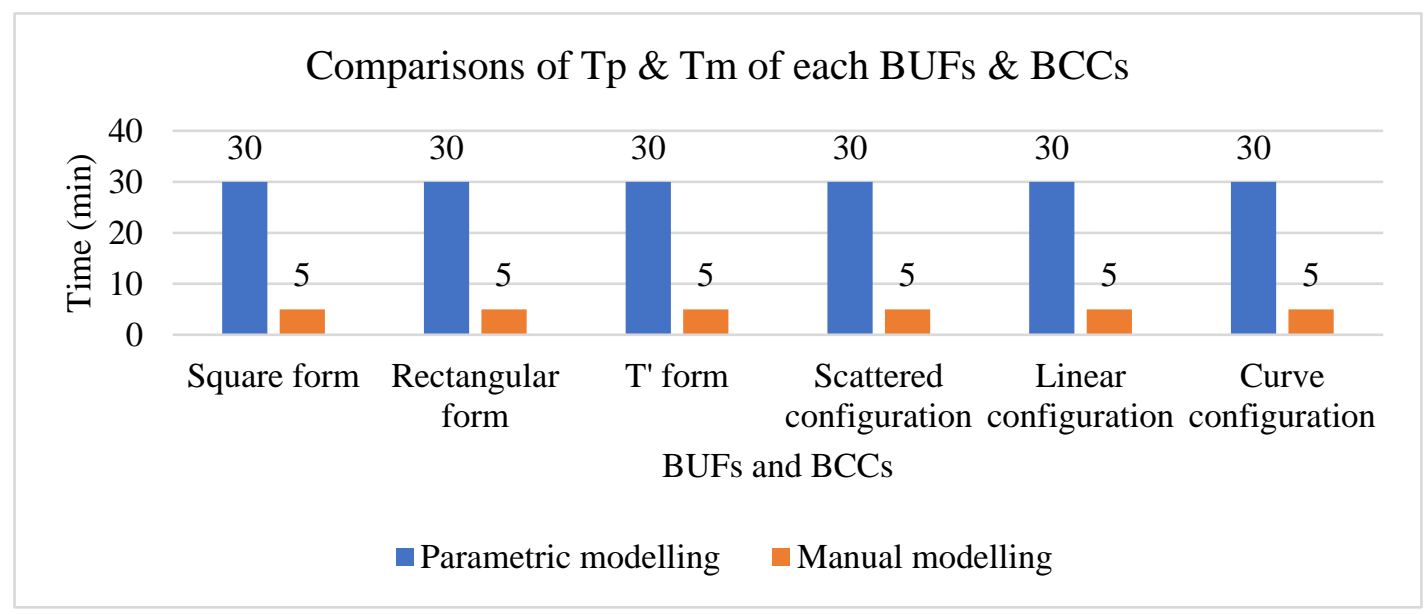

Figure 32. The comparison of times of creating each single parametric and manual models. 


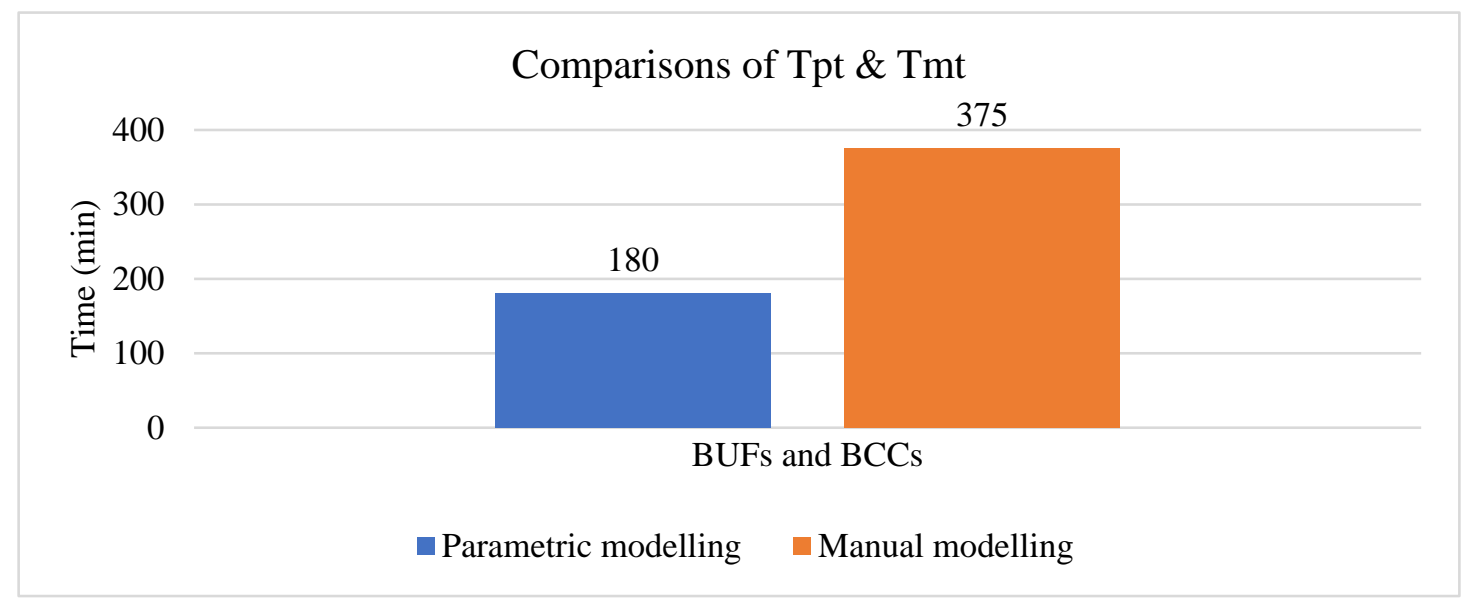

Figure 33. The comparison of total times of parametric modelling and manual modelling.

\subsection{Summary of parametric design}

This chapter has designed a series of representative forms and configurations of high-rise residential buildings in the HSCWA for CFD simulations by establishing parametric design systems based on the building categorization study.

In the building categorization study, definitions of eleven BUFs and four BCCs have been given according to previous studies, site investigations and their geometric attributes. And the numbers of the BUFs and BCCs in the area have been calculated statistically. The BUFs of square form, rectangular form, ' $\mathrm{T}$ ' form and the $\mathrm{BCCs}$ of scattered configuration, linear configuration, curvilinear configuration are selected for this research due to their representativeness. The representative ranges of common building variables of the three BUFs and three BCCs are summarized according to previous studies and the collected building information in the end of the section of 'Statistical analysis of BUFs and BCCs'.

In the parametric modelling, scripts of the representative BUFs and BCCs are developed based on their geometric attributes. The scripts can generate the building models automatically, which largely improves the efficiency of mesh generations for CFD simulations. The parametric models can be adjusted efficiently as the parameters are changed, which enables iterative analysis to study the influences of different building variables. 


\section{Wind environment studies of BUFs}

The overall goal of this chapter is to study the relationships between the influences on wind environments and the building variables of the three BUFs, including square form, rectangular form and ' $\mathrm{T}$ ' form. The chapter focuses on wind environments of the representative BUFs which have been summarized in the parametric design chapter. The research methodology explained in the Third Chapter is used in the studies of BUFs. The CFD simulations, parametric tools and assessment criteria are used in the studies. Influenced areas with different windvelocity magnitudes are compared. The mechanisms are explained by using air pressure magnitudes and wind flow streamlines. An overview of wind environments of BUFs is presented first (Section 5.1.). The specific building variables, results and analysis of the three BUFs are presented in the following three sections (Section 5.2., Section 5.3. and Section 5.4.) respectively. The summary in the end (Section 5.5) summarizes the studies of BUFs briefly.

\subsection{Overview of BUF wind environments}

The overview aims to describe the attributes of wind environments of the three BUFs by analyzing the wind-velocity magnitudes, air-pressure magnitudes and wind-flow streamlines.

According to wind velocity magnitudes on the vertical plane and horizontal plane on the pedestrian level, the wind velocities decline as winds approach to the windward surfaces of the buildings (Figure 34, Figure 35 and Figure 36). The velocities drop from approximately $1.30 \mathrm{~m} / \mathrm{s}$ to $0.50 \mathrm{~m} / \mathrm{s}$ in front of the windward side. As winds flow around the buildings, there are usually two high-velocity areas on the lateral sides of the buildings. The wind velocities in the areas are around $1.50 \mathrm{~m} / \mathrm{s}$ in this research. There is a relatively large low-velocity area behind the leeward side of the building, which is also known as the wind-shadow area (Krishan \& Arvind, 2001). The velocities in the area are in the range of $0.00-0.05 \mathrm{~m} / \mathrm{s}$ in this research. 


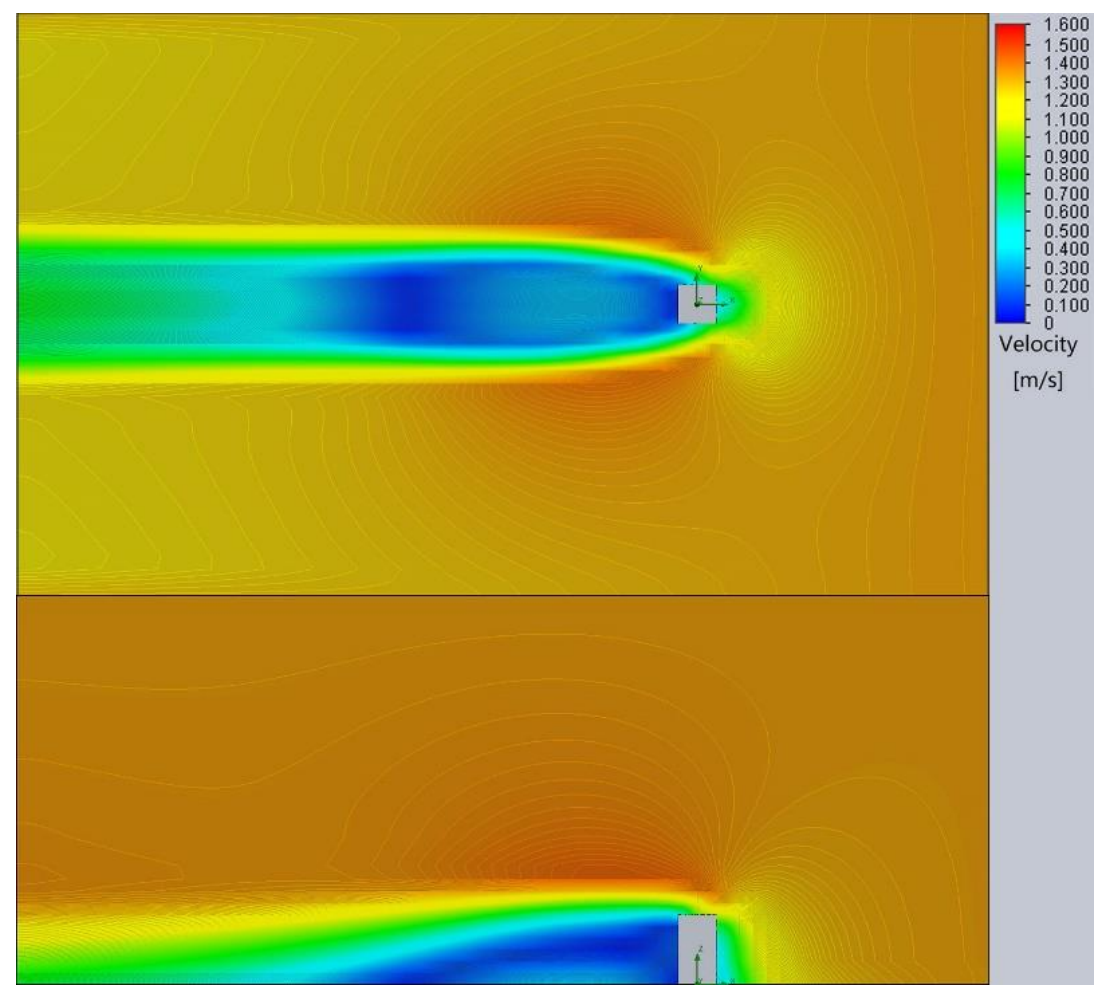

Figure 34. An example of the wind velocity magnitudes on horizontal (1.5m pedestrian level) and vertical planes of the square-form building with the size of $36 \mathrm{~m} * 20 \mathrm{~m} * 20 \mathrm{~m}$.

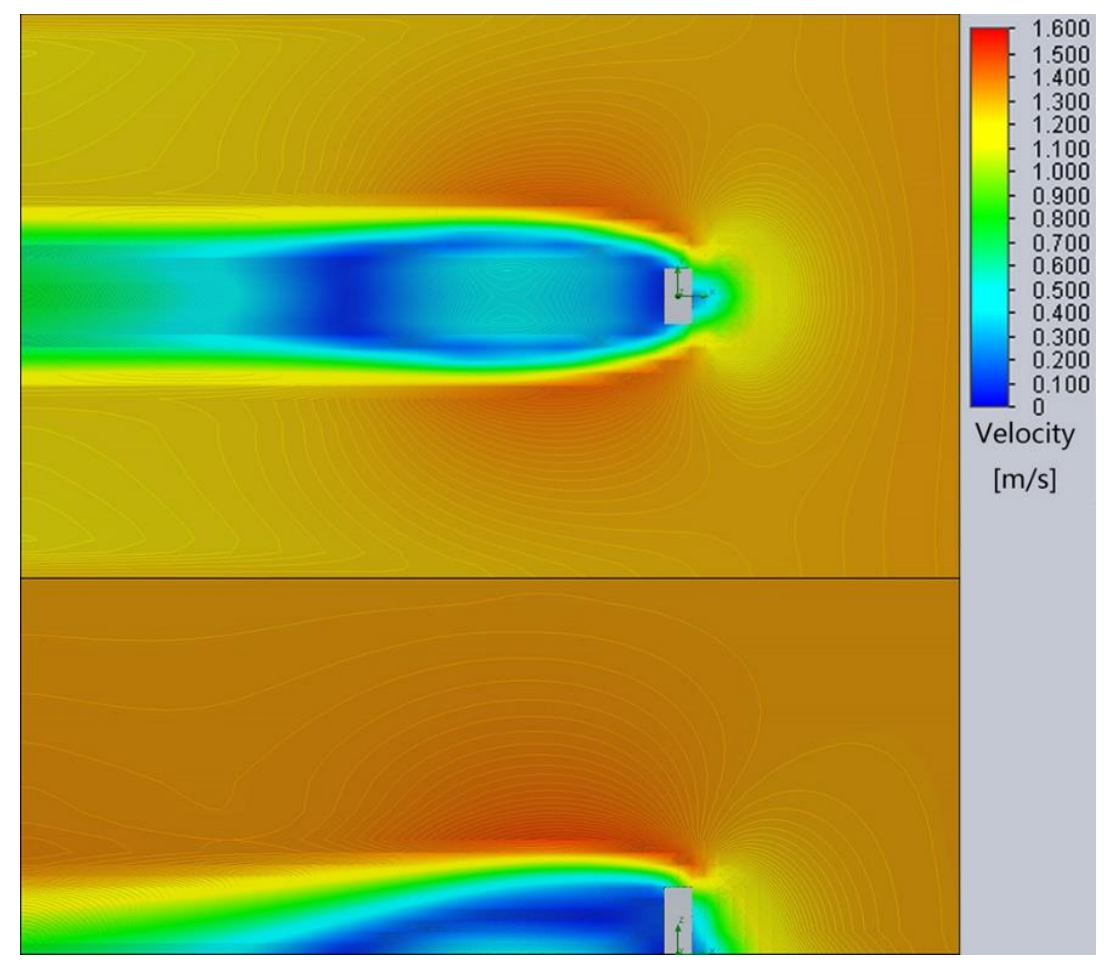

Figure 35 . An example of the wind velocity magnitudes on horizontal (1.5m pedestrian level) and vertical planes of the rectangular-form building with the size of $36 \mathrm{~m} * 15 \mathrm{~m} * 30 \mathrm{~m}$. 


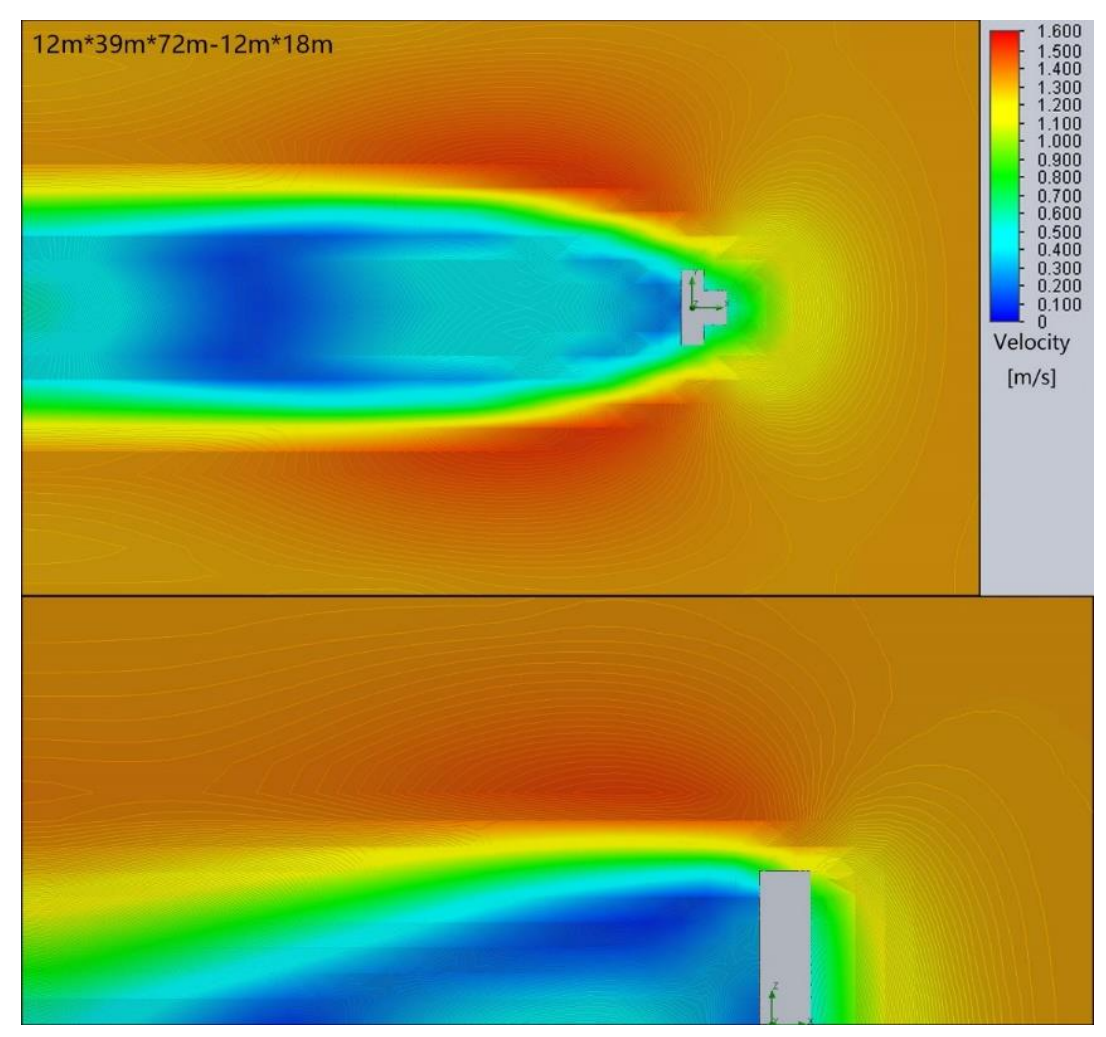

Figure 36 . An example of the wind velocity magnitudes on horizontal (1.5m pedestrian level) and vertical planes of the ' $\mathrm{T}$ '-form building with the major part of $12 \mathrm{~m} * 39 \mathrm{~m} * 72 \mathrm{~m}$ and bulge part of $12 \mathrm{~m} * 18 \mathrm{~m}$.

The air-pressure magnitudes show that the air pressures on the windward sides of the buildings are higher than the air pressures on the leeward sides. As shown in the magnitudes, there are two groups of contour lines of air pressures separated by the windward surfaces of the buildings (Figure 37, Figure 38 and Figure 39). They start from the windward edges of the buildings and go in both windward and leeward directions to surround the buildings with multiple layers in elliptical shapes. Air pressures start to increase from the original value in front of the windward surfaces of the buildings. The air pressures are the highest in the middle of the windward surfaces. The air pressures decline gradually from the middle areas to the edges of the windward surfaces. They continue decreasing on the lateral and top surfaces of the buildings. The air pressures are the lowest on leeward sides of the buildings. A relatively large low-air-pressure areas are created behind the buildings, which are in the similar sizes of the low-velocity areas. 


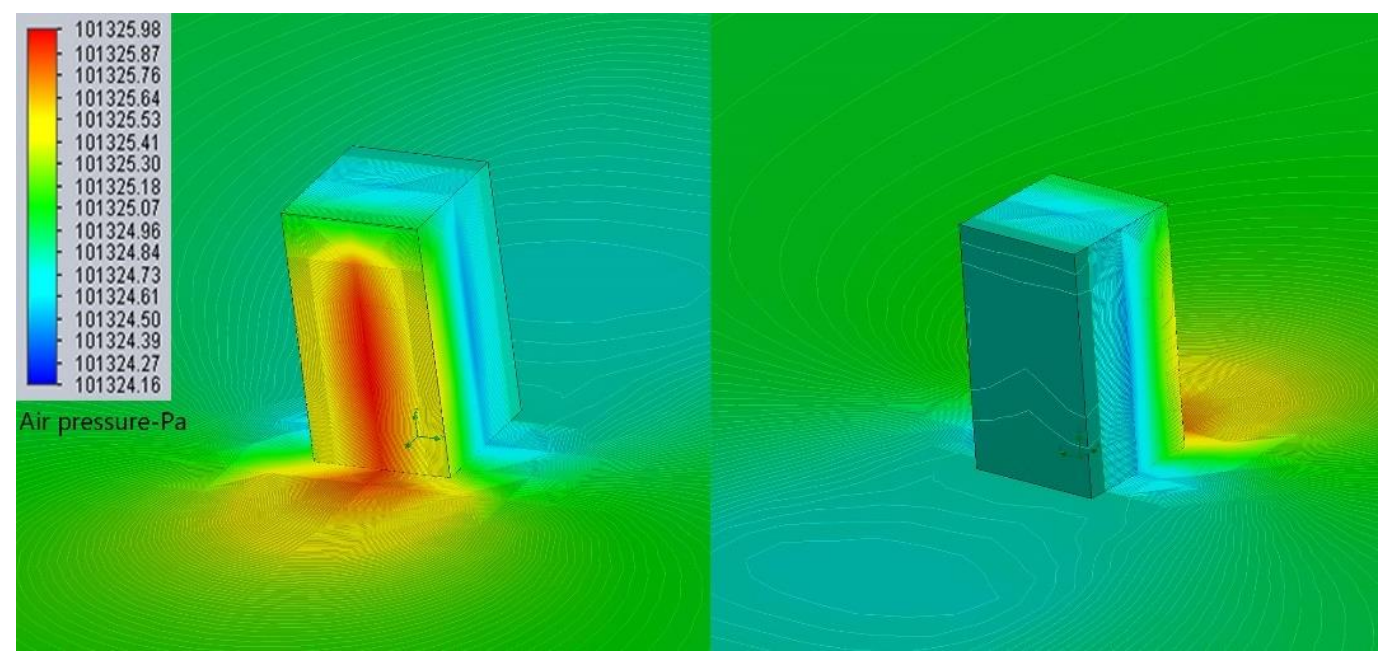

Figure 37. An example of the air-pressure magnitudes of the square-form building with the size of $36 \mathrm{~m} * 20 \mathrm{~m} * 20 \mathrm{~m}$.

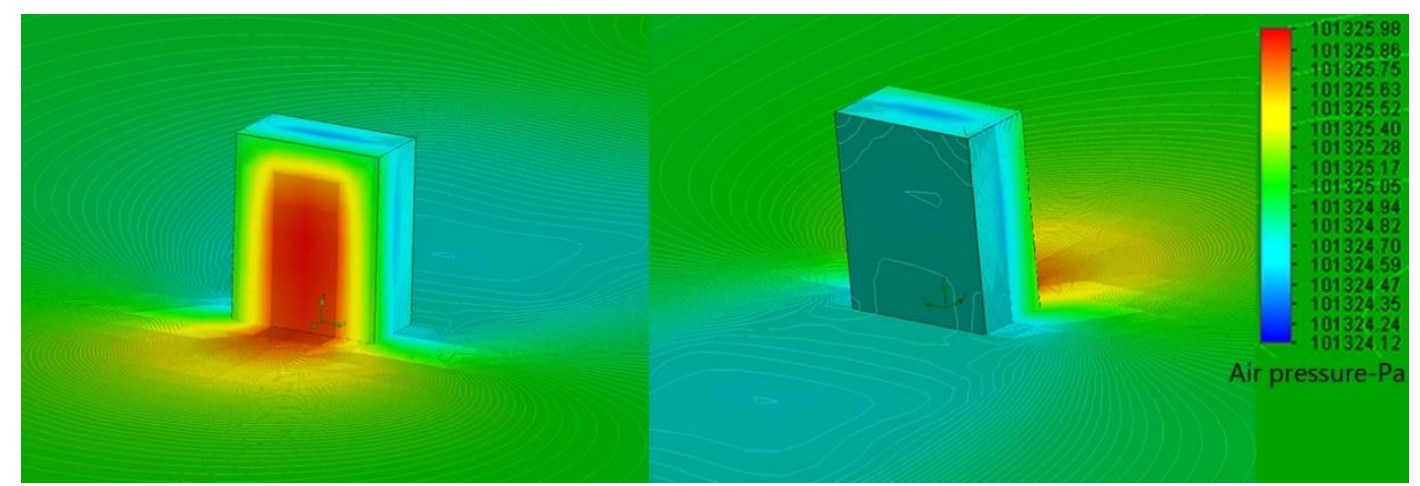

Figure 38. An example of the air-pressure magnitudes of the rectangular-form building with the size of $36 \mathrm{~m} * 15 \mathrm{~m} * 30 \mathrm{~m}$.

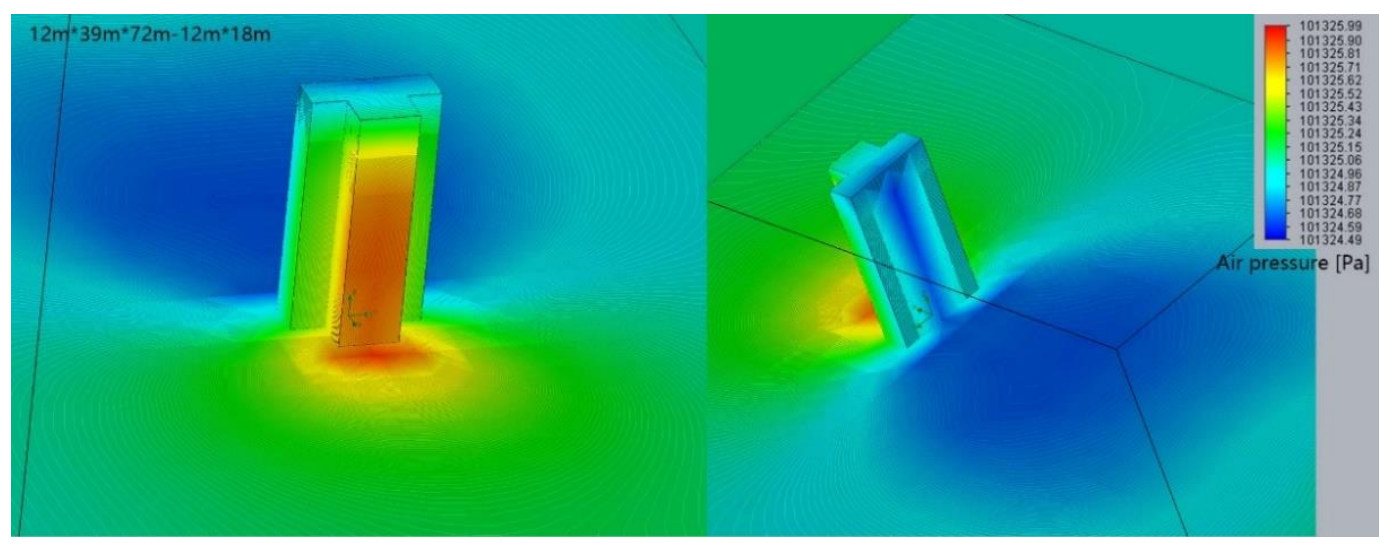

Figure 39. An example of the air-pressure magnitudes of the ' $T$ '-form building with the major part of $12 m * 39 m * 72 m$ and bulge part of $12 m * 18 m$. 
The wind flow streamlines can show how winds flow around the buildings (Figure 40, Figure 41 and Figure 42). After winds meet the buildings, they turn to the edges of the windward surfaces and escape at the lateral sides and top of the buildings. Then they flow into the lowair-pressure areas behind the leeward sides of the buildings. As the air pressures in the areas are lower than the surrounding areas, winds are drawn into the areas. Vortices are created in the low-air-pressure areas as winds flow several rounds behind the buildings. Meanwhile, their velocities decrease significantly in these processes. This is the reason for the low-velocity areas. After winds flow around the tops of the buildings, they descend gradually. Winds from different directions converge again behind the low-air-pressure areas of the buildings.

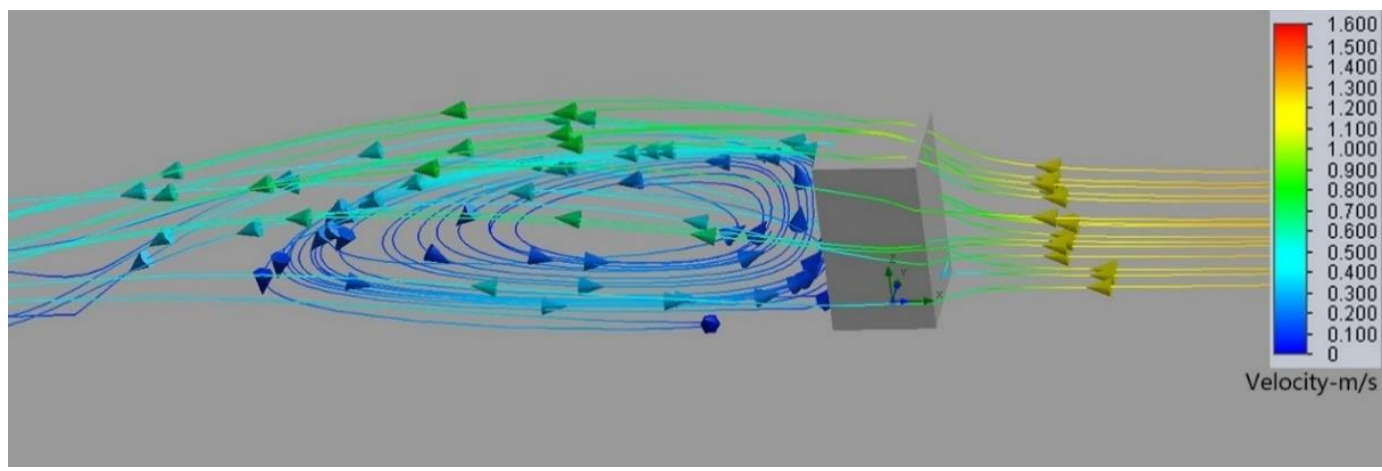

Figure 40. An example of the wind-flow streamlines of the square-form buildings with the size of $36 \mathrm{~m} * 20 \mathrm{~m} * 20 \mathrm{~m}$.

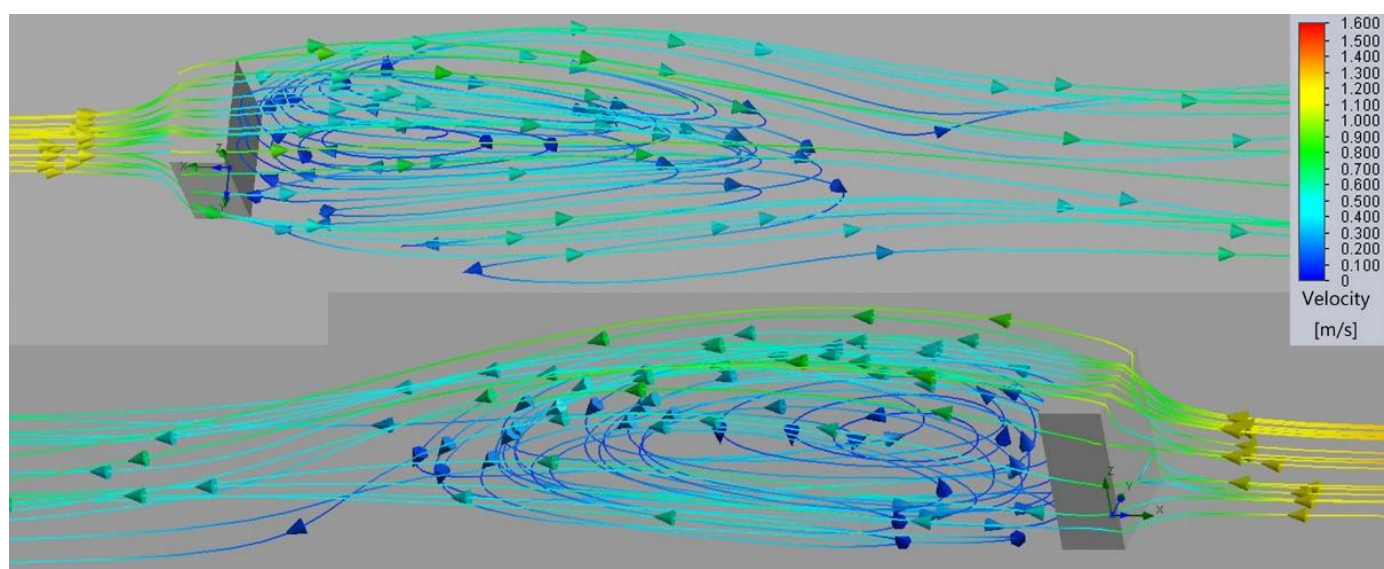

Figure 41. An example of the wind-flow streamlines of the rectangular-form building with the size of $36 \mathrm{~m} * 15 \mathrm{~m} * 30 \mathrm{~m}$. 


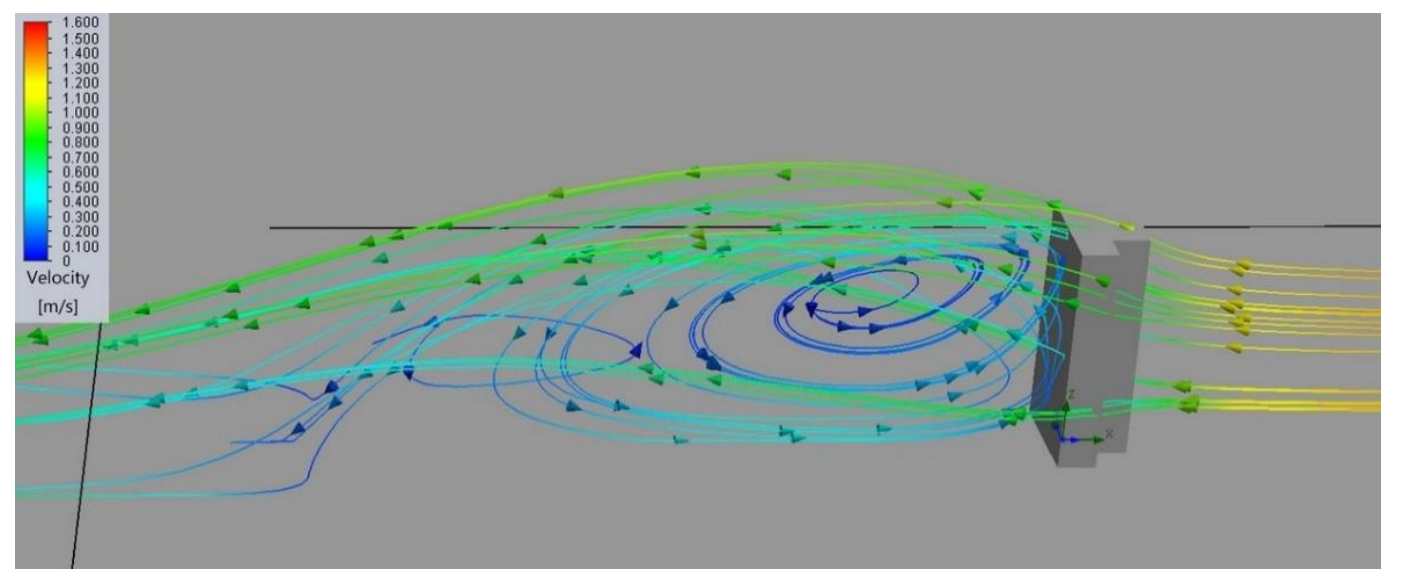

Figure 42. An example of the wind-flow streamlines of the ' $T$ '-form building with the major part of $12 m * 39 m * 72 m$ and bulge part of $12 m * 18 m$.

\subsection{Square form}

This section aims to study the relationships between the influences on wind environments and the building variables of square form, including the edge length and rotation angle (Figure 43). The influences of edge lengths are studied in the first part. The influences of rotation angles are studied in the second part. The building variables setup of the two parts are presented first. Then results and analysis of the two parts are shown.

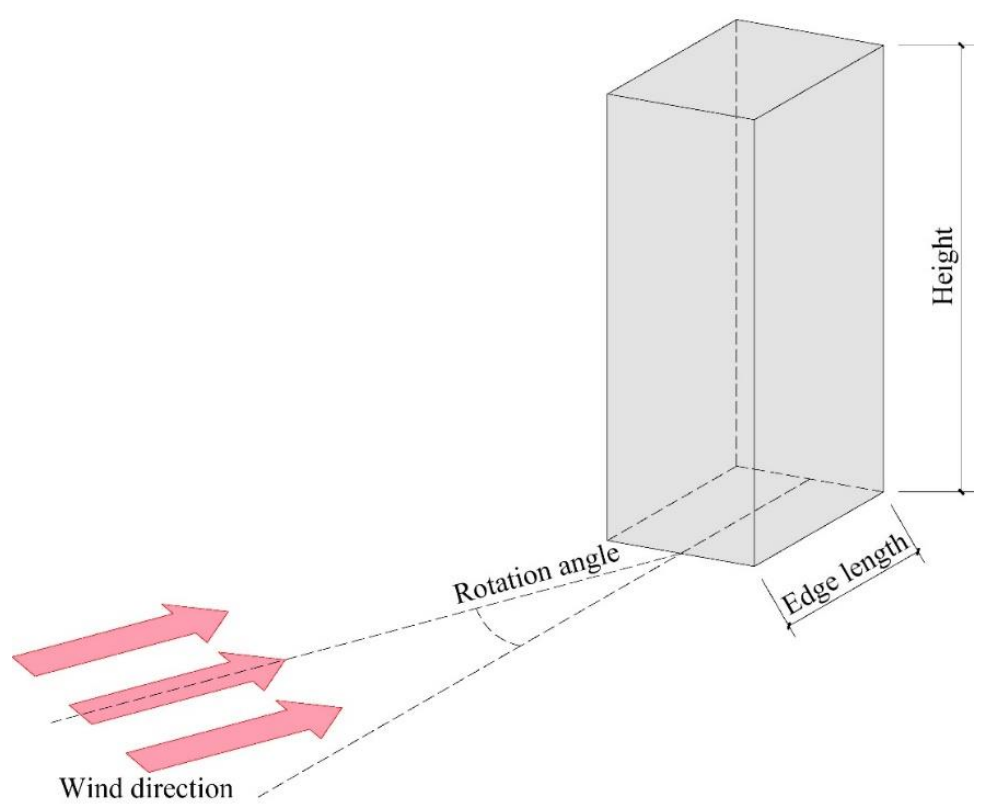

Figure 43. The building variables of square form. 


\subsubsection{Building variables setup of square form}

In the first part, three cases with different edge lengths (named as Group 1) are set up for simulations. According to the summary in the parametric design chapter, common edge lengths are used in the study, including $10 \mathrm{~m}, 20 \mathrm{~m}$ and $30 \mathrm{~m}$; the building height is set as $36 \mathrm{~m}$, which is a common height for a high-rise residential building with twelve floors (Table 7.).

Table 7. The three cases in Group 1 of square form.

\begin{tabular}{|l|l|l|}
\hline Cases & Lengths (of the edge) & Heights \\
\hline Case 1 & $10 \mathrm{~m}$ & $36 \mathrm{~m}$ \\
\hline Case 2 & $20 \mathrm{~m}$ & $36 \mathrm{~m}$ \\
\hline Case 3 & $30 \mathrm{~m}$ & $36 \mathrm{~m}$ \\
\hline
\end{tabular}

In the second part, four cases with different rotation angles (named as Group 2) are set up. The range of rotation angles is $0-45$ degree (Table 8.). This part aims to study the influences of different angles between the wind direction and windward surface of the building. According to the summary in the parametric design chapter, the edge length is set as $30 \mathrm{~m}$, which is a common edge length for a high-rise residential building with 4 or more apartments on each floor; the building height is set as $72 \mathrm{~m}$, which is a common height for a high-rise residential building with 24 floors.

Table 8. The four cases in Group 2 of square form.

\begin{tabular}{|l|l|l|l|}
\hline Cases & Lengths (of the edge) & Heights & Rotation angles \\
\hline Case 1 & $30 \mathrm{~m}$ & $72 \mathrm{~m}$ & 0 degree \\
\hline Case 2 & $30 \mathrm{~m}$ & $72 \mathrm{~m}$ & 15 degree \\
\hline Case 3 & $30 \mathrm{~m}$ & $72 \mathrm{~m}$ & 30 degree \\
\hline Case 4 & $30 \mathrm{~m}$ & $72 \mathrm{~m}$ & 45 degree \\
\hline
\end{tabular}


The parametric modelling script of square form is used to create the building models. In the above two groups, boundaries of CFD simulation results are all set in the same sizes with the width of $500 \mathrm{~m}$, length of $600 \mathrm{~m}$ and height of $200 \mathrm{~m}$ for comparisons of influenced areas.

\subsubsection{Results and analysis of square form}

The CFD simulation results of square form are analyzed in this subsection. Influences on wind environments are compared based on wind-velocity magnitudes. The mechanisms are explained using the air-pressure magnitudes and wind-flow streamlines. In Part 1, the relationship between influences and edge lengths is studied. In Part 2, the relationship between influences and rotation angles is studied.

\section{(1) Part 1: Influences of different edge lengths}

According to the simulation results of Group 1 (Figure 44), the building of Case 1 (edge length: $10 \mathrm{~m}$ ) has the smallest influence on wind environment; the building (edge length: 30m) of Case 3 has the most influence; the influence of Case 2 (edge length: 15m) is between Case 1 and Case 3 (Figure 45). In Case 1, the low-velocity area (velocity below $0.5 \mathrm{~m} / \mathrm{s}$ ) is the smallest and its length is the shortest; as the edge length increases, the low-velocity area increases in Case 2; in Case 3, the low-velocity area is the largest and its length is the longest. In Case 1, the highvelocity areas (velocity over $1.4 \mathrm{~m} / \mathrm{s}$ ) beside the lateral sides are the smallest; as the edge length increases, more winds are found accelerated due to the increase of dark orange areas in Case 2; in Case 3, the areas are the largest. In summary, the influence on wind environment increases as the edge length increases. 


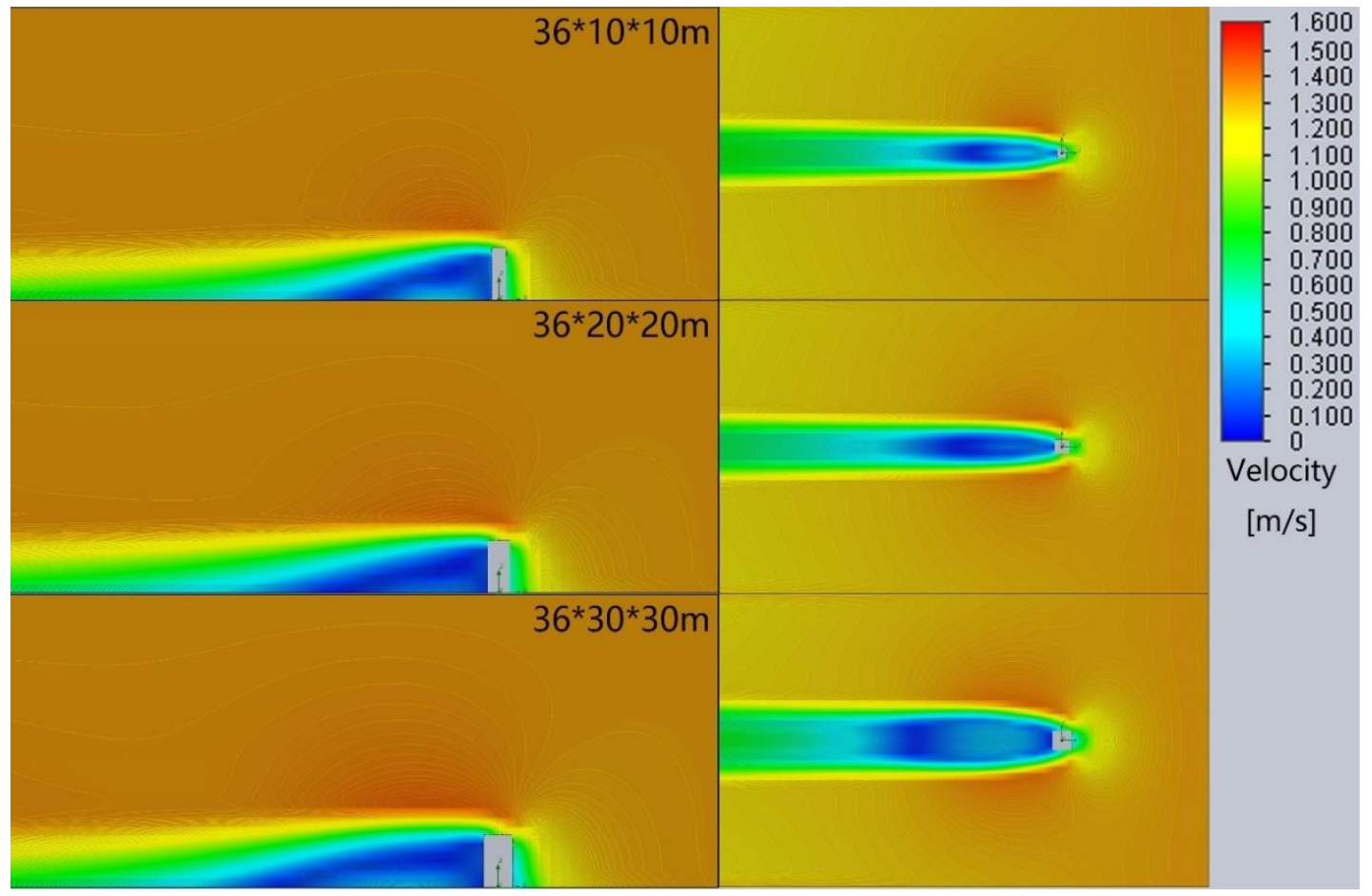

Figure 44. The comparison of wind-velocity magnitudes on horizontal and vertical planes of the square-form buildings with the sizes of $36 \mathrm{~m} *(10 \mathrm{~m} * 10 \mathrm{~m}, 15 \mathrm{~m} * 15 \mathrm{~m}, 20 \mathrm{~m} * 20 \mathrm{~m})$.

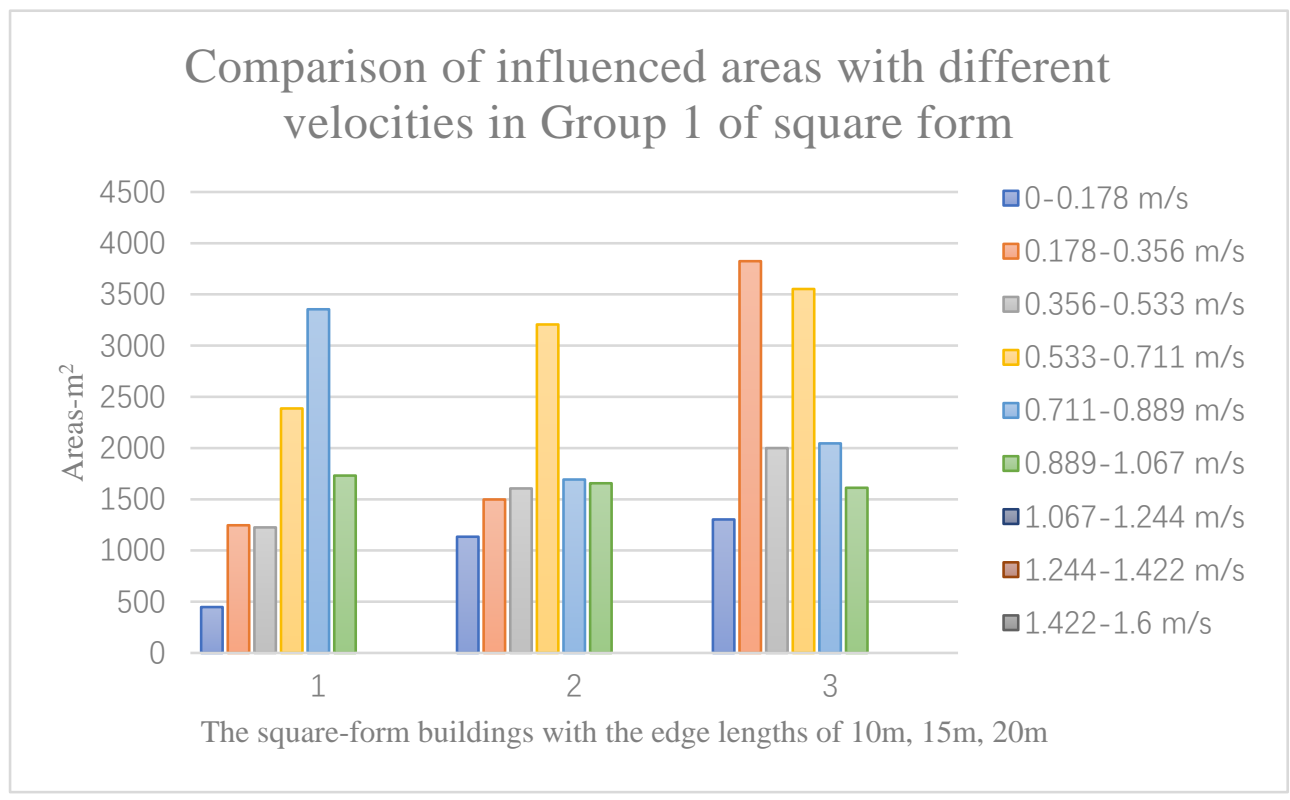

Figure 45. The influenced areas with different velocities in Group 1 of square form.

The changes of influenced areas with different velocities in three cases are closely related to the changes of air pressure and wind flow (Figure 46). As winds approach the buildings, they are obstructed by the windward surfaces. In Case 1, the influence is the smallest because the 
windward surface is the smallest; in Case 2, the influence increases as the windward surface becomes larger; in Case 3, the influences is the largest due to the largest windward surface.

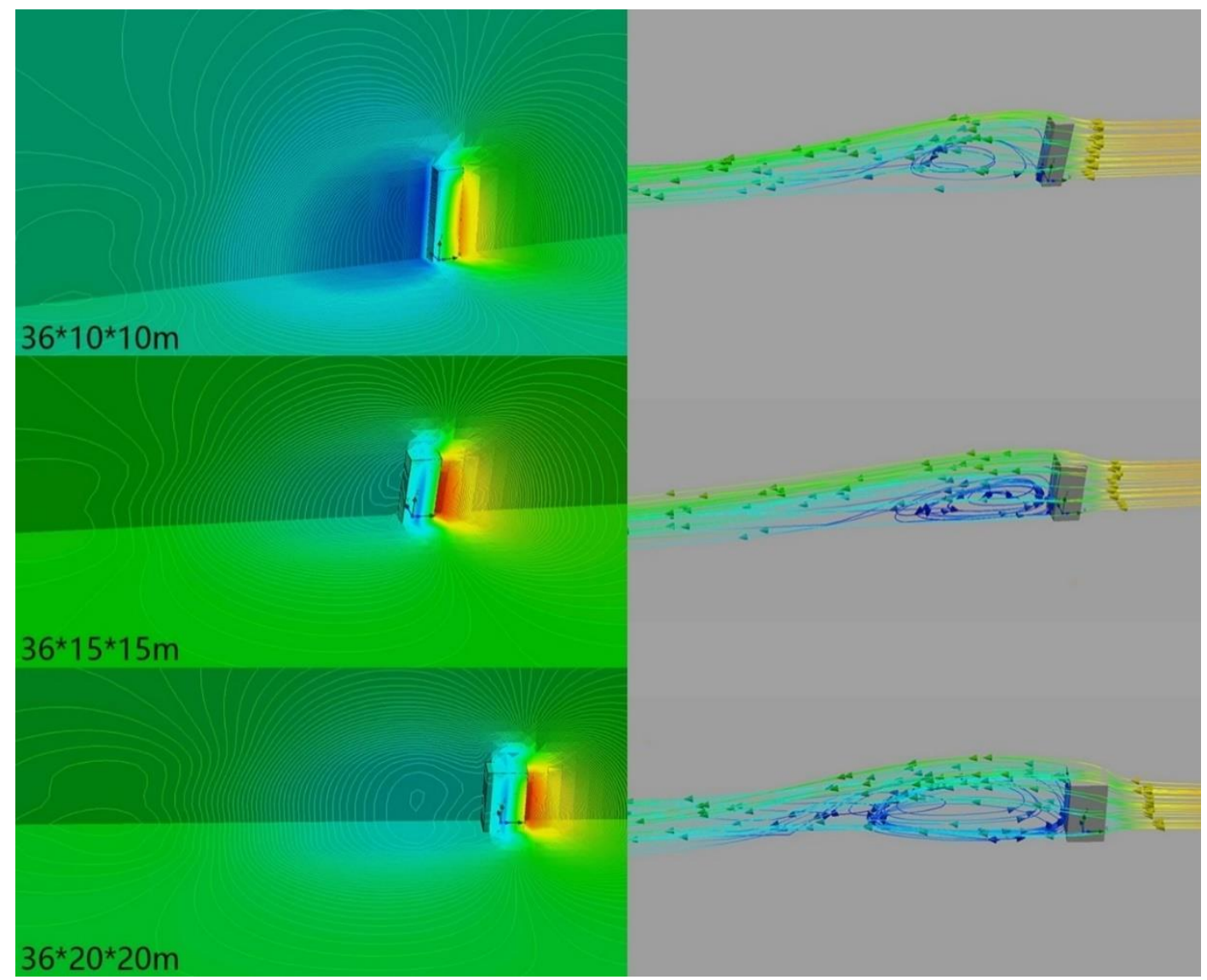

Figure 46. The figure shows the comparisons of streamlines and air pressure magnitudes on horizontal, vertical planes and building surfaces of the square-form buildings with the sizes of $36 \mathrm{~m} *(10 \mathrm{~m} * 10 \mathrm{~m}, 15 \mathrm{~m} * 15 \mathrm{~m}, 20 \mathrm{~m} * 20 \mathrm{~m})$.

\section{(2) Part 2: Influences of different rotation angles}

According to the simulation results of Group 2 (Figure 47), the low-velocity area (velocity below $0.5 \mathrm{~m} / \mathrm{s}$ ) behind the building is the largest and its length is the longest in Case 1 (rotation angle: 0 degree); as the rotation angle increases, the low-velocity area becomes smaller and shorter significantly in Case 2 (rotation angle: 15 degree) and Case 3 (rotation angle: 30 degree); in Case 4, the low-velocity area is the smallest and shortest (rotation angle: 45 degree). From Case 1 to Case 4, the dark-blue part of low-velocity area decreases and the light-blue area increases as the rotation angle increases. The dark-blue area represents extremely low-velocity 
area (velocity below $0.3 \mathrm{~m} / \mathrm{s}$ ). The increase of light-blue area suggests the wind velocity becomes faster in the area. This further indicates the improvement of outdoor ventilation in the low-velocity area behind the building.

In Case 1 (rotation angle: 0 degree), the smallest high-velocity areas (velocity over $1.4 \mathrm{~m} / \mathrm{s}$ ) are found near the lateral sides of the building; as the rotation angle increases, the high-velocity areas increase in Case 2 (rotation angle: 15 degree) and Case 3 (rotation angle: 30 degree); the high-velocity area is the largest in Case 4 (rotation angle: 45 degree). (Figure 48)

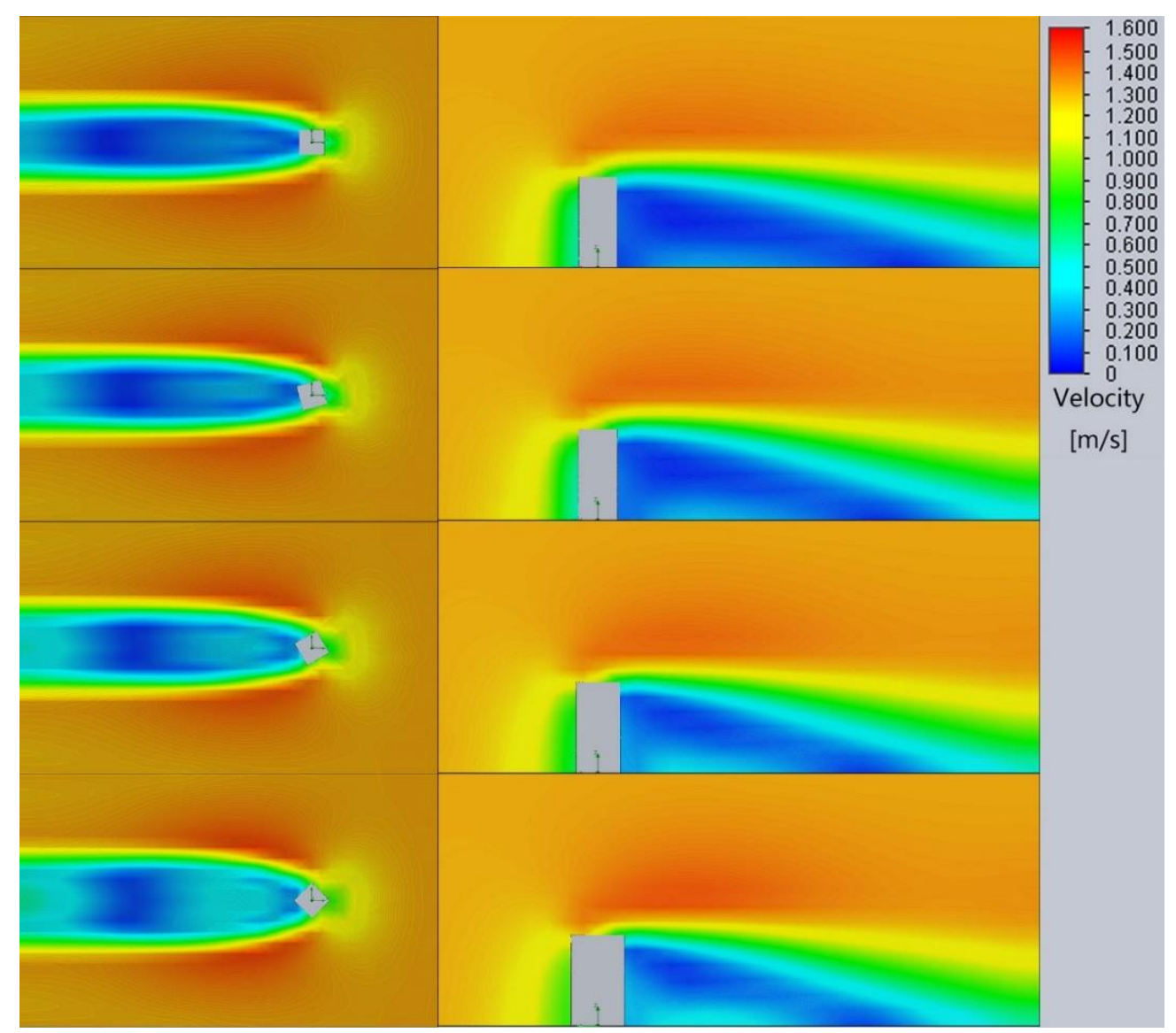

Figure 47. The comparison of wind velocity magnitudes on horizontal and vertical planes of the square-form buildings with different rotation angles of $0,15,30$ and 45 degrees. 


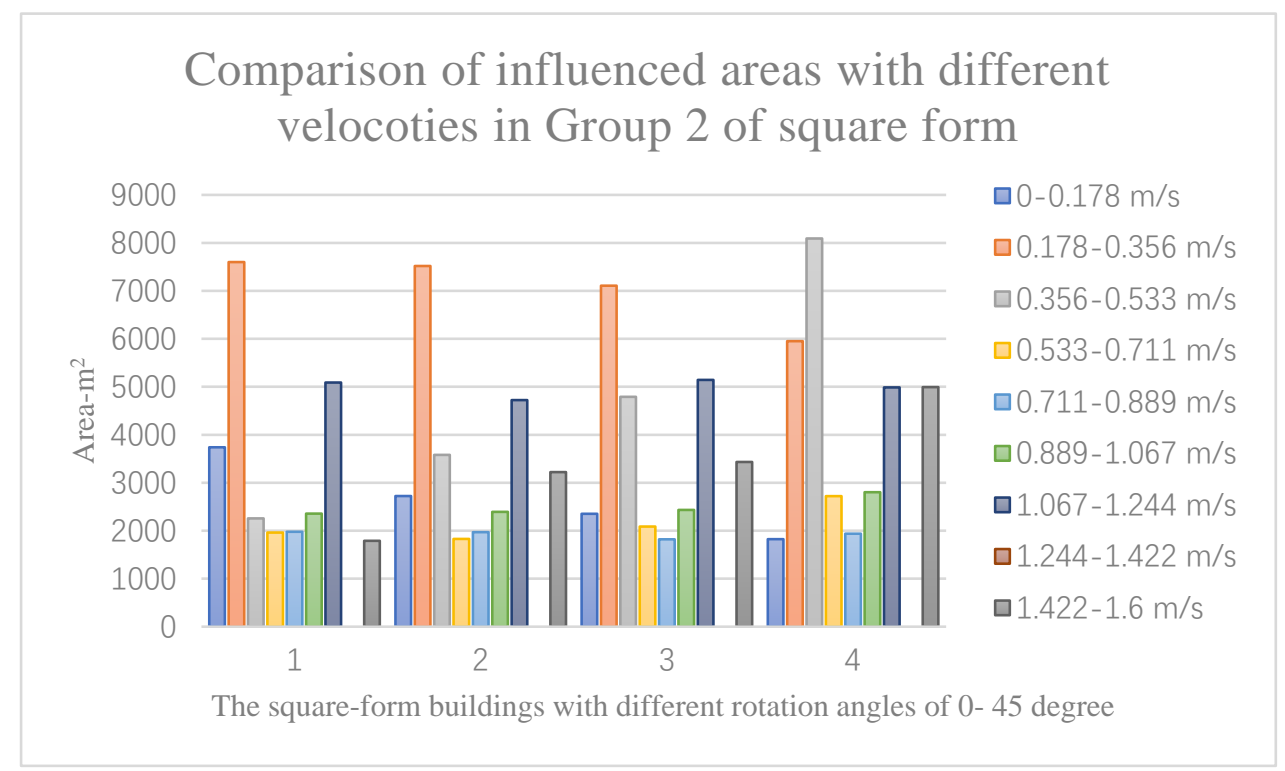

Figure 48. The influenced areas with different velocities in Group 2 of square form.

The changes of influenced areas with different velocities in different cases are related to the changes of air pressure and wind flow (Figure 49). In Case 1, as winds approach the building, they slow down and turn to the edges of the windward surface and escape from the two lateral sides and the top of the building. They are accelerated symmetrically on the lateral sides of the building. Then they flow into the low-air-pressure area on the leeward side of the building. Velocities of winds are decreased as they flow several rounds in the low-air-pressure area behind the building. The low-velocity area is created due to the decrease of wind velocity. In Case 2, as more winds are accelerated on one lateral side, magnitudes of velocity and air pressure are on longer symmetrical on horizontal plane. As the low-air-pressure area is smaller than Case 1, it is easier for winds to escape and the low-velocity area in Case 2 becomes smaller. In Case 3, the changes discovered in Case 2 becomes more obvious. In Case 4, as the center line of the building is parallel with the original wind direction, the magnitudes of velocity and air pressure become symmetrical again. Winds are divided by the windward edge and escape from the two lateral sides more easily than in Case 1. Winds flow round in few circles behind the building and a smaller low-velocity area is generated. 


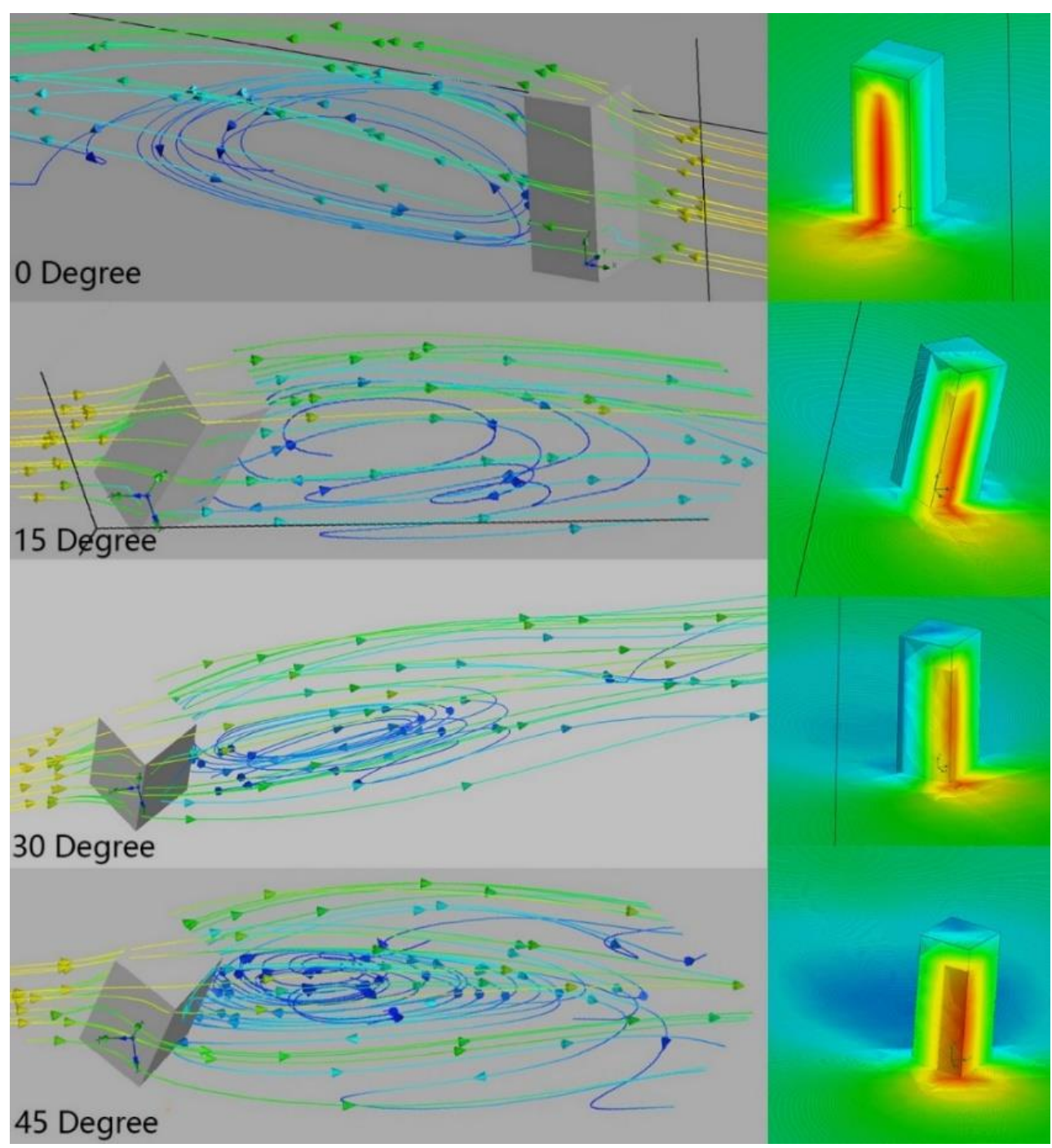

Figure 49. The air pressure magnitudes (right) and streamlines (left) of the square-form buildings with rotation angles of $0,15,30$ and 45 degrees.

\subsection{Rectangular form}

This section aims to study the relationships between the influences on wind environments and the building variables of rectangular form, including the length, width, height and rotation angle (Figure 50). In the first part, the influences of lengths and widths are studied for the rectangularform buildings with the long surfaces (length) on the windward side. In the second part, the influences of heights are studied. In the third part, the influences of lengths and widths are studied for the rectangular-form buildings with the short surfaces (width) on the windward side. In the fourth part, the influences of rotation angles are studied. The building variables setup of 
the four parts are presented first. Then results and analysis of the four parts are presented.

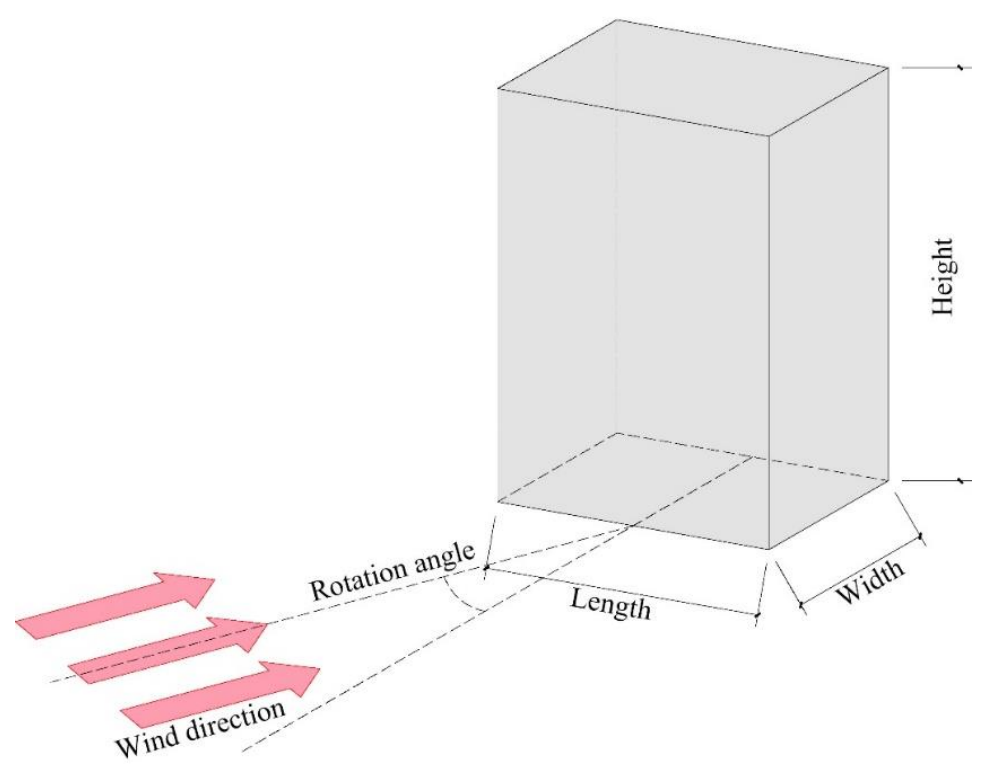

Figure 50. The building variables of rectangular form.

\subsubsection{Building variables setup of rectangular form}

In the first part, eleven cases of three groups with different lengths and widths are set up for simulations (named as Group 1, Group 2 and Group 3). According to the summary in the parametric design chapter, common lengths in the range of $10-40 \mathrm{~m}$ are used in the study; common widths in the range of $10-20 \mathrm{~m}$ are used; the building height of $36 \mathrm{~m}$ is used in all cases, which is a common height for a high-rise residential building with twelve floors. The specific variables of the three groups are presented in the following tables (Table 9., Table 10. and Table 11.).

Table 9. The four cases in Group 1 of rectangular form.

\begin{tabular}{|l|l|l|l|}
\hline Cases & Lengths & Widths & Heights \\
\hline Case 1 & $10 \mathrm{~m}$ & $10 \mathrm{~m}$ & $36 \mathrm{~m}$ \\
\hline Case 2 & $20 \mathrm{~m}$ & $10 \mathrm{~m}$ & $36 \mathrm{~m}$ \\
\hline Case 3 & $30 \mathrm{~m}$ & $10 \mathrm{~m}$ & $36 \mathrm{~m}$ \\
\hline Case 4 & $40 \mathrm{~m}$ & $10 \mathrm{~m}$ & $36 \mathrm{~m}$ \\
\hline
\end{tabular}


Table 10. The four cases in Group 2 of rectangular form.

\begin{tabular}{|l|l|l|l|}
\hline Cases & Lengths & Widths & Heights \\
\hline Case 1 & $10 \mathrm{~m}$ & $15 \mathrm{~m}$ & $36 \mathrm{~m}$ \\
\hline Case 2 & $20 \mathrm{~m}$ & $15 \mathrm{~m}$ & $36 \mathrm{~m}$ \\
\hline Case 3 & $30 \mathrm{~m}$ & $15 \mathrm{~m}$ & $36 \mathrm{~m}$ \\
\hline Case 4 & $40 \mathrm{~m}$ & $15 \mathrm{~m}$ & $36 \mathrm{~m}$ \\
\hline
\end{tabular}

Table 11. The three cases in Group 3 of rectangular form.

\begin{tabular}{|l|l|l|l|}
\hline Cases & Lengths & Widths & Heights \\
\hline Case 1 & $20 \mathrm{~m}$ & $20 \mathrm{~m}$ & $36 \mathrm{~m}$ \\
\hline Case 2 & $30 \mathrm{~m}$ & $20 \mathrm{~m}$ & $36 \mathrm{~m}$ \\
\hline Case 3 & $40 \mathrm{~m}$ & $20 \mathrm{~m}$ & $36 \mathrm{~m}$ \\
\hline
\end{tabular}

In the second part, three cases with different heights are set up (named Group 4). According to the summary in the parametric design chapter, the width and length are set as $15 \mathrm{~m}$ and $30 \mathrm{~m}$ respectively in each case, which is a common size for the floor plane with two apartments; building heights are set as $36 \mathrm{~m}, 54 \mathrm{~m}$ and $72 \mathrm{~m}$ in the three cases (Table 12.). $36 \mathrm{~m}$ is a common height for the residential building with twelve floors. $54 \mathrm{~m}$ is a common height for the residential building with 18 floors. $72 \mathrm{~m}$ is a common height for the residential building with 24 floors.

Table 12. The three cases in Group 4 of rectangular form.

\begin{tabular}{|l|l|l|l|}
\hline Cases & Lengths & Widths & Heights \\
\hline Case 1 & $30 \mathrm{~m}$ & $15 \mathrm{~m}$ & $36 \mathrm{~m}$ \\
\hline Case 2 & $30 \mathrm{~m}$ & $15 \mathrm{~m}$ & $54 \mathrm{~m}$ \\
\hline Case 3 & $30 \mathrm{~m}$ & $15 \mathrm{~m}$ & $72 \mathrm{~m}$ \\
\hline
\end{tabular}

In the third part, eleven cases of three groups with different lengths and widths are set up 
for simulations (named as Group 5, Group 6 and Group 7). This part aims to study the influences of lengths and widths of the rectangular-form buildings with the short surfaces (width) on the windward side. The building variables are the same as the first part. The profiles (short surfaces) of the buildings in the first part are rotated 90 degree toward the windward direction in this part. The specific variables are presented in the following tables (Table 13., Table 14. and Table 15.).

Table 13. The four cases in Group 5 of rectangular form.

\begin{tabular}{|l|l|l|l|l|}
\hline Cases & Lengths & Widths & Heights & Rotation angles \\
\hline Case 1 & $10 \mathrm{~m}$ & $10 \mathrm{~m}$ & $36 \mathrm{~m}$ & 90 degree \\
\hline Case 2 & $20 \mathrm{~m}$ & $10 \mathrm{~m}$ & $36 \mathrm{~m}$ & 90 degree \\
\hline Case 3 & $30 \mathrm{~m}$ & $10 \mathrm{~m}$ & $36 \mathrm{~m}$ & 90 degree \\
\hline Case 4 & $40 \mathrm{~m}$ & $10 \mathrm{~m}$ & $36 \mathrm{~m}$ & 90 degree \\
\hline
\end{tabular}

Table 14. The four cases in Group 6 of rectangular form.

\begin{tabular}{|l|l|l|l|l|}
\hline Cases & Lengths & Widths & Heights & Rotation angles \\
\hline Case 1 & $15 \mathrm{~m}$ & $15 \mathrm{~m}$ & $36 \mathrm{~m}$ & 90 degree \\
\hline Case 2 & $20 \mathrm{~m}$ & $15 \mathrm{~m}$ & $36 \mathrm{~m}$ & 90 degree \\
\hline Case 3 & $30 \mathrm{~m}$ & $15 \mathrm{~m}$ & $36 \mathrm{~m}$ & 90 degree \\
\hline Case 4 & $40 \mathrm{~m}$ & $15 \mathrm{~m}$ & $36 \mathrm{~m}$ & 90 degree \\
\hline
\end{tabular}

Table 15. The three cases in Group 7 of rectangular form.

\begin{tabular}{|l|l|l|l|l|}
\hline Cases & Lengths & Widths & Heights & Rotation angles \\
\hline Case 1 & $20 \mathrm{~m}$ & $20 \mathrm{~m}$ & $36 \mathrm{~m}$ & 90 degree \\
\hline Case 2 & $30 \mathrm{~m}$ & $20 \mathrm{~m}$ & $36 \mathrm{~m}$ & 90 degree \\
\hline Case 3 & $40 \mathrm{~m}$ & $20 \mathrm{~m}$ & $36 \mathrm{~m}$ & 90 degree \\
\hline
\end{tabular}

In the fourth part, twelve cases of three groups with different rotation angels are set up (Table 16., Table 17. and Table 18.). The range of rotation angles is $0-90$ degree; the range of building heights is $36-72 \mathrm{~m}$. This part aims to study the influences of different angles between 
the wind direction and windward surface of the building. According to the summary in the parametric design chapter, the width and length are set as $15 \mathrm{~m}$ and $30 \mathrm{~m}$ respectively in each case; there are four rotation angles: 0,30,60 and 90 degree in each group; the building heights are set as the common heights: $36 \mathrm{~m}, 54 \mathrm{~m}$ and $72 \mathrm{~m}$.

Table 16. The four cases in Group 8 of rectangular form.

\begin{tabular}{|l|l|l|l|l|}
\hline Cases & Lengths & Widths & Heights & Rotation angles \\
\hline Case 1 & $30 \mathrm{~m}$ & $15 \mathrm{~m}$ & $36 \mathrm{~m}$ & 0 degree \\
\hline Case 2 & $30 \mathrm{~m}$ & $15 \mathrm{~m}$ & $36 \mathrm{~m}$ & 30 degree \\
\hline Case 3 & $30 \mathrm{~m}$ & $15 \mathrm{~m}$ & $36 \mathrm{~m}$ & 60 degree \\
\hline Case 4 & $30 \mathrm{~m}$ & $15 \mathrm{~m}$ & $36 \mathrm{~m}$ & 90 degree \\
\hline
\end{tabular}

Table 17. The four cases in Group 9 of rectangular form.

\begin{tabular}{|l|l|l|l|l|}
\hline Cases & Lengths & Widths & Heights & Rotation angles \\
\hline Case 1 & $30 \mathrm{~m}$ & $15 \mathrm{~m}$ & $54 \mathrm{~m}$ & 0 degree \\
\hline Case 2 & $30 \mathrm{~m}$ & $15 \mathrm{~m}$ & $54 \mathrm{~m}$ & 30 degree \\
\hline Case 3 & $30 \mathrm{~m}$ & $15 \mathrm{~m}$ & $54 \mathrm{~m}$ & 60 degree \\
\hline Case 4 & $30 \mathrm{~m}$ & $15 \mathrm{~m}$ & $54 \mathrm{~m}$ & 90 degree \\
\hline
\end{tabular}

Table 18. The four cases in Group 10 of rectangular form.

\begin{tabular}{|l|l|l|l|l|}
\hline Cases & Lengths & Widths & Heights & Rotation angles \\
\hline Case 1 & $30 \mathrm{~m}$ & $15 \mathrm{~m}$ & $72 \mathrm{~m}$ & 0 degree \\
\hline Case 2 & $30 \mathrm{~m}$ & $15 \mathrm{~m}$ & $72 \mathrm{~m}$ & 30 degree \\
\hline Case 3 & $30 \mathrm{~m}$ & $15 \mathrm{~m}$ & $72 \mathrm{~m}$ & 60 degree \\
\hline Case 4 & $30 \mathrm{~m}$ & $15 \mathrm{~m}$ & $72 \mathrm{~m}$ & 90 degree \\
\hline
\end{tabular}

The parametric modelling script of rectangular form is used to create the building models. In the above ten groups, boundaries of CFD simulation results are all set in the same sizes for comparisons of influences on wind environments. 


\subsubsection{Results and analysis of rectangular form}

The CFD simulation results of rectangular form are analyzed in this subsection. Influences on wind environments are compared based on velocity magnitudes. The mechanisms are explained using the air pressure magnitudes and wind flow streamlines. In Part 1, the relationships between influences and lengths and widths are studied. In Part 2, the relationship between influences and heights is studied. In Part 3, the relationships between influences and lengths and widths of the rectangular-form buildings with the short surfaces (width) on the windward side are studied. In Part 4, the relationship between influences and rotation angles is studied.

\section{(1) Part 1. Influences of different lengths and widths (the surface with the length on the windward side)}

The influence of the length is analyzed according to the simulation results in the three groups respectively. In Group 1 (width: 10m) (Figure 51), the building of Case 1 (length: 10m) has the smallest influence on wind environment; the influence of Case 2 (length: $20 \mathrm{~m}$ ) is more than Case 1; and the influence of Case 3 (length: 30m) is more than Case 2; the building of Case 4 (length: 40m) has the most influence (Figure 52). In Case 1, the low-velocity area (velocity below $0.5 \mathrm{~m} / \mathrm{s}$ ) is the smallest and its length is the shortest; as the length increases, the lowvelocity area increases gradually in Case 2 and Case 3; In Case 4, the low-velocity area is the largest and its length is the longest (Figure 53). In Case 1, the high-velocity areas (velocity over $1.4 \mathrm{~m} / \mathrm{s}$ ) beside the lateral sides are the smallest; as the length increases, the areas are increased gradually in Case 2 and Case 3; in Case 4, the areas are the largest (Figure 54).

According to the simulation results in Group 2 (width: 15m) (Figure 55 and Figure 56) and Group 3 (width: 20m) (Figure 57 and Figure 58), the trends of influences on wind environments are the same as Group 1. In summary, the influence on wind environment of rectangular form increases as the length increases.

The influence of the width is analyzed according to the simulation results of Group 1 (width: 10m), Group 2 (width: 15m) and Group 3 (width: 20m) together (Figure 51, Figure 52, 
Figure 53, Figure 54, Figure 55 and Figure 56). Buildings of Group 3 with the longest width (width: $15 \mathrm{~m}$ ) have the smallest influences on wind environments; buildings of Group 1 with the shortest width (width: 10m) have the largest influences; the influences of Group 2 are between Group 1 and Group 3. The low-velocity area and high-velocity areas are decreased as the width increases. The trends can be found clearly in Case 3 and Case 4 in the three Groups. In summary, the influence on wind environment of rectangular form decreases as the width increases.

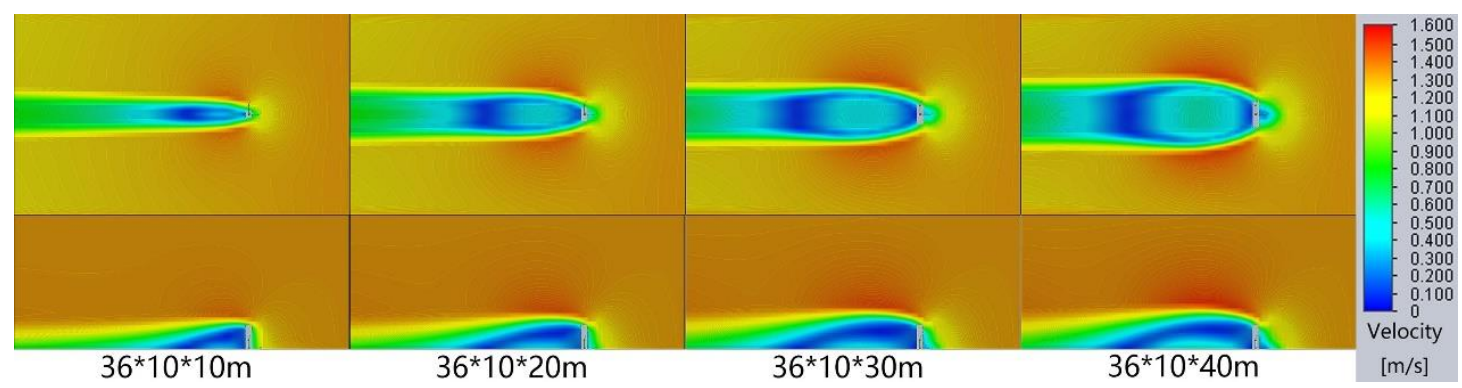

Figure 51. The comparison of wind velocity magnitudes on horizontal and vertical planes of the rectangular-form buildings with the sizes of $36 \mathrm{~m} * 10 \mathrm{~m} *(10 \mathrm{~m}, 20 \mathrm{~m}, 30 \mathrm{~m}, 40 \mathrm{~m})$ in Group 1 .

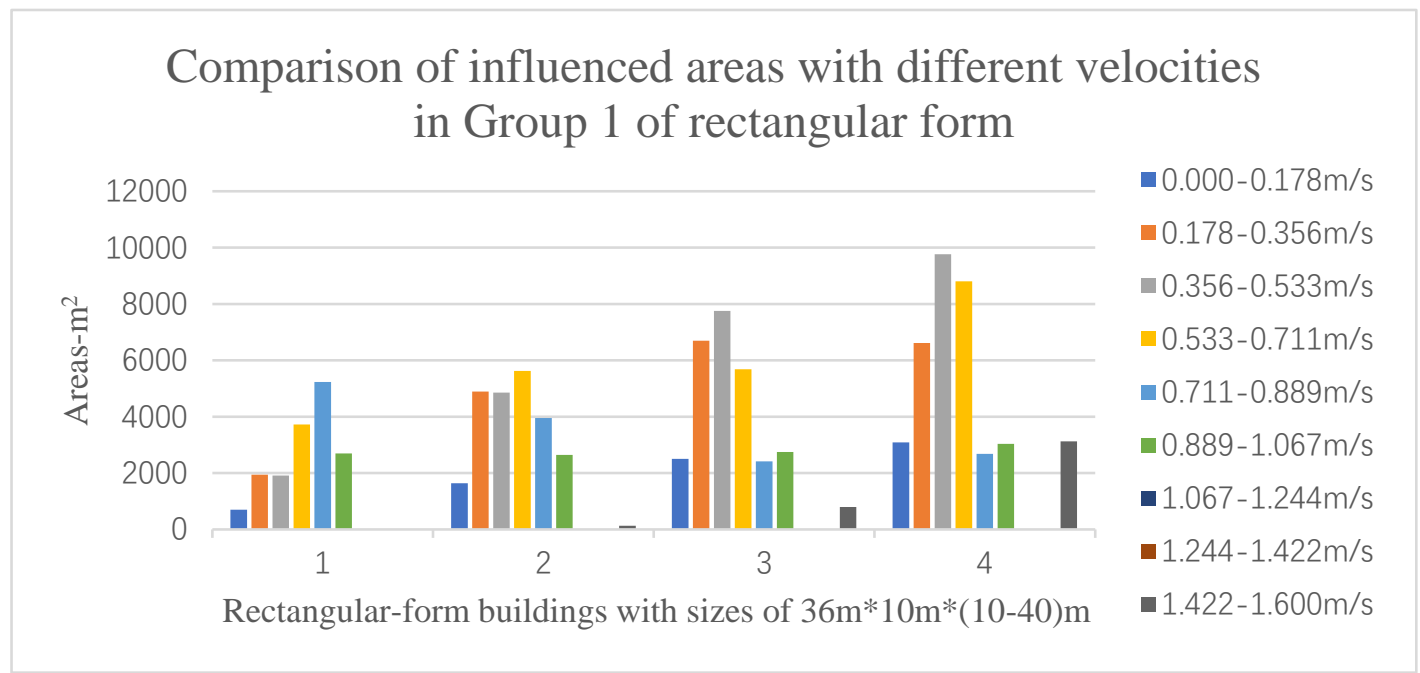

Figure 52. The influenced areas with different velocities in Group 1 of rectangular form. 


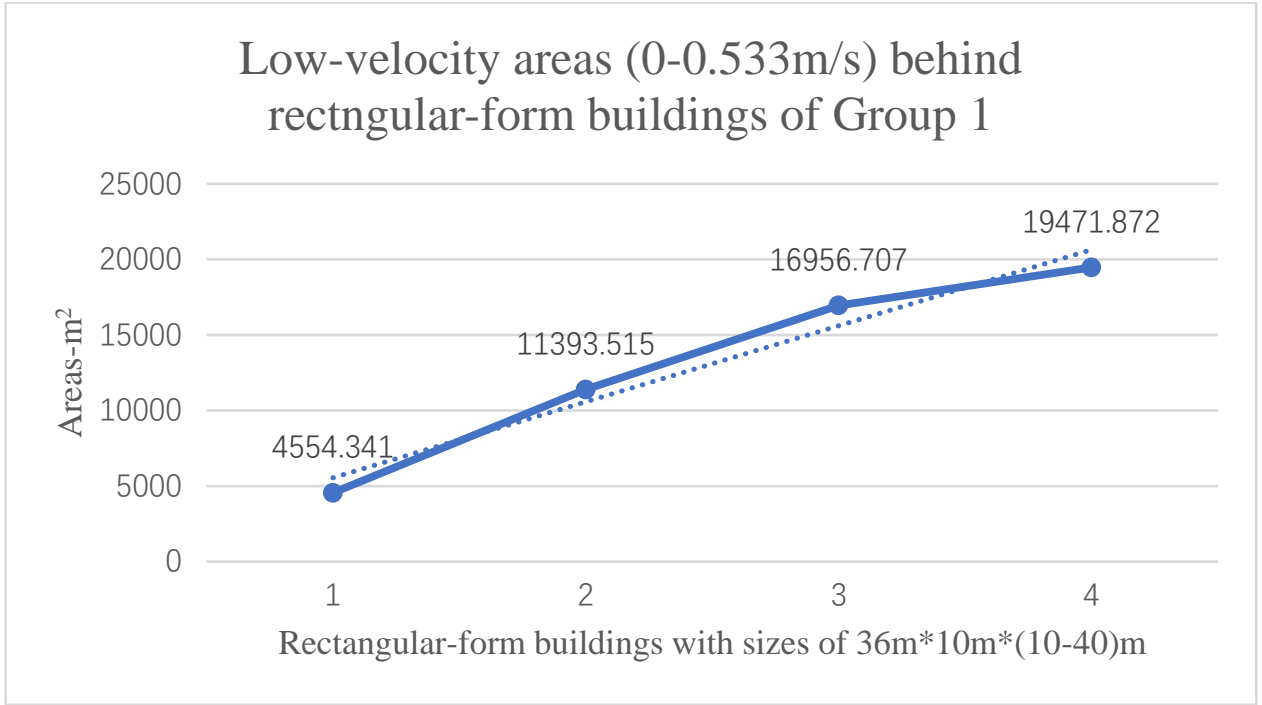

Figure 53 . The comparison of low-velocity areas $(0-0.533 \mathrm{~m} / \mathrm{s})$ of the wind velocity magnitude on horizontal plane of the rectangular-form buildings in Group 1.

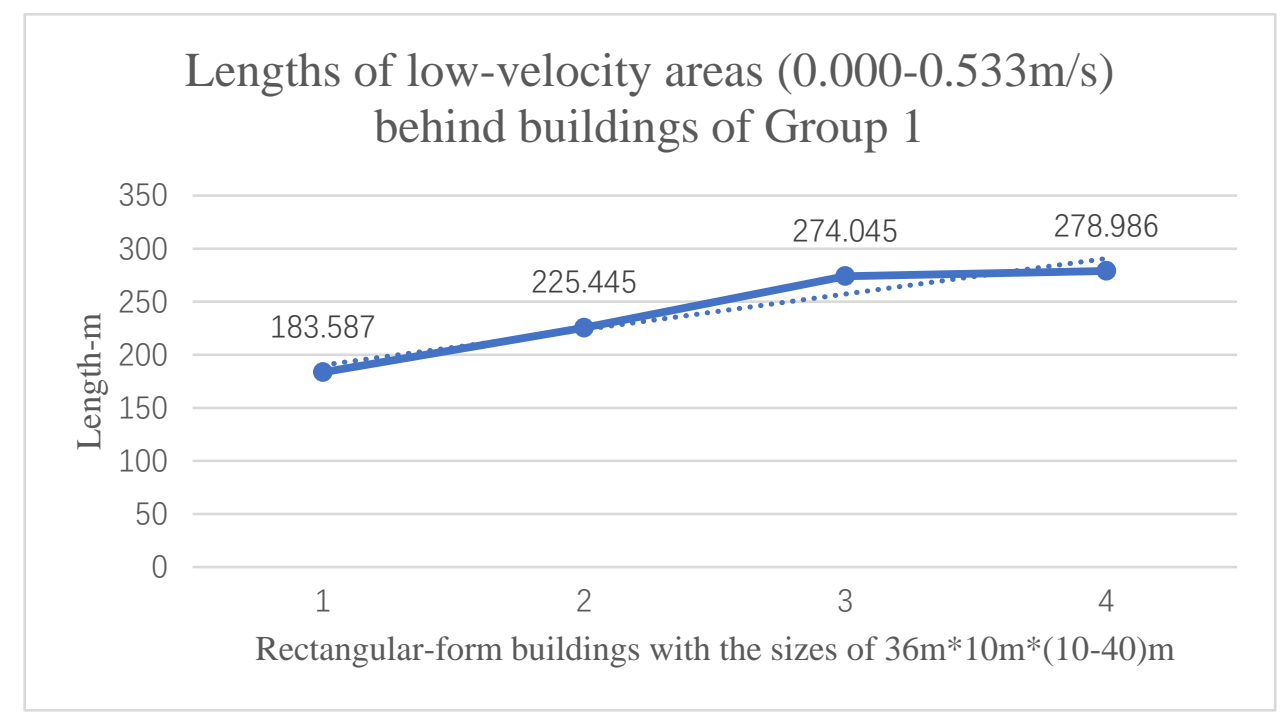

Figure 54 . The trend of the lengths of low-velocity areas $(0-0.533 \mathrm{~m} / \mathrm{s})$ of the wind velocity magnitude on horizontal plane of the rectangular-form buildings in Group 1.

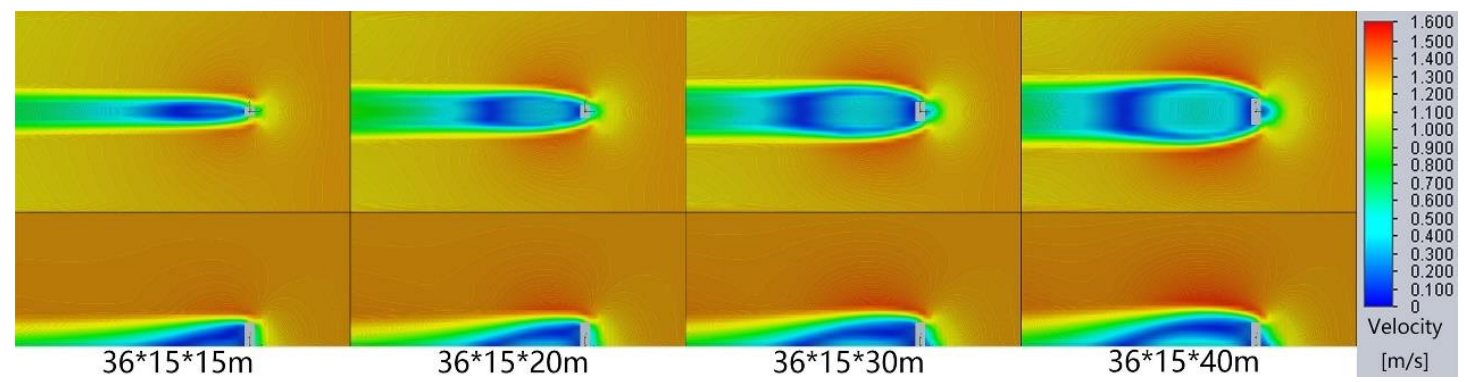

Figure 55. The comparison of wind velocity magnitudes on horizontal and vertical planes of the rectangular-form buildings in Group 2. 


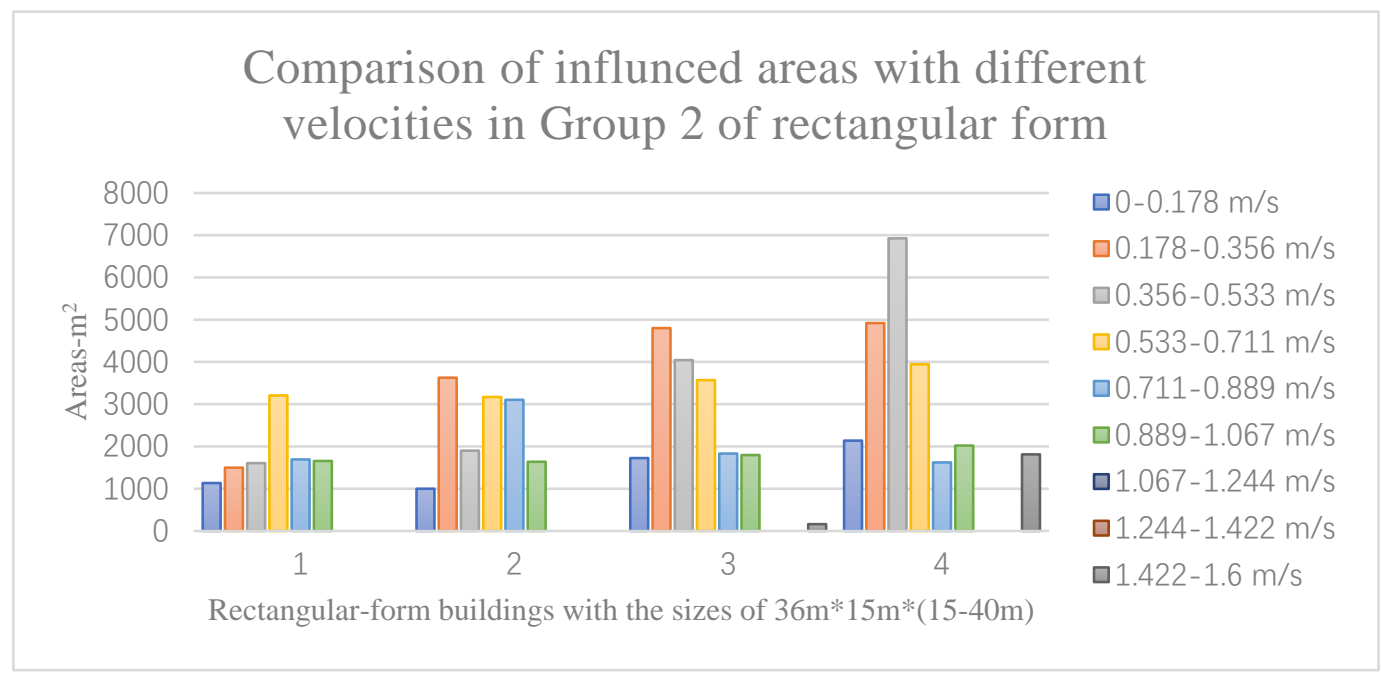

Figure 56. The influenced areas with different velocities in Group 2 of rectangular form.

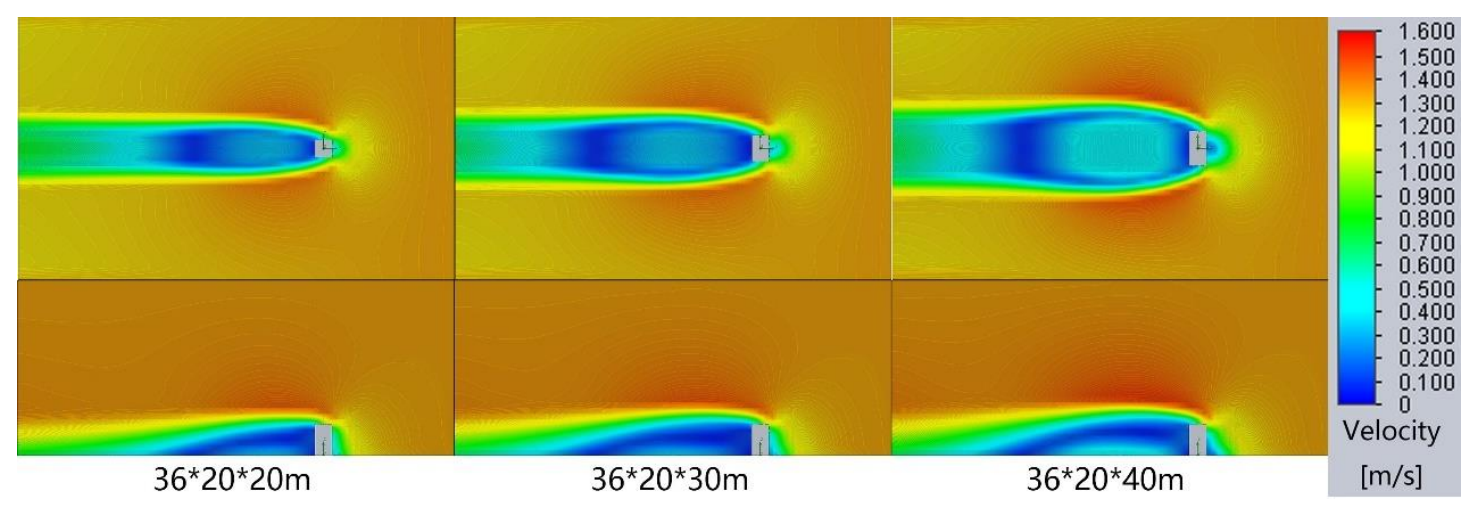

Figure 57. The comparison of wind velocity magnitudes on horizontal and vertical planes of the rectangular-form buildings in Group 3.

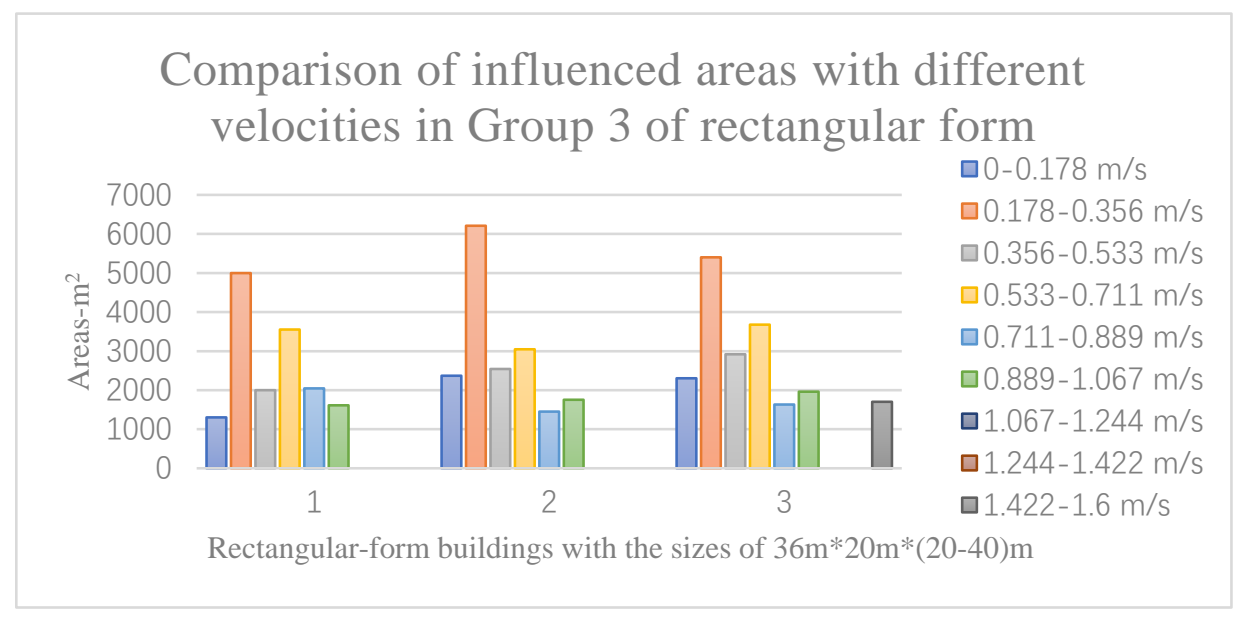

Figure 58. The influenced areas with different velocities in Group 3 of rectangular form. 
The changes of influenced areas are closely related to the changes of air pressure and wind flow (Figure 59). As winds approach the buildings, they are obstructed by the windward surfaces. Takes Group 1 for example, the influence of Case 1 is the smallest because the windward surface is the smallest and narrowest; in Case 2 and Case 3, the influence increases gradually as the windward surface becomes larger and wider; in Case 4, the influence is the largest due to the largest and longest windward surface. The air-pressure magnitudes show that low-air-pressure area behind the building and high-air-pressure area in front of the building are both increased as the length increases (Figure 59). The wind flow streamlines show that more winds are obstructed by the building as the length increases (Figure 59). The situations of Group 2 (Figure 60) and Group 3 (Figure 61) are similar to Group 1.

For the influence of width, the low-air-pressure area and high-air-pressure area are both decreased as the width increases (Figure 59); the wind-flow streamlines show that smaller vortices are created behind the building as the width increases (Figure 59). These correspond with the previous results of influences of the width.

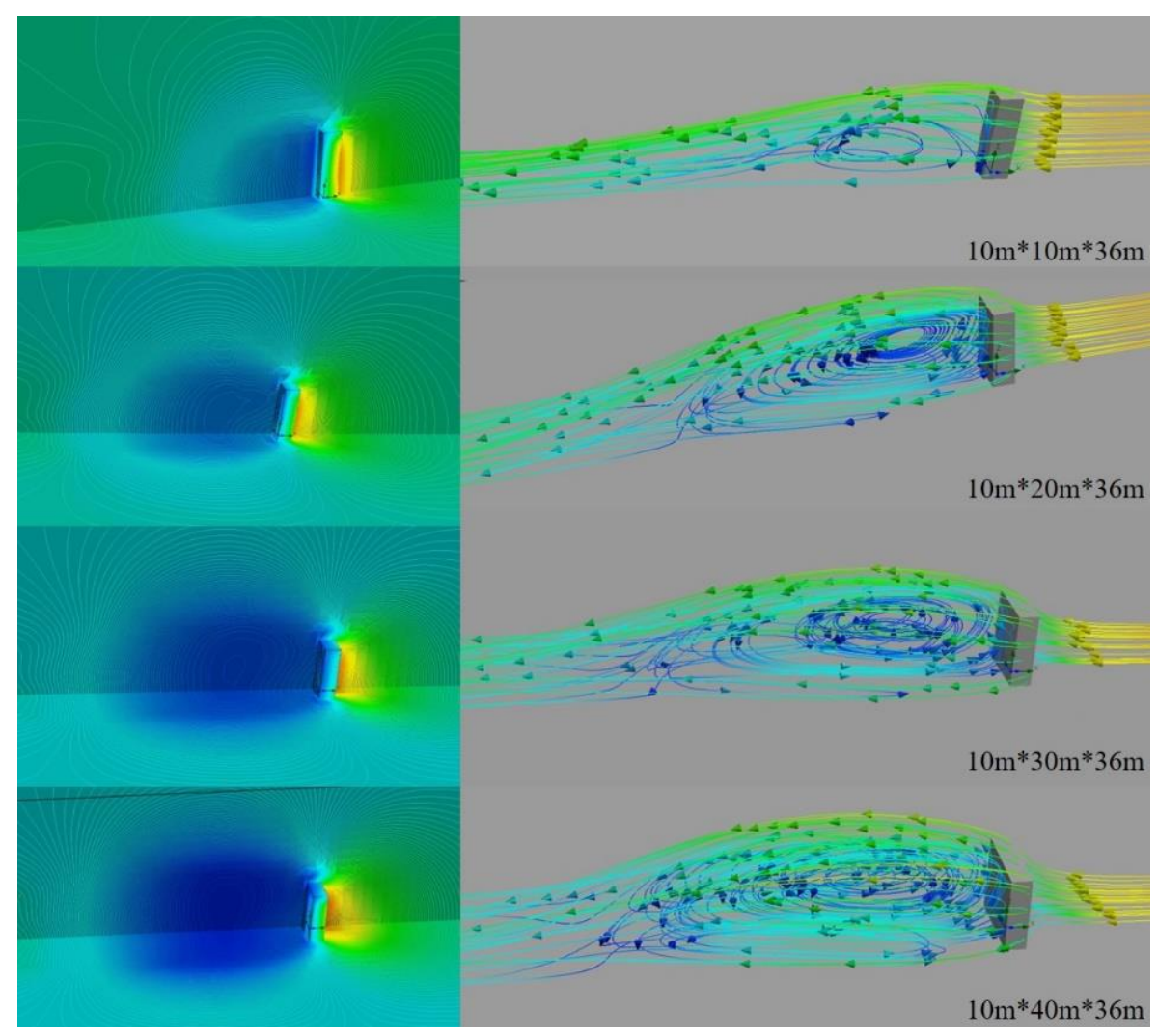

Figure 59. The comparisons of streamlines (right) and air-pressure magnitudes (left) on 
horizontal, vertical planes and building surfaces of the rectangular-form buildings in Group 1.

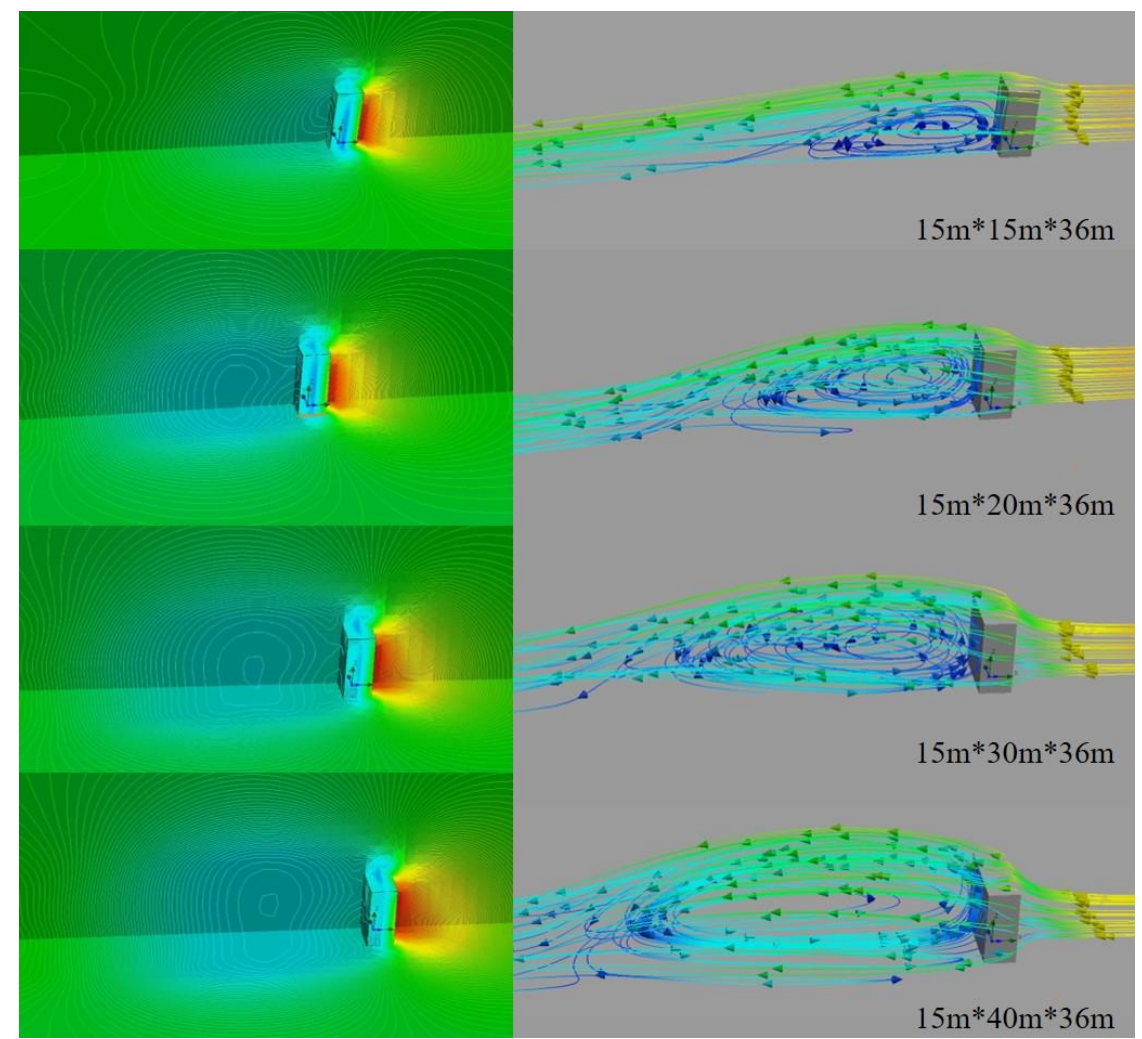

Figure 60. The comparisons of streamlines (right) and air-pressure magnitudes (left) on horizontal, vertical planes and building surfaces of the rectangular-form buildings in Group 2.

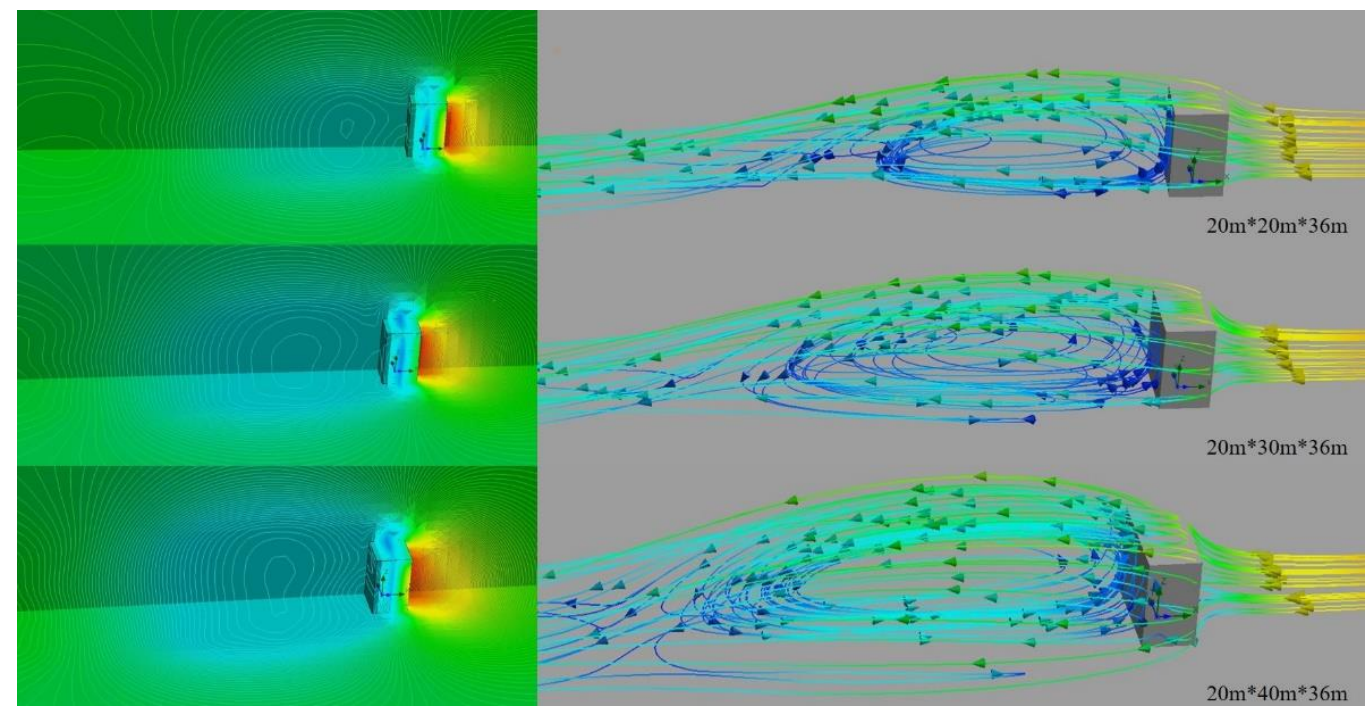

Figure 61. The comparisons of streamlines (right) and air-pressure magnitudes (left) on horizontal, vertical planes and building surfaces of the rectangular-form buildings in Group 3. 


\section{(2) Part 2. Influences of different heights}

According to the simulation results of Group 4 (Figure 62), the building of Case 1 (height: 36m) has the smallest influence on wind environment; the influence of Case 2 (height: $54 \mathrm{~m}$ ) is between Case 1 and Case 3; the building of Case 3 (height: 72m) has the most influence (Figure 63). In Case 1, the low-velocity area (velocity below $0.5 \mathrm{~m} / \mathrm{s}$ ) is the smallest and its length is the shortest; the low-velocity area and its length increase in Case 2; in Case 3, the low-velocity area is the largest and its length is the longest (Figure 62). In Case 1, the high-velocity areas (velocity over $1.4 \mathrm{~m} / \mathrm{s}$ ) on the lateral sides are the smallest; the areas are increased as the height increases in Case 2; in Case 3, the areas are the largest (Figure 62). In summary, the influence on wind environment of rectangular form increases as the height increases.

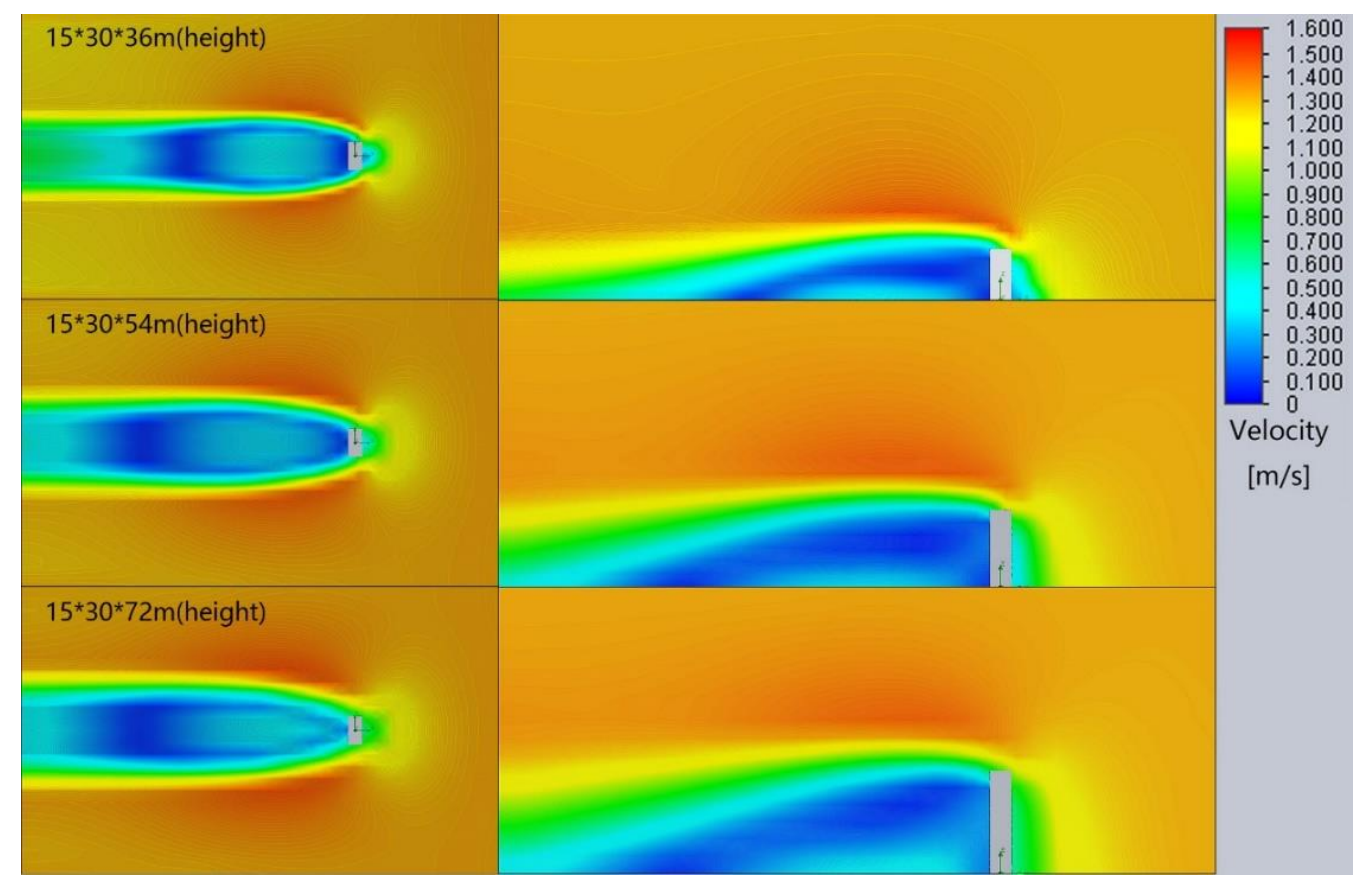

Figure 62. The comparison of wind velocity magnitudes on horizontal and vertical planes of the rectangular-form buildings with different heights in Group 4. 


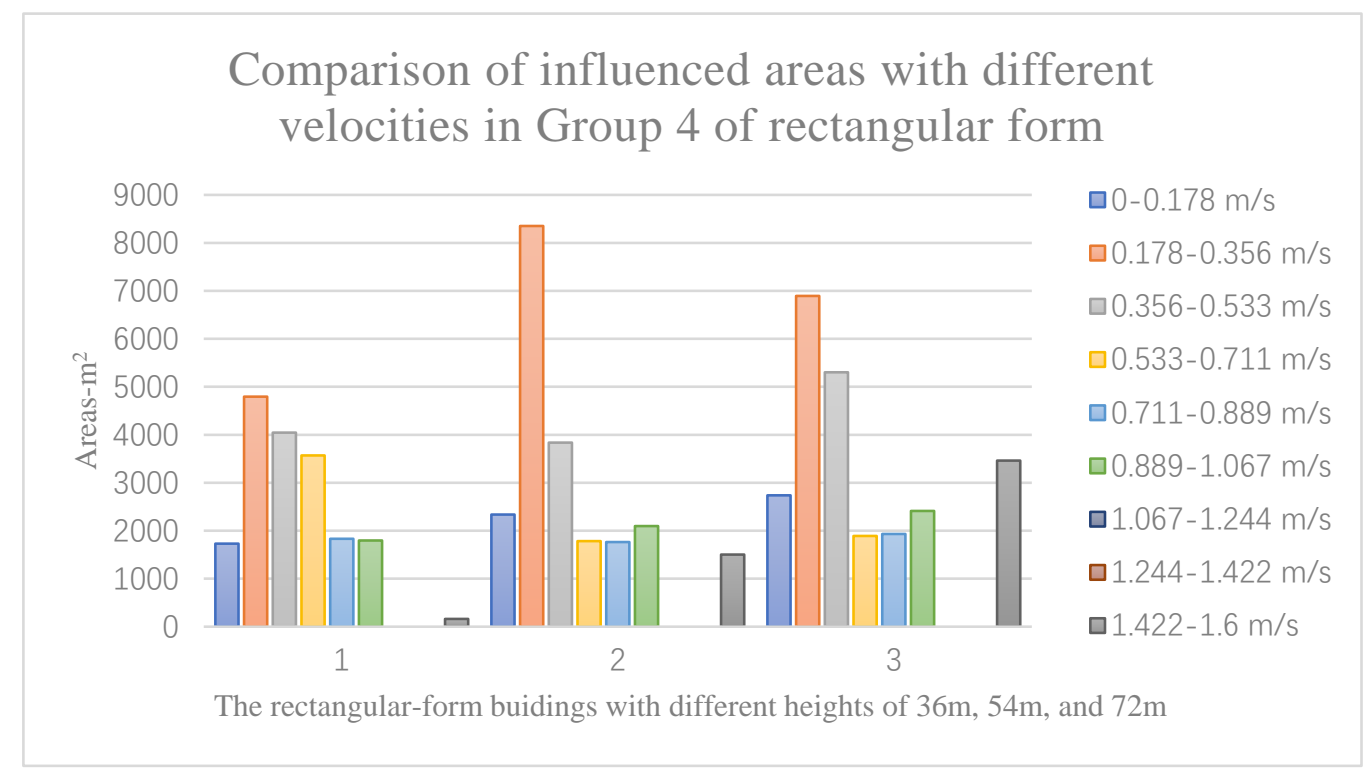

Figure 63. The influenced areas with different velocities in Group 4 of rectangular form.

Changes of the height influence the air pressure and wind flow which are closely related to the changes of influenced areas in Group 4 (Figure 64). As winds approach the buildings, they are obstructed by the windward surfaces. The influence of Case 1 is the smallest because the windward surface is the smallest and lowest; the influence of Case 2 increases as the windward surface becomes larger and taller; the influence of Case 4 is the largest due to the largest and tallest windward surface. The air-pressure magnitudes show that both the low-airpressure area and high-air-pressure area are increased as the height increases (Figure 64). As the height increases, the wind-flow streamlines show that more winds are obstructed by the building; it takes longer distance for winds to go around the building from the top (Figure 64). 


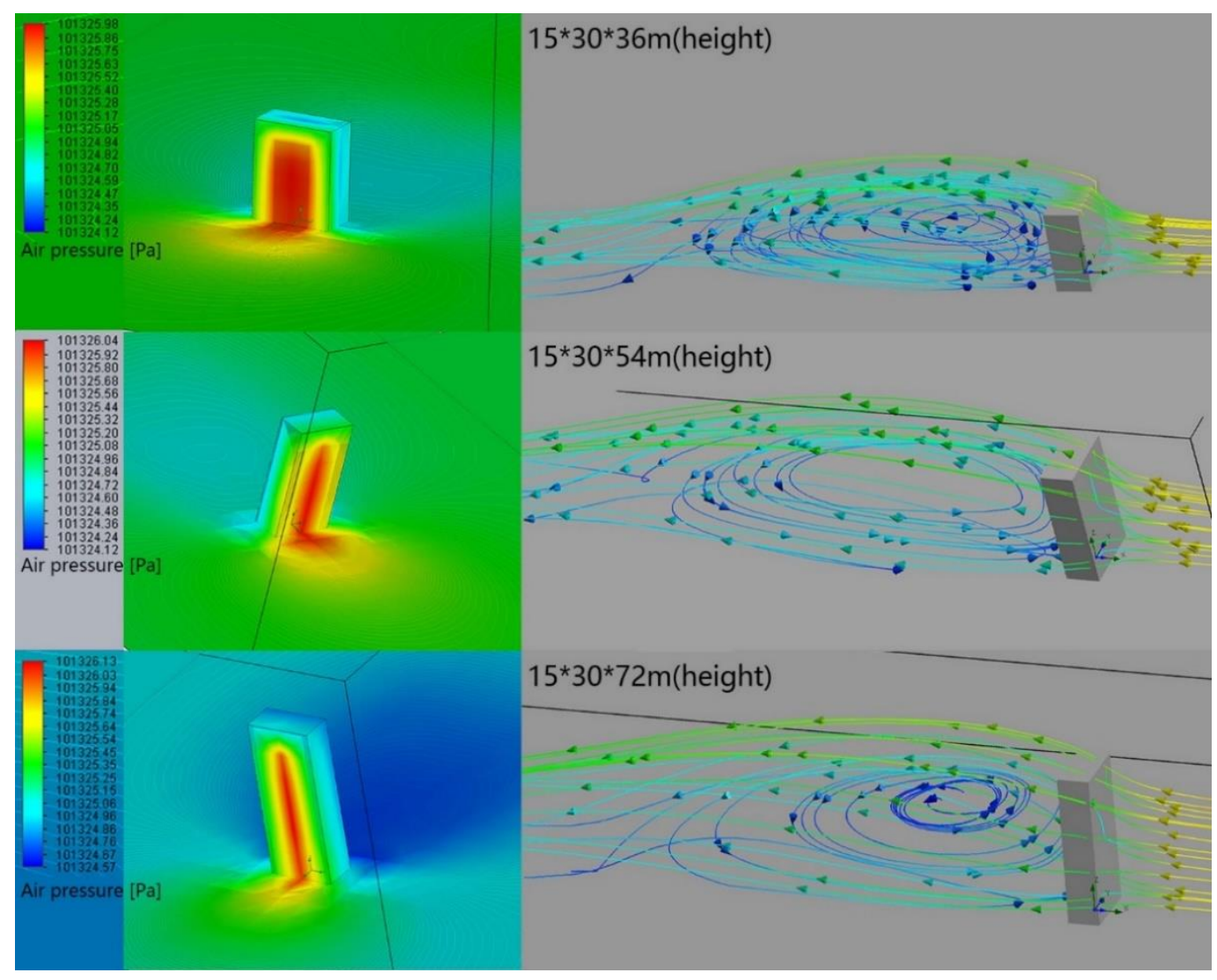

Figure 64. The comparisons of streamlines (right) and air-pressure magnitudes (left) on horizontal, vertical planes and building surfaces of the rectangular-form buildings in Group 4.

\section{(3) Part 3. Influences of different lengths and widths (the surface with the width on the windward side)}

The influence of the length is analyzed according to the simulation results in the three groups respectively. In Group 5 (width: 10m) (Figure 65), the influence of Case 1 (length: 10m) is the most of all; the influence of Case 2 (length: 20m) is less than Case 1; the influence of Case 3 (length: $30 \mathrm{~m}$ ) is the least of all; the influence of Case 4 (length: $40 \mathrm{~m}$ ) is more than Case 3 (Figure 66). In Case 1, the low-velocity area (velocity below $0.5 \mathrm{~m} / \mathrm{s}$ ) is the largest; the lowvelocity area decreases in Case 2; in Case 3, the area is the smallest, and the area with extremely low velocity (velocity below $0.178 \mathrm{~m} / \mathrm{s}$ ) is little; in Case 4 , the low-velocity area is more than Case 3, especially for the large increase of the area with extremely low velocity (velocity below $0.178 \mathrm{~m} / \mathrm{s}$ ), which is the most of all (Figure 66). In Case 1, the high-velocity areas on the lateral sides are the largest; as the length increases, the areas are decreased gradually in Case 2 and Case 3; the areas of Case 3 are the smallest; in Case 4, the areas are more than Case 3 (Figure 
$66)$.

In Group 6 (width: 15m), the results are similar to Group 5 and the influence of Case 3 is the smallest (Figure 67 and Figure 68). In Group 7 (width: 20m), the influence of the building decreases constantly as the length increases (Figure 69 and Figure 70).

The influence of the width is analyzed according to the simulation results of the three groups together (width: 10m, 15m and 20m). For the buildings with the same lengths, buildings of Group 3 with the longest width (width: 20m) have the largest influences on wind environments; buildings of Group 2 are between Group 1 and Group 3; buildings of Group 1 with the shortest width (width: 10m) have the smallest influences. The influence increases constantly as the width increases (Figure 65, Figure 66, Figure 67, Figure 68, Figure 69 and Figure 70).

In summary, the influence of the rectangular-form building increases as the width increases; the least influence of the rectangular-form building with the short surface (width) on the windward side should be decided by a particular ratio of length and width. In the situation of this research, the ratio is approximately $1 / 3$ (width/ length) according to the results analysis of the three groups.

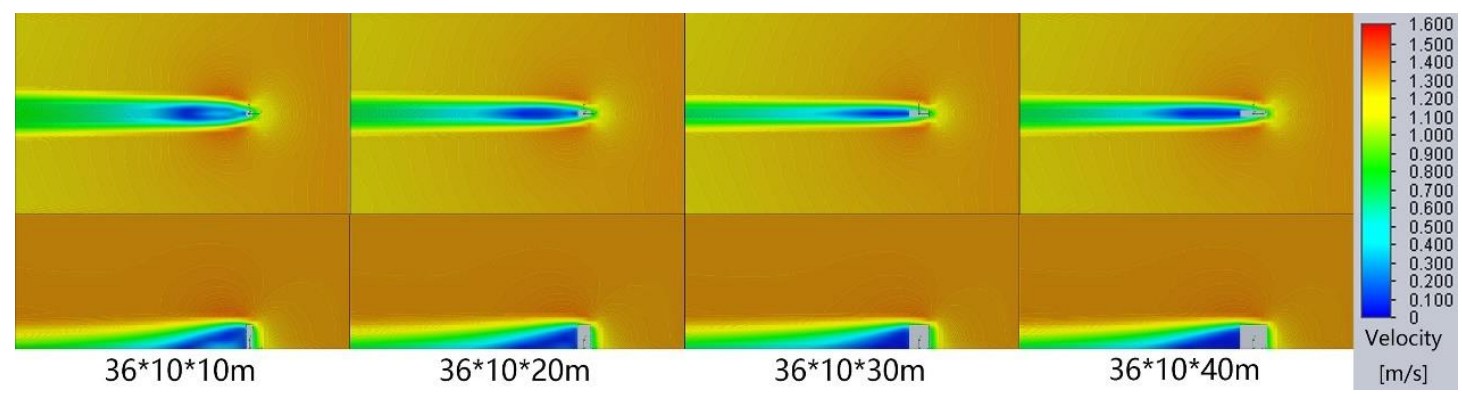

Figure 65 . The comparison of wind velocity magnitudes on horizontal and vertical planes of the rectangular-form buildings with different heights in Group 5. 


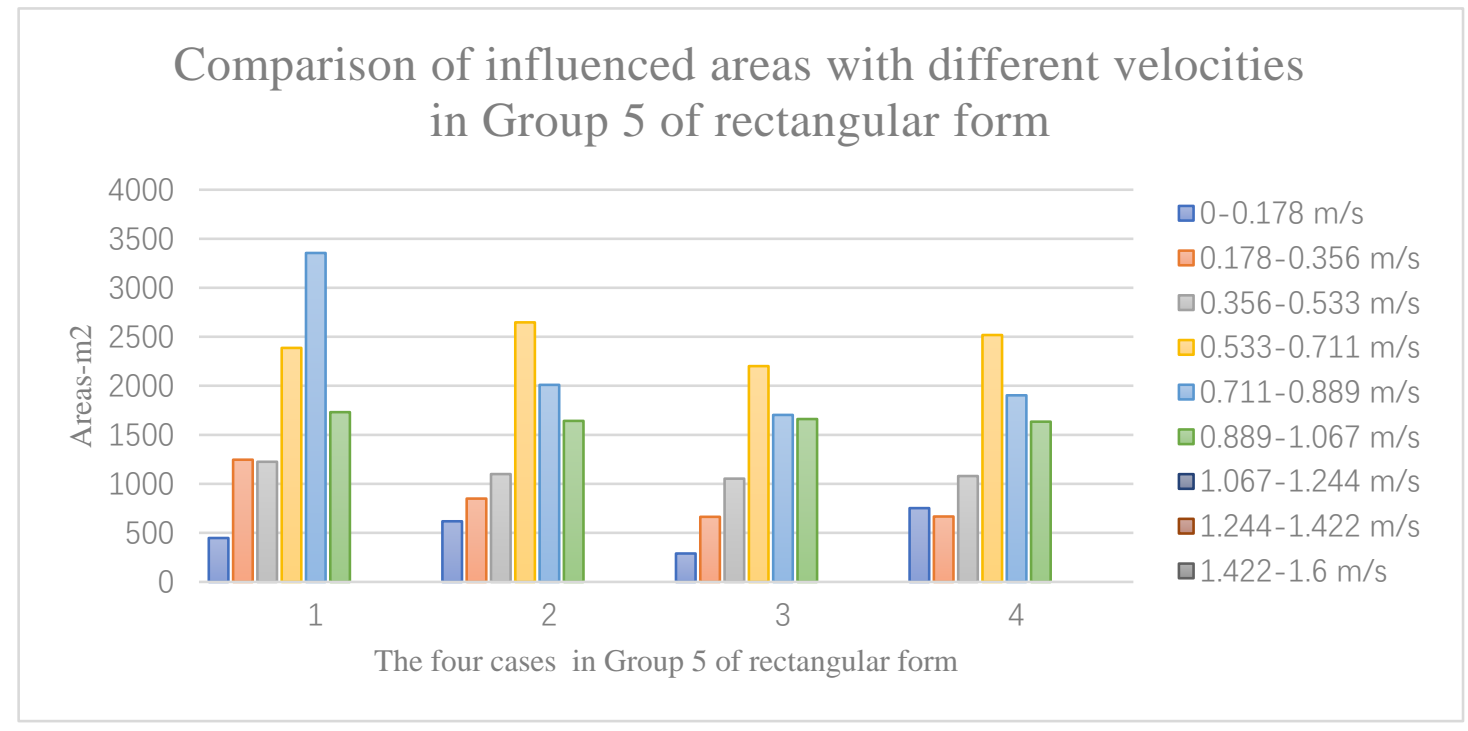

Figure 66. The influenced areas with different velocities in Group 5 of rectangular form.

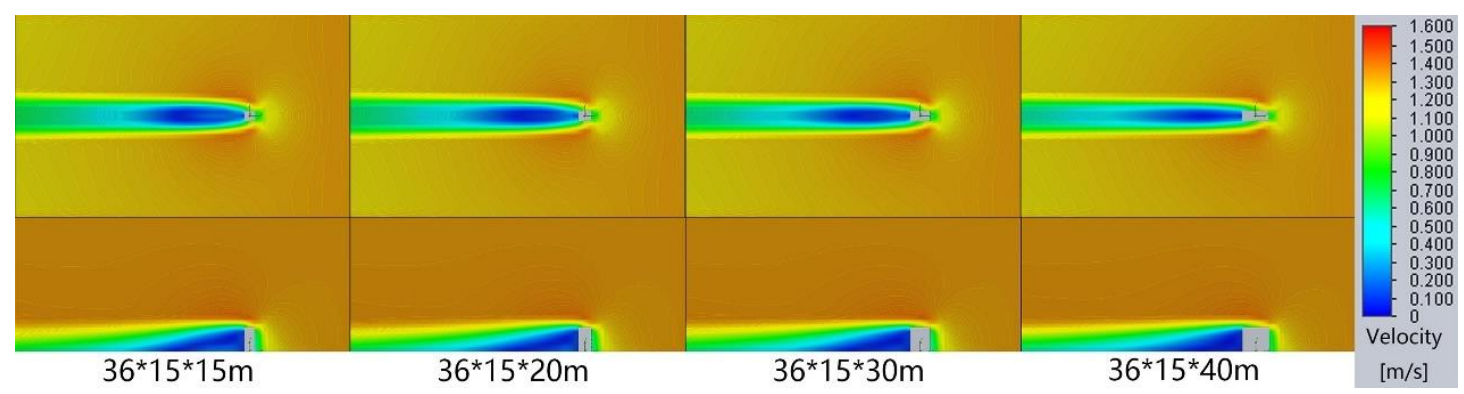

Figure 67. The comparison of wind velocity magnitudes on horizontal and vertical planes of the rectangular-form buildings with different heights in Group 6.

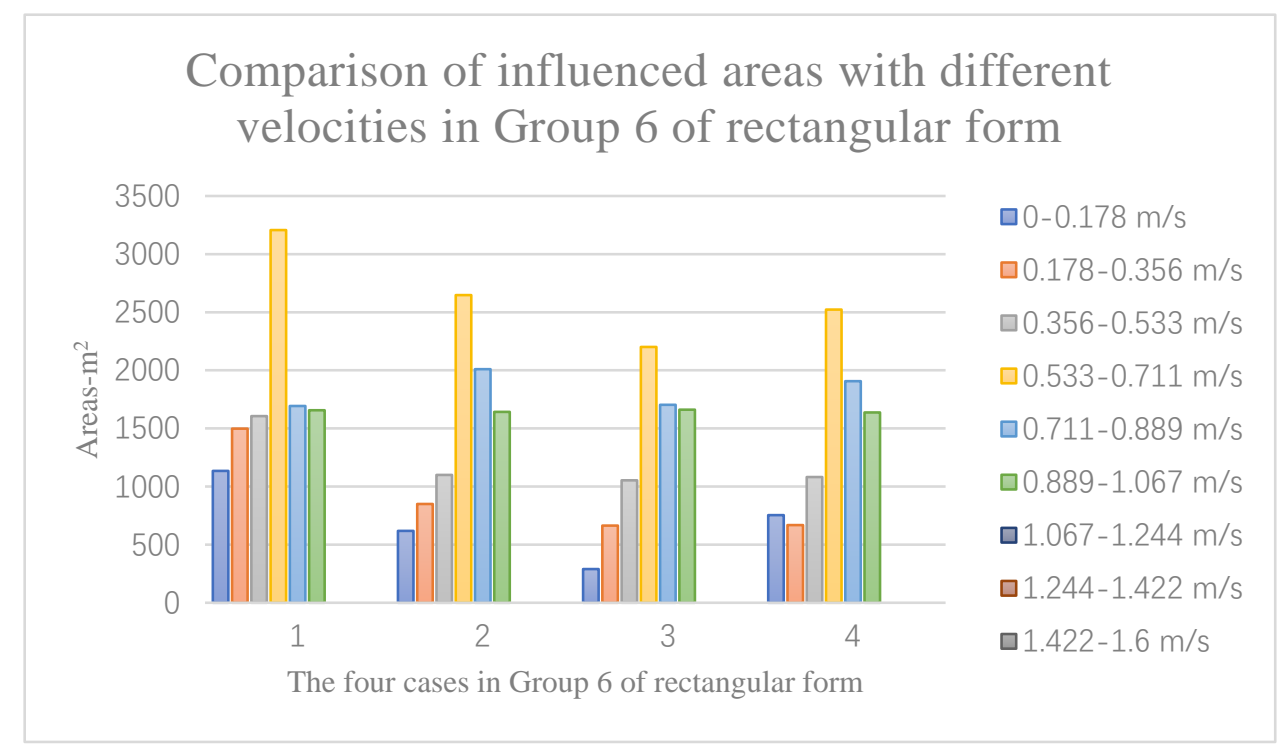

Figure 68. The influenced areas with different velocities in Group 6 of rectangular form. 


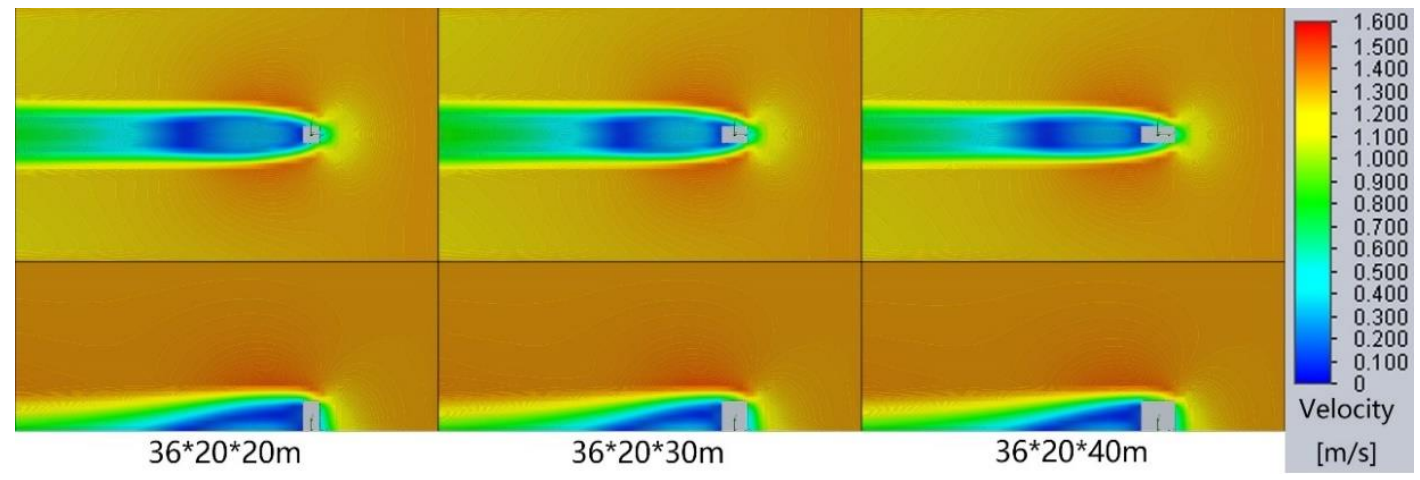

Figure 69. The comparison of wind velocity magnitudes on horizontal and vertical planes of the rectangular-form buildings with different heights in Group 7.

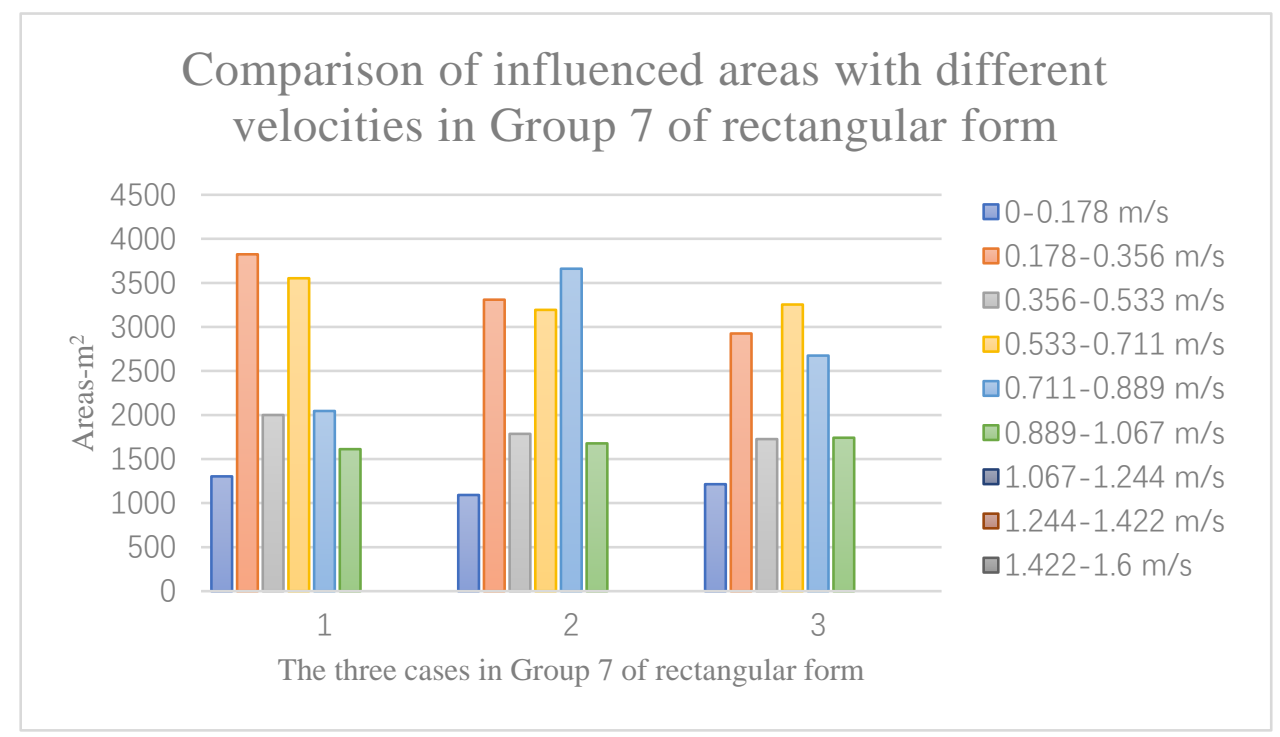

Figure 70. The influenced areas with different velocities in Group 7 of rectangular form.

The changes of influenced areas in Group 5 are closely related to the changes of air pressure and wind flow (Figure 71). As shown in the results of Group 5, the low-air-pressure area behind the building decreases as the length increases; flow of winds becomes smoother, and less vortices are created behind the building. In Case 4, the low-air-pressure area becomes larger and vortices are increased slightly. The situations of Group 6 (Figure 72) and Group 7 (Figure 73) are similar to Group 5. The low-air-pressure area and vortices behind the building decrease constantly as the length increases.

The influence of width corresponds with the air pressure magnitudes and wind flow 
streamlines of the three groups. The low-air-pressure area and high-air-pressure area are increased as the width increases (Figure 71, Figure 72 and Figure 73); more vortices are created behind the building as the width increases (Figure 71, Figure 72 and Figure 73).

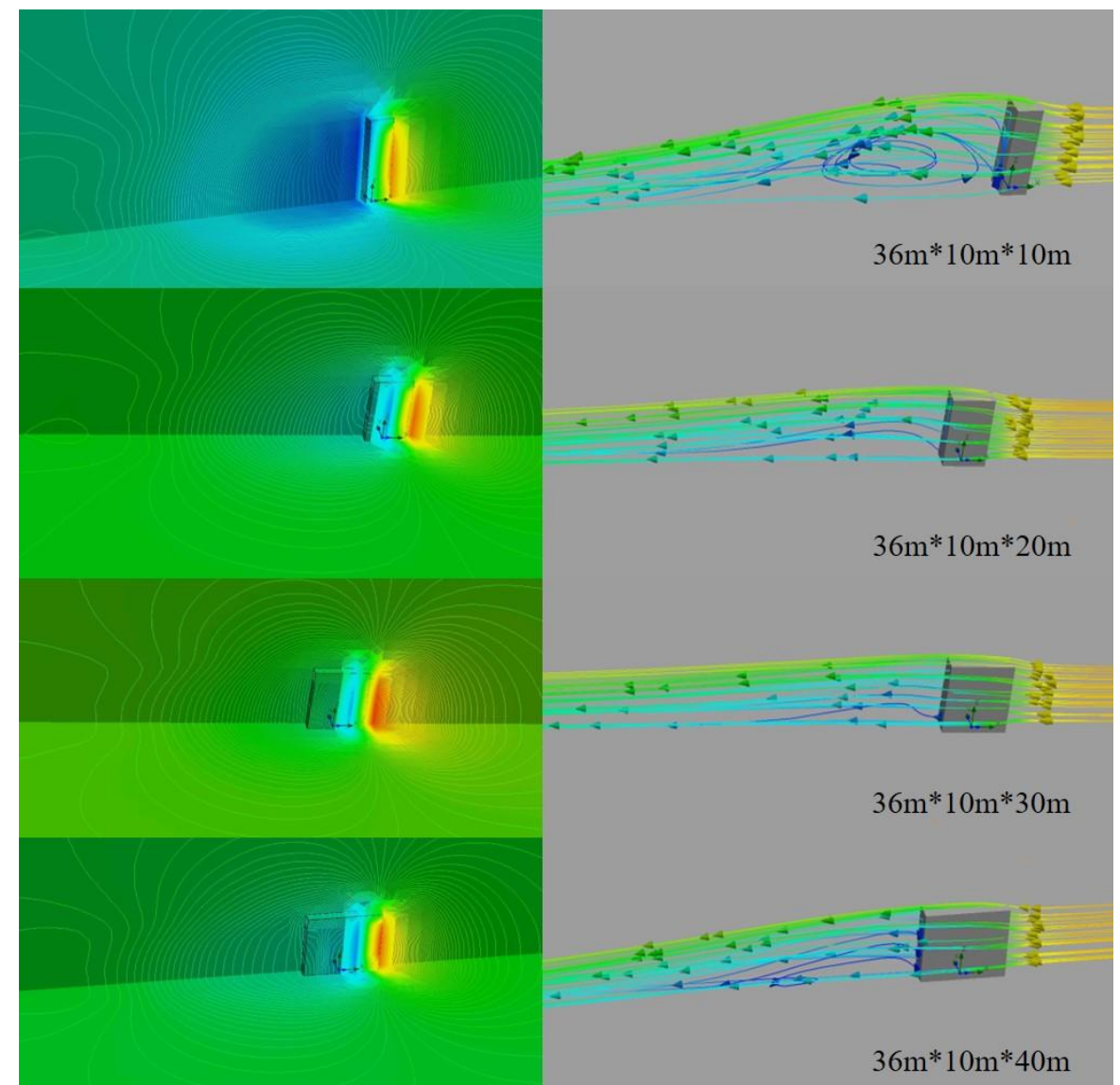

Figure 71. The comparisons of streamlines (right) and air-pressure magnitudes (left) on horizontal, vertical planes and building surfaces of the rectangular-form buildings in Group 5. 


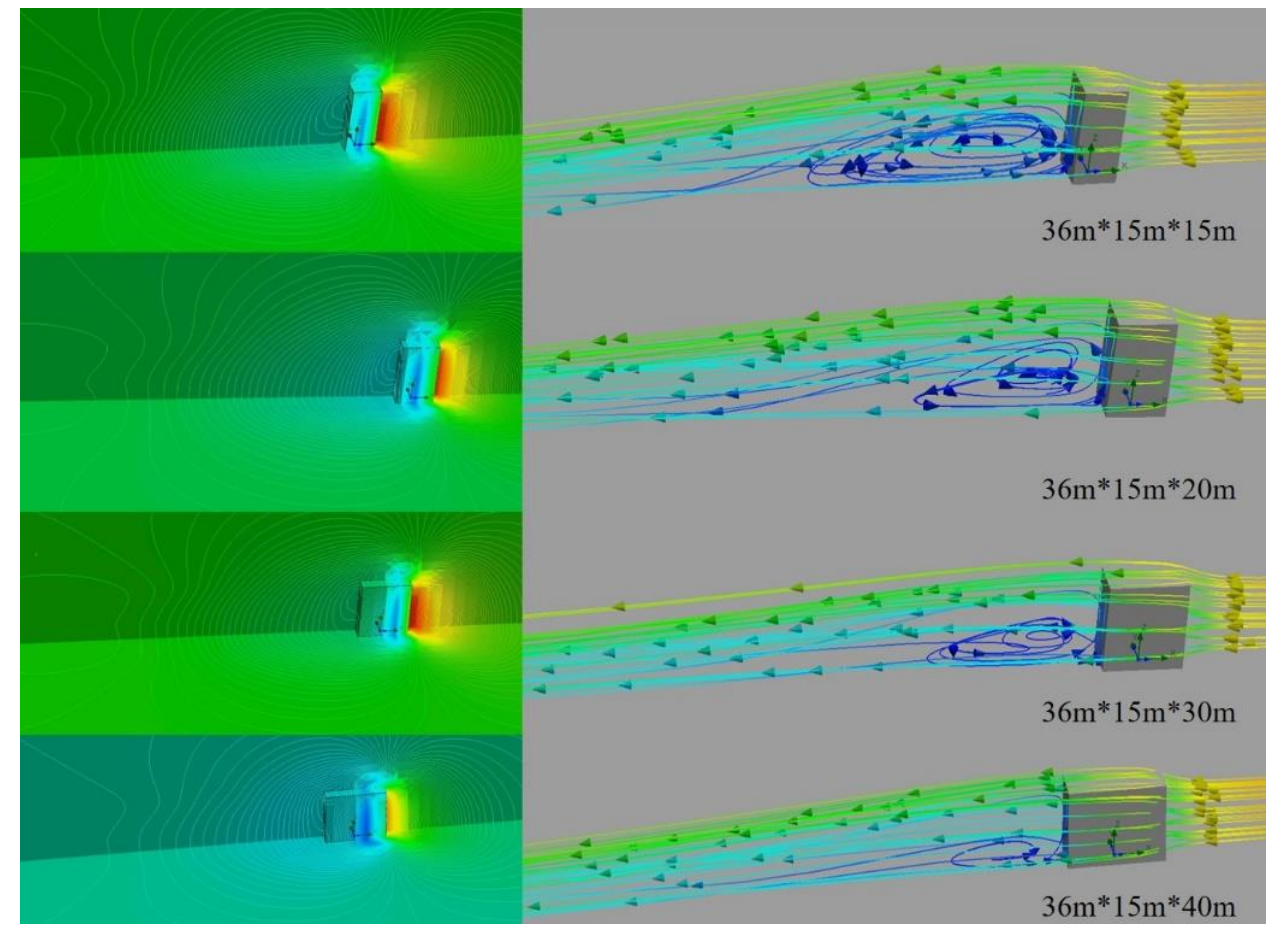

Figure 72. The comparisons of streamlines (right) and air-pressure magnitudes (left) on horizontal, vertical planes and building surfaces of the rectangular-form buildings in Group 6.

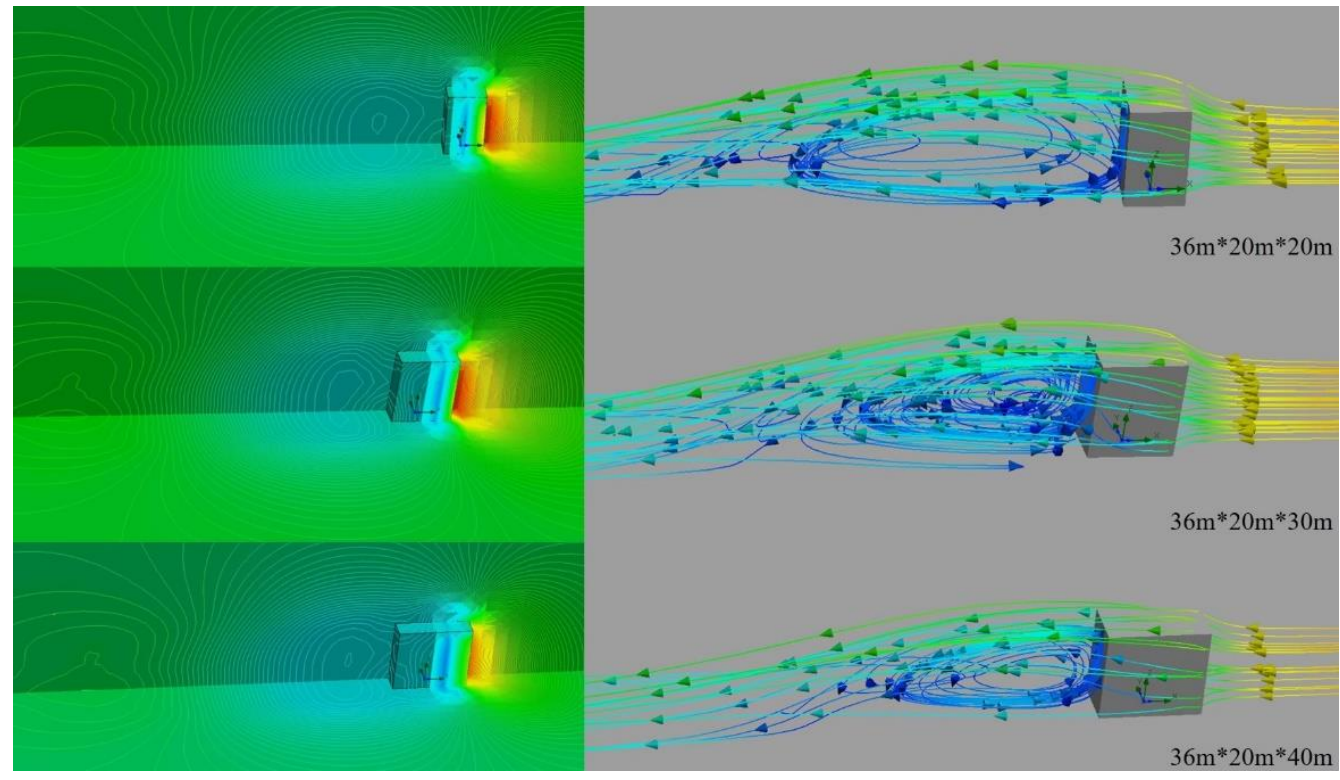

Figure 73. The comparisons of streamlines (right) and air-pressure magnitudes (left) on horizontal, vertical planes and building surfaces of the rectangular-form buildings in Group 7.

\section{(4) Part 4. Influences of different rotation angles}

The simulation results of Group 8 of rectangular form are similar to the Group 2 with different 
rotation angles of square form (Figure 74). In Case 1 (rotation angle: 0 degree), the low-velocity area (velocity below $0.5 \mathrm{~m} / \mathrm{s}$ ) behind the building is the largest and longest; in Case 2 (rotation angle: 30 degree), the low-velocity area is smaller and shorter than Case 1; in Case 3 (rotation angle: 60 degree), the area is smaller and shorter than Case 2; in Case 4 (rotation angle: 90 degree), the low-velocity area is the smallest and shortest of all (Figure 75). The results of Group 9 and Group 10 are similar to Group 8 as trends of the influences are the same (Figure 76, Figure 77, Figure 78 and Figure 79). The influences on wind environments are increased as the building height increases in the two groups. In summary, influences of rectangular-form buildings with the long surfaces on the windward side are decreased constantly as the rotation angles increases.

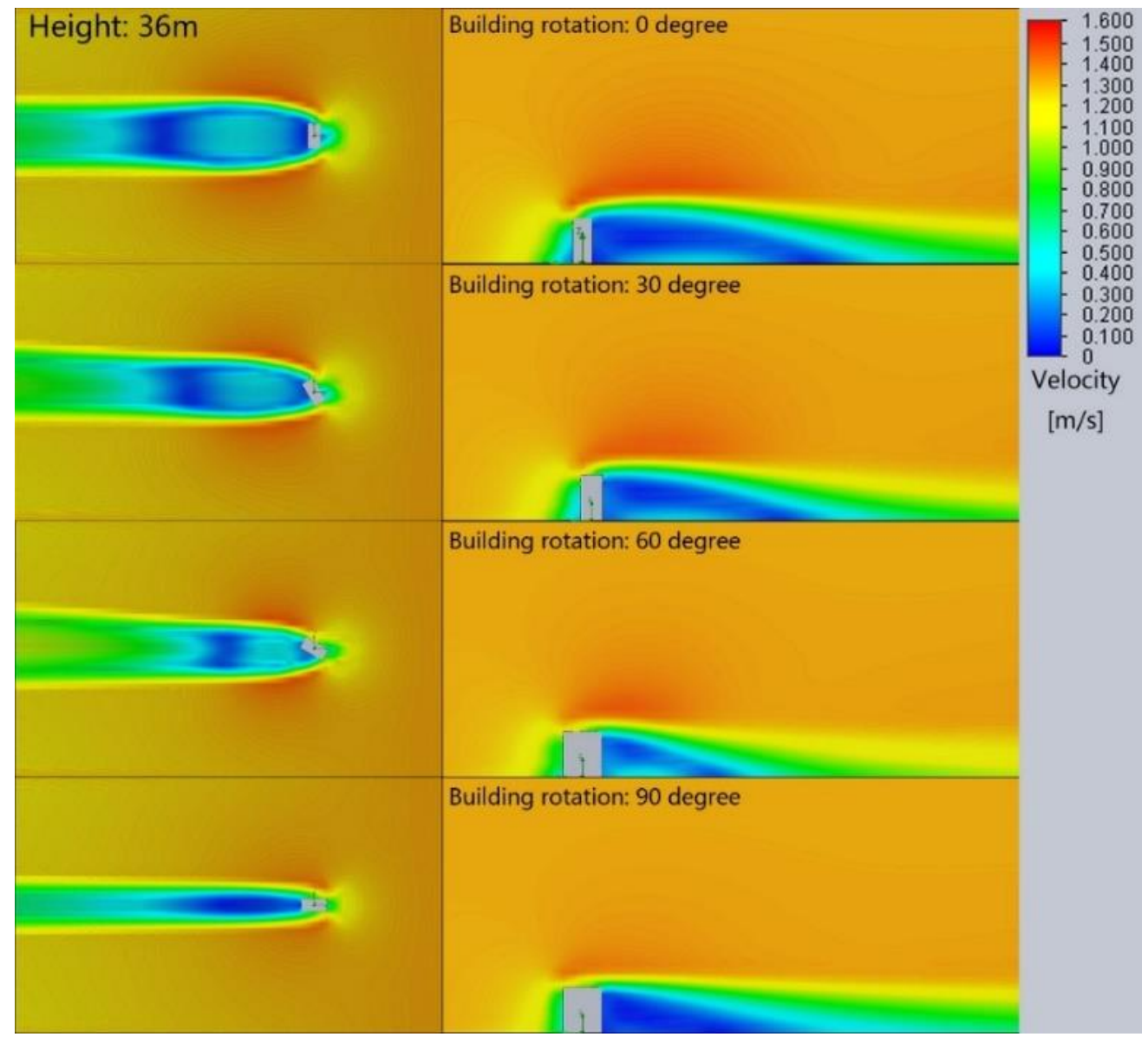

Figure 74. The comparison of wind velocity magnitudes on horizontal and vertical planes of the rectangular-form buildings with different heights in Group 8. 


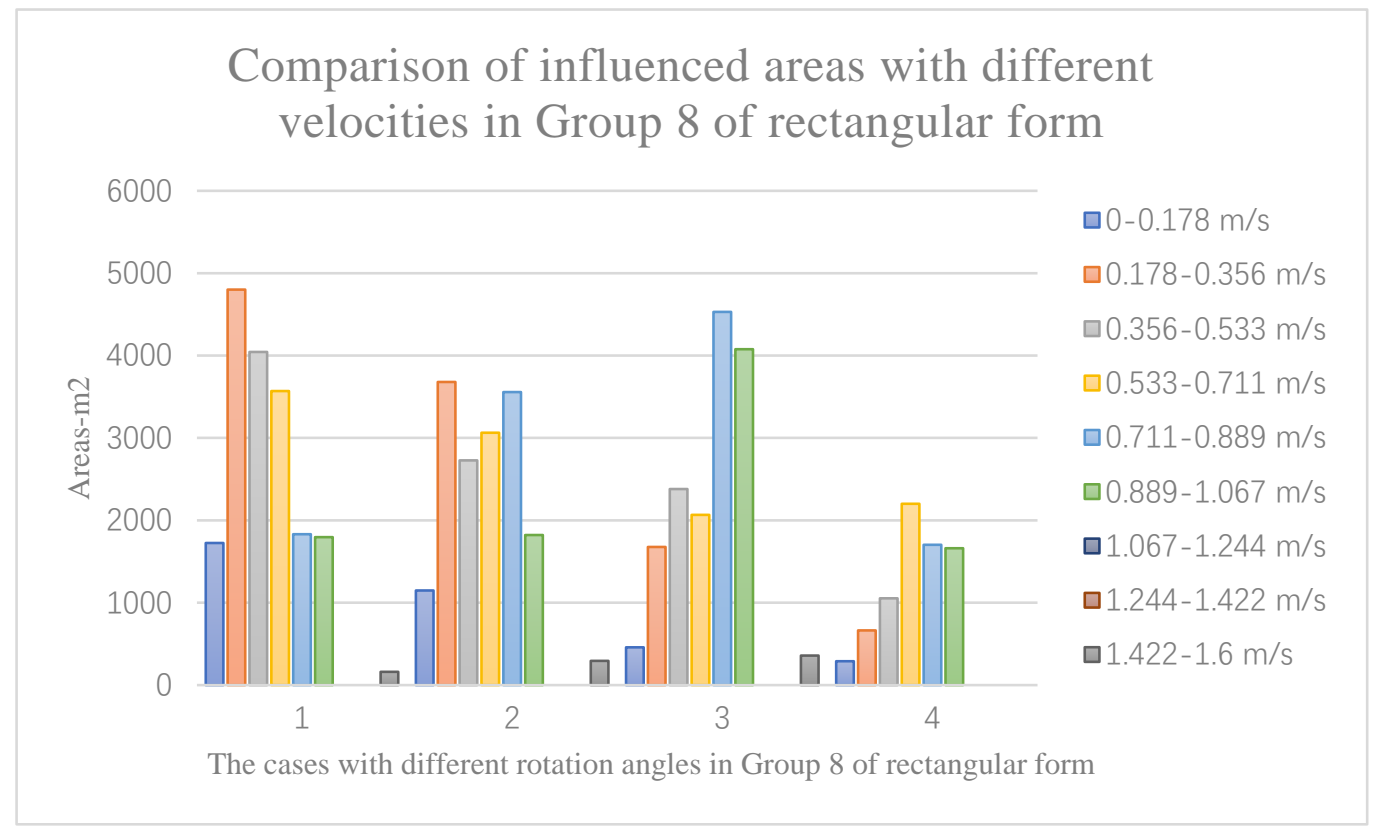

Figure 75. The influenced areas with different velocities in Group 8 of rectangular form.

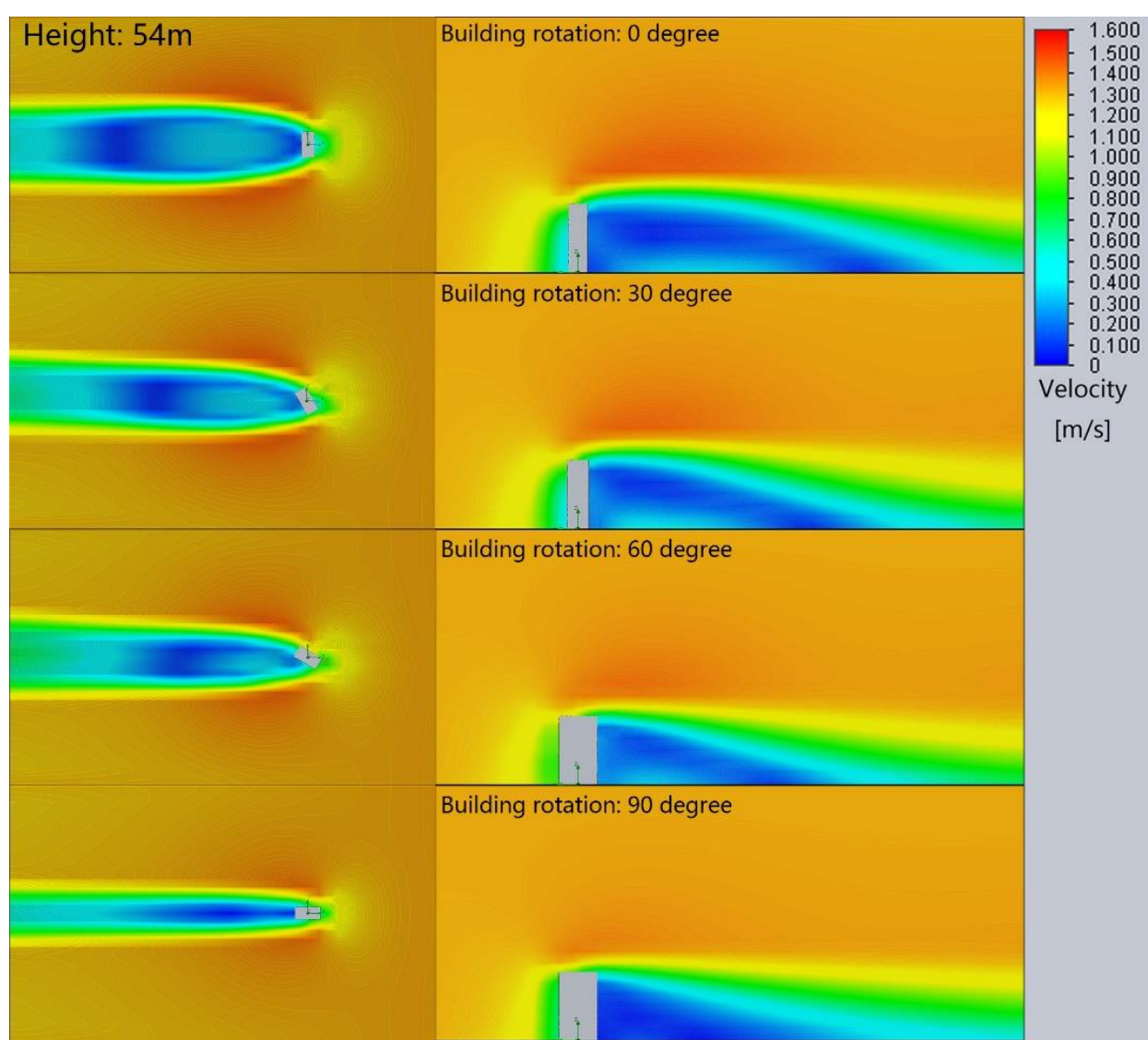

Figure 76. The comparison of wind velocity magnitudes on horizontal and vertical planes of the rectangular-form buildings with different heights in Group 9. 


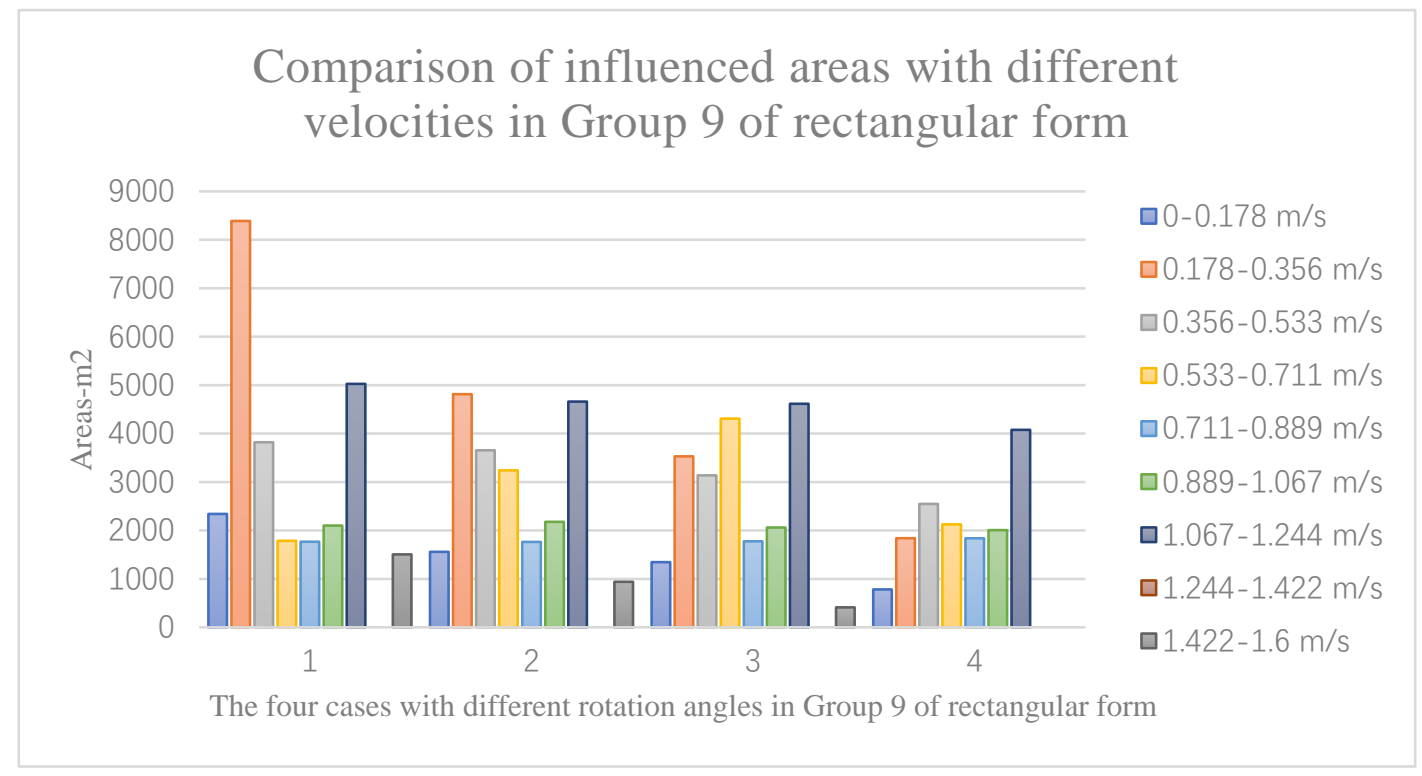

Figure 77. The influenced areas with different velocities in Group 9 of rectangular form.

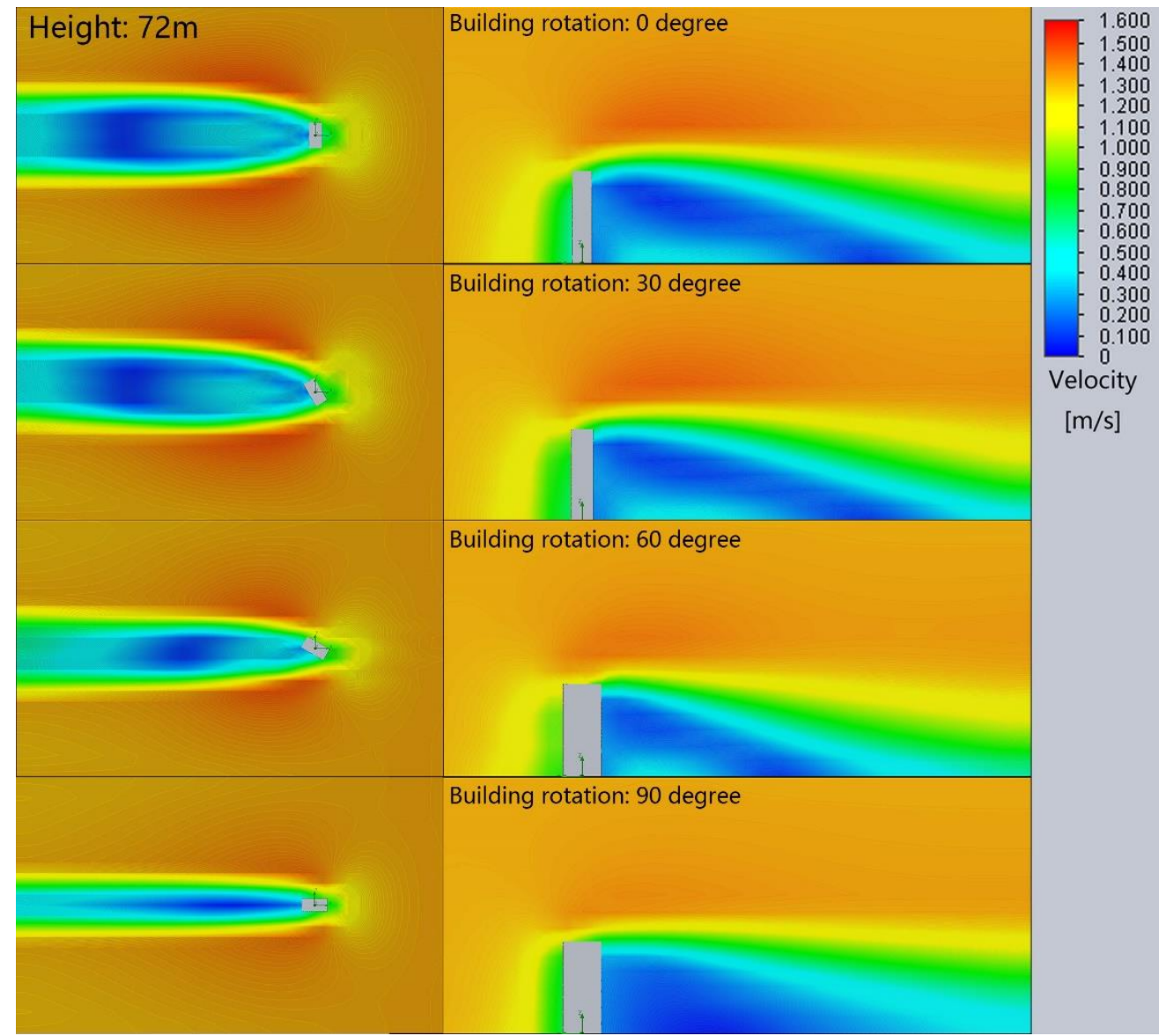

Figure 78. The comparison of wind velocity magnitudes on horizontal and vertical planes of the rectangular-form buildings with different heights in Group 10. 


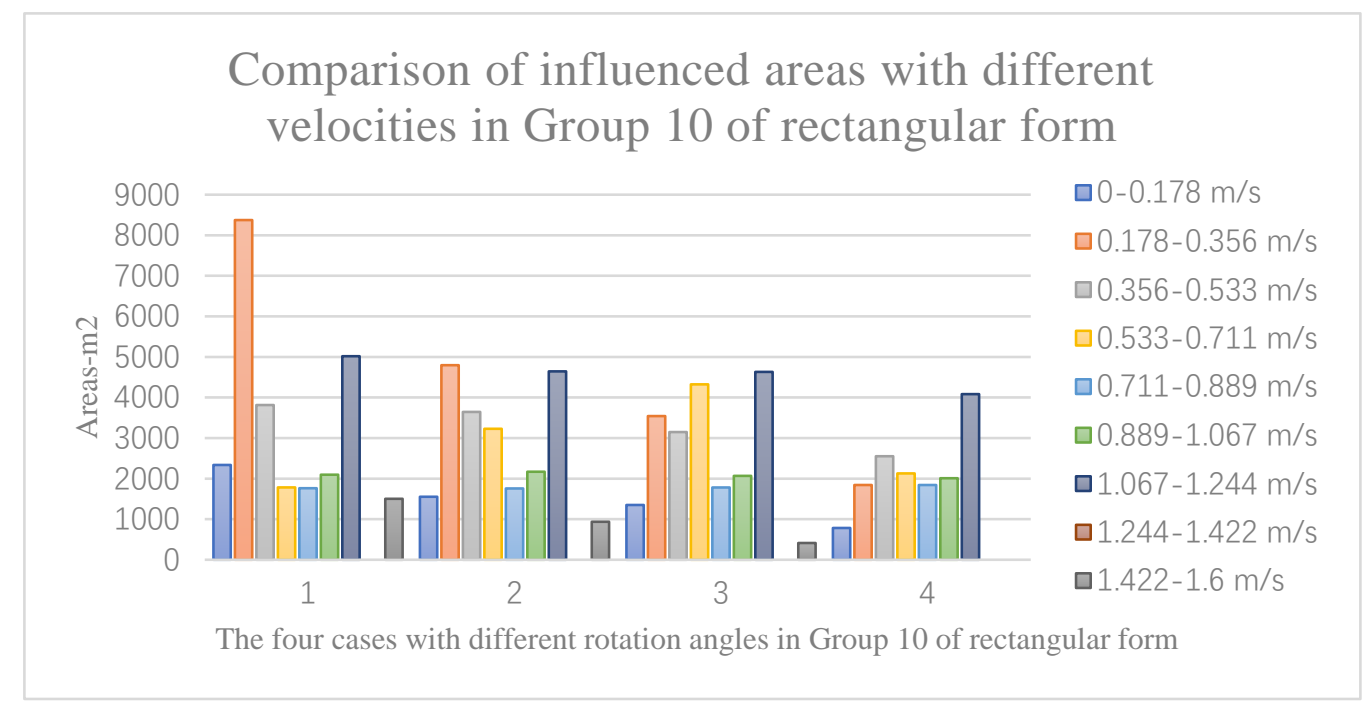

Figure 79. The influenced areas with different velocities in Group 10 of rectangular form.

The changes of influenced areas in different cases of the three groups are related to the changes of air pressure and wind flow (Figure 80, Figure 81 and Figure 82). Winds are obstructed by the windward surfaces as they approach the building. Winds flow to the edges of the windward surface and escape from the two lateral sides and top of the buildings. As the rotation angle increases, the projective area on the windward direction of the building is decreased; winds are divided by the windward edge and the wind flow is smoother on the windward surfaces which are not perpendicular to the wind direction; the low-air-pressure area behind the building and high-air-pressure area in front of the building are both decreased; the vortices behind the building become less and smaller. These can be found in the air-pressure magnitudes and wind flow streamlines of the three groups with different heights (Figure 80, Figure 81 and Figure 82), which are the reasons for the decrease of influence as the rotation angle increases. 


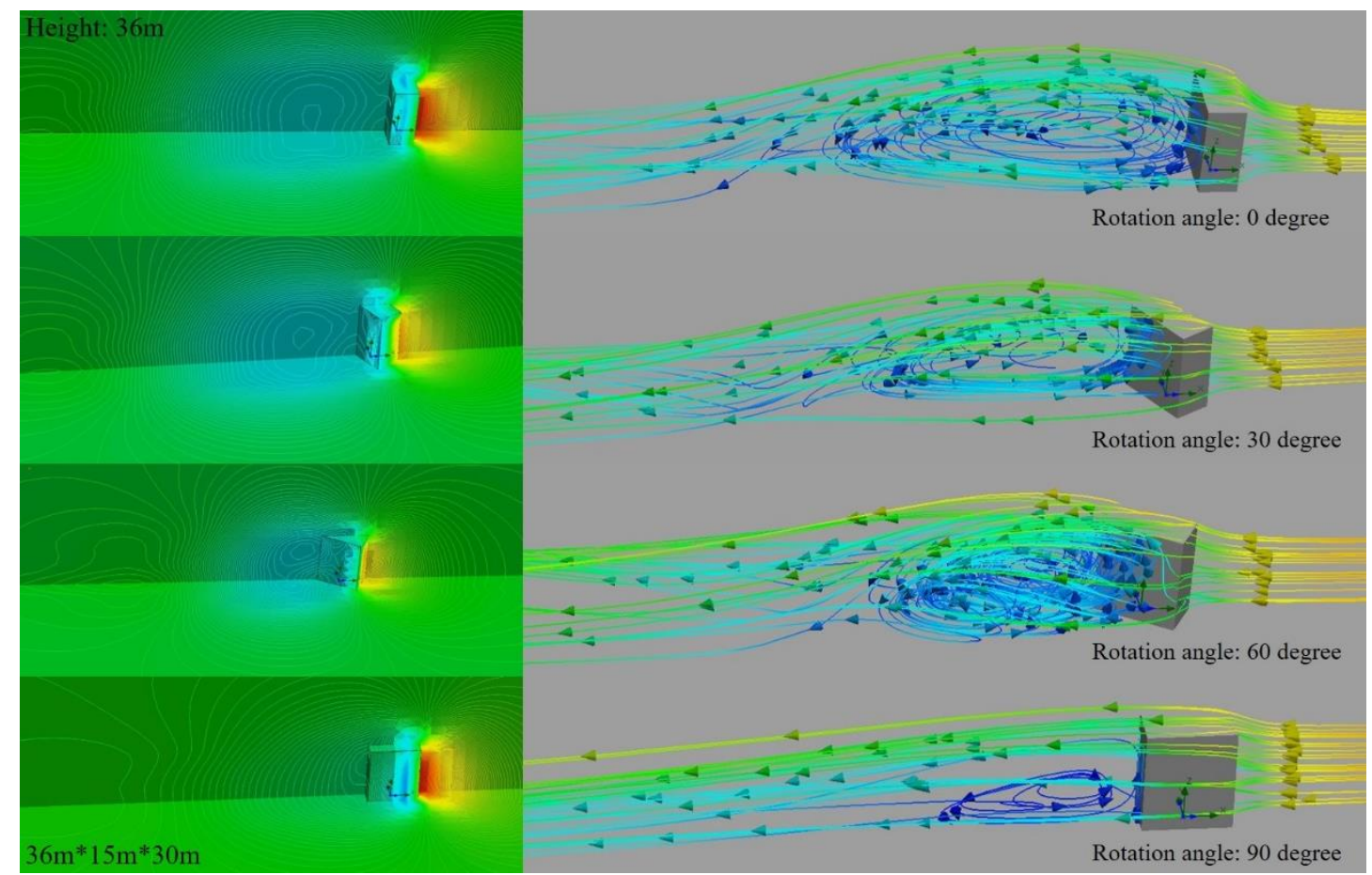

Figure 80 . The comparisons of streamlines (right) and air-pressure magnitudes (left) on horizontal, vertical planes and building surfaces of the rectangular-form buildings in Group 8.

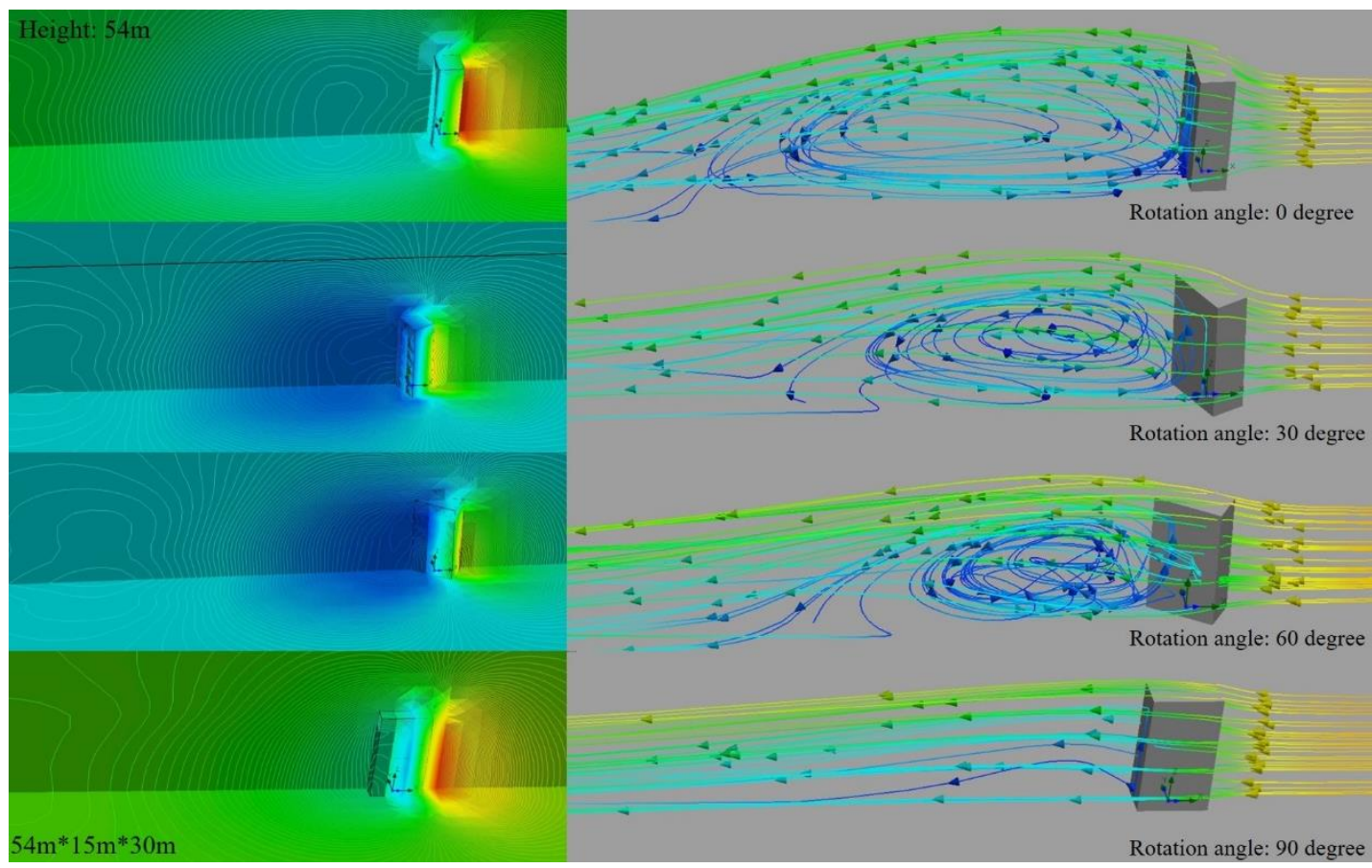

Figure 81 . The comparisons of streamlines (right) and air-pressure magnitudes (left) on horizontal, vertical planes and building surfaces of the rectangular-form buildings in Group 9. 


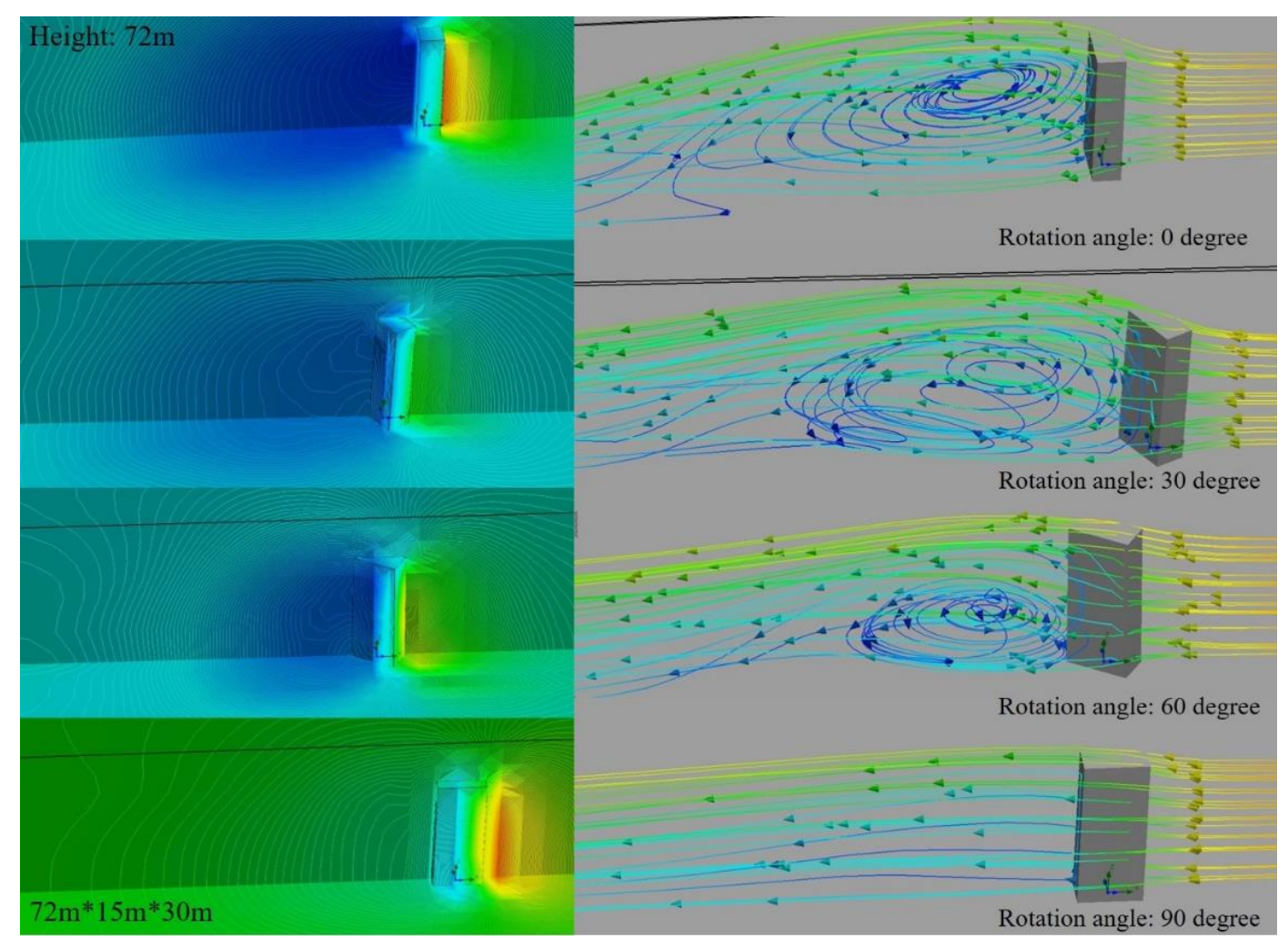

Figure 82. The comparisons of streamlines (right) and air-pressure magnitudes (left) on horizontal, vertical planes and building surfaces of the rectangular-form buildings in Group 10.

\section{4. ' $T$ ' form}

This section aims to study the relationships between the influences on wind environments and the building variables of ' $\mathrm{T}$ ' form, including the length and width of the bulge part (Figure 83). In the first part, the influences of the bulge part with different lengths are studied for the ' $T$ 'form buildings with a typical height of $36 \mathrm{~m}$. The buildings in the first part represent buildings with 12 floors and two apartments on each floor, which are relatively low. In the second part, the influences of the bulge part with different widths and lengths are studied for the ' $\mathrm{T}$ '-form buildings with a typical height of $72 \mathrm{~m}$. The buildings in the second part represent buildings with 24 floors and four apartments on each floor, which are relatively high. The building variables setup of the two parts are shown first. Then results and analysis of the two parts are presented. 


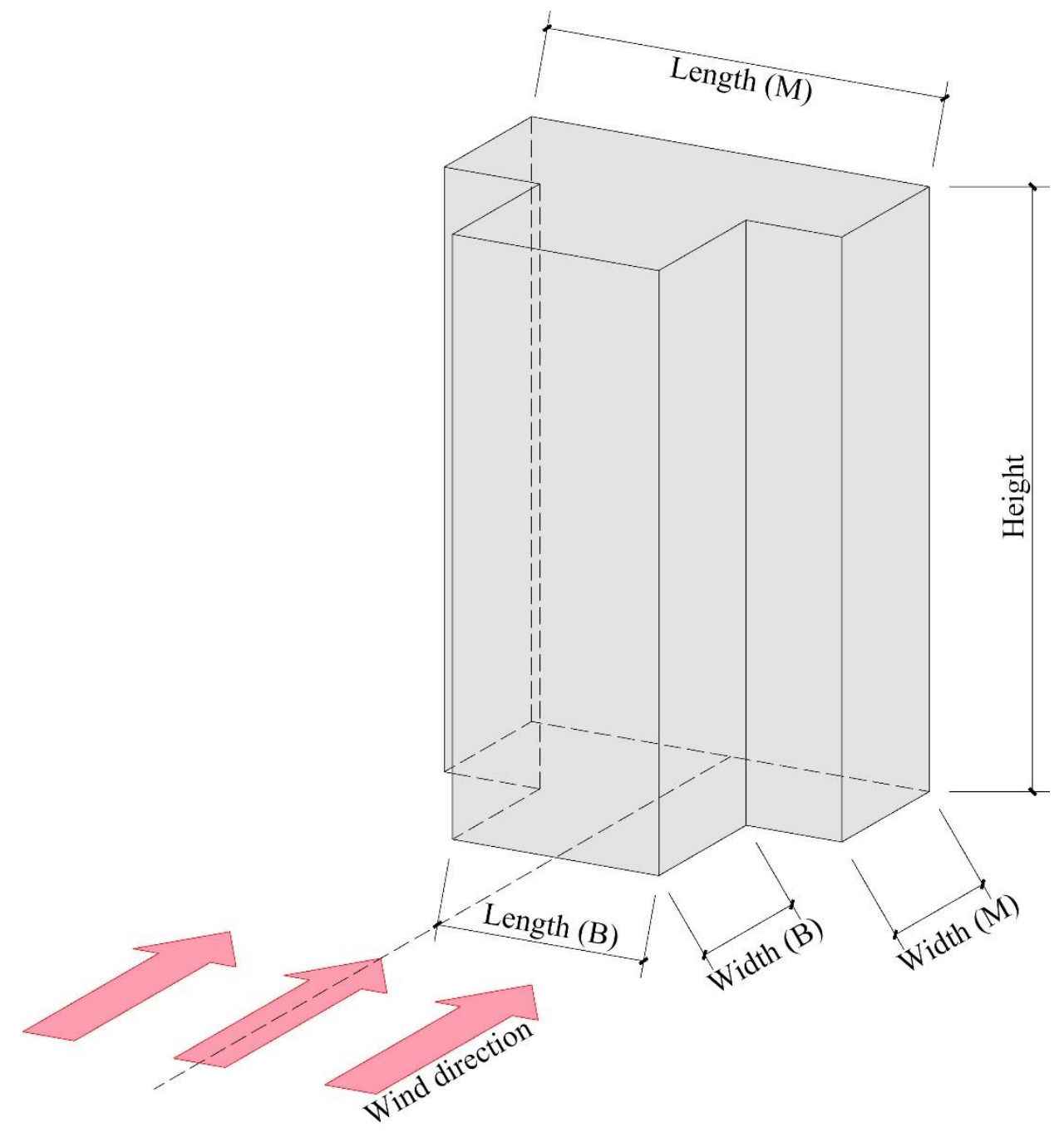

Figure 83. The building variables of ' $\mathrm{T}$ ' form.

\subsubsection{Building variable setup of ' $T$ ' form}

In the first part, five cases with different lengths of the bulge part are set up for simulations (named as Group 1). According to the summary in the parametric design chapter, common lengths in the range of 6-10m and a fixed width of $2 \mathrm{~m}$ are used for the bulge part of the building; the common length of $20 \mathrm{~m}$ and width of $10 \mathrm{~m}$ are used for the major part, which is typical for a residential building with two apartments on each floor; the building height of $36 \mathrm{~m}$ is used in all cases, which is a common height for a high-rise residential building with twelve floors. The specific variables of five cases in Group 1 are presented in the following tables (Table 19.). 
Table 19. The five cases in Group 1 of ' $T$ ' form.

\begin{tabular}{|c|c|c|c|c|c|}
\hline Cases & Lengths (M) & Widths (M) & Heights & Lengths (B) & Widths (B) \\
\hline Case 1 & $20 \mathrm{~m}$ & $10 \mathrm{~m}$ & $36 \mathrm{~m}$ & $0 \mathrm{~m}$ & $0 \mathrm{~m}$ \\
\hline Case 2 & $20 \mathrm{~m}$ & $10 \mathrm{~m}$ & $36 \mathrm{~m}$ & $6 \mathrm{~m}$ & $2 \mathrm{~m}$ \\
\hline Case 3 & $20 \mathrm{~m}$ & $10 \mathrm{~m}$ & $36 \mathrm{~m}$ & $8 \mathrm{~m}$ & $2 \mathrm{~m}$ \\
\hline Case 4 & $20 \mathrm{~m}$ & $10 \mathrm{~m}$ & $36 \mathrm{~m}$ & $10 \mathrm{~m}$ & $2 \mathrm{~m}$ \\
\hline Case 5 & $20 \mathrm{~m}$ & $12 \mathrm{~m}$ & $36 \mathrm{~m}$ & $0 \mathrm{~m}$ & $0 \mathrm{~m}$ \\
\hline \multicolumn{6}{|c|}{$\begin{array}{l}\text { Note: The Length (M) and Width (M) stand for the length and width of the major } \\
\text { part of the building. The Length (B) and Width (B) stand for the length and width }\end{array}$} \\
\hline
\end{tabular}

In the second part, nine cases of three groups are set up for simulations (named as Group 2, Group 3 and Group 4). According to the summary in the parametric design chapter, common lengths in the range of $15-21 \mathrm{~m}$ and widths in the range of $6-18 \mathrm{~m}$ are used for the bulge part of the building; the common length of $39 \mathrm{~m}$ and width of $12 \mathrm{~m}$ are used for the major part, which is typical for a residential building with four apartments on each floor; the building height of $72 \mathrm{~m}$ is used in all cases, which is a common height for a high-rise residential building with 24 floors. The specific variables of nine cases in the three groups are presented in the following tables (Table 20., Table 21. and Table 22.).

Table 20. The three cases in Group 2 of ' $T$ ' form.

\begin{tabular}{|l|l|l|l|l|l|}
\hline Cases & Lengths (M) & Widths (M) & Heights & Lengths (B) & Widths (B) \\
\hline Case 1 & $39 \mathrm{~m}$ & $12 \mathrm{~m}$ & $72 \mathrm{~m}$ & $15 \mathrm{~m}$ & $6 \mathrm{~m}$ \\
\hline Case 2 & $39 \mathrm{~m}$ & $12 \mathrm{~m}$ & $72 \mathrm{~m}$ & $18 \mathrm{~m}$ & $6 \mathrm{~m}$ \\
\hline Case 3 & $39 \mathrm{~m}$ & $12 \mathrm{~m}$ & $72 \mathrm{~m}$ & $21 \mathrm{~m}$ & $6 \mathrm{~m}$ \\
\hline Note: The Length (M) and Width (M) stand for the length and width of the major part \\
of the building. The Length (B) and Width (B) stand for the length and width of the \\
bulge part.
\end{tabular}


Table 21. The three cases in Group 3 of ' $T$ ' form.

\begin{tabular}{|l|l|l|l|l|l|}
\hline Cases & Lengths (M) & Widths (M) & Heights & Lengths (B) & Widths (B) \\
\hline Case 1 & $39 \mathrm{~m}$ & $12 \mathrm{~m}$ & $72 \mathrm{~m}$ & $15 \mathrm{~m}$ & $12 \mathrm{~m}$ \\
\hline Case 2 & $39 \mathrm{~m}$ & $12 \mathrm{~m}$ & $72 \mathrm{~m}$ & $18 \mathrm{~m}$ & $12 \mathrm{~m}$ \\
\hline Case 3 & $39 \mathrm{~m}$ & $12 \mathrm{~m}$ & $72 \mathrm{~m}$ & $21 \mathrm{~m}$ & $12 \mathrm{~m}$ \\
\hline Note: The Length (M) and Width (M) stand for the length and width of the major \\
part of the building. The Length (B) and Width (B) stand for the length and width \\
of the bulge part.
\end{tabular}

Table 22. The three cases in Group 4 of ' $T$ ' form.

\begin{tabular}{|c|c|c|c|c|c|}
\hline Cases & Lengths (M) & Widths (M) & Heights & Lengths (B) & Widths (B) \\
\hline Case 1 & $39 m$ & $12 \mathrm{~m}$ & $72 \mathrm{~m}$ & $15 \mathrm{~m}$ & $18 \mathrm{~m}$ \\
\hline Case 2 & $39 m$ & $12 \mathrm{~m}$ & $72 \mathrm{~m}$ & $18 \mathrm{~m}$ & $18 \mathrm{~m}$ \\
\hline Case 3 & $39 m$ & $12 \mathrm{~m}$ & $72 \mathrm{~m}$ & $21 \mathrm{~m}$ & $18 \mathrm{~m}$ \\
\hline \multicolumn{6}{|c|}{$\begin{array}{l}\text { Note: The Length (M) and Width (M) stand for the length and width of the major } \\
\text { part of the building. The Length (B) and Width (B) stand for the length and width } \\
\text { of the bulge part. }\end{array}$} \\
\hline
\end{tabular}

The parametric modelling script of ' $T$ ' form is used to create the building models. In the above four groups, boundaries of CFD simulation results are all set in the same sizes for comparisons of influences on wind environments.

\subsubsection{Results and analysis of ' $T$ ' form}

The CFD simulation results of ' $T$ ' form are analyzed in this subsection. Influences on wind environments are compared based on velocity magnitudes. The mechanisms are explained using the air pressure magnitudes and wind flow streamlines. In Part 1, the relationship between influences and lengths of the bulge part of the 36m-height building is studied. In Part 2, the relationships between influences and lengths and widths of the bulge part of the $72 \mathrm{~m}$-height 
building are studied.

\section{(1) Part 1. Influences of different bulge-part lengths of 36m-height buildings}

In this part, simulation results are analyzed for the ' $\mathrm{T}$ '-form buildings with different bulge-part lengths in Group 1 (Figure 84). The results include two cases of rectangular-form buildings for comparisons (Case 1: 10m*20m*36m; Case 5: 12m*20m*36m). The building of Case 1 has the largest influence on wind environment; the influence of Case 2 (bulge-part length: $6 \mathrm{~m}$ ) is less than Case 1; the influence of Case 3 (bulge-part length: $8 \mathrm{~m}$ ) is less than Case 2; the influence of Case 4 (bulge-part length: 10m) is less than Case 3; the building of Case 5 has the least influence of all (Figure 85). In Case 1, the low-velocity area (velocity below $0.5 \mathrm{~m} / \mathrm{s}$ ) is the largest and its length is the longest; the low-velocity area decreases gradually in Case 2, Case 3 and Case 4; In Case 5, the low-velocity area is the smallest and its length is the shortest (Figure 85). In Case 1, the high-velocity areas (velocity over 1.4m/s) on the lateral sides are the largest; as the length increases, the areas decrease gradually in Case 2, Case 3 and Case 4; in Case 5, the areas are the smallest (Figure 85). In summary, the influence on wind environment of the ' $\mathrm{T}$ '-form building decreases as the bulge-part length increases. 


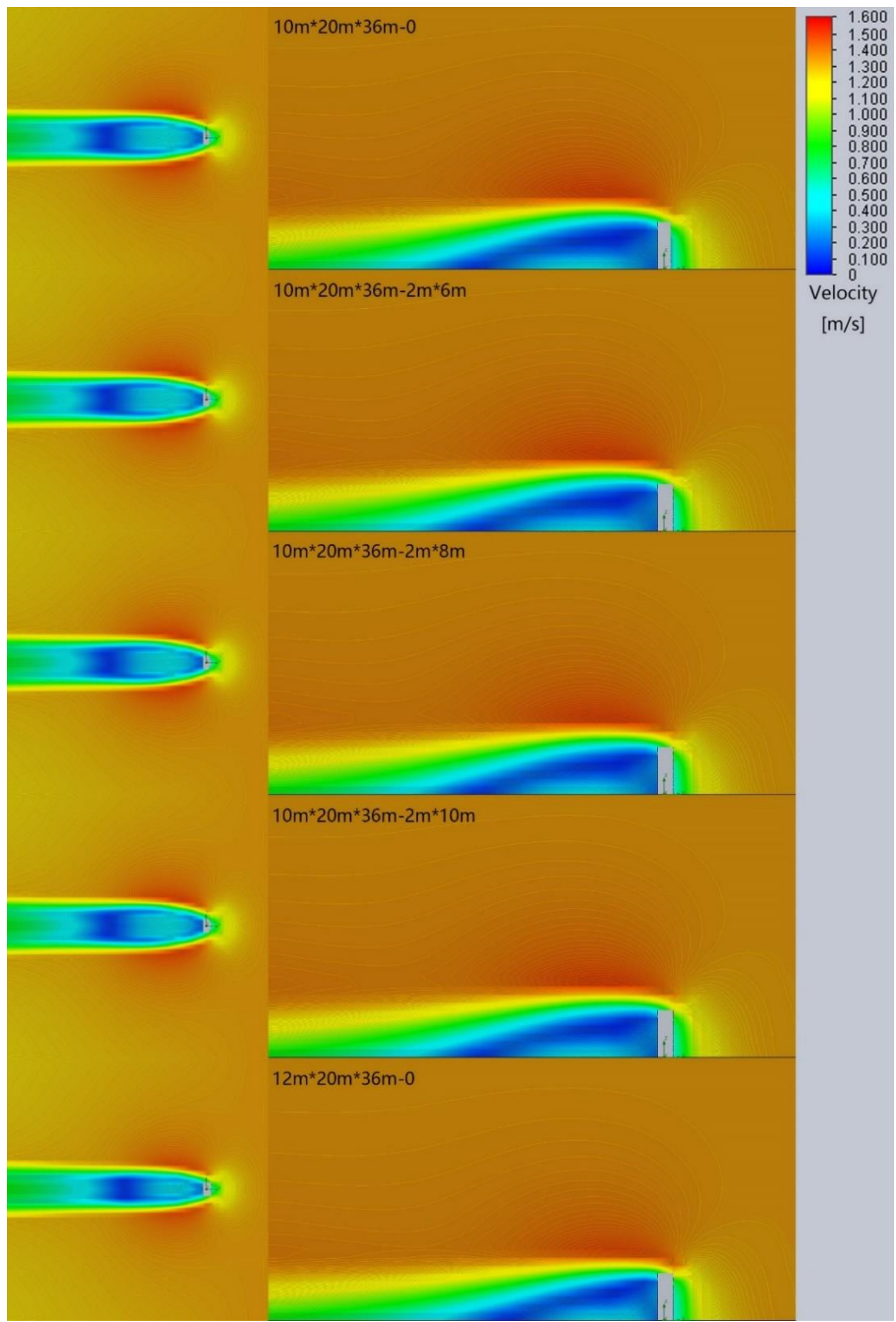

Figure 84 . The comparison of wind velocity magnitudes on horizontal and vertical planes of the ' $\mathrm{T}$ '-form buildings with different heights in Group 1. 


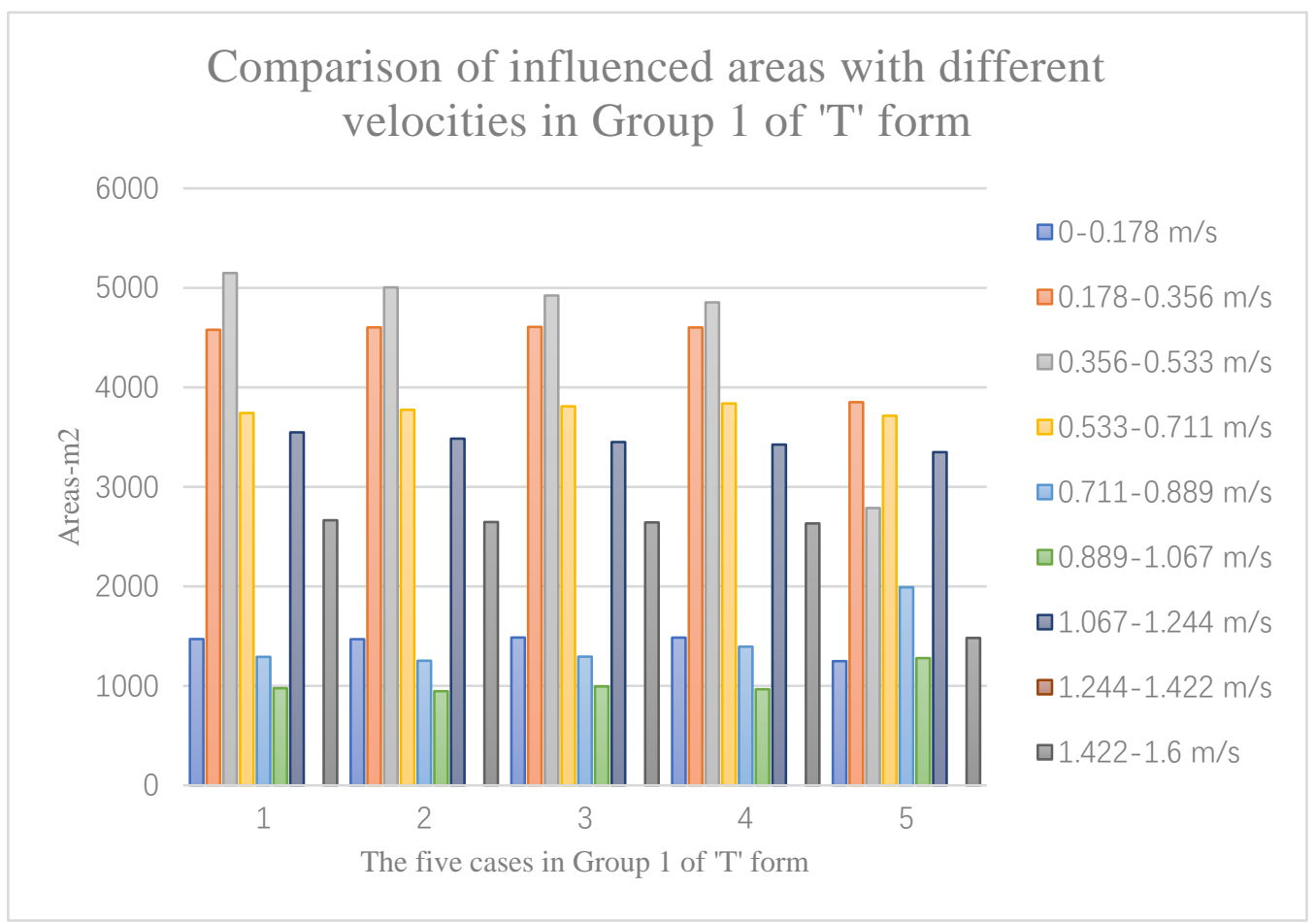

Figure 85. The influenced areas with different velocities in Group 1 of ' $\mathrm{T}$ ' form.

The changes of influenced areas with different velocities in Group 1 are related to the changes of air pressure and wind flow (Figure 86). Usually, the bulge parts are on the windward side according to the common directions of seasonal winds in summer and transitional seasons. Therefore, winds meet the bulge parts first as they approach the buildings. From Case 1 to Case 5, the low-air-pressure area behind the building and the high-air-pressure area in front of the building are decreased as the bulge-part length increases; the vortices on the leeward side of the building become smaller and less as the length increases. These correspond with the results of influenced areas as presented above. 


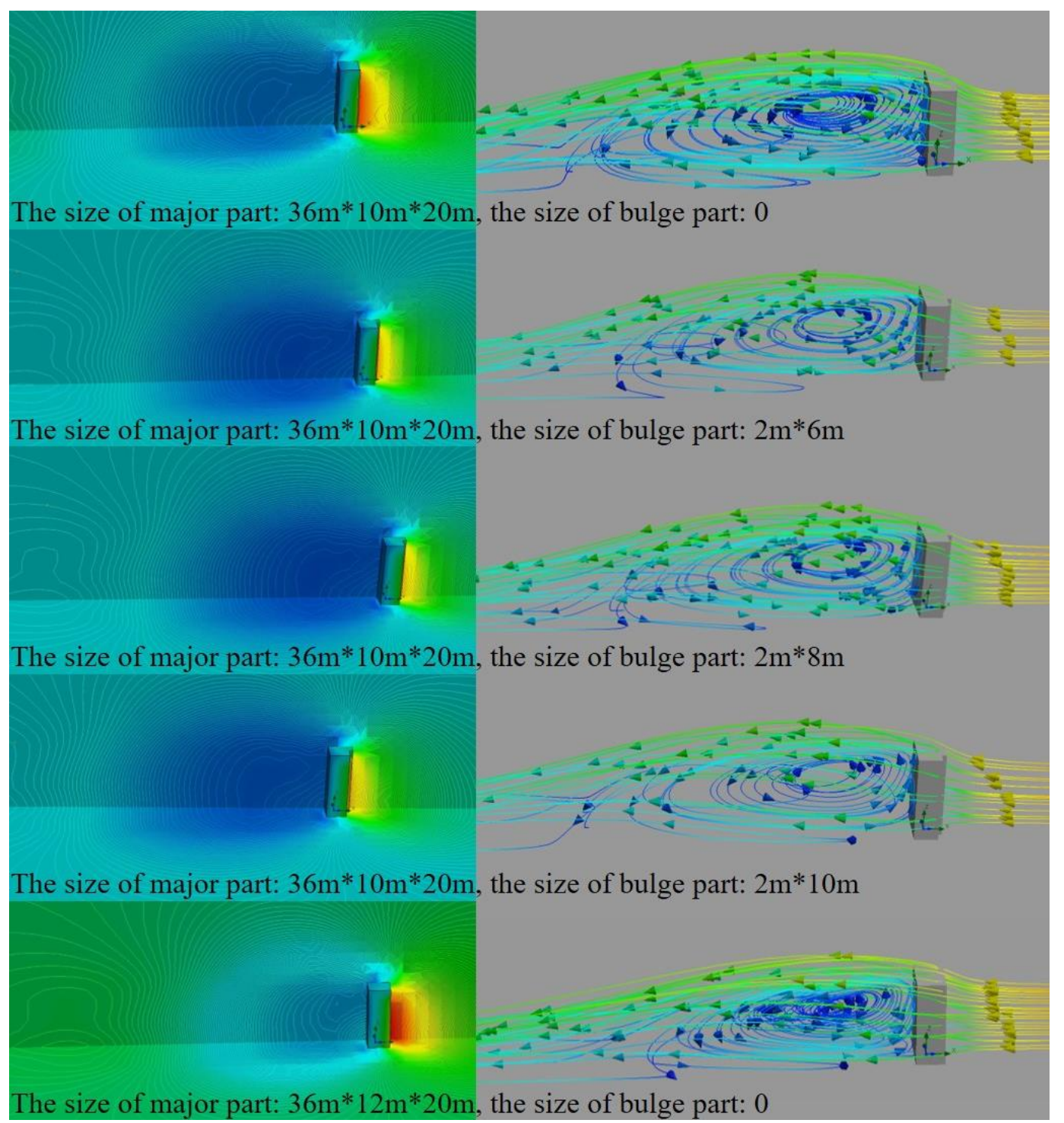

Figure 86. The comparisons of streamlines (right) and air-pressure magnitudes (left) on horizontal, vertical planes and building surfaces of the ' $\mathrm{T}$ '-form buildings in Group 1.

\section{(2) Part 2. Influences of different bulge-part lengths of 72m-height buildings}

In this part, simulation results are analyzed for the buildings with different bulge-part lengths and widths in Group 2, Group 3 and Group 4. In Group 2 (bulge-part width: 6m), the building of Case 1 (bulge-part length: 15m) has the largest influence on wind environment; the influence of Case 2 (bulge-part length: 18m) is between Case 1 and Case 3; the building of Case 3 (bulgepart length: $21 \mathrm{~m}$ ) has the least influence (Figure 87 and Figure 88). In Case 1, the low-velocity area (velocity below $0.5 \mathrm{~m} / \mathrm{s}$ ) is the largest and its length is the longest; the low-velocity area of Case 2 is less than Case 1; In Case 3, the low-velocity area is the smallest and shortest (Figure 87 and Figure 88 ). In Case 1, the high-velocity areas (velocity over $1.4 \mathrm{~m} / \mathrm{s}$ ) on the lateral sides 
are the largest; the areas of Case 2 are less than Case 1; in Case 3, the areas are the smallest (Figure 88). According to the simulation results, the trends of Group 3 (bulge-part width: 12m) and Group 4 (bulge-part width: 18m) are similar to Group 2 (Figure 89, Figure 90, Figure 91, and Figure 92).

Buildings with different bulge-part widths $(6 \mathrm{~m}, 12 \mathrm{~m}$ and $18 \mathrm{~m})$ are also compared in the three groups together. When the bulge-part length has been fixed, the building with the bulgepart width of $6 \mathrm{~m}$ has the most influence; the building with the bulge-part width of $18 \mathrm{~m}$ has the least influence; the influence of the building with the bulge-part width of $12 \mathrm{~m}$ is between the former cases. The low-velocity and high-velocity areas decrease as the width increases. Though differences among the cases are little, the reduction of the influence on wind environment can still be measured. In summary, the influence on wind environment of ' $\mathrm{T}$ ' form decreases as the length and width of the bulge part are increased.

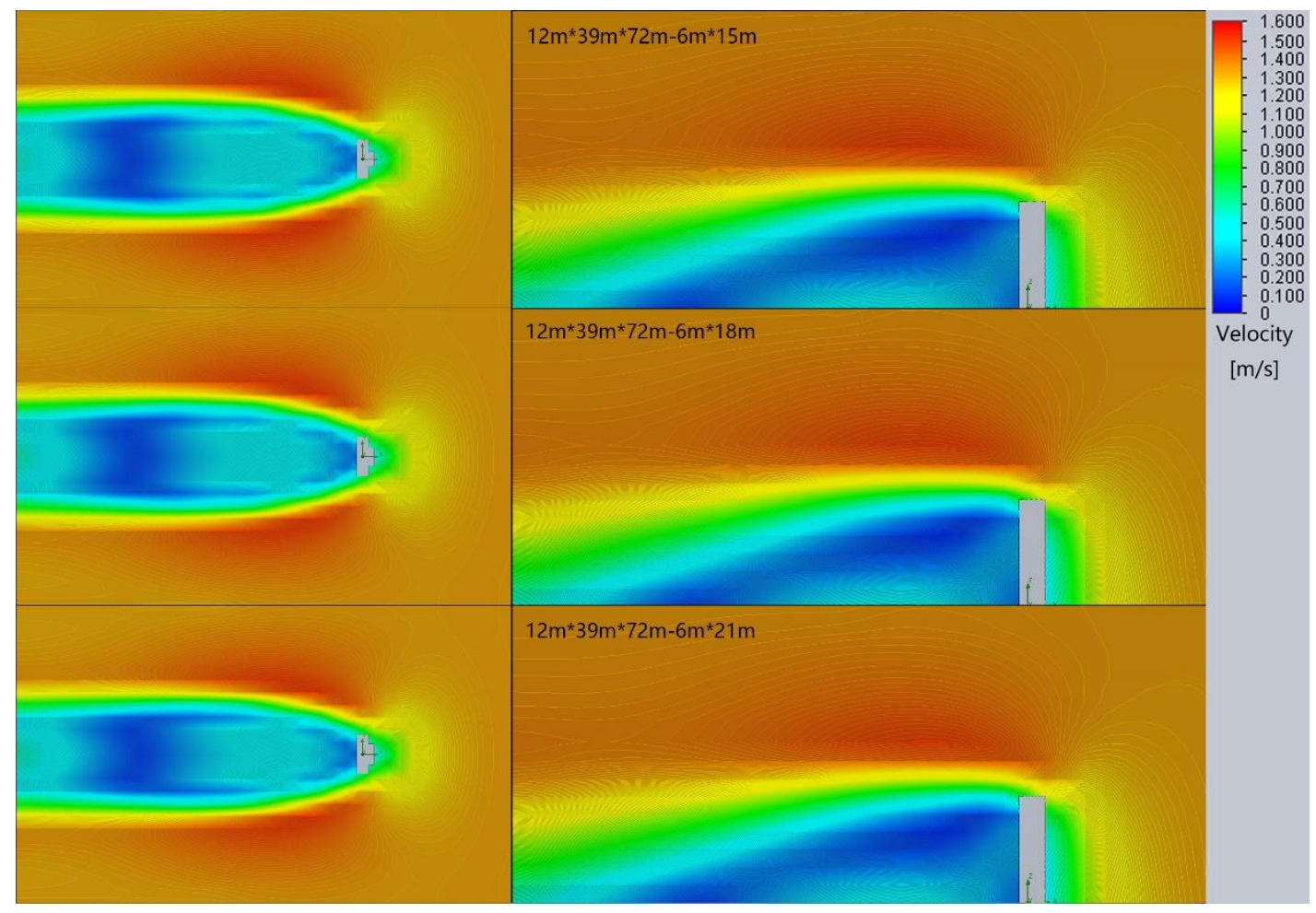

Figure 87 . The comparison of wind velocity magnitudes on horizontal and vertical planes of the ' $\mathrm{T}$ '-form buildings with different heights in Group 2. 


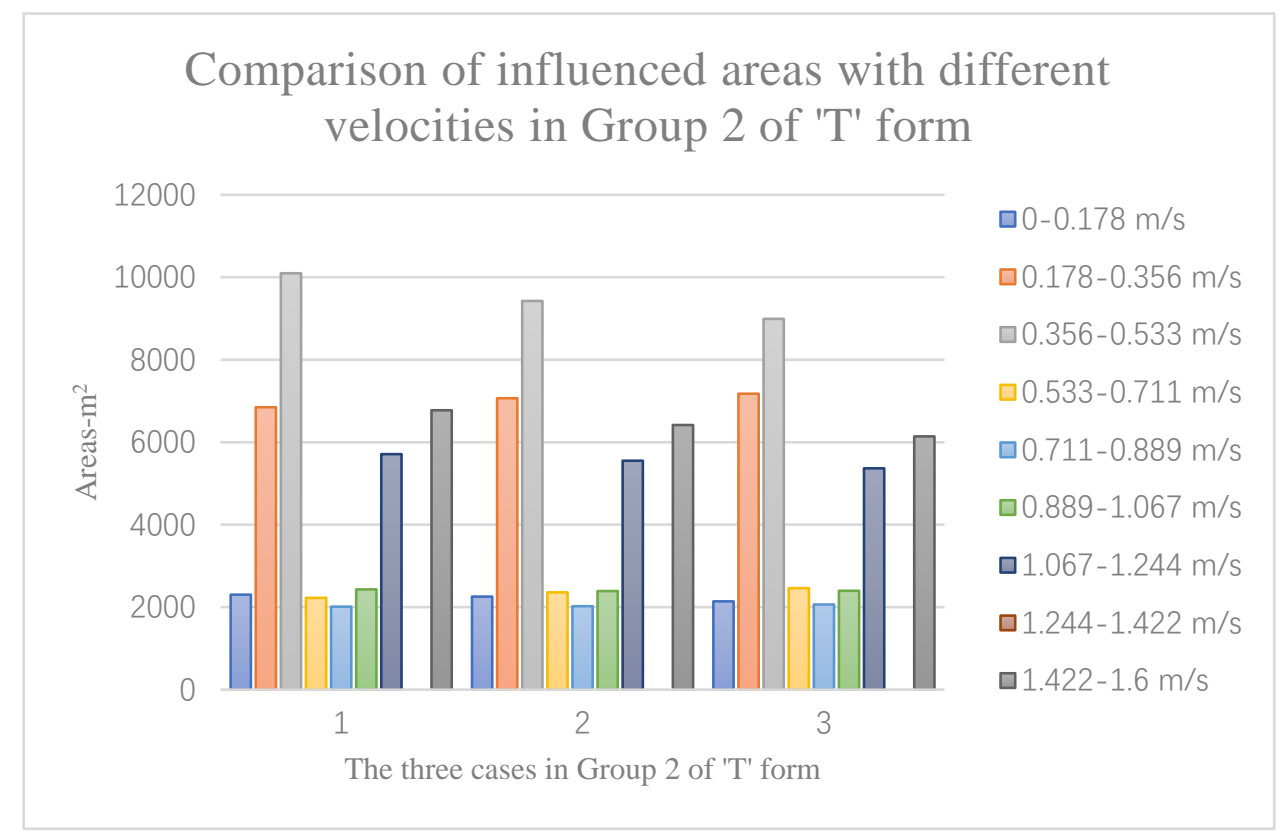

Figure 88. The influenced areas with different velocities in Group 2 of ' $\mathrm{T}$ ' form.

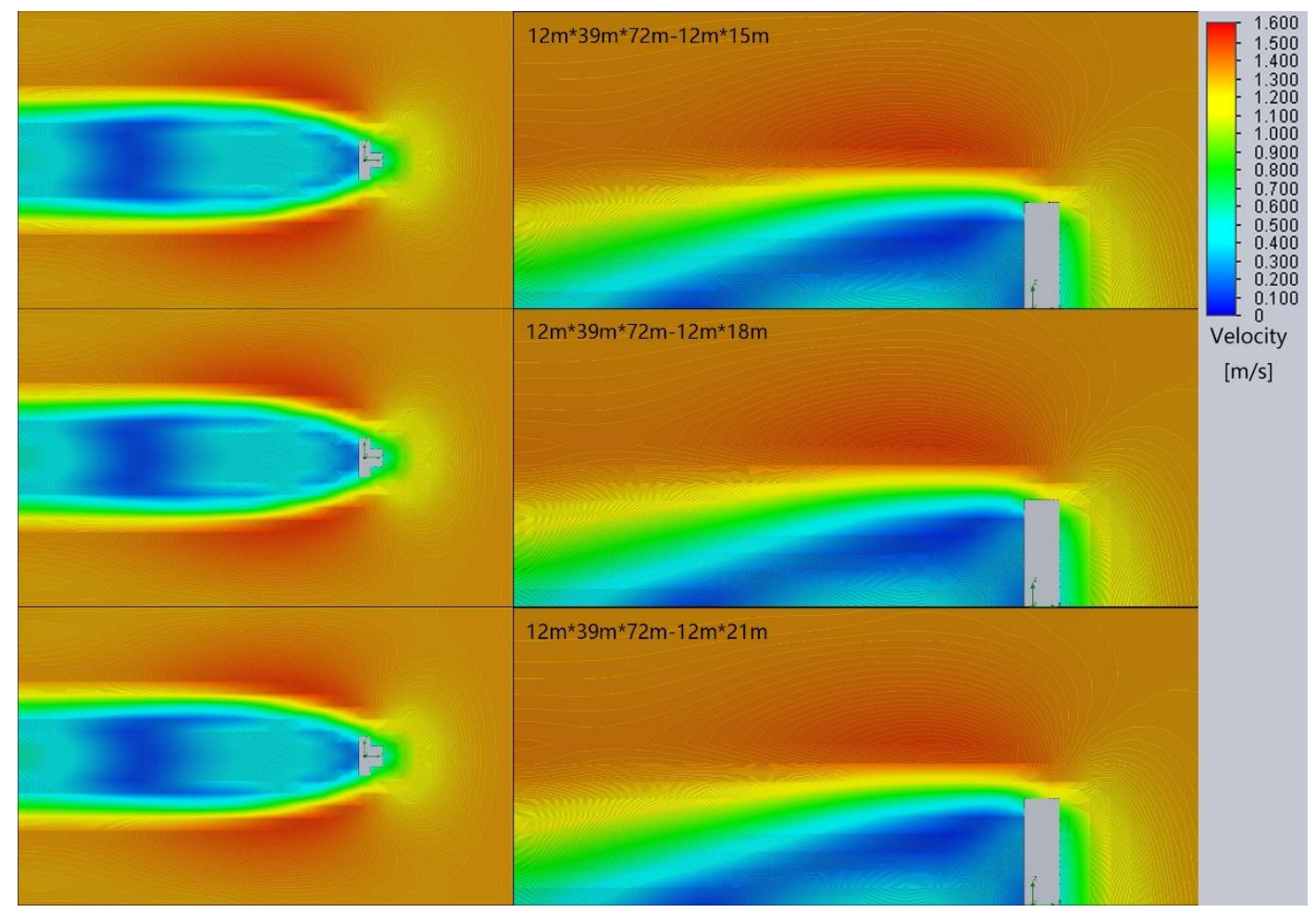

Figure 89 . The comparison of wind velocity magnitudes on horizontal and vertical planes of the ' $\mathrm{T}$ '-form buildings with different heights in Group 3. 


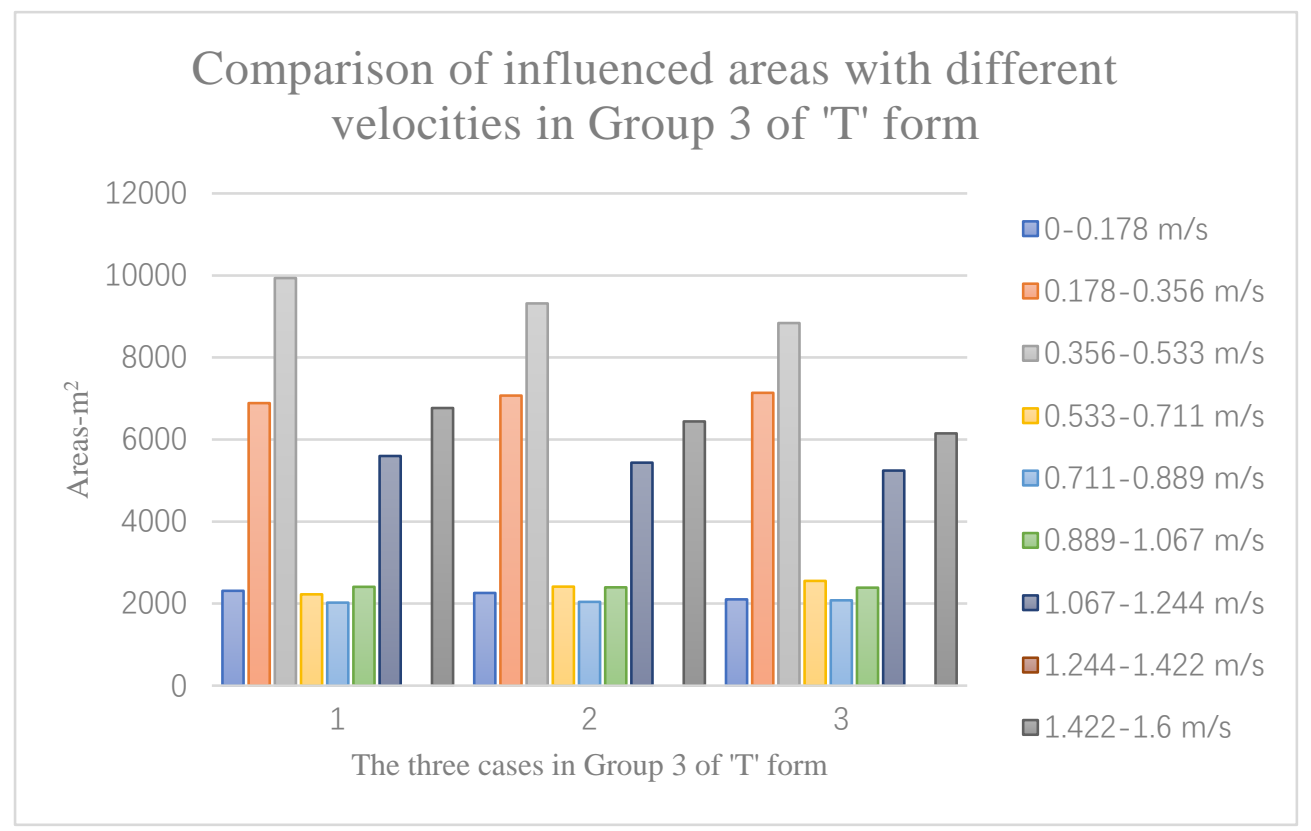

Figure 90. The influenced areas with different velocities in Group 3 of ' $\mathrm{T}$ ' form.

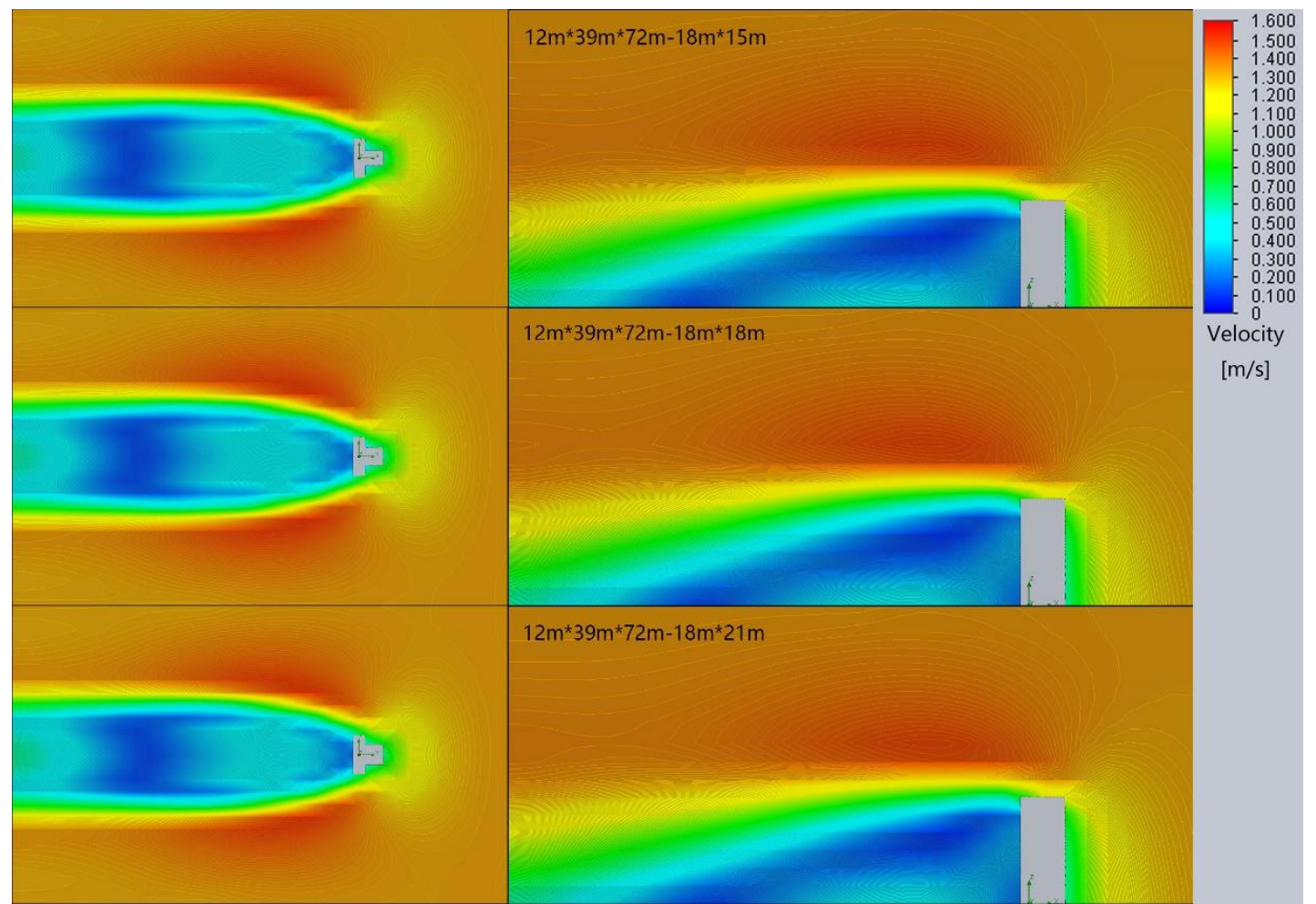

Figure 91. The comparison of wind velocity magnitudes on horizontal and vertical planes of the ' $T$ '-form buildings with different heights in Group 4. 


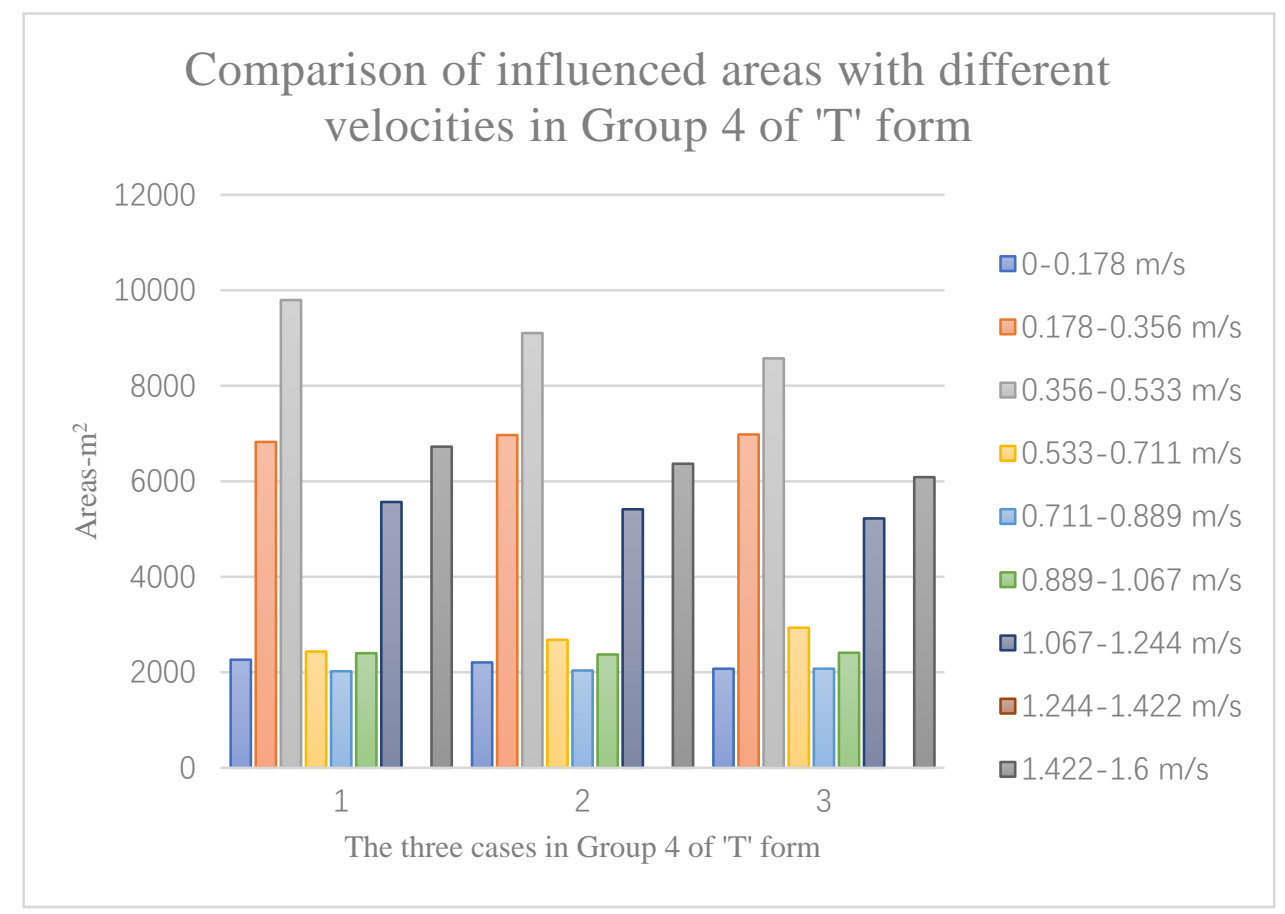

Figure 92. The influenced areas with different velocities in Group 4 of ' $\mathrm{T}$ ' form.

The changes of influenced areas with different velocities correspond with the changes of air pressure and wind flow in the three groups (Figure 93, Figure 94 and Figure 95). Winds first meet the bulge parts on the windward side as they approach the buildings. Take Group 2 for example, the low-air-pressure area behind the building and the high-air-pressure area in front of the building are both reduced due to the increase of bulge-part length from Case 1 to Case 3; the vortices created behind the building become smaller and less due to the increase of bulgepart length. The trends of Group 3 and Group 4 are almost the same to Group 2 according to the simulation results (Figure 93, Figure 94 and Figure 95).

The results of the three groups are compared together for analyzing the influence of bulgepart width. As the width increases, the low-air-pressure and high-air-pressure areas decrease; the vortices become smaller and less.

In summary, the air-pressure difference between the windward and leeward sides of the building becomes less due to the increase of length and width of the bulge part; the bulge part with larger size encourages winds to flow around the building. These can explain the increase of the length and width of the bulge part can cause the decrease of the building influence. 


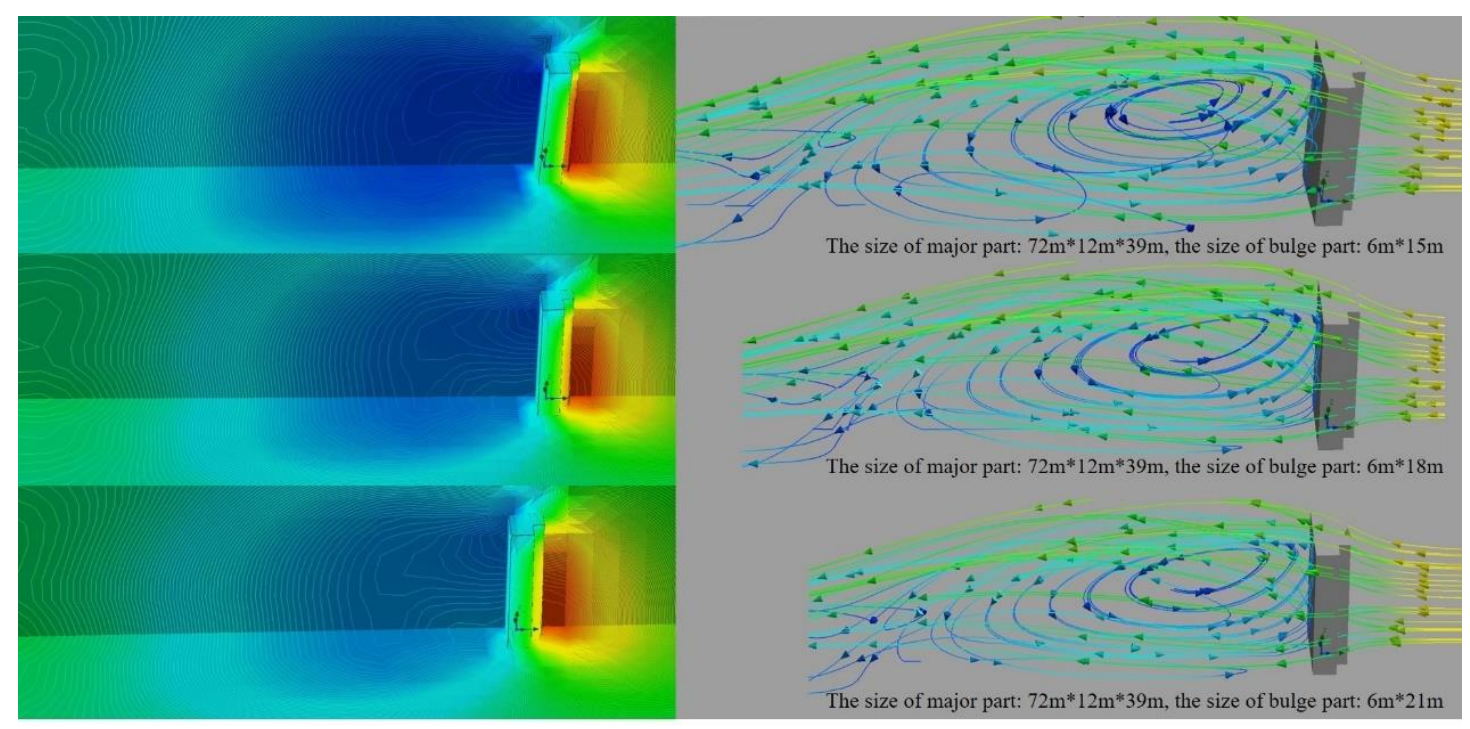

Figure 93. The comparisons of wind-flow streamlines (right) and air-pressure magnitudes (left) on horizontal, vertical planes and building surfaces of the ' $\mathrm{T}$ '-form buildings in Group 2.

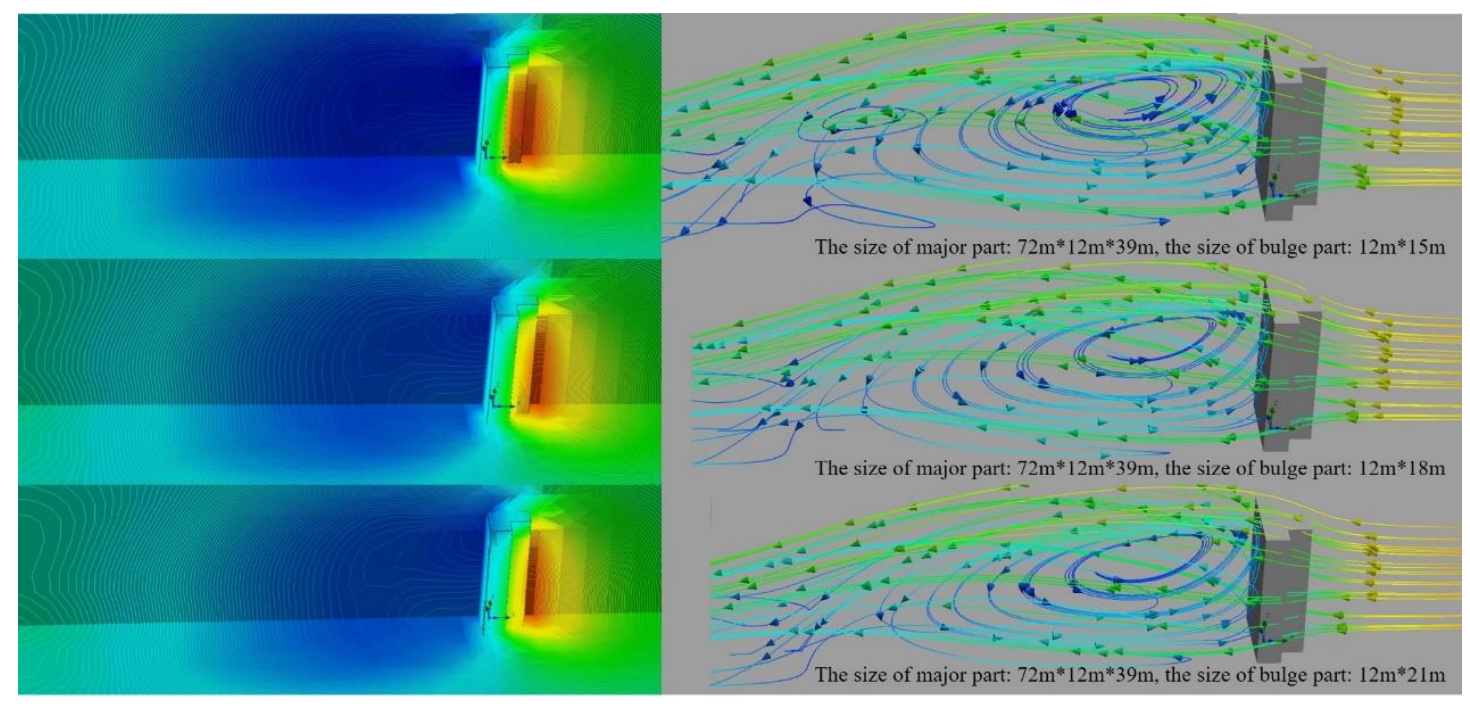

Figure 94. The comparisons of wind-flow streamlines (right) and air-pressure magnitudes (left) on horizontal, vertical planes and building surfaces of the ' $\mathrm{T}$ '-form buildings in Group 3. 


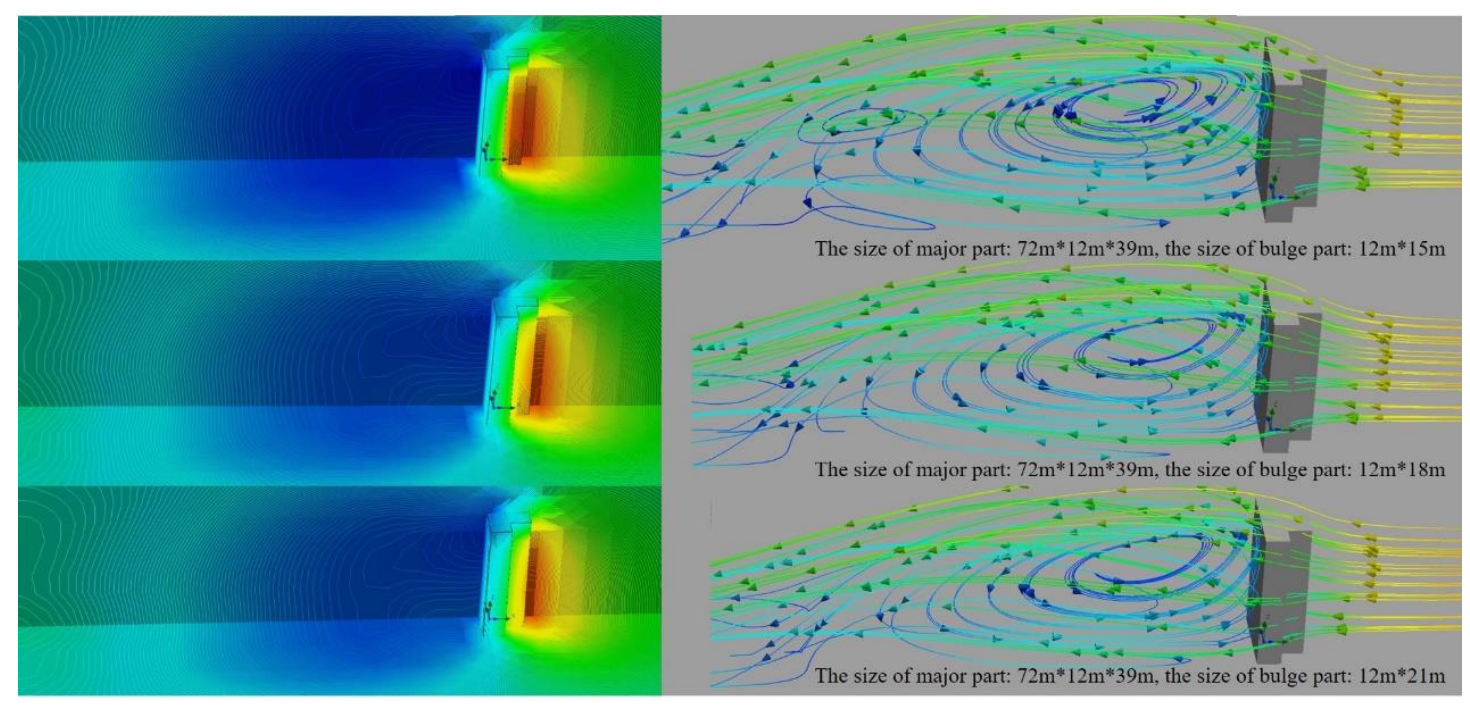

Figure 95. The comparisons of wind-flow streamlines (right) and air-pressure magnitudes (left) on horizontal, vertical planes and building surfaces of the ' $\mathrm{T}$ '-form buildings in Group 4.

\subsection{Summary of BUFs studies}

In this chapter, the relationships between the influences on wind environments and the building variables of the three representative BUFs have been studied, including square form, rectangular form and ' $\mathrm{T}$ ' form. For the influences, total influenced areas with different velocities, low-velocity and high-velocity areas are analyzed. Wind velocity magnitudes are used to compare the influences. Air-pressure magnitudes and wind-streamlines are used to analyze the mechanisms. The BUFs studies are summarized in this section. The outcomes of BUFs studies are discussed and summarized further in the Discussion Chapter.

In the Section 5.1., the overview describes the attributes of wind environments of the three BUFs by explaining the wind-velocity magnitudes, air-pressure magnitudes and wind-flow streamlines. It presents the low-velocity and high-velocity areas, low-air-pressure and high-airpressure areas, and vortices around the buildings.

In the Section 5.2. Square form, the relationships between influences on wind environments and building variables have been studied in two parts, including the edge length and rotation angle. In the first part, three cases with different edge lengths are studied. The influence increases as the edge length increases. In the second part, four cases with different 
rotation angles are studied. The wind flow is promoted as the angle increases.

In the Section 5.3. Rectangular form, the relationships between influences on wind environments and building variables have been studied in four parts, including the length, width, height and rotation angle. In the first part, the long surfaces of the rectangular-form buildings are on the windward side. Eleven cases of three groups with different lengths and widths are studied. The influence increases as the length increases; the influence decreases as the width increases. In the second part, three cases with different heights are studied. The influence increases as the height increases. In the third part, the short surfaces of the rectangular-form buildings are on the windward side. Eleven cases of three groups with different lengths and widths are studied. The influence increases as the width increases; the influence does not always decrease as the length increases, because the least influence is decided by a particular ratio of width and length. In the fourth part, twelve cases of three groups with different rotation angels are studied. For rectangular-form buildings with different lengths, widths and heights, the wind flow is promoted as the angle increases.

In the Section 5.4. ' $\mathrm{T}$ ' form, the relationships between influences on wind environments and building variables have been studied in two parts, including the length and width of the bulge part. The first part is for the short buildings with a relatively small bulge part. Five cases with different lengths of the bulge part are studied. The influence decreases as the length increases. The second part is for the tall buildings with a relatively large bulge part. Nine cases of three groups with different lengths and widths of the bulge-part are studied. The influence decreases as the length and width are increased. 


\section{Wind environment studies of BCCs}

The overall goal of this chapter is to study the relationships between influences on wind environments and building variables of the three BCCs, including scattered configuration, linear configuration and curvilinear configuration. Studies of BCCs are based on the studies of BUFs, as a building cluster is consisted of several building units. The chapter focuses on wind environments of the representative BCCs summarized in the parametric design chapter. The research methodology used in the BCCs studies is the same as the last chapter, which has been explained in the Third Chapter. The computational tools and assessment criteria are used in the studies. Influenced areas are compared by analyzing wind-velocity magnitudes. The mechanisms are explained by using air-pressure magnitudes and wind-flow streamlines. In this chapter, the building units in clusters are all set in the same rectangular form. The specific building variables, results and analysis of the three BCCs are presented in the following three sections respectively (Section 6.1., Section 6.2. and Section 6.3.). The summary in the end (Section 6.4) summarizes the BCCs studies briefly.

\subsection{Scattered configuration}

This section aims to study the relationships between the influences on wind environments and the building variables of scattered configuration, including the building unit number, longitudinal distance, transverse distance and staggered distance (Figure 96). In the first and second parts, the influences of transverse distances are studied for a scattered-configuration building cluster with the building units (unit numbers: 2 and 3) arranged in a building row. In the third and fourth parts, the influences of longitudinal distances are studied for a scatteredconfiguration building cluster with the building units (unit numbers: 2 and 3) arranged in a building column. In the fifth part, the influences of transverse and longitudinal distances are studied for a scattered-configuration building cluster with four building units arranged in two building rows (columns). In the sixth part, the influences of transverse and longitudinal distances are studied for a scattered-configuration building cluster with six building units 
arranged in two building rows. In the seventh part, the influences of transverse and longitudinal distances are studied for a scattered-configuration building cluster with six building units arranged in two building columns. In the eighth part, the influences of transverse and longitudinal distances are studied for a scattered-configuration building cluster with three building units arranged in three building rows (columns). In the ninth part, the influences of staggered distances are studied for a scattered-configuration building cluster with nine building units arranged in three building rows (columns). The building variables setup of the nine parts are shown first. Then results and analysis of the nine parts are presented.

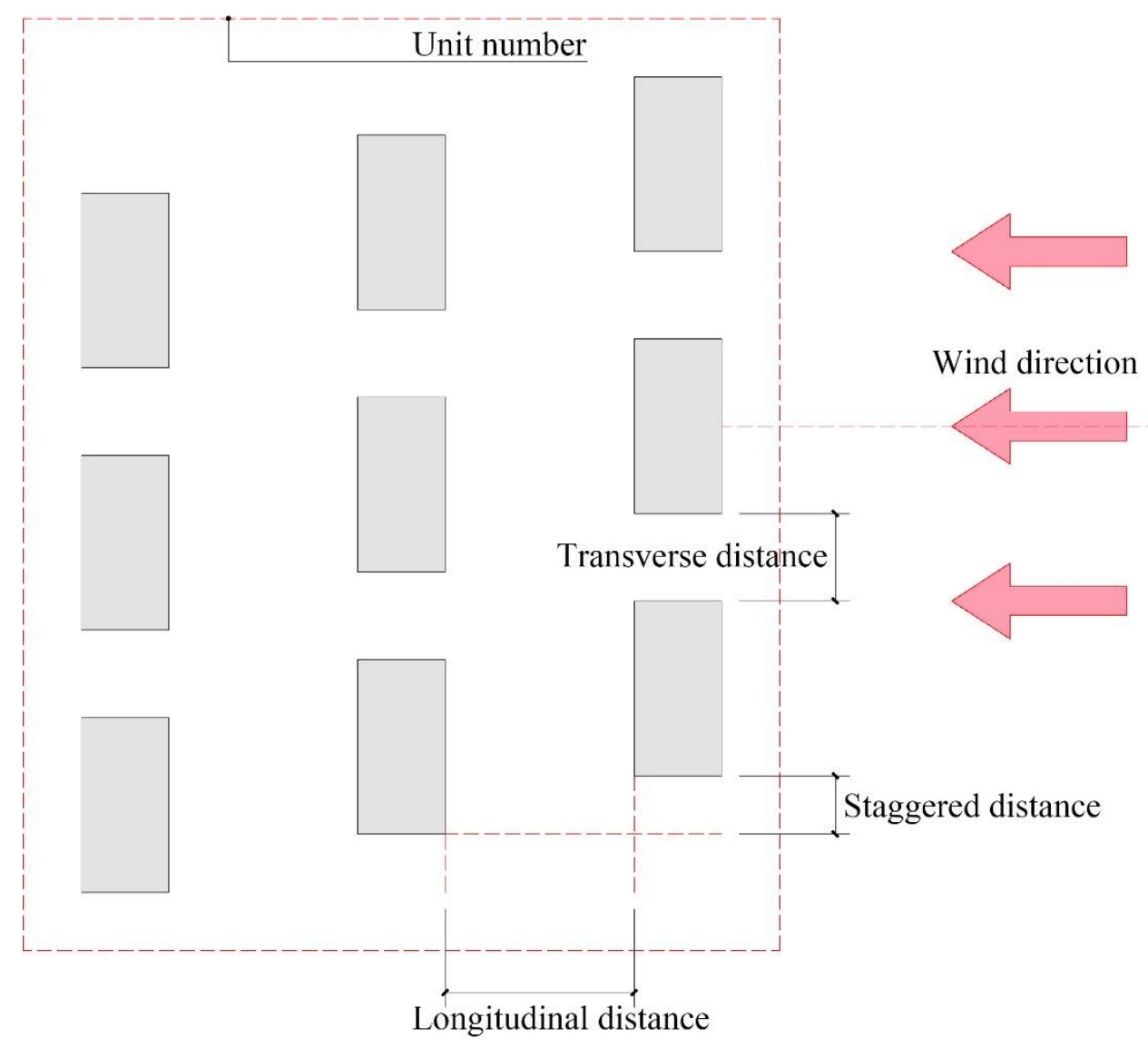

Figure 96. The building variables of staggered configuration.

\subsubsection{Building variable setup of scattered configuration}

In the first part, three cases with different transverse distances are set up in Group 1. According to the summary in the parametric design chapter, common transverse distances in the range of 
$10-20 \mathrm{~m}$ are used in the study; two building units are arranged in a building row. The specific variables of the three cases are presented in the following tables (Table 23.).

Table 23. The three cases in Group 1 of scattered configuration.

\begin{tabular}{|l|l|l|}
\hline Building unit numbers & Rows & Transverse distances \\
\hline 2 & 1 & $10 \mathrm{~m}$ \\
\hline 2 & 1 & $15 \mathrm{~m}$ \\
\hline 2 & 1 & $20 \mathrm{~m}$ \\
\hline
\end{tabular}

In the second part, three cases with different transverse distances are set up in Group 2. According to the summary in the parametric design chapter, common transverse distances in the range of 10-20m are used in the study; three building units are arranged in a building row. The specific variables of the three cases are presented in the following tables (Table 24.).

Table 24. The three cases in Group 2 of scattered configuration.

\begin{tabular}{|l|l|l|}
\hline Building unit numbers & Rows & Transverse distances \\
\hline 3 & 1 & $10 \mathrm{~m}$ \\
\hline 3 & 1 & $15 \mathrm{~m}$ \\
\hline 3 & 1 & $20 \mathrm{~m}$ \\
\hline
\end{tabular}

In the third part, three cases with different longitudinal distances are set up in Group 3. According to the summary in the parametric design chapter, common longitudinal distances in the range of $0.7 * \mathrm{H}-1.1 * \mathrm{H}(25.2-39.6 \mathrm{~m}, \mathrm{H}$ stands for the building height $36 \mathrm{~m})$ are used in the study; two building units are arranged in a building column. The specific variables of the three cases are presented in the following tables (Table 25.).

Table 25. The three cases in Group 3 of scattered configuration.

\begin{tabular}{|l|l|l|}
\hline Building unit numbers & Columns & Longitudinal distances \\
\hline 2 & 1 & $0.7 * \mathrm{H}(25.2 \mathrm{~m})$ \\
\hline
\end{tabular}




\begin{tabular}{|l|l|l|}
\hline 2 & 1 & $0.9 * \mathrm{H}(32.4 \mathrm{~m})$ \\
\hline 2 & 1 & $1.1 * \mathrm{H}(39.6 \mathrm{~m})$ \\
\hline \multicolumn{2}{|l|}{ The ' $\mathrm{H}$ ' in this group stands for the height of the building unit $(36 \mathrm{~m})}$. \\
\hline
\end{tabular}

In the fourth part, three cases with different longitudinal distances are set up in Group 4. According to the summary in the parametric design chapter, common longitudinal distances in the range of $0.7 * \mathrm{H}-1.1 * \mathrm{H}(25.2-39.6 \mathrm{~m}, \mathrm{H}$ stands for the building height $36 \mathrm{~m})$ are used in the study; three building units are arranged in a building column. The specific variables of the three cases are presented in the following tables (Table 26.).

Table 26. The three cases in Group 4 of scattered configuration.

\begin{tabular}{|l|l|l|}
\hline Building unit numbers & Columns & Longitudinal distances \\
\hline 3 & 1 & $0.7 * \mathrm{H}(25.2 \mathrm{~m})$ \\
\hline 3 & 1 & $0.9 * \mathrm{H}(32.4 \mathrm{~m})$ \\
\hline 3 & 1 & $1.1 * \mathrm{H}(39.6 \mathrm{~m})$ \\
\hline \multicolumn{2}{|l|}{ The ' $\mathrm{H}$ ' in this group stands for the height of the building unit $(36 \mathrm{~m})}$. \\
\hline
\end{tabular}

In the fifth part, nine cases with different transverse and longitudinal distances are set up in three groups (Group 5., Group 6., and Group 7.). According to the summary in the parametric design chapter, common transverse distances in the range of $10-20 \mathrm{~m}$ are used in the study; common longitudinal distances in the range of $0.7 * \mathrm{H}-1.1 * \mathrm{H}(25.2-39.6 \mathrm{~m}, \mathrm{H}$ stands for the building height $36 \mathrm{~m}$ ) are used; four building units are arranged in two building rows (columns). The specific variables of the three groups are presented in the following tables (Table 27., Table 28., and Table 29.).

Table 27. The three cases in Group 5 of scattered configuration.

\begin{tabular}{|l|l|l|l|l|}
\hline Unit numbers & Rows & Columns & Transverse distances & Longitudinal distances \\
\hline 4 & 2 & 2 & $10 \mathrm{~m}$ & $0.7 * \mathrm{H}(25.2 \mathrm{~m})$ \\
\hline 4 & 2 & 2 & $10 \mathrm{~m}$ & $0.9 * \mathrm{H}(32.4 \mathrm{~m})$ \\
\hline
\end{tabular}




\begin{tabular}{|l|l|l|l|l|}
\hline 4 & 2 & 2 & $10 \mathrm{~m}$ & $1.1 * \mathrm{H}(39.6 \mathrm{~m})$ \\
\hline \multicolumn{3}{|l|}{ The ' $\mathrm{H}$ ' in this group stands for the height of the building unit $(36 \mathrm{~m})}$. \\
\hline
\end{tabular}

Table 28. The three cases in Group 6 of scattered configuration.

\begin{tabular}{|l|l|l|l|l|}
\hline Unit numbers & Rows & Columns & Transverse distances & Longitudinal distances \\
\hline 4 & 2 & 2 & $15 \mathrm{~m}$ & $0.7 * \mathrm{H}(25.2 \mathrm{~m})$ \\
\hline 4 & 2 & 2 & $15 \mathrm{~m}$ & $0.9 * \mathrm{H}(32.4 \mathrm{~m})$ \\
\hline 4 & 2 & 2 & $15 \mathrm{~m}$ & $1.1 * \mathrm{H}(39.6 \mathrm{~m})$ \\
\hline \multicolumn{7}{|l|}{ The 'H' in this group stands for the height of the building unit (36m). } \\
\hline
\end{tabular}

Table 29. The three cases in Group 7 of scattered configuration.

\begin{tabular}{|l|l|l|l|l|}
\hline Unit numbers & Rows & Columns & Transverse distances & Longitudinal distances \\
\hline 4 & 2 & 2 & $20 \mathrm{~m}$ & $0.7 * \mathrm{H}(25.2 \mathrm{~m})$ \\
\hline 4 & 2 & 2 & $20 \mathrm{~m}$ & $0.9 * \mathrm{H}(32.4 \mathrm{~m})$ \\
\hline 4 & 2 & 2 & $20 \mathrm{~m}$ & $1.1 * \mathrm{H}(39.6 \mathrm{~m})$ \\
\hline
\end{tabular}

In the sixth part, six cases with different transverse and longitudinal distances are set up in two groups (Group 8. and Group 9.). According to the summary in the parametric design chapter, common transverse distances in the range of 10-20m are used in the study; common longitudinal distances in the range of $0.7 * \mathrm{H}-1.1 * \mathrm{H}(25.2-39.6 \mathrm{~m}, \mathrm{H}$ stands for the building height $36 \mathrm{~m}$ ) are used; six building units are arranged in three building rows (two columns). The specific variables of the two groups are presented in the following tables (Table 30. and Table 31.).

Table 30. The three cases in Group 8 of scattered configuration.

\begin{tabular}{|l|l|l|l|l|}
\hline Unit numbers & Rows & Columns & Transverse distances & Longitudinal distances \\
\hline 6 & 3 & 2 & $10 \mathrm{~m}$ & $0.9 * \mathrm{H}(32.4 \mathrm{~m})$ \\
\hline 6 & 3 & 2 & $15 \mathrm{~m}$ & $0.9 * \mathrm{H}(32.4 \mathrm{~m})$ \\
\hline
\end{tabular}




\begin{tabular}{|l|l|l|l|l|}
\hline 6 & 3 & 2 & $20 \mathrm{~m}$ & $0.9 * \mathrm{H}(32.4 \mathrm{~m})$ \\
\hline
\end{tabular}

Table 31. The three cases in Group 9 of scattered configuration.

\begin{tabular}{|l|l|l|l|l|}
\hline Unit numbers & Rows & Columns & Transverse distances & Longitudinal distances \\
\hline 6 & 3 & 2 & $15 \mathrm{~m}$ & $0.7 * \mathrm{H}(25.2 \mathrm{~m})$ \\
\hline 6 & 3 & 2 & $15 \mathrm{~m}$ & $0.9 * \mathrm{H}(32.4 \mathrm{~m})$ \\
\hline 6 & 3 & 2 & $15 \mathrm{~m}$ & $1.1 * \mathrm{H}(39.6 \mathrm{~m})$ \\
\hline \multicolumn{7}{|l|}{ The 'H' in this group stands for the height of the building unit (36m). } \\
\hline
\end{tabular}

In the seventh part, six cases with different transverse and longitudinal distances are set up in two groups (Group 10. and Group 11.). According to the summary in the parametric design chapter, common transverse distances in the range of 10-20m are used in the study; common longitudinal distances in the range of $0.7 * \mathrm{H}-1.1 * \mathrm{H}(25.2-39.6 \mathrm{~m}, \mathrm{H}$ stands for the building height $36 \mathrm{~m}$ ) are used; six building units are arranged in two building rows (three columns). The specific variables of the two groups are presented in the following tables (Table 32. and Table 33.).

Table 32. The three cases in Group 10 of scattered configuration.

\begin{tabular}{|l|l|l|l|l|}
\hline Unit numbers & Rows & Columns & Transverse distances & Longitudinal distances \\
\hline 6 & 2 & 3 & $10 \mathrm{~m}$ & $0.9 * \mathrm{H}(32.4 \mathrm{~m})$ \\
\hline 6 & 2 & 3 & $15 \mathrm{~m}$ & $0.9 * \mathrm{H}(32.4 \mathrm{~m})$ \\
\hline 6 & 2 & 3 & $20 \mathrm{~m}$ & $0.9 * \mathrm{H}(32.4 \mathrm{~m})$ \\
\hline \multicolumn{5}{|l|}{ The ' $\mathrm{H}$ ' in this group stands for the height of the building unit $(36 \mathrm{~m})}$. \\
\hline
\end{tabular}

Table 33. The three cases in Group 11 of scattered configuration.

\begin{tabular}{|l|l|l|l|l|}
\hline Unit numbers & Rows & Columns & Transverse distances & Longitudinal distances \\
\hline 6 & 3 & 2 & $15 \mathrm{~m}$ & $0.7 * \mathrm{H}(25.2 \mathrm{~m})$ \\
\hline 6 & 3 & 2 & $15 \mathrm{~m}$ & $0.9 * \mathrm{H}(32.4 \mathrm{~m})$ \\
\hline
\end{tabular}




\begin{tabular}{|l|l|l|l|l|}
\hline 6 & 3 & 2 & $15 \mathrm{~m}$ & $1.1 * \mathrm{H}(39.6 \mathrm{~m})$ \\
\hline \multicolumn{3}{|l|}{ The ' $\mathrm{H}$ ' in this group stands for the height of the building unit $(36 \mathrm{~m})}$. \\
\hline
\end{tabular}

In the eighth part, six cases with different transverse and longitudinal distances are set up in two groups (Group 12. and Group 13.). According to the summary in the parametric design chapter, common transverse distances in the range of $10-20 \mathrm{~m}$ are used in the study; common longitudinal distances in the range of $0.7 * \mathrm{H}-1.1 * \mathrm{H}(25.2-39.6 \mathrm{~m}, \mathrm{H}$ stands for the building height $36 \mathrm{~m}$ ) are used; nine building units are arranged in three building rows (three columns). The specific variables of the two groups are presented in the following tables (Table 34. and Table 35.).

Table 34. The three cases in Group 12 of scattered configuration.

\begin{tabular}{|l|l|l|l|l|}
\hline Unit numbers & Rows & Columns & Transverse distances & Longitudinal distances \\
\hline 9 & 3 & 3 & $10 \mathrm{~m}$ & $0.9 * \mathrm{H}(32.4 \mathrm{~m})$ \\
\hline 9 & 3 & 3 & $15 \mathrm{~m}$ & $0.9 * \mathrm{H}(32.4 \mathrm{~m})$ \\
\hline 9 & 3 & 3 & $20 \mathrm{~m}$ & $0.9 * \mathrm{H}(32.4 \mathrm{~m})$ \\
\hline \multicolumn{7}{|l|}{ The 'H' in this group stands for the height of the building unit (36m). } \\
\hline
\end{tabular}

Table 35. The three cases in Group 13 of scattered configuration.

\begin{tabular}{|l|l|l|l|l|}
\hline Unit numbers & Rows & Columns & Transverse distances & Longitudinal distances \\
\hline 9 & 3 & 3 & $15 \mathrm{~m}$ & $0.7 * \mathrm{H}(25.2 \mathrm{~m})$ \\
\hline 9 & 3 & 3 & $15 \mathrm{~m}$ & $0.9 * \mathrm{H}(32.4 \mathrm{~m})$ \\
\hline 9 & 3 & 3 & $15 \mathrm{~m}$ & $1.1 * \mathrm{H}(39.6 \mathrm{~m})$ \\
\hline \multicolumn{5}{|l|}{ The ' $\mathrm{H}$ ' in this group stands for the height of the building unit (36m). } \\
\hline
\end{tabular}

In the ninth part, three cases with different staggered distances are set up in Group 14. According to the summary in the parametric design chapter, common staggered distances in the range of $10-30 \mathrm{~m}$ are used in the study; a common transverse distance of $15 \mathrm{~m}$ and a common longitudinal distances of $0.9 * \mathrm{H}(32.4 \mathrm{~m}, \mathrm{H}$ stands for the building height $36 \mathrm{~m})$ are used; nine 
building units are arranged in three building rows. The specific variables of the three cases are presented in the following tables (Table 36.).

Table 36. The three cases in Group 14 of scattered configuration.

\begin{tabular}{|l|l|l|l|l|l|}
\hline Unit numbers & Rows & Columns & $\begin{array}{l}\text { Transverse } \\
\text { distances }\end{array}$ & $\begin{array}{l}\text { Longitudinal } \\
\text { distances }\end{array}$ & $\begin{array}{l}\text { Staggered } \\
\text { distances }\end{array}$ \\
\hline 9 & 3 & 3 & $15 \mathrm{~m}$ & $0.9 * \mathrm{H}(32.4 \mathrm{~m})$ & $10 \mathrm{~m}$ \\
\hline 9 & 3 & 3 & $15 \mathrm{~m}$ & $0.9 * \mathrm{H}(32.4 \mathrm{~m})$ & $20 \mathrm{~m}$ \\
\hline 9 & 3 & 3 & $15 \mathrm{~m}$ & $0.9 * \mathrm{H}(32.4 \mathrm{~m})$ & $30 \mathrm{~m}$ \\
\hline \multicolumn{7}{|l}{ The ' $\mathrm{H}$ ' in this group stands for the height of the building unit $(36 \mathrm{~m})}$. \\
\hline
\end{tabular}

The parametric modelling script of scattered configuration is used to create the building cluster models. The width and length of the building unit are all set as $15 \mathrm{~m}$ and $30 \mathrm{~m}$, which is common for a high-rise residential building with two apartments on each floor. The building height of $36 \mathrm{~m}$ is used in all cases, which is a common height for a high-rise residential building with twelve floors. The range of longitudinal distance $\left(0.7^{*} \mathrm{H}-1.1^{*} \mathrm{H}\right)$ is determined by the architectural standards for requirements of daylighting and fire prevention. In the above fourteen groups, boundaries of CFD simulation results are all set in the same sizes for comparisons of influences on wind environments.

\subsubsection{Results and analysis of scattered configuration}

The CFD simulation results of scattered configuration are analyzed in this subsection. Influences on wind environments are compared based on velocity magnitudes. The mechanisms are explained using the air-pressure magnitudes and wind-flow streamlines. In Part 1, the relationship between the influences and transverse distances is studied for the building cluster with two building units in a building row. In Part 2, the relationship between the influences and transverse distances is studied for the building cluster with three building units in a building row. In Part 3, the relationship between the influences and longitudinal distances is studied for 
the building cluster with two building units in a building column. In Part 4, the relationship between the influences and longitudinal distances is studied for the building cluster with three building units in a building column. In Part 5, the relationships between the influences and transverse and longitudinal distances are studied for the building cluster with four building units in two building rows (columns). In Part 6, the relationships between the influences and transverse and longitudinal distances are studied for the building cluster with six building units in two building rows (and three columns). In Part 7, the relationships between the influences and transverse and longitudinal distances are studied for the building cluster with six building units in three building rows (and two columns). In Part 8, the relationships between the influences and transverse and longitudinal distances are studied for the building cluster with nine building units in three building rows (and three columns). In Part 9, the relationship between the influences and staggered distances is studied for the building cluster with nine building units in three building rows (and three columns).

\section{(1) Part 1. Influences of transverse distance between 2 units in a row}

The influences of different transverse distances of Group 1 are analyzed. The wind-velocity magnitudes on horizontal and vertical planes of the three cases are presented (Figure 97). The total influenced area with different velocities of the three cases is close. Influence of Case 1 with the shortest transverse distance of $10 \mathrm{~m}$ is the least $\left(34543.96 \mathrm{~m}^{2}\right)$; influence of Case 2 with the transverse distance of $15 \mathrm{~m}$ is the most $\left(41867.1 \mathrm{~m}^{2}\right)$; influence of Case 3 with the longest transverse distance of $20 \mathrm{~m}$ is less than Case 2 but more than Case $1\left(37592.35 \mathrm{~m}^{2}\right)$.

In Case 1, the low-velocity area (velocity below $0.2 \mathrm{~m} / \mathrm{s}$ ) behind the building row is the largest and longest; In Case 2, the low-velocity area becomes less than Case 1; in Case 3, the low-velocity area is the smallest of all. The low-velocity area behind the building row decreases obviously as the transverse distance increases.

In all three cases, there are high-velocity areas (velocity range: $1.4-1.8 \mathrm{~m} / \mathrm{s}$ ) on the lateral sides of the building cluster and in the gap between the building units. The high-velocity areas of the three cases are close. In Case 1, the high-velocity areas $(2759.126 \mathrm{~m} 2)$ are the smallest; in Case 2, the high-velocity areas (2942.572m2) become more than Case 1; in Case 3, the highvelocity areas (2866.125m2) are less than Case 2 but more than Case 1. The high-low velocity 
area in the gap increases constantly as the transverse distance increases from Case 1 to Case 3 .

(Figure 98)

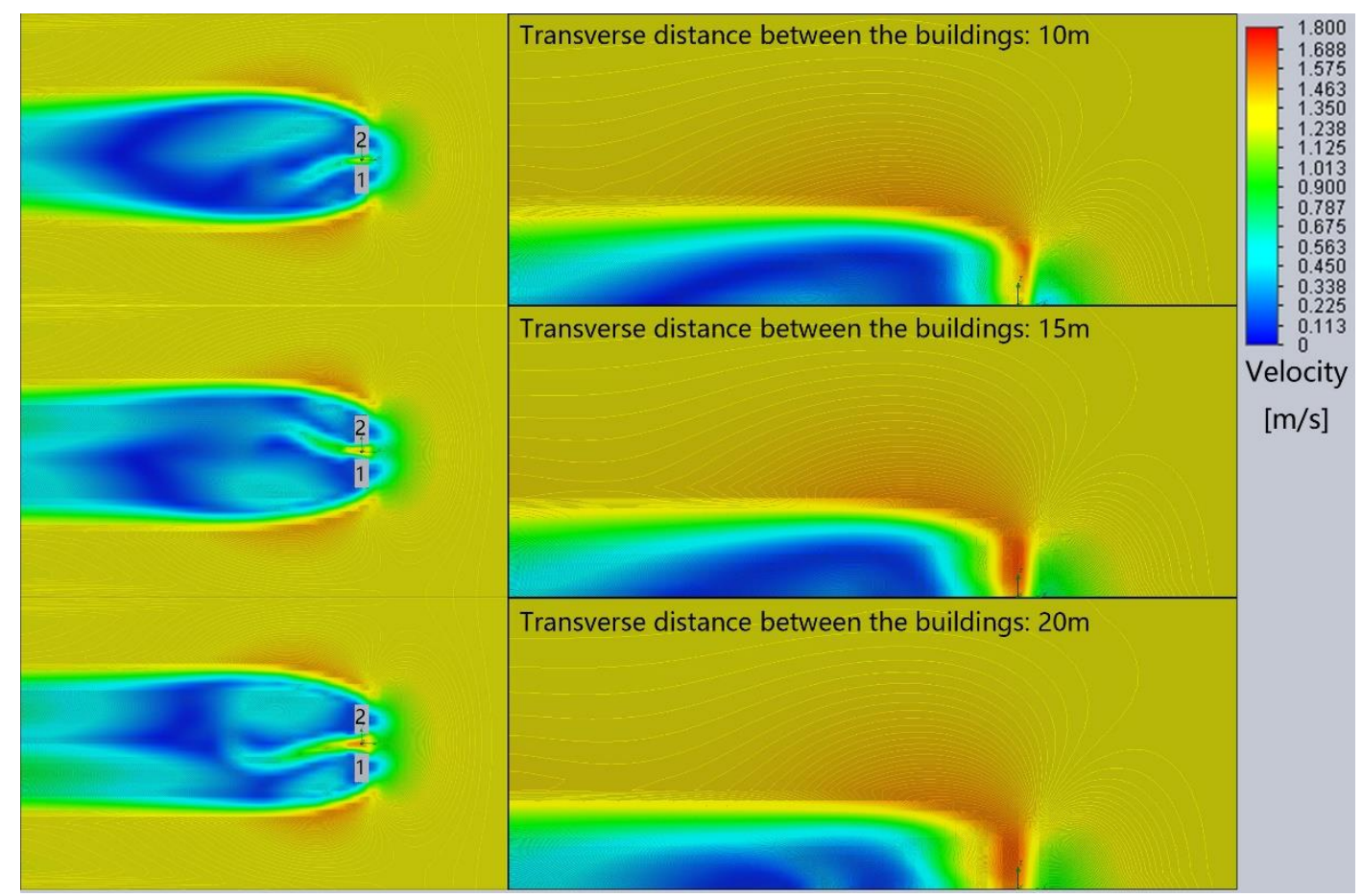

Figure 97. The comparison of wind velocity magnitudes on horizontal and vertical planes of the building clusters in Group 1 of scattered configuration.

In general, it was thought that the influence on wind environment of the building cluster decreases as the transverse distance increases (in the range of 10-20m). Actually, the influenced areas of the three cases are close in Group 1. Because the windward projective area of the entire building cluster increases as the transverse distance increases by regarding the two building units and gap between them as a whole. However, the increase of transverse distance promotes wind flow in the gap between the two building units. As the gap becomes larger, more winds are introduced to flow into the low-velocity area behind the buildings. Therefore, the lowvelocity area decreases and the outdoor ventilation behind the building cluster is improved significantly. 


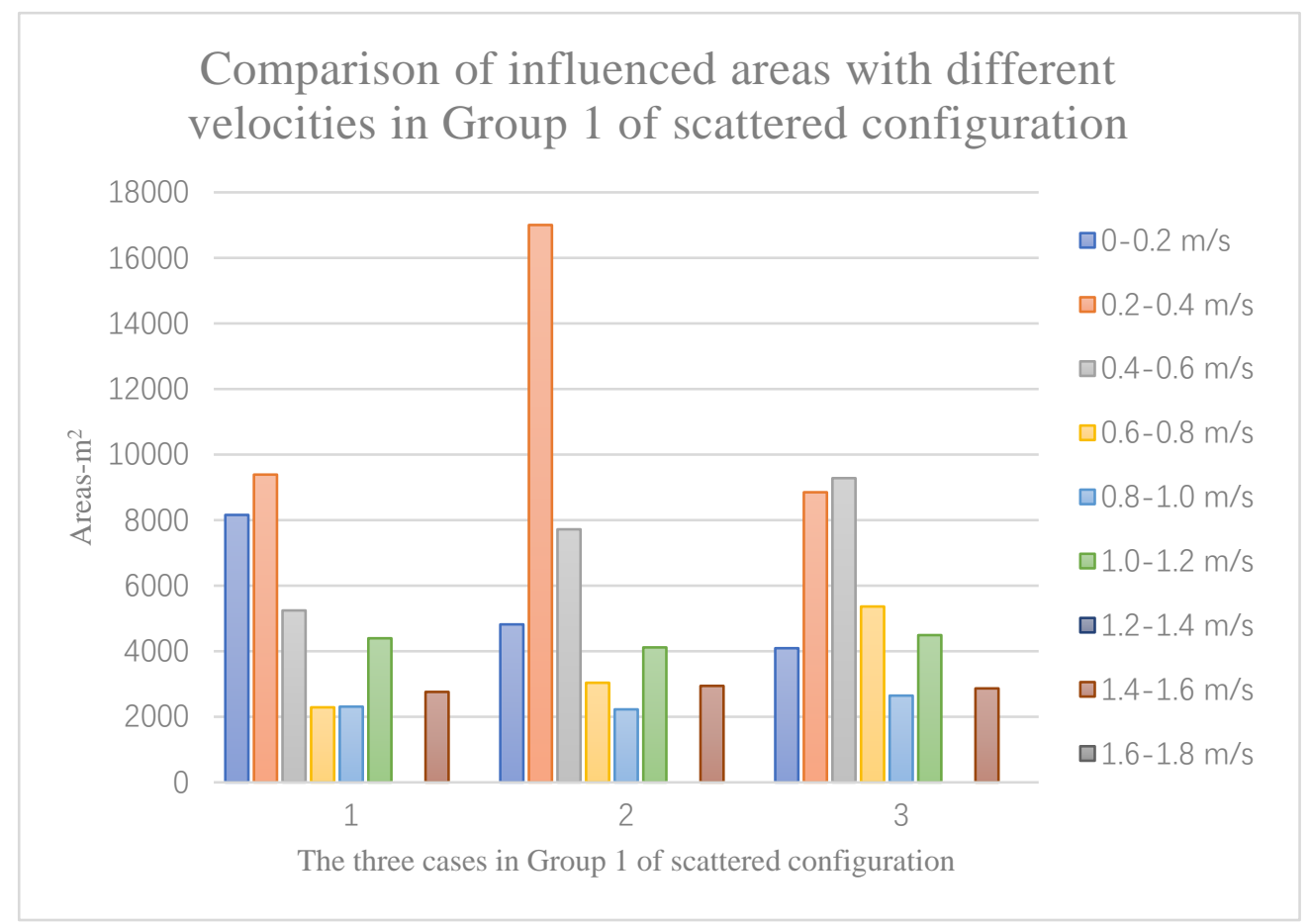

Figure 98. The influenced areas with different velocities in Group 1 of scattered configuration.

The changes of influenced areas in the three cases are related to the changes of air pressure (Figure 99) and wind flow (Figure 100). Because wind flow is caused by air-pressure differences. And winds usually flow from high-air-pressure areas to low-air-pressure areas. In Case 1, the low-velocity area is the largest due to the largest low-air-pressure area behind the building cluster; in Case 2, the low-velocity area decreases as the low-air-pressure area becomes less; in Case 3, the low-velocity area is the least as the low-air-pressure area is the smallest. The streamlines show that winds are able to escape in the larger building interval more easily. Less vortices are created behind the leeward side of the building. 


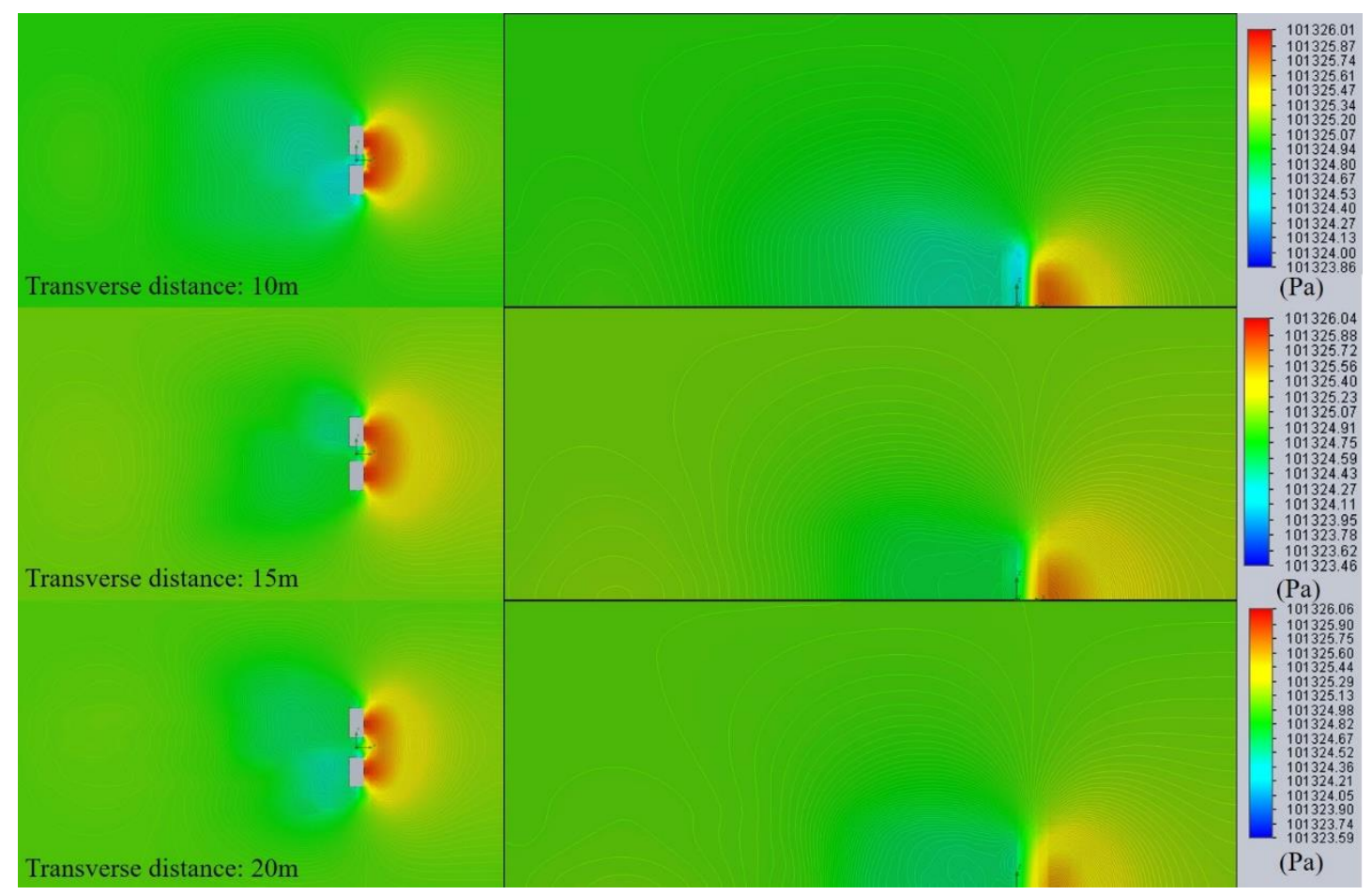

Figure 99. The comparison of air-pressure magnitudes on the horizontal and vertical planes of the building clusters in Group 1 of scattered configuration.

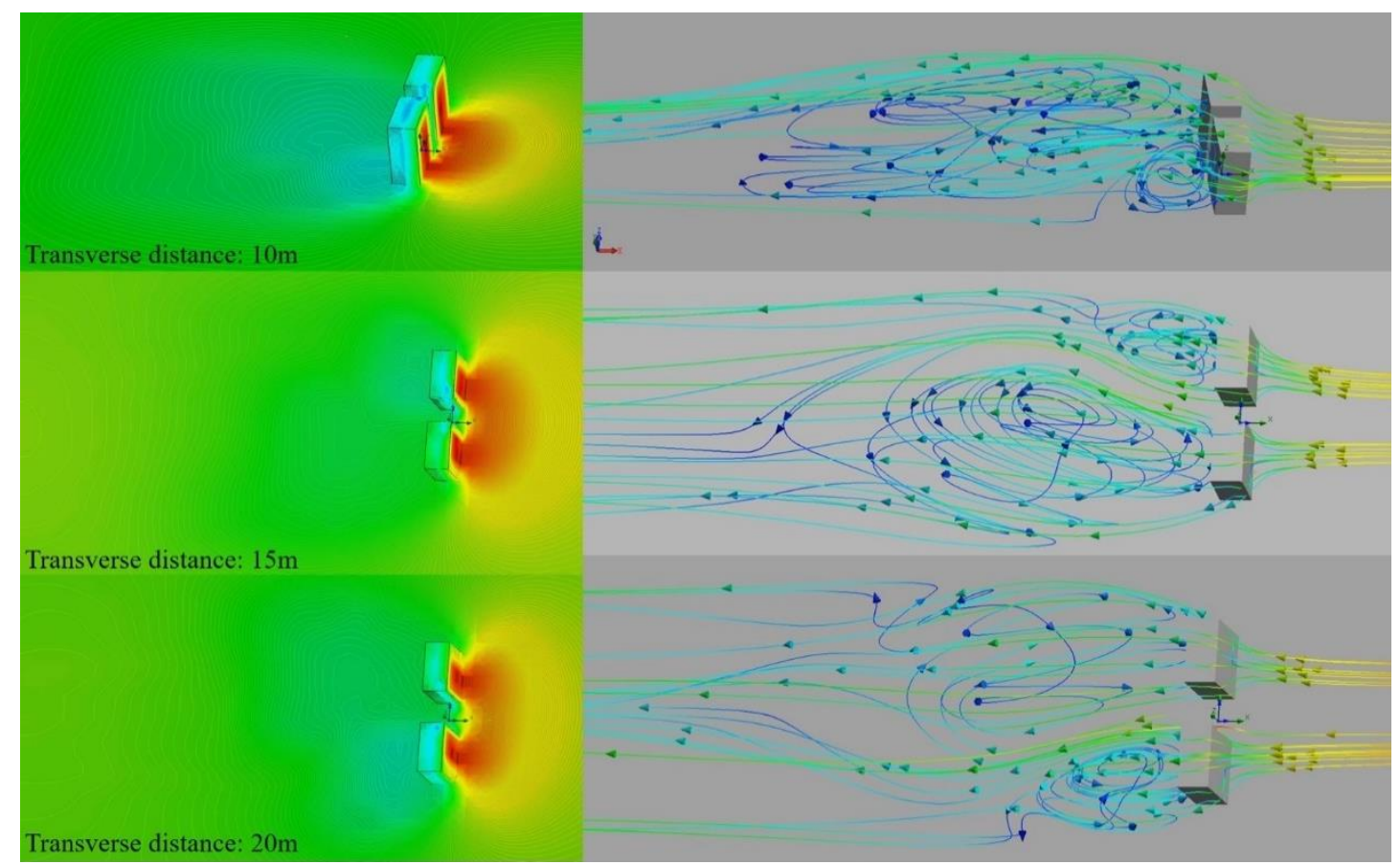

Figure 100. The comparison of wind-flow streamlines (right) and air-pressure magnitudes (left) on the horizontal plane and building surfaces in Group 1 of scattered configuration. 


\section{(2) Part 2. Influences of transverse distance among 3 units in a row}

In this part, the influences of different transverse distances of Group 2 are analyzed. The windvelocity magnitudes on horizontal and vertical planes of three cases are presented in the figure (Figure 101). In Case $1(10 \mathrm{~m})$, the total influence of the building cluster is the least (influenced area: $\left.47451.51 \mathrm{~m}^{2}\right)$; in Case $2(15 \mathrm{~m})$, the influence of the building cluster is more than Case 1 (influenced area: $\left.49182.64 \mathrm{~m}^{2}\right)$; in Case $3(20 \mathrm{~m})$, the influence of the building cluster is the most of all (influenced area: $50980.16 \mathrm{~m}^{2}$ ).

In Case 1, the low-velocity area (velocity below $0.2 \mathrm{~m} / \mathrm{s}$ ) behind the building row is the largest; In Case 2, the low-velocity area becomes much less than Case 1; in Case 3, the lowvelocity area is the smallest of all. The low-velocity area behind the building row decreases obviously as the transverse distance increases.

In all the cases, there are high-velocity areas (velocity: $1.4-1.8 \mathrm{~m} / \mathrm{s}$ ) on the lateral sides of the building cluster and in the gaps among the building units. In Case 1, the high-velocity areas are the largest; in Case 2, the high-velocity areas become less than Case 1; in Case 3, the highvelocity areas are the least of all (Figure 102). However, the high-velocity areas in the two gaps increase constantly as the transverse distance increases from Case 1 to Case 3.

In summary, the total influenced area with different velocities is increased as the transverse distance increases. As explained in Part 1, this is because the windward projective area of the entire building cluster increases as the transverse distance increases. The increase of transverse distance also promotes wind flow in the gaps among the three building units. And the lowvelocity area decreases significantly behind the building cluster. 


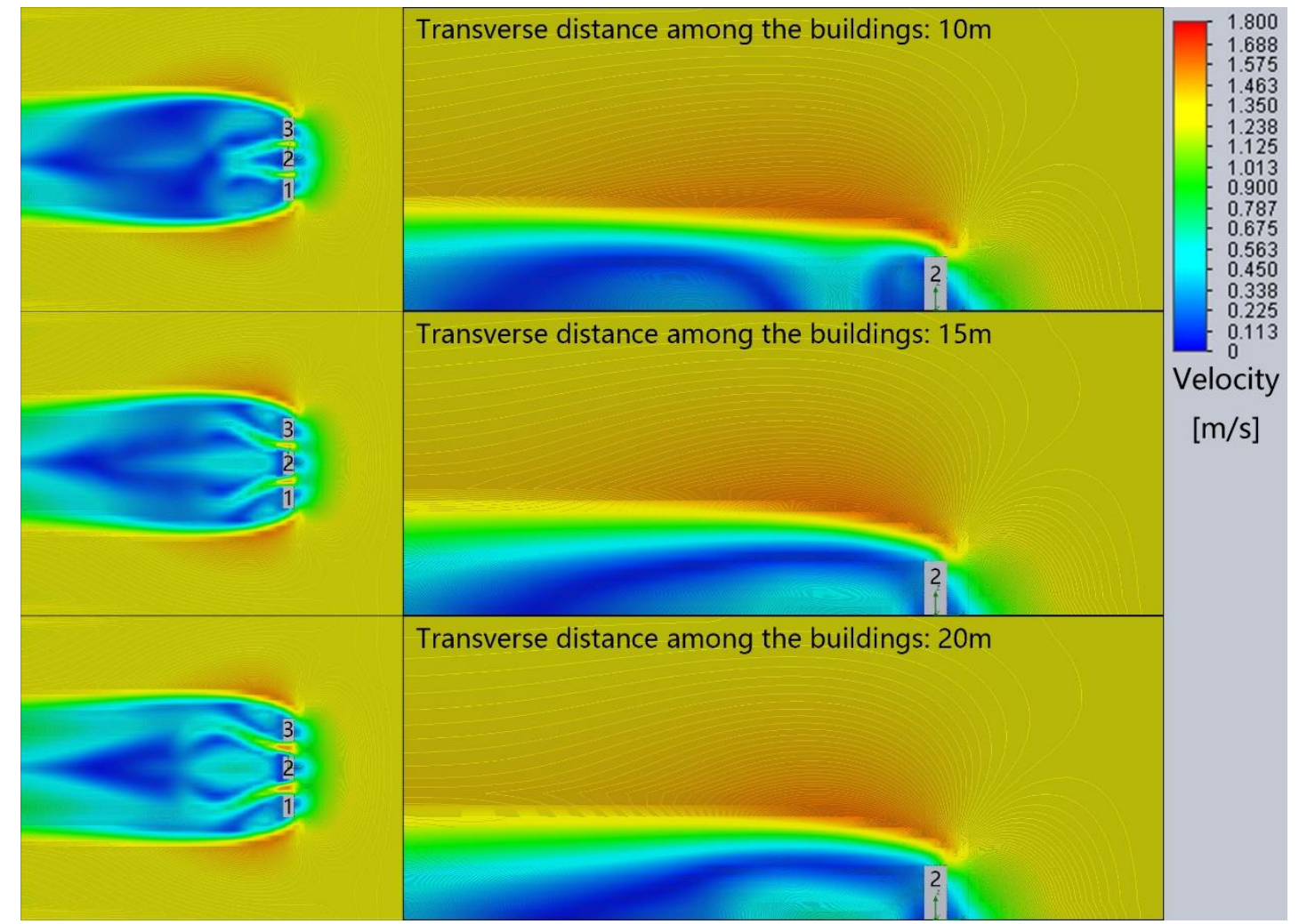

Figure 101. The comparison of wind-velocity magnitudes on horizontal and vertical planes of the building clusters in Group 2 of scattered configuration.

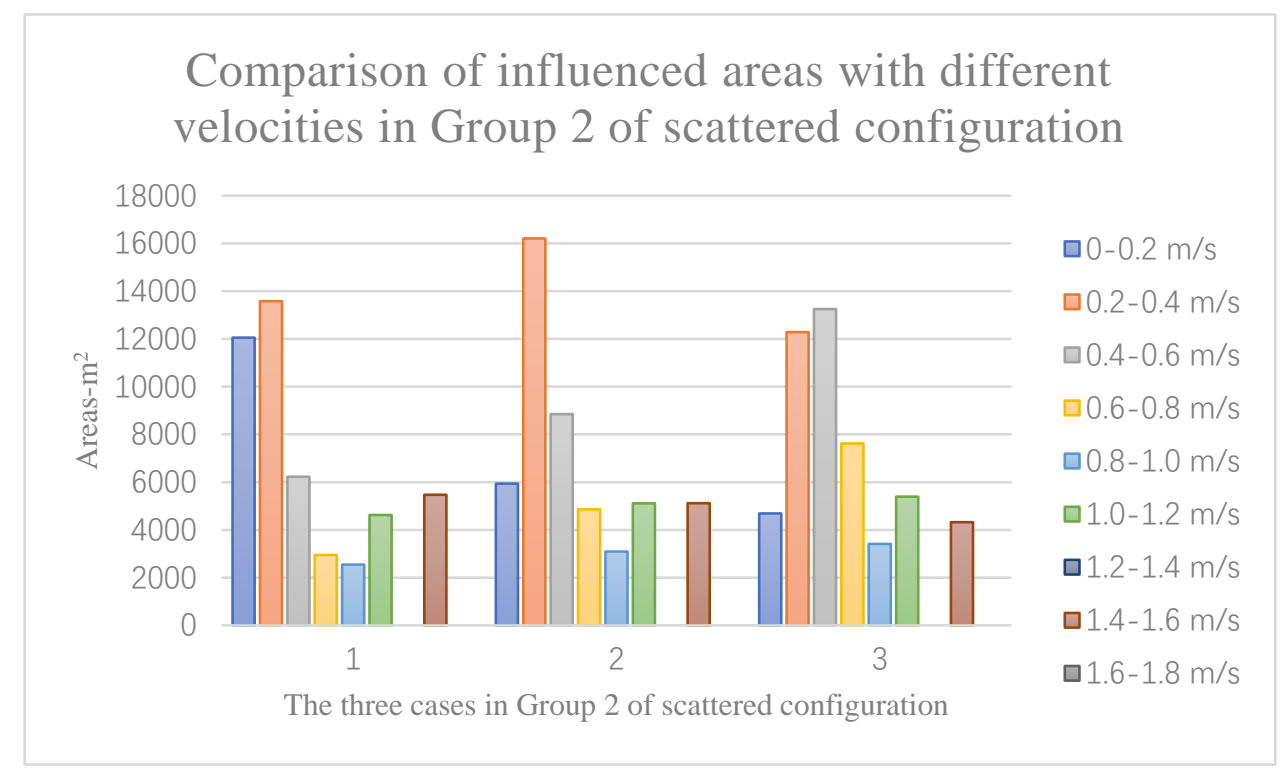

Figure 102. The influenced areas with different velocities in Group 2 of scattered configuration.

The changes of influenced areas with different velocities in Group 2 are related to the changes of air pressure and wind flow (Figure 103 and Figure 104). Winds usually flow from high-air-pressure areas to low-air-pressure areas. The air-pressure magnitudes show the trend 
of changes is similar to Group 1. The low-velocity area of Case 1 is the largest due to the largest low-air-pressure area; the low-velocity area of Case 2 decreases as the low-air-pressure area becomes less; the low-velocity area of Case 3 is the least as the low-air-pressure area is the smallest. The streamlines show that winds escape more easily in the larger gaps among building units. Most vortices are created behind the two lateral building units. The vortices are reduced significantly as the transverse distance increases.

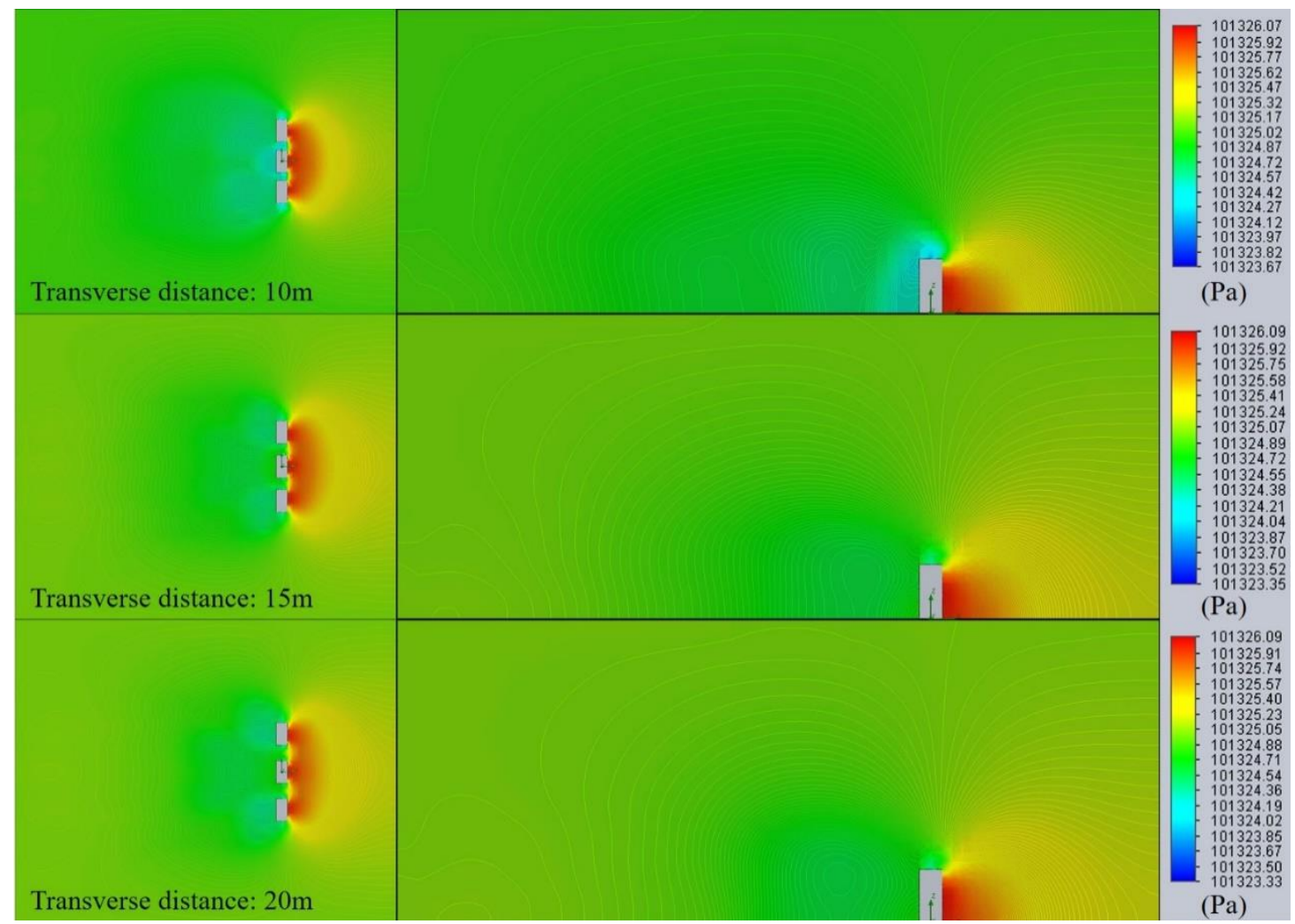

Figure 103. The comparison of air-pressure magnitudes on the horizontal and vertical planes of the building clusters in Group 2 of scattered configuration. 


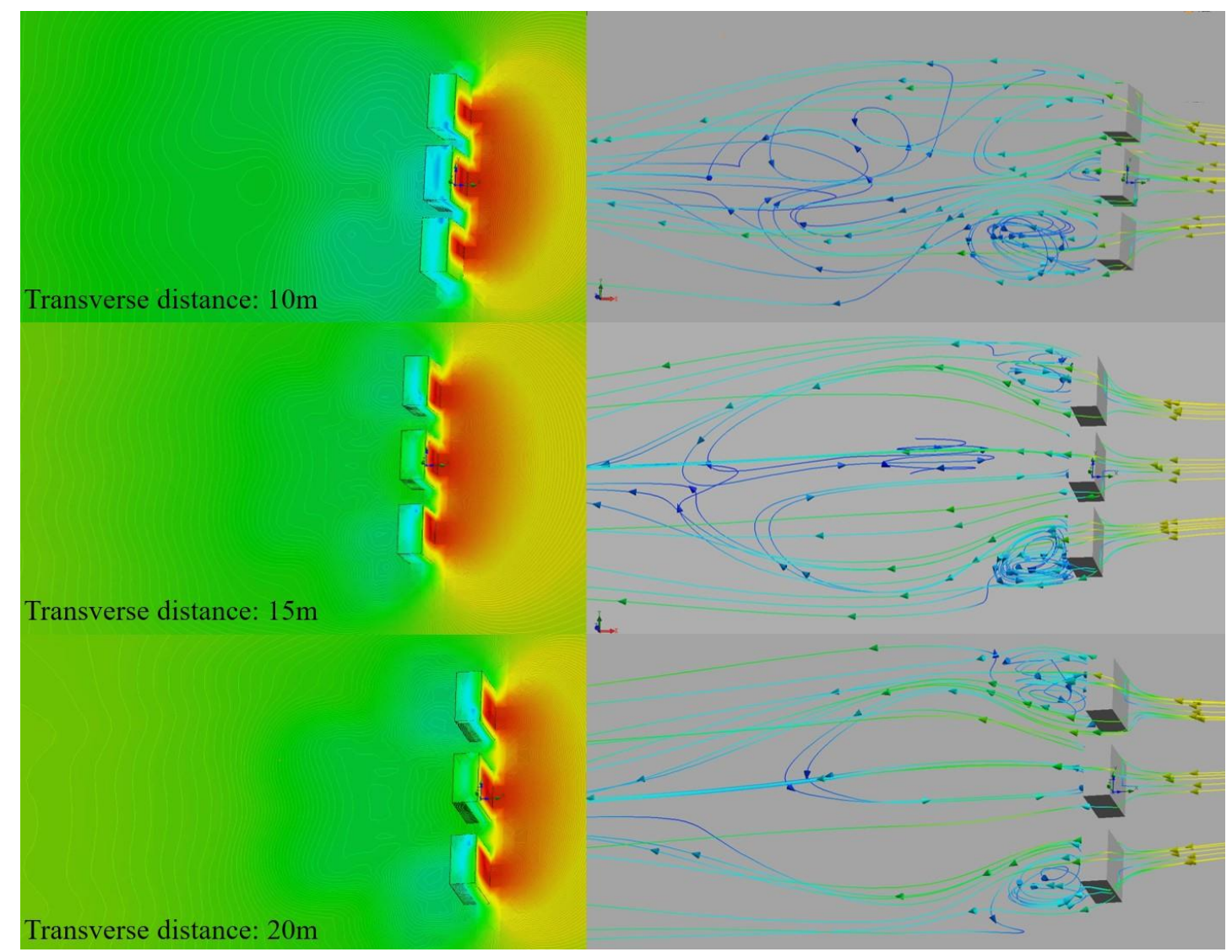

Figure 104. The comparison of wind-flow streamlines (right) and air-pressure magnitudes (left) on the horizontal plane and building surfaces in Group 2 of scattered configuration.

\section{(3) Part 3. Influences of longitudinal distance between 2 units in a column}

The influences of different longitudinal distances of Group 3 are analyzed in this part. The wind-velocity magnitudes on horizontal and vertical planes of three cases are presented (Figure 105). The total influenced areas with different velocities of the three cases are almost the same. In Case $1(0.7 * \mathrm{H}: 25.2 \mathrm{~m})$, the influence of the building column is the least (influenced area: $\left.21920.62 \mathrm{~m}^{2}\right)$; in Case $2(0.9 * \mathrm{H}: 32.4 \mathrm{~m})$, the influence of the building column is the largest (influenced area: $\left.22833 \mathrm{~m}^{2}\right)$; in Case $3(1.1 * \mathrm{H}: 39.6 \mathrm{~m})$, the influence of the building cluster is less than Case 2 but more than Case 1 (influenced area: 22188.33m²). (Figure 106)

The total low-velocity area decreases as the longitudinal distance increases. The lowvelocity area behind the leeward side of the building cluster becomes smaller and shorter as the longitudinal distance increases. In Case 1 , the low-velocity area (velocity below $0.3 \mathrm{~m} / \mathrm{s}$ ) behind the building column is the largest and longest; In Case 2, the low-velocity area is less and shorter than Case 1; in Case 3, the low-velocity area is the smallest and shortest of all. However, 
the low-velocity area in the space between the building units increases as the longitudinal distance increases. The low velocity between the units are nearly unchanged in the three cases. In all the cases, there are high-velocity areas (velocity: $1.4-1.8 \mathrm{~m} / \mathrm{s}$ ) on the lateral sides of the building column. The areas are increased as the longitudinal distance increases. In Case 1, the high-velocity areas are the smallest; in Case 2, the high-velocity areas are more than Case 1; in Case 3, the high-velocity areas are the most of all. (Figure 105)

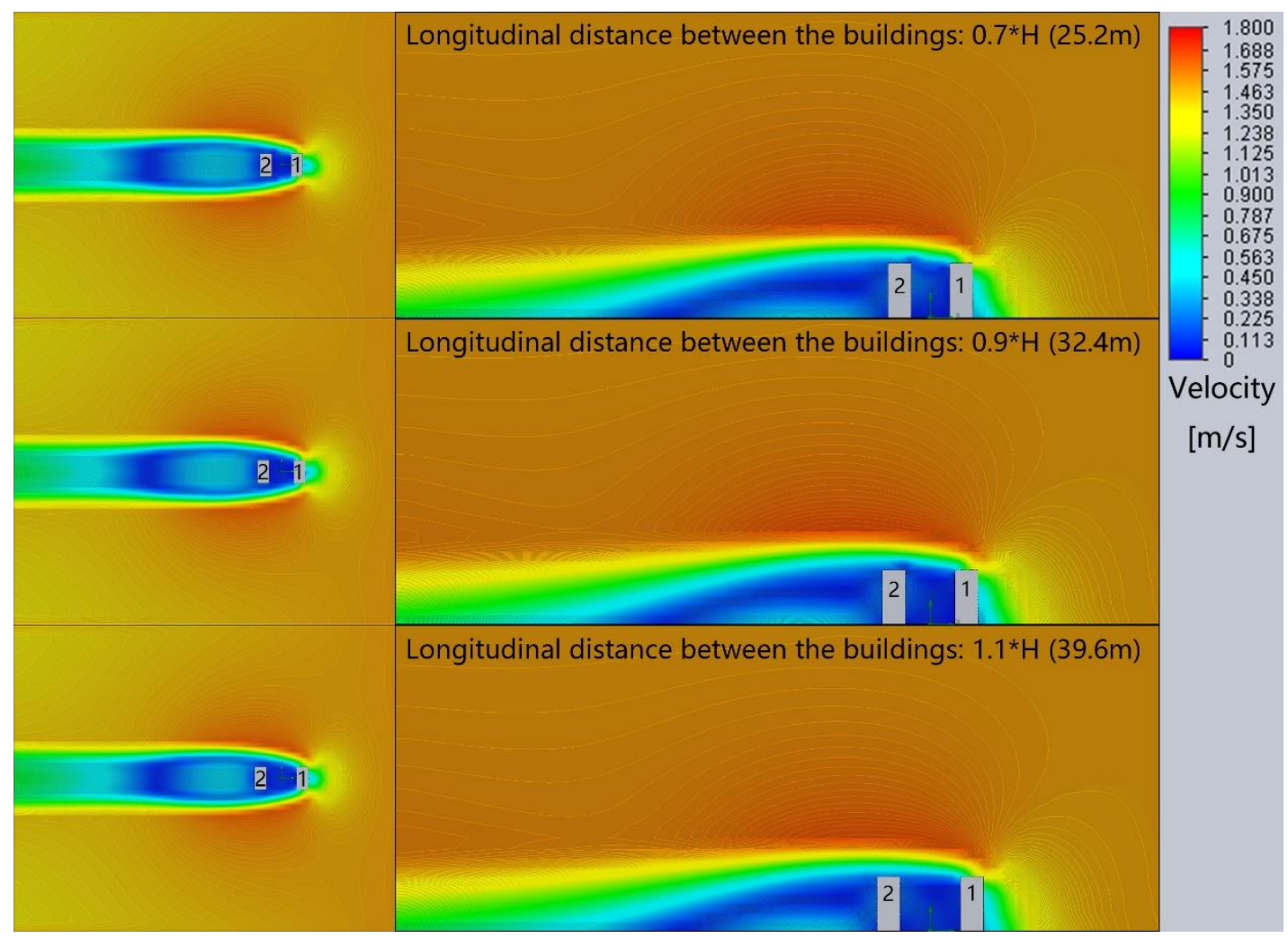

Figure 105. The comparison of wind-velocity magnitudes on horizontal and vertical planes of the building clusters in Group 3 of scattered configuration. 


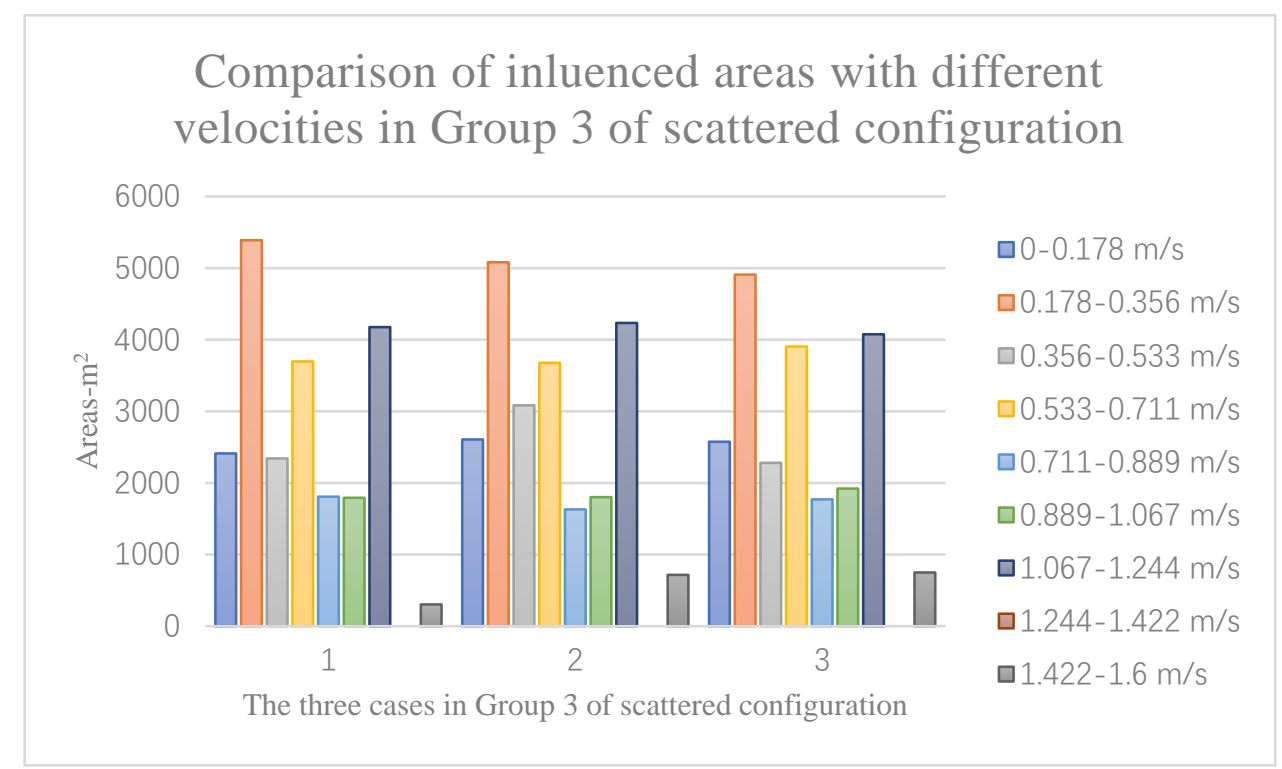

Figure 106. The influenced areas with different velocities in Group 3 of scattered configuration.

The changes of influenced areas with different velocities are related to the changes of air pressure and wind flow in Group 3 (Figure 107). The air-pressure magnitudes show that the low-air-pressure and high-air-pressure areas are almost the same in the three cases. The windward surface of the building is the boundary of the two separate areas. The building unit on the leeward side is in the low-air-pressure area. The low-air-pressure area behind the leeward unit becomes shorter as the longitudinal distance increases. The wind flow streamlines show that the vortices behind the building column are reduced as the longitudinal distance increases. And the sizes of the vortices become smaller and shorter. There are a lot of vortices created between the building units in the three cases. The increase of longitudinal distance has limited effect in improving outdoor ventilation of the space between the building units, though more winds flow into the space. The high-velocity areas on the lateral sides are increased as wind flow is promoted due to the increase of longitudinal distance. Therefore, the air-pressure magnitudes and wind flow streamlines correspond with the wind velocity magnitudes of Group 3. The increase of longitudinal distance can improve the surrounding outdoor ventilation, though there are some limitations. 


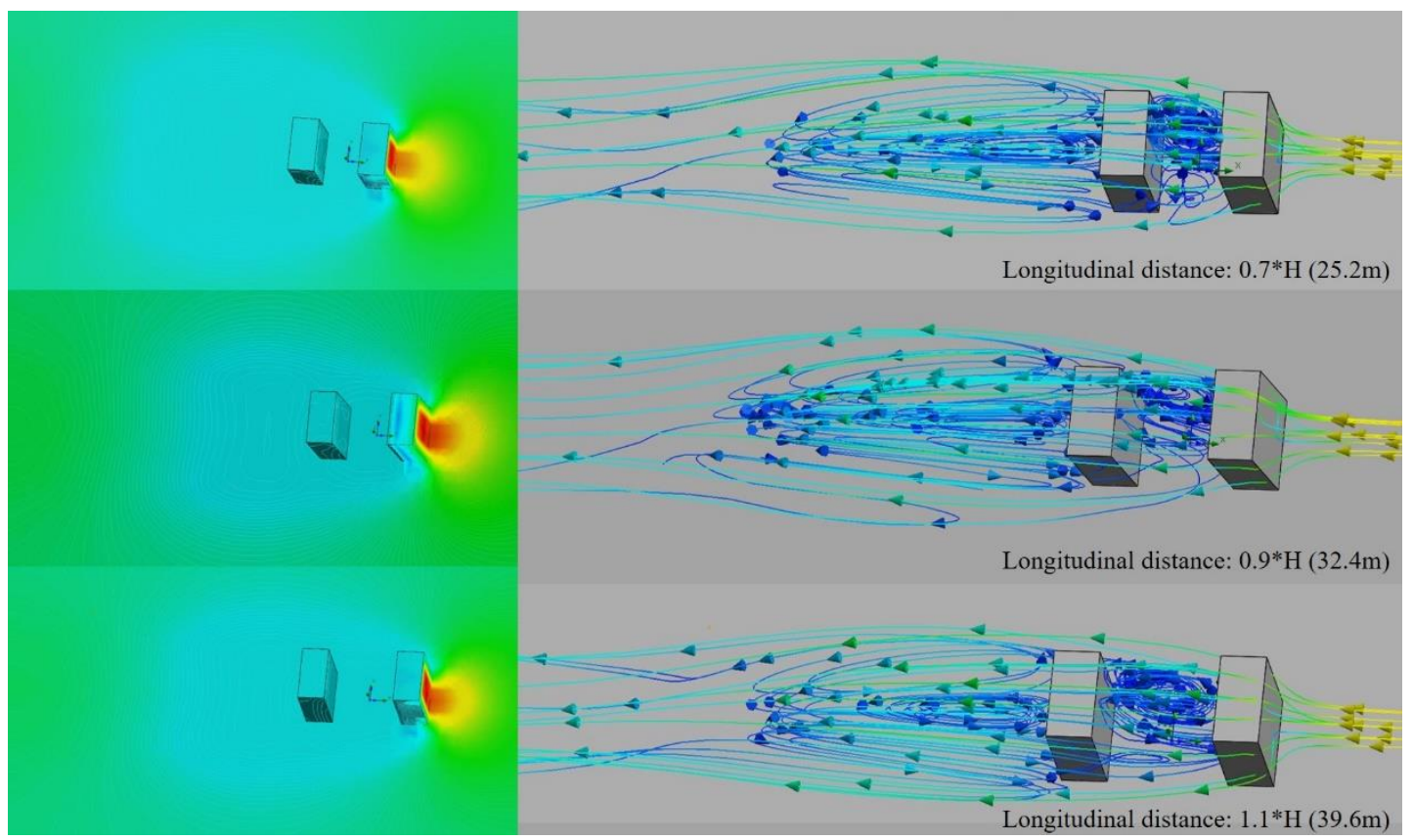

Figure 107. The comparison of wind-flow streamlines (right) and air-pressure magnitudes (left) on the horizontal plane and building surfaces in Group 3 of scattered configuration.

\section{(4) Part 4. Influences of longitudinal distance among 3 units in a column}

In this part, the influences of different longitudinal distances of Group 4 are analyzed. The wind-velocity magnitudes on horizontal and vertical planes of three cases are presented in the figure (Figure 108). The results of Group 4 are similar to Group 3. The total influenced areas of the three cases are close in Group 4 (Figure 109). In Case $1\left(0.7^{*} \mathrm{H}: 25.2 \mathrm{~m}\right)$, the influence of the building column is between Case 2 and Case 3 (influenced area: $23264.55 \mathrm{~m}^{2}$ ); in Case 2 $(0.9 * \mathrm{H}: 32.4 \mathrm{~m})$, the influence of the building column is the largest (influenced area: $\left.23697.27 \mathrm{~m}^{2}\right)$; in Case $3\left(1.1^{*} \mathrm{H}: 39.6 \mathrm{~m}\right)$, the influence of the building cluster is the least of all (influenced area: $22713.06 \mathrm{~m}^{2}$ ).

The total low-velocity area (velocity range: $0-0.533 \mathrm{~m} / \mathrm{s}$ ) decreases as the longitudinal distance increases (Figure 109). The low-velocity area (velocity below $0.533 \mathrm{~m} / \mathrm{s}$ ) of Case 1 is the largest $\left(10019.21 \mathrm{~m}^{2}\right)$; the low-velocity area of Case 2 is less than Case $1\left(9876.049 \mathrm{~m}^{2}\right)$; the low-velocity area of Case 3 is the smallest of all $\left(8691.743 \mathrm{~m}^{2}\right)$. The low-velocity area with the velocity in the range of $0.178-0.356 \mathrm{~m} / \mathrm{s}$ decreases constantly in Case $1\left(4236.642 \mathrm{~m}^{2}\right)$, Case 2 $\left(3981.918 \mathrm{~m}^{2}\right)$ and Case $3\left(2675.278 \mathrm{~m}^{2}\right)$. Similarly, the low-velocity area with the velocity in the range of $0.356-0.533 \mathrm{~m} / \mathrm{s}$ decreases constantly in Case $1\left(4236.642 \mathrm{~m}^{2}\right)$, Case $2\left(3981.918 \mathrm{~m}^{2}\right)$ 
and Case $3\left(2675.278 \mathrm{~m}^{2}\right)$. However, The low-velocity area with the lowest velocity in the range of $0-0.178 \mathrm{~m} / \mathrm{s}$ increases constantly in Case $1\left(3099.211 \mathrm{~m}^{2}\right)$, Case $2\left(3423.773 \mathrm{~m}^{2}\right)$ and Case 3 $\left(3732.498 \mathrm{~m}^{2}\right)$. From Case 1 to Case 3, the low-velocity area behind the leeward side of the building column becomes smaller and shorter as the longitudinal distance increases; the lowvelocity area in the space among the three building units are increased as the longitudinal distance increases. This should be the reason for the increase of the low-velocity area with the velocity in range of $0-0.178 \mathrm{~m} / \mathrm{s}$. The wind velocity magnitudes show that the velocity in the space between the first and second units is extremely low; the velocity in the space between the second and third units is higher than the former building interval.

In the three cases, there are high-velocity areas (velocity range: $1.422-1.6 \mathrm{~m} / \mathrm{s}$ ) on the lateral sides of the building column. The areas are increased constantly as the longitudinal distance increases (Figure 102.). The high-velocity areas of Case 1 are the smallest $\left(1718.537 \mathrm{~m}^{2}\right)$; the areas of Case $2\left(2289.034 \mathrm{~m}^{2}\right)$ are between Case 1 and Case 3 ; the areas of Case 3 are the largest of all $\left(2781.855 \mathrm{~m}^{2}\right)$.

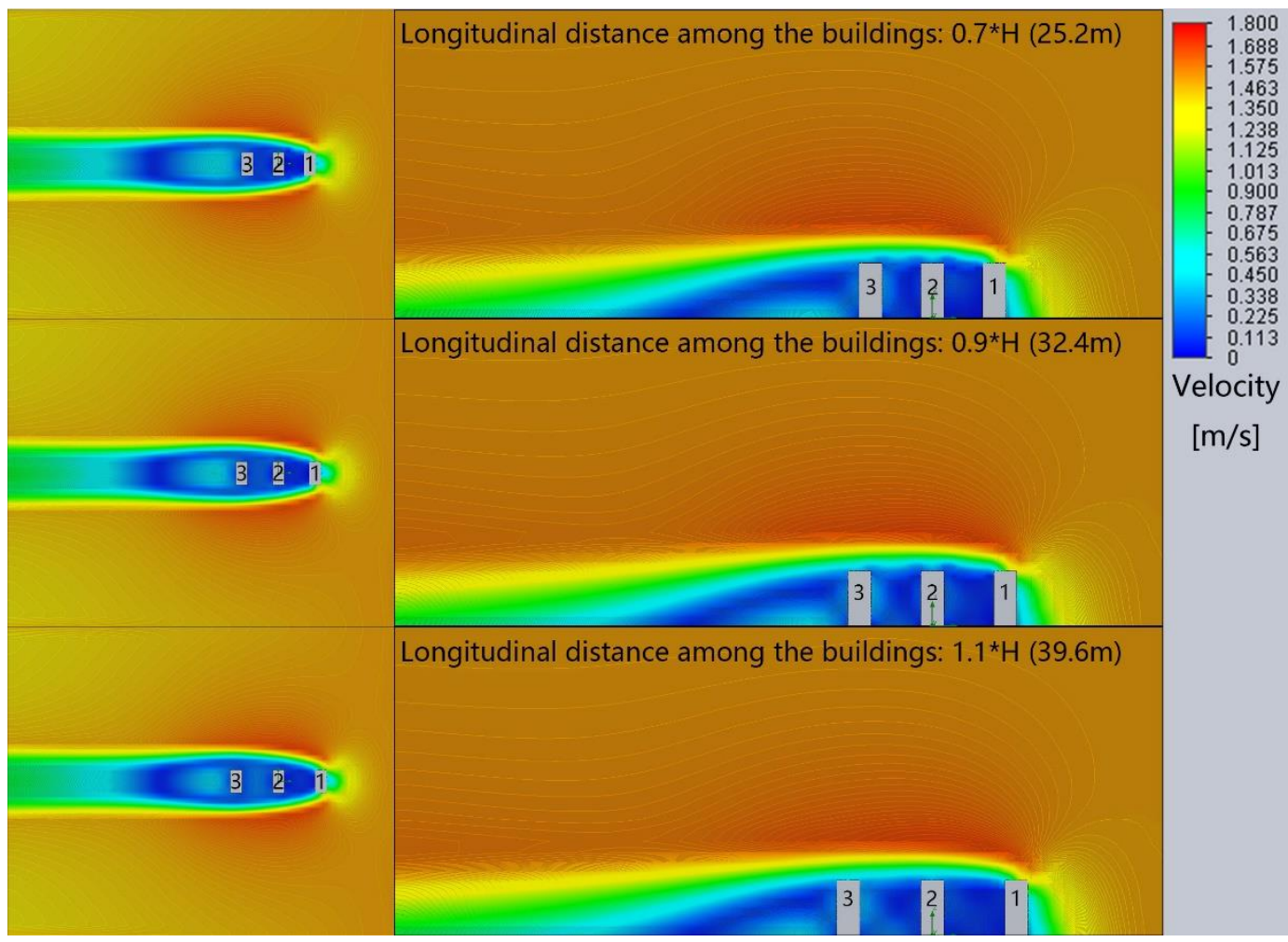

Figure 108. The comparison of wind-velocity magnitudes on horizontal and vertical planes of the building clusters in Group 4 of scattered configuration. 


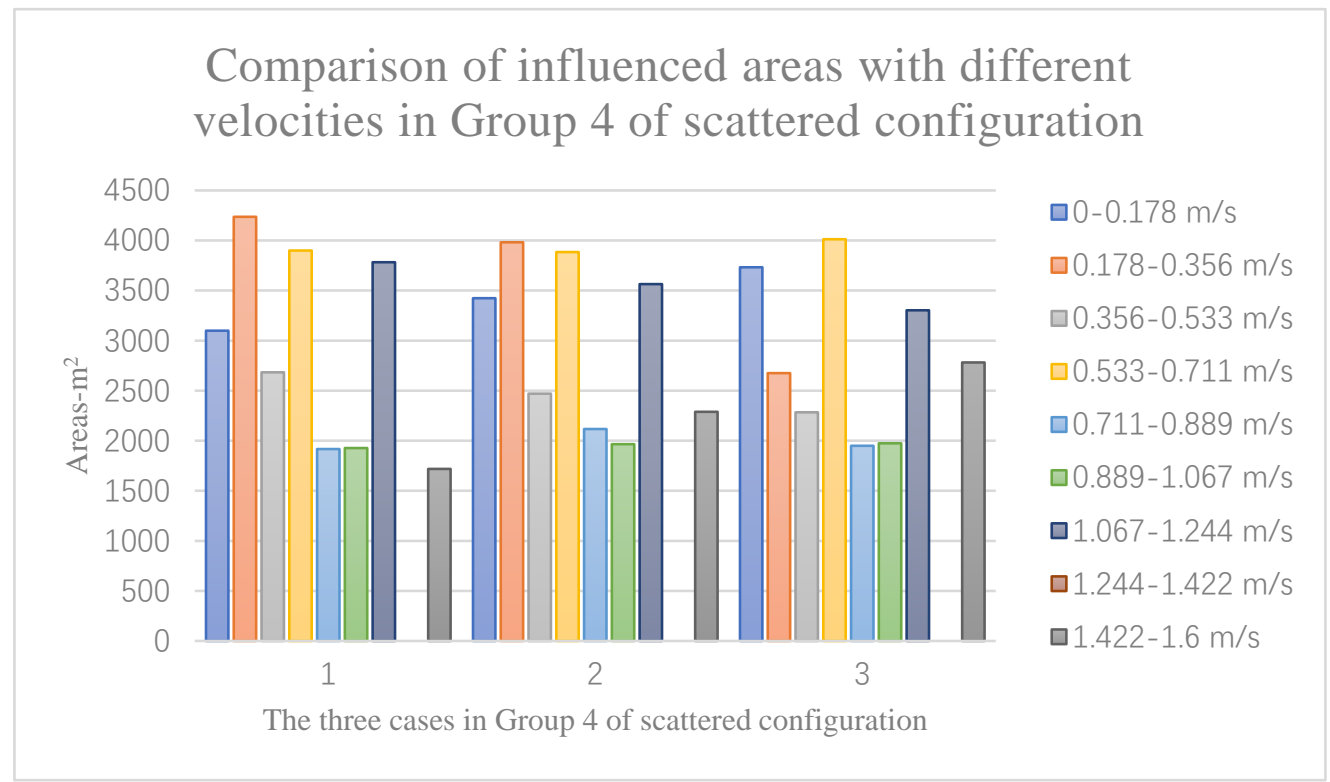

Figure 109. The influenced areas with different velocities in Group 4 of scattered configuration.

The changes of influenced areas with different velocities in Group 4 are related to the changes of air pressure and wind flow (Figure 110). The low-air-pressure area and high-airpressure area are separate at the windward surface of the first building unit. The air-pressure magnitudes show that the two areas are reduced as the longitudinal distance increases. The difference between the two areas increases slightly as the longitudinal distance increases, which should be the reason for the increase of the high-velocity areas on the lateral sides of the building column. The two building units on the leeward side are in the low-air-pressure area. The length of the low-air-pressure area behind the last unit decreases as the longitudinal distance increases, which is the reason for the decrease of the low-velocity area.

The wind flow streamlines show that there are vortices among the building units and behind the leeward side of the building column. As the longitudinal distance increases, the vortices behind the leeward side of the building column become smaller and shorter; the vortices among the building units become less. More winds can flow into the space among the units. The decrease of the vortices should be the reason for the decrease of the low-velocity area. The streamlines also show that vortices between the first and second units are more than the vortices between the second and third units. This can explain the wind velocity in the space between the second and third units is higher than the velocity in the space between the first and 
second units. As the longitudinal distance increases, the low-air-pressure area decreases; and the sizes and numbers of vortices are reduced. Therefore, the increase of the longitudinal distance can promote the outdoor ventilation around the building cluster.

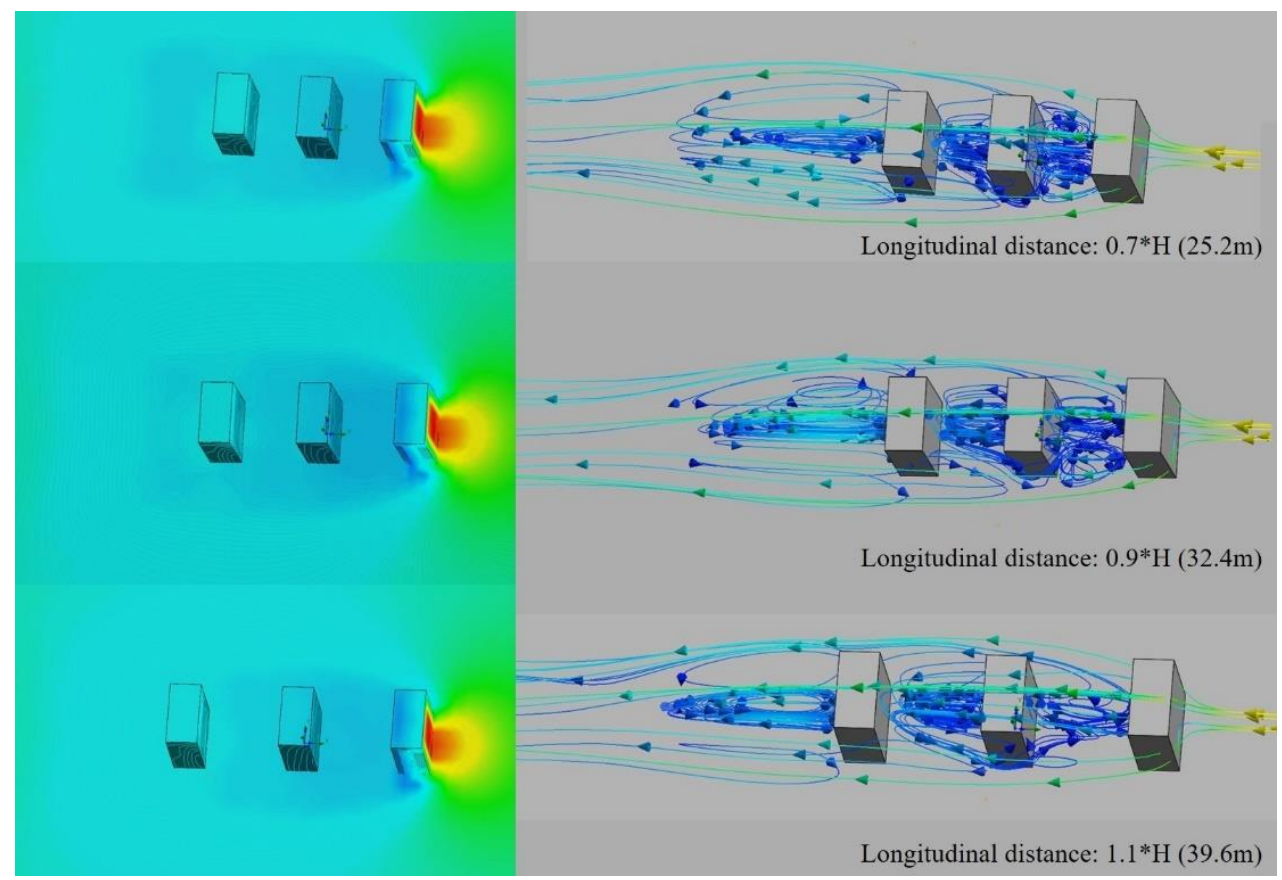

Figure 110. The comparison of wind-flow streamlines (right) and air-pressure magnitudes (left) on the horizontal plane and building surfaces in Group 4 of scattered configuration.

\section{(5) Part 5. Influences of longitudinal and transverse distances among 4 units in 2 rows}

In this part, the influences of different longitudinal distances of Group 5 (transverse distance: $10 \mathrm{~m})$ are analyzed first. The wind-velocity magnitudes on horizontal and vertical planes of three cases are analyzed (Figure 111). In Group 5, the total influenced area with different velocities increases as the longitudinal distance increases, though the differences among the three cases are relatively small (Figure 112$)$. In Case $1(0.7 * \mathrm{H}: 25.2 \mathrm{~m})$, the influence of the building cluster is the least (influenced area: $\left.39567.69 \mathrm{~m}^{2}\right)$; in Case $2(0.9 * \mathrm{H}: 32.4 \mathrm{~m})$, the influence of the building cluster is between Case 1 and Case 3 (influenced area: $40457.8 \mathrm{~m}^{2}$ ); in Case $3(1.1 * \mathrm{H}: 39.6 \mathrm{~m})$, the influence of the building cluster is the most of all (influenced area: $\left.41117.34 \mathrm{~m}^{2}\right)$.

The low-velocity areas with the velocity ranges of $0-0.2 \mathrm{~m} / \mathrm{s}$ and $0.2-0.4 \mathrm{~m} / \mathrm{s}$ are both decreased constantly as the longitudinal distance decreases (Figure 112). Therefore, the total 
low-velocity area (velocity range: $0-0.4 \mathrm{~m} / \mathrm{s}$ ) decreases as the longitudinal distance increases. In Case 1, the total low-velocity area is the largest $\left(17398.69 \mathrm{~m}^{2}\right)$; in Case 2, the area is less than Case $1\left(17019.86 \mathrm{~m}^{2}\right)$; in Case 3, the area is the least of all $\left(16372.9 \mathrm{~m}^{2}\right)$. The wind velocity magnitudes show that the velocity in the space between the building rows is relatively lower than the other low-velocity area; the wind velocity in the middle gap between the building columns is higher than the low-velocity area. As the longitudinal distance increases, the lowvelocity area behind the leeward side of the building cluster becomes smaller and shorter; the wind velocity is slightly increased in the space between the two building rows, though the lowvelocity area between the two building rows are also increased.

There are high-velocity areas (velocity range: $1.4-1.6 \mathrm{~m} / \mathrm{s}$ ) on the lateral sides of the building cluster. The areas increase constantly as the longitudinal distance increases (Figure 105.). In Case 1, the high-velocity areas are the smallest of all $\left(9221.722 \mathrm{~m}^{2}\right)$; in Case 2, the areas $\left(17019.86 \mathrm{~m}^{2}\right)$ are more than Case 1 ; in Case 3, the areas are the largest $\left(16372.9 \mathrm{~m}^{2}\right)$. Also, winds are accelerated in the middle gap between the building columns. But the wind velocity is lower than the high-velocity areas on the lateral sides.

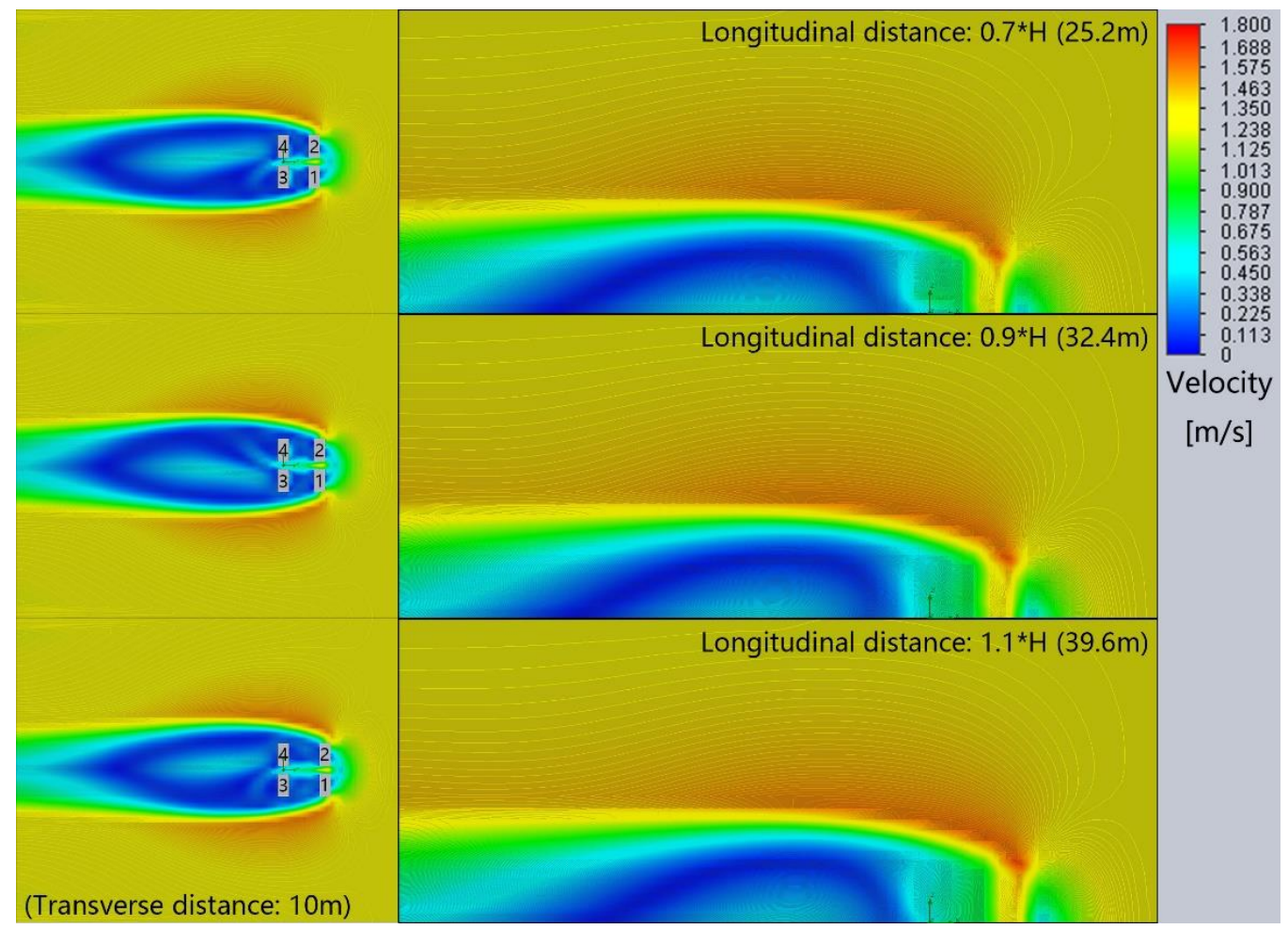

Figure 111. The comparison of wind-velocity magnitudes on horizontal and vertical planes of the building clusters in Group 5 of scattered configuration. 


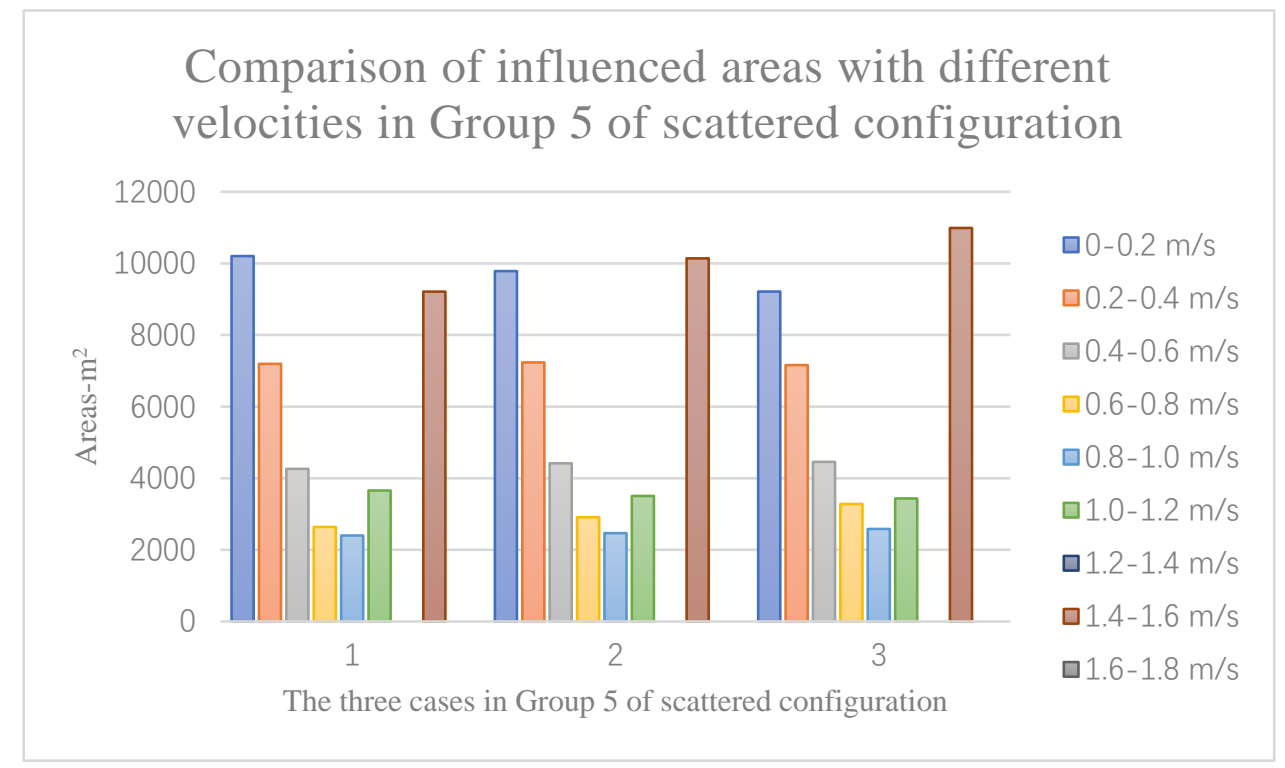

Figure 112. The influenced areas with different velocities in Group 5 of scattered configuration.

According to the analysis of the wind-velocity magnitudes, the results of the influences of longitudinal distances in Group 6 (transverse distance: $15 \mathrm{~m}$ ) and Group 7 (transverse distance: 20m) are similar to former Group 5 (Figure 113, Figure 114, Figure 115 and Figure 116). In Group 6, the total influenced areas of the three cases are close (Figure 114). The influence of Case $1(0.7 * \mathrm{H}: 25.2 \mathrm{~m})$ is the most (influenced area: $\left.39776.97 \mathrm{~m}^{2}\right)$; the influence of Case 2 $(0.9 * \mathrm{H}: 32.4 \mathrm{~m})$ is the least (influenced area: $\left.39442.69 \mathrm{~m}^{2}\right)$; the influence of Case $3(1.1 * \mathrm{H}$ : 39.6m) is between Case 1 and Case 2 (influenced area: $41117.34 \mathrm{~m}^{2}$ ). In Group 7, the total influenced area decreases gradually as the longitudinal distance increases, though the areas of the three cases are close (Figure 116). The influence of Case 1 is the most (influenced area: $41373.66 \mathrm{~m}^{2}$ ); the influence of Case 2 (influenced area: $41695.25 \mathrm{~m}^{2}$ ) is less than Case 1; the influence of Case 3 is the least (influenced area: $42210.74 \mathrm{~m}^{2}$ ).

In Group 6, the low-velocity area (velocity range: $0-0.4 \mathrm{~m} / \mathrm{s}$ ) decreases constantly in Case 1 (influenced area: $14293.22 \mathrm{~m}^{2}$ ), Case 2 (influenced area: $13414.48 \mathrm{~m}^{2}$ ) and Case 3 (influenced area: $12499.7 \mathrm{~m}^{2}$ ). The low-velocity areas with the velocity ranges of $0-0.2 \mathrm{~m} / \mathrm{s}$ and $0.2-0.4 \mathrm{~m} / \mathrm{s}$ are both decreased as the longitudinal distance increases (Figure 114). In Group 7, the lowvelocity area (velocity range: $0-0.4 \mathrm{~m} / \mathrm{s}$ ) decreases constantly in Case 1 (influenced area: $14024.87 \mathrm{~m}^{2}$ ), Case 2 (influenced area: $13336.33 \mathrm{~m}^{2}$ ) and Case 3 (influenced area: $12912.81 \mathrm{~m}^{2}$ ). 
Also, the low-velocity areas with the velocity ranges of $0-0.2 \mathrm{~m} / \mathrm{s}$ and $0.2-0.4 \mathrm{~m} / \mathrm{s}$ are both decreased as the longitudinal distance increases. In addition, the low-velocity area with the velocity range of $0.4-0.6 \mathrm{~m} / \mathrm{s}$ decreases significantly in Case 1 (influenced area: $7081.35 \mathrm{~m}^{2}$ ), Case 2 (influenced area: $6253.88 \mathrm{~m}^{2}$ ) and Case 3 (influenced area: $5464.36 \mathrm{~m}^{2}$ ) (Figure 116).

In Group 6 and Group 7, the trends of the high-velocity areas (velocity range: $1.4-1.6 \mathrm{~m} / \mathrm{s}$ ) are the same as Group 5 (Figure 114 and Figure 116). In Group 6, the high-velocity areas increase constantly in Case 1 (influenced area: $7228.25 \mathrm{~m}^{2}$ ), Case 2 (influenced area: $8135.24 \mathrm{~m}^{2}$ ) and Case 3 (influenced area: $8872.75 \mathrm{~m}^{2}$ ). In Group 7, the high-velocity areas also increase in Case 1 (influenced area: $7369.66 \mathrm{~m}^{2}$ ), Case 2 (influenced area: $8028.17 \mathrm{~m}^{2}$ ) and Case 3 (influenced area: $8761.12 \mathrm{~m}^{2}$ ). There are high-velocity areas on the lateral sides and in the middle gap between the building column.

According to the velocity magnitudes of Group 5, Group 6 and Group 7, the total influence of the building cluster increases gradually as the transverse distance increases, though the differences among cases with different transverse distances are close. However, the lowvelocity area and high-velocity areas decrease as the transverse distance increases. But the decreases of high-velocity areas are little in Group 6 and Group 7. The wind velocity increases in the gap between the building columns as the transverse distance increases. As the gap becomes larger, more winds are able to flow through the building cluster. This is the reason for the reduction of the low-velocity area behind the building cluster and high-velocity areas on lateral sides. In addition, the wind velocity between the building rows is increased as the transverse distance increases. Therefore, the increase of the transverse distance between the two building columns can improve the surrounding outdoor ventilation generally. 


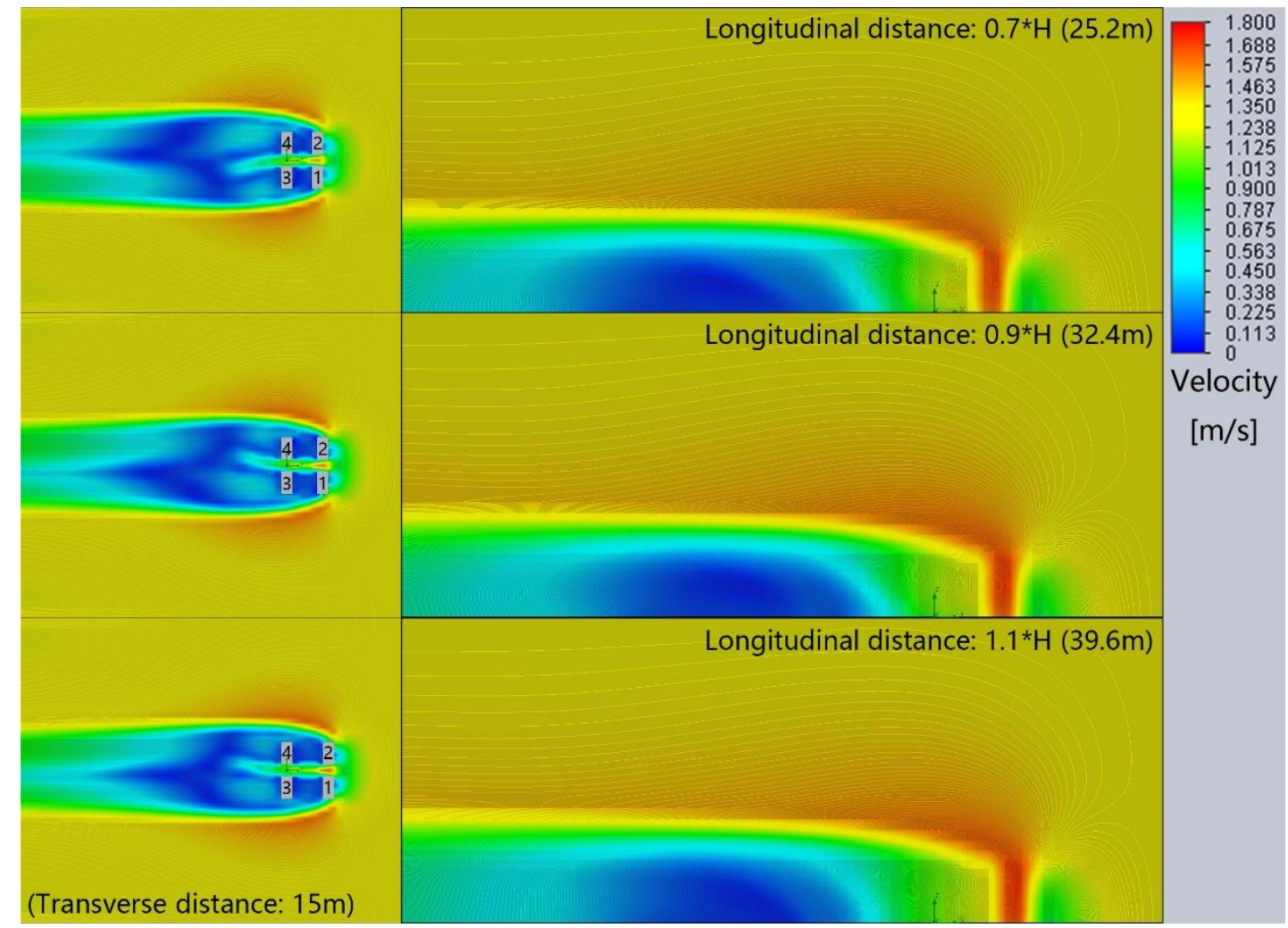

Figure 113. The comparison of wind-velocity magnitudes on horizontal and vertical planes of the building clusters in Group 6 of scattered configuration.

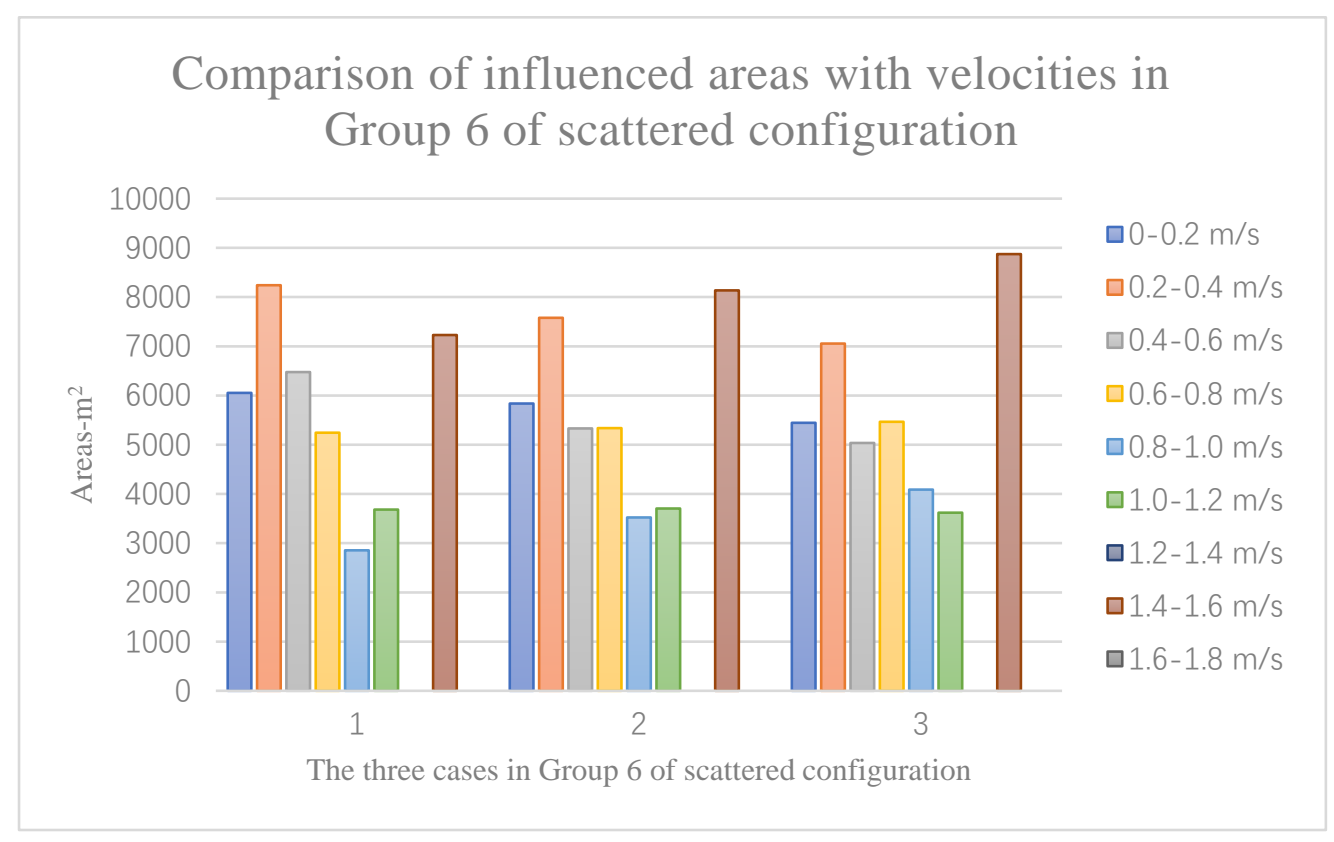

Figure 114. The influenced areas with different velocities in Group 6 of scattered configuration. 


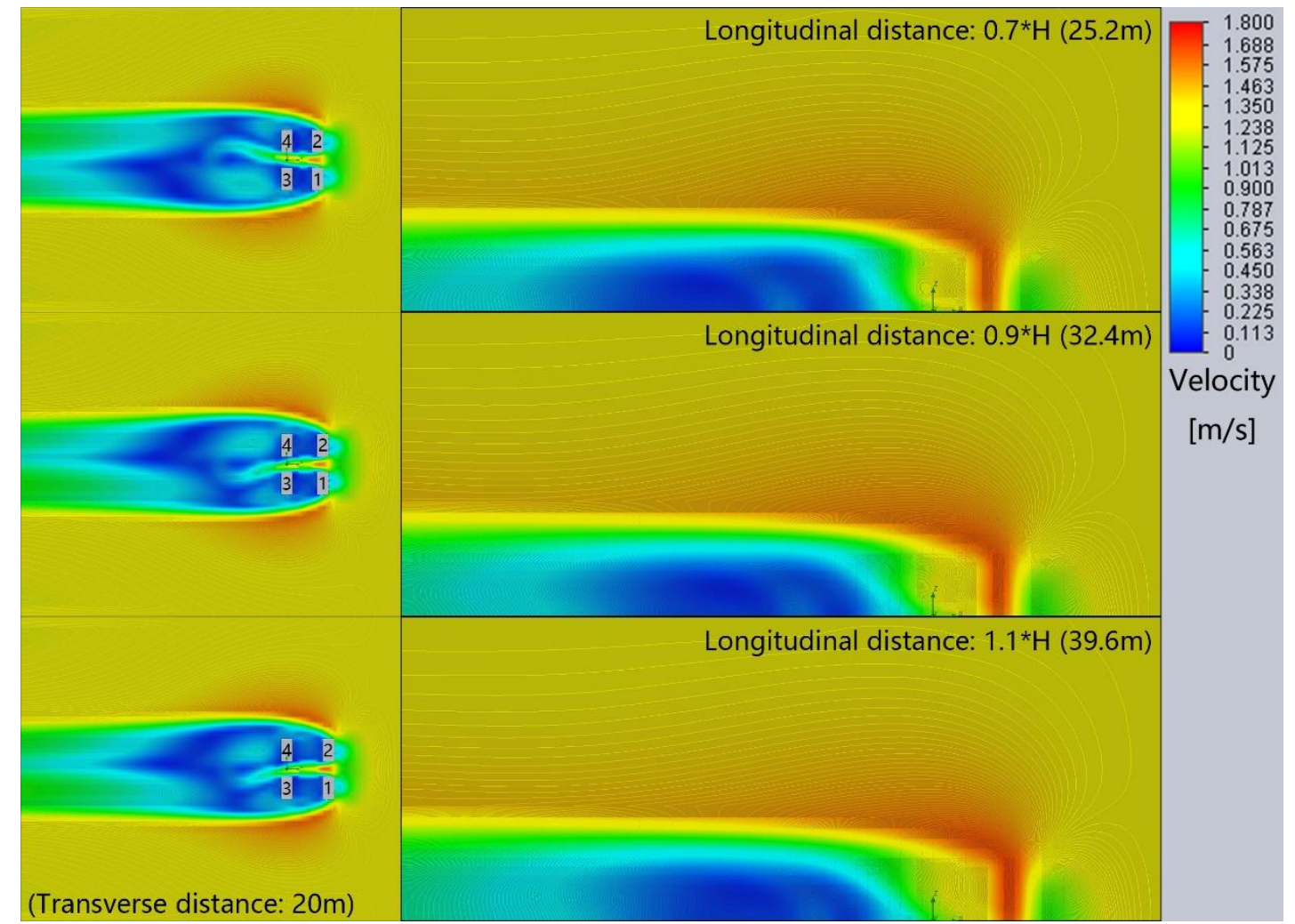

Figure 115. The comparison of wind-velocity magnitudes on horizontal and vertical planes of the building clusters in Group 7 of scattered configuration.

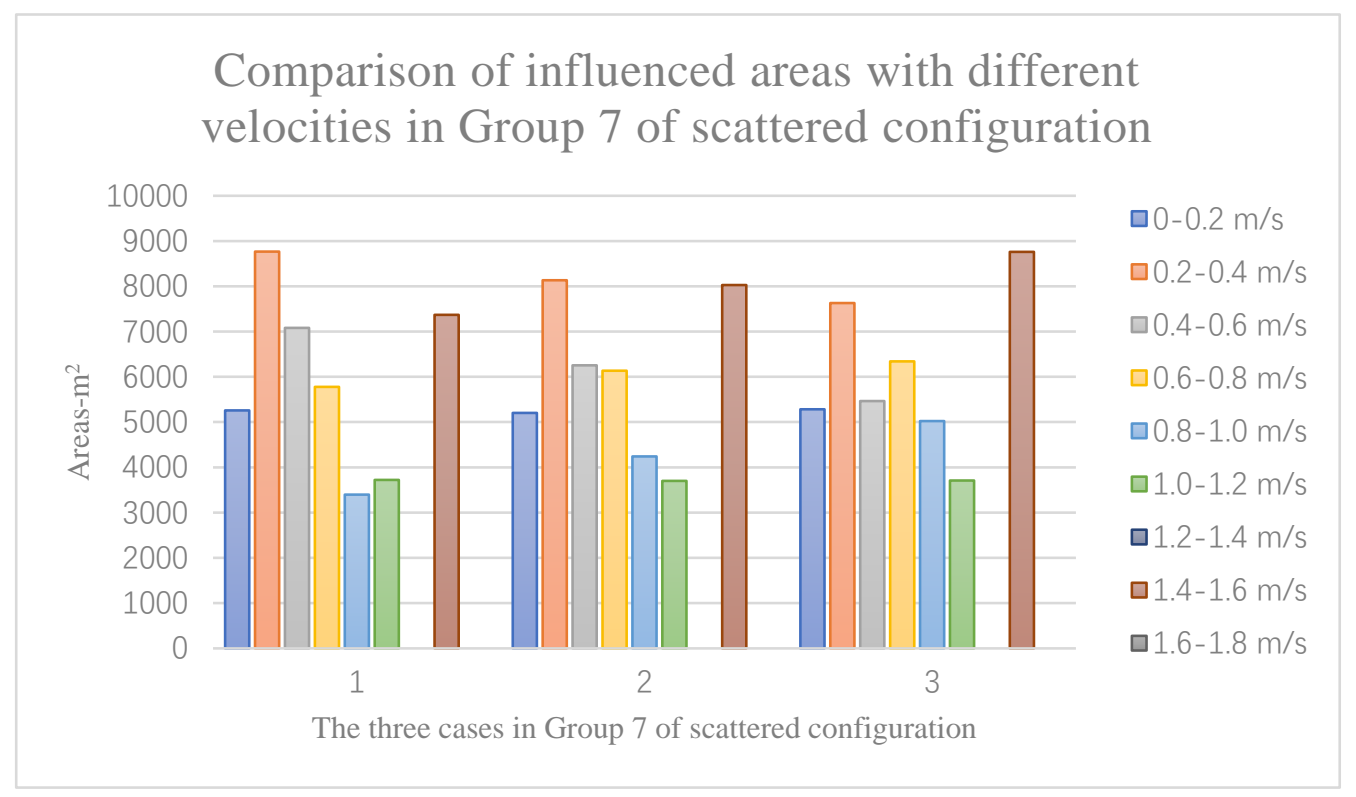

Figure 116. The influenced areas with different velocities in Group 7 of scattered configuration.

The changes of influenced areas with different velocities are related to the changes of air pressure and wind flow. The trends of air-pressure magnitudes and wind-flow streamlines of 
Group 6, Group 7 and Group 5 are similar (Figure 117, Figure 118, Figure 119, Figure 120, Figure 121 and Figure 122).

As the longitudinal distance increases, the low-air-pressure area behind the building cluster and high-air-pressure area in front of the building cluster are reduced; the difference between the highest and lowest air-pressure values increases slightly, which causes the increase of the high-velocity areas; the air pressure (in the low-air-pressure area) between the building rows is almost the same in the three cases; the length of the low-air-pressure area behind the building row on the leeward side is decreased, which makes the low-velocity area become shorter; the vortices between the building rows become less; the vortices on the leeward side of the building cluster become smaller and shorter.

The increase of transverse distance has similar influences as the increase of longitudinal distance. Both the low-air-pressure and high-air-pressure areas are reduced and the overall airpressure magnitudes on the horizontal plane become more even. And the decrease of the lowair-pressure area makes the low-velocity area smaller and shorter. Also, the air-pressure difference is increased. The wind flow streamlines show that the increase of transverse distance makes winds go through the building cluster more easily. Less vortices are created between the building rows and behind the building cluster. The vortices behind the building cluster become smaller and shorter significantly. Therefore, these are the reason for the decrease of the entire low-velocity area. 


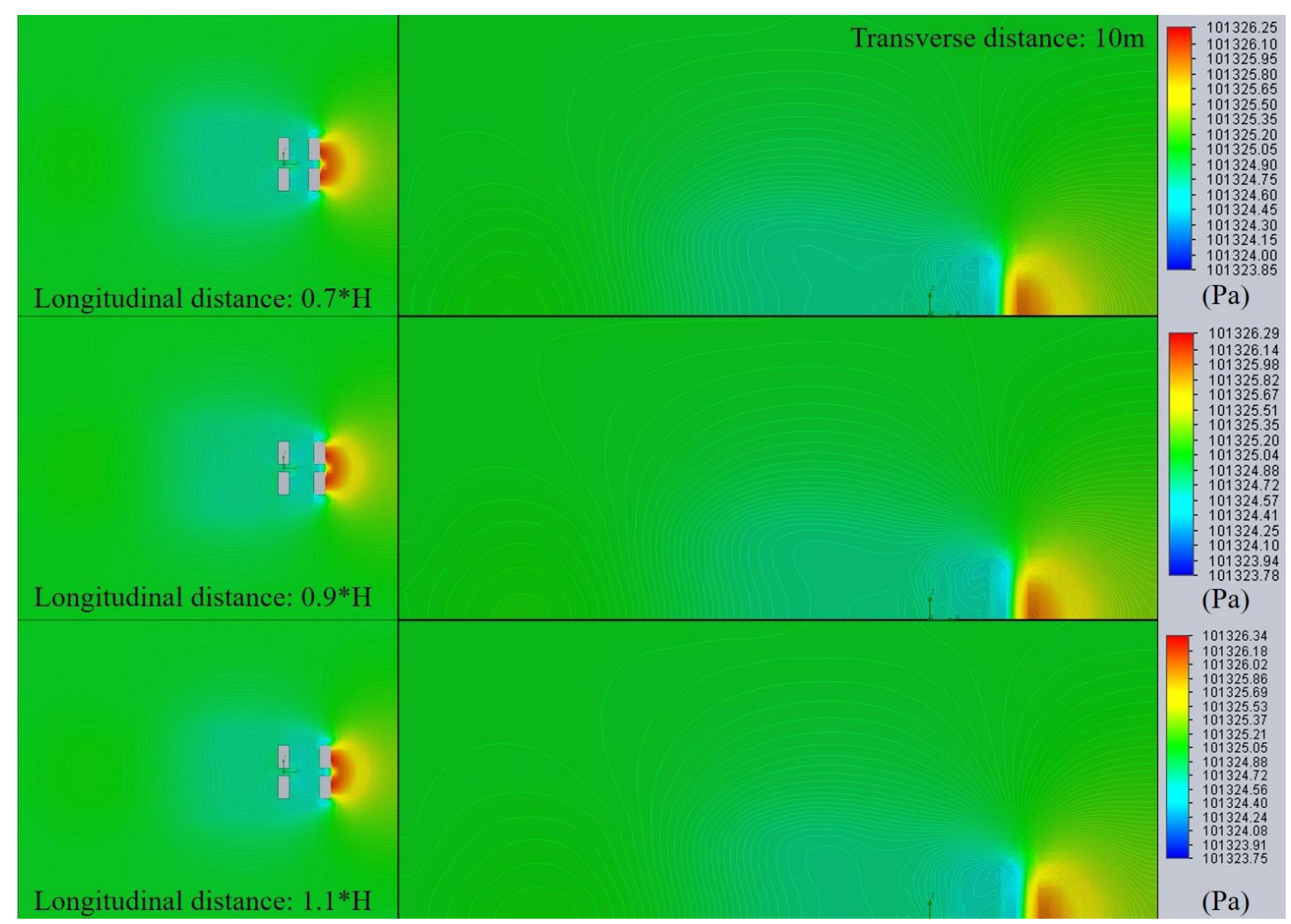

Figure 117. The comparison of air-pressure magnitudes on the horizontal and vertical planes of the building clusters in Group 5 of scattered configuration.

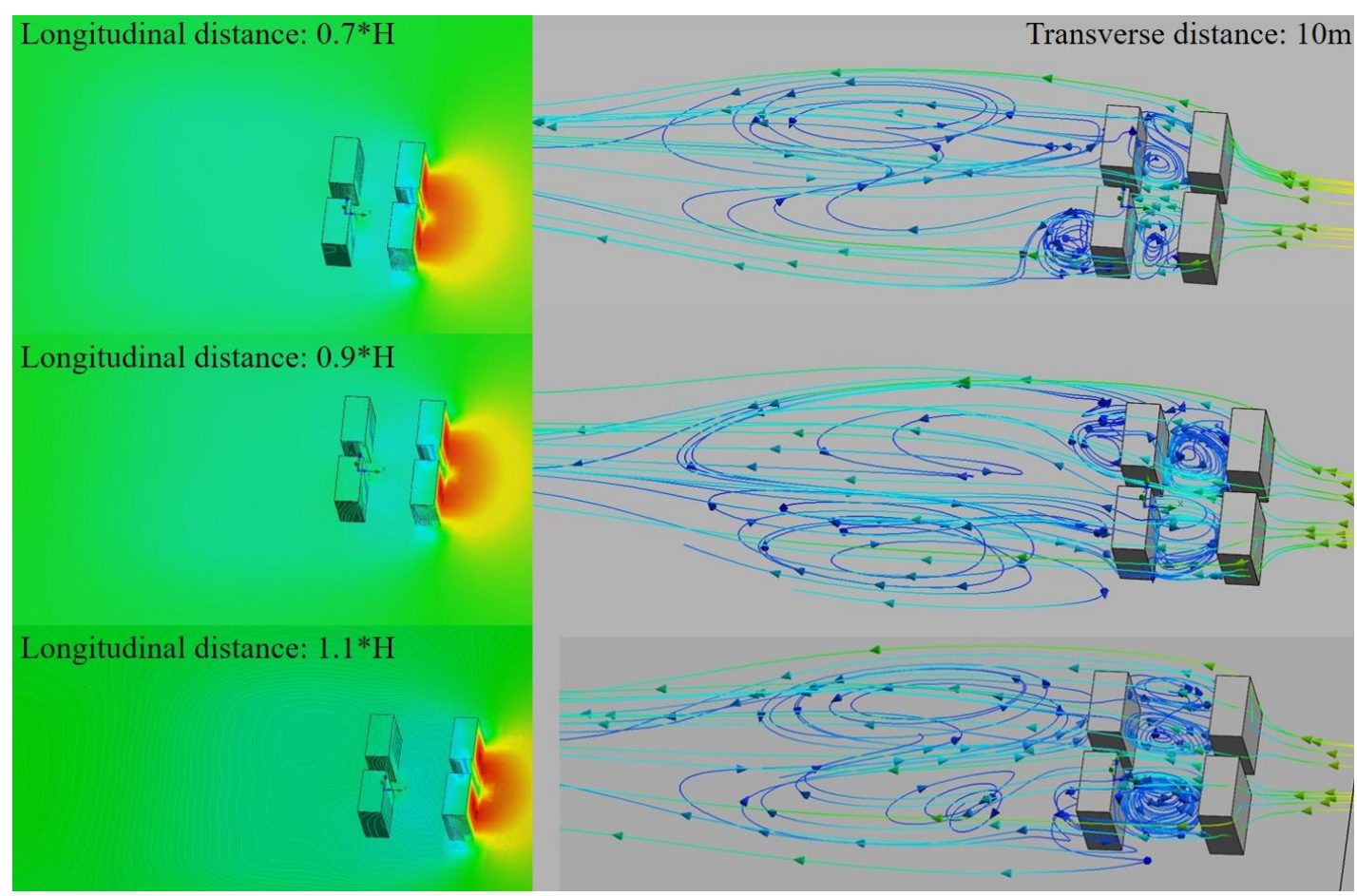

Figure 118. The comparison of wind-flow streamlines (right) and air-pressure magnitudes (left) on the horizontal plane and building surfaces in Group 5 of scattered configuration. 


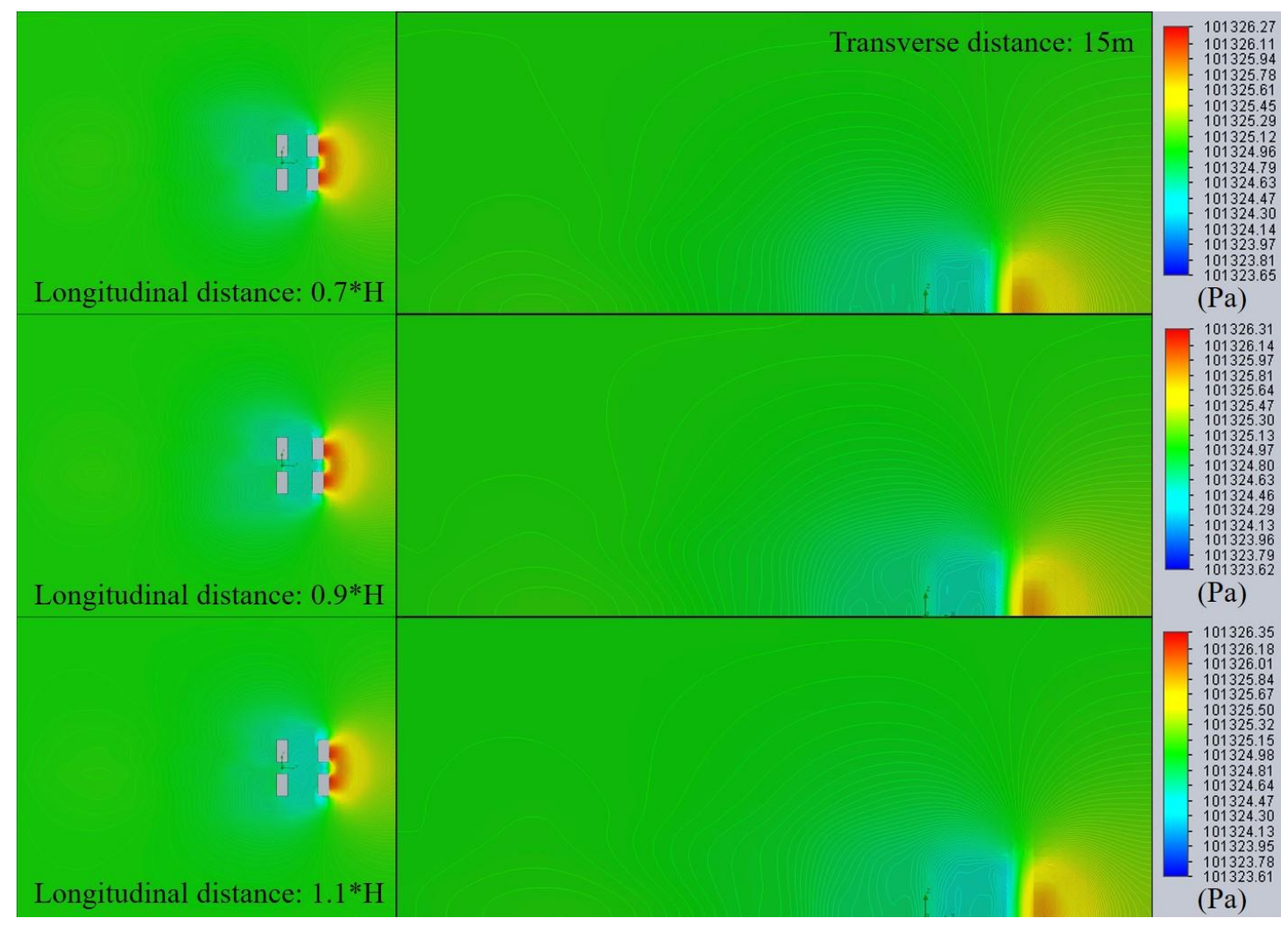

Figure 119. The comparison of air-pressure magnitudes on the horizontal and vertical planes of the building clusters in Group 6 of scattered configuration.

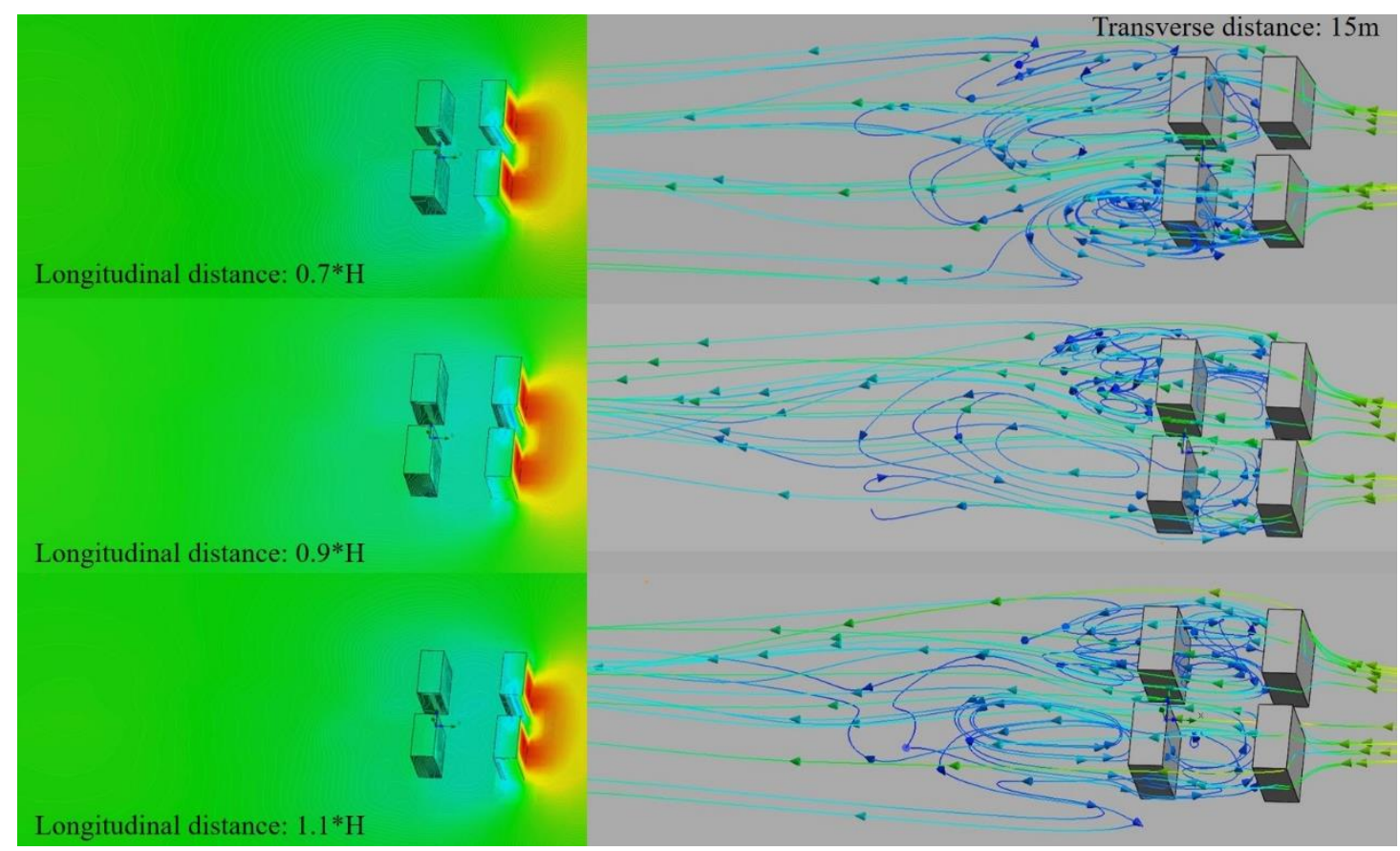

Figure 120. The comparison of wind-flow streamlines (right) and air-pressure magnitudes (left) 
on the horizontal plane and building surfaces in Group 6 of scattered configuration.

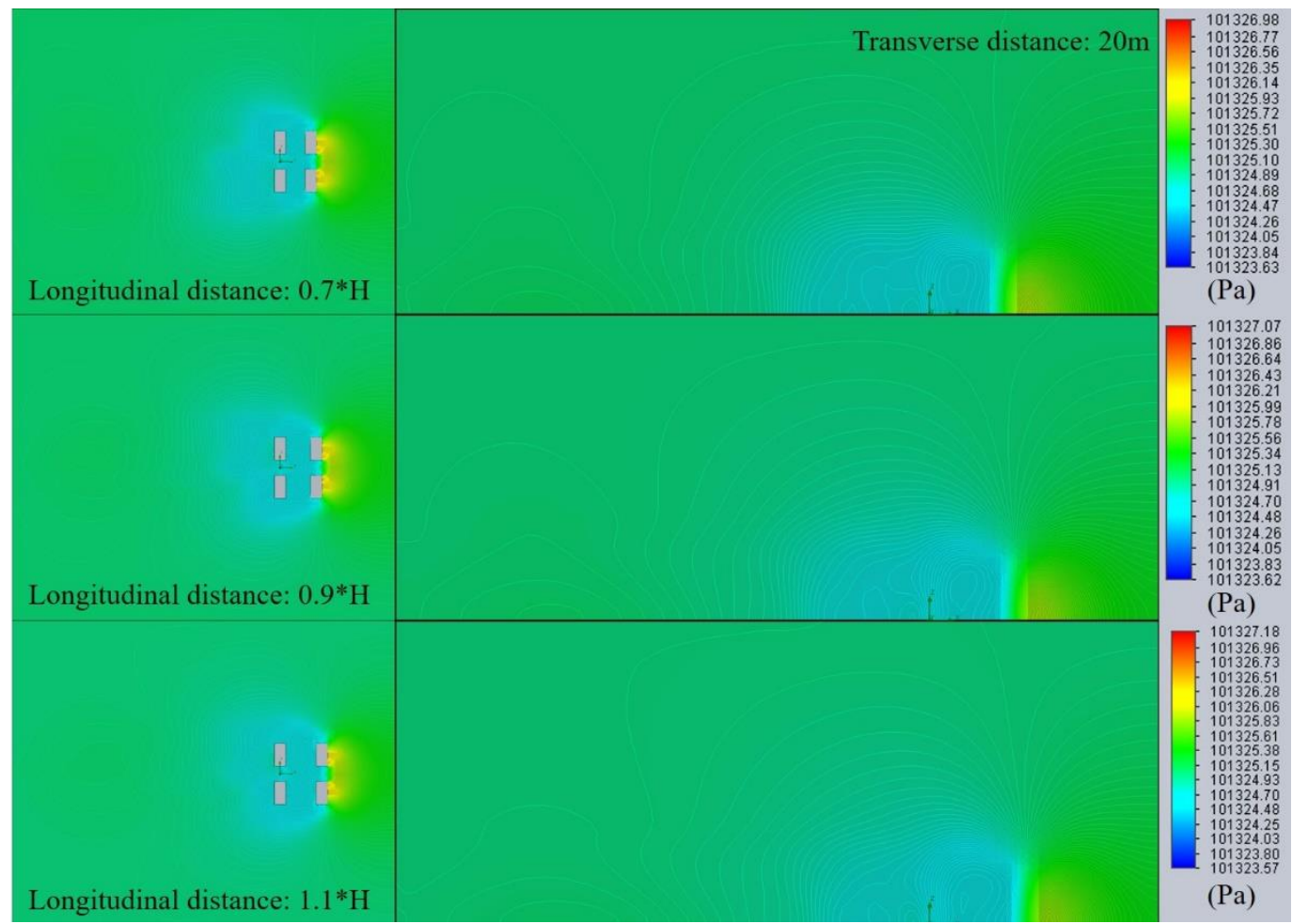

Figure 121. The comparison of air-pressure magnitudes on the horizontal and vertical planes of the building clusters in Group 7 of scattered configuration.

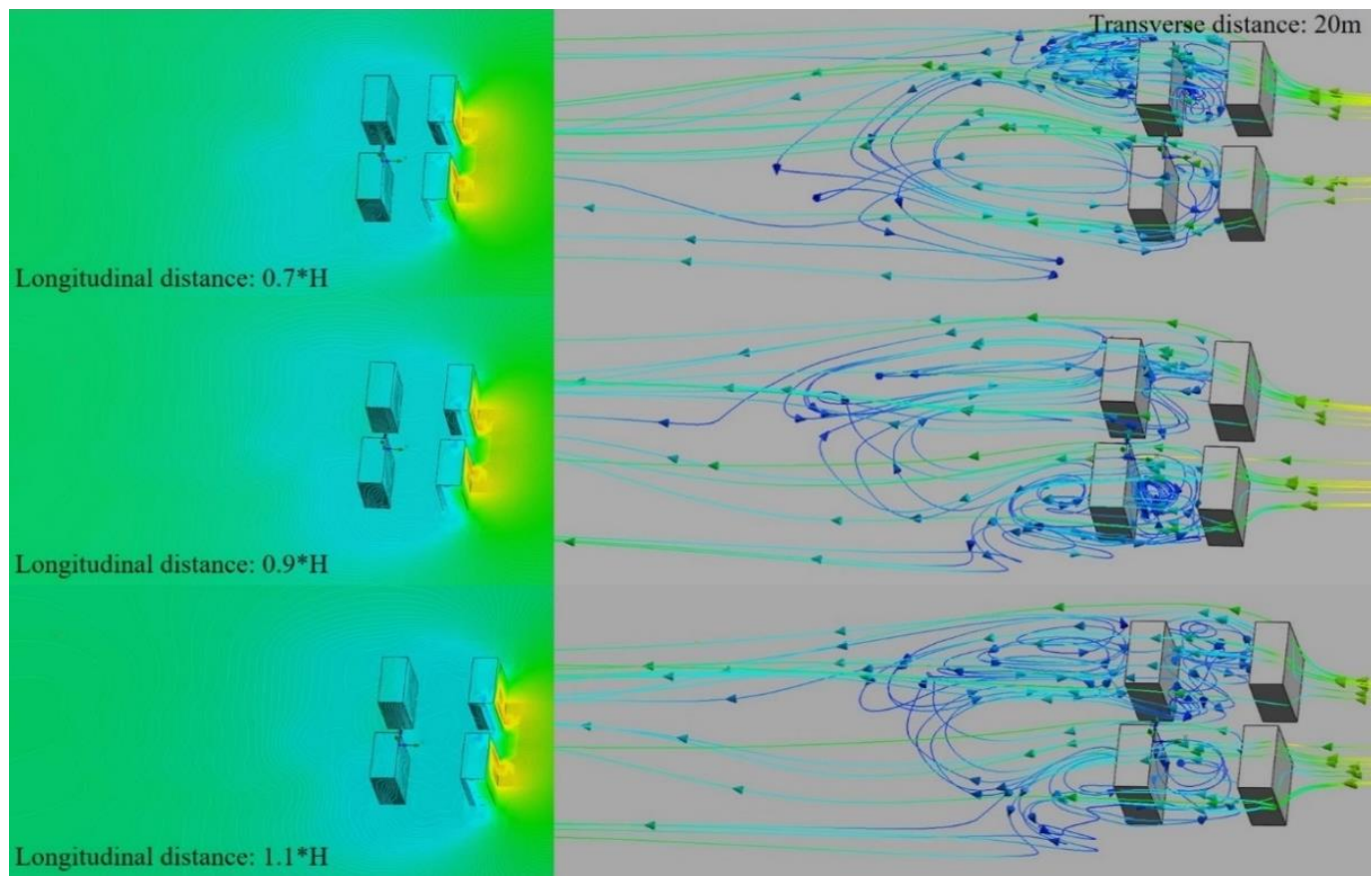

Figure 122. The comparison of wind-flow streamlines (right) and air-pressure magnitudes (left) 
on the horizontal plane and building surfaces in Group 7 of scattered configuration.

\section{(6) Part 6. Influences of longitudinal and transverse distances of 6 units in 3 rows}

In this part, the influences of different longitudinal distances of Group 8 (transverse distance: $15 \mathrm{~m})$ are analyzed first. The wind-velocity magnitudes on horizontal and vertical planes of three cases are analyzed (Figure 123 and Figure 124). In Group 8, the influence of Case 1 $\left(0.7^{*} \mathrm{H}: 25.2 \mathrm{~m}\right)$ is the most (influenced area: $\left.43635.56 \mathrm{~m}^{2}\right)$; the influence of Case $2(0.9 * \mathrm{H}$ : $32.4 \mathrm{~m}$ ) is the least (influenced area: $\left.38418.65 \mathrm{~m}^{2}\right)$; the influence of Case $3(1.1 * \mathrm{H}: 39.6 \mathrm{~m}$ ) is between Case 1 and Case 2 (influenced area: $41164.75 \mathrm{~m}^{2}$ ). Generally, the total influenced area of Group 8 decreases and the outdoor ventilation is promoted as the longitudinal distance increases. However, the influence of Case 3 is more than Case 2. The increase is largely due to the increase of the influenced areas with the velocity ranges of $0.6-0.8 \mathrm{~m} / \mathrm{s}$ and $0.8-1.0 \mathrm{~m} / \mathrm{s}$.

The low-velocity area with the velocity range of $0-0.4 \mathrm{~m} / \mathrm{s}$ is the largest in Case 1 $\left(19740.73 \mathrm{~m}^{2}\right)$; the area of Case 2 is the least of all $\left(13164.83 \mathrm{~m}^{2}\right)$; the area of Case 3 is between Case 1 and Case $2\left(13849.19 \mathrm{~m}^{2}\right)$. Therefore, the low-velocity areas are generally decreased as the longitudinal distance increases (Figure 124). But the low-velocity area of Case 3 is slightly more than Case 2. As the longitudinal distance increases, the wind velocities in the spaces among the building rows are increased; the area with extremely low velocities (velocity below $0.1 \mathrm{~m} / \mathrm{s}$ ) is reduced; the low-velocity area on the leeward side of the building cluster decreases, though the differences of the three cases are marginal.

There are high-velocity areas (velocity range: $1.4-1.6 \mathrm{~m} / \mathrm{s}$ ) on the lateral sides of the building clusters and in the middle gap between the building columns. The high-velocity areas are the largest of all in Case $1\left(10544.55 \mathrm{~m}^{2}\right)$; the areas of Case $2\left(8046.319 \mathrm{~m}^{2}\right)$ are the least of all; in Case 3, the areas are slightly larger than Case $2\left(8120.031 \mathrm{~m}^{2}\right)$. Therefore, the highvelocity areas are also decreased as the longitudinal distance decreases (Figure 124). But the areas of Case 3 are slightly larger than Case 2 . And the velocity magnitudes show that the highvelocity area in the middle gap between the building columns increases as the longitudinal distance increases. 


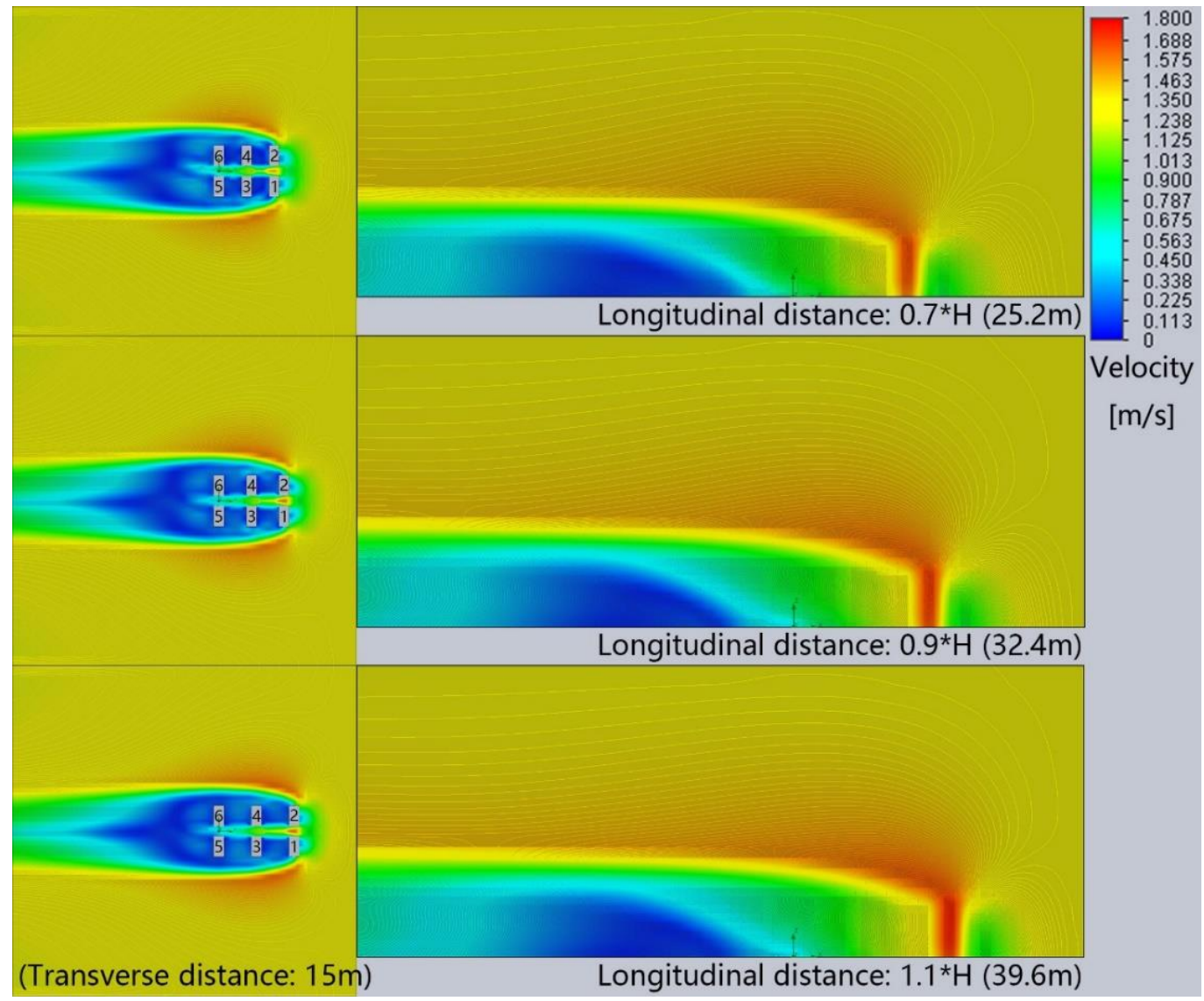

Figure 123. The comparison of wind-velocity magnitudes on horizontal and vertical planes of the building clusters in Group 8 of scattered configuration.

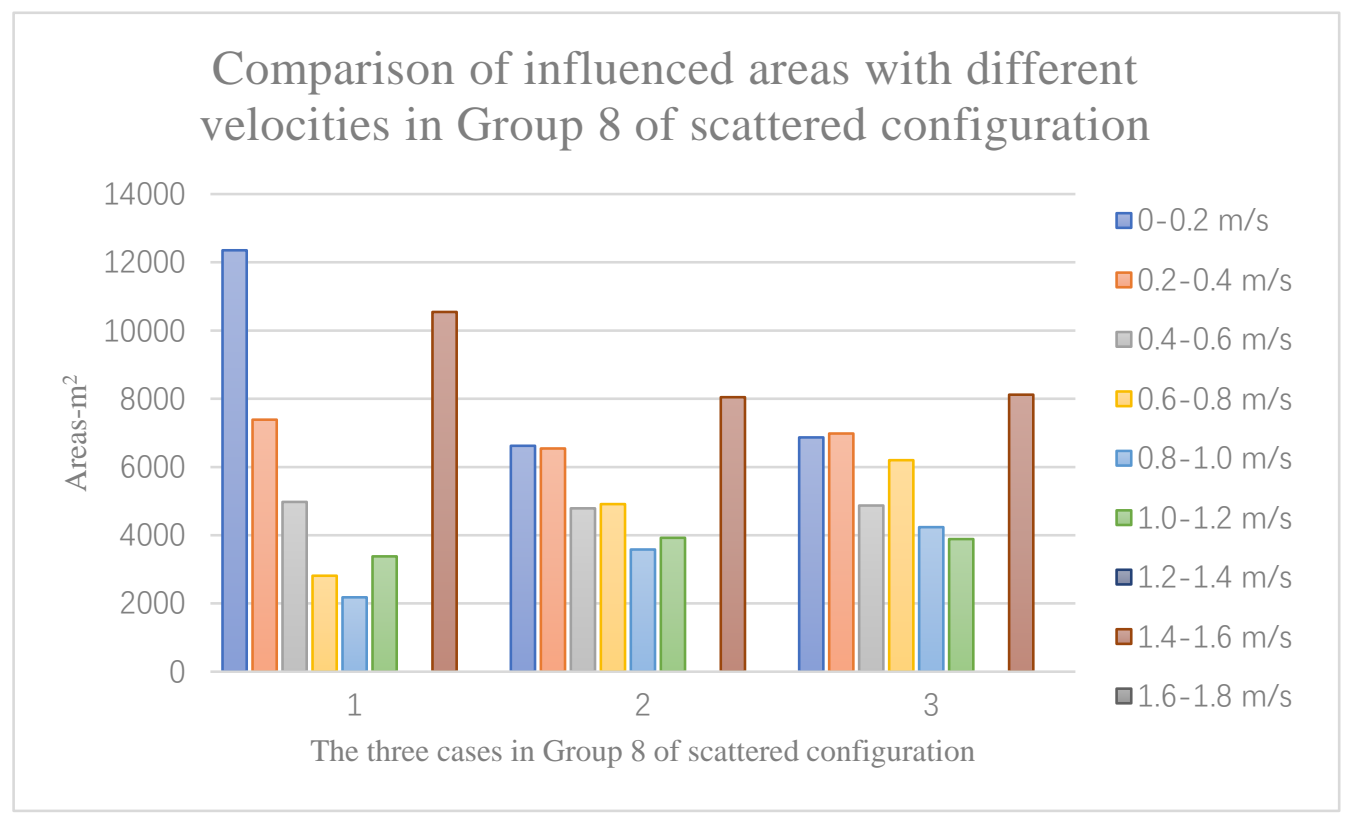

Figure 124. The influenced areas with different velocities in Group 8 of scattered configuration.

Second, the influences of different transverse distances of Group 9 (longitudinal distance: 
$0.9 * \mathrm{H}=32.4 \mathrm{~m}$ ) are studied. The wind-velocity magnitudes on horizontal and vertical planes of three cases are analyzed (Figure 125 and Figure 126). In Group 9, the influence of Case 1 (transverse distance: $10 \mathrm{~m}$ ) is between Case 2 and Case 3 (influenced area: $39844.24 \mathrm{~m}^{2}$ ); the influence of Case 2 (transverse distance: $15 \mathrm{~m}$ ) is the least (influenced area: $38469.74 \mathrm{~m}^{2}$ ); the influence of Case 3 (transverse distance: $20 \mathrm{~m}$ ) is the most of all (influenced area: $40228.98 \mathrm{~m}^{2}$ ). Generally, the total influenced area of Group 9 increases as the transverse distance increases. The width of the entire building cluster increases as the transverse distance increases. Therefore, the increase of the windward projective area of the entire building cluster should be the reason for the increase of the total influenced area. Though the influence of Case 2 is the least, the differences among the three cases are relatively small. The decrease is largely due to the decrease of the influenced areas with the velocity ranges of $0.2-0.4 \mathrm{~m} / \mathrm{s}$ and $0.8-1.0 \mathrm{~m} / \mathrm{s}$.

In Group 9, the low-velocity area (velocity range: $0-0.6 \mathrm{~m} / \mathrm{s}$ ) decreases constantly as the transverse distance increases (Figure 126). The low-velocity area is the largest in Case 1 $\left(20782.08 \mathrm{~m}^{2}\right)$; the area of Case 2 is less than Case $1\left(17953.11 \mathrm{~m}^{2}\right)$; the area of Case 3 is the least $\left(17442.73 \mathrm{~m}^{2}\right)$. Also, the area with the velocity range of $0.6-0.8 \mathrm{~m} / \mathrm{s}$ is decreased as the transverse distance increases. The low-velocity area on the leeward side of the building cluster decreases significantly as the transverse distance increases. And its length becomes much shorter. According to the velocity magnitudes of the three cases, results of Case 2 and Case 3 are largely different from Case 1; results of Case 2 and Case 3 are relatively close. These suggests that the decrease of the low-velocity area becomes limited if the transverse distance exceeds $15 \mathrm{~m}$ approximately.

There are high-velocity areas (velocity range: $1.4-1.6 \mathrm{~m} / \mathrm{s}$ ) on the lateral sides of the building clusters and in the middle gap between the building columns. The high-velocity areas are increased constantly as the transverse distance increases (Figure 126). In Case 1, the highvelocity areas are the largest of all $\left(7245.84 \mathrm{~m}^{2}\right)$; in Case 2, the areas are larger than Case 1 $\left(8066.55 \mathrm{~m}^{2}\right)$; in Case 3 , the areas are the largest $\left(10106.67 \mathrm{~m}^{2}\right)$. The high-velocity area increases significantly in the middle gap between the building columns from Case 1 to Case 3 . Because more winds are able to flow through the gap as the transverse distance increases. In summary, the outdoor ventilation can be promoted as the transverse distance increases. 


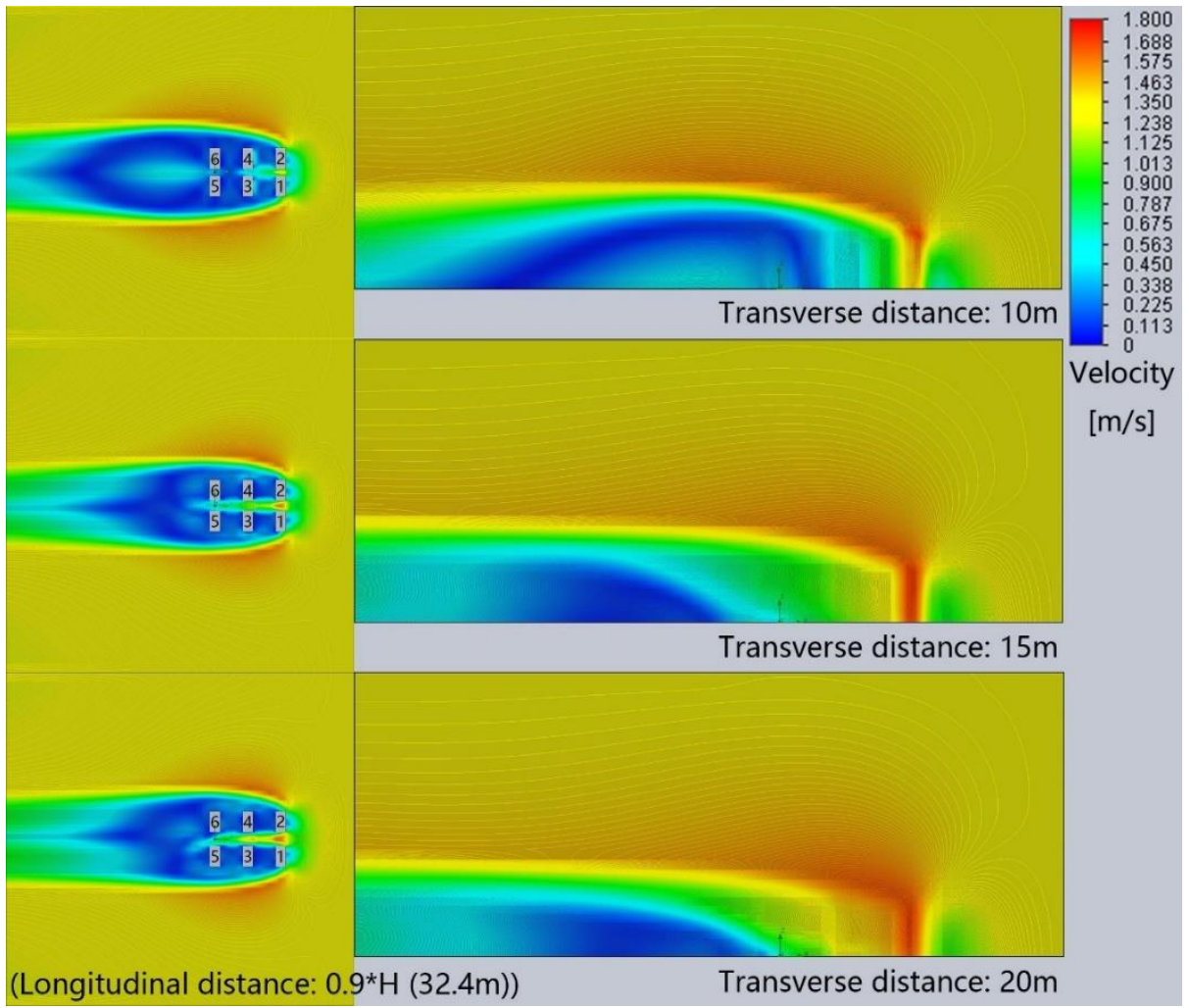

Figure 125. The comparison of wind-velocity magnitudes on horizontal and vertical planes of the building clusters in Group 9 of scattered configuration.

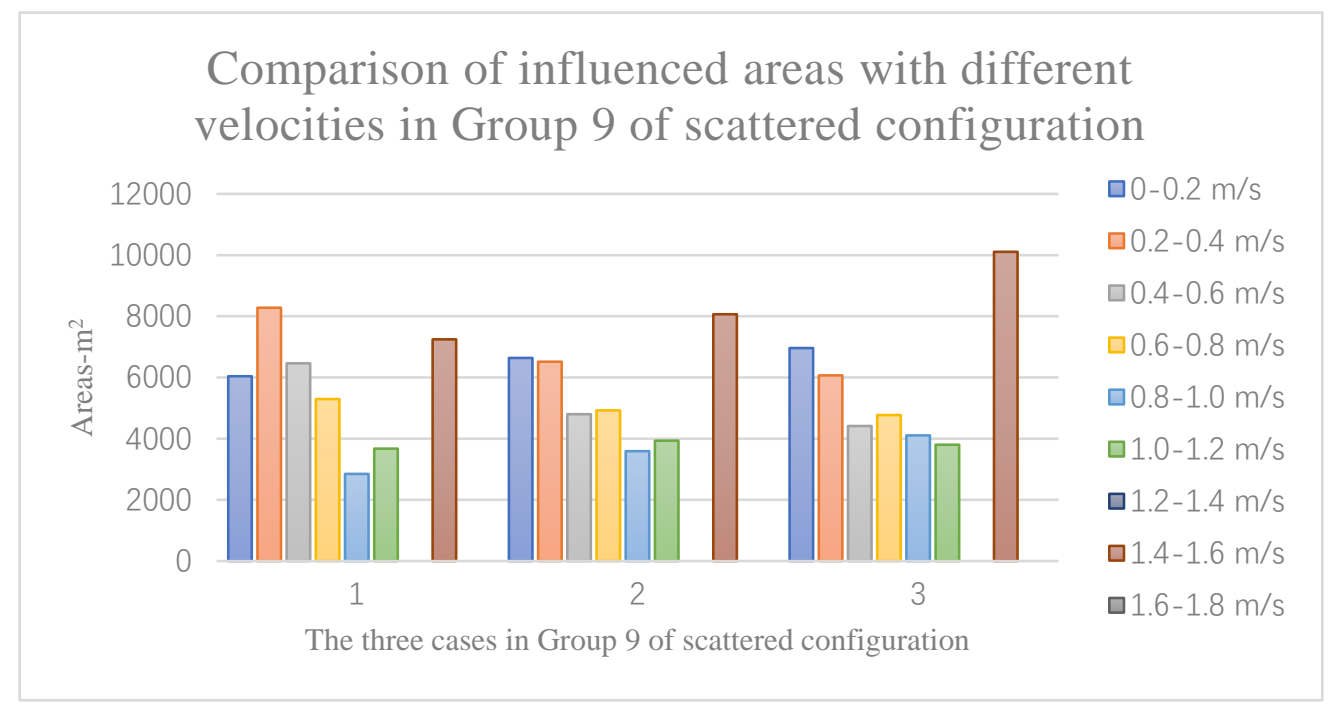

Figure 126. The influenced areas with different velocities in Group 9 of scattered configuration.

The changes of influenced areas with different velocities in Group 8 and Group 9 are related to the changes of air pressure and wind flow (Figure 127 and Figure 128). The airpressure magnitudes show that the high-air-pressure and low-air-pressure areas are separated 
by the windward surfaces of the first building row. The air pressure between the first and second building rows is the lowest; the air pressure between the second and third building rows is higher than the former one. As the longitudinal and transverse distances increases, both the lowair-pressure and high-air-pressure areas are reduced; the difference between the highest and lowest air-pressure values slightly increases, and the extent of air-pressure changes increases, which should be the reasons for the increase of the high-velocity areas; the low-air-pressure areas between the adjacent building rows are decreased, which is the reason for the decrease of the low-velocity areas; the low-air-pressure area on the leeward side of the building cluster decreases, which reduces the length of the low-velocity area.

The wind flow streamlines show that winds flow around the building cluster on the lateral sides; and they flow through the building cluster in the middle gap; lots of vortices are created among the building rows and behind the building cluster. As the longitudinal and transverse distances increases, the vortices among the building rows become less, which causes the decrease of the low-velocity areas among the building rows; the vortices on the leeward side of the building cluster become significantly smaller and shorter, which corresponds with the decrease of the low-velocity area behind the building cluster. The streamlines show that the vortices reduction caused by the increase of the transverse distance is more obvious than the increase of the longitudinal distance. The wind velocity magnitudes also show that the reduction of the low-velocity area in the increase of transverse distance is more than the reduction in the increase of longitudinal distance, especially for the low-velocity area behind the building cluster. Because the increase of transverse distance allows more winds to flow through the building cluster. The vortices and the low-velocity area decrease as the gap between the building columns becomes larger. However, vortices among the building rows decrease significantly as the longitudinal distance increases; vortices reduced by the increase of the transverse distance is slightly less. 


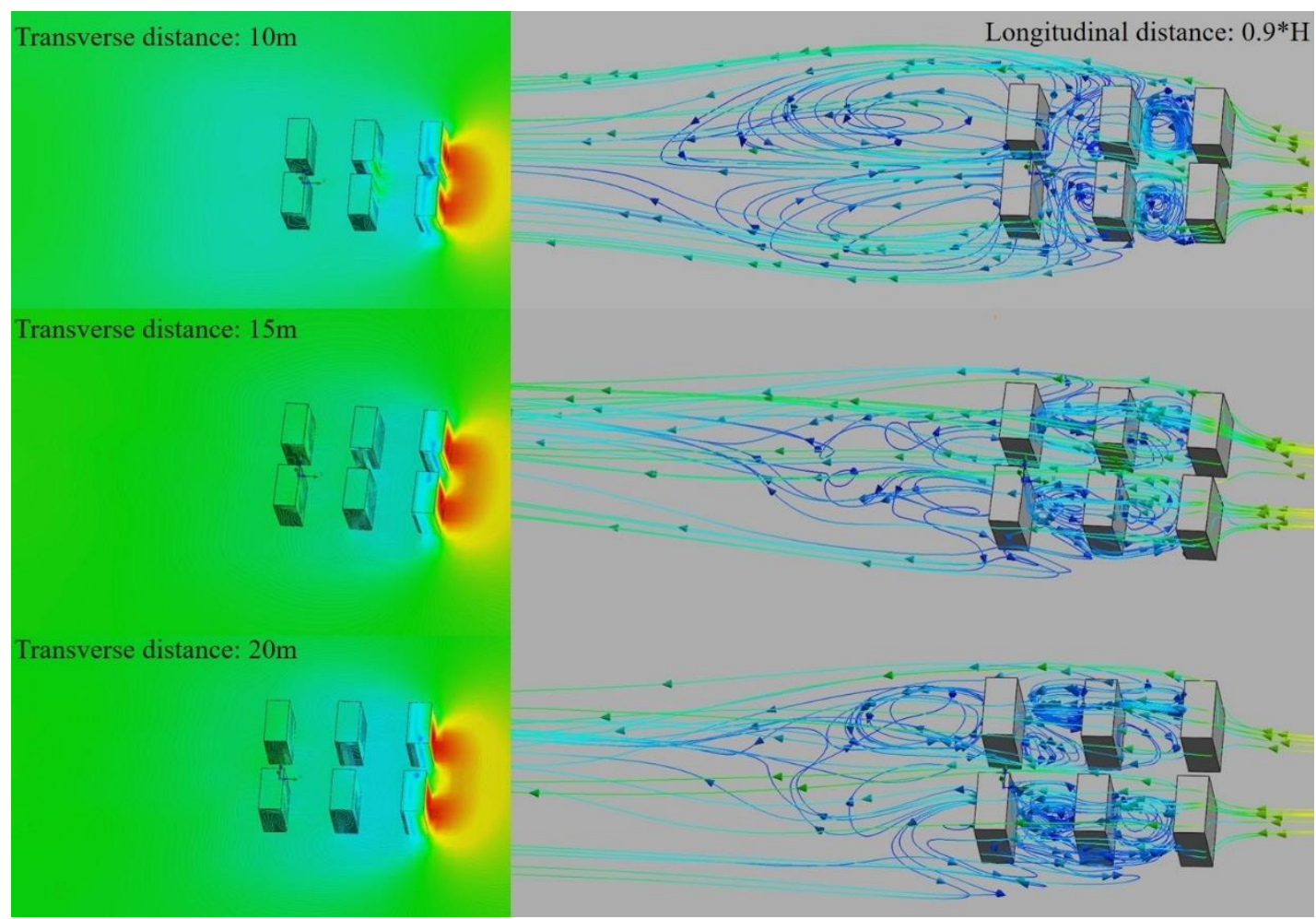

Figure 127. The comparison of wind-flow streamlines (right) and air-pressure magnitudes (left) on the horizontal plane and building surfaces in Group 8 of scattered configuration.

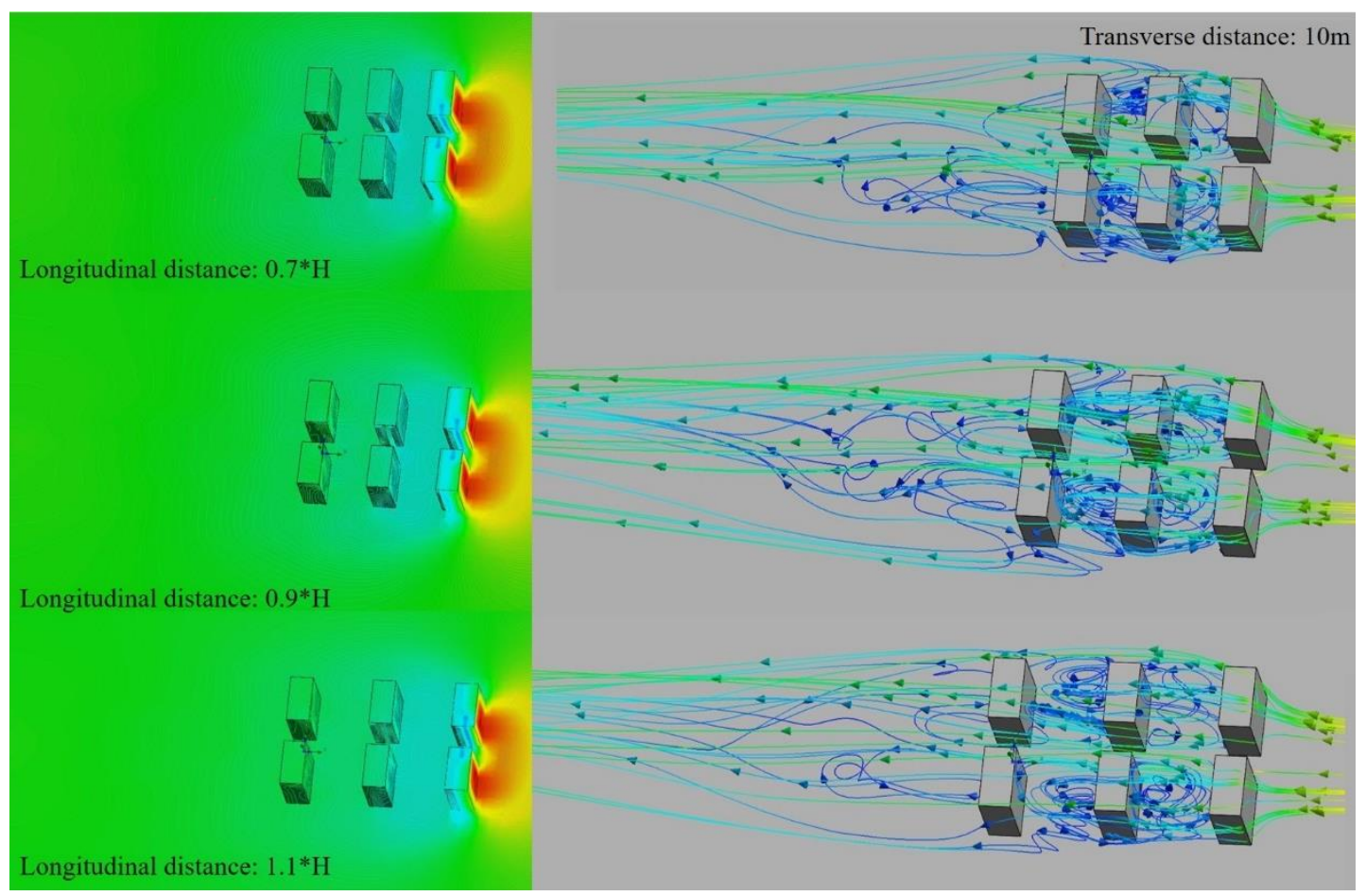

Figure 128. The comparison of wind-flow streamlines (right) and air-pressure magnitudes (left) on the horizontal plane and building surfaces in Group 9 of scattered configuration. 
(7) Part 7. Influences of longitudinal and transverse distances among 6 buildings in 2 rows

In this part, the influences of different transverse distances of Group 10 (longitudinal distance: $0.9 * \mathrm{H}=32.4 \mathrm{~m}$ ) are studied first. The wind-velocity magnitudes on horizontal and vertical planes of the three cases are analyzed. The results show that the influence of the building cluster increases constantly as the transverse distance increases, though the differences among the cases are relatively small (Figure 129 and Figure 130). In Case 1 (transverse distance: 10m), the influence of the building cluster is the most (influenced area: $47373.26 \mathrm{~m}^{2}$ ); in Case 2 (transverse distance: $15 \mathrm{~m}$ ), the influence is less than Case 1 (influenced area: $47404.44 \mathrm{~m}^{2}$ ); in Case 3 (transverse distance: $20 \mathrm{~m}$ ), the influence is the least (influenced area: $47603.55 \mathrm{~m}^{2}$ ). The increase of transverse distance increases the width of the entire building cluster. Therefore, the windward projective area of the building cluster is increased, which increases the influence on wind environment.

The velocity magnitudes show that the low-velocity area (velocity range: $0-0.4 \mathrm{~m} / \mathrm{s}$ ) decreases constantly as the transverse distance increases (Figure 129 and Figure 130). In Case 1, the low-velocity area is the largest $\left(26818.79 \mathrm{~m}^{2}\right)$; in Case 2, the area is less than Case 1 $\left(22588.66 \mathrm{~m}^{2}\right)$; in Case 3, the area is the least $\left(19494.76 \mathrm{~m}^{2}\right)$. The decreases of the low-velocity area are relatively large as the transverse distance increases. The velocity magnitudes also show that the area with the velocity range of $0.4-0.8 \mathrm{~m} / \mathrm{s}$ increases in Case $1\left(6757.19 \mathrm{~m}^{2}\right)$, Case 2 $\left(12018.69 \mathrm{~m}^{2}\right)$ and Cas3 $\left(14699.90 \mathrm{~m}^{2}\right)$. This suggests that the relatively low velocities (in the low-velocity area with the velocity range of $0-0.4 \mathrm{~m} / \mathrm{s}$ ) are increased gradually as the transverse distance increases. However, the velocity increase in the low-velocity area between the building rows is relatively small, which indicates the outdoor ventilation promotion in the space between the building rows is limited as the transverse distance increases.

The velocity magnitudes also show that there are high-velocity areas (velocity range: 1.4$1.6 \mathrm{~m} / \mathrm{s}$ ) on the lateral sides of the building cluster and in the gaps among the building columns. The high-velocity area decreases constantly as the transverse distance increases (Figure 130). In Case 1, the high-velocity areas are the largest $\left(6730.01 \mathrm{~m}^{2}\right)$; in Case 2, the areas are less than Case $1\left(5329.35 \mathrm{~m}^{2}\right)$; in Case 3, the areas are the least of all $\left(4628.37 \mathrm{~m}^{2}\right)$. The high-velocity 
areas in the gaps among the building columns increase as the transverse distance increases.

Because more winds are able to flow through the gaps with increasing widths.

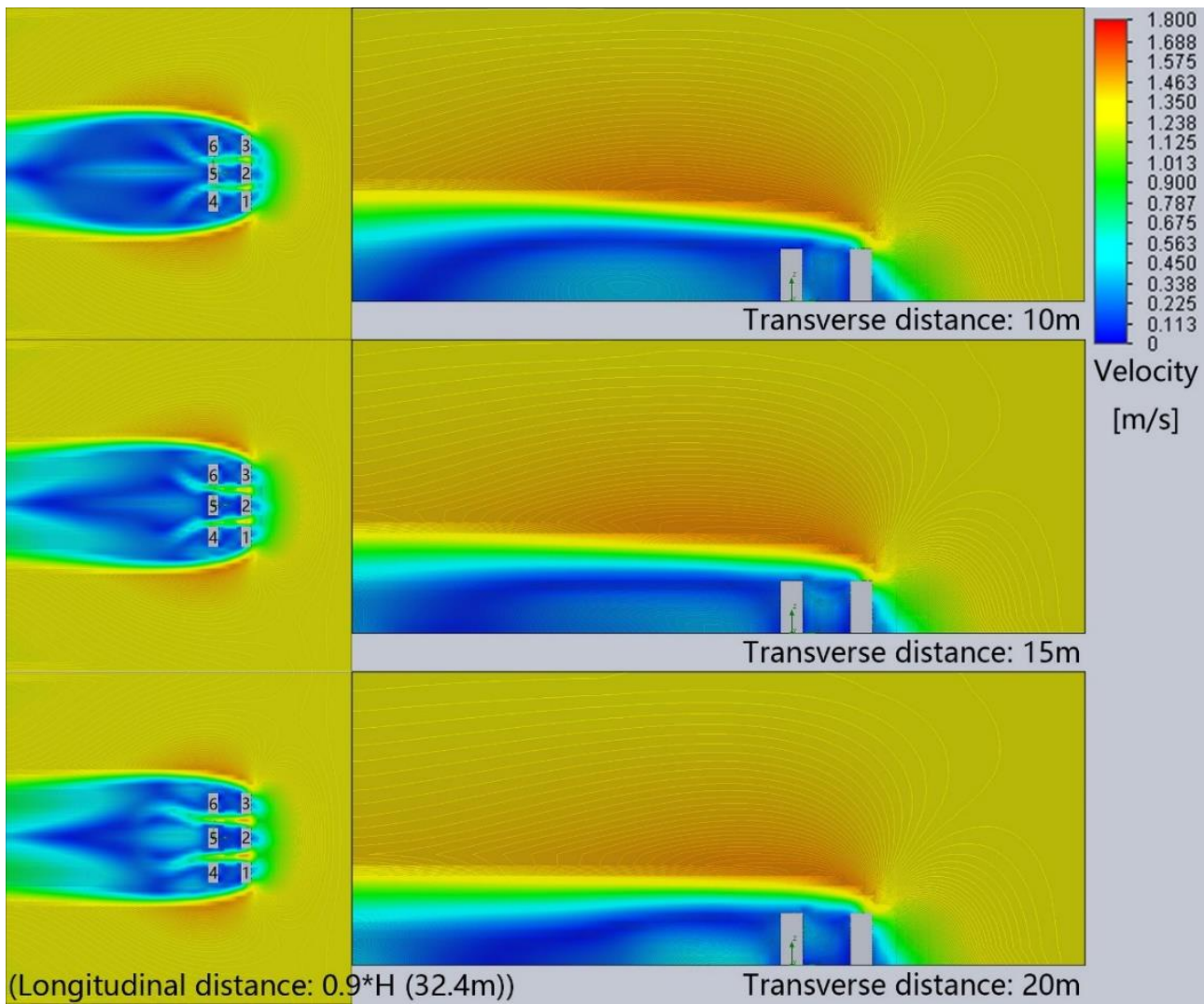

Figure 129. The comparison of wind-velocity magnitudes on horizontal and vertical planes of the building clusters in Group 10 of scattered configuration.

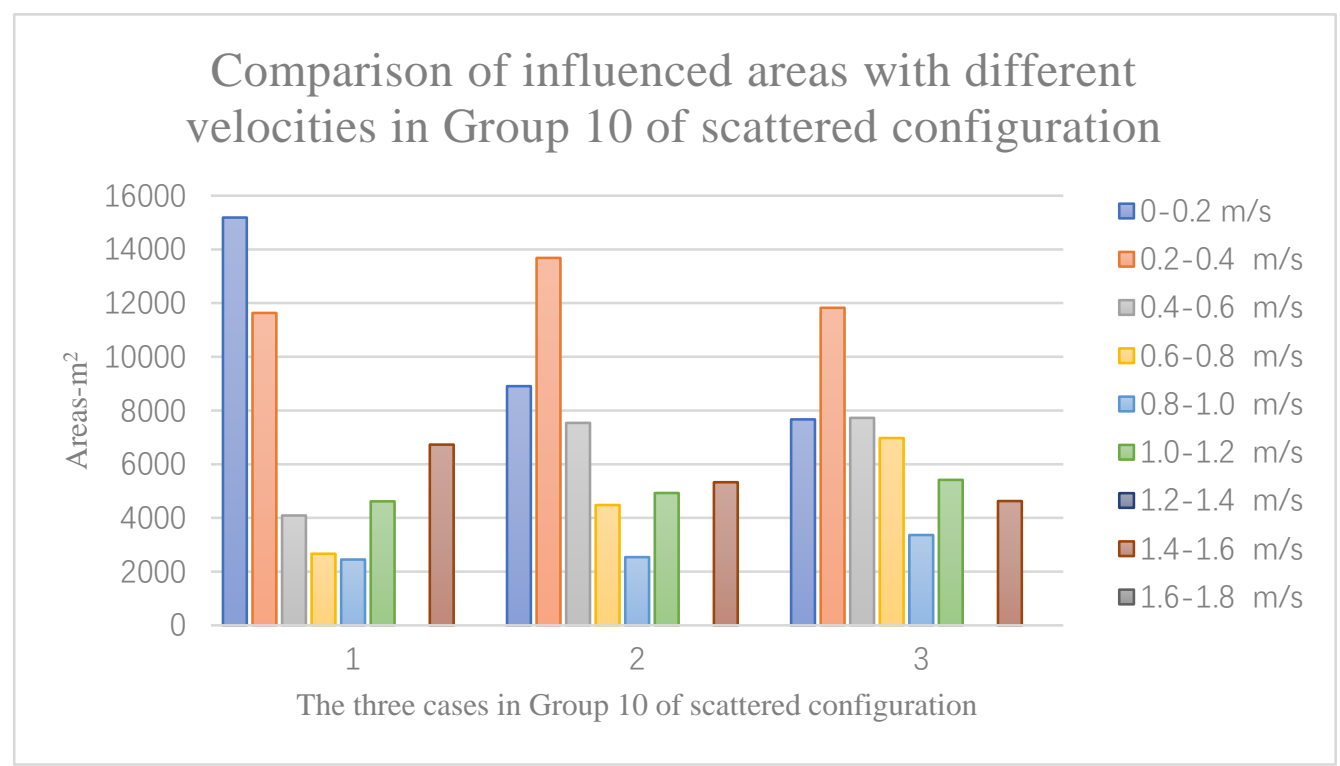

Figure 130. The influenced areas with different velocities in Group 10 of scattered configuration. 
Second, the influences of different longitudinal distances of Group 11 (transverse distance: $15 \mathrm{~m})$ are analyzed. The wind-velocity magnitudes on horizontal and vertical planes of the three cases show that the total influence of the building cluster is increased as the longitudinal distance increases, though the differences among the three cases are marginal (Figure 131 and Figure 132). In Case 1 (longitudinal distance: $0.9 * \mathrm{H}$ ), the influence is the least (influenced area: $46552.26 \mathrm{~m}^{2}$ ); in Case 2 (longitudinal distance: $0.9^{*} \mathrm{H}$ ), the influence is slightly more than Case 1 (influenced area: $47262.36 \mathrm{~m}^{2}$ ); in Case 3 (longitudinal distance: $1.1 * \mathrm{H}$ ), the influence is the most of all (influenced area: $47536.72 \mathrm{~m}^{2}$ ). The increase of longitudinal distance increases the length of the entire building cluster. The total influence increases because the building cluster occupies larger area.

The velocity magnitudes show that the low-velocity area (velocity range: $0-0.4 \mathrm{~m} / \mathrm{s}$ ) decreases constantly as the longitudinal distance increases (Figure 131 and Figure 132). In Case 1, the low-velocity area is the largest $\left(23233.65 \mathrm{~m}^{2}\right)$; in Case 2, the area is less than Case 1 $\left(22532.01 \mathrm{~m}^{2}\right)$; in Case 3, the area is the least $\left(21262.57 \mathrm{~m}^{2}\right)$. The area with the velocity range of $0-0.2 \mathrm{~m} / \mathrm{s}$ increases slightly as the longitudinal distance increases. The area with the velocity range of $0.2-0.4 \mathrm{~m} / \mathrm{s}$ decreases gradually as the longitudinal distance increases. The area with the velocity ranges of $0.4-0.6 \mathrm{~m} / \mathrm{s}$ and $0.6-0.8 \mathrm{~m} / \mathrm{s}$ increases gradually as the longitudinal distance increases. Because there are low-velocity areas between the building rows. The increase of the space between the building rows should be the reason for the increase of the area with the velocity range of $0-0.2 \mathrm{~m} / \mathrm{s}$. The decrease of the area with the velocity range of $0.2-0.4 \mathrm{~m} / \mathrm{s}$ and increases of areas with the velocity ranges of $0.4-0.6 \mathrm{~m} / \mathrm{s}$ and $0.6-0.8 \mathrm{~m} / \mathrm{s}$ all suggest the outdoor ventilation is promoted in the low-velocity area. The velocities are increased in the low-velocity area between the building rows and on the leeward side of the building cluster.

The velocity magnitudes show that high-velocity areas (velocity range: $1.4-1.6 \mathrm{~m} / \mathrm{s}$ ) increase constantly on the lateral sides and in the gaps of the building cluster as the longitudinal distance increases (Figure 131 and Figure 132). In Case 1, the high-velocity areas are the least $\left(4601.00 \mathrm{~m}^{2}\right)$; in Case 2, the areas are larger than Case $1\left(5315.98 \mathrm{~m}^{2}\right)$; in Case 3, the areas are the largest $\left(5953.89 \mathrm{~m}^{2}\right)$. 


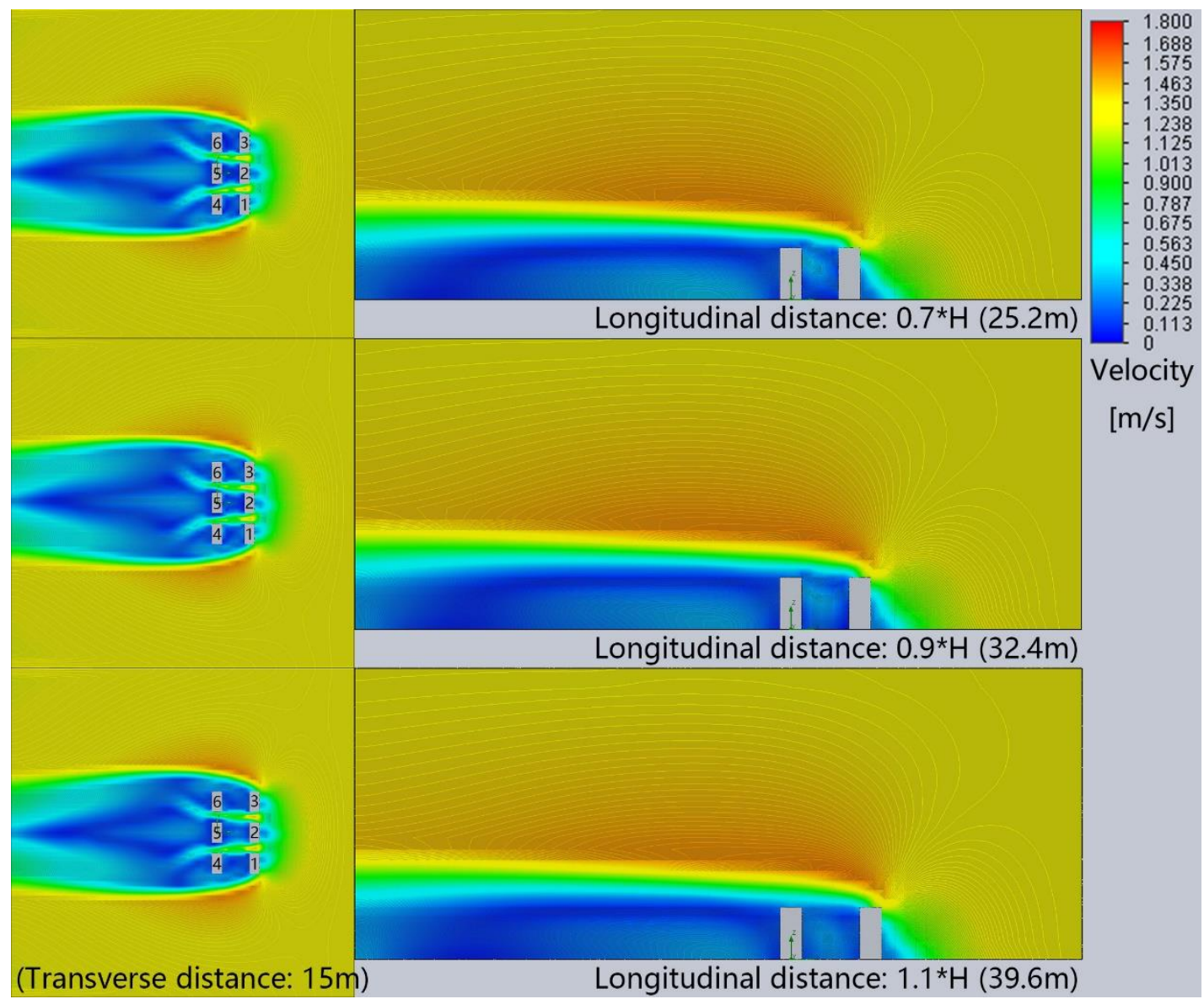

Figure 131. The comparison of wind-velocity magnitudes on horizontal and vertical planes of the building clusters in Group 11 of scattered configuration.

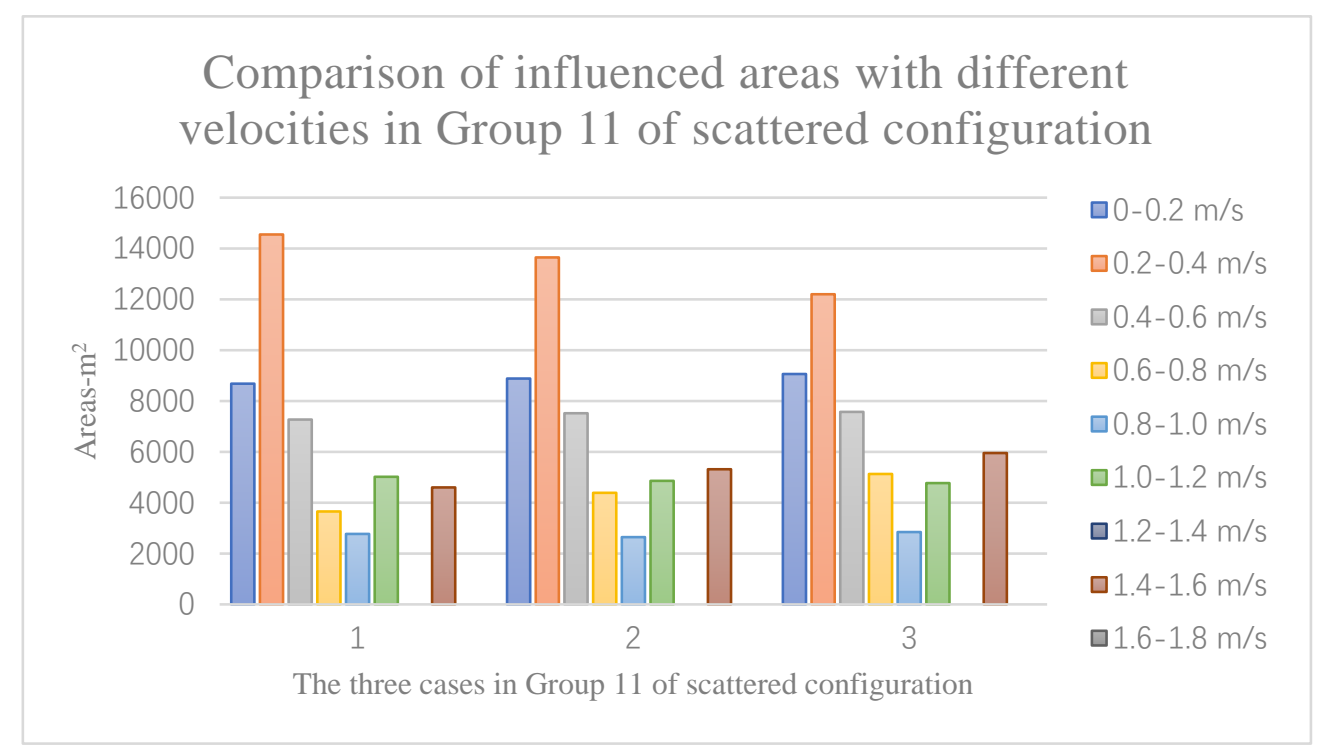

Figure 132. The influenced areas with different velocities in Group 11 of scattered configuration.

The changes of influenced areas with different velocities in Group 10 and Group 11 are 
related to the changes of air pressure and wind flow (Figure 133 and Figure 134). The airpressure magnitudes show that the high-air-pressure and low-air-pressure areas are separated by the windward surfaces of the first building row. The outdoor ventilation can be promoted as the longitudinal and transverse distances increase.

As the transverse distance increases, the air-pressure magnitudes show that low-airpressure area on the leeward side of the building cluster decreases significantly; the wind-flow streamlines show that the vortices behind the building cluster become less and smaller. In addition, the relatively low air pressures in the space between the building rows are decreased; vortices created in the space are reduced. These are the reasons for the decrease of the lowvelocity area, which prove the outdoor ventilation is promoted as the transverse distance increases.

As the longitudinal distance increases, the air-pressure magnitudes show that both the lowair-pressure and low-air-pressure areas decrease slightly; the difference between the highest and lowest air pressures becomes larger, and the extent of air-pressure changes is increased, which cause the increase of high-velocity area; the wind-flow streamlines show that the vortices between the building rows and behind the building cluster are reduced. These can explain the decrease of the low-velocity area. Especially for the space between the building rows, the significant reduction of vortices suggests the promotion of outdoor ventilation.

In addition, the air-pressure magnitudes and wind-flow streamlines also suggest that the increase of the transverse distance mainly focuses on the reduction of the low-velocity area on the leeward side of the building cluster; the increase of the longitudinal distance mainly focuses on the reduction of the low-velocity area between the building rows. It is relatively easier for winds to flow through the building cluster as the transverse distance increases. 


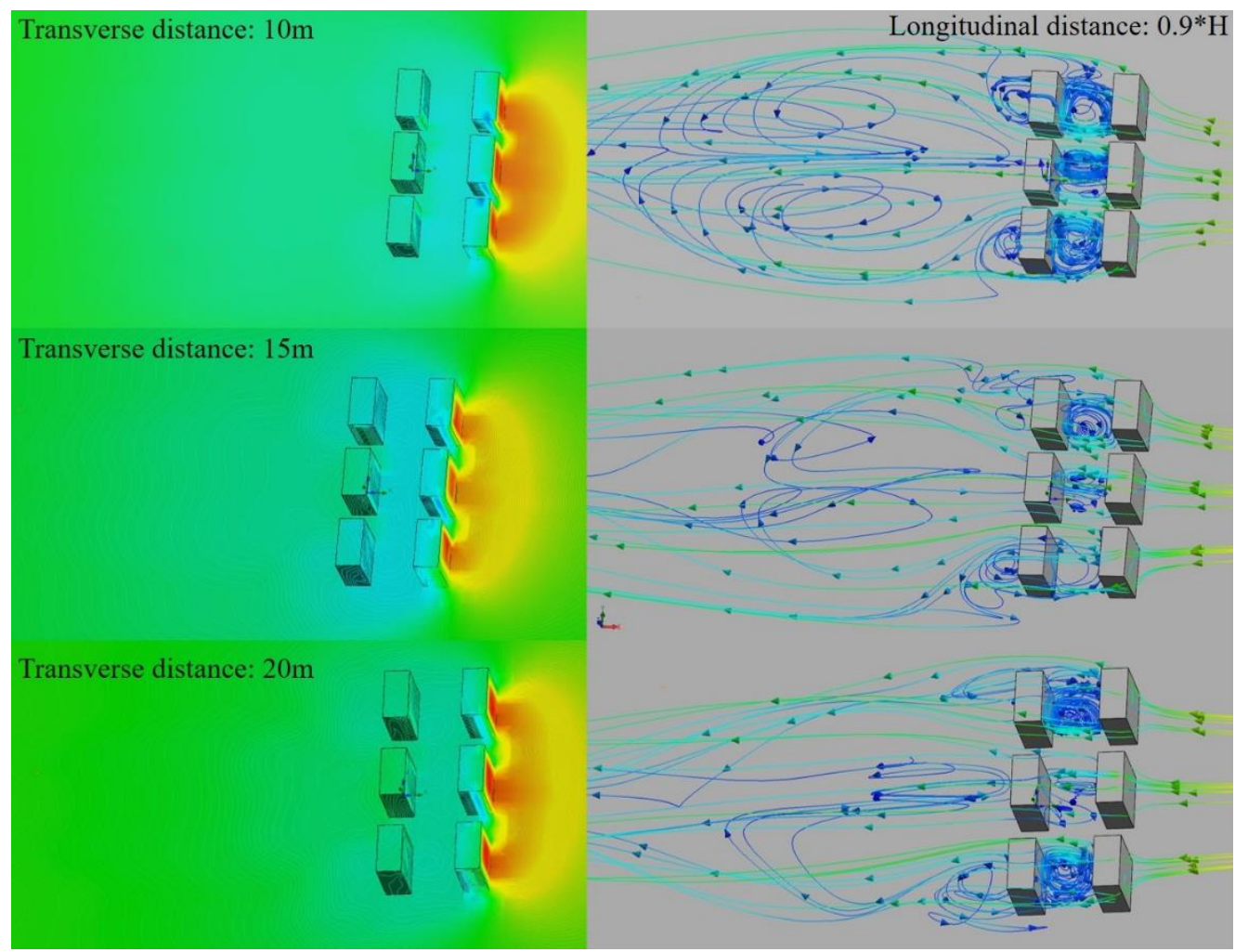

Figure 133. The comparison of wind-flow streamlines (right) and air-pressure magnitudes (left) on the horizontal plane and building surfaces in Group 10 of scattered configuration.

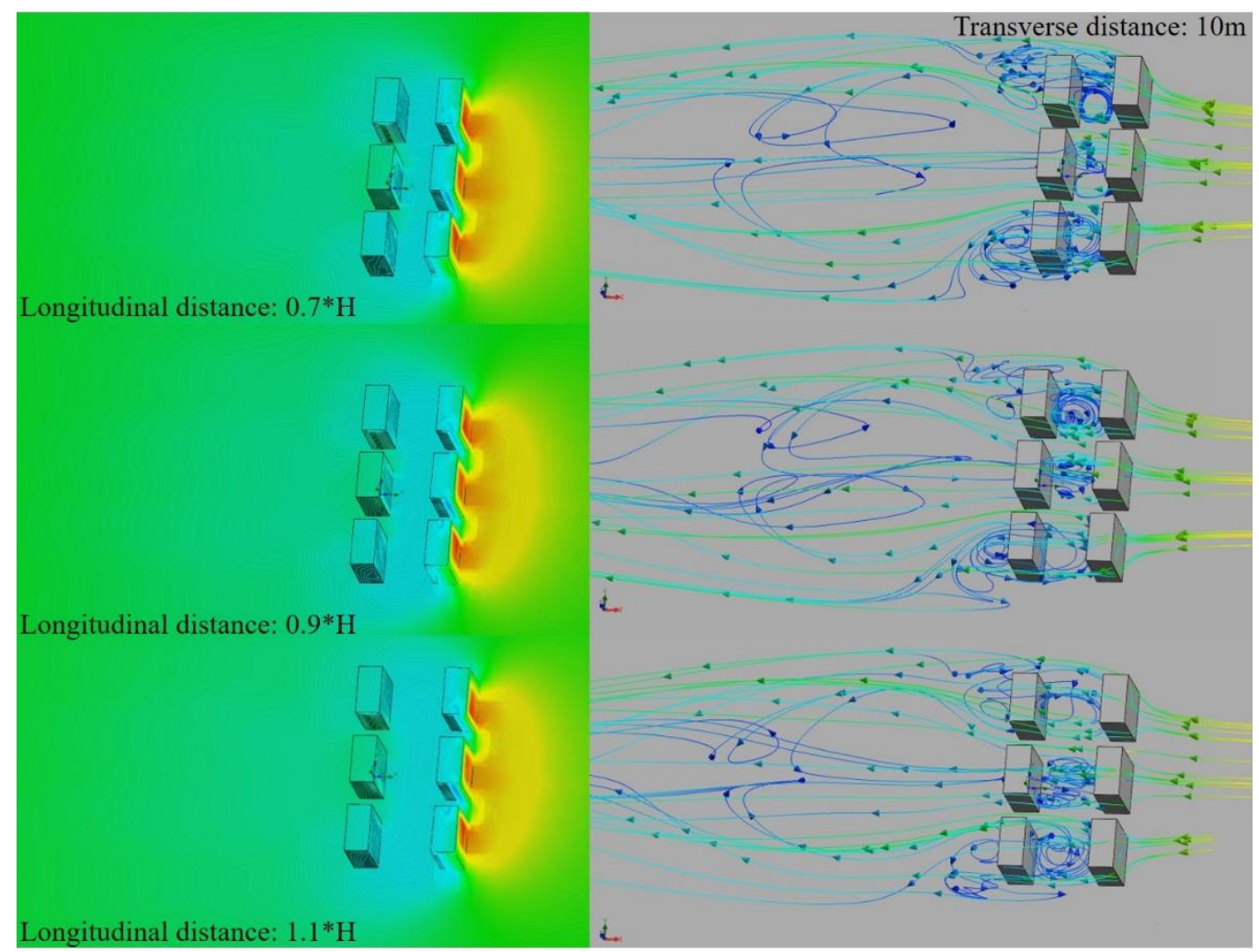

Figure 134. The comparison of wind-flow streamlines (right) and air-pressure magnitudes (left) 
on the horizontal plane and building surfaces in Group 11 of scattered configuration.

\section{(8) Part 8. Influences of longitudinal and transverse distances of Group 12}

First, the influences of different transverse distances of Group 12 are analyzed. The windvelocity magnitudes on horizontal and vertical planes of three cases are presented in the figure (Figure 135 and Figure 136). In Case 1, the building cluster with the shortest transverse distance of $10 \mathrm{~m}$ have the largest influence on surrounding wind environment; in Case 2, the influence of the building cluster with the transverse distance of $15 \mathrm{~m}$ is less than Case 1; in Case 3, the building cluster with the longest transverse distance of $20 \mathrm{~m}$ has the least influence.

In Case 1, the low-velocity area (velocity below $0.5 \mathrm{~m} / \mathrm{s}$ ) behind the building cluster is the largest and its length is the longest; In Case 2, the low-velocity area is less than Case 1; in Case 3 , the low-velocity area is the smallest of all. The low-velocity area behind the building cluster decreases obviously as the transverse distance increases (Figure 136). The outdoor ventilation in the space among the three building rows is improved significantly from Case 1 to Case 3 .

In all three cases, there are high-velocity areas (velocity over $1.4 \mathrm{~m} / \mathrm{s}$ ) on the lateral sides of the building cluster and in the gaps among building columns. In Case 1, the high-velocity areas on the lateral sides are the largest and the high-velocity area in the gaps among building columns is the smallest; in Case 2, the high-velocity areas on the lateral sides are less than Case 1 and the high-velocity area in the gaps among building columns is larger than Case 1; in Case 3, the high-velocity areas on the lateral sides are the smallest and the high-velocity area in the gaps among building columns is the largest (Figure 136). 


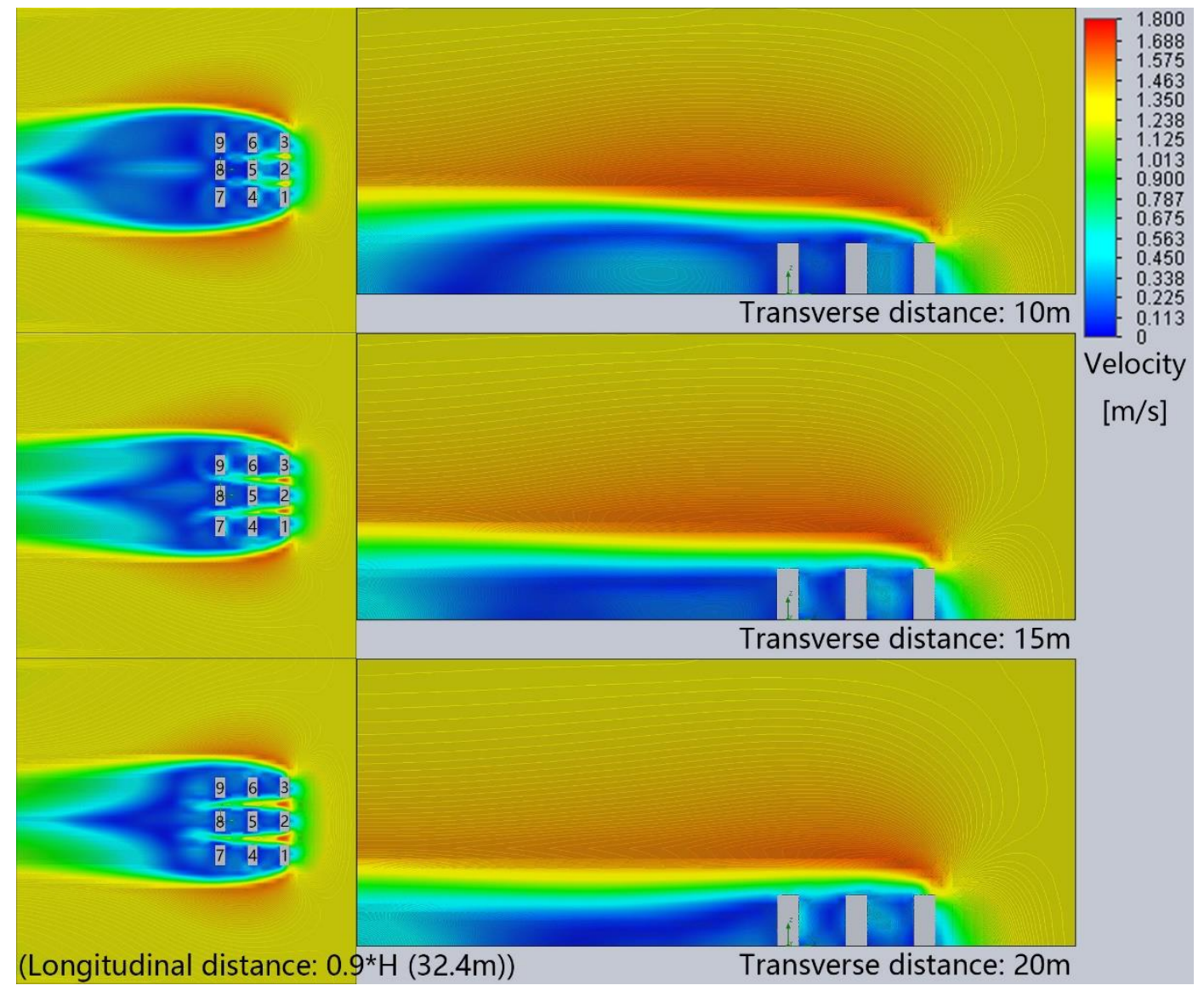

Figure 135. The comparison of wind-velocity magnitudes on horizontal and vertical planes of the building clusters in Group 12 of scattered configuration.

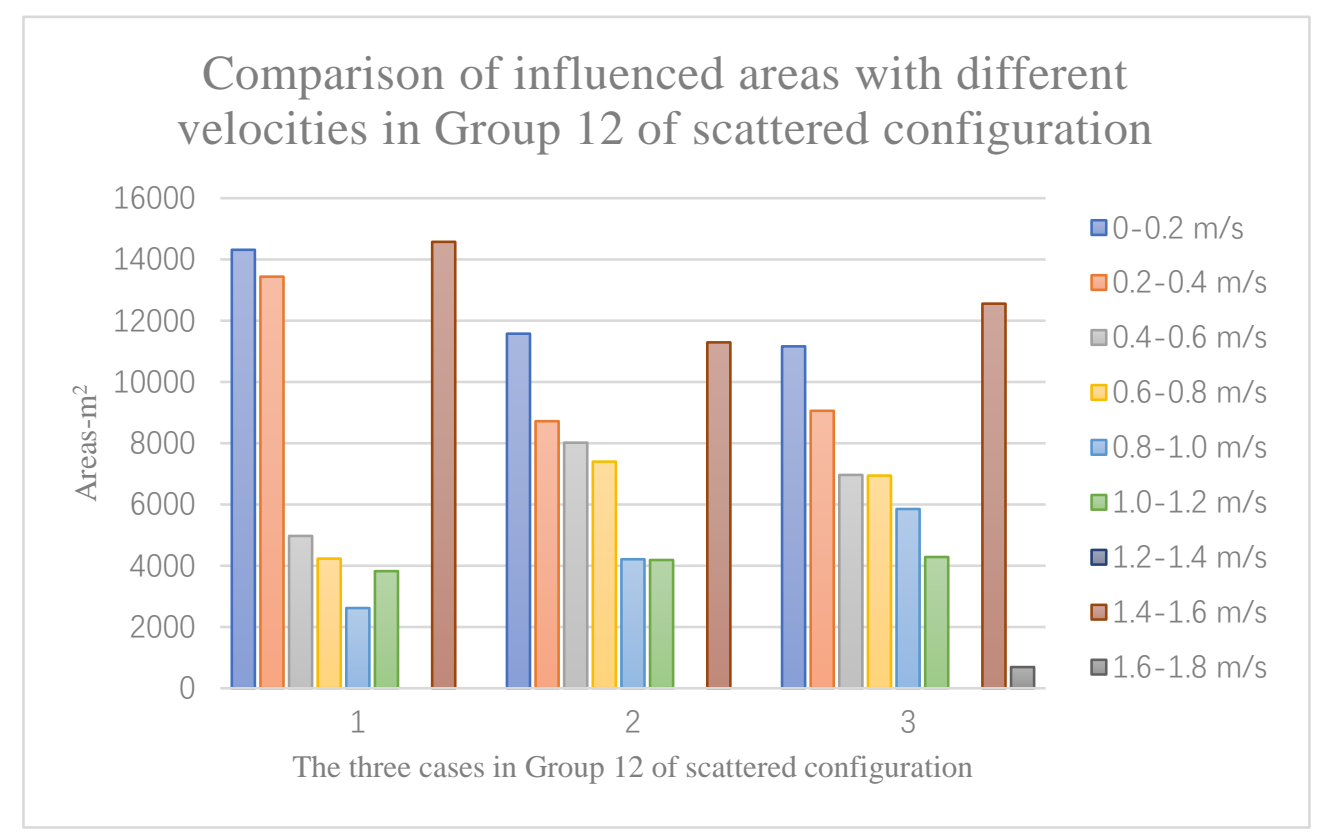

Figure 136. The influenced areas with different velocities in Group 12 of scattered configuration. 
The changes of influenced areas in different cases of Group 12 are related to the changes of air pressure and wind flow (Figure 137 and Figure 138). Wind flow is caused by air-pressure differences. Winds usually flow from high-air-pressure areas to low-air-pressure areas. In Case 1 , the influence is the largest due to the largest high-air-pressure area and low-air-pressure area; in Case 2, the influence decreases as the high-air-pressure area and low-air-pressure area become less; in Case 3, the influence is the least as high-air-pressure area and low-air-pressure area are the smallest. The streamlines show less winds are obstructed as transverse distance increases. Winds are able to escape in the larger building intervals more easily.

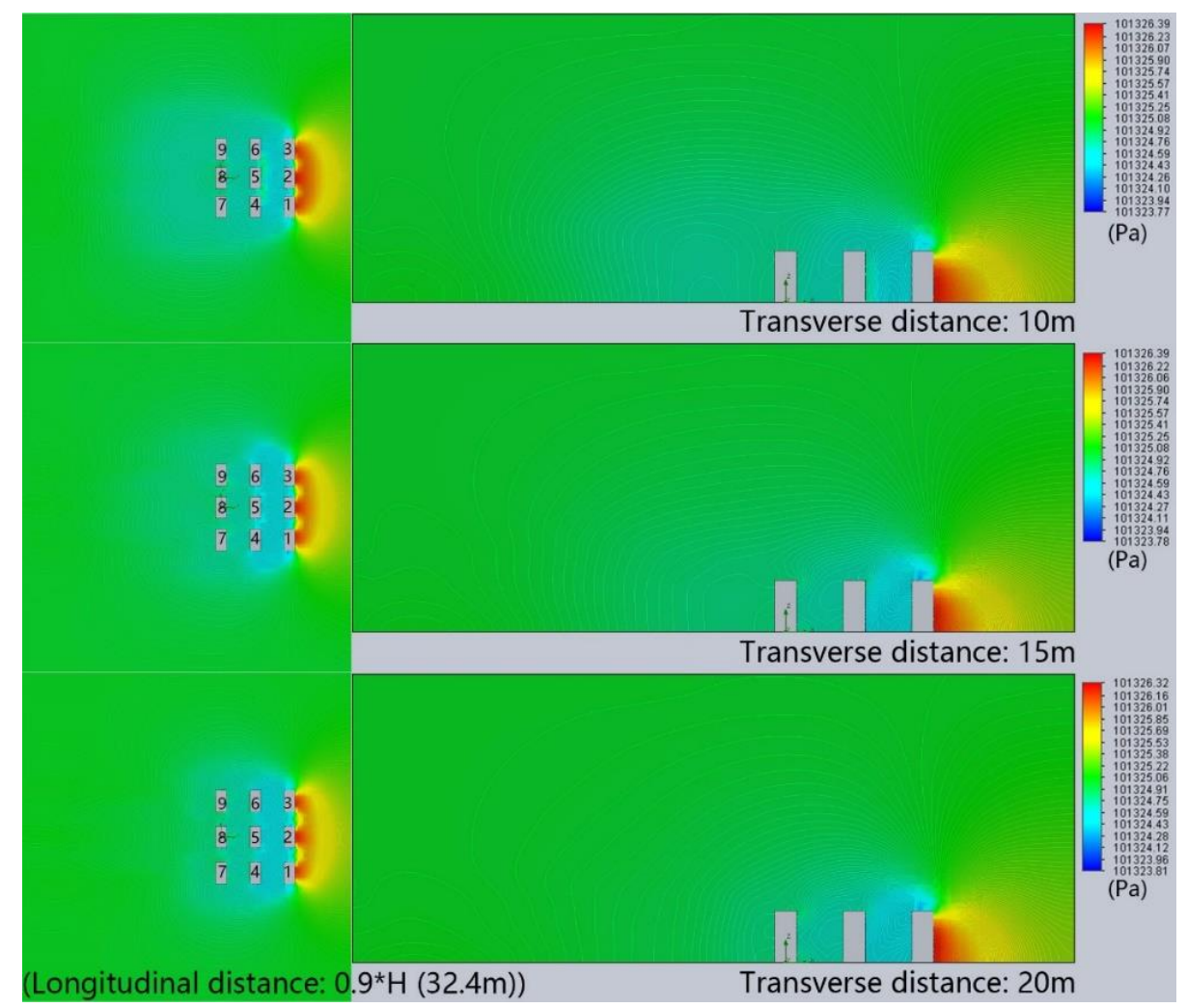

Figure 137. The comparison of air-pressure magnitudes on the horizontal and vertical planes of the building clusters in Group 12 of scattered configuration. 


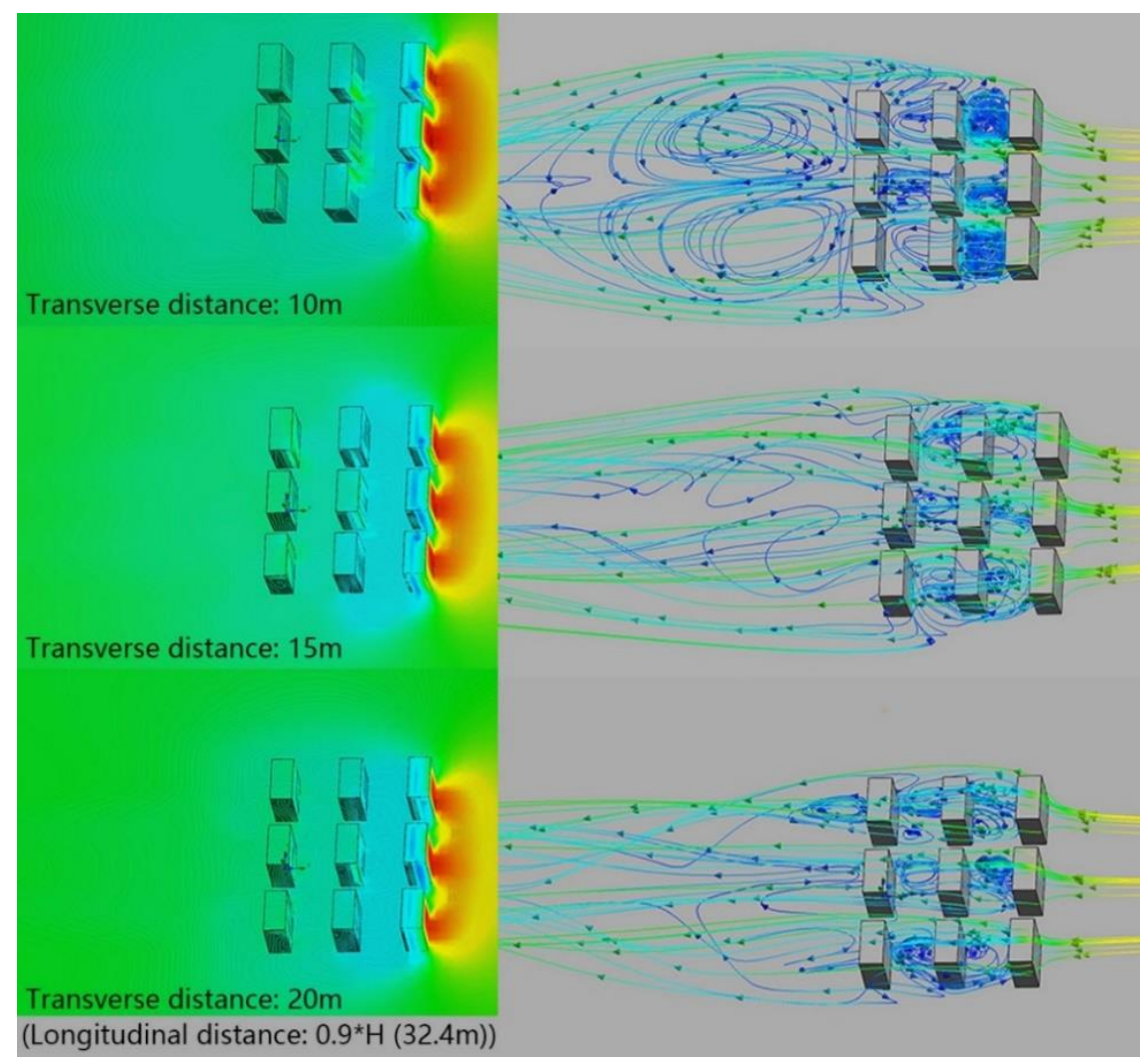

Figure 138. The comparison of wind-flow streamlines (right) and air-pressure magnitudes (left) on the horizontal plane and building surfaces in Group 12 of scattered configuration.

Second, the influences of different longitudinal distances of Group 13 are analyzed. The wind-velocity magnitudes on horizontal and vertical planes of three cases are presented in the figure (Figure 139 and Figure 140). In the three cases, influences of building clusters are close. In Case 1, the building cluster with the shortest longitudinal distance of $0.7^{*} \mathrm{H}(25.2 \mathrm{~m})$ has the largest influence on wind environment; in Case 2, the influence of the building cluster with the longitudinal distance of $0.9^{*} \mathrm{H}(32.4 \mathrm{~m})$ is less than Case 1; in Case 3, the building cluster with the longest longitudinal distance of $1.1^{*} \mathrm{H}(39.6 \mathrm{~m})$ has the least influence (Figure 140).

The low-velocity areas (velocity below $0.5 \mathrm{~m} / \mathrm{s}$ ) behind the building clusters of three cases are close (Figure 139). However, outdoor ventilation among the building rows is improved significantly due to the increase of the wind velocity from Case 1 to Case 3 .

In all three cases, there are high-velocity areas (velocity over $1.4 \mathrm{~m} / \mathrm{s}$ ) on the lateral sides of the building cluster and in the gaps among building columns. In Case 1, the high-velocity areas on the lateral sides are the smallest; in Case 2, the high-velocity areas on the lateral sides 
are the larger than Case 1; in Case 3, the high-velocity areas on the lateral sides are the largest. The high-velocity areas in the gaps among building columns are close in the three cases (Figure 140).

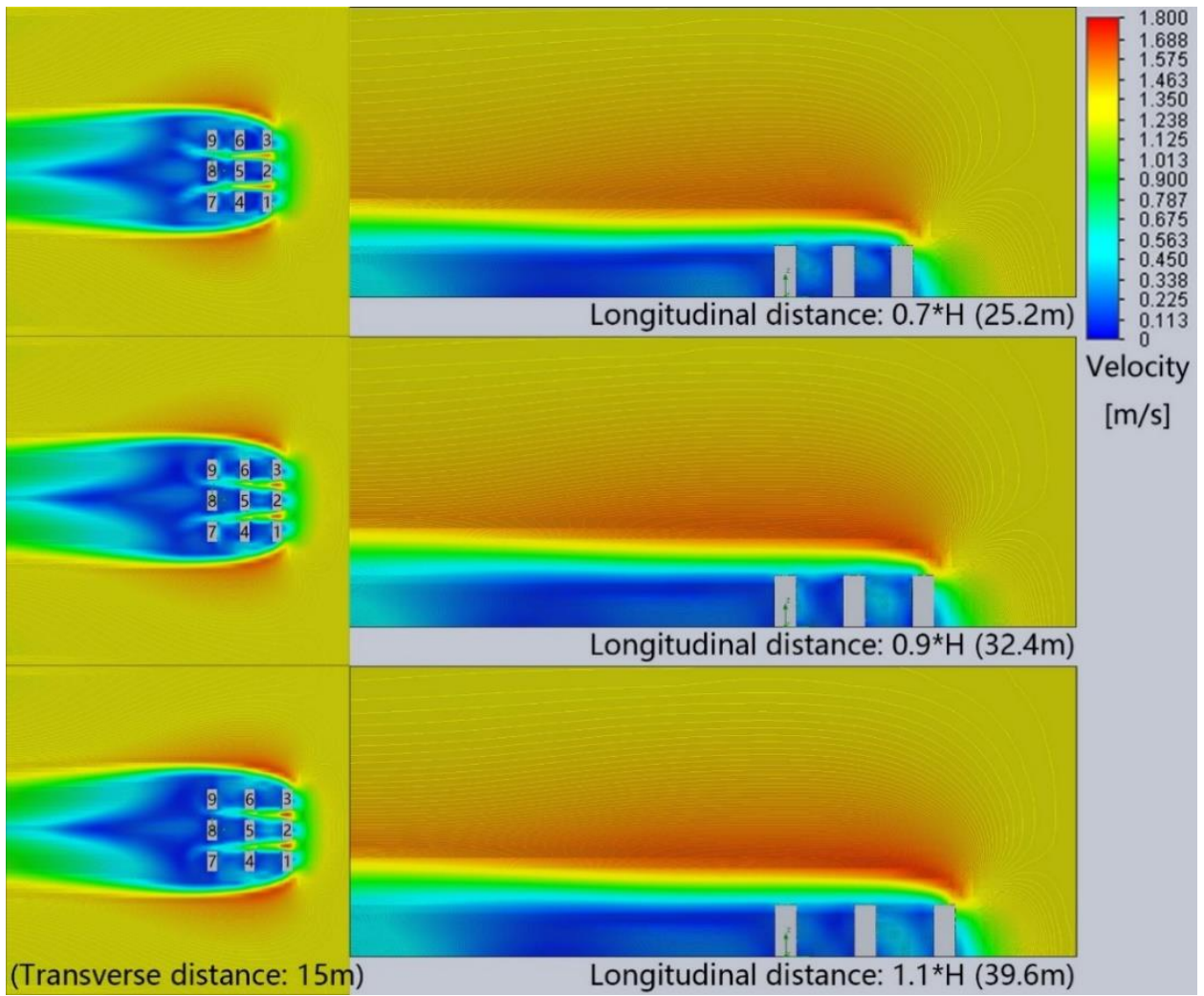

Figure 139. The comparison of wind-velocity magnitudes on horizontal and vertical planes of the building clusters in Group 13 of scattered configuration.

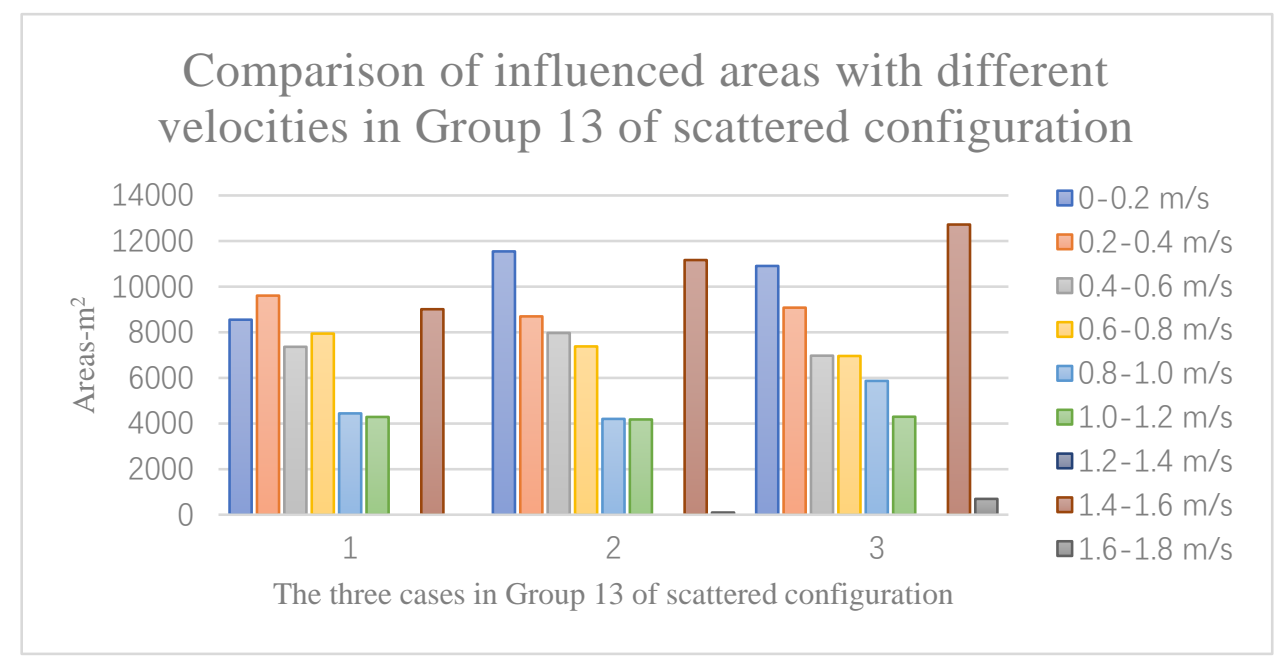

Figure 140. The influenced areas with different velocities in Group 13 of scattered configuration. 
The influenced areas in different cases are related to air pressure and wind flow (Figure 141 and Figure 142). The high-air-pressure area and low-air-pressure area becomes larger as the longitudinal distance increases. The streamlines show less winds are obstructed as longitudinal distance increases. Similar to previous groups, winds are able to escape more easily from the building cluster when the building interval becomes larger.

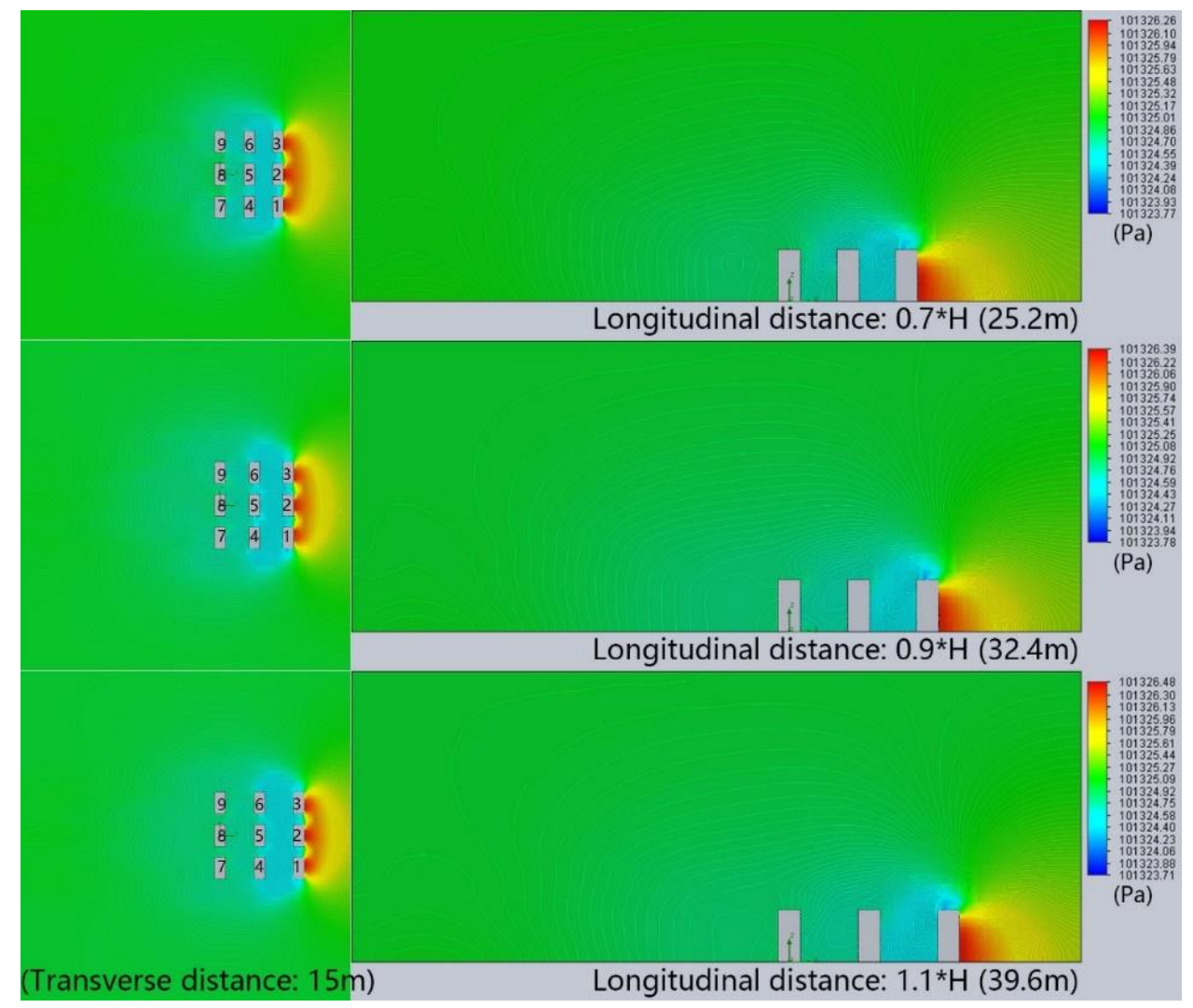

Figure 141. The comparison of air-pressure magnitudes on the horizontal and vertical planes of the building clusters in Group 13 of scattered configuration. 


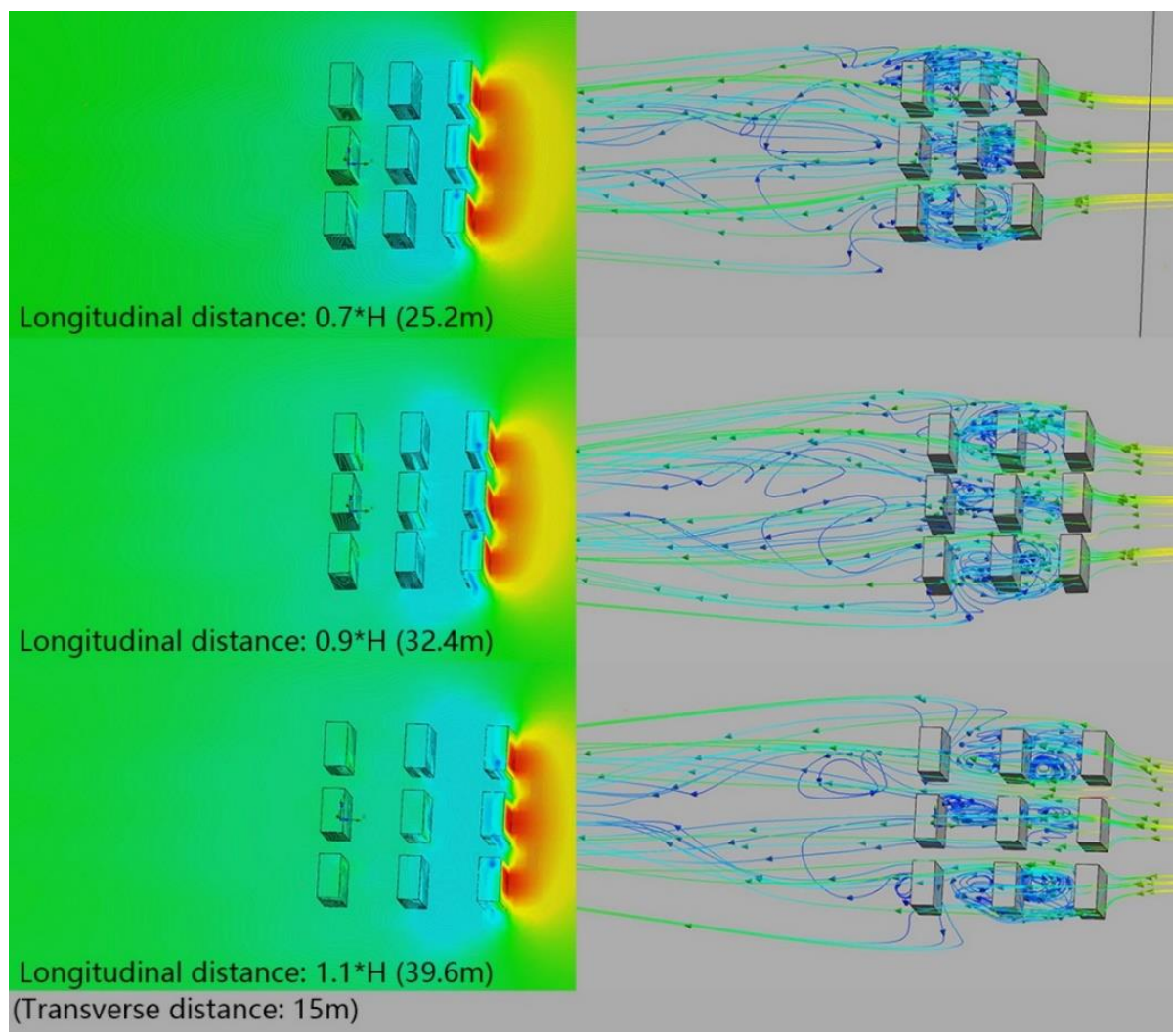

Figure 142. The comparison of wind-flow streamlines (right) and air-pressure magnitudes (left) on the horizontal plane and building surfaces in Group 13 of scattered configuration.

\section{(9) Part 9. Influences of staggered distances among 9 buildings in 3 rows}

In this part, the influences of different staggered distances of Group 14 (transverse distance: $15 \mathrm{~m}$; longitudinal distance: $0.9 * \mathrm{H}=32.4 \mathrm{~m}$ ) are analyzed. The wind-velocity magnitudes on horizontal and vertical planes of the three cases are analyzed. The results show that the total influence of the building cluster increases constantly as the staggered distance increases (staggered distance: 10m, 20m and 30m). And the increases of influence are significant (Figure 143 and Figure 144). The influence of the building cluster is the least in Case 1(influenced area: $59978.94 \mathrm{~m}^{2}$ ); the influence of Case 2 is much more than Case 1 (influenced area: $64833.51 \mathrm{~m}^{2}$ ); the influence of Case 3 is the most of all (influenced area: $76324.4 \mathrm{~m}^{2}$ ). The significant increases of the total influence are due to the increases of the windward projective area of the building cluster. Because the increase of staggered distance increases the width of the entire building cluster.

The velocity magnitudes show that the low-velocity area (velocity range: $0-0.4 \mathrm{~m} / \mathrm{s}$ ) 
increases constantly as the staggered distance increases. The significant increase of the lowvelocity area takes the major part of the increase of the total influence. The low-velocity area of Case 1 is the least $\left(29580.69 \mathrm{~m}^{2}\right)$; the area of Case 2 is much more than Case $1\left(36453.17 \mathrm{~m}^{2}\right)$; the area Case 3 is the most $\left(56339.47 \mathrm{~m}^{2}\right)$. The most low-velocity area is created on the leeward side of the building cluster. The increase of the area is due to the increase of the width of the entire building cluster. Though the entire low-velocity area increases as the staggered distance increases, the area with extremely low wind velocities (below $0.1 \mathrm{~m} / \mathrm{s}$ ) behind the building cluster is reduced; the low-velocity areas among the building rows decrease significantly, which suggests the outdoor ventilation among the building rows is promoted (Figure 143 and Figure 144).

There are high-velocity areas (velocity range: $1.4-1.6 \mathrm{~m} / \mathrm{s}$ ) on the lateral sides of the building cluster and in the gaps among the building units of the first building row (Figure 143). The high-velocity areas are fluctuated as the staggered distance increases. The high-velocity areas of Case 1 are between Case 2 and Case $3\left(14337.72 \mathrm{~m}^{2}\right)$; the areas of Case 2 are the most $\left(14378.88 \mathrm{~m}^{2}\right)$; the areas of Case 3 are the least of all $\left(7090.14 \mathrm{~m}^{2}\right)$. The high-velocity areas in the gaps among the building rows are close in the three cases, especially for the areas in the gaps among the first building row. Because the gaps are the same in the three cases and the amount of winds in the gaps are almost same. The high-velocity area on one lateral side becomes less, especially for the significant decrease in Case 3. The increase of the windward surfaces of the second and third building rows reduces the high-velocity area on the lateral side of the building cluster. 


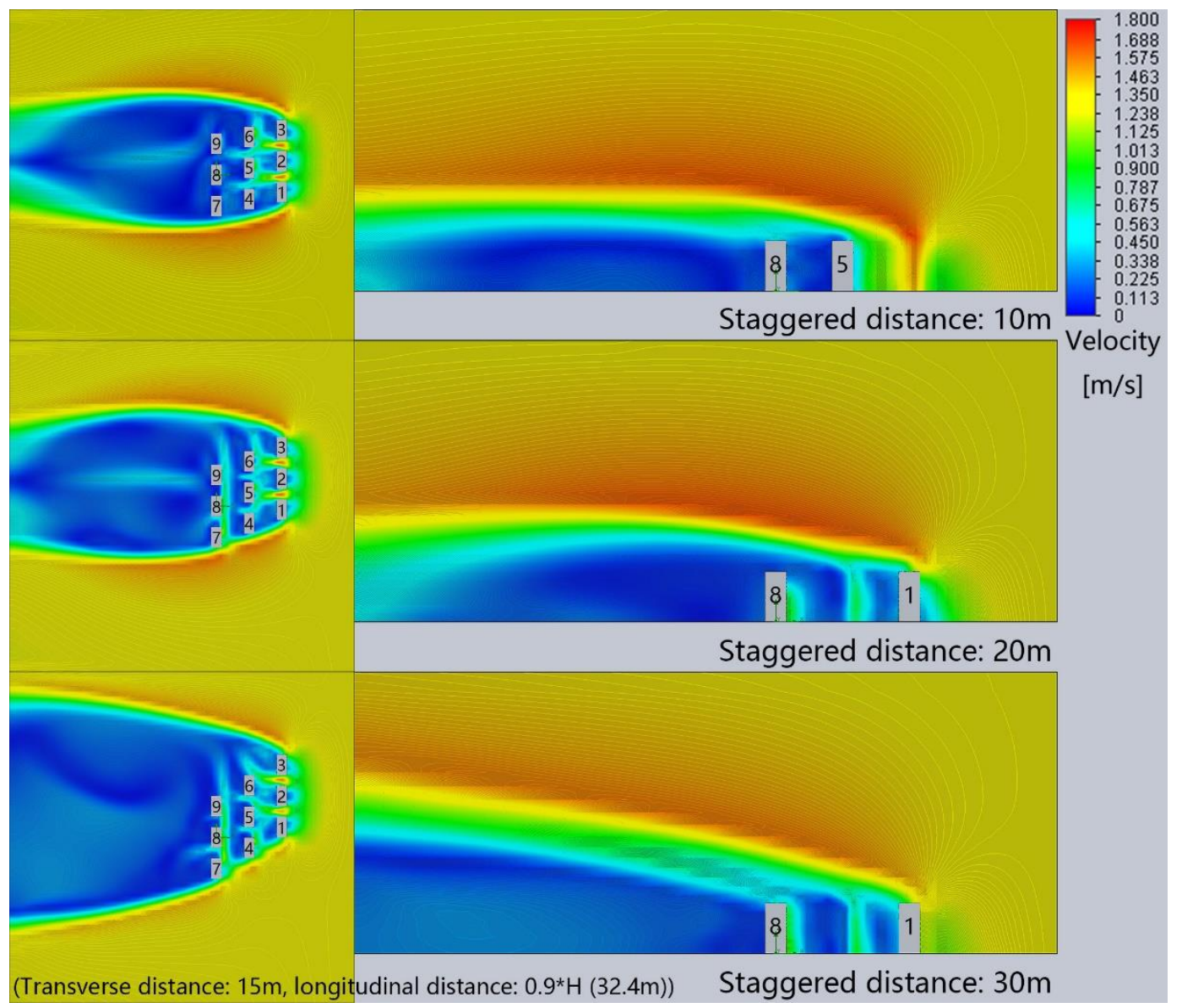

Figure 143. The comparison of wind-velocity magnitudes on horizontal and vertical planes of the building clusters in Group 14 of scattered configuration.

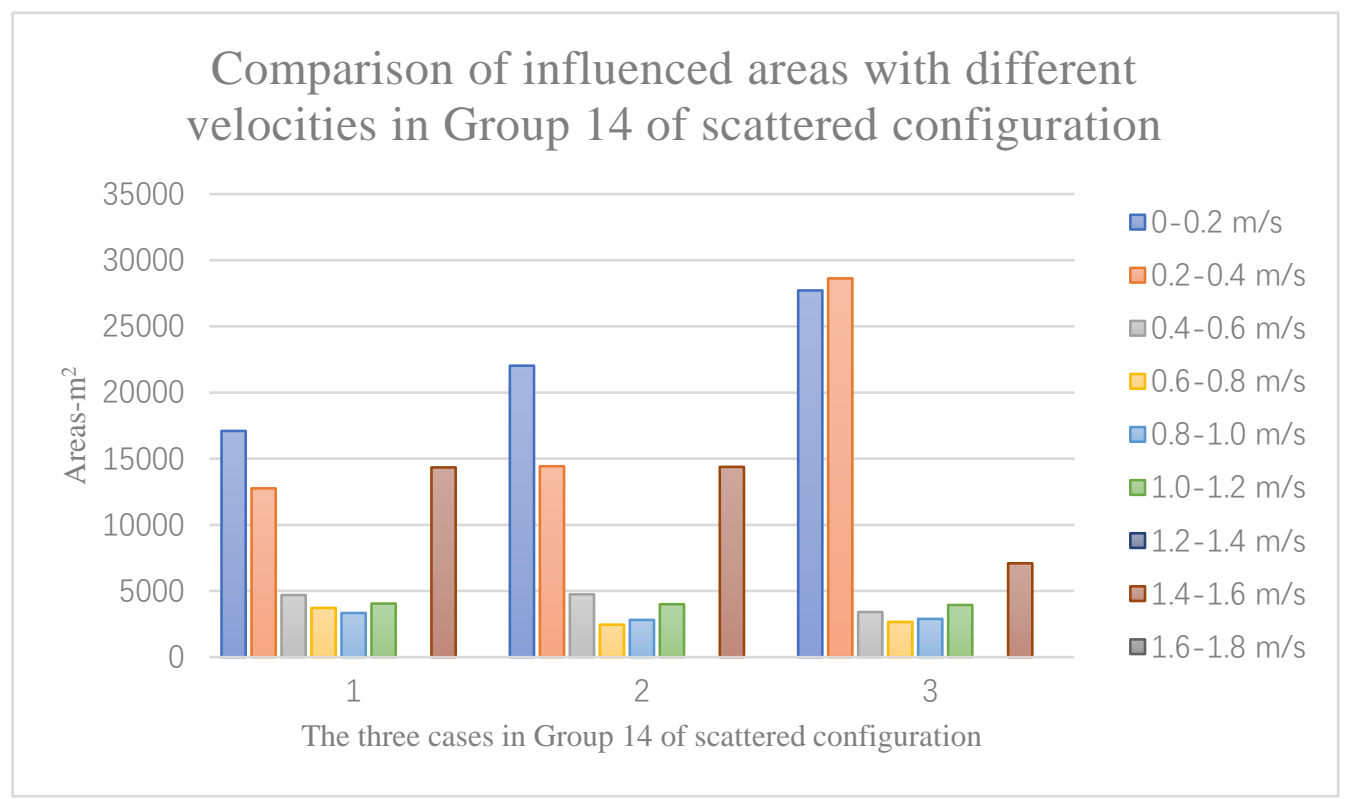

Figure 144. The influenced areas with different velocities in Group 14 of scattered configuration. 
The changes of influenced areas with different velocities in Group 14 are related to the changes of air pressure and wind flow (Figure 145 and Figure 146). As the staggered distance increases, the influenced areas with different air pressures are increased; however, the air pressures on the horizontal plane of the entire area become more even, which is the reason for the increase of influenced areas behind the building cluster shown on the velocity magnitudes; the low-air-pressure areas among the building rows are decreased, which is the reason for the decrease of the low-velocity areas among the building rows; the low-air-pressure area on the leeward side of the building cluster increases, which increases the low-velocity area behind the building cluster; vortices among the building rows are reduced, which proves the increases of wind velocities among the building rows; vortices behind the building cluster are reduced, which suggests the velocities in the low-velocity area are increased.

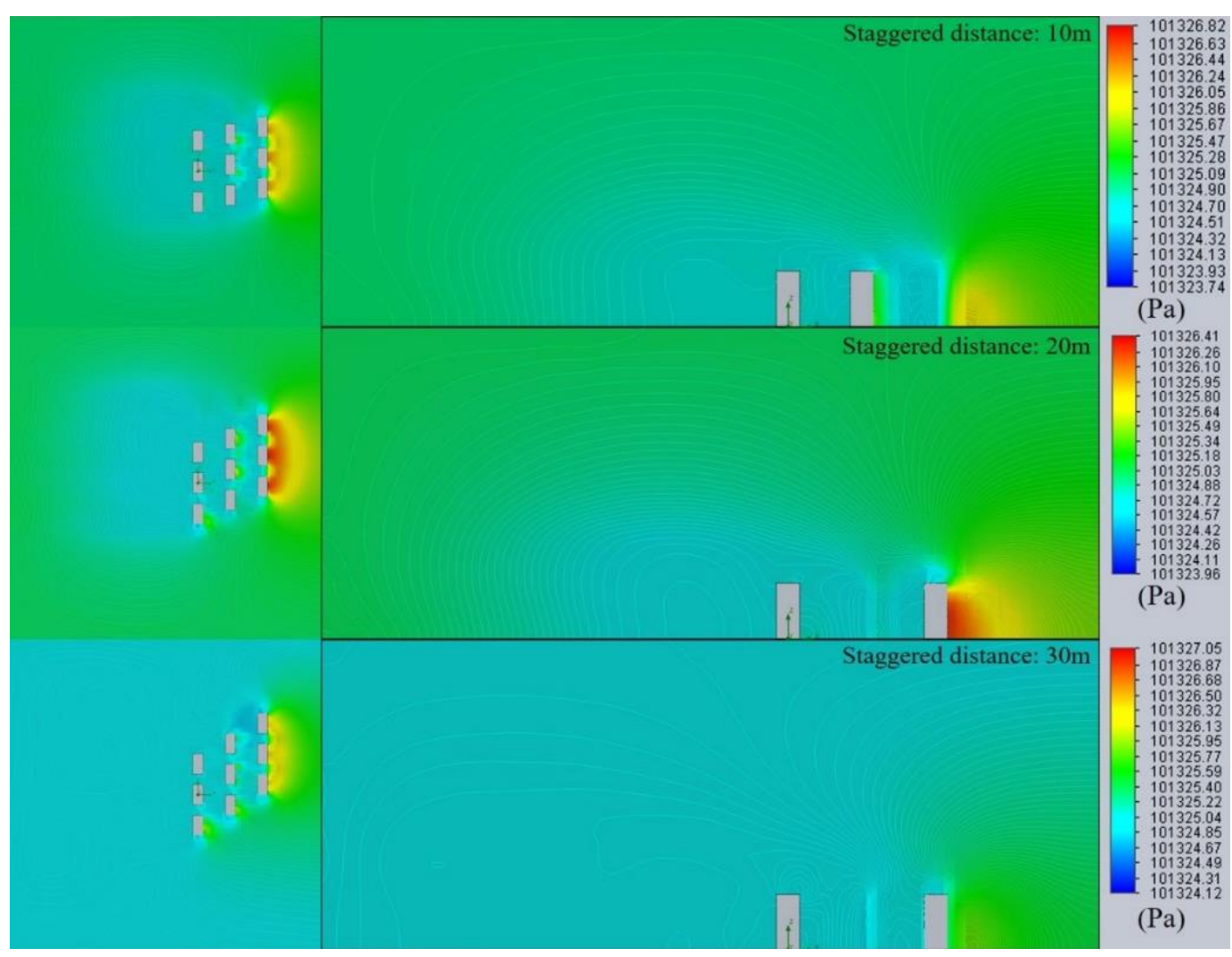

Figure 145. The comparison of air-pressure magnitudes on the horizontal and vertical planes of the building clusters in Group 14 of scattered configuration. 


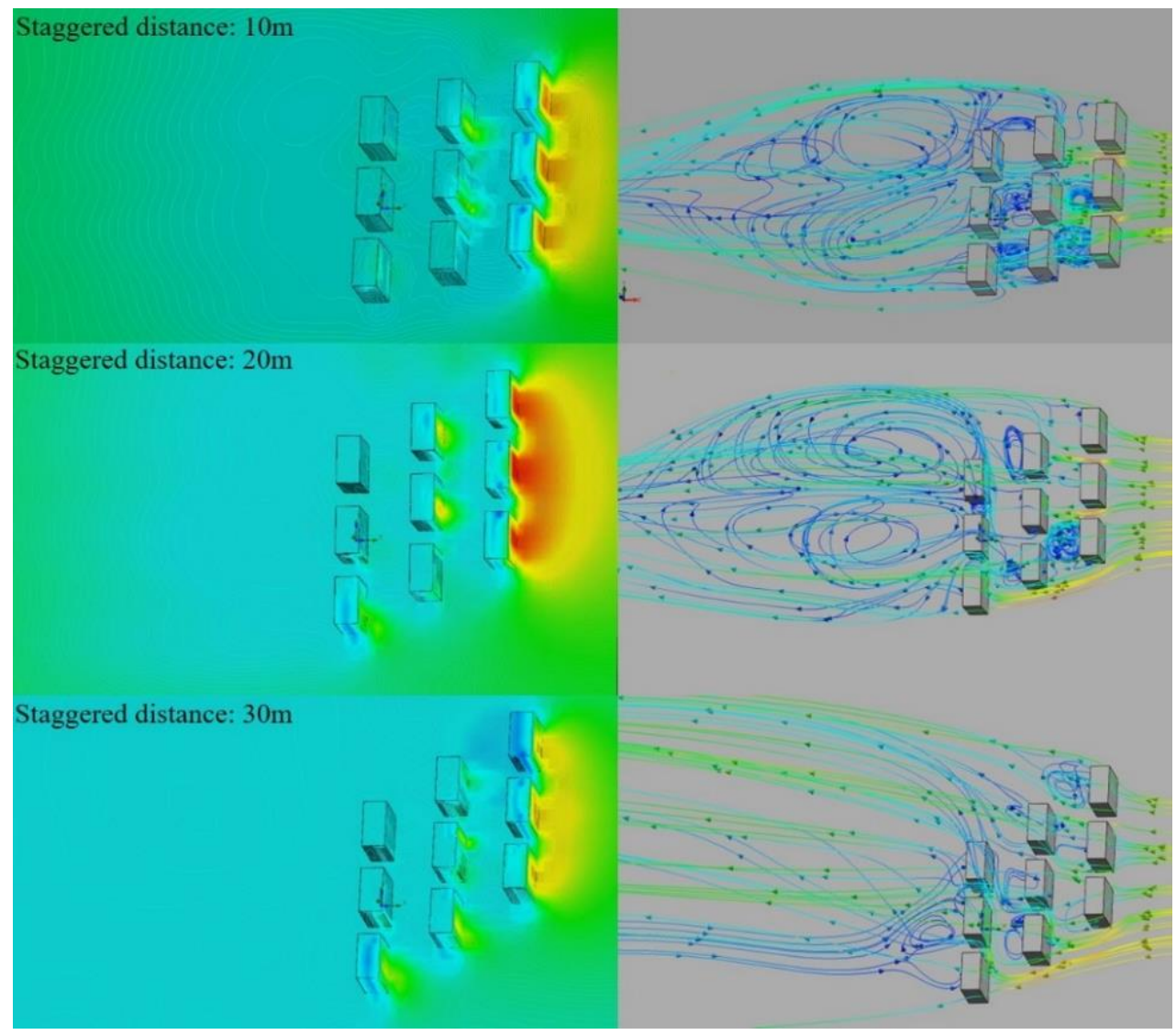

Figure 146. The comparison of wind-flow streamlines (right) and air-pressure magnitudes (left) on the horizontal plane and building surfaces in Group 14 of scattered configuration.

Therefore, the air-pressure magnitudes and wind-flow streamlines suggest that the increase of the staggered distance can improve the overall outdoor ventilation of the building cluster, especially for the spaces among the building rows. Though the low-velocity area is increased as the staggered distance increases, the velocities in the area is actually increased because less vortices are created. However, the increase of the total influenced area is relatively large due to the increase of the windward width of the entire building cluster.

\subsection{Linear configuration}

This section aims to study the relationships between the influences on wind environments and the building variables of linear configuration, including the building unit number, longitudinal distance and staggered distance (Figure 147). In the first part, the influences of building-unit 
numbers are studied for a linear-configuration building cluster with several units arranged in a building row. In the second part, the influences of building-unit numbers and longitudinal distances are studied for a linear-configuration building cluster with building units arranged in two building rows. In the third part, the influences of building-unit numbers and longitudinal distances are studied for a linear-configuration building cluster with building units arranged in three building rows. In the fourth part, the influences of staggered distances are studied for a linear-configuration building cluster with nine building units arranged in three building rows. The building variables setup of the four parts are shown first. Then results and analysis of the four parts are presented.

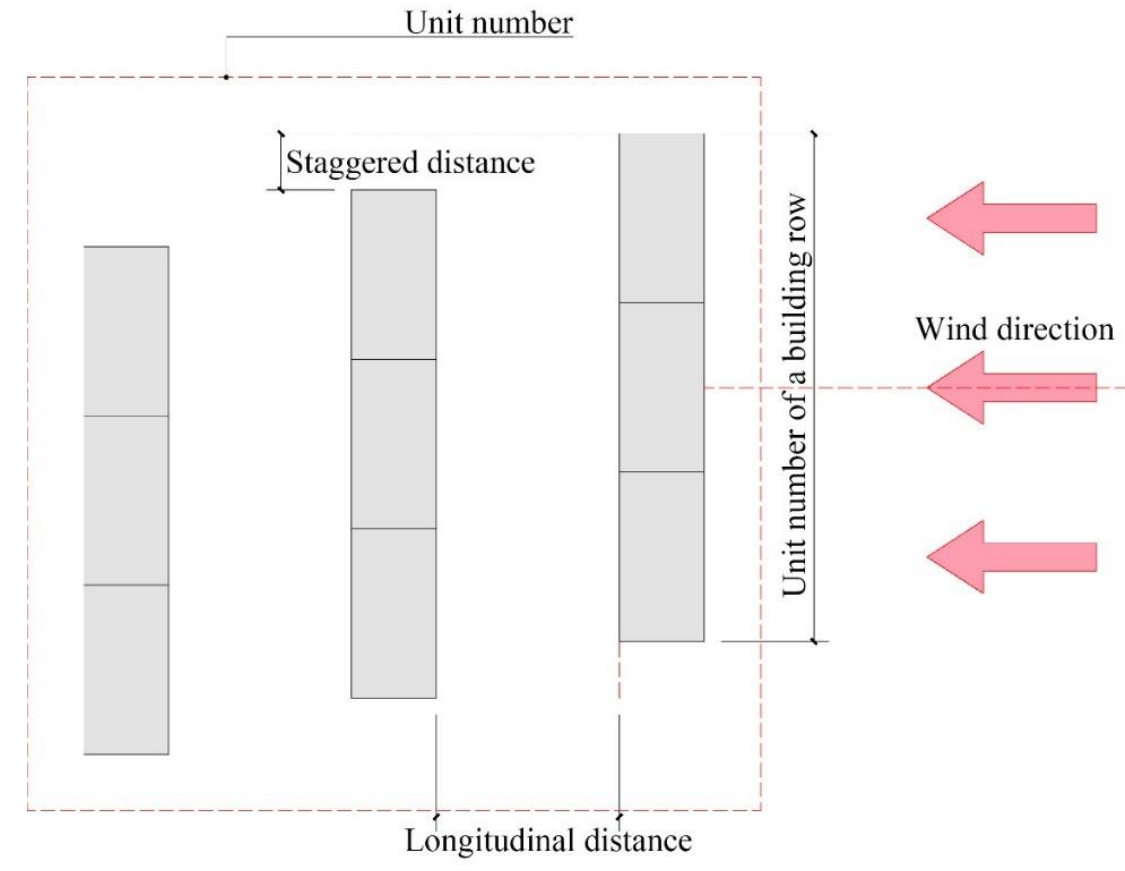

Figure 147. The building variables of linear configuration.

\subsubsection{Building variable setup of linear configuration}

In the first part, three cases with different building-unit numbers are set up in Group 1. According to the summary in the parametric design chapter, common unit numbers in the range of 2-4 are used in the study; building units are arranged in a linear building row. The specific variables of the three cases are presented in the following tables (Table 37.). 
Table 37. The three cases in Group 1 of linear configuration.

\begin{tabular}{|l|l|}
\hline Unit numbers & Rows \\
\hline 2 & 1 \\
\hline 3 & 1 \\
\hline 4 & 1 \\
\hline
\end{tabular}

In the second part, six cases with different unit numbers and longitudinal distances are set up in two groups (Group 2. and Group 3.). According to the summary in the parametric design chapter, common unit numbers in the range of 4-8 are used in the study; common longitudinal distances in the range of $0.7 * \mathrm{H}-1.1 * \mathrm{H}(25.2-39.6 \mathrm{~m}, \mathrm{H}$ stands for the building height $36 \mathrm{~m})$ are used; the building units are arranged in two building rows. The specific variables of the two groups are presented in the following tables (Table 38. and Table 39.).

Table 38. The three cases in Group 2 of linear configuration.

\begin{tabular}{|l|l|l|}
\hline Unit numbers & Rows & Longitudinal distances \\
\hline 4 & 2 & $0.9 * \mathrm{H}(32.4 \mathrm{~m})$ \\
\hline 6 & 2 & $0.9 * \mathrm{H}(32.4 \mathrm{~m})$ \\
\hline 8 & 2 & $0.9 * \mathrm{H}(32.4 \mathrm{~m})$ \\
\hline \multicolumn{2}{|l}{ The 'H' in this group stands for the height of the building $(36 \mathrm{~m})}$. \\
\hline
\end{tabular}

Table 39. The three cases in Group 3 of linear configuration.

\begin{tabular}{|l|l|l|}
\hline Unit numbers & Rows & Longitudinal distances \\
\hline 6 & 2 & $0.7 * \mathrm{H}(25.2 \mathrm{~m})$ \\
\hline 6 & 2 & $0.9 * \mathrm{H}(32.4 \mathrm{~m})$ \\
\hline 6 & 2 & $1.1 * \mathrm{H}(39.6 \mathrm{~m})$ \\
\hline \multicolumn{2}{|l|}{ The 'H' in this group stands for the height of the building (36m). } \\
\hline
\end{tabular}

In the third part, six cases with different unit numbers and longitudinal distances are set 
up in two groups (Group 4. and Group 5.). According to the summary in the parametric design chapter, common unit numbers in the range of 6-12 are used in the study; common longitudinal distances in the range of $0.7 * \mathrm{H}-1.1 * \mathrm{H}(25.2-39.6 \mathrm{~m}, \mathrm{H}$ stands for the building height $36 \mathrm{~m})$ are used; the building units are arranged in three building rows. The specific variables of the two groups are presented in the following tables (Table 40. and Table 41.).

Table 40. The three cases in Group 4 of linear configuration.

\begin{tabular}{|l|l|l|}
\hline Unit numbers & Rows & Longitudinal distances \\
\hline 6 & 3 & $0.9 * \mathrm{H}(32.4 \mathrm{~m})$ \\
\hline 9 & 3 & $0.9 * \mathrm{H}(32.4 \mathrm{~m})$ \\
\hline 12 & 3 & $0.9 * \mathrm{H}(32.4 \mathrm{~m})$ \\
\hline \multicolumn{2}{|l}{ The 'H' in this group stands for the height of the building $(36 \mathrm{~m})}$. \\
\hline
\end{tabular}

Table 41. The three cases in Group 5 of linear configuration.

\begin{tabular}{|l|l|l|}
\hline Unit numbers & Rows & Longitudinal distances \\
\hline 9 & 3 & $0.7 * \mathrm{H}(25.2 \mathrm{~m})$ \\
\hline 9 & 3 & $0.9 * \mathrm{H}(32.4 \mathrm{~m})$ \\
\hline 9 & 3 & $1.1 * \mathrm{H}(39.6 \mathrm{~m})$ \\
\hline \multicolumn{2}{|l}{ The 'H' in this group stands for the height of the building (36m). } \\
\hline
\end{tabular}

In the fourth part, three cases with different staggered distances are set up in Group 6 . According to the summary in the parametric design chapter, common staggered distances in the range of $10-30 \mathrm{~m}$ are used in the study; a common longitudinal distance of $0.9^{*} \mathrm{H}(32.4 \mathrm{~m}, \mathrm{H}$ stands for the building height $36 \mathrm{~m}$ ) is used; nine building units are arranged in three building rows. The specific variables of the three cases are presented in the following tables (Table 42).

Table 42. The three cases in Group 6 of linear configuration.

\begin{tabular}{|l|l|l|l|}
\hline Unit numbers & Rows & Longitudinal distances & Staggered distances \\
\hline 9 & 3 & $0.9 * \mathrm{H}(32.4 \mathrm{~m})$ & $10 \mathrm{~m}$ \\
\hline
\end{tabular}




\begin{tabular}{|l|l|l|l|}
\hline 9 & 3 & $0.9 * \mathrm{H}(32.4 \mathrm{~m})$ & $20 \mathrm{~m}$ \\
\hline 9 & 3 & $0.9 * \mathrm{H}(32.4 \mathrm{~m})$ & $30 \mathrm{~m}$ \\
\hline \multicolumn{4}{|l|}{ The ' $\mathrm{H}$ ' in this group stands for the height of the building $(36 \mathrm{~m})}$. \\
\hline
\end{tabular}

The parametric modelling script of linear configuration is used to create the building cluster models. The setting of building units is the same as the setting in the study of scattered configuration. The width and length of the building unit are all set as $15 \mathrm{~m}$ and $30 \mathrm{~m}$, which is common for a high-rise residential building with two apartments on each floor. The building height of $36 \mathrm{~m}$ is used in all cases, which is a common height for a high-rise residential building with twelve floors. The range of longitudinal distance $\left(0.7^{*} \mathrm{H}-1.1^{*} \mathrm{H}\right)$ is determined by the architectural standards. In the above four groups, boundaries of CFD simulation results are all set in the same sizes for comparisons of influences on wind environments.

\subsubsection{Results and analysis of linear configuration}

The CFD simulation results of linear configuration are analyzed in this subsection. Influences on wind environments are compared based on velocity magnitudes. The mechanisms are explained using the air-pressure magnitudes and wind-flow streamlines. In Part 1 , the relationship between influences and building-unit numbers of a linear building row is studied. Part 2 focuses on the building cluster with two linear building rows: the relationship between influences and building-unit numbers is studied; and the relationship between influences and longitudinal distances is studied. Part 3 focuses on the building cluster with three linear building rows: the relationship between influences and building-unit numbers is studied; and the relationship between influences and longitudinal distances is studied. In Part 4, the relationship between influences and staggered distances are studied for the building cluster with nine units in three rows.

\section{(1) Part 1. Influences of building unit numbers in a row}

In this part, the influences of different building-unit numbers of Group 1 are studied. The wind- 
velocity magnitudes on horizontal and vertical planes of three cases are analyzed (Figure 148 and Figure 149). In Group 1, the total influenced area with different velocities increases constantly as the building-unit number increases. In Case 1 (unit number: 2), the influence of the building cluster is the least (influenced area: $29582.42 \mathrm{~m}^{2}$ ); in Case 2 (unit number: 3 ), the influence is more than Case 1 (influenced area: $38696.43 \mathrm{~m}^{2}$ ); in Case 3 (unit number: 4), the influence is the most of all (influenced area: $48752.25 \mathrm{~m}^{2}$ ).

There is a low-velocity area (velocity range: $0-0.4 \mathrm{~m} / \mathrm{s}$ ) on the leeward side of the building cluster. The low-velocity area is increased constantly as the unit number decreases (Figure 148 and Figure 149). In Case 1, the low-velocity area is the least $\left(11391.83 \mathrm{~m}^{2}\right)$; in Case 2, the area is less than Case $1\left(15033.74 \mathrm{~m}^{2}\right)$; in Case 3, the area is the most of all $\left(21804.47 \mathrm{~m}^{2}\right)$.

There are high-velocity areas (velocity range: $1.4-1.6 \mathrm{~m} / \mathrm{s}$ ) on the lateral sides of the building clusters in the three cases. The high-velocity areas increase constantly as the unit number increases (Figure 148 and Figure 149). In Case 1, the high-velocity areas are the smallest of all $\left(2897.99 \mathrm{~m}^{2}\right)$; in Case 2, the areas are more than Case $1\left(5557.47 \mathrm{~m}^{2}\right.$; in Case 3 , the areas are the largest $\left(7310.47 \mathrm{~m}^{2}\right)$.

In summary, the total influenced area, low-velocity area and high-velocity areas are all increased as the unit number increases. The linear building row with an increasing buildingunit number can be judged as a rectangular-form building with an increasing length. The increases of the influenced areas are due to the increased windward projective area of the building cluster. The velocity magnitudes also show the trend is similar to the results of rectangular form. 


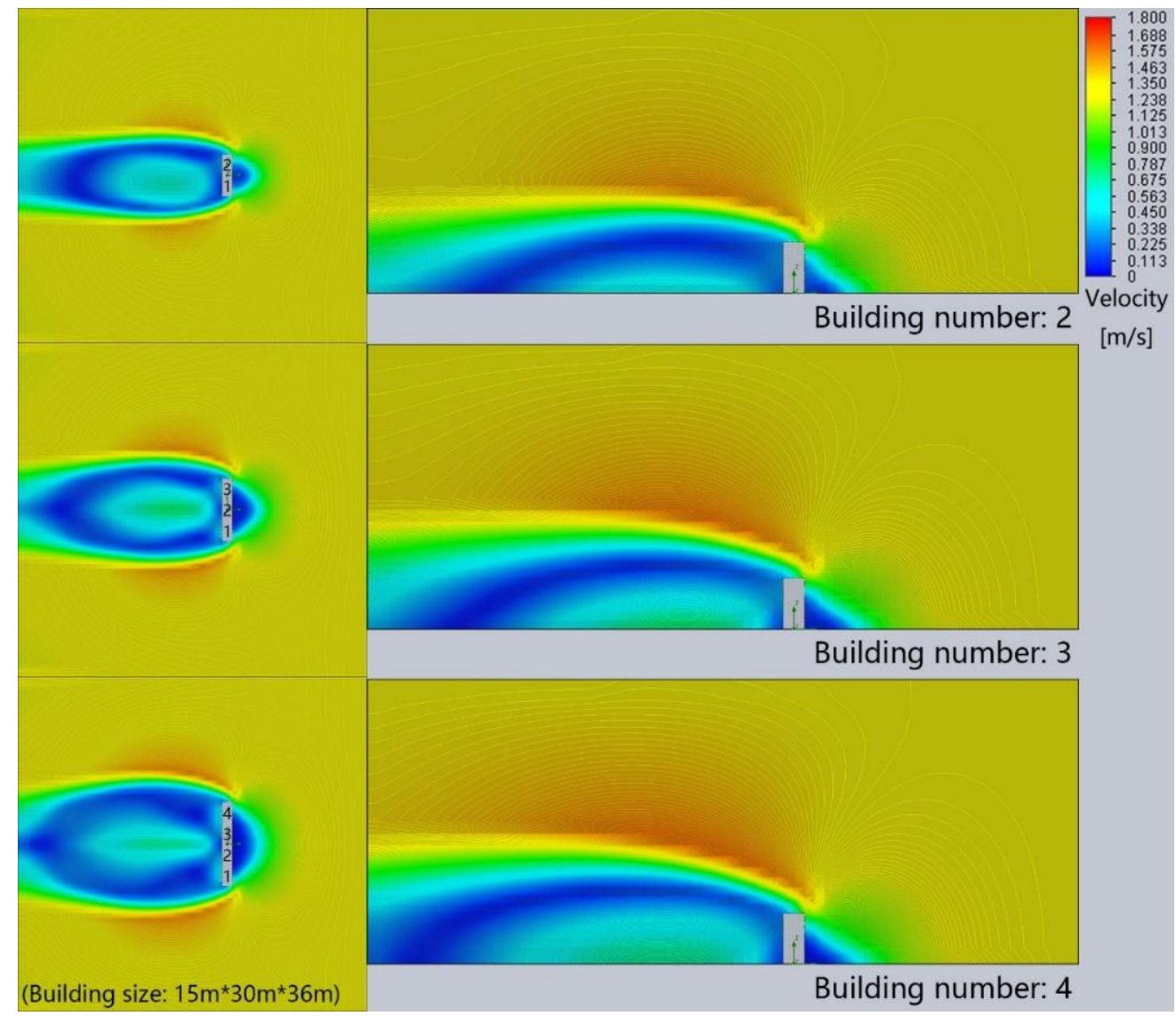

Figure 148. The comparison of wind-velocity magnitudes on horizontal and vertical planes of the building clusters in Group 1 of linear configuration.

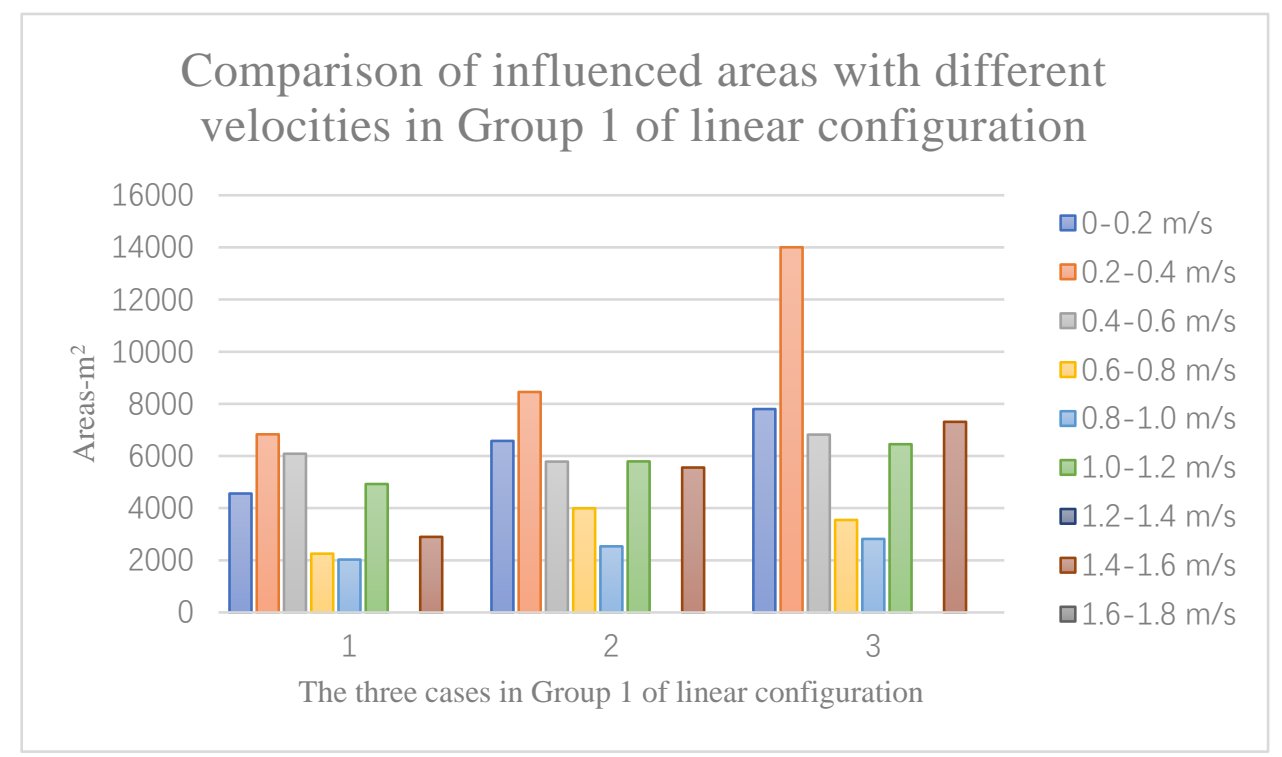

Figure 149. The influenced areas with different velocities in Group 1 of linear configuration.

The changes of influenced areas with different velocities in Group 1 are related to the 
changes of air pressure and wind flow (Figure 150 and Figure 151). As the unit number increases, the low-air-pressure area behind the building cluster and high-air-pressure area in front of the building cluster are both increased significantly, which is the reason for the increase of total influenced area; the air-pressure difference between the areas increases, and the extent of air-pressure changes is increased, which causes the increase of the high-velocity areas; the vortices on the leeward side of the building cluster increase and become larger, which corresponds to the increase of the low-velocity area on the leeward side. The windward projective area of the linear building row increases as the building-unit number increases. Therefore, more winds are obstructed by the building cluster.

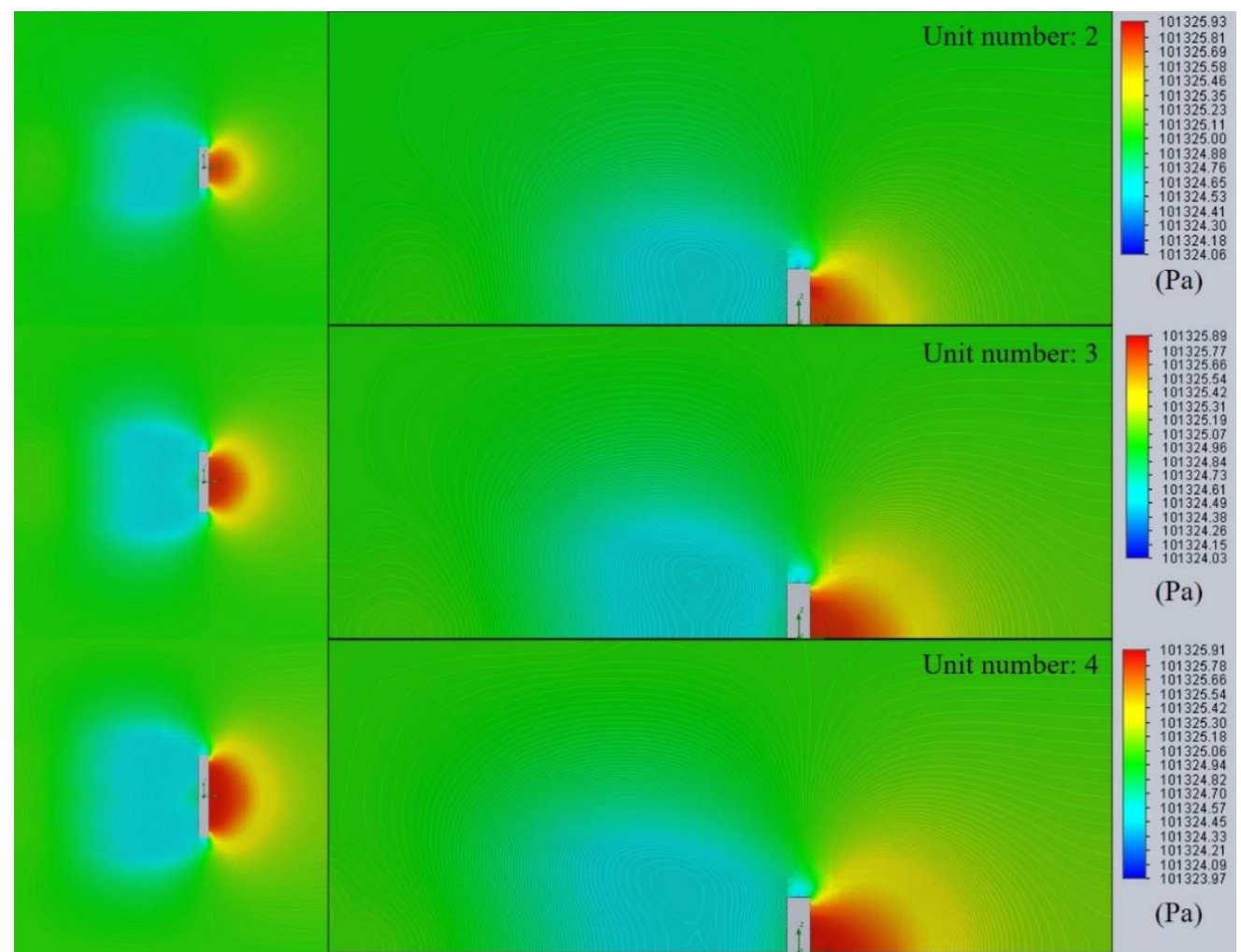

Figure 150. The comparison of air-pressure magnitudes on the horizontal and vertical planes of the building clusters in Group 1 of linear configuration. 


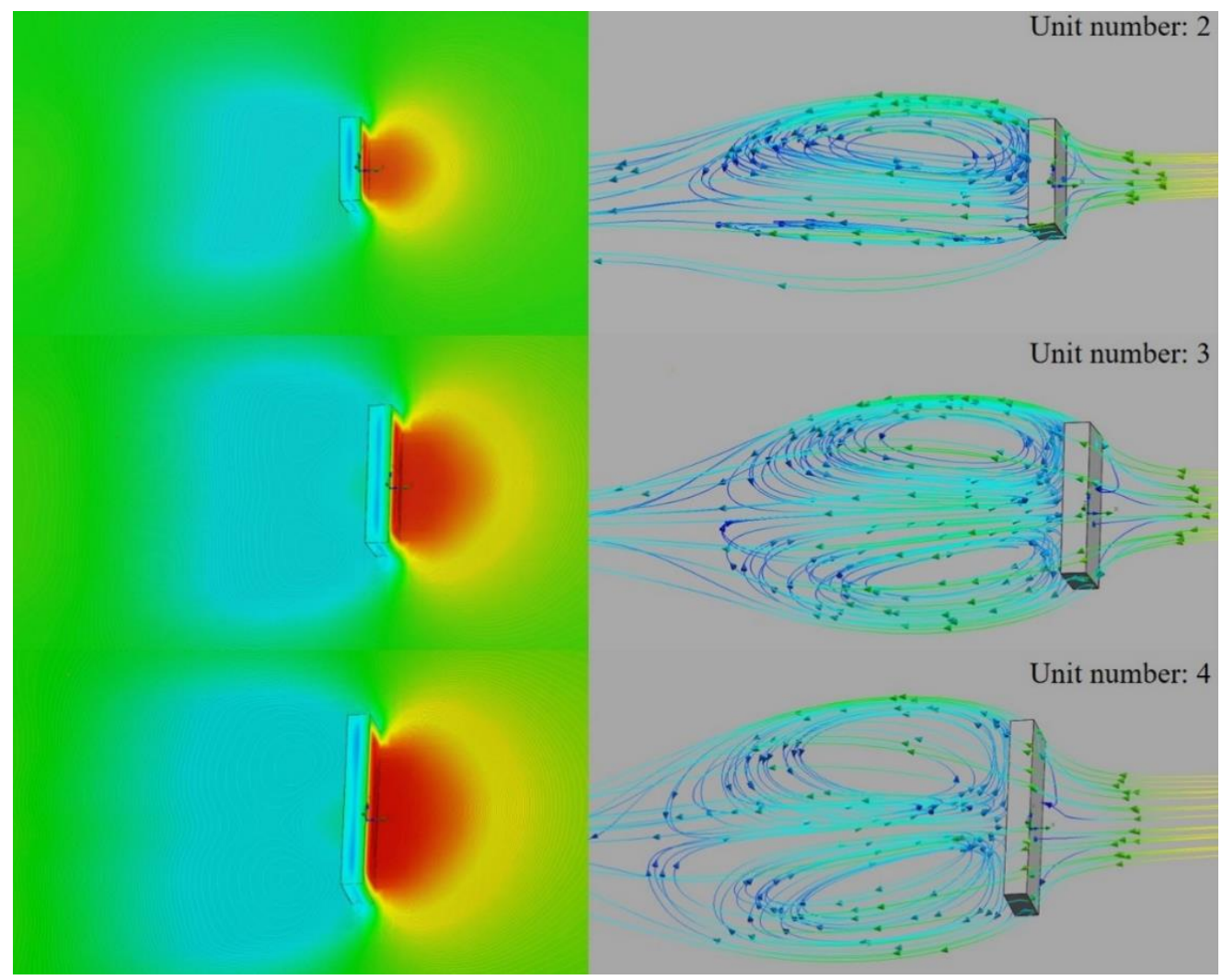

Figure 151. The comparison of wind-flow streamlines (right) and air-pressure magnitudes (left) on the horizontal plane and building surfaces in Group 1 of linear configuration.

\section{(2) Part 2. Influences of building unit numbers and longitudinal distances in 2 rows}

In this part, the influences of different building-unit numbers of Group 2 (longitudinal distance: $0.9 * \mathrm{H}=32.4 \mathrm{~m}$ ) are studied first. The wind-velocity magnitudes on horizontal and vertical planes of three cases are analyzed (Figure 152 and Figure 153). In Group 2, the total influenced area increases constantly as the building-unit number increases. The trend is significant, which is similar to Group 1. In Case 1, the influence is the least (influenced area: $33487.97 \mathrm{~m}^{2}$ ); in Case 2, the influence is more than Case 1 (influenced area: $45094.87 \mathrm{~m}^{2}$ ); in Case 3, the influence is the most of all (influenced area: $55356.11 \mathrm{~m}^{2}$ ).

There are low-velocity areas (velocity range: $0-0.4 \mathrm{~m} / \mathrm{s}$ ) on the leeward side of the building cluster and between the adjacent building rows. As the unit number increases, the total lowvelocity area increases constantly; the low-velocity area on the leeward side increases; the lowvelocity area between the building rows also increases. In Case 1, the total low-velocity area is the least $\left(13864.36 \mathrm{~m}^{2}\right)$; in Case 2, the area is more than Case $1\left(18215.52 \mathrm{~m}^{2}\right)$; the area of Case 
3 is the most of all $\left(24408.89 \mathrm{~m}^{2}\right)$. (Figure 152 and Figure 153)

There are high-velocity areas (velocity range: $1.4-1.6 \mathrm{~m} / \mathrm{s}$ ) on the lateral sides of the building clusters. The high-velocity areas increase constantly as the unit number increases. In Case 1, the high-velocity areas are the least $\left(10544.55 \mathrm{~m}^{2}\right)$; in Case 2, the areas are more than Case $1\left(8046.319 \mathrm{~m}^{2}\right)$; in Case 3, the areas are the largest $\left(8120.031 \mathrm{~m}^{2}\right)$. (Figure 152 and Figure 153)

In summary, the influences of the building cluster are increased as the unit number increases. Especially for the low-velocity area between the building rows, the area with extremely low velocities increases significantly. The reason is the increase of windward projective area of the building cluster, which is similar to Group 1.

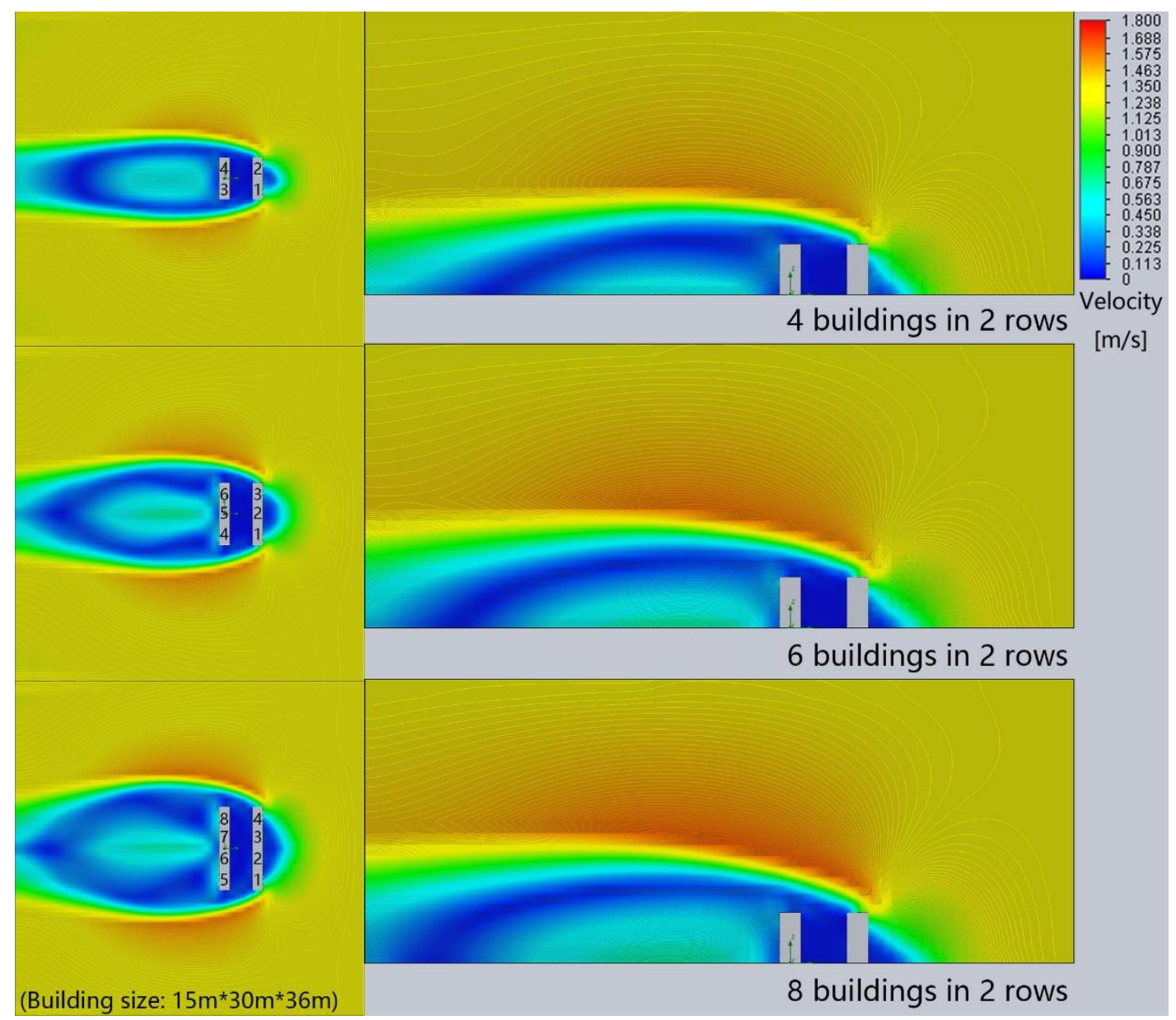

Figure 152. The comparison of wind-velocity magnitudes on horizontal and vertical planes of the building clusters in Group 2 of linear configuration. 


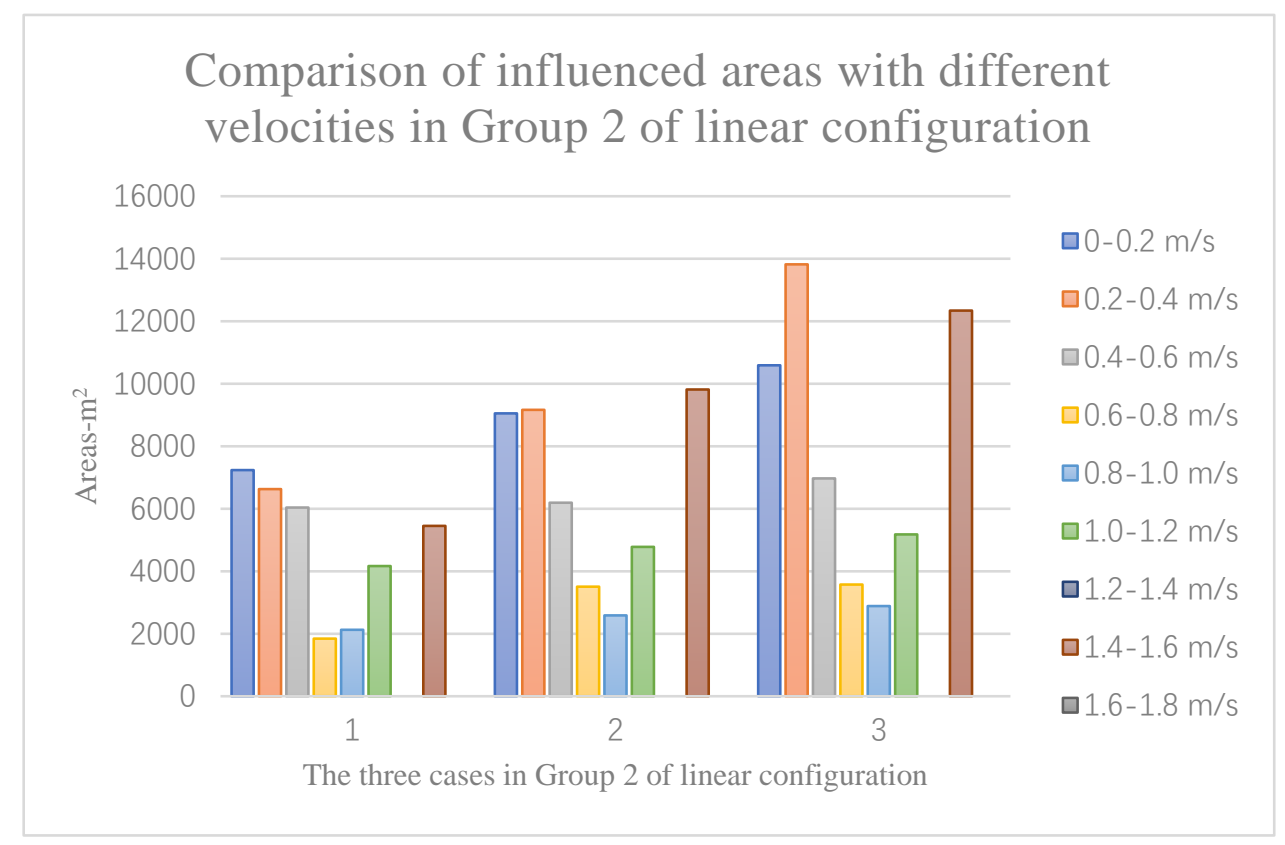

Figure 153. The influenced areas with different velocities in Group 2 of linear configuration.

The changes of influenced areas with different velocities in Group 2 are related to the changes of air pressure and wind flow (Figure 154 and Figure 155). As the unit number increases, the influenced areas with different air pressures are increased significantly, which is the reason for the increase of the influenced areas with different velocities; the air-pressure difference between the low-air-pressure and high-air-pressure areas increases, which causes the increase of the high-velocity areas on the lateral sides; the vortices between the building rows increase and become wider, which lead to the increase of the low-velocity area between the building rows; the vortices behind the building cluster become larger and longer, which lead to the increase of the low-velocity area behind the building cluster. These are because more winds are obstructed as the windward projective area of the building cluster increases. 


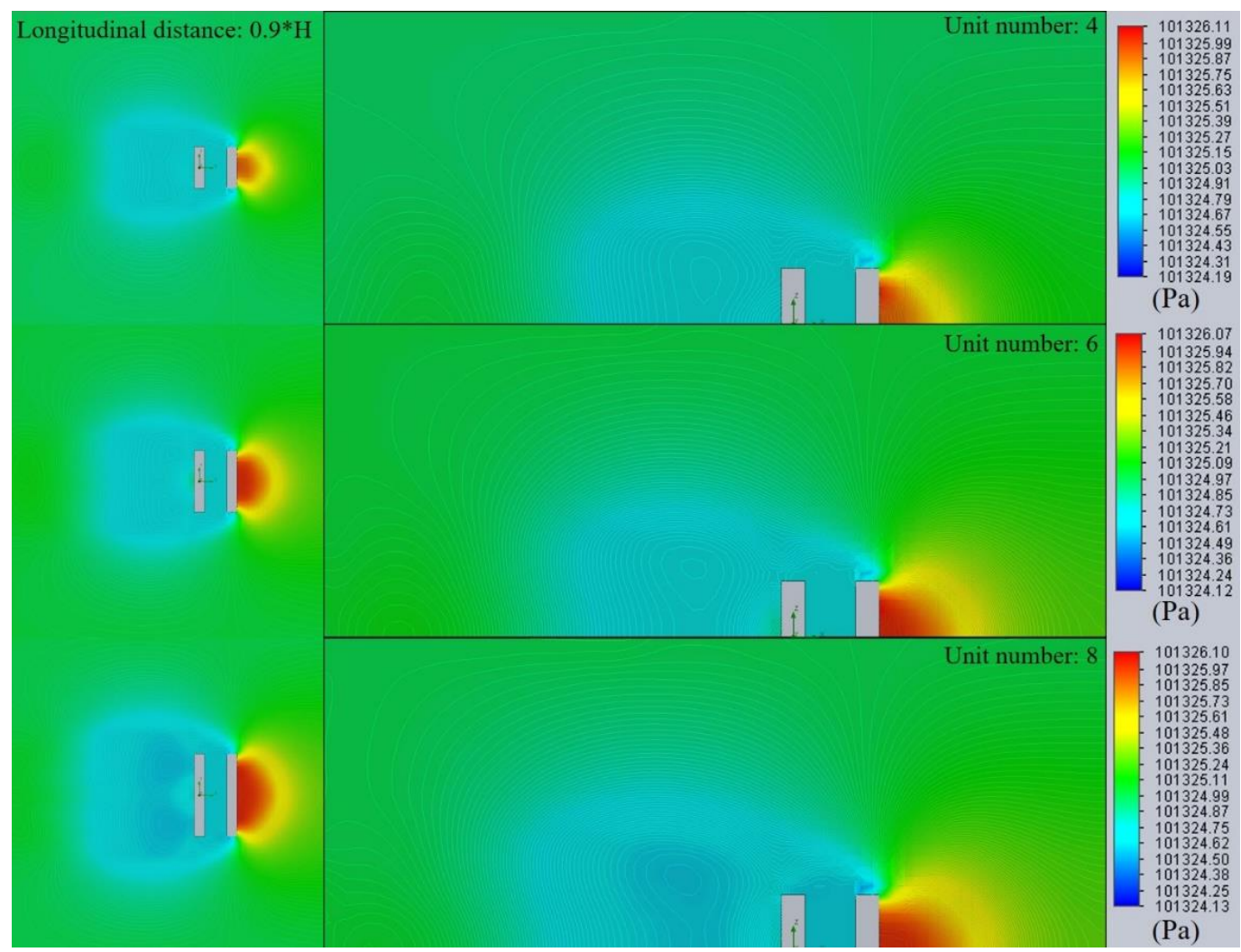

Figure 154. The comparison of air-pressure magnitudes on the horizontal and vertical planes of the building clusters in Group 1 of linear configuration.

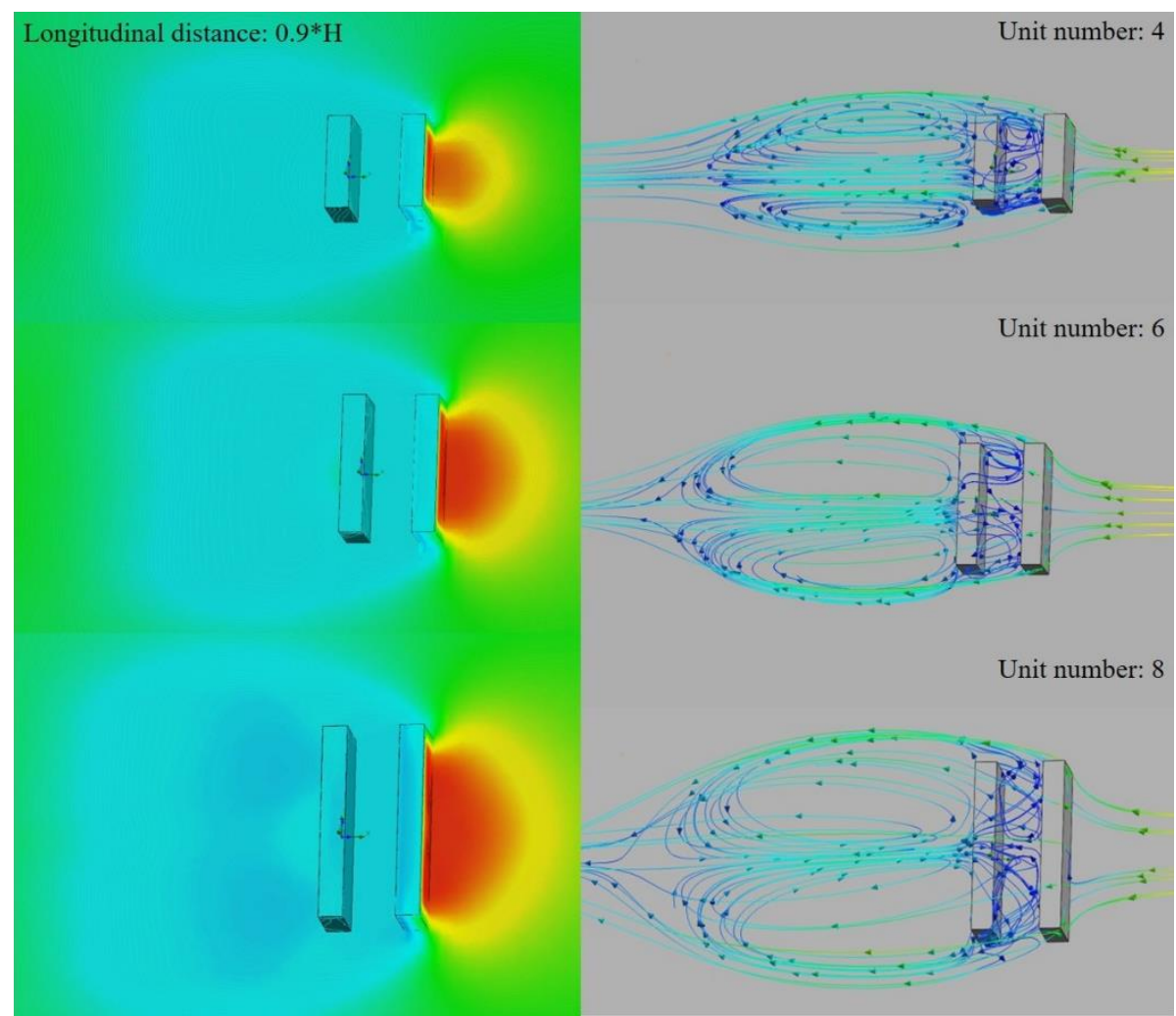

Figure 155. The comparison of wind-flow streamlines (right) and air-pressure magnitudes (left) 
on the horizontal plane and building surfaces in Group 2 of linear configuration.

Second, the influences of different longitudinal distances of Group 3 (unit number: 6) are studied. The wind-velocity magnitudes on horizontal and vertical planes of three cases are analyzed (Figure 156 and Figure 157). In Group 3, the total influence of Case 1 is the least (influenced area: $43002.89 \mathrm{~m}^{2}$ ); the influence of Case 2 is more than Case 1 (influenced area: $45094.87 \mathrm{~m}^{2}$ ); the influence of Case 3 is the most of all (influenced area: $45830.98 \mathrm{~m}^{2}$ ). Therefore, the total influence increases as the longitudinal distance increases. However, the increases are relatively small, especially for the results of Case 2 and Case 3 . The later analysis shows that the increases are largely due to the increases of the low-velocity area. Because the low-velocity area between the building rows increases as the longitudinal distance increases. But the low-velocity area behind the building cluster becomes smaller and shorter.

There are low-velocity areas (velocity range: $0-0.4 \mathrm{~m} / \mathrm{s}$ ) on the leeward side of the building cluster and between the building rows. In Case 1, the total low-velocity area is the least $\left(18198.63 \mathrm{~m}^{2}\right)$; in Case 2, the area is more than Case $1\left(18215.52 \mathrm{~m}^{2}\right)$; the area of Case 3 is the most of all $\left(18431.15 \mathrm{~m}^{2}\right)$. Therefore, the total low-velocity area increases as the longitudinal distance increases, though the increases are relatively small (Figure 156 and Figure 157). The low-velocity area between the adjacent building rows increases, especially for the area with extremely low velocities (velocity range: $0-0.2 \mathrm{~m} / \mathrm{s}$ ). However, the low-velocity area on the leeward side decreases and becomes shorter slightly.

There are high-velocity areas (velocity range: $1.4-1.6 \mathrm{~m} / \mathrm{s}$ ) on the lateral sides of the building clusters. In Case 1, the high-velocity areas are the least $\left(8607.99 \mathrm{~m}^{2}\right)$; in Case 2, the areas are more than Case $1\left(9815.19 \mathrm{~m}^{2}\right)$; in Case 3, the areas are the largest $\left(10720.73 \mathrm{~m}^{2}\right)$. Therefore, the high-velocity areas increase constantly as the longitudinal distance increases (Figure 156 and Figure 157).

In summary, as the longitudinal distance increases, the total influence of the building cluster increases; the low-velocity and high-velocity areas are all increased. However, the increases of the total influence and low-velocity area are relatively small. The high-velocity areas are increased; and the low-velocity area on the leeward side of the building cluster becomes shorter and smaller. Therefore, the increase of the longitudinal distance can promote 
the overall outdoor ventilation. But the promotion of the outdoor ventilation between the building rows is limited because of the relatively long building row.

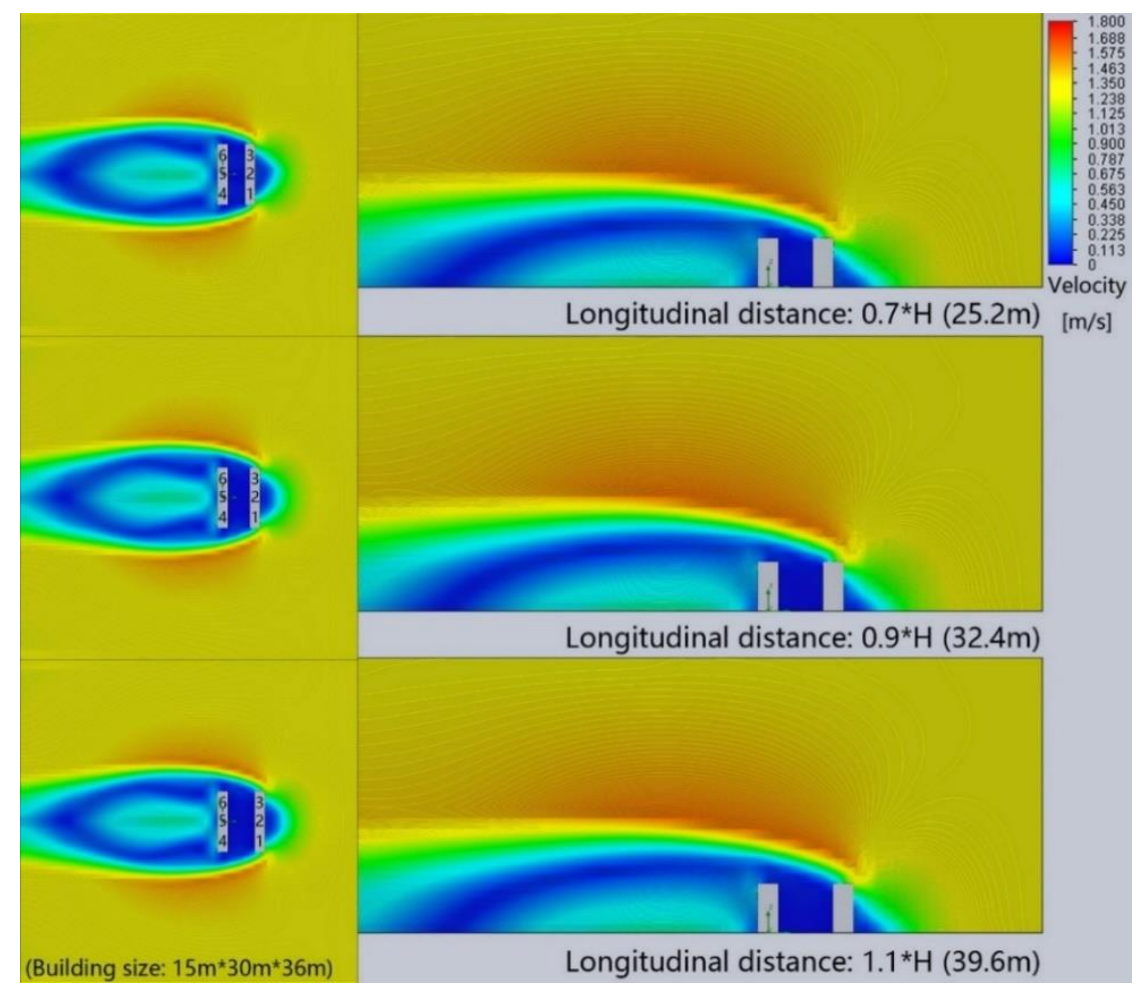

Figure 156. The comparison of wind-velocity magnitudes on horizontal and vertical planes of the building clusters in Group 3 of linear configuration.

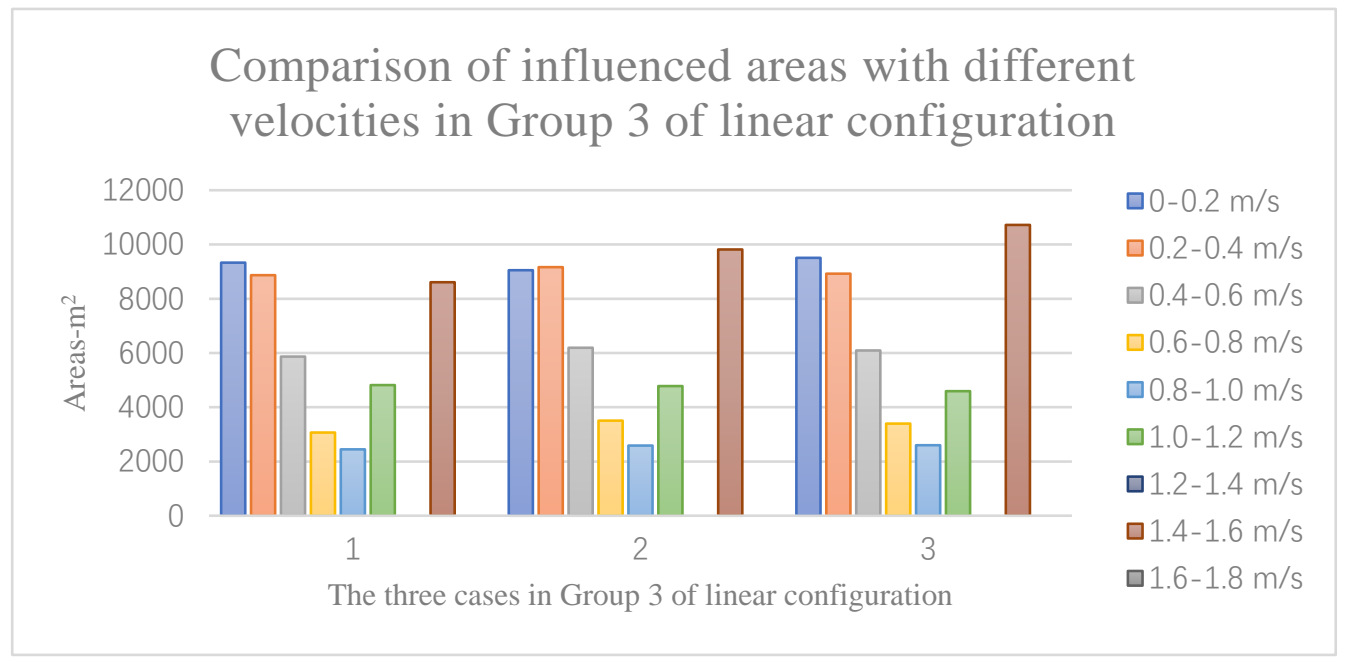

Figure 157. The influenced areas with different velocities in Group 3 of linear configuration.

The changes of influenced areas with different velocities in Group 3 are related to the changes of air pressure and wind flow (Figure 158 and Figure 159). The high-air-pressure and 
low-air-pressure areas of the three cases are almost the same. This is the reason for the marginal differences among the total influenced areas of the three cases. As the longitudinal distance increases, the low-air-pressure area behind the building cluster decreases and becomes shorter slightly, which corresponds with the decreases of the low-velocity area behind the building cluster. The increases of the high-velocity areas are due to the increases of the air-pressure difference.

The vortices are less and smaller as the longitudinal distance increases. This can also explain the slight decreases of the low-velocity area behind the building cluster. But the differences of the three cases are marginal, especially for the vortices between the building rows. These suggests that it is limited to promote the outdoor ventilation promoted by increasing the longitudinal distance.

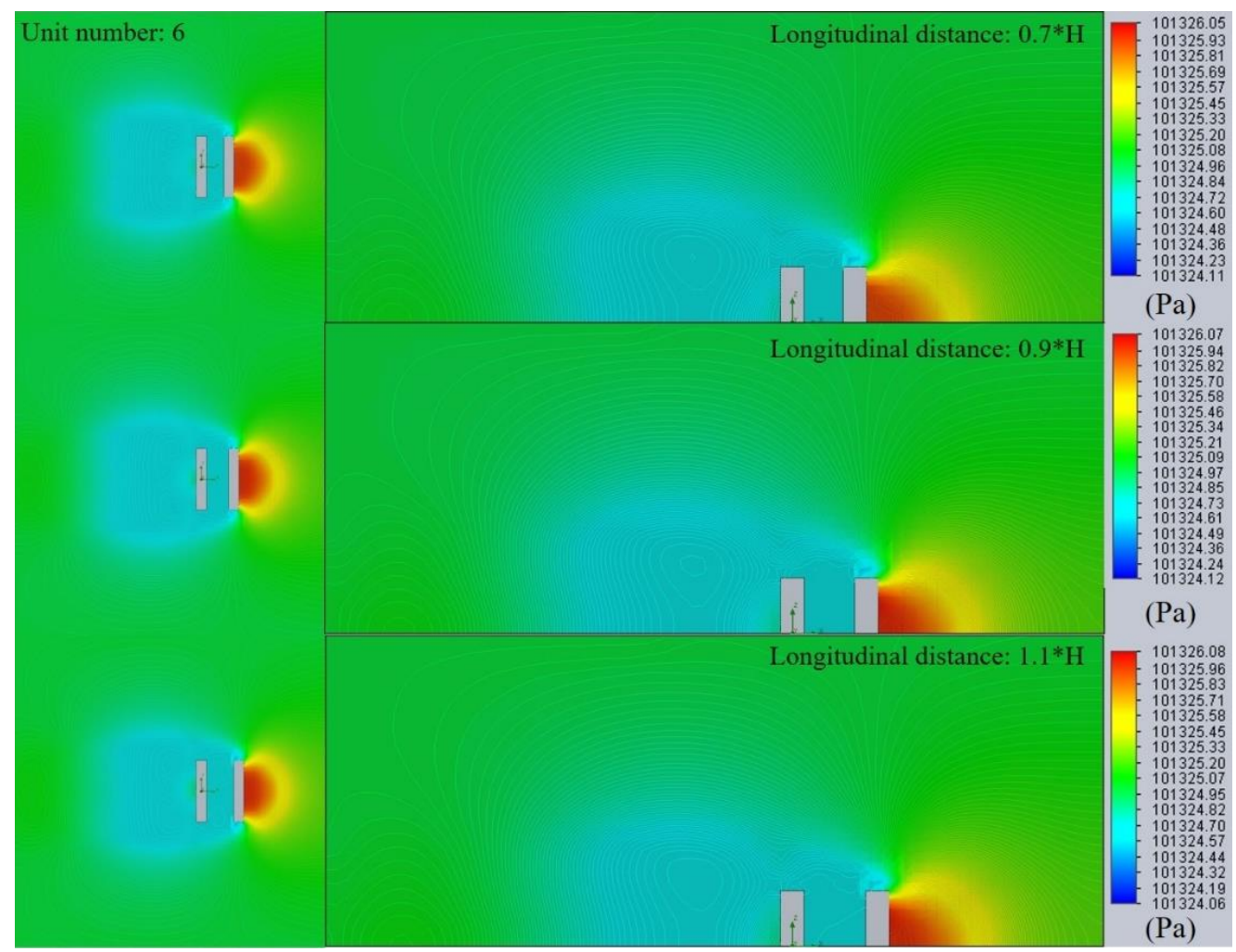

Figure 158. The comparison of air-pressure magnitudes on the horizontal and vertical planes of the building clusters in Group 3 of linear configuration. 


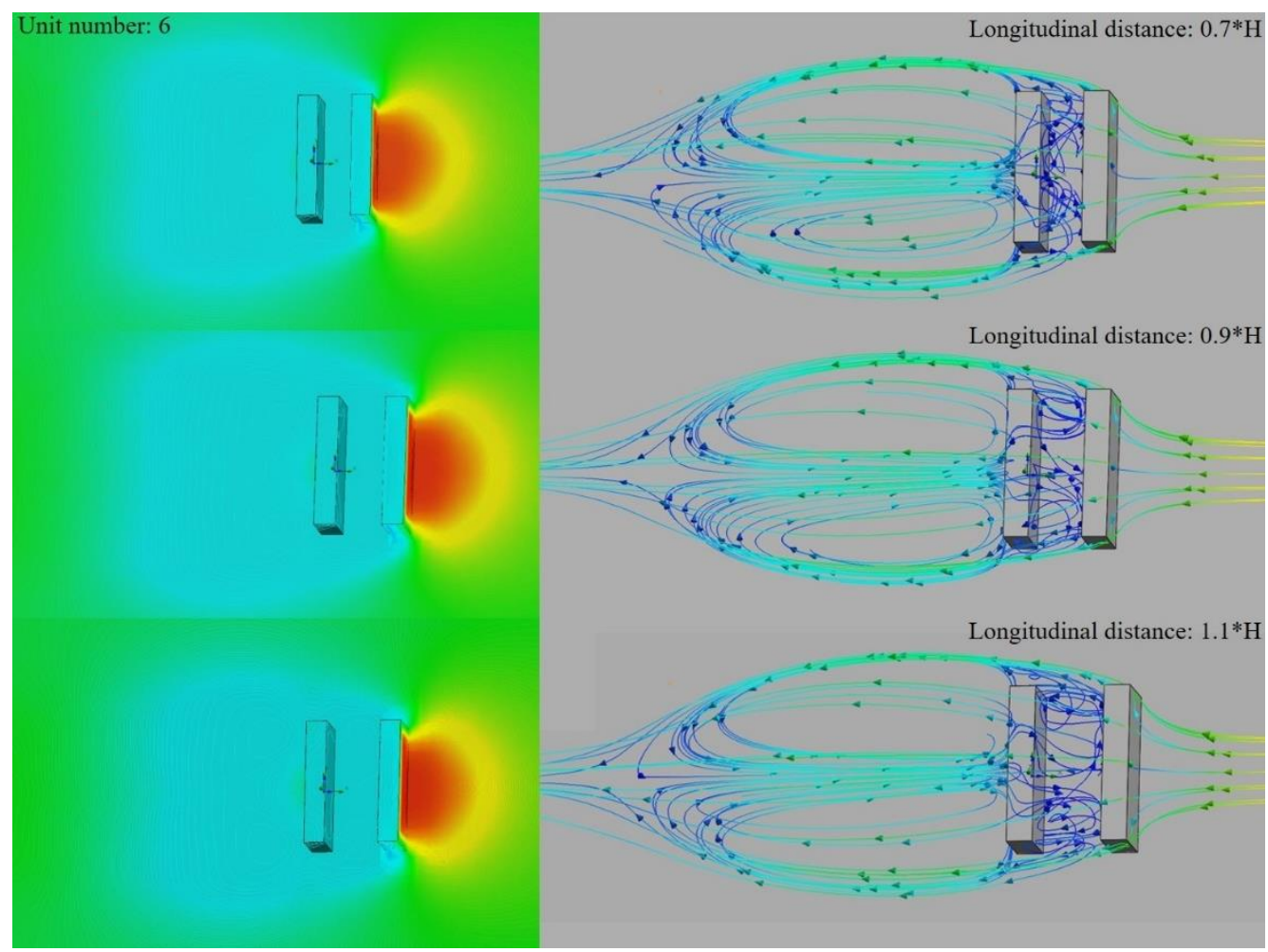

Figure 159. The comparison of wind-flow streamlines (right) and air-pressure magnitudes (left) on the horizontal plane and building surfaces in Group 3 of linear configuration.

\section{(3) Part 3. Influences of building unit numbers and longitudinal distances in 3 rows}

In this part, the influences of different building-unit numbers of Group 4 (longitudinal distance: $0.9 * \mathrm{H}=32.4 \mathrm{~m}$ ) are studied first. The wind-velocity magnitudes on horizontal and vertical planes of the three cases are analyzed (Figure 160 and Figure 161). The results of Group 4 are similar to Group 2. The total influenced area increases constantly as the building-unit number increases. The total influence of Case 1 is the least (influenced area: $41129.65 \mathrm{~m}^{2}$ ); the influence of Case 2 is more than Case 1 (influenced area: $54960.45 \mathrm{~m}^{2}$ ); the influence of Case 3 is the most of all (influenced area: $66288.65 \mathrm{~m}^{2}$ ).

There are low-velocity areas (velocity range: $0-0.4 \mathrm{~m} / \mathrm{s}$ ) on the leeward side of the building cluster and among the building rows. The total low-velocity area is the least in Case 1 $\left(13864.36 \mathrm{~m}^{2}\right)$; the area of Case 2 is more than Case $1\left(18215.52 \mathrm{~m}^{2}\right)$; the area of Case 3 is the most of all $\left(24408.89 \mathrm{~m}^{2}\right)$. Therefore, the low-velocity area increases constantly as the unit number increases (Figure 160 and Figure 161).

There are high-velocity areas (velocity range: $1.4-1.8 \mathrm{~m} / \mathrm{s}$ ) on the lateral sides of the 
building cluster. The high-velocity areas of Case 1 are the least $\left(11807.11 \mathrm{~m}^{2}\right)$; the areas of Case 2 are more than Case $1\left(17927.68 \mathrm{~m}^{2}\right)$; the areas of Case 3 are the largest of all $\left(22337.21 \mathrm{~m}^{2}\right)$. Therefore, the high-velocity areas increase constantly as the unit number increases. The increases are significant, especially for the areas with the velocity range of $1.6-1.8 \mathrm{~m} / \mathrm{s}$ (Figure 160 and Figure 161).

In summary, the influences of the building cluster increase significantly as the unit number increases. Because the area on the horizontal plane and the windward projective area of the entire building cluster are both increased. This is similar to Group 1 and Group 2 .

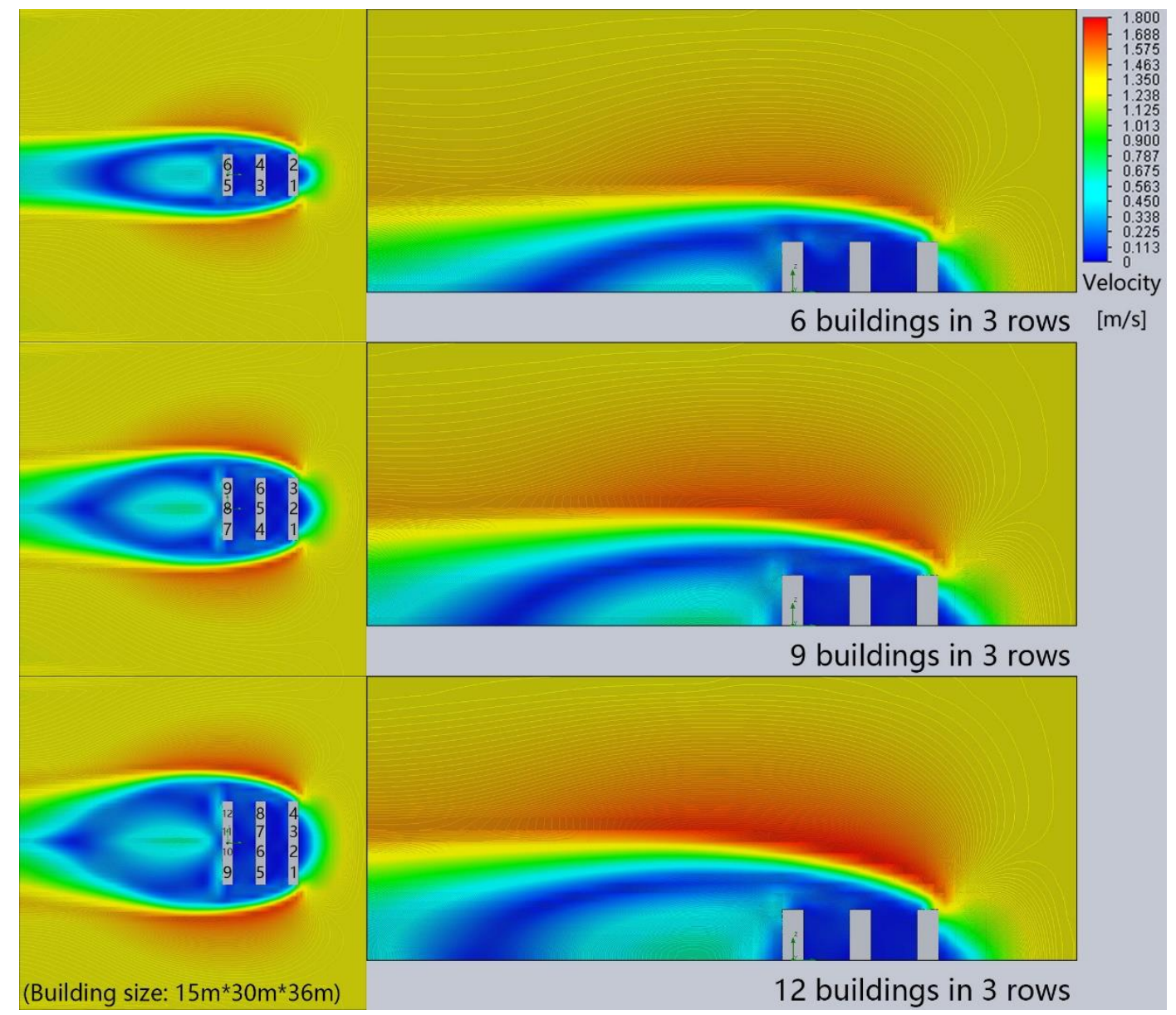

Figure 160. The comparison of wind-velocity magnitudes on horizontal and vertical planes of the building clusters in Group 4 of linear configuration. 


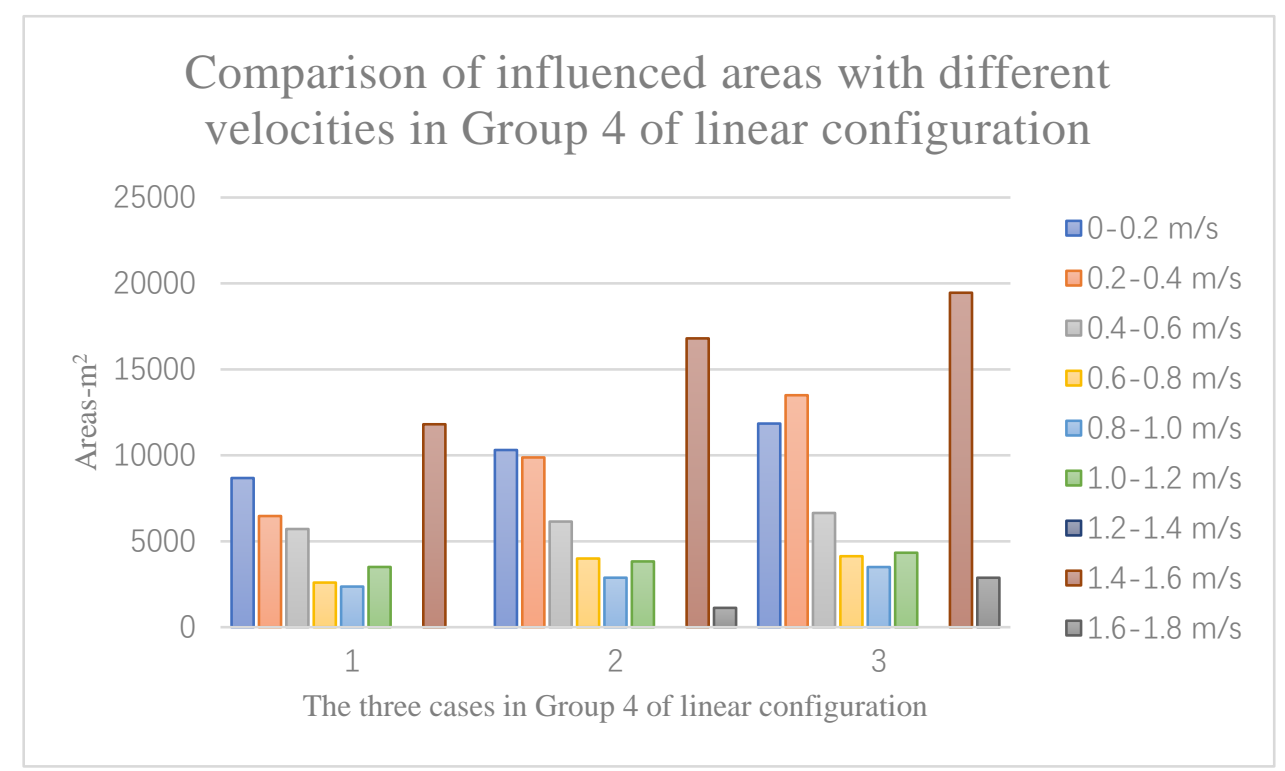

Figure 161. The influenced areas with different velocities in Group 4 of linear configuration.

The changes of influenced areas with different velocities in Group 4 are related to the changes of air pressure and wind flow (Figure 162 and Figure 163). The trends of the air pressure magnitudes and wind flow streamlines are similar to Group 2. As the unit number increases, more winds are obstructed by the larger windward projective area of the building cluster. Therefore, the influenced areas with different air pressures increase, which lead to the increase of the total influence of wind velocities; the air-pressure difference increases, which lead to the increase of high-velocity areas; the vortices among the building rows and behind the building cluster are increased, which lead to the increase of the low-velocity area. 


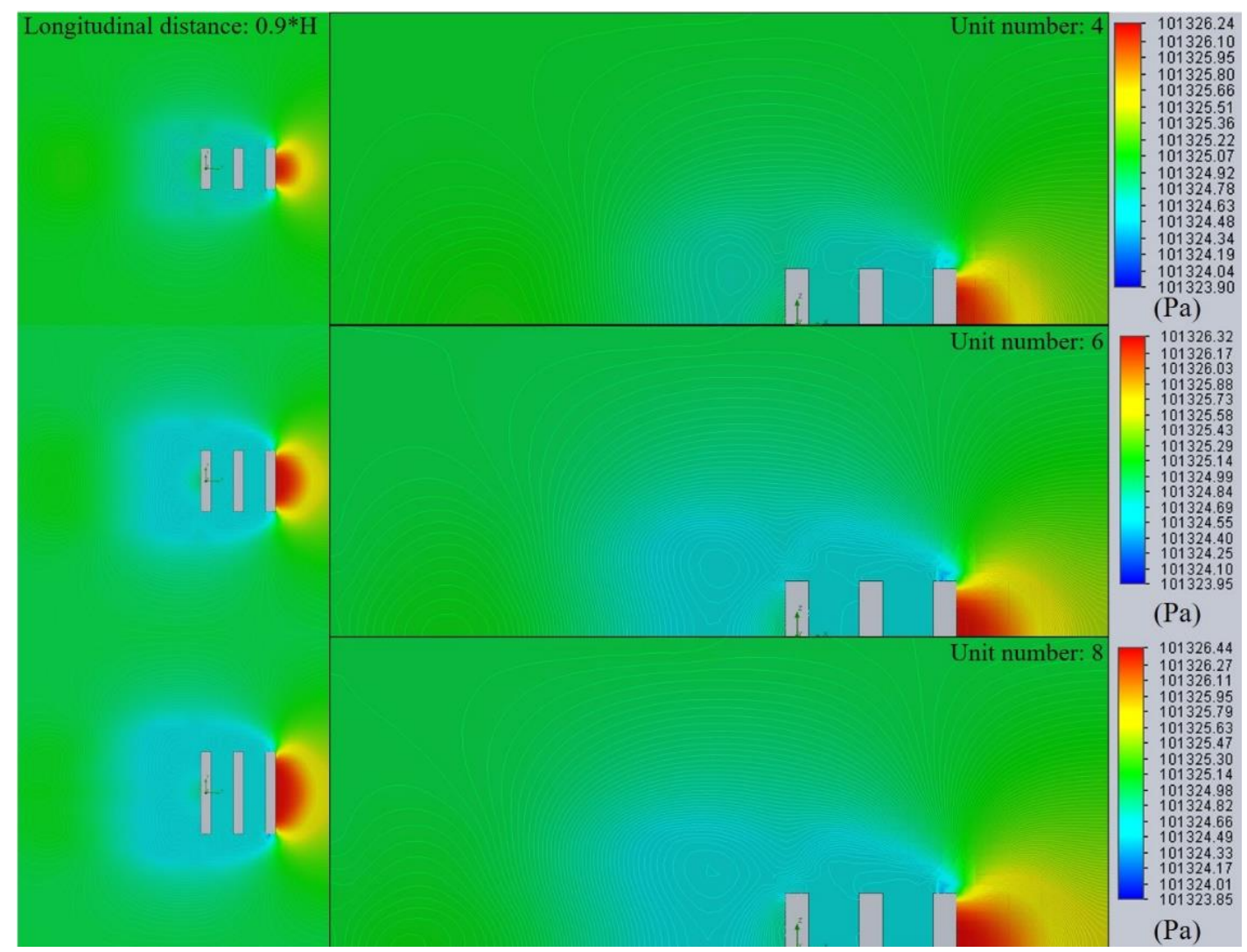

Figure 162. The comparison of air-pressure magnitudes on the horizontal and vertical planes of the building clusters in Group 4 of linear configuration.

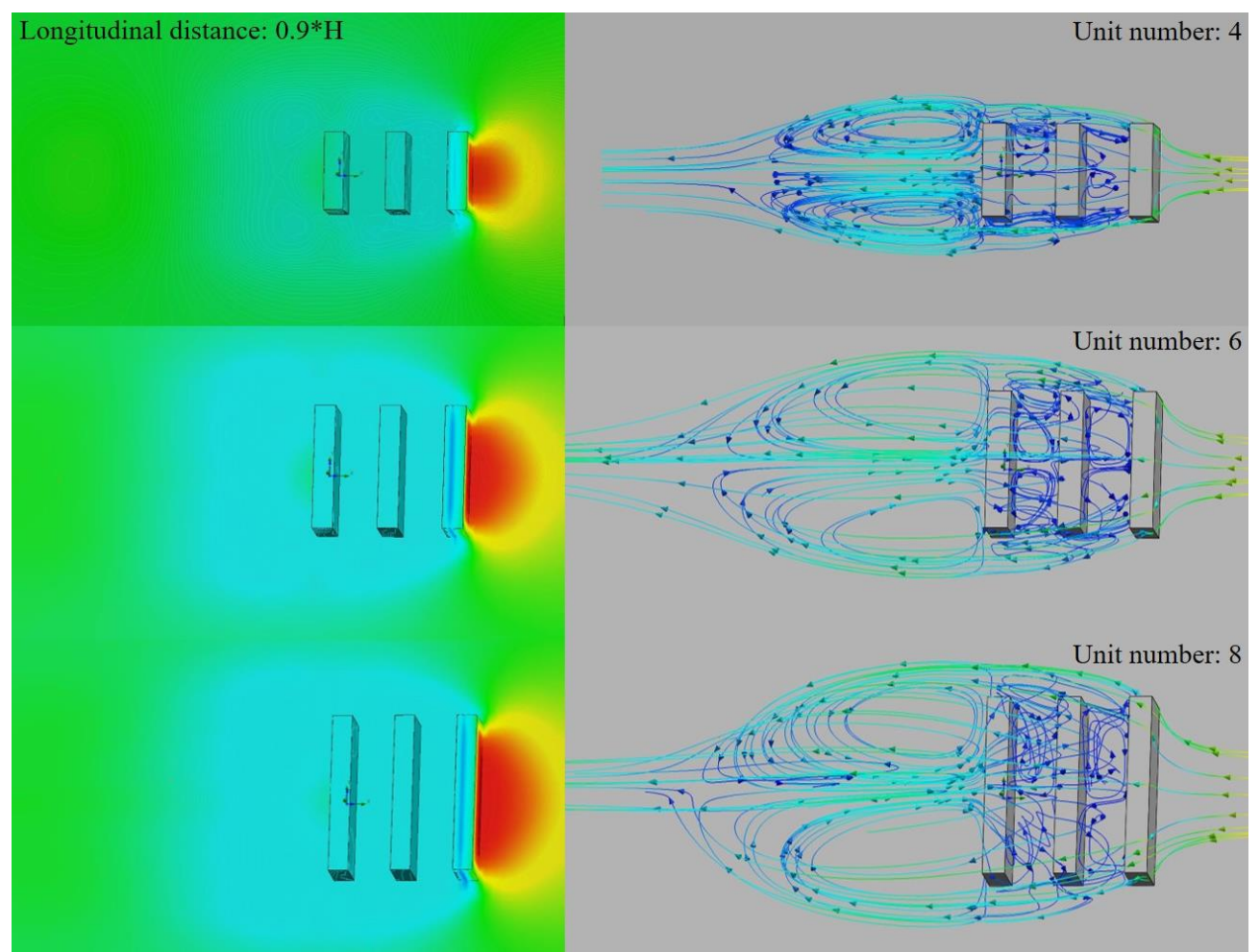

Figure 163. The comparison of wind-flow streamlines (right) and air-pressure magnitudes (left) 
on the horizontal plane and building surfaces in Group 4 of linear configuration.

Second, the influences of different longitudinal distances of Group 5 (unit number: 9) are studied. The wind-velocity magnitudes on horizontal and vertical planes of three cases are analyzed (Figure 164 and Figure 165). The results of Group 5 are similar to Group 3. In Case 1, the total influence of is the least (influenced area: $52249.94 \mathrm{~m}^{2}$ ); in Case 2, the influence is more than Case 1 (influenced area: $54960.45 \mathrm{~m}^{2}$ ); in Case 3, the influence is the most of all (influenced area: $56599.6 \mathrm{~m}^{2}$ ). Therefore, the total influence increases as the longitudinal distance increases. According to the later analysis, the increases are largely due to the increase of the high-velocity area.

The changes of the low-velocity area (velocity range: $0-0.4 \mathrm{~m} / \mathrm{s}$ ) are relatively small in Group 5. The total low-velocity area is the least in Case $1\left(19779.04 \mathrm{~m}^{2}\right)$; the area of Case 2 is the most of all $\left(20185.25 \mathrm{~m}^{2}\right)$; the area of Case 3 is between Case 1 and Case $2\left(20106.79 \mathrm{~m}^{2}\right)$. Therefore, the total low-velocity area is fluctuated as the longitudinal distance increases (Figure 164 and Figure 165). Though the low-velocity area among the building rows increases, the lowvelocity area on the leeward side of the building cluster becomes smaller and shorter.

The high-velocity areas (velocity range: $1.4-1.8 \mathrm{~m} / \mathrm{s}$ ) on the lateral sides of the building clusters are increased constantly as the longitudinal distance increases (Figure 164 and Figure 165). The high-velocity areas are the least in Case $1\left(15431.41 \mathrm{~m}^{2}\right)$; the areas of Case 2 are more than Case $1\left(17927.68 \mathrm{~m}^{2}\right)$; the areas of Case 3 are the largest $\left(20774.47 \mathrm{~m}^{2}\right)$.

In summary, the total influence of the building cluster increases as the longitudinal distance increases. However, the differences among the total influences of the three cases are relatively small. The low-velocity areas of the three cases are relatively close, because the area behind the building cluster decreases and the area among the building rows increases. The lowvelocity area behind the building cluster decreases and becomes shorter. The increases of the high-velocity areas are significant, especially for the areas with the velocity range of $1.6-1.8 \mathrm{~m} / \mathrm{s}$. Therefore, the increase of the longitudinal distance can promote the overall outdoor ventilation. But the promotion of the outdoor ventilation among the building rows is limited. 


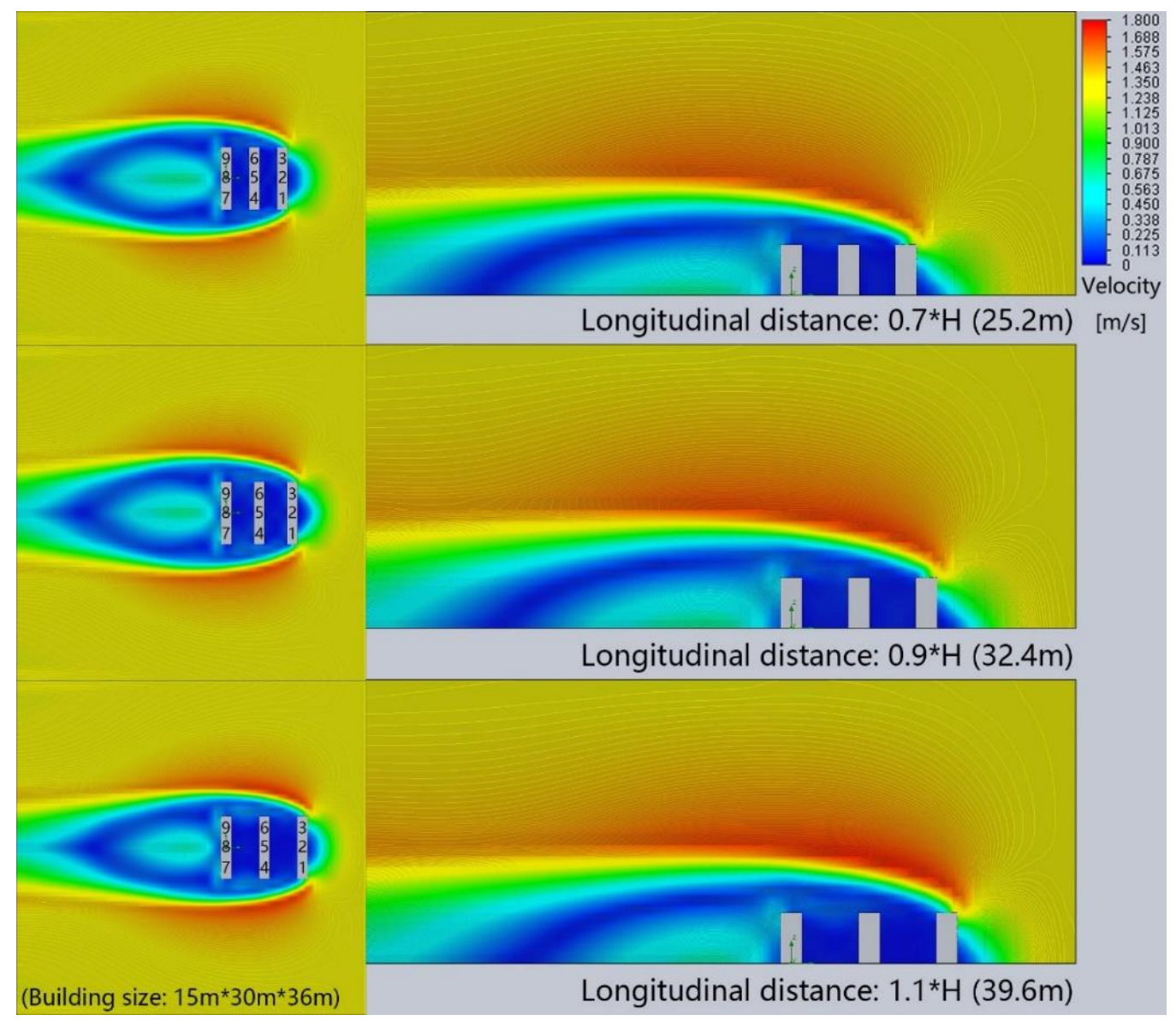

Figure 164. The comparison of wind-velocity magnitudes on horizontal and vertical planes of the building clusters in Group 5 of linear configuration.

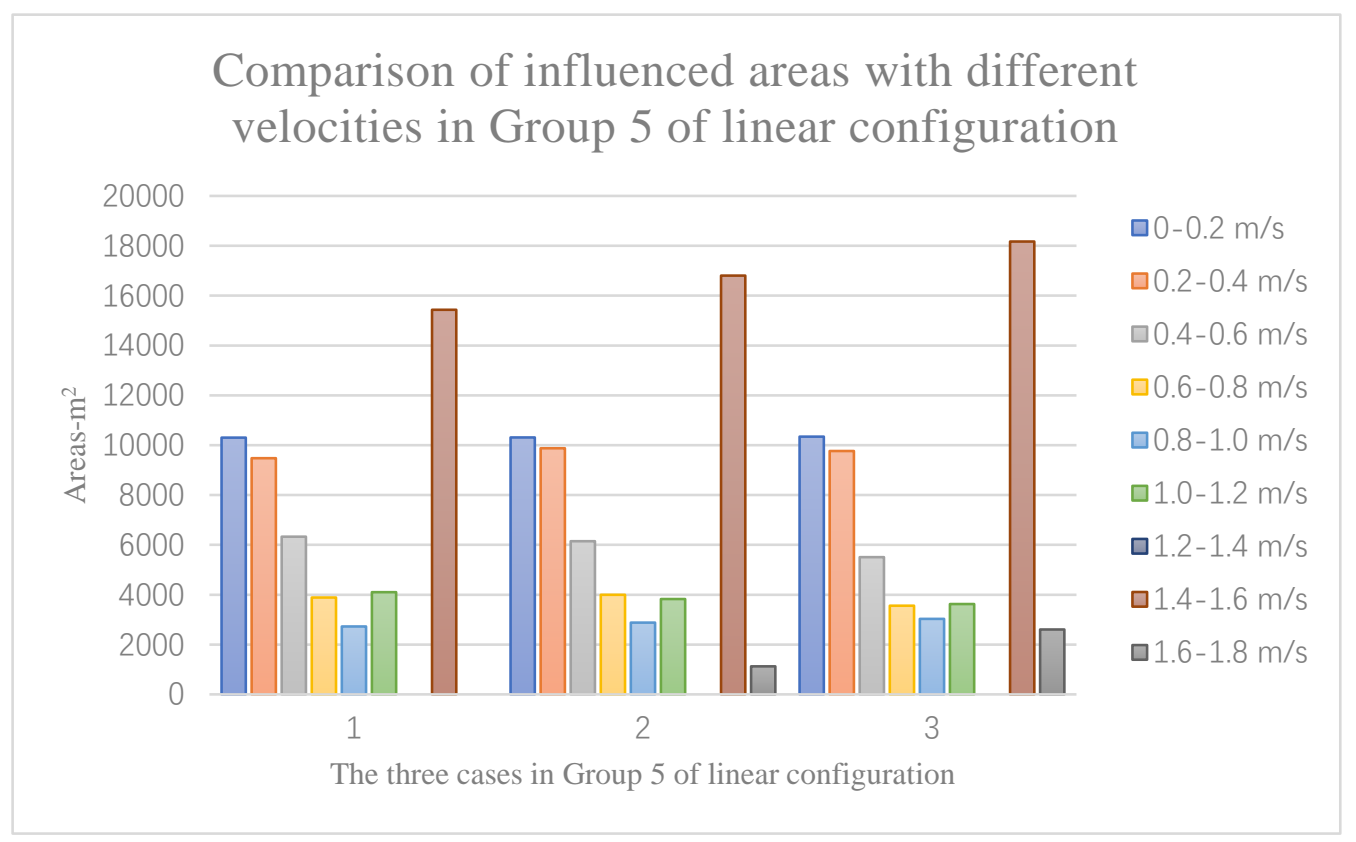

Figure 165. The influenced areas with different velocities in Group 5 of linear configuration. 
The changes of influenced areas with different velocities in Group 5 are related to the changes of air pressure and wind flow (Figure 166 and Figure 167). The influenced areas with different air pressures of the three cases are almost the same. The low-air-pressure area behind the building cluster becomes smaller and shorter as the longitudinal distance increases. This is the reason for the decreases of the low-velocity area behind the building cluster. The increases of the air-pressure difference lead to the increases of the high-velocity areas. The vortices behind the building cluster become smaller and shorter as the longitudinal distance increases. This leads to the decrease of the low-velocity area behind the building cluster. Therefore, the air-pressure magnitudes and wind flow streamlines can prove the overall outdoor ventilation is improved, though the promotion of the outdoor ventilation among the building rows is limited.

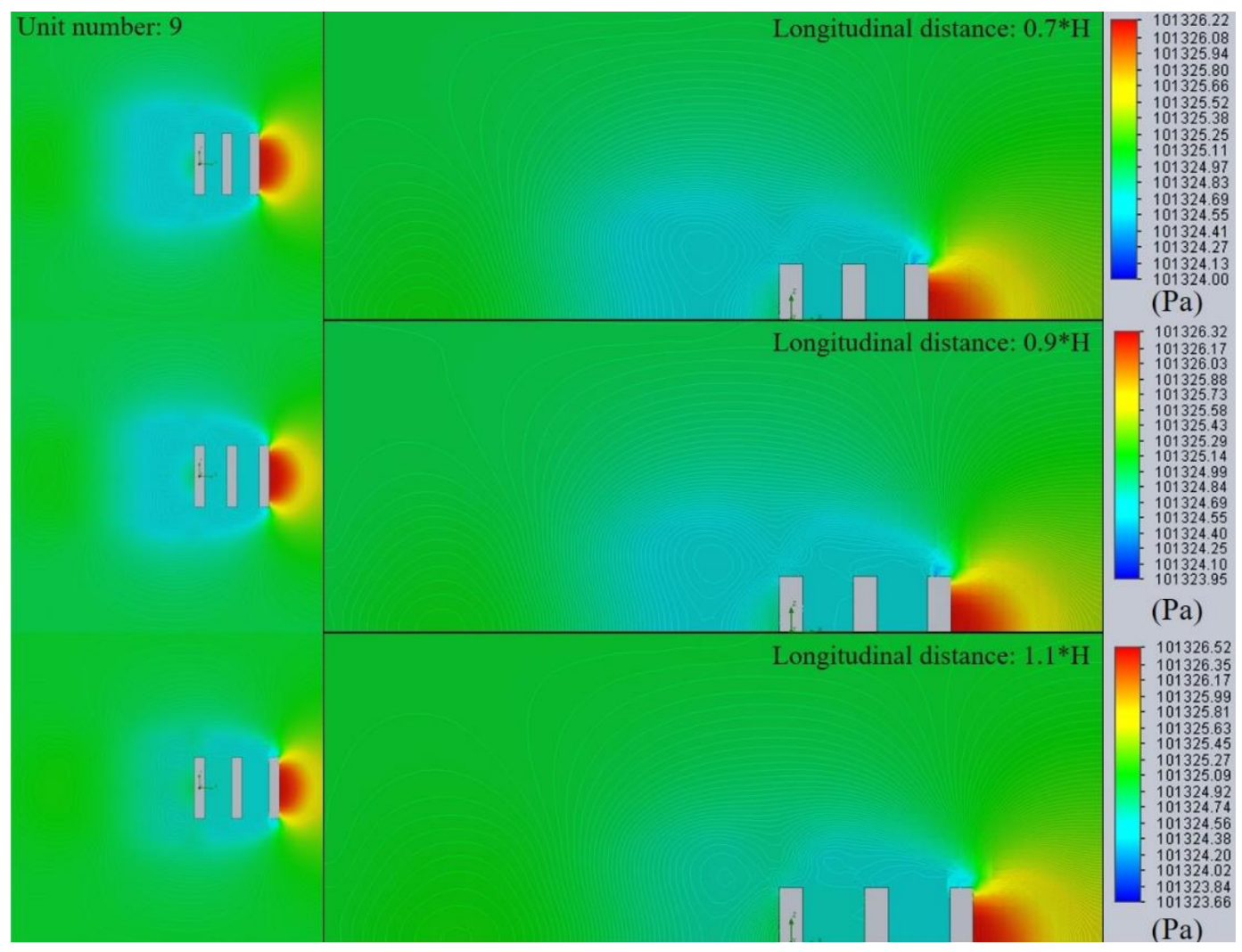

Figure 166. The comparison of air-pressure magnitudes on the horizontal and vertical planes of the building clusters in Group 5 of linear configuration. 


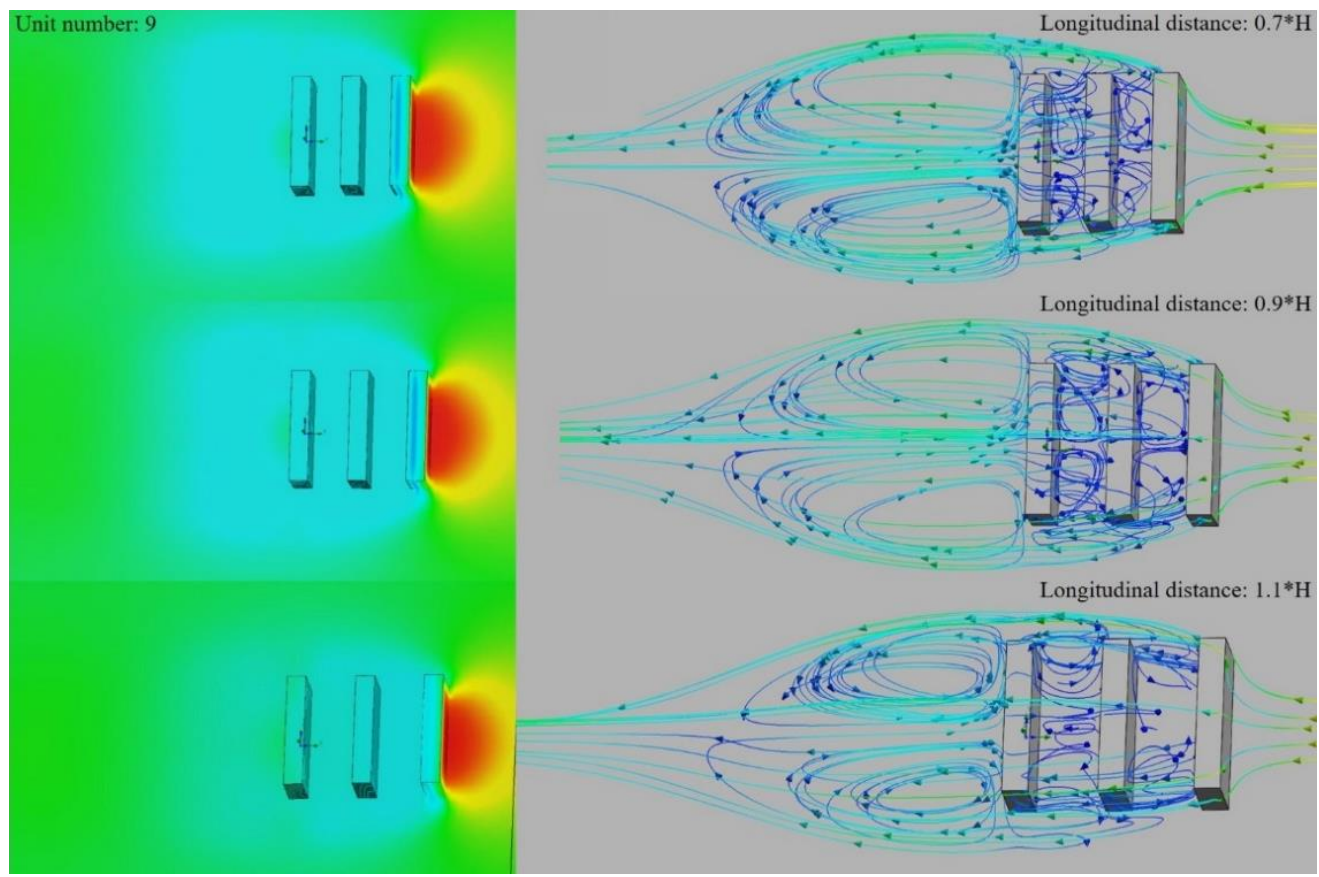

Figure 167. The comparison of wind-flow streamlines (right) and air-pressure magnitudes (left) on the horizontal plane and building surfaces in Group 5 of linear configuration.

\section{(4) Part 4. Influences of staggered distances among 3 rows}

In this part, the influences of different staggered distances of Group 6 are studied (unit number: 9; longitudinal distance: $0.9 * \mathrm{H}=32.4 \mathrm{~m})$. The wind-velocity magnitudes on horizontal and vertical planes of the three cases are analyzed. The results of Group 6 are similar to Group 14 of the scattered configuration. The total influenced area of the building cluster increases constantly as the staggered distance increases (staggered distance: 10m, 20m and 30m) (Figure 168 and Figure 169). In Case 1, the total influenced area is the least (influenced area: $53571.49 \mathrm{~m}^{2}$ ); in Case 2, the area is more than Case 1 (influenced area: $59782.80 \mathrm{~m}^{2}$ ); in Case 3, the area is the most of all (influenced area: $67090.10 \mathrm{~m}^{2}$ ). Because the width of the building cluster increases as the staggered distance increases, the increase of the windward projective area of the building cluster is the reason for the increase of the total influenced area.

The low-velocity area (velocity range: $0-0.4 \mathrm{~m} / \mathrm{s}$ ) increases constantly as the staggered distance increases (Figure 168 and Figure 169). The increase of low-velocity area is significant, which takes the major part of the increase of total influence. In Case 1, the low-velocity area is the least $\left(19648.57 \mathrm{~m}^{2}\right)$; in Case 2, the area is much more than Case $1\left(23300.25 \mathrm{~m}^{2}\right)$; in Case 3, the area is the most of all $\left(39713.99 \mathrm{~m}^{2}\right)$. The low-velocity area is generated among the building 
rows and behind the building cluster. Though the entire low-velocity area increases as the staggered distance increases, the wind velocities are increased (from the range of $0-0.2 \mathrm{~m} / \mathrm{s}$ to the range of $0.2-0.4 \mathrm{~m} / \mathrm{s}$ ) in the low-velocity area among the building rows. The velocity magnitudes show that the area with the velocity range of $0-0.2 \mathrm{~m} / \mathrm{s}$ is reduced among the building rows; and the area with the velocity range of $0.2-0.4 \mathrm{~m} / \mathrm{s}$ is increased. These suggests the outdoor ventilation among the building rows can be improved by increasing the staggered distance.

The high-velocity areas (velocity range: $1.4-1.8 \mathrm{~m} / \mathrm{s}$ ) on the lateral sides of the building cluster are fluctuated as the staggered distance increases (Figure 168 and Figure 169). In Case 1, the high-velocity areas are between Case 2 and Case $3\left(17680.59 \mathrm{~m}^{2}\right)$; in Case 2, the areas are the most $\left(18279.71 \mathrm{~m}^{2}\right)$; in Case 3, the areas are the least of all $\left(11227.05 \mathrm{~m}^{2}\right)$. The areas of Case 1 and Case 2 are close. There is a significant decrease in Case 3. Because more winds are obstructed by the larger windward surfaces of the second and third building rows on the lateral side of the building cluster. 


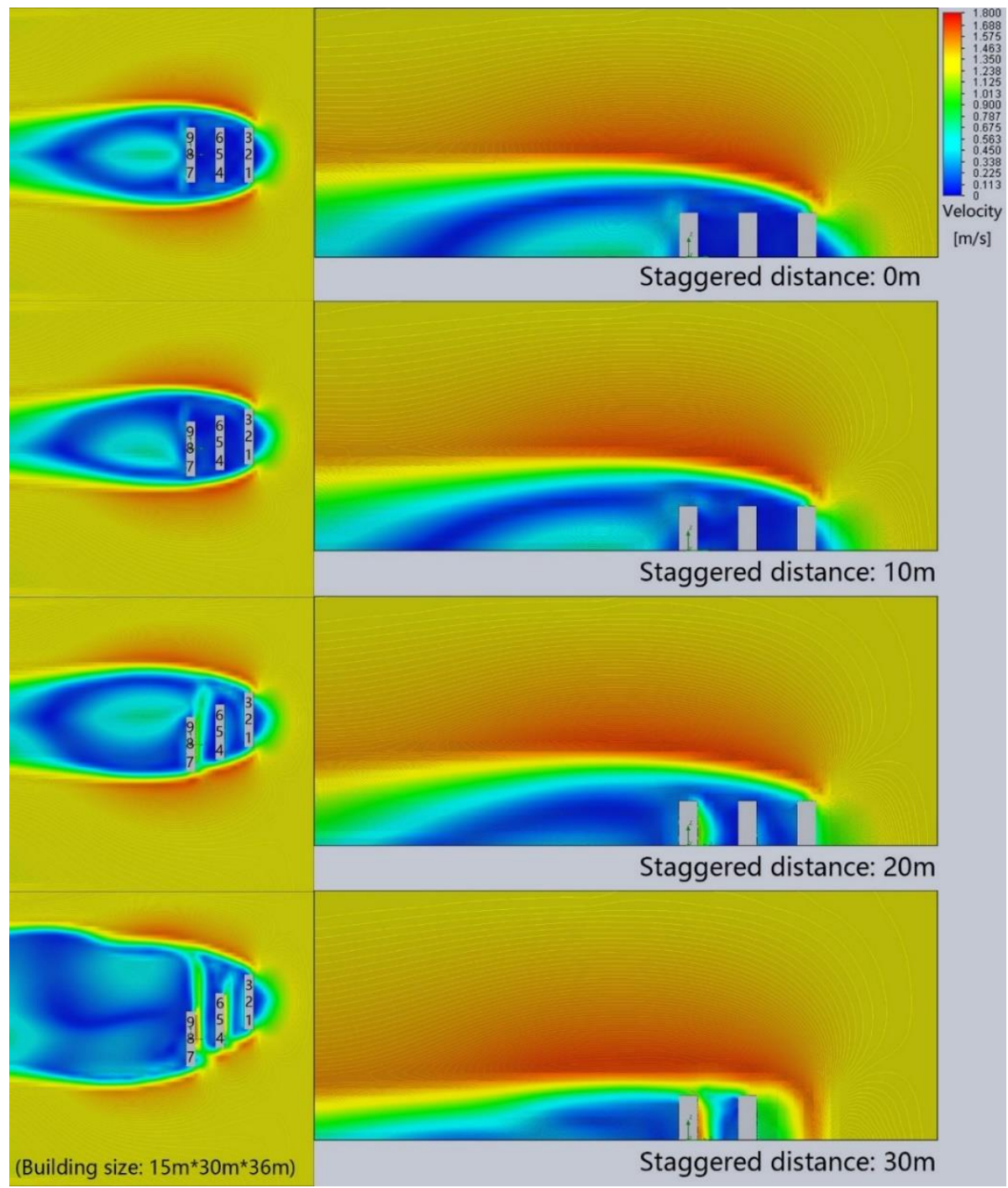

Figure 168. The comparison of wind-velocity magnitudes on horizontal and vertical planes of the building clusters in Group 6 of linear configuration.

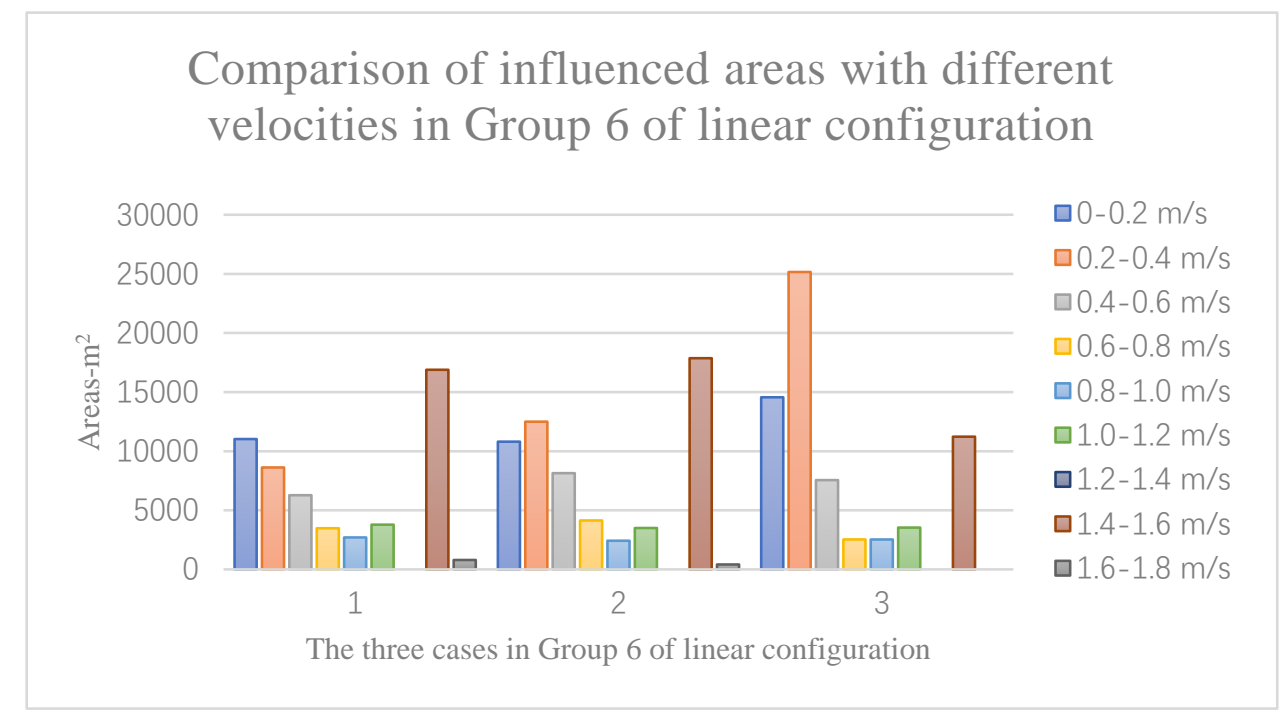

Figure 169. The influenced areas with different velocities in Group 6 of linear configuration. 
The changes of influenced areas with different velocities in Group 6 are related to the changes of air pressure and wind flow (Figure 170 and Figure 171). The influenced areas with different air pressures are increased as the staggered distance increases. This is the reason for the increase of influenced areas with different velocities. Especially for the increase of the lowvelocity area behind the building cluster, it is due to the increase of the low-air-pressure area behind the building cluster. However, the air pressures among the building rows are increased as the staggered distance increases. The vortices among the building rows are also reduced. This increases the velocities in the low-velocity area among the building rows. The decrease of the air-pressure difference on the lateral side of the building cluster reduces the high-velocity area. The reduction of the vortices behind the building cluster corresponds to the increases of velocities in the low-velocity area. Therefore, the air-pressure magnitudes and wind-flow streamlines also suggest that the increase of the staggered distance can improve the overall outdoor ventilation, especially for the low-velocity area among the building rows.

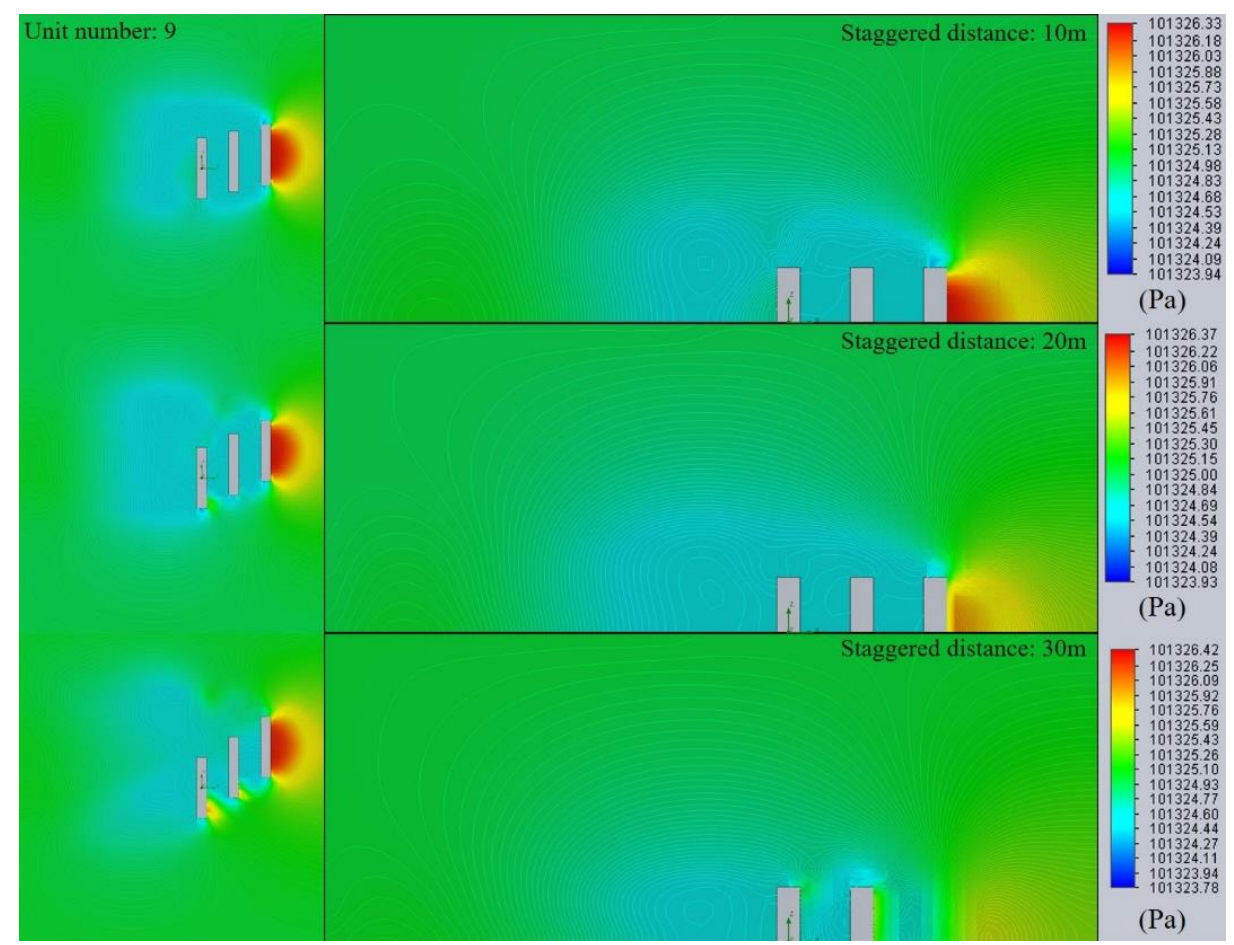

Figure 170. The comparison of air-pressure magnitudes on the horizontal and vertical planes of the building clusters in Group 6 of linear configuration. 


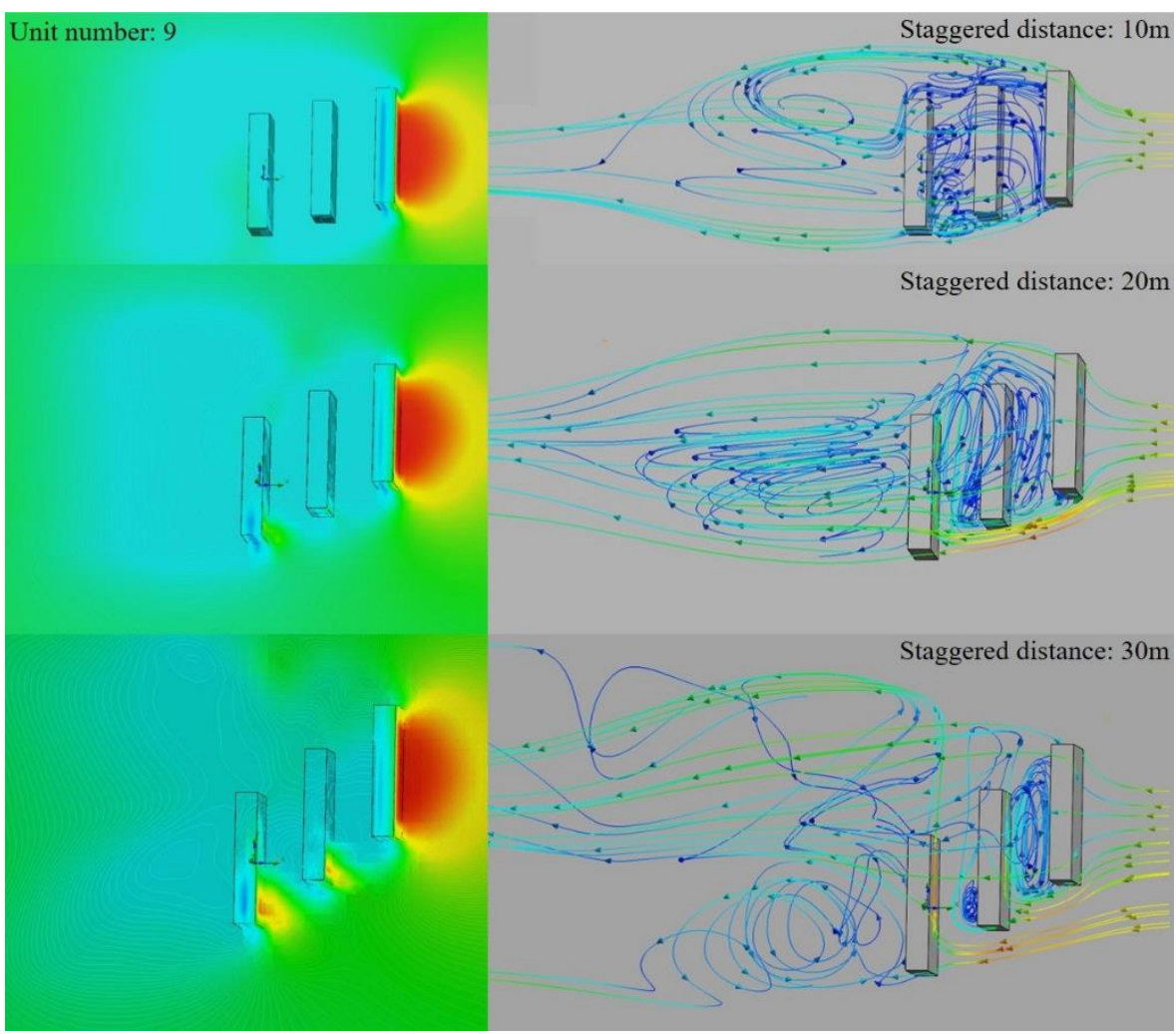

Figure 171. The comparison of wind-flow streamlines (right) and air-pressure magnitudes (left) on the horizontal plane and building surfaces in Group 6 of linear configuration.

\subsection{Curvilinear configuration}

This section aims to study the relationship between the influence on wind environments and the building variable of curvilinear configuration. The study focuses on the central angle of the arc, which is a variable related to the curvature of the curvilinear building row (Figure 172). Because the arc length of the curvilinear building row is fixed in the study, the curvature can be determined by the central angle. In the first and second parts, the influences of central angles are studied for a curvilinear-configuration building cluster. In the first part, the building units are arranged in a curvilinear building row with the convex surface on the windward side. In the second part, the building units are arranged in a curvilinear building row with the concave surface on the windward side. In this section, the building variables setup of the two parts are shown first. Then results and analysis of the two parts are presented. 


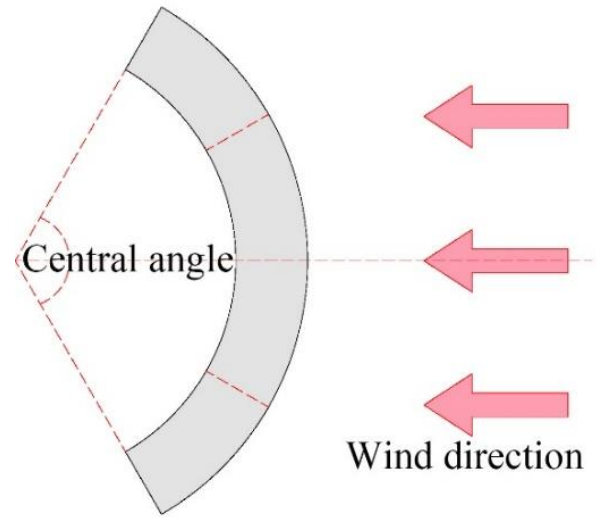

The convex surface on windward side

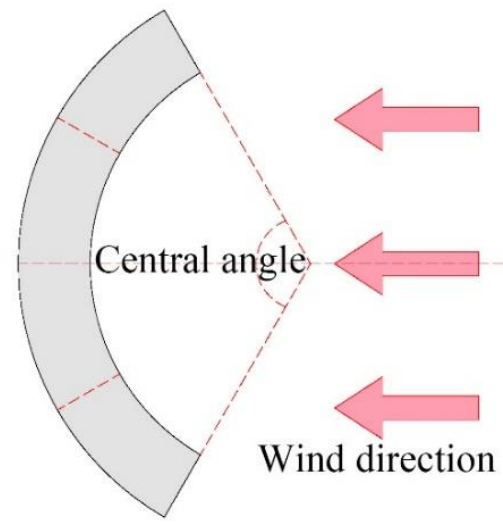

The concave surface on windward side

Figure 172. The building variables of curvilinear configuration.

\subsubsection{Building-variable setup of curvilinear configuration}

In the first part, nine cases with different central angles are set up in Group 1. According to the summary in the parametric design chapter, common central angles in the range of 10-90degree are used in the study; building units are arranged in a curvilinear building row with the arc length of $40 \mathrm{~m}$, width of $10 \mathrm{~m}$ and height of $36 \mathrm{~m}$; the convex surface of the building row is on the windward side. In the second part, variables of nine cases in Group 2 are the same as Group 1. The difference is that the concave surface of the building row is on the windward side. The specific variables of the two groups are presented in the following table (Table 43.).

Table 43. The cases in Group 1 and Group 2 of curvilinear configuration.

\begin{tabular}{|l|l|}
\hline Central angles & Row numbers \\
\hline 10 degree & 1 \\
\hline 20 degree & 1 \\
\hline 30 degree & 1 \\
\hline 40 degree & 1 \\
\hline 50 degree & 1 \\
\hline 60 degree & 1 \\
\hline 70 degree & 1 \\
\hline
\end{tabular}




\begin{tabular}{|l|l|}
\hline 80 degree & 1 \\
\hline 90 degree & 1 \\
\hline The curvilinear building rows of Group 1 and Group 2 are all set \\
up with the arc length of $40 \mathrm{~m}$, width of $10 \mathrm{~m}$ and height of $36 \mathrm{~m}$. \\
In Group 1, the convex surface of the building row is on the \\
windward side; in Group 2, the concave surface of the building \\
row is on the windward side.
\end{tabular}

\subsubsection{Results and analysis of curvilinear configuration}

The CFD simulation results of curvilinear configuration are analyzed in this subsection. Influences on wind environments are compared based on wind-velocity magnitudes. The mechanisms are explained using the air-pressure magnitudes and wind-flow streamlines. In Part 1 and Part 2, the relationship between influences and central angles of a curvilinear building row are studied. In Part 1, the concave surface of the building row is on the windward side; in Part 2, the convex surface of the building row is on the windward side.

\section{(1) Part 1. Influences of the central angle of the curvilinear building row with the convex surface on the windward side in Group 1}

This part studies the influences of different central angles of Group 1. The wind-velocity magnitudes on horizontal and vertical planes of nine cases are analyzed (Figure 173 and Figure 174). In general, the total influence of the curvilinear building row is increased as the central angle increases. The nine cases are close and the results fluctuate irregularly (Figure 174). In Case 1, the total influence of the curvilinear building row is the least (influenced areas: 34302.27m2). The influence increases with some fluctuations from Case 1 to Case 8 . In Case 8 , the total influenced area is the largest of all (influenced areas: $40414.13 \mathrm{~m} 2$ ). The influenced area of Case 9 becomes less than Case 8 (influenced areas: $37321.25 \mathrm{~m} 2$ ).

There is a low-velocity area (velocity range: $0.00-0.36 \mathrm{~m} / \mathrm{s}$ ) on the leeward side of the building row. The low-velocity area decreases constantly as the central angle increases (Figure 
175, Figure 176, Figure 177 and Figure 178). The low-velocity area of Case 1 is the largest $\left(12980.82 \mathrm{~m}^{2}\right)$. From Case 2 to Case 8 , the area decreases gradually. The low-velocity area of Case 9 is the least of all $\left(10344.25 \mathrm{~m}^{2}\right)$.

There are high-velocity areas (velocity range: $1.42-1.60 \mathrm{~m} / \mathrm{s}$ ) on the lateral sides of the building clusters in the nine cases. In General, the high-velocity areas increase as the central angle increases (Figure 173, Figure 174 and Figure 179). The high-velocity areas of Case 1 are the smallest $\left(1700.01 \mathrm{~m}^{2}\right)$. From Case 2 to Case 8, the areas increase gradually. The areas of Case 8 are the largest of all $\left(3654.01 \mathrm{~m}^{2}\right)$. The areas of Case 9 are decreased (influenced areas: $\left.3281.11 \mathrm{~m}^{2}\right)$.

In summary, as the central angle increases, the total influenced area and high-velocity areas increase; and the low-velocity area is decreased. The increases of the total influenced area are mainly due to the increases of the high-velocity areas. In addition, the velocity magnitudes show that the other areas with the velocities over $0.36 \mathrm{~m} / \mathrm{s}$ are increased (Figure 178 and Figure 179). This suggests the overall wind velocities are increased around the building cluster. Therefore, the increase of the central angle promotes the wind flow and improves the outdoor ventilation. 


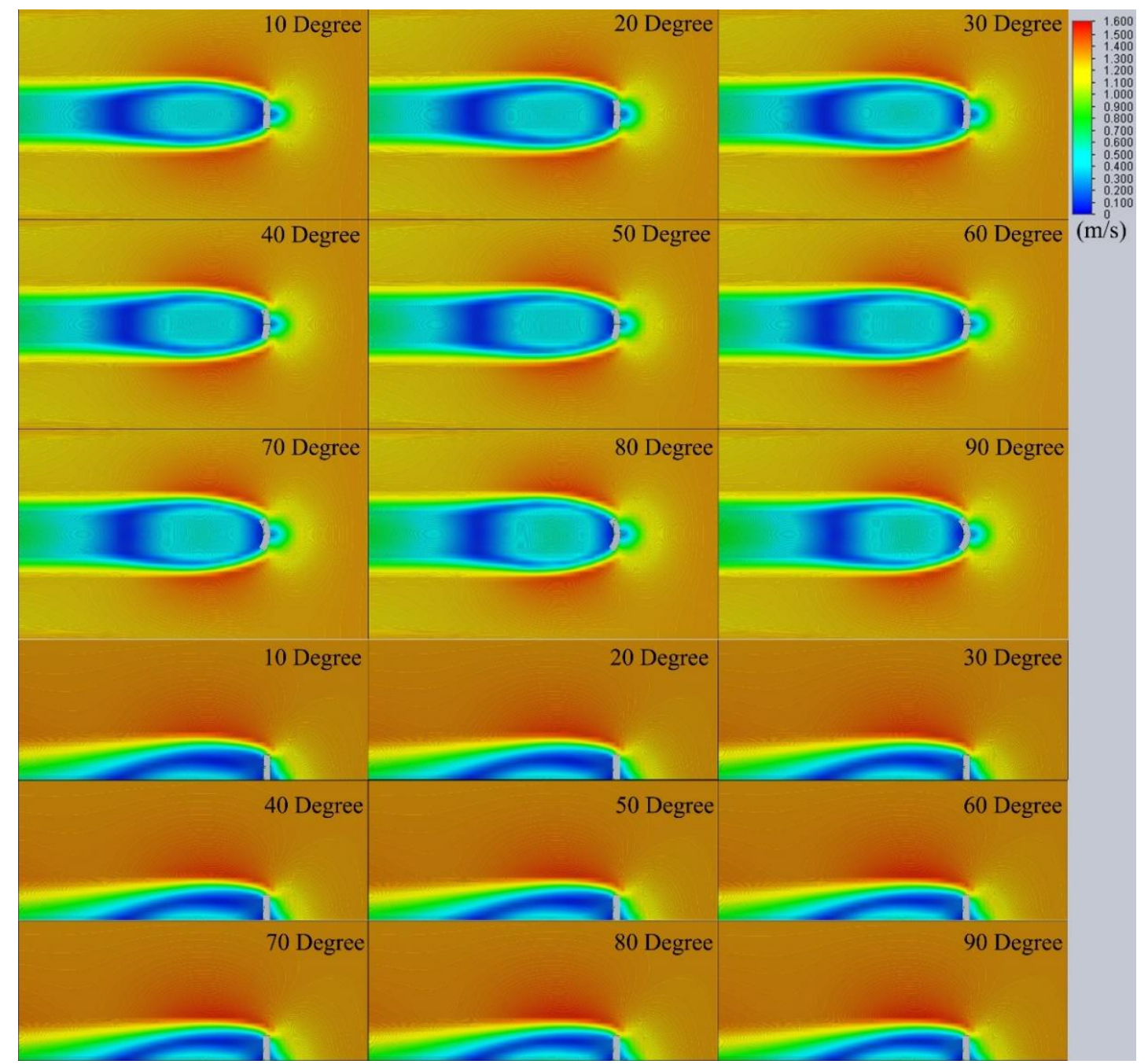

Figure 173. The comparison of wind-velocity magnitudes on horizontal and vertical planes of the building clusters in Group 1 of curvilinear configuration.

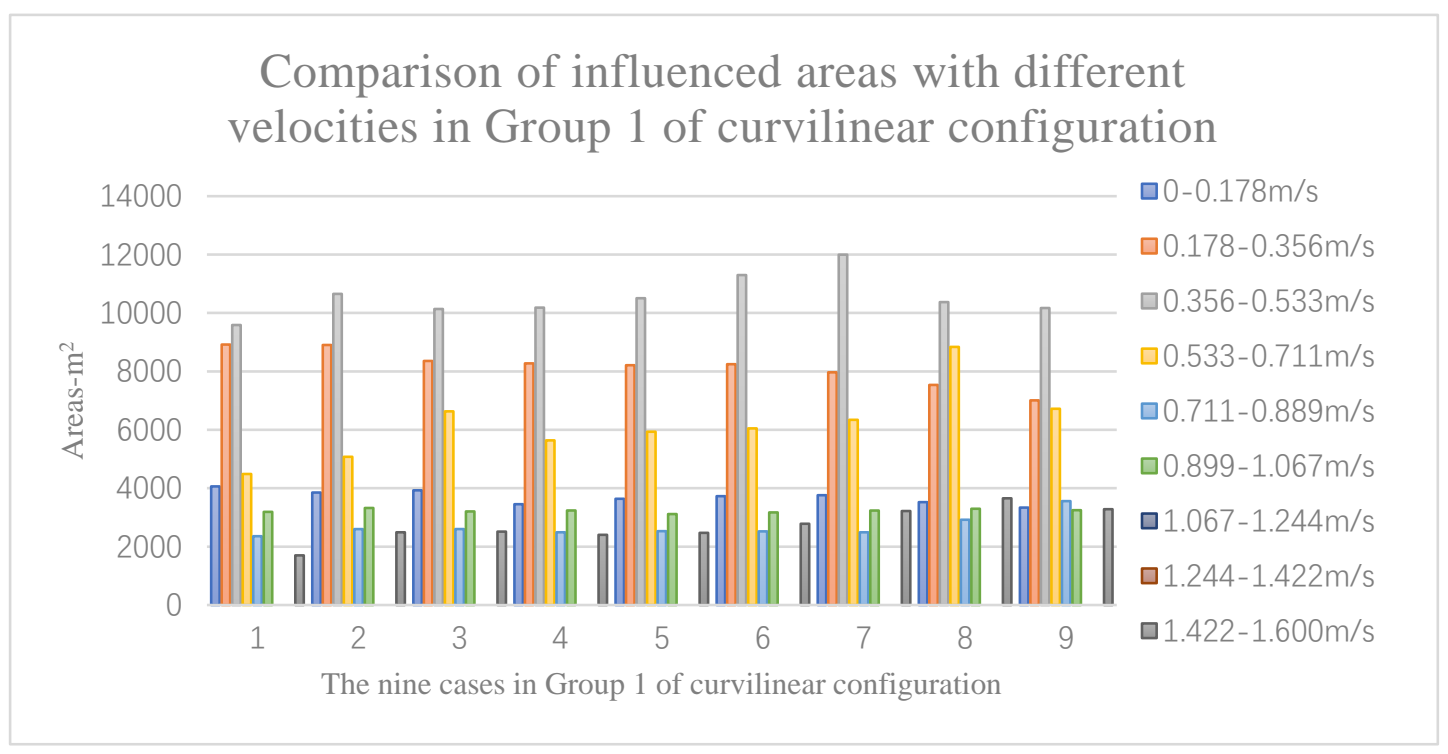

Figure 174. The influenced areas with different velocities in Group 1 of curvilinear 
configuration.

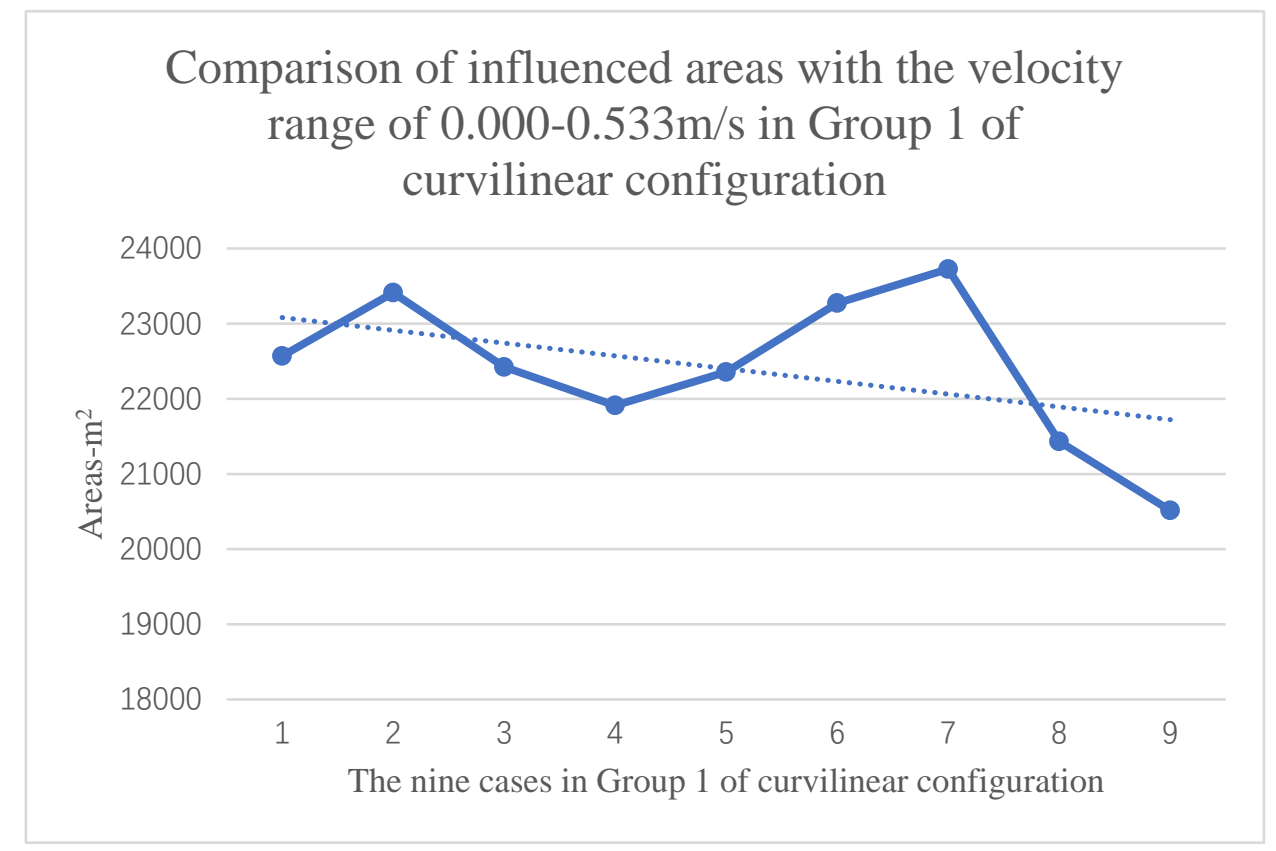

Figure 175 . The influenced areas with the velocity range of $0.000-0.533 \mathrm{~m} / \mathrm{s}$ in Group 1 of curvilinear configuration.

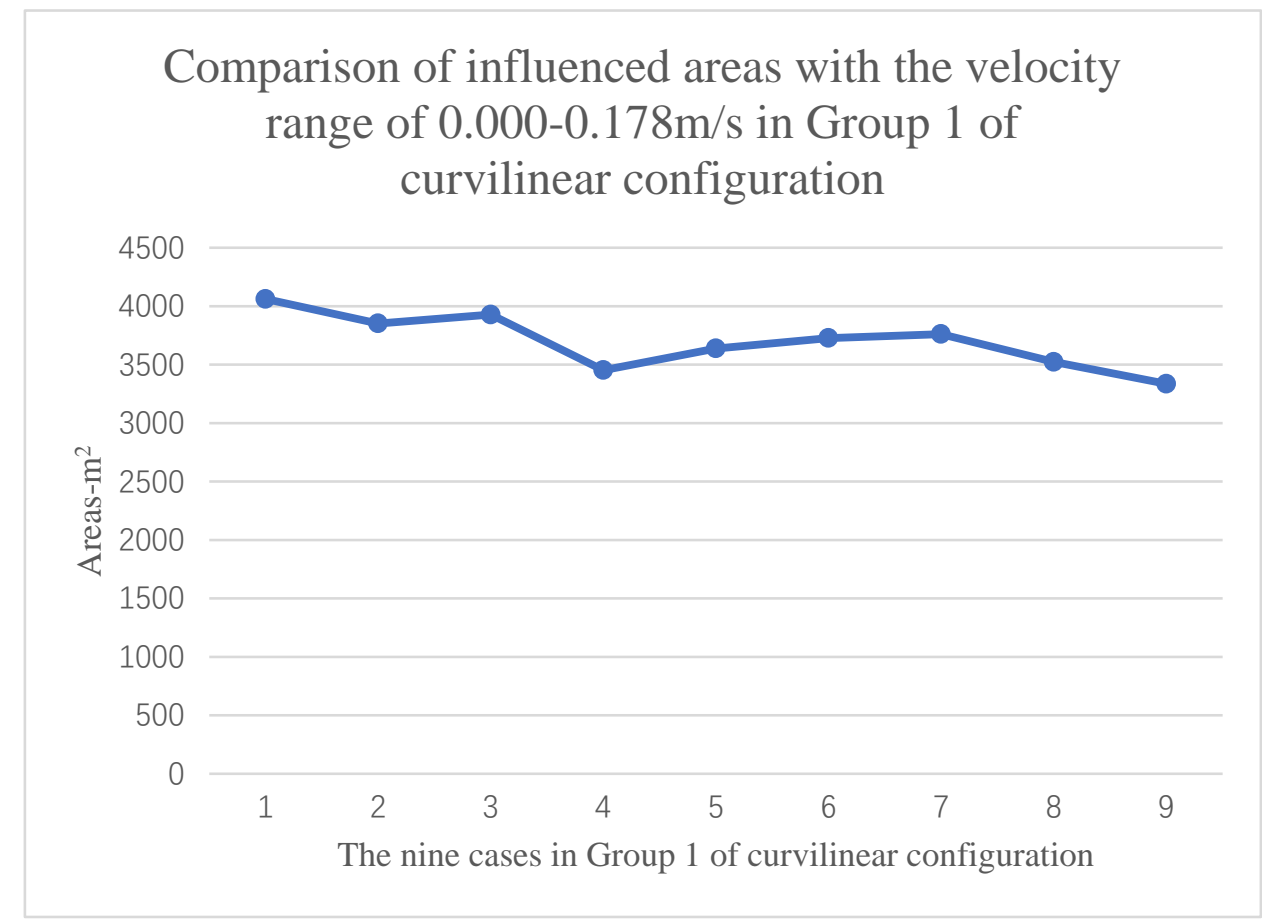

Figure 176. The influenced areas with the velocity range of $0.000-0.178 \mathrm{~m} / \mathrm{s}$ in Group 1 of curvilinear configuration. 


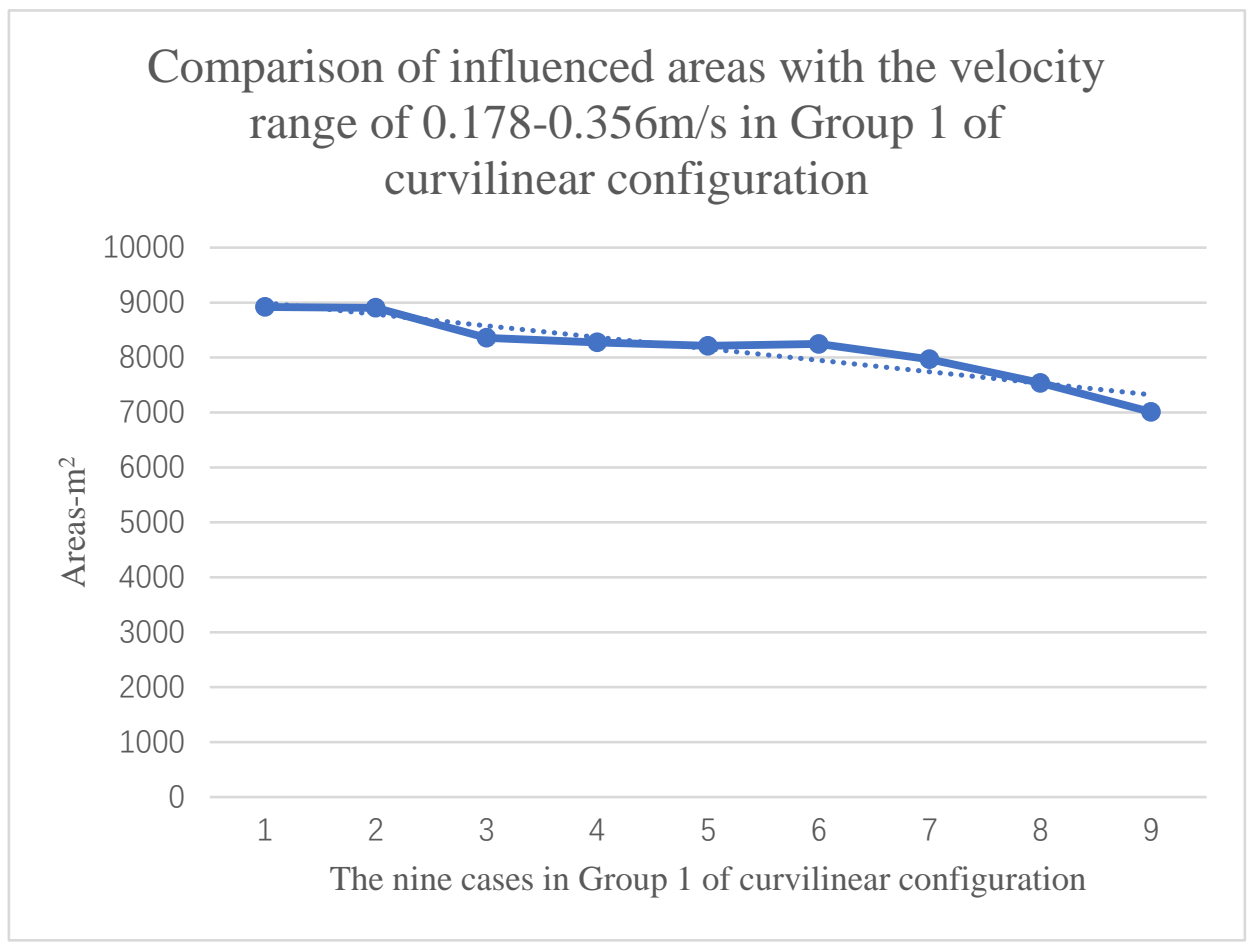

Figure 177. The influenced areas with the velocity range of $0.178-0.356 \mathrm{~m} / \mathrm{s}$ in Group 1 of curvilinear configuration.

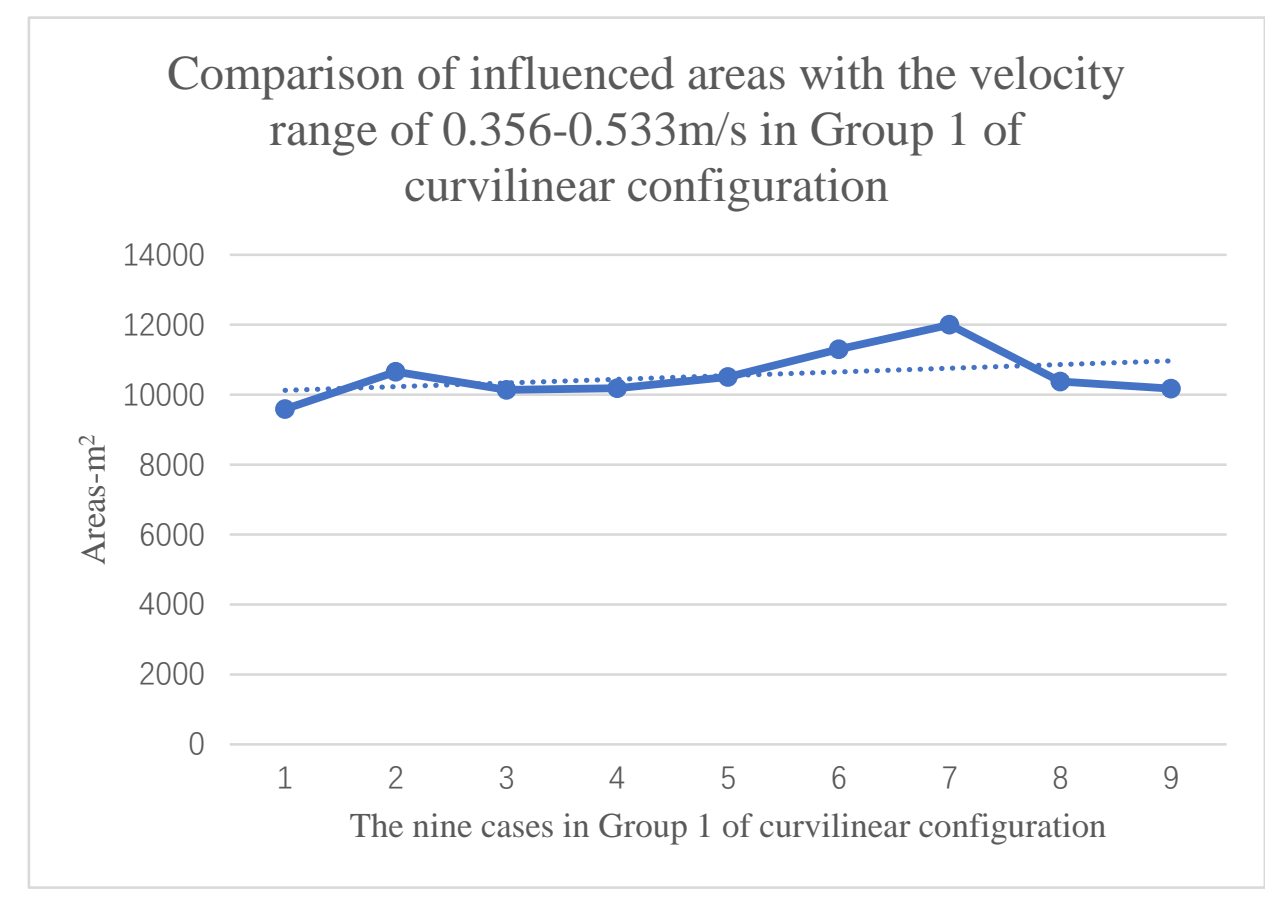

Figure 178. The influenced areas with the velocity range of $0.356-0.533 \mathrm{~m} / \mathrm{s}$ in Group 1 of curvilinear configuration. 


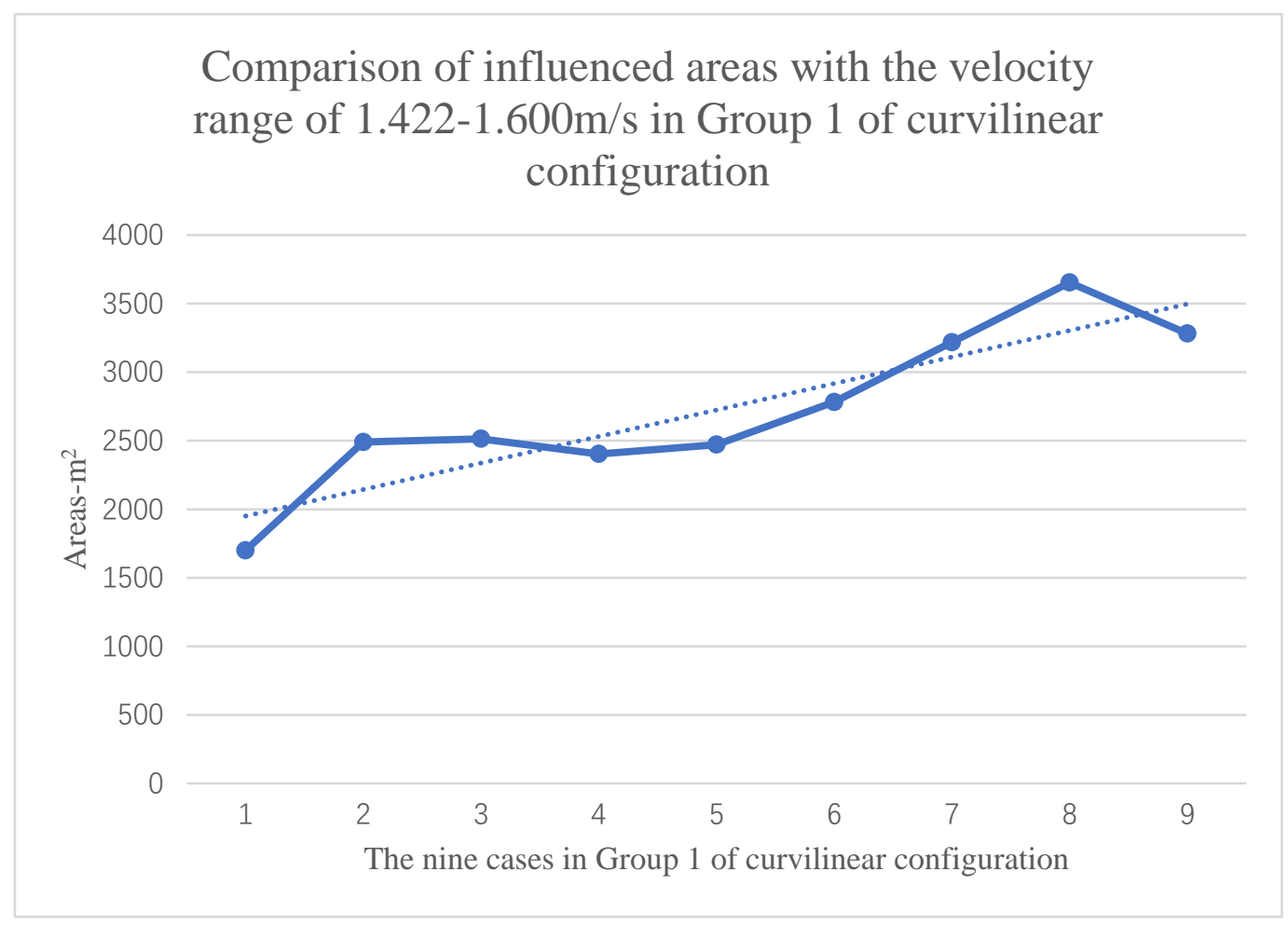

Figure 179. The influenced areas with the velocity range of $1.422-1.600 \mathrm{~m} / \mathrm{s}$ in Group 1 of curvilinear configuration.

The changes of influenced areas with different velocities in Group 1 are related to the changes of air pressure and wind flow (Figure 180 and Figure 181). There are high-air-pressure and low-air-pressure areas in front of windward side and behind the windward side. The influenced air-pressure areas are close in the nine cases. As the central angle increases, the lowair-pressure and high-air-pressure areas are decreased. Especially for the low-air-pressure area behind the building cluster, the decrease lead to the decrease of the low-velocity area. Also, the vortices behind the building cluster become shorter and smaller, which lead to the decreases of the low-velocity area.

The air-pressure magnitudes and wind-flow streamlines all suggest the increase of the central angle of a curvilinear building row with the convex surface on windward side can promote the wind flow. The convex surface can be regarded as a surface consisted of countless small flat surfaces. The angles between the flat surfaces and wind direction are different from each other. The increase of the central angle can reduce the angle between the windward surfaces and wind direction. Therefore, it is easier for winds to flow around the surfaces. Winds are accelerated on the lateral sides which increases the high-velocity areas. And the low- 
velocity area is also reduced. The surrounding outdoor ventilation of the building cluster is improved.

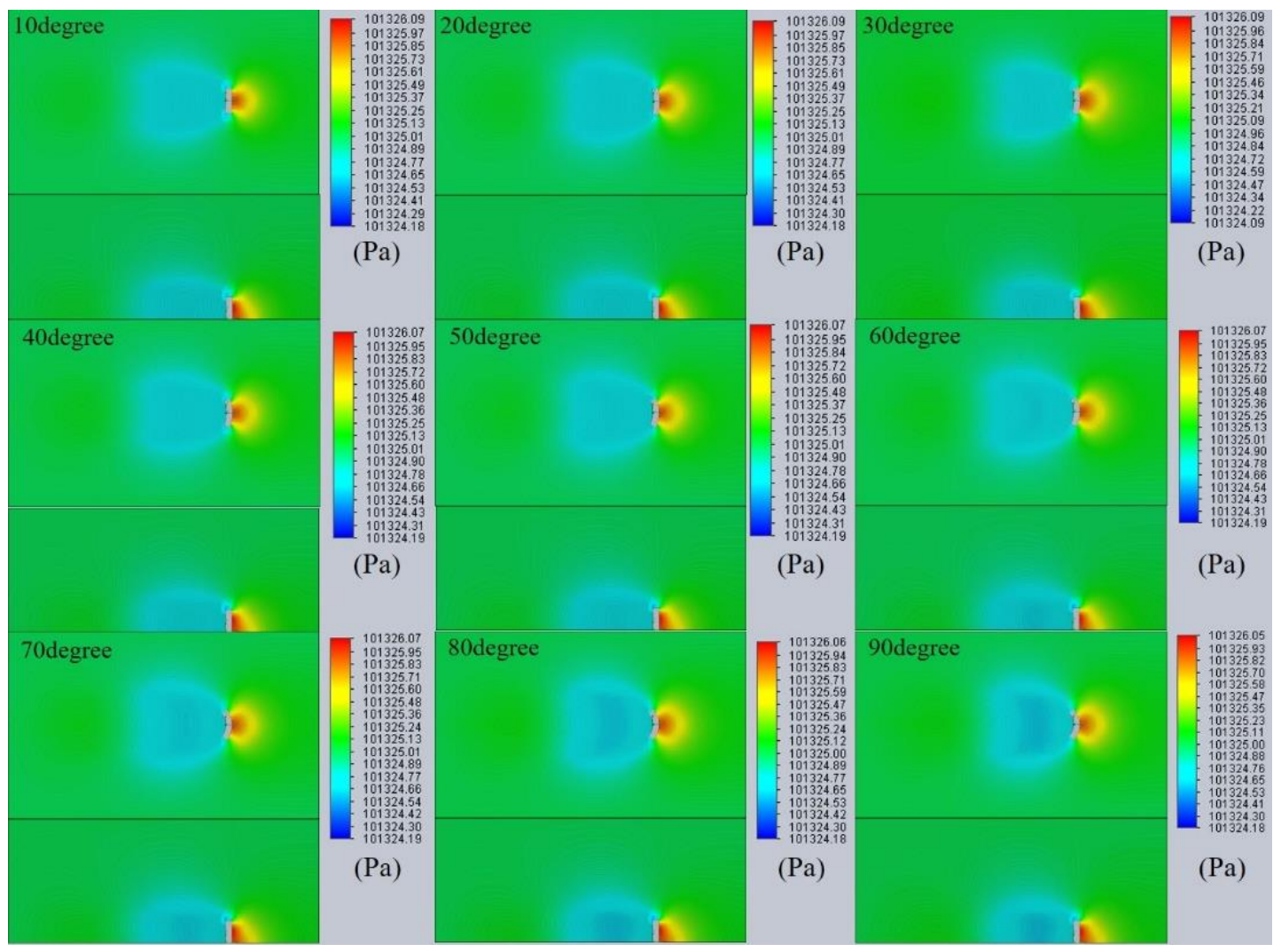

Figure 180. The comparison of air-pressure magnitudes on the horizontal and vertical planes of the building clusters in Group 1 of curvilinear configuration. 


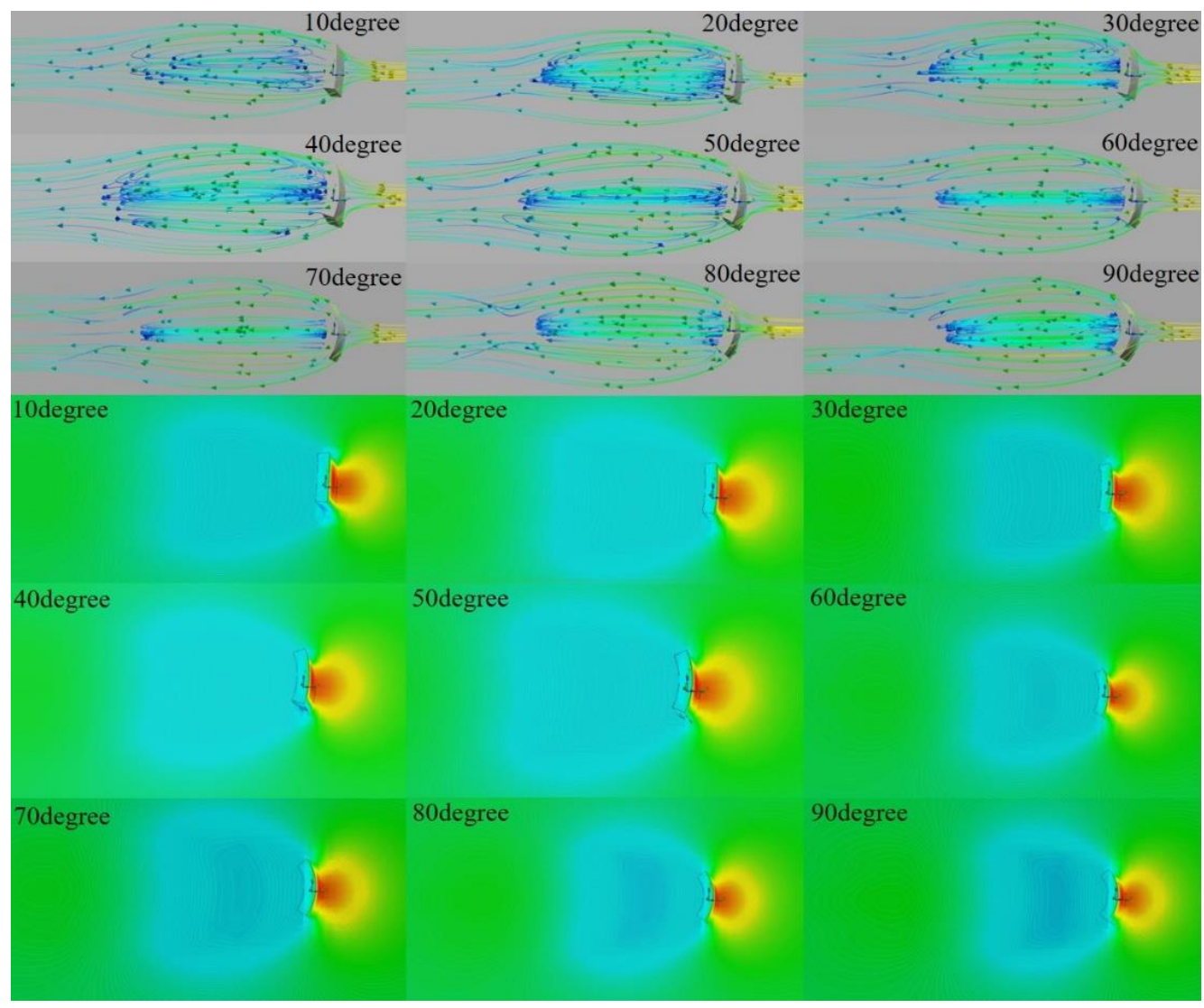

Figure 181. The comparison of wind-flow streamlines (top) and air-pressure magnitudes (bottom) on the horizontal plane and building surfaces in Group 1 of curvilinear configuration.

\section{(2) Part 2. Influences of the central angle of the curvilinear building row with the concave} surface on the windward side in Group 2

This part studies the influences of different central angles of Group 2. The wind-velocity magnitudes on horizontal and vertical planes of nine cases are analyzed (Figure 182 and Figure 183). The total influenced area increases constantly from Case 1 (influenced area: $41570.65 \mathrm{~m}^{2}$ ) to Case 8 (influenced area: $48646.18 \mathrm{~m}^{2}$ ) (Figure 182 and Figure 183). The area only decreases in Case 9 (influenced area: $46202.46 \mathrm{~m}^{2}$ ). Therefore, in general, the total influenced area increases as the central angle increases.

The low-velocity area (velocity range: $0.00-0.36 \mathrm{~m} / \mathrm{s}$ ) on the leeward side increases at first and then decreases (Figure 182 and Figure 183). As the central angle increases, the low-velocity area increases constantly from Case 1 (influenced area: $11484.17 \mathrm{~m}^{2}$ ) to Case 4 (influenced area: $12635.38 \mathrm{~m}^{2}$ ); the area decreases from Case 5 (influenced area: $12461.46 \mathrm{~m}^{2}$ ) to Case 9 (influenced area: $10785.55 \mathrm{~m}^{2}$ ). The low-velocity area of Case 4 is the largest; and the low- 
velocity area of Case 9 is the least of all.

The high-velocity areas (velocity range: $1.42-1.60 \mathrm{~m} / \mathrm{s}$ ) on the lateral sides increase constantly as the central angle increases (Figure 188). The high-velocity areas of Case 1 are the smallest $\left(1440.66 \mathrm{~m}^{2}\right)$. The areas increase from Case 2 to Case 8 . The areas of Case 8 are the largest of all $\left(3745.20 \mathrm{~m}^{2}\right)$. Though the high-velocity areas are increased in Group 2, they are smaller than Group 1.

As the central angle increases, the total influenced area increases slightly with some fluctuations; the high-velocity areas increase; and the low-velocity area is fluctuated; the areas with the other velocity ranges are also fluctuated (Figure 184, Figure 185, Figure 186 and Figure 187). In general, the overall wind velocities are increased slightly. The promotion of the outdoor ventilation should be due to the decrease of the windward projective area of the entire building cluster.

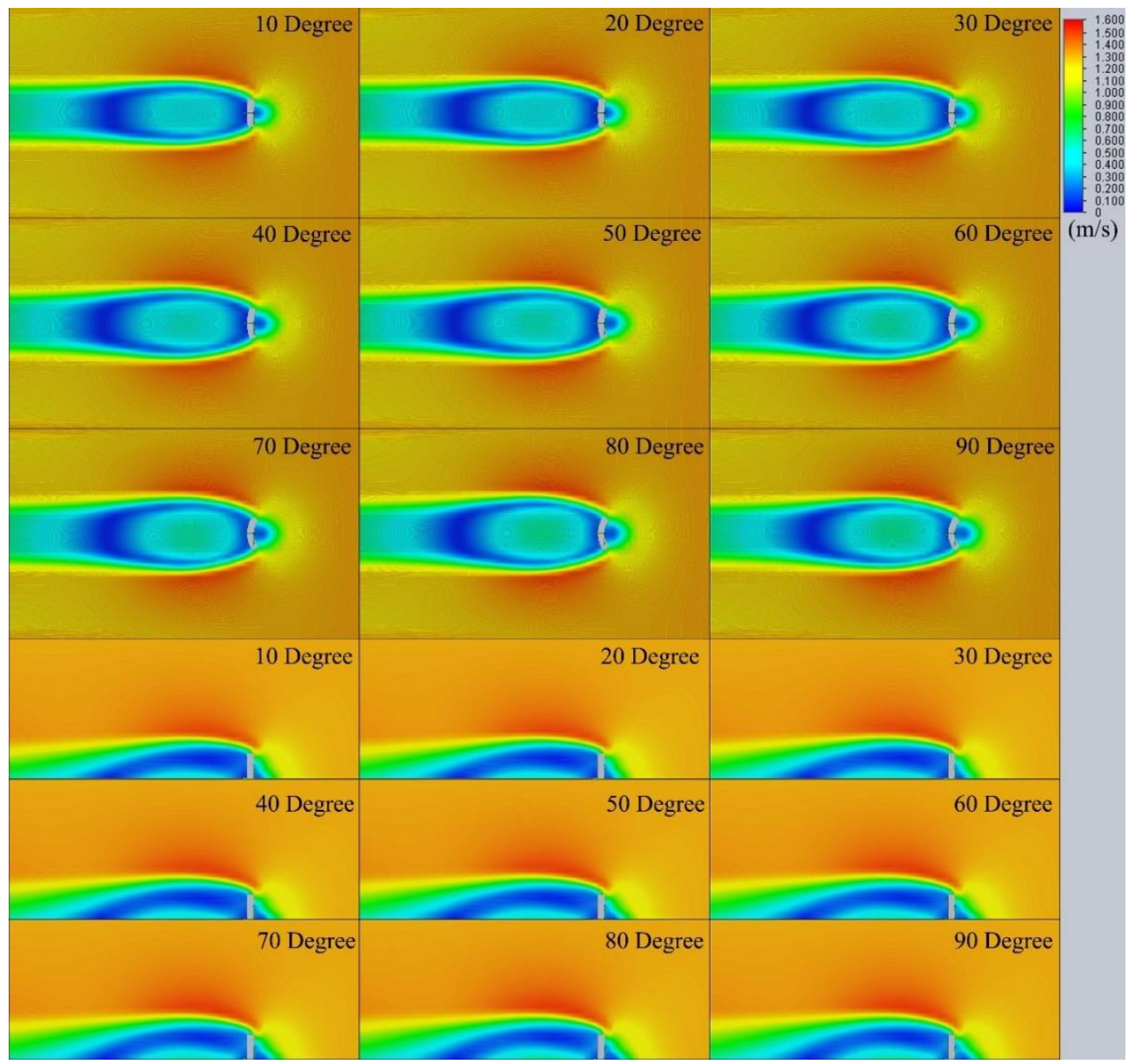

Figure 182. The comparison of wind-velocity magnitudes on horizontal and vertical planes of 
the building clusters in Group 2 of curvilinear configuration.

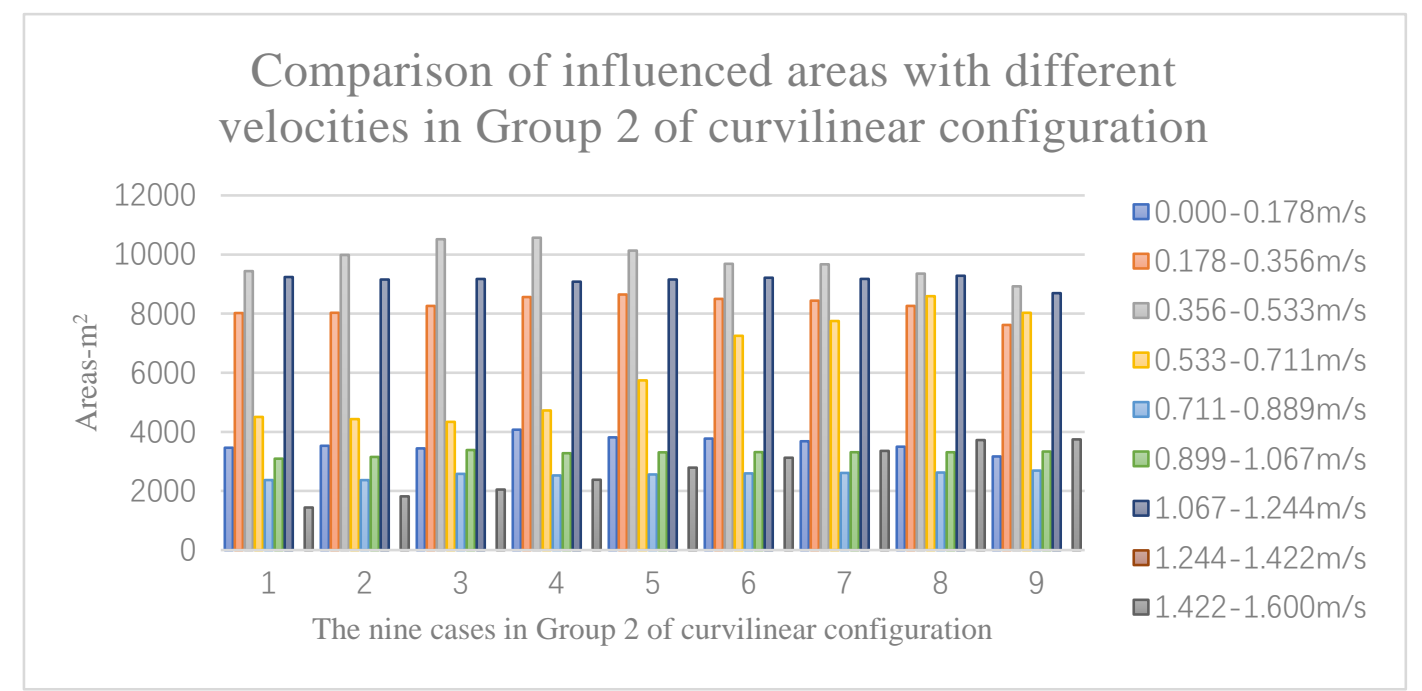

Figure 183. The influenced areas with different velocities in Group 2 of curvilinear configuration.

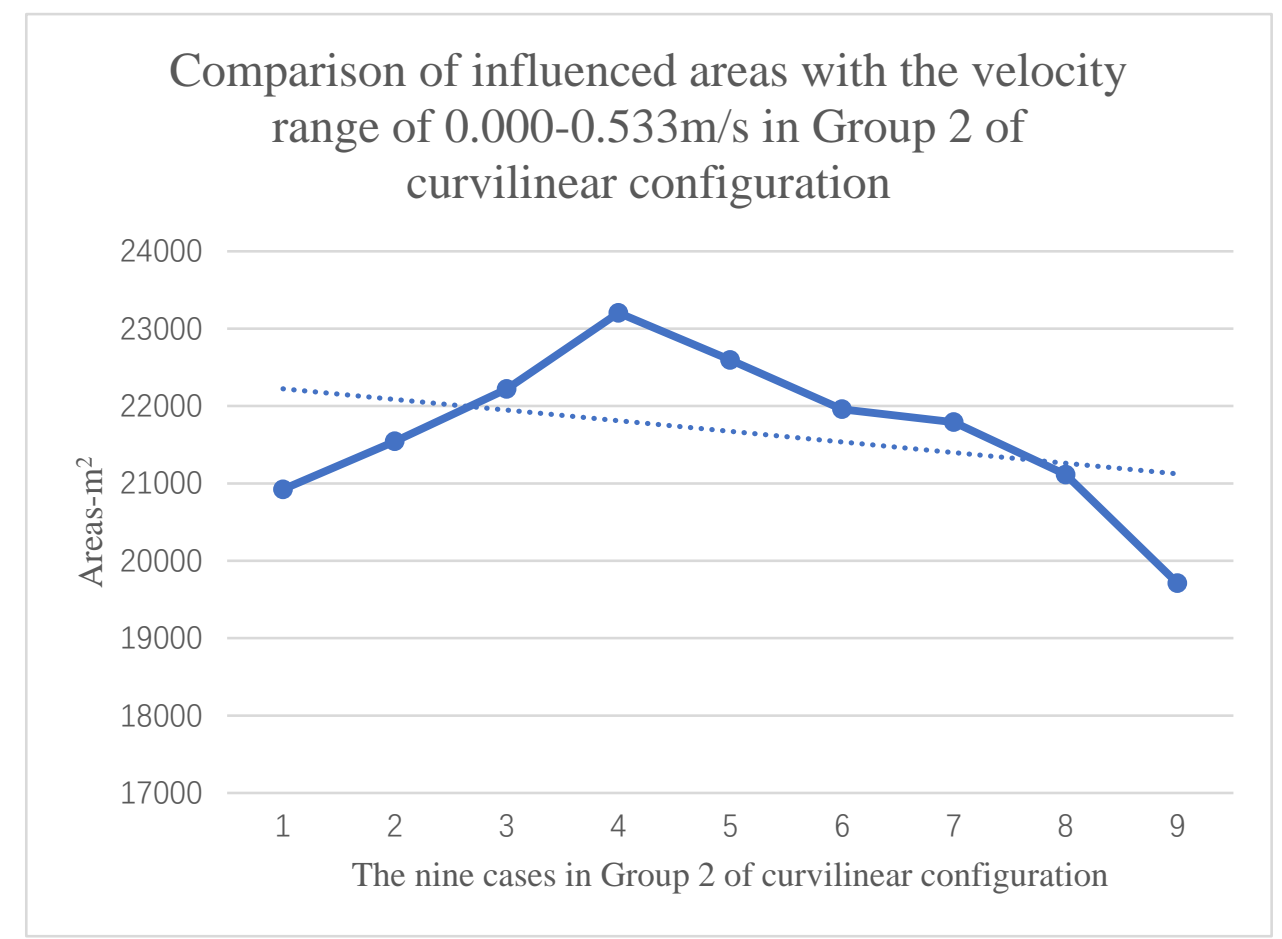

Figure 184. The influenced areas with the velocity range of $0.000-0.533 \mathrm{~m} / \mathrm{s}$ in Group 2 of curvilinear configuration. 


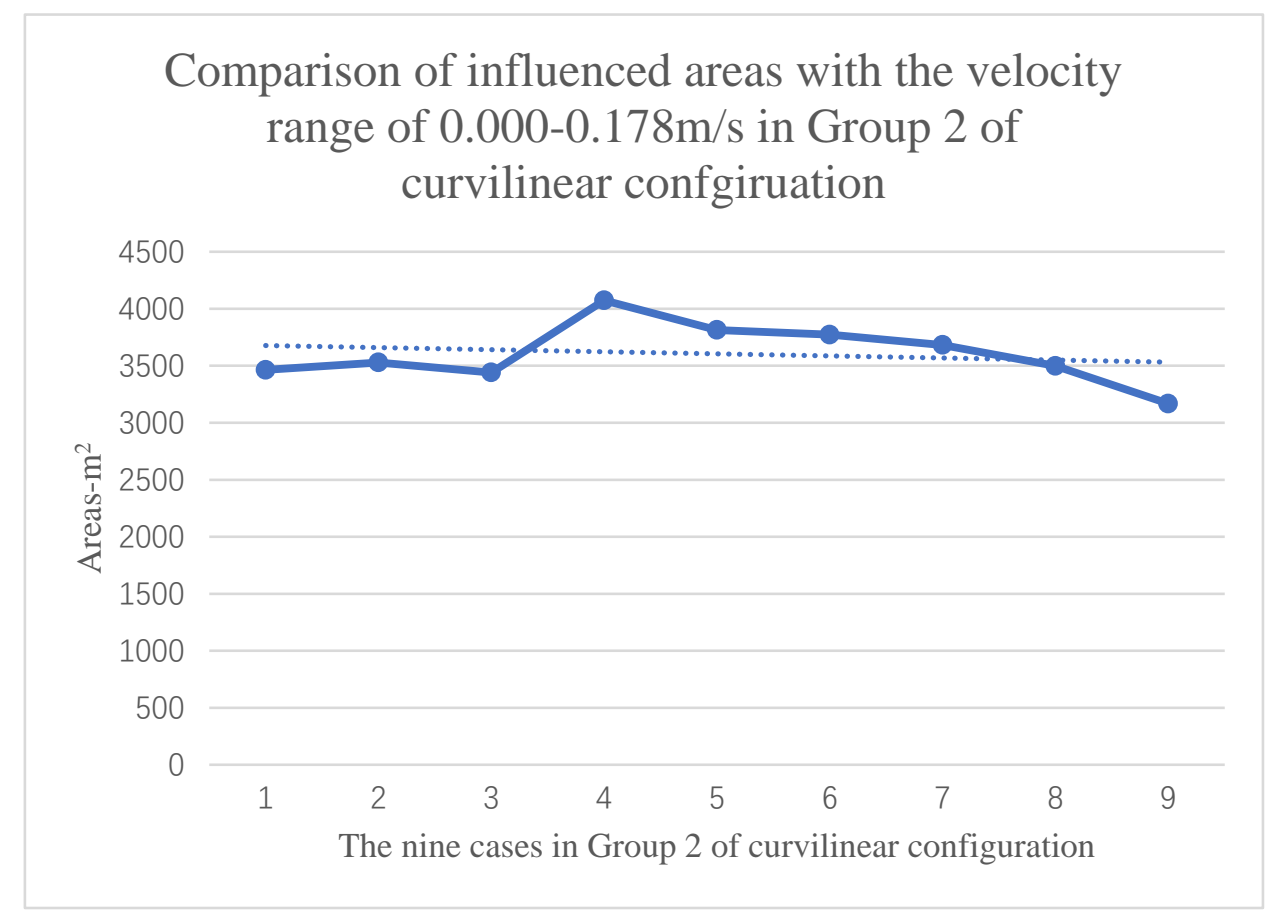

Figure 185. The influenced areas with the velocity range of $0.000-0.178 \mathrm{~m} / \mathrm{s}$ in Group 2 of curvilinear configuration.

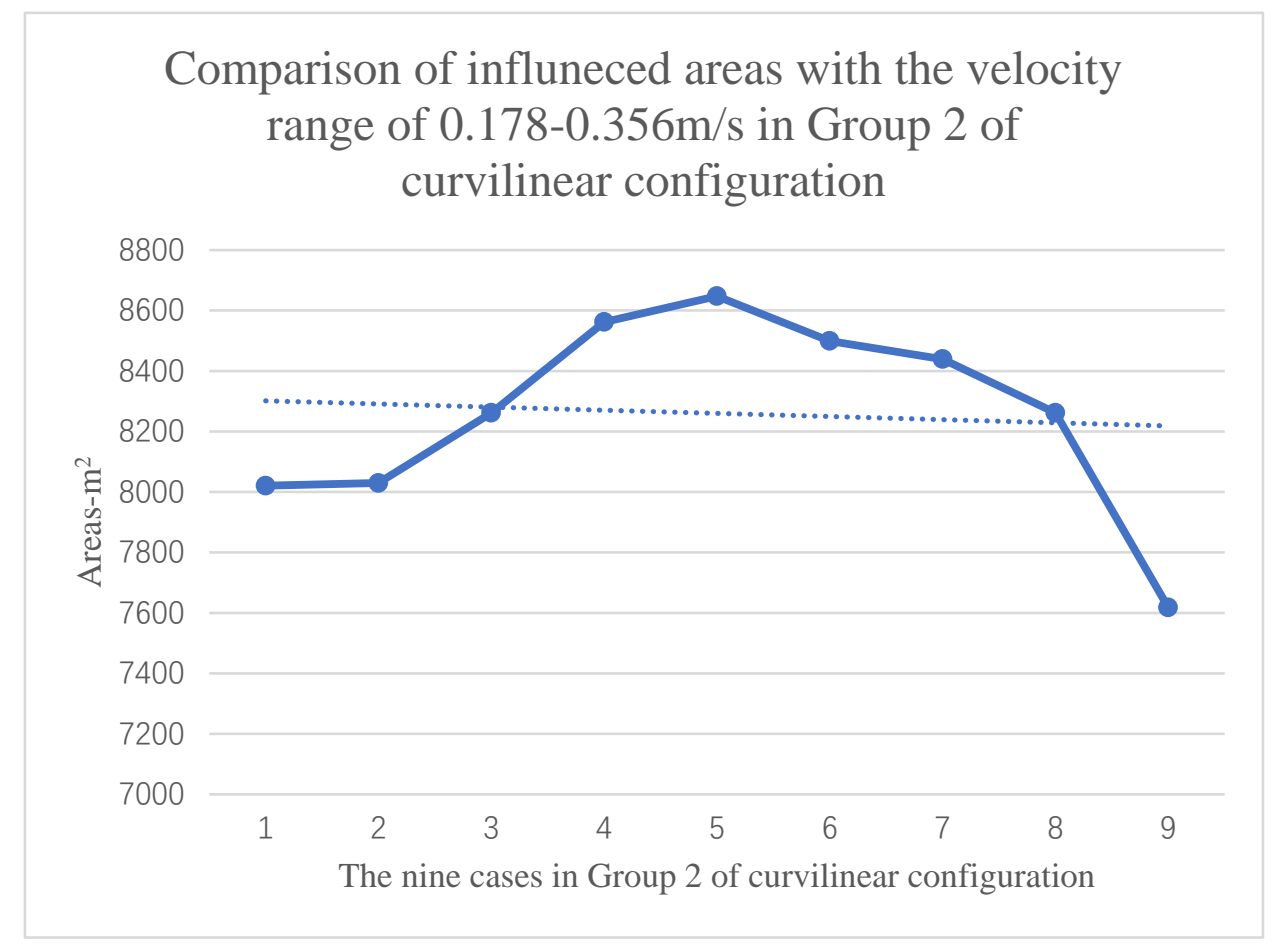

Figure 186. The influenced areas with the velocity range of $0.178-0.356 \mathrm{~m} / \mathrm{s}$ in Group 2 of curvilinear configuration. 


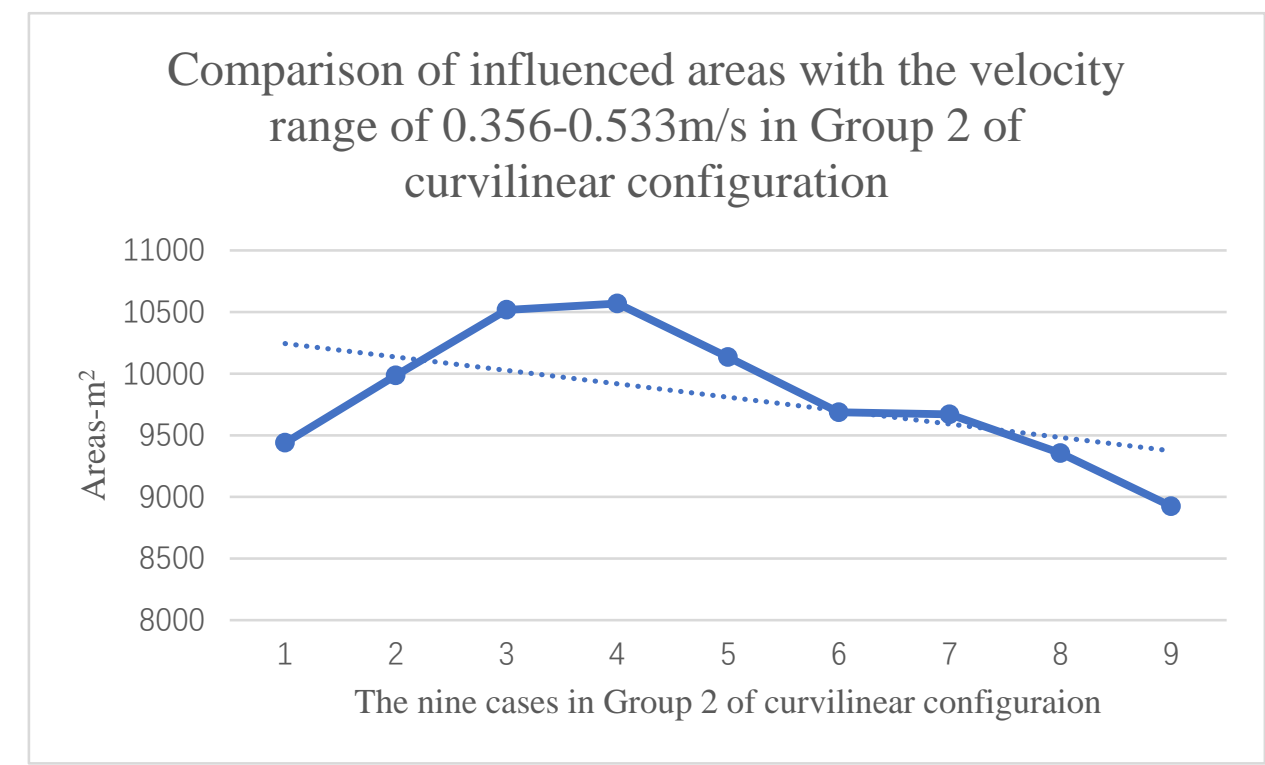

Figure 187. The influenced areas with the velocity range of $0.356-0.533 \mathrm{~m} / \mathrm{s}$ in Group 2 of curvilinear configuration.

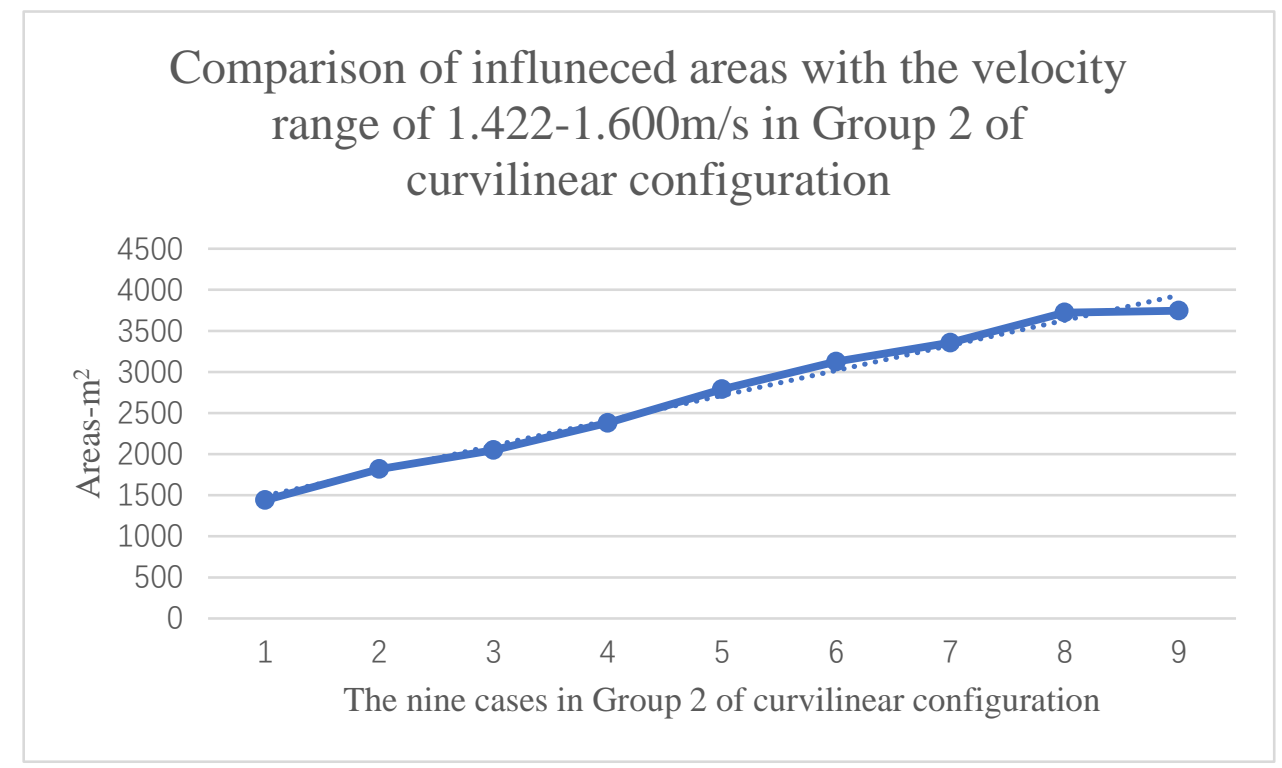

Figure 188. The influenced areas with the velocity range of $1.422-1.600 \mathrm{~m} / \mathrm{s}$ in Group 2 of curvilinear configuration.

The changes of influenced areas with different velocities in Group 2 are related to the changes of air pressure and wind flow (Figure 189 and Figure 190). The trend of Group 2 is contrary to Group 1. As the central angle increases, the low-air-pressure behind the building cluster and high-air-pressure areas in front of the building cluster are all increased. Air-pressure magnitudes show the air pressures in the low-air-pressure area is decreased. And the low-air- 
pressure area becomes larger. In addition, the vortices behind the building cluster become longer and larger. These are the reason for the increase of low-velocity area.

The air-pressure magnitudes and wind flow streamlines suggest the increase of the central angle of a curvilinear building row with the concave surface on windward side obstructs the wind flow. The concave surface is similar to a parachute, which increases the resistance of air flow. Therefore, it is more difficult for wind to flow around the surfaces. Because of the obstruction of the wind flow, the low-velocity area is increased and surrounding outdoor ventilation of the building cluster is discouraged.

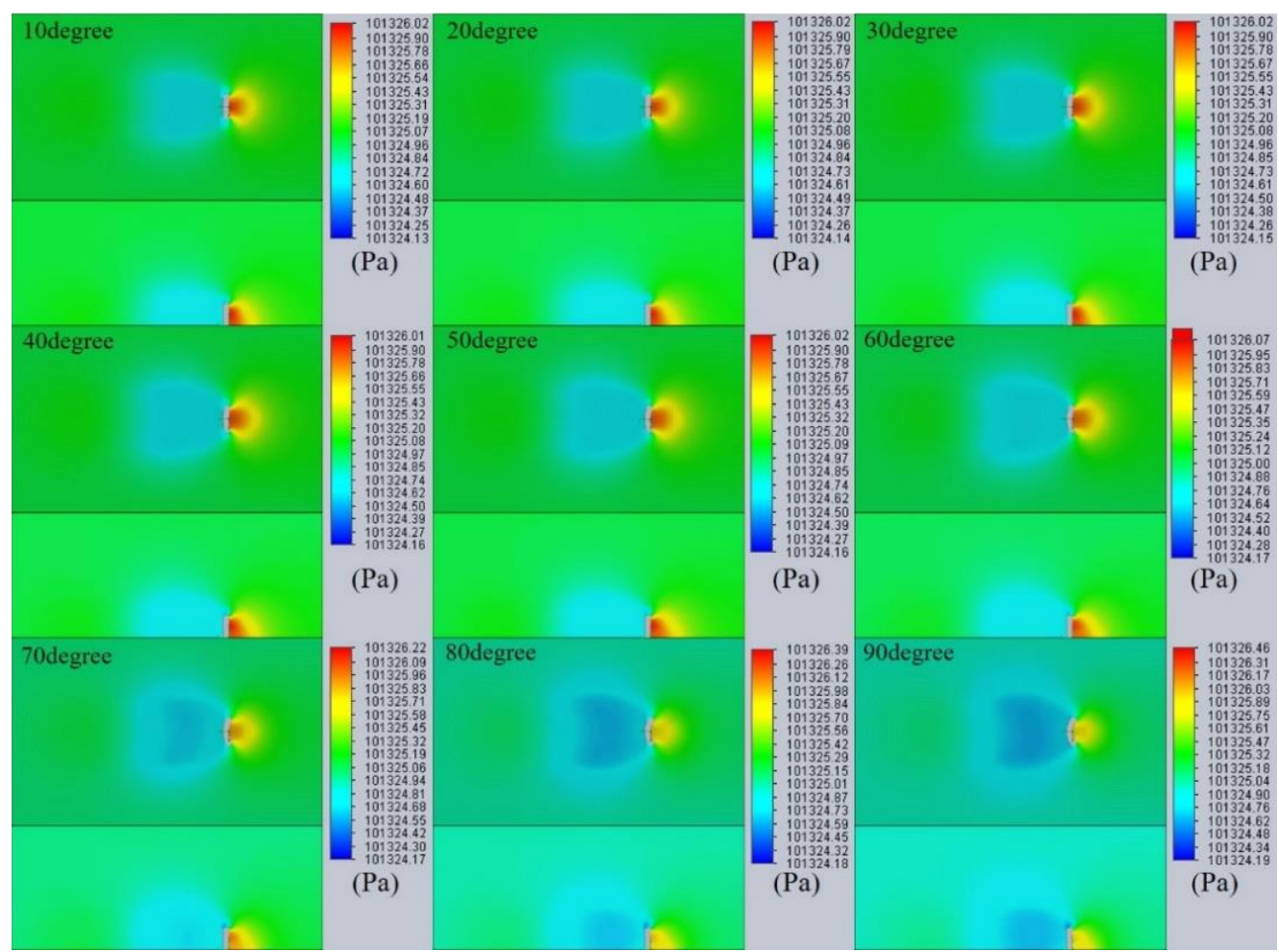

Figure 189. The comparison of air-pressure magnitudes on the horizontal and vertical planes of the building clusters in Group 2 of curvilinear configuration. 


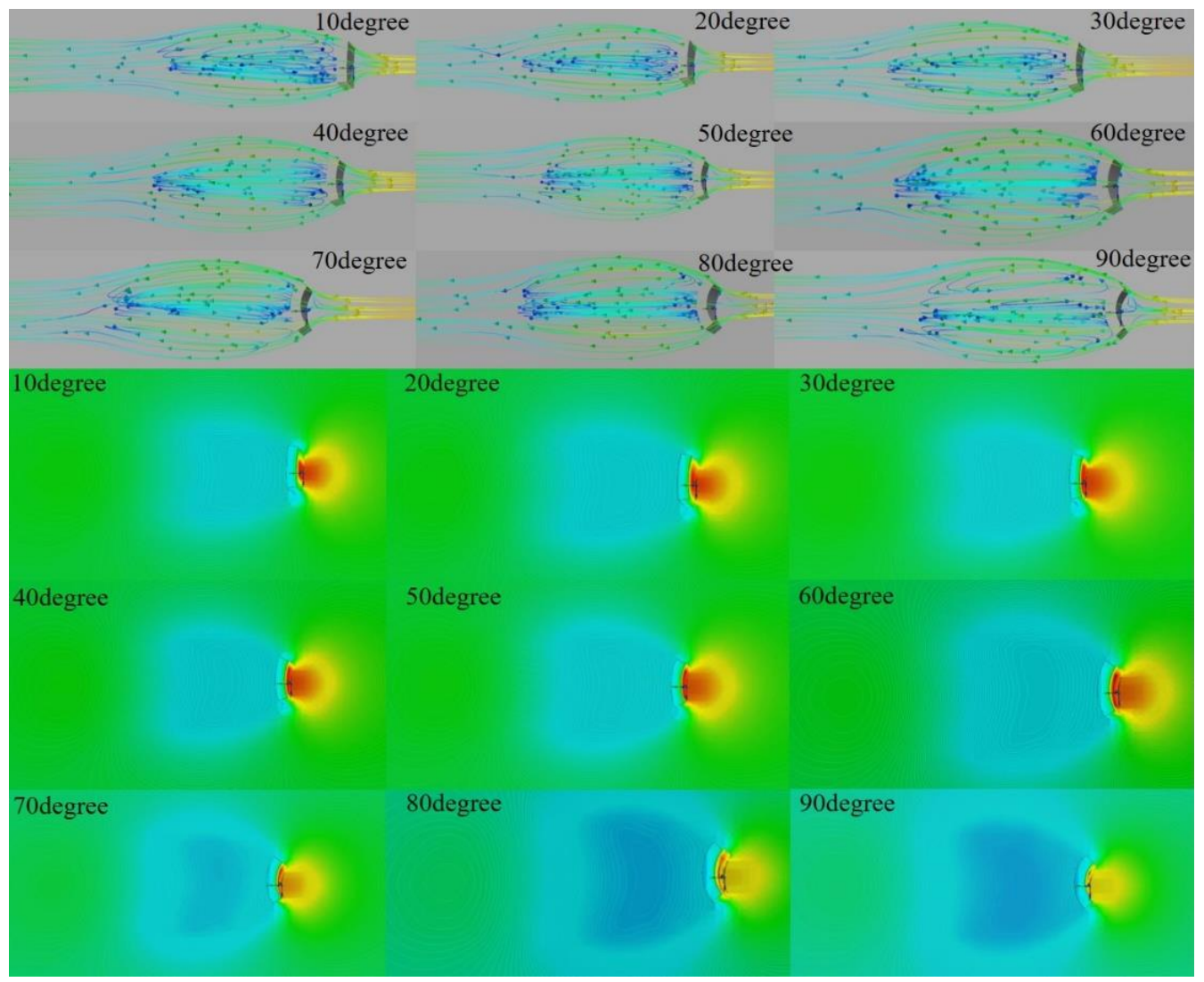

Figure 190. The comparison of wind-flow streamlines (top) and air-pressure magnitudes (bottom) on the horizontal plane and building surfaces in Group 2 of curvilinear configuration.

\subsection{Summary of BCCs studies}

In this chapter, the relationships between the influences on wind environments and the building variables of the three BCCs have been studied, including scattered configuration, linear configuration and curvilinear configuration. The building units in clusters are all set in the same rectangular form. For the influences, total influenced areas with different velocities, lowvelocity and high-velocity areas are calculated. Wind velocity magnitudes are used to compare the influences quantitatively. Air-pressure magnitudes and wind-streamlines are used to analyze the mechanisms. The BCCs studies are summarized in this section. The outcomes of BCCs studies are discussed and summarized further in the Discussion Chapter.

In the Section 6.1. Scattered configuration, the relationships between influences on wind environments and building variables have been studied in nine parts, including the building unit 
number, longitudinal distance, transverse distance and staggered distance. In the first part, three cases with different transverse distances are studied for the building cluster with two building units arranged in a building row. In the second part, three cases with different transverse distances are studied for the building cluster with three building units arranged in a building row. In the two parts, the wind flow and outdoor ventilation are promoted as the transverse distance increases. In the third part, three cases with different longitudinal distances are studied for the building cluster with two building units arranged in a building column. In the fourth part, three cases with different longitudinal distances are studied for the building cluster with three building units arranged in a building column. In the two parts, the influence decreases as the longitudinal distance increases. In the fifth part, nine cases with different transverse and longitudinal distances are studied for the building cluster with four building units arranged in two building rows (two columns). The influence increases as the transverse and longitudinal distances increase; but the wind flow is promoted as the transverse and longitudinal distances are increased. In the sixth part, six cases with different transverse and longitudinal distances are studied for the building cluster with six building units arranged in three building rows (two columns). The influence increases as the transverse distance increases; the influence decreases as the longitudinal distance increases. In the seventh part, six cases with different transverse and longitudinal distances are studied for the building cluster with six building units arranged in two building rows (three columns). The influence increases as the transverse and longitudinal distances are increased. In the eighth part, six cases with different transverse and longitudinal distances are studied for the building cluster with nine building units arranged in three building rows (three columns). The influence decreases as the transverse distance increases; the influence is nearly unchanged as the longitudinal increases. In the ninth part, three cases with different staggered distances are studied for the building cluster with nine building units arranged in three building rows. The influence increases as the staggered distance increases. In all the parts, the overall outdoor ventilation is promoted as the transverse and longitudinal distances are increased.

In the Section 6.2. Linear configuration, the relationships between influences on wind environments and building variables have been studied in four parts, including the building unit number, longitudinal distance and staggered distance. In the first part, three cases with different 
building-unit numbers are studied for the building cluster with building units arranged in one building row. In the second part, six cases with different unit numbers and longitudinal distances are studied for the building cluster with building units arranged in two building rows. In the third part, six cases with different unit numbers and longitudinal distances are studied for the building cluster with building units arranged in three building rows. In the fourth part, three cases with different staggered distances are studied for the building cluster with nine building units arranged in three building rows. Generally, the influence increases as the unit numbers and longitudinal and staggered distances are increased in all the parts. The overall wind flow is promoted as the longitudinal and staggered distances increase.

In the Section 6.3. Curvilinear configuration, the relationship between the influence on wind environments and the building variable of central angle has been studied in two parts. The central angle of the arc is related to the curvature of the curvilinear building row. In the first part, nine cases with different central angles are studied for the building cluster with building units arranged in a curvilinear building row. The convex surface of the building row is on the windward side. The influence increases with some fluctuations as the central angle increases. In the second part, nine cases with different central angles are studied for the building cluster with building units arranged in a curvilinear building row. The concave surface of the building row is on the windward side. Generally, the influence increases as the central angle increases. In summary, the effects on winds are contrary in the two parts. 


\section{Discussion}

By answering the research question, this research has studied how wind environments are influenced by contemporary high-rise residential buildings, especially for novel forms and configurations in the HSCWA. The research outcomes are discussed in this chapter, including the research methodology, parametric design, BUFs studies and BCCs studies. Then limitations and future works are discussed afterwards.

\subsection{Discussion of research methodology}

The research methodology consists of the parametric design method, CFD simulation method, and analysis method. The methodology is developed by making multiple computational tools work in coordination. This research requires a large amount of comparison studies for building designs with different variables. The integration of multiple tools can improve the efficiency of comparison studies. The computational tools can provide abundant functions in the parametric modelling, CFD simulations and results analysis.

Based on the investigation in building morphology, the parametric design sets up a bridge between building variables and CFD simulations to study building forms and configurations which are representative for generic high-rise residential buildings in the area. After finding the most representative BUFs and BCCs in the categorization study, parametric design systems are established to link building variables and simulations. The building models are used to help mesh generation for CFD simulations. Parametric modelling is used due to its high efficiency in modelling. Software tools of Rhino and its plugin Grasshopper are applied on the modelling stage. Building variables and attributes of building forms and configurations are considered in the modelling strategy. Most models can be divided into several sub models created by modelling script modules. Different algorithms are developed by using abundant components of the software tools.

The CFD simulation method has been used to study the wind environments of building forms and configurations comprehensively. Wind conditions of simulations are set according 
to the local climate data (CSWD). The computational domains are created in cuboid shapes by following the guidelines in the CFD software (Frank et al., 2007). The mesh creation technology is based upon the use of Cartesian-based meshes. The RANS method is used in simulations. A modified k- $\varepsilon$ model with damping functions has been explained in the governing equations. Simulation strategies are explained for different purposes. The quick simulation uses a rough geometry and coarse grids, and the comprehensive simulation uses a detailed geometry and fine grids. The simulation method has been validated by the wind tunnel experiment (Brown, Lawson, Decroix \& Lee, 2001). The grid-independent analysis has been performed by constructing coarse gird, medium grid and fine grid. The GCI has been calculated to ensure grid accuracy. The PRMSD method, NMSE, CC and FB are considered to evaluate the statistical discrepancies between simulation and experimental results. The calculation results of wind velocity and turbulent kinetic energy have demonstrated the accuracy of the method is acceptable.

In the analysis of simulation results, reasonable criteria are established to evaluate the wind environments of buildings, including three aspects of wind-velocity magnitudes, air-pressure magnitudes, wind-flow streamlines. By using the analysis tools, the simulation results can be visualized through images of the three aspects; the images are processed to distinguish slight differences of different CFD simulation results of wind environments. Two analysis strategies are used in this research, including categorized analysis and iterative analysis.

The development of the research methodology contributes to the knowledge of wind environment study of contemporary buildings related to urban microclimates. The methodology shows that making the multiple computational tools working in coordination can provide efficient iterative analysis and implement quantitative comparisons for studying influences of buildings on winds, especially for wind environment studies of large amount of buildings and their modifications. The parametric modelling highly improved modelling efficiency for iterative analysis. Though the development of parametric modelling scripts is time-consuming, modifications of building models with different parameters are convenient and quick. The image processing tool also helps in distinguishing differences of influences by converting calculated pixel numbers to actual influenced areas. The CFD simulation method is accurate and efficient enough to study wind environments of buildings with multiple variables for 
iterative analysis. Based on the criteria, the relationships between influences on wind environments and building variables are discovered. In the Chapter 5 and Chapter 6, influences on wind environments of BUFs and BCCs are studied by using the research methodology. On the other hand, the research methodology can be used for new studies of wind environments. Especially for applications in architectural practices, the methodology can give immediate analysis of wind environments on early stage. It is easy to change any functional modular or even the tools if there are better alternatives. Because more advanced modelling scripts or CFD software may be developed in the future. The parametric modelling can seamlessly be employed to analyze other factors of sustainable design on computational platforms.

\subsection{Discussion of parametric design}

In the parametric design, the parametric design systems have been established for a series of representative forms and configurations of high-rise residential buildings in the HSCWA for CFD simulations based on the building categorization study. The systems connect the building objects and the simulations.

In the building categorization study, definitions of eleven BUFs and four BCCs have been given according to the previous studies, site investigations and their geometric attributes. The definitions are clearer and more accurate than the previous vague definitions of high-rise residential buildings. The definitions can cover almost all the forms and configurations of highrise residential buildings in the area. And numbers of BUFs and BCCs in the area have been calculated statistically. The statistical analysis of the large numbers of buildings shows the popularities of the BUFs and BCCs. The square form, rectangular form and ' $\mathrm{T}$ ' form are the most popular BUFs in the area; and scattered configuration, linear configuration and curvilinear configuration are the most popular BCCs in the area. The three BUFs and three BCCs are selected for the study due to their representativeness. The representative ranges of common building variables of the three BUFs and three BCCs are summarized according to previous studies and collected building information in the end of the section of 'Statistical analysis of BUFs and BCCs'. These all make the study outcomes more applicable. 
In the parametric modelling, scripts of the representative BUFs and BCCs are developed based on their geometric attributes. The logics of geometries are expressed by using scripts. Modelling strategies are based on the architectural essence, inheritance of conventional drawing expression and actual construction process. The scripts can generate the building models automatically, which largely improves the efficiency of mesh generations for CFD simulations. The parametric models can be adjusted efficiently as the parameters are changed, which enables iterative analysis to study the influences of different building variables. In the parametric modelling scripts of square form, rectangular form and ' $\mathrm{T}$ ' form, the building models are created from their planes and facades. A floor-plane surface is created based on several control points first and then the height is defined to generate the entire building. The parametric models of square and rectangular forms are defined by the widths, lengths and heights. For the parametric model of ' $\mathrm{T}$ ' form, the major part is defined using a group of length and width; the bulge part is defined using another group of length and width. In the parametric modelling of scattered and linear configurations, the modelling scripts are created with the modelling modules of BUFs. The relationships and positions of building units are arranged by the scripts. In the parametric modelling of curvilinear configuration, the connected building units in a curvilinear building row are simplified as a unity. The four parameters of height, width, length and central angle are used to create the curvilinear building row model. The simulation boundaries are defined by the other group of parameters.

The categorization of typical BUFs and BCCs to enable parametric assessments is an original attempt. The study presents the preference of contemporary high-rise residential buildings. Because the parametric modelling system can generate the building models automatically. There are potentials to use the parametric model systems in other studies and designs. It can also be modified to generate different models or even expand its functions.

\subsection{Discussion of BUFs studies}

In the BUFs studies, relationships between the influences on wind environments and building variables are studied for the square form, rectangular form and ' $\mathrm{T}$ ' form. 
In the overview, the attributes of wind environments of the three BUFs are described by explaining the wind-velocity magnitudes, air-pressure magnitudes and wind-flow streamlines. When winds approach a building, they are obstructed and they flow around the building. There is a relatively large low-velocity area generated on the leeward side of the building. There are high-velocity areas generated on the lateral sides of the building. The influences of buildings on wind-velocity magnitudes can be explained by using air-pressure magnitudes and wind-flow streamlines. The air-pressure magnitudes show that the low-air-pressure area behind the building is usually the reason for the low-velocity area. Because winds usually flow from the high-air-pressure area to the low-air-pressure area. It is relatively difficult for winds to escape from the low-air-pressure area. Wind streamlines show that there are vortices generated in the area. The high-velocity areas are due to the increases of air-pressure differences and the extents of air-pressure changes. The air-pressure magnitudes show the increases are obvious on the lateral sides. The wind-flow streamlines show winds are accelerated when they go around the edges of the windward surfaces of buildings.

\subsubsection{Discussion of square form}

In the study of square form, the relationships between influences on wind environments and building variables are studied in two parts, including the edge length (range: 10-30m) and rotation angle (range: 0-45 degree).

In the first part, three cases with different edge lengths are studied. The influence increases as the edge length increases. Because more winds are obstructed by the windward surface as the edge length increases. The low-velocity area increases as the edge length increases. And more vortices are generated on the leeward side. Though the high-velocity areas are increased as the edge length increases, they are relatively small compared to the low-velocity area. Therefore, the outdoor ventilation is weakened as the edge length increases.

In the second part, four cases with different rotation angles are studied. Winds are divided by the windward edge and escape from the lateral sides more easily as the rotation angle increases. The low-velocity area decreases as the rotation angle increases, and less vortices are 
generated on the leeward side. The high-velocity areas on the lateral sides increase as the rotation angle increases. The outdoor ventilation is promoted as the angle increases.

Take a square-form building design for example. The outdoor ventilation can be promoted by encouraging the wind flow around the building. The edge length should be as short as possible. This reduces the low-velocity area behind building. The building should be rotated as much as possible up to 45 degree. The edge on the windward side can help to divide the winds. And it is easier for winds to flow around the non-vertical surfaces towards the wind direction. (Figure 191) (Table 44.)

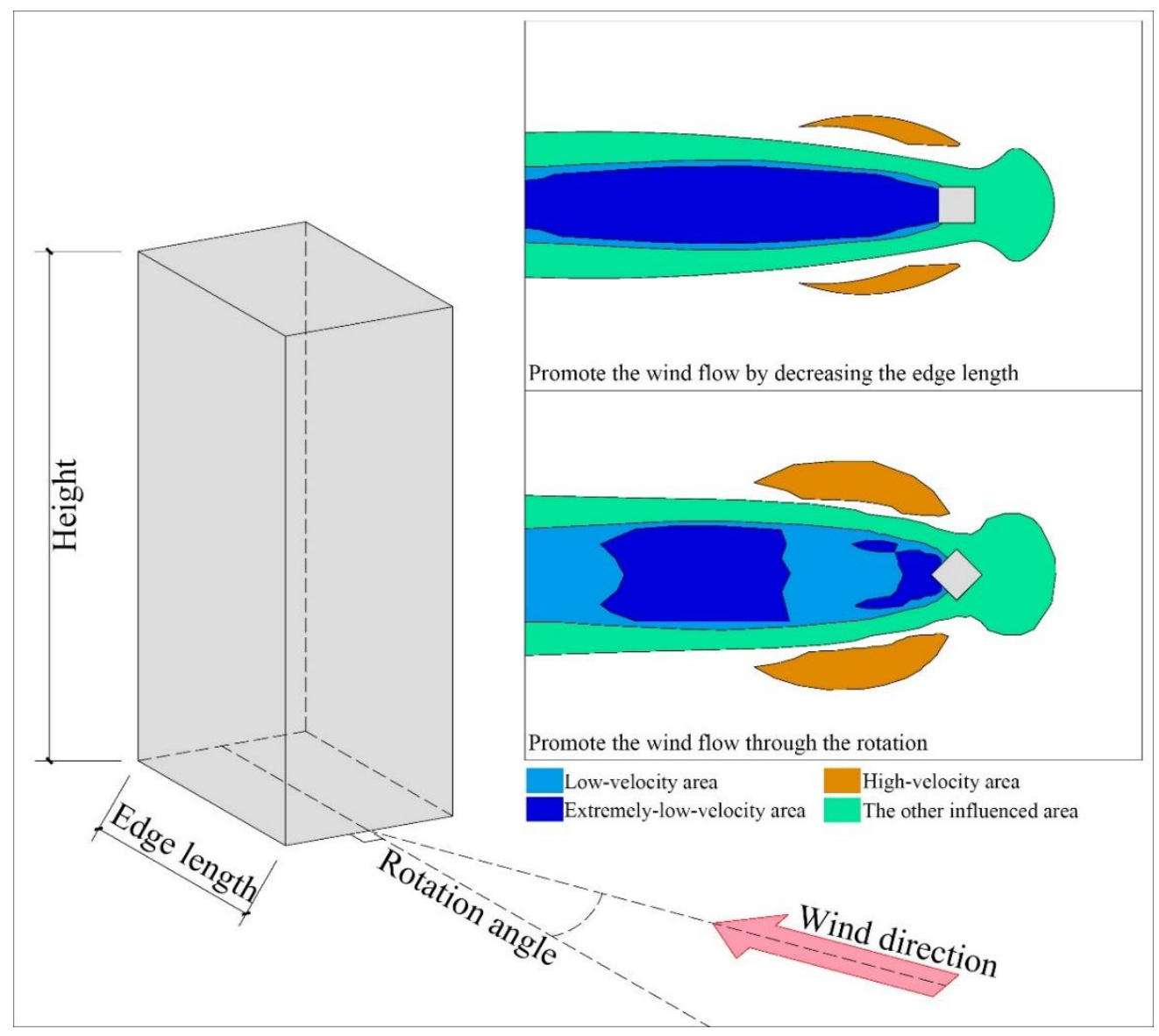

Figure 191. The wind environment influenced by the variables of square form.

Table 44. Relationships between the influences and building variables of square form

\begin{tabular}{|l|l|}
\hline Increases of building variables & Influences on outdoor ventilation \\
\hline Edge length & Weakened \\
\hline Rotation angle & Promoted \\
\hline
\end{tabular}




\subsubsection{Discussion of rectangular form}

In the study of rectangular form, the relationships between influences on wind environments and building variables are studied in four parts, including the length (range: 10-40m), width (range: 10-20m), height (range: 36-72m) and rotation angle (range: 0-90 degree).

In the first part, the surfaces with the lengths of the rectangular-form buildings are on the windward side. Eleven cases of three groups with different lengths and widths are studied. The influence increases as the length increases. The low-velocity area and vortices are increased as the length increases. The high-velocity areas on the lateral sides are also increased. But they are smaller than the low-velocity area. Because more winds are obstructed due to the increase of the windward surface. The low-air-pressure and high-air-pressure areas are increased due to the increase of length. Therefore, the outdoor ventilation is weakened as the length increases. The influence decreases as the width increases. The low-velocity and high-velocity areas decrease as the width increases. Because the low-air-pressure and high-air-pressure areas decrease as the width increases. Less vortices are generated on the leeward side. Therefore, the outdoor ventilation is promoted as the width increases.

In the second part, three cases with different heights are studied. The influence increases as the height increases. The low-velocity and high-velocity areas decrease as the width increases. The low-air-pressure and high-air-pressure areas increase as the height increases. Because more winds are obstructed by the larger windward surface caused by the increase of height. The outdoor ventilation is weakened as the height increases.

In the third part, the surfaces with the widths of the rectangular-form buildings are on the windward side. Eleven cases of three groups with different lengths and widths are studied. As the length increases, the influence decreases at first and then increases slightly (especially obvious in Group 1 with the width of $10 \mathrm{~m}$ ). The least influence is decided by a particular ratio of width and length. The ratio should be related to the wind velocity. Faster the velocity it is, smaller the ratio it might be. In this research, the ratio of width and length is approximately $1 / 3$. The low-velocity and high-velocity areas have the similar trends. Their changes are relevant to 
the changes of air-pressure magnitudes and vortices. Therefore, outdoor ventilation is promoted as the length increases in a range; the promotion is the most when the ratio of width and length reaches a particular value. The influence increases as the width increases. The low-velocity and high-velocity areas are increased as the width increases. More vortices are generated as the width increases. Because more winds are obstructed by the increasing windward surface. Therefore, the outdoor ventilation is weakened as the width increases.

In the fourth part, twelve cases of three groups with different rotation angels are studied for rectangular-form buildings (with different lengths, widths and heights). The influence decreases as the rotation angle increases. The low-velocity and high-velocity areas are decreased as the rotation angle increases. And less vortices are generated on the leeward side. Because the low-air-pressure and high-air-pressure areas decrease as the rotation angle increases. The windward projective area of the building is decreased. Winds are divided by the windward edge and flow more smoothly on the windward surfaces which are not vertical from the wind directions. Therefore, the outdoor ventilation is promoted as the angle increases.

Take a rectangular-form building design for example. When the surface with the length is on the windward side, the outdoor ventilation surrounds the building can be promoted by decreasing the length, increasing the width, decreasing the building height and rotating the building (up to 90 degree). When the surface with the width is on the windward side, the outdoor ventilation can be promoted by increasing the length and decreasing the width. But the best performance of outdoor ventilation is decided by a particular ratio of width and length. According to the wind-velocity condition in this research, the ratio is around 1/3. (Figure 192) (Table 45.) 


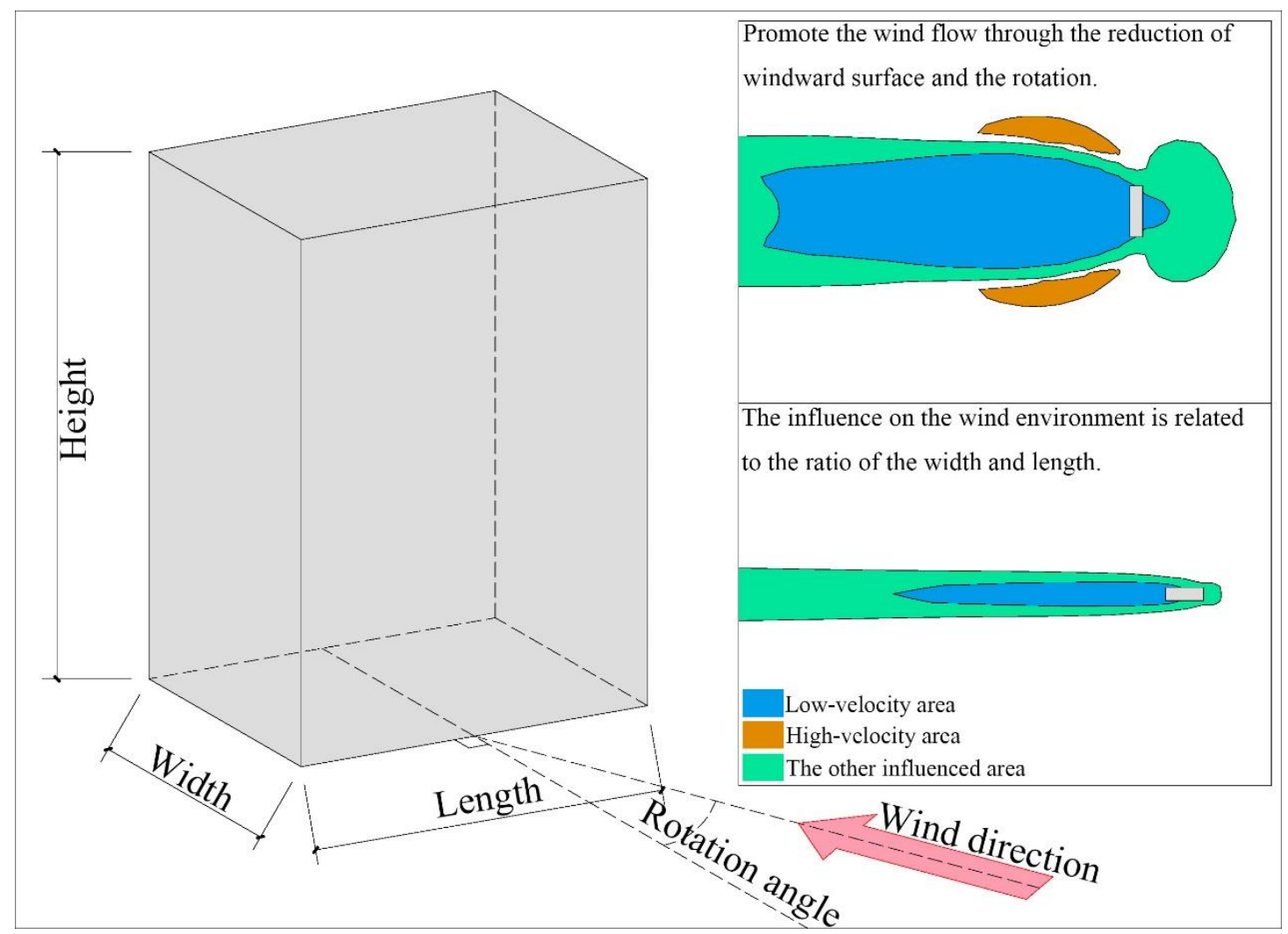

Figure 192. The wind environment influenced by the variables of the rectangular form.

Table 45. Relationships between the influences and building variables of rectangular form

\begin{tabular}{|l|l|}
\hline Increases of building variables & Influences on outdoor ventilation \\
\hline Length & Weakened \\
\hline Width & Promoted \\
\hline Height & Weakened \\
\hline Length (short surfaces on the windward side) & Promoted (in a range) \\
\hline Width (short surface on the windward side) & Weakened \\
\hline Rotation angle & Promoted \\
\hline
\end{tabular}

\subsubsection{Discussion of ' $T$ ' form}

In the study of ' $\mathrm{T}$ ' form, the relationships between the influences on wind environments and the building variables are studied in two parts, including the length and width of the bulge part. The first part is for the short buildings with a relatively small bulge part. The buildings are $36 \mathrm{~m}$ 
in height, which are common for buildings with twelve floors and two apartments on each floor. The bulge-part length is in the range of $6-10 \mathrm{~m}$. The second part is for the tall buildings with a relatively large bulge part. The buildings are $72 \mathrm{~m}$ in height, which are common for buildings with 24 floors and two apartments on each floor. The bulge-part length is in the range of 15$21 \mathrm{~m}$. The bulge-part width is in the range of $6-18 \mathrm{~m}$.

In the first part, five cases with different bulge-part lengths are studied. The influence decreases as the length increases. The high-velocity and low-velocity areas are decreased as the length increases. Because the low-air-pressure and high-air-pressure areas are decreased as the length increases. The vortices on the leeward side decrease as the length increases. Therefore, the outdoor ventilation is promoted as the bulge-part length increases.

In the second part, nine cases of three groups with different lengths and widths of the bulge-part are studied. The influence decreases as the length and width are increased. The highvelocity and low-velocity areas are decreased as the length and width increase. Because the low-air-pressure and high-air-pressure areas are decreased as the length and width increase. And the vortices on the leeward side also decrease. The effect of the increase of length is slightly larger than the increase of width. Because the increase of length causes more reductions of highvelocity and low-velocity areas. Therefore, the outdoor ventilation is promoted as the length and width of the bulge part are increased. And the promotion is slightly more as the length increases.

Take a 'T'-form building design for example. The outdoor ventilation of the building can be promoted by increasing the bulge part size. Because the increase of bulge part size encourages wind flow. And the increase of bulge-part length has larger effect in outdoor ventilation promotion than the increase of bulge-part width. (Figure 193) (Table 46.) 


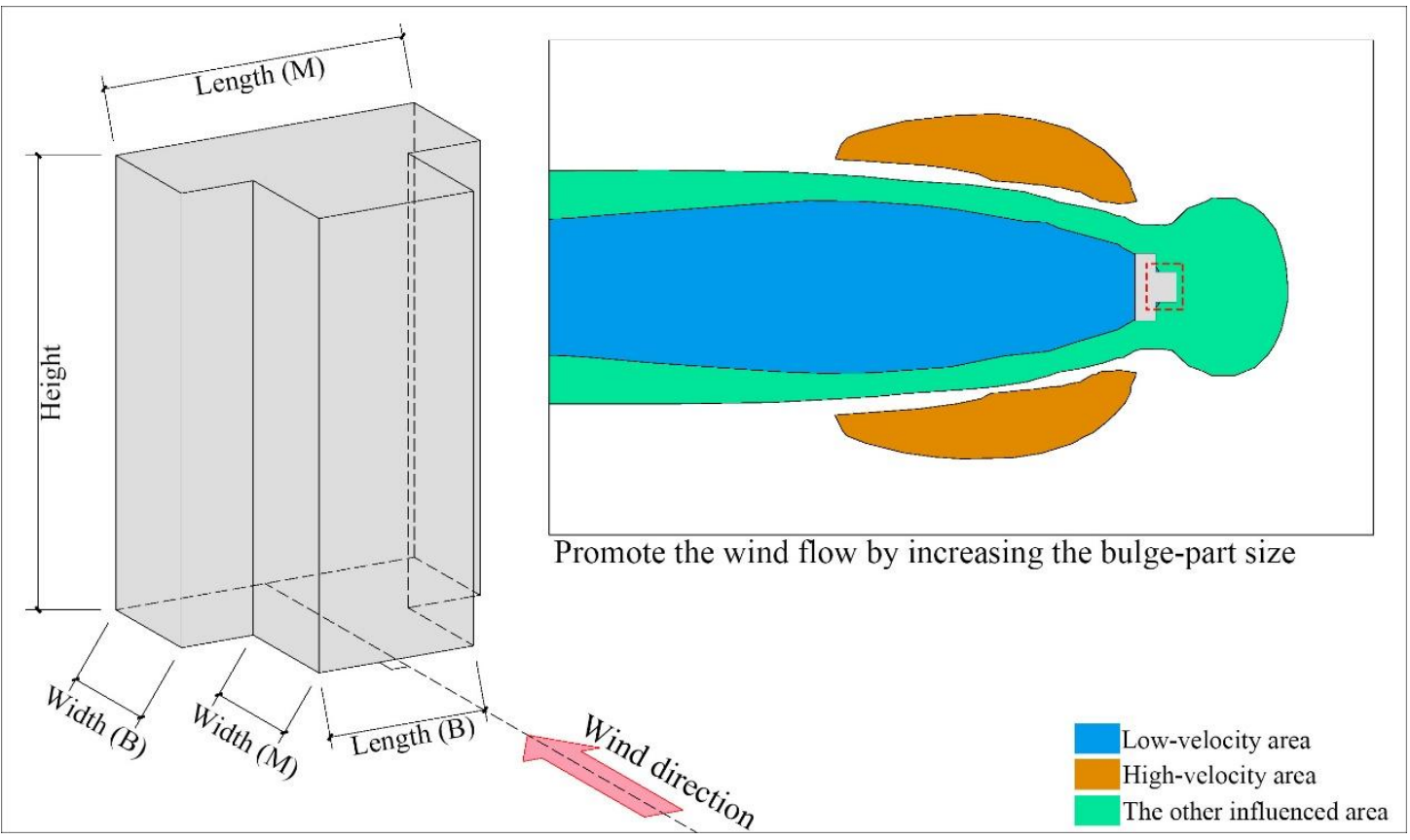

Figure 193. The wind environment influenced by the variables of ' $T$ ' form.

Table 46. Relationships between the influences and building variables of ' $T$ ' form

\begin{tabular}{|l|l|}
\hline Increases of building variables & Influences on outdoor ventilation \\
\hline Bulge-part length (of the short buildings) & Promoted \\
\hline Bulge-part length (of the tall buildings) & Promoted \\
\hline Bulge-part width (of the tall buildings) & Promoted \\
\hline
\end{tabular}

\subsubsection{Summary of the discussion of BUFs}

In summary, the influences on wind environments increase as the height, windward width and length of BUFs are increased, because more winds are obstructed by the increasing windward surfaces. For square and rectangular forms, the outdoor ventilation is improved as the rotation angle increases, because the windward surfaces which are not vertical from the wind directions promote wind flow. When the surface with the width of a rectangular-form building is on the windward side, the influence on the wind environment decreases as the ratio of length and width increases before it reaches a particular value, because the influenced air-pressure area is decreased. For a ' $\mathrm{T}$ '-form building, the influence on the wind environment decreases as the bulge-part size increases, because the increase of bulge-part helps to divide winds and leads the 
winds to flow around the building. The results and analysis of BUFs show that the increase of windward projective area can increase the influence on wind environment, because more winds are obstructed.

The studies of BUFs contributes original knowledge to wind environments of the BUFs of square form, rectangular form and ' $\mathrm{T}$ ' form. The relationships between the influences on wind environments and building variables have been explored. In the study of square form, the influences of the edge length and rotation angle have been studied. In the study of rectangular form, the influences of the length, width, height and rotation angle have been studied. In the study of ' $\mathrm{T}$ ' form, the influences of the bulge-part sizes have been studied. The mechanisms of the influences have been explained by using the air-pressure magnitudes and wind-flow streamlines. On the other hand, there is the potential for the study outcomes to be used for helping in relevant researches and designs of BUFs. The study can give information to the improvement of outdoor wind environments of BUFs.

\subsection{Discussion of BCCs studies}

In the BCCs studies, the relationships between influences on wind environments and building variables are studied for the scattered configuration, linear configuration and curvilinear configuration. The building units in clusters are all set in the same rectangular form. The influences are compared in wind velocity magnitudes, especially for the low-velocity and highvelocity areas. The mechanisms are explained by analyzing air-pressure magnitudes and windstreamlines.

\subsubsection{Discussion of scattered configuration}

In the study of scattered configuration, the relationships between influences on wind environments and building variables are studied in nine parts, including the building unit number (range: 2-9), longitudinal distance (range: 0.7H-1.1H(25.2-39.6m)), transverse distance (range: 10-20m) and staggered distance (range: $10-30 \mathrm{~m}$ ). 
In the first part, three cases with different transverse distances are studied for the building cluster with two building units arranged in a building row. The influence is nearly unchanged. The high-velocity areas are fluctuated as the transverse distance increases. The fluctuations are due to the contrary effects of the increases of windward projective area of the entire building cluster and gap between the adjacent building units. The low-velocity area decreases as the transverse distance increases. Because the low-air-pressure and high-air-pressure areas decrease as the transverse distance increases. And the vortices on the leeward side are reduced. Therefore, the outdoor ventilation is promoted as the transverse distance increases.

In the second part, three cases with different transverse distances are studied for the building cluster with three building units arranged in a building row. The influence increases as the transverse distance increases. The increase of influence is due to the increase of the windward projective area of the entire building cluster. The high-velocity and low-velocity areas are decreased as the transverse distance increases. Because the low-air-pressure and highair-pressure areas decrease as the transverse distance increases. And vortices on the leeward side are reduced. It is easier for more winds to flow through the wider gaps among building units. The outdoor ventilation is promoted as the transverse distance increases.

In the third part, three cases with different longitudinal distances are studied for the building cluster with two building units arranged in a building column. The influence is nearly unchanged. The low-velocity and high-velocity areas increase as the longitudinal distance increases. The low-velocity area decreases because the low-air-pressure area on the leeward side of the building cluster decreases. And less vortices are generated. Therefore, the outdoor ventilation is promoted as the longitudinal distance increases.

In the fourth part, three cases with different longitudinal distances are studied for the building cluster with three building units arranged in a building column. The result is similar to the third part. The changes of influence are marginal. The low-velocity area decreases as the longitudinal distance increases. The high-velocity areas increase as the longitudinal distance increases. The decrease of low-velocity area is due to the increase of low-air-pressure area on the leeward side of the building cluster. And less vortices are generated. The outdoor ventilation is promoted as the longitudinal distance increases.

In the fifth part, nine cases with different transverse and longitudinal distances are studied 
for the building cluster with four building units arranged in two building rows (two columns). As the longitudinal distance increases, the influence increases; the low-velocity area decreases; the high-velocity areas increase. As the transverse distance increases, the influence increases; the low-velocity and high-velocity areas decrease. These are because the low-air-pressure and high-air-pressure areas decrease as the longitudinal and transverse distances increases. And the vortices are reduced. Therefore, the outdoor ventilation is promoted as the transverse and longitudinal distances are increased.

In the sixth part, six cases with different transverse and longitudinal distances are studied for the building cluster with six building units arranged in three building rows (and two columns). As the longitudinal distance increases, the total influence, low-velocity and highvelocity areas all decrease first and then increase slightly; the low-velocity area on the leeward side of the building cluster becomes shorter as the longitudinal distance increases; the velocity increases in the low-velocity area between the building rows. These are because the low-airpressure areas decrease and less vortices are generated. As the transverse distance increases, the influence decreases slightly first and then increases. The differences among the changes are marginal. The increase of the influence is due to the increase of the windward projective area of the entire building cluster. The low-velocity area decreases as the transverse distance increases. The high-velocity areas increase as the transverse distance increases. Because more winds are able to flow through the gap between the building columns. The increase of transverse distance can reduce more vortices and low-velocity area than the increase of longitudinal distance, especially for the low-velocity area behind the building cluster. Therefore, the outdoor ventilation is promoted as the transverse and longitudinal distances increase. And there is more promotion in the increase of transverse distance.

In the seventh part, six cases with different transverse and longitudinal distances are studied for the building cluster with six building units arranged in two building rows (and three columns). As the transverse distance increases, the influence increases; the low-velocity area decreases; the high-velocity area increases. The increase of influence is due to the increased area of the entire building cluster. The decrease of low-velocity area and increase of highvelocity areas are due to the larger gaps among building units which allow more winds to flow through. Because the high-air-pressure and low-air-pressure areas are decreased as the 
transverse distance increases. And the vortices are also reduced. Therefore, the outdoor ventilation is promoted as the transverse distance increases. As the longitudinal distances increases, the influence increases; the low-velocity area decreases; the high-velocity areas increase. The increase of the influence is due to the increase of length of the entire building cluster, which occupies a larger area. The influenced air-pressure areas are reduced slightly. And the vortices are reduced. Therefore, the outdoor ventilation is promoted as the transverse and longitudinal distances increase. The increase of transverse distance mainly focuses on the promotion of outdoor ventilation on the leeward side of the building cluster; the increase of longitudinal distance mainly focuses on the promotion of outdoor ventilation between the building rows.

In the eighth part, six cases with different transverse and longitudinal distances are studied for the building cluster with nine building units arranged in three building rows (and three columns). As the transverse distance increases, the influence increases; the low-velocity and high-velocity areas decrease. The increase of influence is due to the increase of windward projective area of the entire building cluster. Because it is easier for winds to flow through larger gaps among building columns, the high-velocity areas are decreased. The influenced airpressure areas and vortices are reduced as the transverse distance increases. As the longitudinal distance increases, the influence and the low-velocity area are nearly unchanged; the highvelocity areas increase. The velocities in the low-velocity area among building rows are increased. Winds are able to escape more easily from the building cluster as the longitudinal distance increases. Less vortices are generated. Therefore, the outdoor ventilation is promoted as the transverse and longitudinal distances increase.

In the ninth part, three cases with different staggered distances are studied for the building cluster with nine building units arranged in three building rows. The influence and the lowvelocity area increase as the staggered distance increases. Because the windward projective area of the building cluster is increased as the staggered distance increases. The high-velocity areas are fluctuated. Because the increase of windward surfaces of the second and third building rows reduces the high-velocity area on one lateral side of the building cluster. The overall outdoor ventilation is promoted as the staggered distance increases, especially for the spaces among building units. However, the low-velocity area is relatively large on the leeward side of 
the building cluster.

Take a design of scattered-configuration building cluster for example. The outdoor ventilation can be promoted by reducing building-unit numbers, increasing the longitudinal, transverse and staggered distances (Table 47.) (Figure 194). The increase of transverse distance usually has the larger effect of outdoor ventilation promotion than the increase of longitudinal distance, especially for a building cluster with many building units. The increase of transverse distance usually reduces more low-velocity area behind the building cluster. The reduction of low-velocity area between the adjacent building rows caused by the increase of longitudinal distance becomes relatively small as the building columns increases. The outdoor ventilation among building rows is promoted as the staggered distance increases. But the low-velocity area behind the building cluster is relatively large because of the increased windward surface of the building cluster.

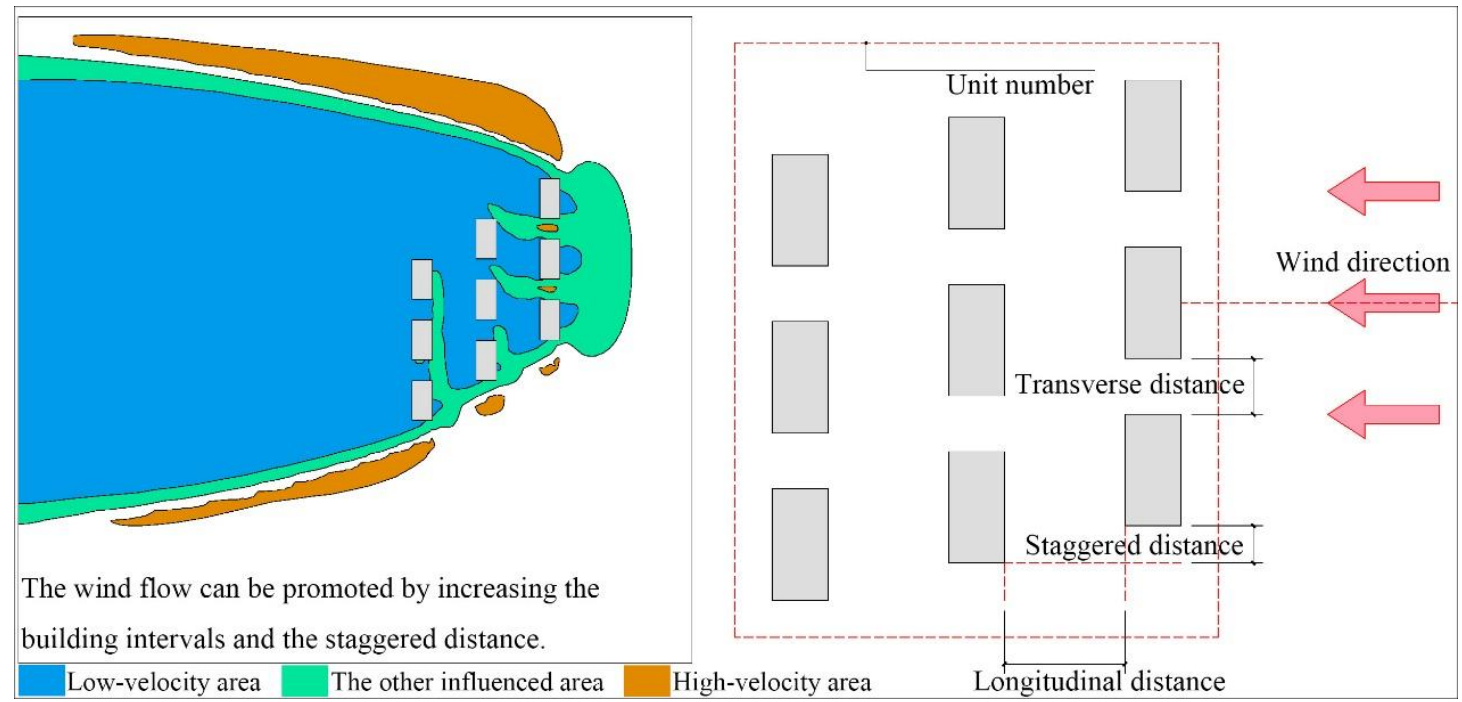

Figure 194. The wind environment influenced by the variables of scattered configuration.

Table 47. Relationships between the influences and variables of scattered configuration

\begin{tabular}{|l|l|}
\hline Increases of building variables & Influences on outdoor ventilation \\
\hline Building-unit number & Weakened \\
\hline Transverse distance & Promoted \\
\hline Longitudinal distance & Promoted \\
\hline Staggered distance & Promoted \\
\hline
\end{tabular}




\subsubsection{Discussion of linear configuration}

In the study of linear configuration, the relationships between the influences on wind environments and the building variables are studied in four parts, including the building unit number (range: 2-9), longitudinal distance (range: 0.7H-1.1H (25.2-39.6m)) and staggered distance (10-30m).

In the first part, three cases with different building-unit numbers are studied for the building cluster with building units arranged in one building row. The influence, low-velocity and high-velocity areas all increase as the unit number increases. The increases of the influence are due to the increased windward projective area of the building cluster. The low-air-pressure and high-air-pressure areas increase as the unit number increases. And vortices are increased. Therefore, the outdoor ventilation is weakened as the unit number increases.

In the second part, six cases with different unit numbers and longitudinal distances are studied for the building cluster with building units arranged in two building rows. As the building-unit number increases, the influence, low-velocity and high-velocity areas all increase. Because windward projective area of the building cluster increases and more winds are obstructed. The low-air-pressure and high-air-pressure areas are increased. And more vortices are generated, especially for the space between the building rows. As the longitudinal distance increases, the influence, low-velocity and high-velocity areas are all increased. But the increases of the influence and low-velocity area are marginal. The increase of influence is largely due to the increase of low-velocity area. Because the low-velocity area between the building rows increases as the longitudinal distance increases. However, the velocity increases in the low-velocity area between the building rows; the low-velocity area on the leeward side of the building cluster are decreased. And less vortices are generated. The low-air-pressure and high-air-pressure areas are nearly unchanged. Therefore, the outdoor ventilation is promoted as the longitudinal distance increases. But the promotion is limited, especially for the low-velocity area between the building rows with a relatively long length.

In the third part, six cases with different unit numbers and longitudinal distances are 
studied for the building cluster with building units arranged in three building rows. As the building-unit number increases, the influence, low-velocity and high-velocity areas are increased. The increases are due to the increase of the windward projective area of the building cluster surface. The low-air-pressure and high-air-pressure areas are also increased. And more vortices are generated. Therefore, the outdoor ventilation is weakened as the building-unit number increases. As the longitudinal distance increases, the influence and high-velocity areas increase; the low-velocity area is nearly unchanged. The increase of influence is largely due to the increase of high-velocity areas. And the low-velocity area on the leeward side of the building cluster decreases. Because the low-air-pressure area decreases as the longitudinal distance increases. The increase of the air-pressure difference lead to the increases of highvelocity areas. And less vortices are generated. Therefore, the outdoor ventilation is promoted as the longitudinal distance increases. But the promotion of outdoor ventilation is limited among the building rows.

In the fourth part, three cases with different staggered distances are studied for the building cluster with nine building units arranged in three building rows. As the staggered distance increases, the influence and low-velocity area increase. Because the windward projective area of the building cluster is increased. The high-velocity areas are fluctuated as the staggered distance increases. Because more winds are obstructed by the larger windward surfaces of the second and third building rows on one lateral side of the building cluster. The high-air-pressure and low-air-pressure areas increase as the staggered distance increases. The increase of the lowair-pressure area on the leeward side of the building cluster leads to the increase of low-velocity area on the leeward side. This is due to the increase of the low-air-pressure area behind the building cluster. But the low-air-pressure area among the building rows decreases and the velocity of the area increases. Vortices are reduced as the staggered distance increases. The overall outdoor ventilation is promoted as the staggered distance increases, especially for the spaces among building rows. But the low-velocity area on the leeward side of the building cluster is relatively large.

Take a design of linear-configuration building cluster for example. The outdoor ventilation can be promoted by reducing the unit number of building row, increasing the longitudinal and staggered distances (Table 48.) (Figure 195). The decrease of the unit number can promote the 
outdoor ventilation significantly, because the windward area decreases as the length of building row is reduced. The outdoor ventilation promotion caused by the increase of longitudinal distance is relatively limited due to the long length of building row. The outdoor ventilation among building rows is promoted as the staggered distance increases. But the low-velocity area behind the building cluster is relatively large due to the increase of the windward surface of the building cluster.

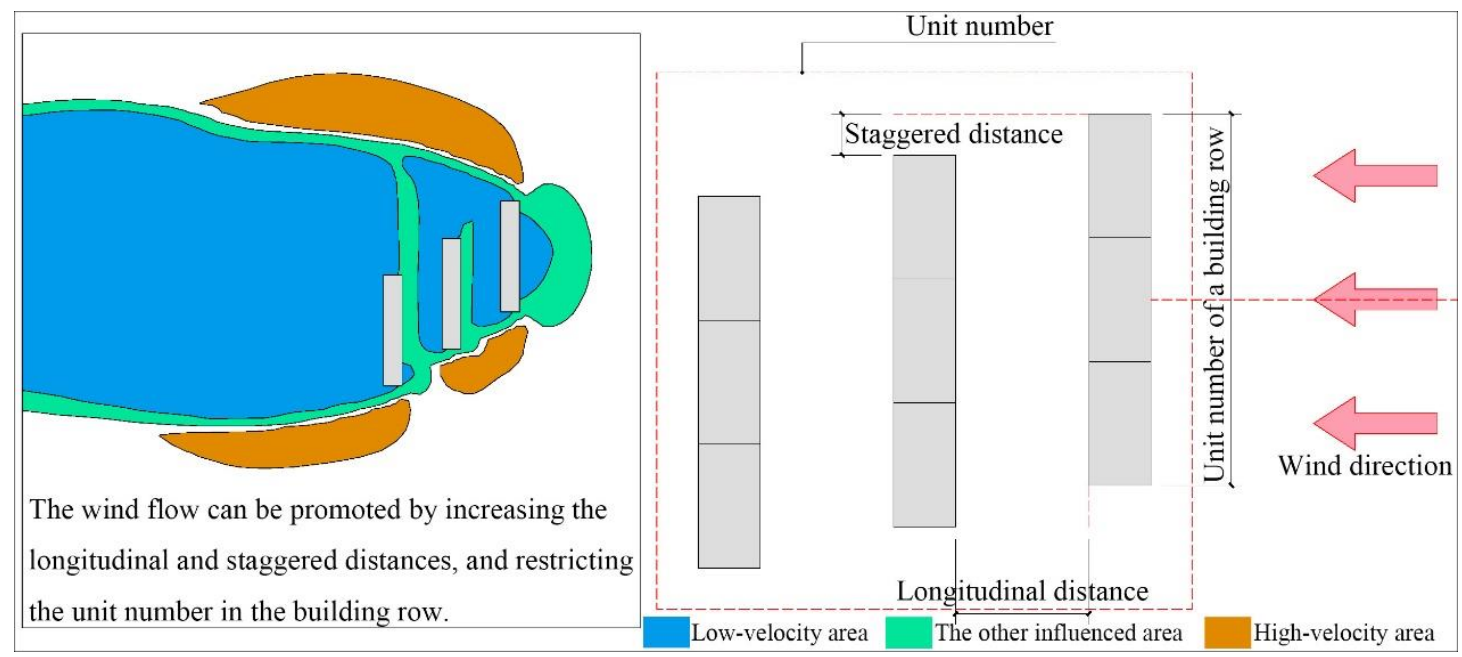

Figure 195. The wind environment influenced by the variables of linear configuration.

Table 48. Relationships between the influences and variables of linear configuration

\begin{tabular}{|l|l|}
\hline Increases of building variables & Influences on outdoor ventilation \\
\hline Building-unit number & Weakened \\
\hline Longitudinal distance & Promoted \\
\hline Staggered distance & Promoted \\
\hline
\end{tabular}

\subsubsection{Discussion of curvilinear configuration}

In the study of curvilinear configuration, the relationship between the influence on wind environments and the building variable of central angle (range: 10-90 degree) is studied in two parts. The central angle of the arc is related to the curvature of the curvilinear building row.

In the first part, nine cases with different central angles are studied for the building cluster 
with building units arranged in a curvilinear building row. The convex surface of the building row is on the windward side. As the central angle increases, the influence increases with some fluctuations; the low-velocity area decreases; the high-velocity areas increase. The increase of influence is largely due to the increase of high-velocity areas. As the central angle increases, the low-air-pressure and high-air-pressure areas are decreased, especially for the low-airpressure area behind the building cluster. And the vortices are reduced. These leads to the decrease of the low-velocity area. It is easier for winds to flow around the convex surface as the central angle increases. Winds are accelerated on the lateral sides which increases the highvelocity areas. Therefore, the outdoor ventilation is promoted as the central angle increases.

In the second part, nine cases with different central angles are studied for the building cluster with building units arranged in a curvilinear building row. The concave surface of the building row is on the windward side. As the central angle increases, the influence increases slightly; the low-velocity area increases first and then decreases; the high-velocity areas increase. In general, the overall wind velocities increase slightly. The reason should be the decrease of the windward projective area of the entire building cluster. As the central angle increases, the low-air-pressure areas on leeward side and high-air-pressure areas on windward side are increased. The air pressures in the low-air-pressure area is decreased. The sizes of vortices increase. The concave surface on the windward side obstructs the wind flow. It increases the resistance of air flow which is similar to a parachute. Therefore, the outdoor ventilation is weakened as the central angel increases. The effects on winds are contrary in the two parts with different surfaces on the windward side.

Take a design of curvilinear-configuration building cluster for example. When the convex surface of the curvilinear building row is on the windward side, the outdoor ventilation can be promoted by increasing the central angle. Because the convex surface can be regarded as a surface consisted of countless small flat surfaces. The increase of the central angle can reduce the angle between the windward surfaces and the wind direction, which makes it easier for winds to flow around the convex surface. When the concave surface of the curvilinear building row is on the windward side, the outdoor ventilation can be promoted by reducing the central angle. The concave surface is similar to a parachute which increases the resistance of air flow. Therefore, it is more difficult for wind to flow around the concave surface (Figure 196) (Table 


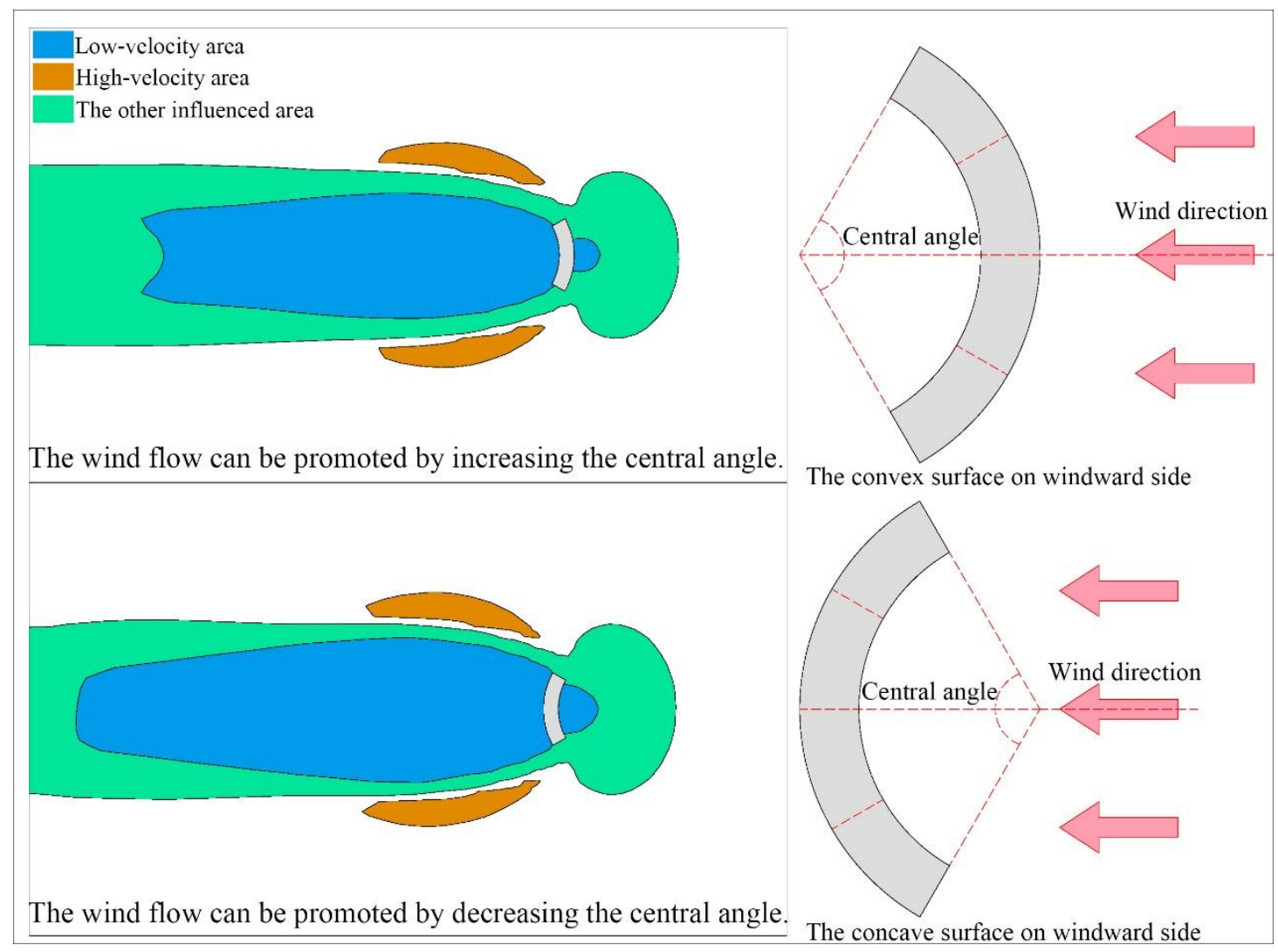

Figure 196. The wind environment influenced by the variables of curvilinear configuration.

Table 49. Relationships between the influences and variables of curvilinear configuration

\begin{tabular}{|l|l|}
\hline Increases of building variables & Influences on outdoor ventilation \\
\hline Central angle (the convex surface on the windward side) & Promoted \\
\hline Central angle (the concave surface on the windward side) & Weakened \\
\hline
\end{tabular}

\subsubsection{Summary of the discussion of BCCs}

In summary, the outdoor ventilation of scattered configuration should be the best, because it is relatively easy for winds to flow around scattered building units. For building rows, the outdoor ventilation can be improved as the longitudinal distance and the staggered distance are increased, because the larger building intervals promote winds to flow through the building cluster. For curvilinear configuration, the convex surface on the windward side can promote the 
wind flow and the concave surface on the windward side can obstruct the wind flow. As the central angle of the curvilinear building row increases, the effects of both situations are increased. The results of BCCs show that the increase of windward projective area of buildings can increase the influences on wind environments, because more winds are obstructed.

The study of the BCCs contributes original knowledge of wind environments of the building clusters of scattered configuration, linear configuration and curvilinear configuration. The relationships between the influences on wind environments and the building variables have been explored. In the study of scattered configuration, the influences of the unit number, longitudinal distance, transverse distance and staggered distance have been studied. In the study of linear configuration, the influences of the unit number, longitudinal distance and staggered distance have been studied. In the study of curvilinear configuration, the influence of the central angle has been studied. The mechanisms of the influences have been explained by using the air-pressure magnitudes and wind-flow streamlines. On the other hand, there is the potential for the study outcomes to be used for helping in relevant researches and designs of BCCs. The study can give information to the improvement of outdoor wind environments of BCCs.

\subsection{Limitations}

In the methodology, parametric tools mainly help in collecting building information, mesh generation for CFD simulation and result analysis. There are limitations in direct improvement of algorithms in mesh generation and CFD simulation due to differences among platforms. The mesh generation is to create grids for finite element analysis. The parametric modelling only generates building models automatically. It does not improve the method of grid creation. In this research, the parametric models have not been tested in multiple platforms such as other CFD software. Though RANS method is only tested in CFD simulations for the methodology, it is believed other CFD methods can be used as well.

For parametric design, the building information are only collected in the Optic Valley Area. Though a large amount of buildings is collected, the situations of other cities in the HSCWA might be different. The parametric design system is only developed for square form, rectangular 
form, ' $\mathrm{T}$ ' form, scattered configuration, linear configuration and curvilinear configuration. The parametric models are simplified for CFD simulations. But the simplification makes the research outcome more applicable to general buildings instead for specific buildings.

In the study of BUFs, the study only focuses on the wind environments of square form, rectangular form and ' $\mathrm{T}$ ' form. For the three BUFs, the study only considers the building variables as mentioned in the previous Section 7.3.. The building variables are in the certain ranges and the cases in some groups are limited. The study of ' $\mathrm{T}$ '-form building focuses on the influences of different sizes of the bulge part. Other influences such as the sizes of the major part have not been considered.

In the study of BCCs, the study only focuses on the wind environments of scattered configuration, linear configuration and curvilinear configuration. For the three BCCs, the study only considers the building variables as mentioned in the previous Section 7.4.. And the building variables are in the certain ranges and the cases in some groups are limited. The building unit in the building cluster are in the same shape as rectangular form. Other building forms which may have different influences have not been studied. Experiments may be needed to validate the simulation results in the future, especially for the curvilinear configuration which is different from the other BCCs.

\subsection{Future works}

For research methodology, other numerical models can be tested to further enhance accuracy and save time of simulations. Scripts of suitable computational method can be created to adapt to specific studies. Other novel tools can be integrated for different study purposes. The methodology can be applied for studying different BUFs and BCCs in other regions. Future research can consider residential buildings in different cities. There are potentials to apply the methodology for other sustainable studies by using different analysis tools. Such as daylighting and sound performance, they are all relevant to building forms and configurations. For parametric design, building information in more cities can be collected and analyzed. Architectural morphology can be expanded including more BUFs and BCCs in the 
categorization study. The parametric modelling system can be expanded by developing modelling scripts of more BUFs and BCCs. For the future study of BUFs, the other BUFs and more variables can be studied. The study of ' $\mathrm{T}$ '-form building can be expanded by considering other influences such as the sizes of the major part and rotation angles. More building details can be considered in future studies. Influences of the other specific designs or architectural components such as balcony, open floor and some decorations can be studied. For the future study of BCCs, the other BCCs and more building variables can be studied. The building clusters with building units in the other BUFs can be studied. There is a need to study the composite configuration with a combination of different BCCs. The other civil buildings in the urban area can also be studied. They may be similar in forms and configurations, but the architectural scales should be different from residential buildings.

\subsection{Summary of discussion}

In the Discussion Chapter, the research outcomes including the research methodology, the parametric design, the BUFs studies and the BCCs studies have been discussed respectively. In the Discussion of BUFs Studies, the outcomes of the square form, rectangular form, and ' $T$ ' form have been discussed; in the Discussion of BCCs Studies, the scattered configuration, linear configuration, and curvilinear configuration have been discussed. Then the limitations of the research have been discussed. The future works have been discussed at the last. Based on the discussions, the findings and the contributions of the research will be concluded in the following Conclusion Chapter. 


\section{Conclusions}

In this research, the relationships between influences on wind environments and variables related to the representative forms and configurations of high-rise residential buildings in the HSCWA are discovered. It illustrates how wind environments are influenced by the contemporary high-rise residential buildings. The research covers the BUFs of square form, rectangular form and ' $\mathrm{T}$ ' form, and the $\mathrm{BCC}$ of scattered configuration, linear configuration and curvilinear configuration. A novel research methodology has been developed to enable high-efficient iterative analysis to evaluate the building influences on wind environments quantitatively. A parametric design system is established for the representative BUFs and BCCs, which makes the research outcome applicable to general architectural practices. The influences of building variables of BUFs and BCCs have been compared in the iterative analysis, which illustrates the trends and mechanisms of the influences. In this way, the relationships between the influences on wind environments and building variables are discovered. In the conclusion chapter, findings and contributions of this research are presented respectively.

\section{1. $\quad$ Findings}

In this section, findings of this research are presented, including a novel research methodology, a parametric design system, and relationships between the influences on wind environments and building variables of the BUFs and BCCs.

\subsubsection{A novel research methodology}

In this research, a novel research methodology was developed by integrating multiple computational tools work in coordination. Its effectiveness and correctness have been demonstrated in the wind environment studies of BUFs and BCCs. It is consisted of parametric design, simulation, and analysis. First, parametric modelling scripts are developed based on categorization study to create building models. Second, architectural wind environment is 
simulated by using the CFD method. Third, simulation results are analyzed based on the criteria. The accuracy of reliability of simulation results was validated by grid independent study and experimental data. In the methodology, the multiple computational tools enable a high-efficient modelling-simulation-analysis process for iterative analysis by helping in statistics of building categorization, parametric modelling and analyzing results. The CFD simulation provides results of wind-velocity magnitudes, air-pressure magnitudes and wind-flow streamlines. The influences on wind environments are evaluated by calculating the pixel numbers of windvelocity magnitude maps and converting them into actual influenced areas. The further wind environment studies show the methodology are accurate and efficient for iterative analysis of wind environments of contemporary buildings with different parameters related to forms and configurations. With the aid of the novel methodology, new knowledge that has not been found by existing methods is discovered.

\subsubsection{A parametric design system}

The establishment of the parametric design system bridges the building variables and computational simulations. The system was established based on the building categorization study. In the building categorization study, morphological attributes of the buildings are studied. A series of representative forms and configurations of high-rise residential buildings in the HSCWA are designed for CFD simulations. Clear definitions of eleven BUFs and four BCCs are given based on architectural morphology. The popularity of BUFs and BCCs in the area are studied. The numbers of BUFs and BCCs are calculated statistically. It is discovered that the most popular three BUFs are square form, rectangular form and ' $\mathrm{T}$ ' form; and the most popular three BCCs are scattered configuration, linear configuration and curvilinear configuration. Those BUFs and three BCCs are selected for study due to their representativeness. The representative ranges of common building variables of the three BUFs and three BCCs are summarized based on building information collection and previous studies. In the parametric modelling of the BUFs and BCCs, the modelling scripts are developed using logical algorithms based on their geometric attributes. In the end, the building models can be created automatically 
and adjusted easily to improve the efficiency of mesh generation for CFD simulations. In the iterative analysis of this research, using the parametric design system saved more than half time than using conventional manual modelling.

\subsubsection{Relationships between the influences and variables of the BUFs}

In the BUFs studies, the methodology was applied to studying the relationships between influences on winds and building variables of square form, rectangular form and ' $\mathrm{T}$ ' form. The relationships are discovered after the analysis of results. The overview study shows how winds are obstructed by buildings. There are high-velocity areas on the lateral sides and a low-velocity area on the leeward side. And vortices are created behind the building. The studies of the three BUFs show how those influences were changed as the building variables were changed. In particular, the influences on wind-velocity magnitudes on pedestrian level were analyzed quantitatively. It was found that the wind-velocity magnitudes were closely related to airpressure magnitudes and wind-flow streamlines.

\section{(1) Square form}

For square form, influences of edge lengths (range: 10-30m) and rotation angles (angle: 0-45 degree) have been studied respectively.

The increase of edge length increases the influence on wind environment, and the decrease has the contrary effect. Because the obstruction of winds is directly related to windward surfaces. The increase of rotation angle encourages wind flow. Because it is easier for winds to go around windward surfaces which are not vertical from the wind directions. The outdoor ventilation can be promoted by increasing the edge length and rotation angle.

\section{(2) Rectangular form}

For rectangular form, influences of lengths (range: 10-40m), widths (range: 10-20m), heights (range: $36-72 \mathrm{~m}$ ) and rotation angles (range: 0-90 degree) have been studied respectively.

When the surface with the length is on the windward side, the increases of length increase 
the total influences on wind environments. Both the low-velocity and high-velocity areas are increased. Because more winds are obstructed by the larger windward surfaces. The increase of width reduces the influence on wind environment, especially for the low-velocity area. Because the low-air-pressure area decreases and vortices become less behind the building.

When the surface with the width is on the windward side, the results are contrary to the situation with the length on the windward side. The influences become much less due to the reduction of windward projective area. The increases of length and decrease of width can reduce the influence. Because the influenced air-pressure areas are decreased. However, the reduction is not constant as the length increases. The least influence is decided by a particular ratio of width and length. For the wind condition in this research, the ratio is approximately 1/3.

In the both situations above, the increase of height increases the total influence. Because more winds are obstructed by the larger windward surfaces. Similar to the square from, the influence decreases as the rotation angle increases. Wind flow is promoted because it is easier for winds to go around windward surfaces which are not vertical from the wind directions.

Therefore, recommendations of rectangular-form building design can be summarized for wind-flow promotion: (1) when the surface with the length is on the windward side, the outdoor ventilation can be promoted by decreasing the length, increasing the width, decreasing building height and rotating the building (up to 90 degree); (2) when the surface with the width is on the windward side, the outdoor ventilation can be promoted by increasing the length and decreasing the width. But the most promotion of outdoor ventilation is decided by a particular ratio of width and length (approximately $1 / 3$ in this research).

\section{(3) ' $T$ ' form}

The study of ' $T$ ' form focuses on the influences of various sizes of the bulge parts. Two kinds of ' $\mathrm{T}$ '-form buildings with two typical heights $(36 \mathrm{~m}$ and $72 \mathrm{~m})$ are studied. The bulge parts are on the windward side and their windward surfaces are vertical from the wind direction. Both the increases of length and width of the bulge part can reduce the influences on wind environments. The low-velocity and high-velocity areas are decreased. The analysis of windflow streamlines suggests that the bulge part helps to divide the winds and guide them to go around the building. As the bulge-part size increases, the low-air-pressure and high-air-pressure 
areas decrease; and the vortices become smaller on the leeward side. Therefore, the outdoor ventilation can be promoted by increasing the bulge-part sizes of ' $\mathrm{T}$ '-form buildings, including the length and width of the bulge-part.

\section{(4) Summary of BUFs}

The findings of the BUFs studies can be summarized as follows:

(1) Generally, the increases of windward projective surface areas of buildings increase the influence on wind environment (Figure 197). As shown in the BUFs studies, the influences increase as the heights and lengths (or widths) on windward sides are increased. Especially, the low-velocity areas on the leeward sides and high-velocity areas on the lateral sides are increased significantly. Because more winds are obstructed by the increased windward surface of the buildings.

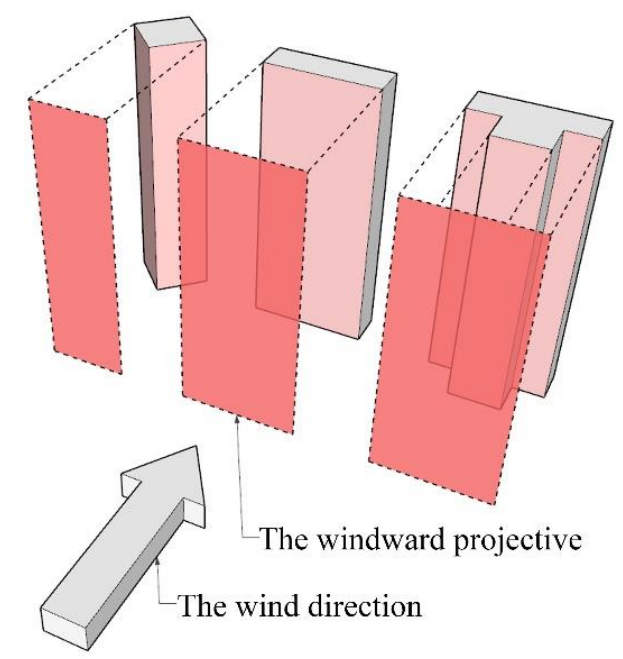

Figure 197. The influences on wind environments are relevant to the windward projective areas.

(2) The influence on wind environment is relevant to the ratio of length and width of a rectangular-form building (Figure 198). The minimum influence is decided by a certain ratio. As shown in the study of rectangular form, when the surface with the width is on windward side, the least influence is decided by a particular ratio of width and length. The ratio is approximately $1 / 3$ in this research. 


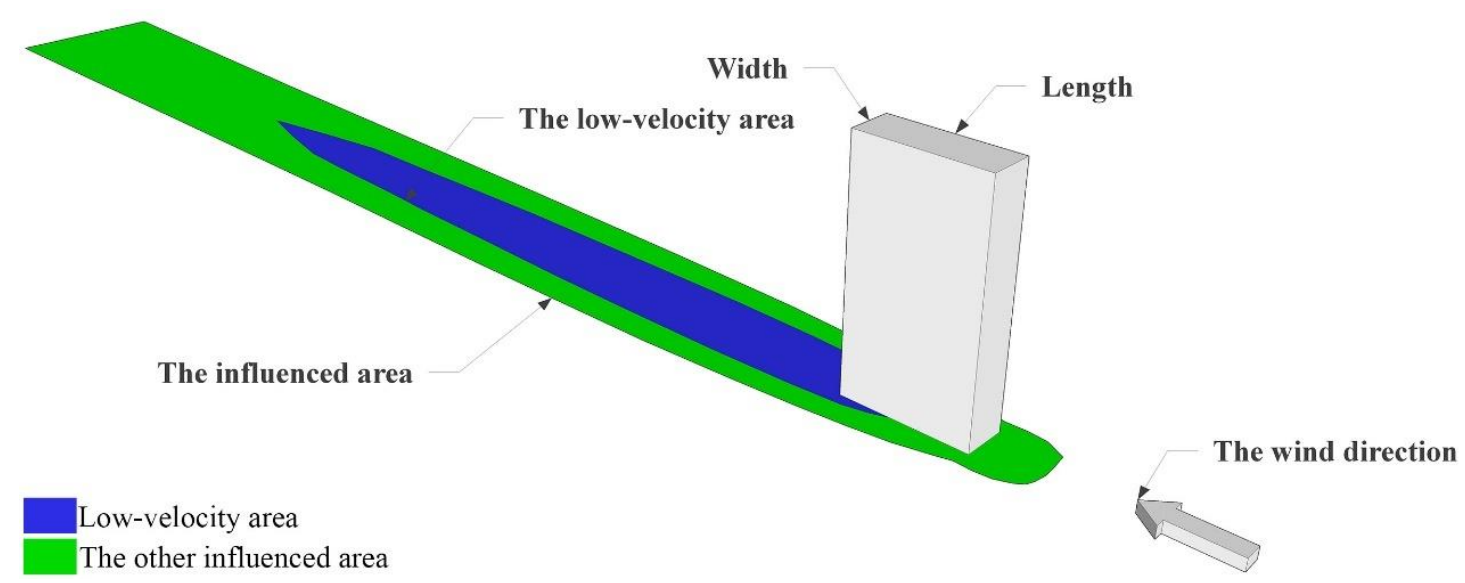

Figure 198. The wind environment influenced by the rectangular-form building (the surface with the width on the windward side).

(3) Studies of BUFs with various rotation angles (wind directions) show that it is relatively easier for winds to go around windward surfaces which are not vertical from wind directions (Figure 199). The outdoor ventilation can be improved by rotating the buildings appropriately. Sometimes the increase of influence is due to the increase of high-velocity areas. The study of square-form building with increasing rotation angle is such an example.

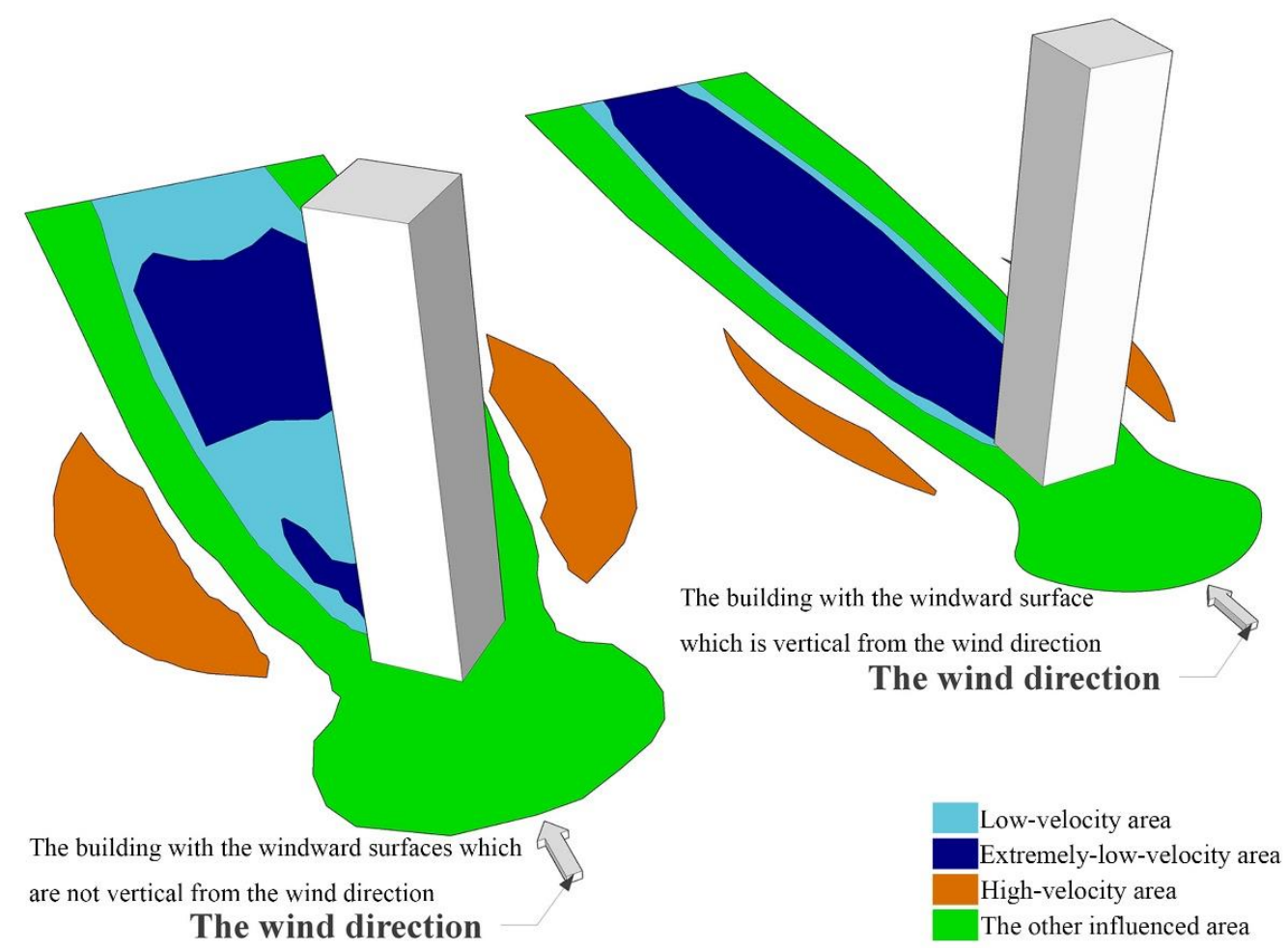

Figure 199. The comparison of the square-form buildings with the surfaces which are vertical 
and not vertical from the wind direction.

(4) The influences on wind environments decreases as the bulge-part sizes of ' $T$ '-form buildings increase (Figure 200). Especially the low-velocity area on the leeward side drops significantly. Because the increase of bulge-part size helps to divide winds and lead them to flow around the building.

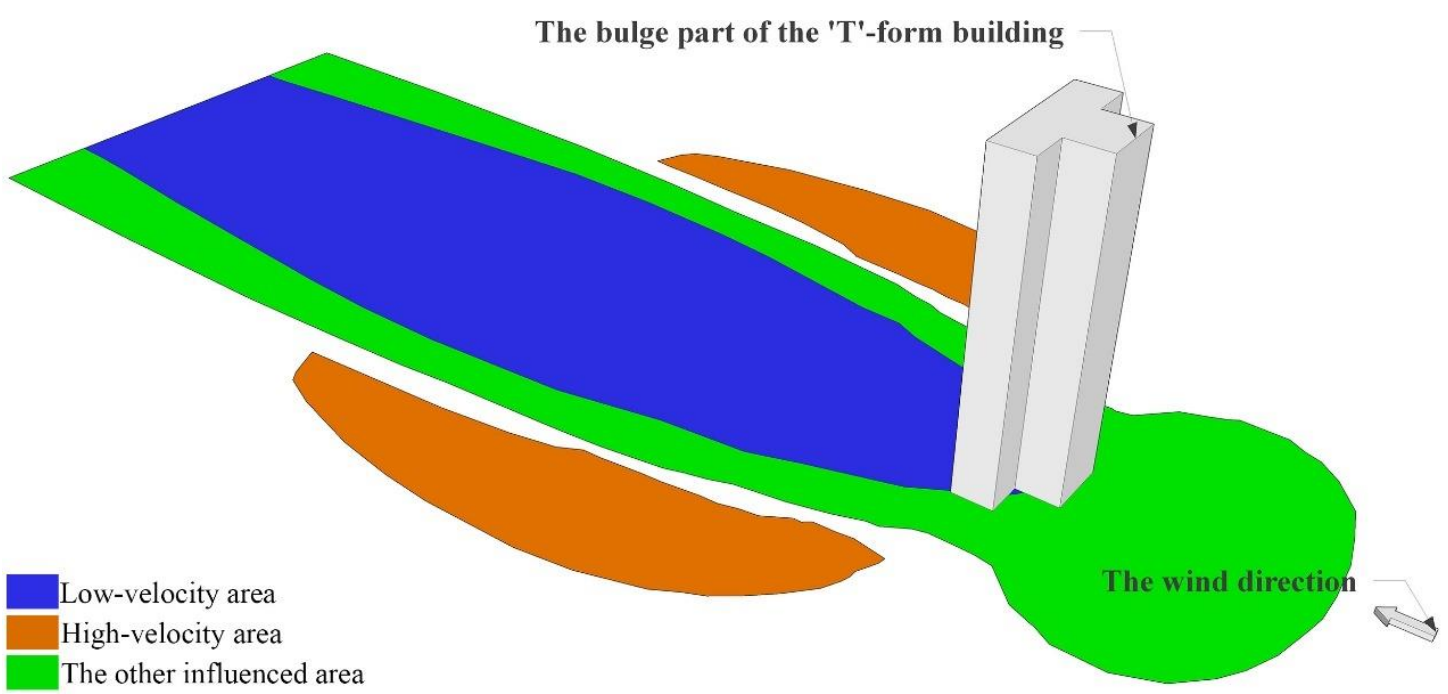

Figure 200. The wind environment influenced by the ' $T$ '-form building.

\subsubsection{Relationships between the influences and variables of the BCCs}

In the BCCs studies, the methodology was applied to studying the relationships between influences on winds and building variables of scattered configuration, linear configuration and curvilinear configuration. There are rectangular-form building units in all the building clusters. The studies of the three BCCs show how the influences were changed as the building variables were changed. Similar to BUFs studies, the influences on wind-velocity magnitudes on pedestrian level were analyzed quantitatively. The air-pressure magnitudes and wind-flow streamlines are used to explain the mechanisms.

\section{(1) Scattered configuration}

For scattered configuration, influences of the building-unit numbers (2-9), transverse distances 
(range: $10-20 \mathrm{~m}$ ), longitudinal distances (range: $0.7 \mathrm{H}-1.1 \mathrm{H}(25.2-39.6 \mathrm{~m})$ ) and staggered distances (range: 10-30m) have been studied respectively.

It is found that the overall influence is directly proportional to the windward projective area of the entire building cluster. The influence increases as the area increases. This is aligned with previous research, which demonstrates the correctness and effectiveness of the novel methodology used in this research. With the aid of the methodology, there is new knowledge discovered for the scattered configuration.

Generally, the increase of building unit increases the influenced area. The number of building row or column will increase as the unit number increases. Because the area on horizontal plane of the entire building cluster is increased.

Larger building interval promotes winds to flow through the building cluster. But the promotion of wind flow caused by the increase of longitudinal distance becomes limited when the unit numbers are increased (especially for the increase of building rows). According to the analysis of the wind velocity magnitudes, air pressure magnitudes and wind flow streamlines, it is relatively difficult for the fresh winds to flow into the low-velocity areas between the adjacent building rows as the unit number increases. In the building cluster with many units, it is recommended to increase the transverse distance to promote the outdoor ventilation.

The increase of staggered distance can make the wind velocity faster among building units. But the total influence and low-velocity areas increases at the same time. This is due to the increase of the windward projective area of the entire building cluster. In the practical design, further tests are needed for specific cases of staggered building rows to ensure the effect of wind flow promotion.

\section{(2) Linear configuration}

For linear configuration, influences of unit numbers (2-9), longitudinal distances (range: $0.7 \mathrm{H}$ $1.1 \mathrm{H}(25.2-39.6 \mathrm{~m}))$ and staggered distances (range: 10-30m) have been studied respectively.

Similar to the scattered configuration, the overall influence is directly proportional to the windward projective area of the entire building cluster. The increase of influence is more significant than the scattered configuration as the area increases. Because there are not any gaps in a linear building row for winds to pass through. The increase of building unit generally 
increases the influenced area. Because the area on horizontal plane of the entire building cluster is increased; or the building-row length is increased. This is aligned with previous research, and again demonstrates the correctness and effectiveness of the methodology. With the aid of the methodology, some new knowledge was discovered for the linear configuration.

The increase of longitudinal distance focuses on the reduction of low-velocity area behind the building cluster. Because the low-air-pressure area becomes shorter as the longitudinal distance increases. The increase of longitudinal distance can promote winds to flow through the space(s) between the adjacent building rows. Compared with the scattered configuration, the promotion is very limited. According to the analysis, it is difficult for the fresh winds to flow into the low-velocity areas between the adjacent building rows as the unit number increases. Therefore, the unit number of a building row should be controlled for the linear configuration. It is recommended to restrict the unit number to two in a linear building row.

The increase of staggered distance can reduce the low-velocity areas in the space(s) between the adjacent building rows. Because the winds are guided into the low-velocity areas by the staggered building rows. And the promotion of wind flow in the areas is more significant than the scattered configuration. But the total influence and low-velocity areas behind the building cluster increases at the same time due to the increase of the windward projective area of the entire building cluster. It is recommended to use the staggered building rows in linearconfiguration building clusters. Further tests are needed for specific cases in the practical design to ensure the overall effect of wind flow promotion.

\section{(3) Curvilinear configuration}

The study of curvilinear configuration focuses on the influences of various central angles (range: 10-90 degree). The curvilinear building rows with the convex surfaces on the windward side and the curvilinear building rows with the concave surfaces on the windward side have been compared. This is an original study that contributes new knowledge for wind-environment studies of curvilinear configuration.

When the convex surface of the curvilinear building row is on the windward side, the outdoor ventilation is generally promoted as the central angle increases. Because the lowvelocity area decreases and high-velocity areas increase. The total influence increases with 
some fluctuations because of the increase of the areas with relatively high velocities. According to the analysis of air-pressure magnitudes and wind-flow streamlines, the low-air-pressure area on the leeward side decreases and vortices becomes smaller as the central angle increases. Therefore, the increase of central angle promotes winds to flow around the convex surface.

When the concave surface of the curvilinear building row is on the windward side, the outdoor ventilation is generally weakened as the central angle increases. Because the lowvelocity area is larger than the curvilinear building row with the concave surface on the windward side; the increase of low-velocity area is more than the increase of the high-velocity areas. There are fluctuations of the low-velocity area, which are due to the decrease of windward projective area and influence of lateral surfaces of the curvilinear building row. According to the analysis of air-pressure magnitudes and wind-flow streamlines, the low-airpressure area is increased and the air pressure is decreased on the leeward side. The increase of the central angle makes more winds filled in the concave surface, which is similar to a parachute.

In summary, the wind flow and outdoor ventilation are promoted by the convex surface; the wind flow is obstructed by the concave surface and the outdoor ventilation is weakened; the increase of the central angle increases the effects respectively in two situations.

\section{(4) Summary of BCCs}

The findings of the BCCs studies can be summarized as follows:

(1) The increase of the windward projective area of the entire building cluster usually increases the total influence. The area covers the windward surfaces of buildings and the gaps among the buildings (Figure 201). As shown in the BCCs studies, the influences increase as the building units in the building row on the windward side are increased. The influences increase as the staggered distances are increased. Because the low-velocity areas behind the building clusters increase significantly. The influences sometimes increase as the transverse distances increase in the scattered-configuration building clusters. Though the low-velocity areas behind the building clusters are decreased, the high-velocity areas in the gaps among the buildings are increased. 


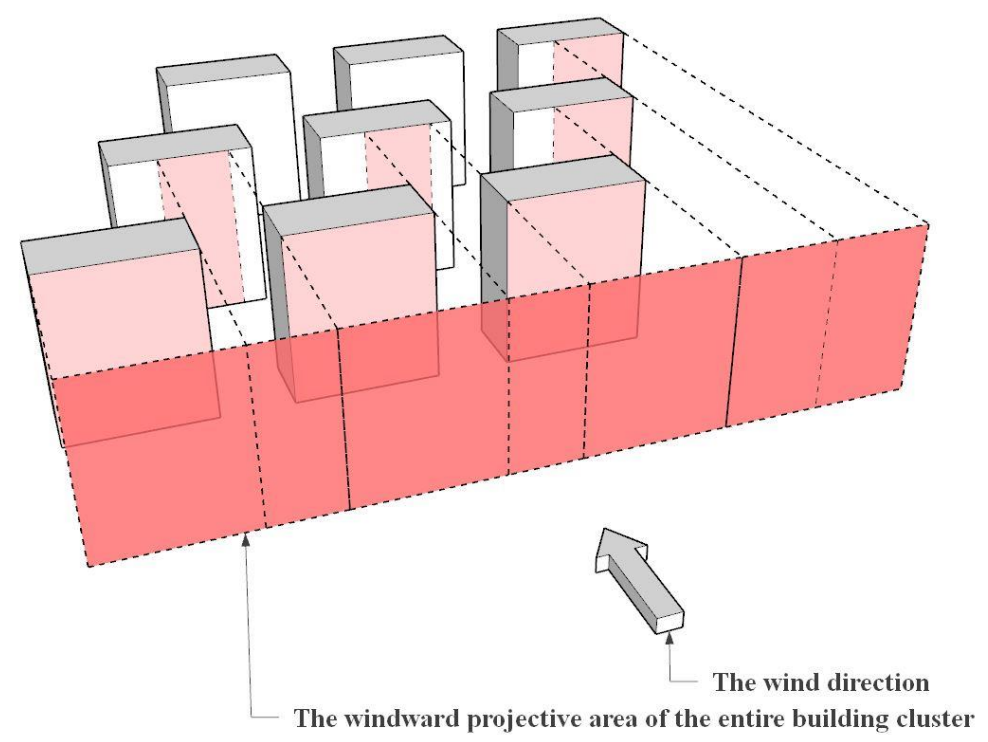

Figure 201. The windward projective area of the entire building cluster.

(2) Larger building intervals (such as the increases of the transverse distance and the longitudinal distance) promote winds to flow through the scattered-configuration building clusters. However, the promotion of wind flow caused by the increase of longitudinal distance becomes limited when unit numbers are increased, especially for the increase of building rows (Figure 202). It is recommended to increase the transverse distance to promote the outdoor ventilation for the building clusters with many units.

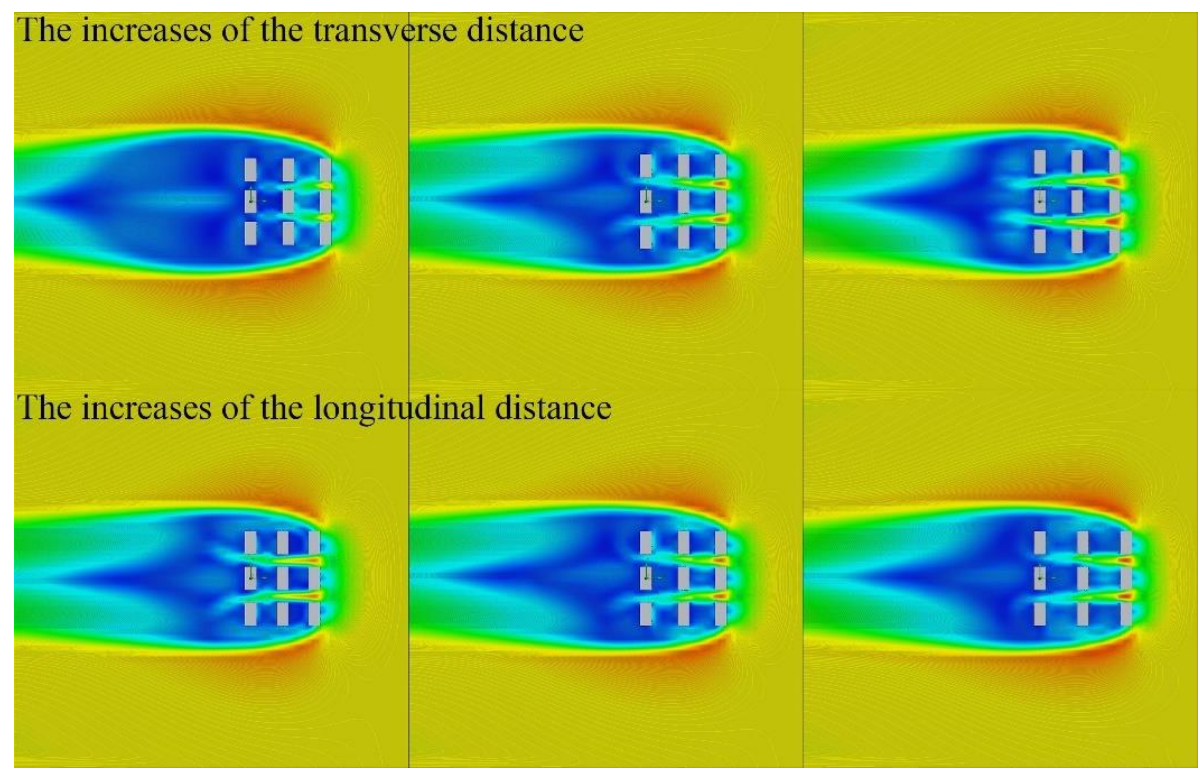

Figure 202. The comparison of the influences of the increases of the longitudinal and transverse distances of the scattered-configuration building cluster. 
(3) The increase of staggered distance can promote the wind flow among the building units. Especially for linear configuration, an appropriate staggered distance can significantly increase the wind velocities in the spaces among the linear building rows. However, the total influence and the low-velocity area are increased at the same time (Figure 203). This is due to the increase of the windward projective area of the entire building cluster. It is recommended to further test specific cases for practical designs to ensure the overall windflow promotion, especially for the scattered-configuration building clusters.
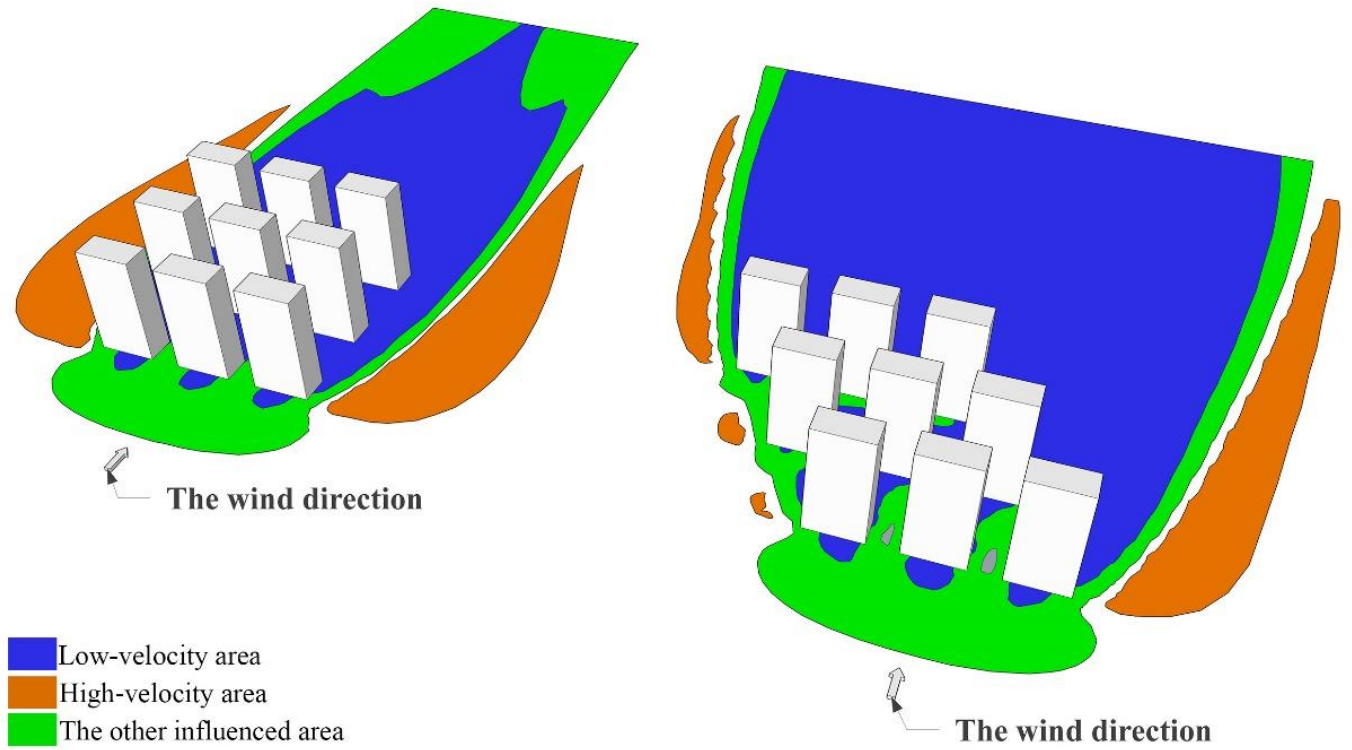

Figure 203. The comparison of the influences of the normal scattered configuration and the staggered configuration.

(4) For curvilinear configuration, the convex surface on the windward side can promote wind flow; the concave surface on the windward side can obstruct wind flow (Figure 204). The promotion and obstruction are directly proportional to the central angle. It is recommended to design the curvilinear building row with the convex surface on the windward side and the concave surface on the leeward side for better outdoor ventilation. 


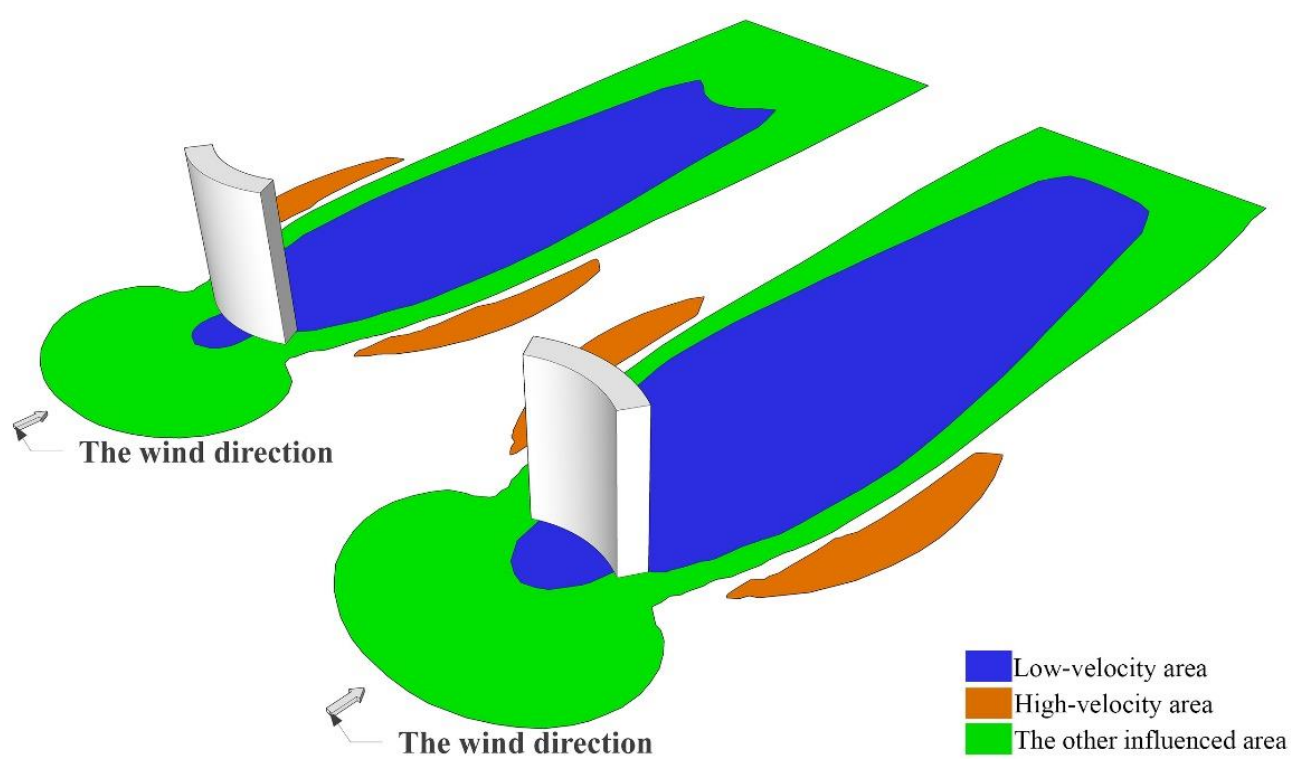

Figure 204. The comparison of the influences of the curvilinear building rows with the convex and concave surfaces on the windward side.

In the comparison of the three $\mathrm{BCCs}$, the outdoor ventilation of the scattered configuration is usually the best of all. It is relatively easy for winds to flow through the gap(s) between the adjacent building units. The scattered configuration is recommended for the high-rise residential communities. For the linear configuration, it is recommended to restrict the unit number to two in a linear building row and to use the staggered building rows in linearconfiguration building clusters.

\subsection{Contributions}

The research contributions can be summarized in the three aspects, including theoretical contribution, practical contribution and social contribution.

\subsubsection{Theoretical contribution}

This research contributes original knowledge to the wind environment studies of contemporary high-rise residential buildings in response to the major trend of urbanizations around world.

It is an original attempt to develop the research methodology integrated by multiple 
computational tools that can provide efficient iterative analysis and implement quantitative comparisons for studying the influences on wind environments. It can give immediate analysis of wind environments of buildings on early stage for comparisons of many design alternatives. It is an original attempt to calculate the influenced areas with different velocity ranges by using the image processing method. The slight differences of the influences on wind environments of different buildings can be distinguished through the quantitative analysis. New knowledge that has not been found by existing methods is discovered by using the novel methodology. The research shows great potentials for integration of multiple computational tools for sustainable and environmental researches.

The parametric design study contributes to the original knowledge of architectural morphonology of contemporary high-rise residential buildings in the HSCWA. It is the first time to implement such a study in the Optic Valley Area. The categorization of representative BUFs and BCCs to enable computational assessment is an original attempt. In addition, the statistical analysis of BUFs and BCCs presents the preference of contemporary high-rise residential buildings in the developing region. It bridges building variables and computational simulations through the parametric transition of the morphological information of the buildings.

The wind environments of representative building forms and configurations have been studied with the aid of the methodology. The studies of BUFs include the square form, rectangular form and ' $\mathrm{T}$ ' form. The studies of BCCs include the scattered configuration, linear configuration and curvilinear configuration. The influences on wind environments are analyzed in the quantitative comparisons of velocity magnitudes. The iterative analysis presents how the influences are changed as the building variables are changed. The mechanisms of the influences are illustrated by analyzing air-pressure magnitudes and wind-flow streamlines together. In this way, the relationships between the influences and building variables of BUFs and BCCs are discovered. The studies of ' $\mathrm{T}$ ' form and curvilinear configuration are totally original. Though some similar forms and configurations have been studied before, there are some new findings as mentioned in the above section. And the building variables in this research are different. It is the first time to quantitively analyze the influenced areas with different velocities and compare all these BUFs and BCCs systematically. The research outcome can give information to the optimization of outdoor wind environments of high-rise residential communities and the 
improvement of microclimates in urban areas.

\subsubsection{Practical contribution}

There are great potentials for the research outcome to be used for helping in relevant researches and designs of BUFs and BCCs. Because the outcome can give information to the improvement of outdoor wind environments of BUFs and BCCs. Researchers can use the findings to study sustainable habitat and related topics. The research methodology can be used or modified for new studies. Architects and urban planners can use the findings to improve their designs on early stages. Because sometimes there are many design alternatives needed for comparisons in a relatively short time. The methodology can give immediate analysis of wind environments of initial building designs. As presented in BUFs and BCCs studies, the quantitative analysis of influenced areas with different velocity ranges is intuitive for architects and urban planners to better understand the intensities of the influences on wind environments of various specific building cases. The explanations of the mechanisms help them to understand that how the building variables of BUFs and BCCs influence the wind environments. In addition, city administrators and local authorities can use the findings to help in making urban development strategies and defining design guidelines.

\subsubsection{Social contribution}

The research outcome can give information to building designs and modifications for creating appropriate wind environments in urban areas. Especially for the increasing high-rise residential communities in the developing regions, the surrounding environments are important. Keeping wind velocity in an appropriate range can improve the thermal comfort. The promotion of outdoor ventilation can reduce air pollution. Appropriate wind environments can potentially promote building performance for saving energy of cooling (and heating). The reduction of extremely strong winds can prevent safety issues. As the changes of microclimates are accumulated, there are great potentials to influence the climates of large urban areas. 


\subsection{Summary}

In the Conclusion Chapter, the findings and contributions of the research have been presented respectively. The findings include the novel research methodology, the parametric design system, the relationships between the influences and variables of the BUFs, and the relationships between the influences and variables of the BCCs. The contributions include the theoretical contribution, the practical contribution, and the social contribution.

The thesis has presented a research that studies the relationships between the influences on wind environments and the variables of BUFs and BCCs of contemporary high-rise residential buildings. The research starts initially in the Optic Valley Area of Wuhan City due to its representativeness of the buildings in HSCWA and other developing regions. The findings and the contributions of the research are universal. The methodology has been developed by integrating multiple computational tools and the evaluation criteria, which provides an efficient modelling-simulation-analysis solution for iterative comparison studies. The establishment of the parametric design system has bridged the building variables and CFD simulations. The methodology has been successfully applied to studying the wind environments of the BUFs and the BCCs. The new findings of the BUFs studies and the BCCs studies have been presented and summarized. 


\section{References}

Allegrini, J., Dorer, V., \& Carmeliet, J.. (2014). Buoyant flows in street canyons: validation of CFD simulations with wind tunnel measurements. Building \& Environment, 72(2), 63-74.

Angel S., Sheppard S. C. \& Civco D. L.. (2005). The Dynamics of Global Urban Expansion. Transport and Urban Development Department. The World Bank. Washington D. C..

Aynsley. R. M.. (1989). Politics of pedestrian level urban wind control. Building and Environment. 24(4), 291-295.

Aynsley, R. M.. (1999). Shape and Flow: The Essence of Architectural Aerodynamics. Architectural Science Review, 42(2), 69-74.

Baniotopoulos, C. C., Borri, C., \& Stathopoulos, T.. (2011). Environmental Wind Engineering and Design of Wind Energy Structures. Springer Vienna.

Blocken, B., \& Carmeliet, J.. (2004). Pedestrian wind environment around buildings: Literature review and practical examples. Journal of Thermal Envelope and Building Science, 28(2), 107159.

Bourbia, F., \& Boucheriba, F.. (2010). Impact of street design on urban microclimate for semi arid climate (Constantine). Renewable Energy, 35(2), 343-347.

Cermak, J. E.. (2003). Wind-tunnel development and trends in applications to civil engineering. Journal of Wind Engineering \& Industrial Aerodynamics, 91(3), 355-370.

Chatzidimitriou, A., \& Axarli, K.. (2017). Street canyon geometry effects on microclimate and comfort; a case study in Thessaloniki. Procedia Environmental Sciences, 38, 643-650. 
Chen, F.. (2008). Wind environment study of high-rise building. Architectural Journal, (2), 7277.

Chen, J., Liu, Y., Yang, R., \& Shen, S.. (2007). Three dimensional numerical investigations of wind environment around Lanqiying Buildings. Building Simulation 2007, 1146-1149.

Chen, K., He, L., \& Wu, L.. (2012). Evaluation method of wind environment around buildings based on daily maximum wind records. Journal of Experiments in Fluid Mechanics, 26(5), 47$51,78$.

China Meteorological Bureau, Climate Information Center, Climate Data Office and Tsinghua University, Department of Building Science and Technology. (2005). China Standard Weather Data for Analyzing Building Thermal Conditions, April 2005. Beijing: China Building Industry Publishing House, ISBN 7-112-07273-3 (13228).

Chow, W. K., \& Gao, Y.. (2002). Numerical simulation on the wind action and fire-induced flow field of an international finance building in Beijing. Acta Aerodynamica Sinica, 63 Suppl 1, S108-13.

Chu, C. R., \& Chiang, B. F.. (2014). Wind-driven cross ventilation in long buildings. Building and Environment, 80(7), 150-158.

Cochran, L.. (2004). Design features to change and/ or ameliorate pedestrian wind conditions. In Structures 2004: Building on the Past, Securing the Future, Nashville, Tennessee, ASCE/ SEI Structure Congress. (pp. 1-8).

Cox, W. M., \& Tikvart, J. A.. (1990). A statistical procedure for determining the best performing air quality simulation model. Atmospheric Environment. Part A. General Topics, 24(9), 23872395. 
Cui, W. L., Cao, G. G., Qin, O. Y., \& Zhu, Y.. (2013). Influence of dynamic environment with different airflows on human performance. Building and Environment, 62, 124-132.

Davenport, A. G., Grimmond, C. S. B., Oke, T. R., \& Wieringa, J.. (2000). Estimating the roughness of cities and sheltered country. In Proceedings 12th Conference on Applied Climatology, Asheville, NC, American Meteorological Society, Boston (pp. 96-99).

Dimitroulopoulou, C.. (2012). Ventilation in European dwellings: a review. Building and Environment, 47(47), 109-125.

Dowson, D.. (1962). A generalized Reynolds equation for fluid-film lubrication. International Journal of Mechanical Sciences, 4(2), 159-170.

Du, Y., Mak, C. M., Huang, T., \& Niu, J.. (2017). Towards an integrated method to assess effects of lift-up design on outdoor thermal comfort in Hong Kong. Building and Environment, 125, 261-272.

Franke, J., Hellsten, A., Schlünzen, H., Carissimo, B.. (2007). Best Practice Guideline for the CFD Simulation of Flows in the Urban Environment, COST Office, Hamburg.

Gan, Y., Peng, B., Li, B., Chen, H.. (2013). The strategy of climate adapt design of the existing building: a case study of the Wuhan Jianshe Dasha Comprehensive Reconstruction. South Architecture, (6), 43-45.

Germano, M., Pomelli, U., Moin, P., Cabot., W. H.. (1991). A dynamic sub-grid scale eddy viscosity model. Physics Fluids A, 3(3), pp. 1760-1765

Givoni, B.. (1994). Passive and Low Energy Cooling of Buildings. Van Nosterand Reinhold, New York. 
Grimmond, C. S. B., \& Oke, T. R.. (1999). Aerodynamic properties of urban areas derived from analysis of surface form. Journal of Applied Meteorology, 38(9), 1262-1292.

Gromke, C., \& Blocken, B.. (2015). Influence of avenue-trees on air quality at the urban neighborhood scale. part ii: traffic pollutant concentrations at pedestrian level. Environmental Pollution, 196, 176-184.

Gromke, C., Buccolieri, R., Sabatino, S. \& Ruck, R.. (2008). Dispersion study in a street canyon with tree planting by means of wind tunnel and numerical investigations - evaluation of CFD data with experimental data. Atmospheric Environment, 42, 8640-8650.

Hang, J., \& Li, Y.. (2011). Age of air and air exchange efficiency in high-rise urban areas and its link to pollutant dilution. Atmospheric Environment, 45(31), 5572-5585.

Hong, L., Yu, Z., Li, K.. (2011). Study on grand urban windway planning in hot summer and cold winter area-case study of the city design of Sixin Area of Wuhan. Chinese Landscape Architecture, 27(2), 39-43.

Kaijima, S., Bouffanais, R., Willcox, K., \& Naidu, S.. (2013). Computational fluid dynamics for architectural design. Architectural Design, 83(2), 118-123.

Katzschner, L.. (2000). Urban climate maps-a tool for calculations of thermal conditions in outdoor spaces. In Architecture, City, Environment: Proceedings of PLEA 2000, Cambridge, United Kingdom (p. 453). Earthscan.

Kawamoto, H., Yoshida, H., \& Oryuu, T.. (2000). 2-Dimensional steady turbulent flow simulation of static aerodynamic forces of bridge girder sections. Proceedings of 16 th National Symposium on Wind Engineering, (pp. 191-196). Tokyo, Japan: Meteorological Society of Japan. 
Khonsari, M. M.. (2013). Reynolds Equation. Springer US.

Kondo, H., Asahi, K., Tomizuka, T., \& Suzuki, M.. (2006). Numerical analysis of diffusion around a suspended expressway by a multi-scale CFD model. Atmospheric Environment, 40(16), 2852-2859.

Kondo, K., Murakami, S., \& Mochida, A.. (1997). Generation of velocity fluctuations for inflow boundary condition of LES. Journal of Wind Engineering \& Industrial Aerodynamics, 67, 51-64.

Krishan, A., Baker, N., Yannas, S., Szokolay, S.. (2001). Climate Responsive Architecture: a design handbook for energy efficient buildings. Tata McGraw-Hill Pub. Com. Limited.

Kubota, T., Miura, M., Tominaga, Y., \& Mochida, A.. (2008). Wind tunnel tests on the relationship between building density and pedestrian-level wind velocity: development of guidelines for realizing acceptable wind environment in residential neighborhoods. Building and Environment, 43(10), 1699-1708.

Landsberg, H.E.. (1981). The Urban Climate. Academic Press, Inc. (London) Ltd., London.

Launder, B. E., \& Kato, M.. (1993). Modelling flow-induced oscillations in turbulent flow around a square cylinder. ASME-PUBLICATIONS-FED, 157, 189-189.

Lee, R. X., Jusuf, S. K., \& Wong, N. H.. (2015). The study of height variation on outdoor ventilation for Singapore's high-rise residential housing estates. International Journal of LowCarbon Technologies, 10(1), 15-33.

Li, X. X., \& Leung, L. D. Y. C.. (2005). Development of a k- $\varepsilon$ model for the determination of air exchange rates for street canyons. Atmospheric Environment, 39(38), 7285-7296. 
Li, Z., Luo, D., Shi, W., Li, Z., \& Liang, X.. (2011). Field measurement of wind-induced stress on glass facade of a coastal high-rise building. Science China Technological Sciences, 54(10), 2587-2596.

Lin, M., Hang, J., Li, Y., Luo, Z., \& Sandberg, M.. (2014). Quantitative ventilation assessments of idealized urban canopy layers with various urban layouts and the same building packing density. Building and Environment, 79, 152-167.

Liu, J. L., \& Niu, J. L.. (2016). CFD simulation of the wind environment around an isolated high-rise building: an evaluation of SRANS, LES and DES models. Building and Environment, 96, 91-106.

Liu, J. L., Niu, J. L., Mak, C. M., \& Xia, Q.. (2017). Detached eddy simulation of pedestrianlevel wind and gust around an elevated building. Building and Environment, 125, 168-179.

Liu, S., Mak, C. M., \& Niu, J. L.. (2011). Numerical evaluation of louver configuration and ventilation strategies for the windcatcher system. Building and Environment, 46(8), 1600-1616.

Loftness, V., \& Haase, D.. (2013). Sustainable Built Environments. New York, NY: Springer.

Mak, C. M., Cheng, C., \& Niu, J. L.. (2005). The application of computational fluid dynamics to the assessment of green features in buildings: part 1: wing walls. Architectural Science Review, 48(2), 121-134.

Martos, A., Pacheco-Torres R., Ordóñez J., \& Jadraque-Gago, E.. (2016). Towards successful environmental performance of sustainable cities: intervening sectors. a review. Renewable and Sustainable Energy Reviews, 2016, 57, 479-495.

Middel, A., Häb, K., Brazel, A. J., Martin, C. A., \& Guhathakurta, S.. (2014). Impact of urban 
form and design on mid-afternoon microclimate in phoenix local climate zones. Landscape and Urban Planning, 122, 16-28.

Ministry of Housing and Urban-Rural Development of China. (2014). Green Building Evaluation Standard. China Architecture \& Building Express.

Murakami, S.. (1997). Current status and future trends in computational wind engineering. Journal of Wind Engineering \& Industrial Aerodynamics, 67-68(1), 3-34.

Murakami, S.. (1998). Overview of turbulence models applied in CWE-1997. Journal of Wind Engineering \& Industrial Aerodynamics, 74, 1-24.

Murakami, S., Ooka, R., Mochida, A., Yoshida, S., \& Kim, S.. (1999). CFD analysis of wind climate from human scale to urban scale. Journal of Wind Engineering \& Industrial Aerodynamics, 81(1-3), 57-81.

Ng, E.. (2009). Policies and technical guidelines for urban planning of high-density cities-air ventilation assessment (AVA) of Hong Kong. Building and Environment, 44(7), 1478-1488.

Ng, E., Yuan, C., Chen, L., Ren, C., \& Fung, J. C. H.. (2011). Improving the wind environment in high-density cities by understanding urban morphology and surface roughness: a study in Hong Kong. Landscape \& Urban Planning, 101(1), 59-74.

Niu, J. L., Tang, Y. M., \& Mak, C. M.. (2005). The application of computational fluid dynamics to the assessment of green features in buildings: part 2: communal sky gardens. Architectural Science Review,48(4), 337-344.

Oke, T. R.. (1987). Boundary layer climates. Methuen, Inc, USA.

Oke, T. R.. (2006). Initial guidance to obtain representative meteorological observations at 
urban sites. Geog.ubc.ca.

Oke, T. R.. (2006). The energetic basis of the urban heat island. Quarterly Journal of the Royal Meteorological Society, 108(455), 1-24.

Paas, B., \& Schneider, C.. (2016). A comparison of model performance between ENVI-met and Austal2000 for particulate matter. Atmospheric Environment, 145, 392-404.

Penwarden, A. D., \& Wise, A. F. E.. (1975). Wind Environment Around Buildings. London, England: HMSO.

Raupach, M.. (1992). Drag and drag partition on rough surfaces. Boundary-Layer Meteorology, 60(4), 375-395.

Richards, P. J., Mallinson, G. D., Mcmillan, D., \& Li, Y. F.. (2002). Pedestrian level wind speeds in downtown Auckland. Wind \& Structures, 5(234), 151-164.

Richardson, L. F.. (1911). The approximate arithmetical solution by finite differences of physical problems involving differential equations, with an application to the stresses in a masonry dam. Philosophical Transactions of the Royal Society of London. Series A, Containing Papers of a Mathematical or Physical Character, 210, 307-357.

Roache, P. J.. (1994). Perspective: a method for uniform reporting of grid refinement studies. Trans.asme J.fluids Eng,116(3), 405-413.

Scotti, A., Meneveau, C., \& Lilly, D. K.. (1993). Generalized Smagorinsky model for anisotropic grids. Physics of Fluids A (Fluid Dynamics), 5(9), 2306-2308.

Selvam, R. P.. (1996). Computation of flow around Texas tech building using k- $\varepsilon$ and KatoLaunder k-ع turbulence model. Engineering Structures, 18(11), 856-860. 
Shao, Y., \& Yang, Y.. (2005). A theory for drag partition over rough surfaces. Atmospheric Environment, 39(38), 7351-7361.

Simiu, E., \& Scanlan, R. H.. (1978). Wind Effects on Structures: An Introduction to Wind Engineering. New York, NY: John Wiley.

Sobachkin, A., Dumnov, G.. (2014). Numerical Basis of CAD-Embedded CFD White Paper.

Solecki, W. D., Rosenzweig, C., Parshall, L., Pope, G., Clark, M., \& Cox, J., et al. (2005). Mitigation of the heat island effect in urban New Jersey. Environmental Hazards, 6(1), 39-49.

Stathopoulos, T., \& Baniotopoulos, C. C.. (2007). Wind Effects on Buildings and Design of Wind-Sensitive Structures. Vol. 493. Springer Science \& Business Media.

Surhone, L. M., Timpledon, M. T., \& Marseken, S. F.. (2010). Urban Heat Island. Hong Kong, China: Betascript Publishing.

Su, M., \& Tang, G.. (1996). Numerical simulation of wind-loading on high building. Acta Aerodynamica Sinica, 4.

Tan, Z., Dong, J., Xiao, Y., \& Tu, J.. (2015). A numerical study of diurnally varying surface temperature on flow patterns and pollutant dispersion in street canyons. Atmospheric Environment, 104, 217-227.

Tang, Y., \& Meng, Q.. (2001). Study on the simulative outdoor wind environment of high-rise residential area in Guangzhou. Journal of Xi'an University of Architecture \& technology, 4, $352-356$.

Vinchurkar, S., \& Longest, P. W.. (2008). Evaluation of hexahedral, prismatic and hybrid 
mesh styles for simulating respiratory aerosol dynamics. Computers and Fluids, 37(3), 317331.

United Nations. (2011). World urbanization prospects: The 2011 revision. New York.

United Nations. (2014). World urbanization prospects: The 2014 revision. New York.

United Nations. (2015). Transforming our world: The 2030 agenda for sustainable development. New York.

Walton, D., Dravitzki, V., \& Donn, M.. (2007). The relative influence of wind, sunlight and temperature on user comfort in urban outdoor spaces. Building and Environment, 42(9), 31663175 .

Wang, H., Wu, H., Ding, Y., Feng, J., \& Wang, S.. (2014). Feasibility and optimization of aerogel glazing system for building energy efficiency in different climates. International Journal of Low-Carbon Technologies, 10(4), 412-419.

Watson, D. \& Labs, K.. (1983). Climatic design: energy-efficient building principles and practices. McGraw-Hill Book Co. New York.

Wu, T., \& Chen, Q.. (1997). Wind tunnel test for pressure on wall and roof of extension project of Shenzhen airport building. Journal of Building Structures, 18(04), 44-50.

Xie, X., Liu, C. H., Leung, D. Y. C., \& Leung, M. K. H.. (2006). Characteristics of air exchange in a street canyon with ground heating. Atmospheric Environment, 40(33), 6396-6409.

Yim, S., Fung, J., Lau, A., \& Kot, S.. (2009). Air ventilation impacts of the "wall effect" resulting from the alignment of high-rise buildings. Atmospheric Environment, 43, 4982-4994.

Yuan, C., \& Ng, E.. (2014). Practical application of CFD on environmentally sensitive architectural design at high density cities: a case study in Hong Kong. Urban Climate, 8, 5777. 
Yuan, C., Ren, C., \& Ng, E.. (2014). GIS-based surface roughness evaluation in the urban planning system to improve the wind environment-A study in Wuhan, China. Urban Climate, 10, 585-593.

Yu, H., \& Thé, J.. (2016). Validation and optimization of SST k- $\omega$ turbulence model for pollutant dispersion within a building array. Atmospheric Environment, 145, 225-238.

Zhang, A., \& Gu, M.. (2008). Wind tunnel tests and numerical simulations of wind pressures on buildings in staggered arrangement. Journal of Wind Engineering \& Industrial Aerodynamics, 96(10), 2067-2079.

Zhang, Q. Y., Huang, J.. (2004). Chinese Typical Year Weather Data for Architectural Use. ISBN 7-111-14810-X. Beijing: China Machine Press.

Zhang, X.. (2012). A Collection of Residential District Planning. Nanjing, China: Phoenix Publishing \& Media.

Zhu, C. L., Wei, H. Y., \& Long, H.. (2011). Design Theories of Residential Architecture, China Architecture \& Building Press. 


\section{Publications}

(Relevant publications in the context of the $\mathrm{PhD}$ research)

He, Y. \& Schnabel, M. A.. (2016). A CFD analysis of wall-effect building groups in a curved layout: a study in Sha Tin, Hong Kong. Proceedings of 11th International Symposium on Architectural Interchanges in Asia (ISAIA), Sendai, Japan: Architectural Institute of Japan. pp. 1771-1776.

He, Y., Schnabel, M. A., Chen, R., \& Wang, N. (2017). A parametric analysis process for daylight illuminance: The influence of perforated facade panels on the indoor illuminance. Protocols, Flows, and Glitches - Proceedings of the 22nd International Conference of the Association for Computer-Aided Architectural Design Research in Asia (CAADRIA) 2017, pp 417-424.

He, Y., Schnabel, M. A., Chen, R., \& Wang, N. (2017). A comprehensive application of BIM modeling for semi-underground public architecture: a study for the Tiantian Square Complex, Wuhan, China. Future Trajectories of computation in design: CAAD Futures 2017, pp 297-308.

He, Y. \& Schnabel, M. A. (2017). Computational architectural integrated implementation: A digital process from initial design to fabrication. Challenges for Technology Innovation: An Agenda for the Future, CRC Press, Taylor \& Francis, London, UK, pp 435-439, DOI: 10.1201/9781315198101-87.

He, Y. \& Schnabel, M. A. (2018). An approach for daylight calculation of a building integrated photovoltaics (BIPV) façade, Architectural Science Review, 61:4, 226-233, DOI: 10.1080/00038628.2018.1470964.

He, Y., Schnabel, M. A. \& Mei, Y.. (2019). A novel methodology for architectural wind 
environment study by integrating CFD simulation, multiple parametric tools and evaluation criteria. Building Simulation. 50 pgs. (accepted) 


\section{Appendix}

The appendix presents the initial categorization record of BUFs and BCCs for high-rise residential buildings in the Optics Valley area, Wuhan, China. The codes used in the record are defined for twelve BUFs and four BCCs respectively in the following two tables (Table 50. and Table 51.). The names of residential districts, BUFs, BCCs and building numbers recorded as presented in Table 52..

Table 50. Categorization of BUFs.

\begin{tabular}{|c|c|}
\hline Code & Name \\
\hline B01 & Square form \\
\hline B02 & Rectangular form \\
\hline B03 & ' $T$ ' form \\
\hline B04 & Butterfly form \\
\hline B05 & Flog form \\
\hline B06 & Hash form ('\#' form) \\
\hline B07 & Cross form, 'X' form, Windmill form \\
\hline B08 & ' $Y$ ' form \\
\hline B09 & 'H' form \\
\hline B10 & ' $V$ ' form \\
\hline B11 & Circular form \\
\hline B12 & Irregular form \\
\hline
\end{tabular}

Table 51. Categorization of BCCs.

\begin{tabular}{|c|c|}
\hline Code & Name \\
\hline A01 & Scattered configuration \\
\hline A02 & Linear configuration \\
\hline A03 & Curvilinear configuration \\
\hline A04 & Compositive configuration \\
\hline
\end{tabular}


Table 52. Categorization record of BUFs and BCCs for high-rise residential buildings in the Optics Valley area, Wuhan, China.

\begin{tabular}{|c|c|c|c|c|c|}
\hline \multicolumn{2}{|c|}{ Residential district names } & BUFs & BCCs & $\begin{array}{l}\text { Building } \\
\text { number }\end{array}$ & $\begin{array}{l}\text { Note (Chinese names } \\
\text { of the district) }\end{array}$ \\
\hline & Senlinhuayuan & $\mathrm{B} 02, \mathrm{~B} 03$ & A03, (A04) & N19 & 森林花园 \\
\hline 2) & Xinjiayuan & B02 & A03 & N05 & 新嘉园 \\
\hline & Xuefujiayuan & B02, B03 & $\begin{array}{l}\text { A02, A03, } \\
\text { A04 }\end{array}$ & $\mathrm{N} 26$ & 学府家园 \\
\hline 4) & Balihaoting & B03 & $\begin{array}{l}\mathrm{A} 01, \mathrm{~A} 02, \\
(\mathrm{~A} 04)\end{array}$ & N18 & 巴黎豪庭 \\
\hline 5) & Kangjuyuanxiaoqu & $\begin{array}{l}\mathrm{B} 01, \mathrm{~B} 02, \\
\mathrm{~B} 03\end{array}$ & A02, A04 & N33 & 康居园小区 \\
\hline 6) & Xinshijiehengdahuafu & B03 & $\begin{array}{l}\mathrm{A} 02, \mathrm{~A} 03, \\
\text { (A04) }\end{array}$ & N40 & 新世界恒大华府 \\
\hline 7) & Gezhoubashijihuayuan & B03 & $\begin{array}{l}\mathrm{A} 02, \mathrm{~A} 03, \\
(\mathrm{~A} 04)\end{array}$ & N41 & 葛洲坝世纪花园 \\
\hline 8) & Wankejiayuan & $\begin{array}{l}\mathrm{B} 01, \mathrm{~B} 02, \\
\mathrm{~B} 12\end{array}$ & $\begin{array}{l}\text { A01, A02, } \\
(\mathrm{A} 04)\end{array}$ & N11 & 万科嘉园 \\
\hline 9) & Wenhuajiaoshixiaoqu & B03 & $\begin{array}{l}\text { A01, A02, } \\
\text { (A04) }\end{array}$ & N03 & 文华教师小区 \\
\hline 10) & LanguangCOCOshidai & B03, B04 & $\begin{array}{l}\text { A01, A02, } \\
\text { A03, (A04) }\end{array}$ & N08 & 蓝光 COCO 时代 \\
\hline 11) & Heyeshanshequ & B03 & $\begin{array}{l}\text { A02, A03, } \\
\text { (A04) }\end{array}$ & N60 & 荷叶山社区 \\
\hline 12) & Jiufengjie & B03, B05 & $\mathrm{A} 01, \mathrm{~A} 02$ & N90 & 九峰街 (片区) \\
\hline 13) & $\begin{array}{l}\text { Biguiyuanshengtaiche } \\
\text { ng }\end{array}$ & $\begin{array}{l}\text { B03, } \\
\text { B12(B03) }\end{array}$ & $\begin{array}{l}\mathrm{A} 01, \mathrm{~A} 02, \\
\mathrm{~A} 03,(\mathrm{~A} 04)\end{array}$ & N14 & 碧桂园生态城 \\
\hline 14) & Wankehuashanziyuew & B03 & $\mathrm{A} 02$ & N10 & 万科花山紫悦湾 \\
\hline
\end{tabular}




\begin{tabular}{|c|c|c|c|c|c|}
\hline & an & & & & \\
\hline 15) & $\begin{array}{l}\text { Huashanshengtaixinch } \\
\text { eng }\end{array}$ & B02, B03 & $\begin{array}{l}\text { A01, A02, } \\
\text { (A04) }\end{array}$ & $\mathrm{N} 24$ & 花山生态新城 \\
\hline 16) & Yuyuanxiaoquyiqierqi & B03 & $\begin{array}{l}\mathrm{A} 01, \mathrm{~A} 02, \\
\mathrm{~A} 03,(\mathrm{~A} 04)\end{array}$ & N35 & 喻园小区一期二期 \\
\hline 17) & Huakexibiangaoceng & $\begin{array}{l}\text { B12 } \\
\text { (B03) }\end{array}$ & A01 & N06 & 华科西边高层 \\
\hline 18) & Yujiashanzhuang & B03 & $\begin{array}{l}\mathrm{A} 01, \mathrm{~A} 02, \\
\text { (A04) }\end{array}$ & N06 & 喻家山庄 \\
\hline 19) & $\begin{array}{l}\text { Zhongjiansanjuergong } \\
\text { sixiaoqu }\end{array}$ & B03 & $\begin{array}{l}\mathrm{A} 01, \mathrm{~A} 02, \\
\text { (A04) }\end{array}$ & N02 & 中建三局二公司小区 \\
\hline 20) & Huakekangyuanxiaoqu & B03 & $\begin{array}{l}\mathrm{A} 01, \mathrm{~A} 02, \\
(\mathrm{~A} 04)\end{array}$ & N05 & 华科康园小区 \\
\hline 21) & Zisongjiaoshigongyu & B02, B03 & A02, A04 & N03 & 紫菘教师公寓 \\
\hline 22) & $\begin{array}{l}\text { Guangguzhongxinhuay } \\
\text { uan }\end{array}$ & $\mathrm{B} 10$ & $\begin{array}{l}\mathrm{A} 01, \mathrm{~A} 02, \\
\text { (A04) }\end{array}$ & N03 & 光谷中心花园 \\
\hline 23) & Xinduhui & B07 & A01 & N03 & 新都汇 \\
\hline 24) & Dongguyinzuo & $\mathrm{B} 12$ & A01 & N02 & 东谷银座 \\
\hline 25) & Jiazhouyangguang & B03, B12 & $\begin{array}{l}\mathrm{A} 01, \mathrm{~A} 02, \\
\text { (A04) }\end{array}$ & N04 & 加州阳光 \\
\hline 26) & Milanyingxiang & B03 & $\begin{array}{l}\mathrm{A} 01, \mathrm{~A} 02, \\
\text { (A04) }\end{array}$ & N05 & 米兰映像 \\
\hline 27) & Guangshahuating & $\begin{array}{l}\text { B05 } \\
\text { (B03) }\end{array}$ & A01 & N07 & 广厦华庭 \\
\hline 28) & Lanchaoyipin & B03 & $\begin{array}{l}\mathrm{A} 01, \mathrm{~A} 02, \\
(\mathrm{~A} 04)\end{array}$ & N03 & 蓝巢逸品 \\
\hline 29) & $\begin{array}{l}\text { Changqingtengmingyu } \\
\text { an }\end{array}$ & $\begin{array}{l}\text { B02, B03, } \\
\text { B05 }\end{array}$ & $\begin{array}{l}\text { A01, A02, } \\
\text { A04 }\end{array}$ & N07 & 常青藤名苑 \\
\hline 30) & Hubeizhongyiyuanxiao & B05 & A01 & N03 & 湖北中医院小区 \\
\hline
\end{tabular}




\begin{tabular}{|c|c|c|c|c|c|}
\hline & $\mathrm{qu}$ & (B03) & & & \\
\hline 31) & Fengdubolin & B02, B04 & $\begin{array}{l}\text { A01, A02, } \\
(\mathrm{A} 04)\end{array}$ & N06 & 风度柏林 \\
\hline 32) & Baolihuayuan & B03, B08 & $\begin{array}{l}\text { A01, A02, } \\
\text { A03, A04 }\end{array}$ & N36 & 保利花园 \\
\hline 33) & Baolimoligongguan & B02, B03 & $\mathrm{A} 01, \mathrm{~A} 02$ & N08 & 保利茉莉公馆 \\
\hline 34) & $\begin{array}{l}\text { Guangguchuangyejieb } \\
\text { uxingjie }\end{array}$ & $\begin{array}{l}\mathrm{B} 02 \\
\text { (B03) }\end{array}$ & A01 & N07 & 光谷创业街步行街 \\
\hline 35) & Guanggubahao & B03 & $\begin{array}{l}\mathrm{A} 01, \mathrm{~A} 02, \\
(\mathrm{~A} 04)\end{array}$ & N10 & 光谷 8 号 \\
\hline 36) & $\begin{array}{l}\text { Wolongjianqiaochuntia } \\
\mathrm{n}\end{array}$ & $\begin{array}{l}\text { B01, B03, } \\
\text { B12 }\end{array}$ & $\begin{array}{l}\text { A01, A02, } \\
\text { A04 }\end{array}$ & N30 & 卧龙剑桥春天 \\
\hline 37) & Donghuguangchang & B03, B05 & $\mathrm{A} 01, \mathrm{~A} 02$ & N02 & 东湖广场 \\
\hline 38) & Hualehuayuan & B02, В03 & $\begin{array}{l}\text { A01, A02, } \\
\text { A03, A04 }\end{array}$ & $\mathrm{N} 10$ & 华乐花园 \\
\hline 39) & Jindihuagongguan & B02, В03 & $\begin{array}{l}\text { A01, A02, } \\
\text { A03, (A04) }\end{array}$ & N09 & 金地华工馆 \\
\hline 40) & Liantouyuyuan & B03 & A01 & N05 & 联投喻园 \\
\hline 41) & Hongjinghuayuan & $\mathrm{B} 02, \mathrm{~B} 03$ & $\mathrm{~A} 01, \mathrm{~A} 02$ & N03 & 虹景花园 \\
\hline 42) & Jinxinguoji & $\begin{array}{l}\text { B03, B05, } \\
\text { B12 }\end{array}$ & $\mathrm{A} 01, \mathrm{~A} 02$ & N07 & 金金国际 \\
\hline 43) & Guanggulujingyuan & $\begin{array}{l}\mathrm{B} 02, \mathrm{~B} 03, \\
\mathrm{~B} 12\end{array}$ & $\begin{array}{l}\mathrm{A} 01, \mathrm{~A} 02, \\
(\mathrm{~A} 04)\end{array}$ & N16 & 光谷陆景苑 \\
\hline 44) & Zizhouhuayuan & B03 & B02 & N03 & 梓洲花苑 \\
\hline 45) & Lidaomancheng & B03 & $\begin{array}{l}\mathrm{A} 01, \mathrm{~A} 02, \\
\mathrm{~A} 03,(\mathrm{~A} 04)\end{array}$ & N24 & 丽岛漫城 \\
\hline 46) & Yingbinjiayuan & B02, B03 & $\begin{array}{l}\mathrm{A} 01, \mathrm{~A} 02, \\
(\mathrm{~A} 04)\end{array}$ & N10 & 迎宾家园 \\
\hline 47) & Tongjiantiancheng & B01, B03 & $\mathrm{A} 01, \mathrm{~A} 02$, & N18 & 统建天成 \\
\hline
\end{tabular}




\begin{tabular}{|c|c|c|c|c|c|}
\hline & & & $\mathrm{A} 03,(\mathrm{~A} 04)$ & & \\
\hline 48) & Hukoushequ & $\begin{array}{l}\text { B03, B12 } \\
\text { (B02) }\end{array}$ & $\begin{array}{l}\text { A01, A02, } \\
\text { (A04) }\end{array}$ & N33 & 湖口社区 \\
\hline & Chengshizhiguang & B02, B03 & $\begin{array}{l}\mathrm{A} 01, \mathrm{~A} 02, \\
\text { (A04) }\end{array}$ & $\mathrm{N} 21$ & 城市之光 \\
\hline 50$)$ & Jiayuanhuadu & B02, B03 & $\begin{array}{l}\text { A01, A02, } \\
\text { A03, A04 }\end{array}$ & N49 & 佳源花都 \\
\hline 51) & $\begin{array}{l}\text { Guangguhangtianchen } \\
\text { g }\end{array}$ & B03 & $\begin{array}{l}\mathrm{A} 01, \mathrm{~A} 02, \\
(\mathrm{~A} 04)\end{array}$ & N09 & 光谷航天城 \\
\hline 52) & Guanggumantingchun & B03 & $\begin{array}{l}\text { A01, A02, } \\
\text { (A04) }\end{array}$ & N12 & 光谷满庭春 \\
\hline 53) & Yishenghuayuan & B02, B03 & $\begin{array}{l}\mathrm{A} 01, \mathrm{~A} 02, \\
(\mathrm{~A} 04)\end{array}$ & N12 & 宜盛花园 \\
\hline 54) & $\begin{array}{l}\text { Changhanglanjingguoj } \\
\text { i }\end{array}$ & B03 & $\begin{array}{l}\mathrm{A} 01, \mathrm{~A} 02, \\
\mathrm{~A} 03,(\mathrm{~A} 04)\end{array}$ & N09 & 长航蓝晶国际 \\
\hline 55$)$ & Zisongyijinghuating & $\mathrm{B} 03$ & $\begin{array}{l}\mathrm{A} 01, \mathrm{~A} 02, \\
(\mathrm{~A} 04)\end{array}$ & N07 & 紫菘逸景华庭 \\
\hline 56$)$ & Guangguxinshijie & B03, B08 & $\begin{array}{l}\mathrm{A} 01, \mathrm{~A} 02, \\
\mathrm{~A} 03,(\mathrm{~A} 04)\end{array}$ & N15 & 光谷新世界 \\
\hline 57$)$ & Jinditaiyangcheng & B02, B03 & $\begin{array}{l}\mathrm{A} 01, \mathrm{~A} 02, \\
\mathrm{~A} 03,(\mathrm{~A} 04)\end{array}$ & N51 & 金地太阳城 \\
\hline 58$)$ & Baolishidai & $\mathrm{B} 03$ & $\begin{array}{l}\mathrm{A} 01, \mathrm{~A} 02, \\
\text { (A04) }\end{array}$ & N17 & 保利时代 \\
\hline 59) & $\begin{array}{l}\text { Guangguqingnianchen } \\
\mathrm{g}\end{array}$ & $\begin{array}{l}\text { B01, B02, } \\
\text { B03 }\end{array}$ & $\begin{array}{l}\mathrm{A} 01, \mathrm{~A} 02, \\
\mathrm{~A} 03,(\mathrm{~A} 04)\end{array}$ & N13 & 光谷青年城 \\
\hline 60$)$ & $\begin{array}{l}\text { Zisongfenglinshangche } \\
\text { ng }\end{array}$ & $\begin{array}{l}\text { B03, B11, } \\
\text { B12 }\end{array}$ & $\begin{array}{l}\mathrm{A} 01, \mathrm{~A} 02, \\
\mathrm{~A} 03,(\mathrm{~A} 04)\end{array}$ & N08 & 紫菘枫林上城 \\
\hline 61) & Hongfujiayuan & $\begin{array}{l}\text { B02, B03, } \\
\text { B12 }\end{array}$ & $\begin{array}{l}\mathrm{A} 01, \mathrm{~A} 02, \\
\mathrm{~A} 03,(\mathrm{~A} 04)\end{array}$ & N12 & 洪福家园 \\
\hline
\end{tabular}




\begin{tabular}{|c|c|c|c|c|c|}
\hline 62) & Lvzhiyuan & $\begin{array}{l}\text { B02, B03, } \\
\text { B07 }\end{array}$ & $\begin{array}{l}\mathrm{A} 01, \mathrm{~A} 02, \\
\mathrm{~A} 03,(\mathrm{~A} 04)\end{array}$ & N10 & 绿之苑 \\
\hline 63) & Wenhaoyuan & B03, B12 & $\begin{array}{l}\mathrm{A} 02, \mathrm{~A} 03, \\
(\mathrm{~A} 04)\end{array}$ & N07 & 文豪苑 \\
\hline 64) & Jindizhongxincheng & B03 & $\begin{array}{l}\mathrm{A} 01, \mathrm{~A} 02, \\
\mathrm{~A} 03,(\mathrm{~A} 04)\end{array}$ & N14 & 金地中心城 \\
\hline 65$)$ & Jindixiongchuyihao & B03 & $\begin{array}{l}\mathrm{A} 01, \mathrm{~A} 02, \\
(\mathrm{~A} 04)\end{array}$ & N14 & 金地雄楚一号 \\
\hline 66) & Jinditianyue & B03 & $\begin{array}{l}\mathrm{A} 01, \mathrm{~A} 02, \\
(\mathrm{~A} 04)\end{array}$ & N11 & 金地天悦 \\
\hline 67) & Bayihuayuan & B02, B05 & $\begin{array}{l}\mathrm{A} 01, \mathrm{~A} 02, \\
(\mathrm{~A} 04)\end{array}$ & N10 & 八一花园 \\
\hline 68) & Shuguangxincheng & B02, B03 & $\begin{array}{l}\mathrm{A} 01, \mathrm{~A} 02, \\
\mathrm{~A} 04\end{array}$ & $\mathrm{~N} 21$ & 曙光新城 \\
\hline 69) & Jindigelindongjun & B03, B12 & $\begin{array}{l}\mathrm{A} 01, \mathrm{~A} 02, \\
\mathrm{~A} 03,(\mathrm{~A} 04)\end{array}$ & N15 & 金地格林东郡 \\
\hline 70) & $\begin{array}{l}\text { Changchengzuobiaoch } \\
\text { eng }\end{array}$ & $\begin{array}{l}\text { B02, B03, } \\
\text { B12 }\end{array}$ & $\begin{array}{l}\mathrm{A} 01, \mathrm{~A} 02, \\
\mathrm{~A} 04\end{array}$ & N69 & 长城坐标城 \\
\hline 71) & Guangguzuobiaocheng & $\begin{array}{l}\text { B03, B04 } \\
\text { (B08) }\end{array}$ & A01 & N10 & 光谷坐标城 \\
\hline 72) & Wankehuajingyuan & $\begin{array}{l}\text { B02, B03, } \\
\text { B12 }\end{array}$ & $\begin{array}{l}\mathrm{A} 01, \mathrm{~A} 02, \\
\mathrm{~A} 04\end{array}$ & N14 & 万科花璟苑 \\
\hline 73) & Guanggulixiangcheng & B03 & $\begin{array}{l}\mathrm{A} 01, \mathrm{~A} 02, \\
(\mathrm{~A} 04)\end{array}$ & $\mathrm{N} 10$ & 光谷理想城 \\
\hline 74) & Zhongjiankangcheng & B03, B04 & $\begin{array}{l}\mathrm{A} 01, \mathrm{~A} 02, \\
(\mathrm{~A} 04)\end{array}$ & N17 & 中建康城 \\
\hline 75) & Babilunbao & B02, B03 & $\begin{array}{l}\mathrm{A} 02, \mathrm{~A} 03, \\
\mathrm{~A} 04\end{array}$ & $\mathrm{~N} 12$ & 芭比伦堡 \\
\hline 76) & Qingjiangshanshui & B02, B03, & $\mathrm{A} 01, \mathrm{~A} 02$, & N79 & 清江山水 \\
\hline
\end{tabular}




\begin{tabular}{|c|c|c|c|c|c|}
\hline & & $\mathrm{B} 12$ & $\mathrm{~A} 03, \mathrm{~A} 04$ & & \\
\hline 77) & Donglinwailu & $\begin{array}{l}\mathrm{B} 03, \mathrm{~B} 05 \\
\mathrm{~B} 12\end{array}$ & $\begin{array}{l}\text { A01, A02, } \\
\text { A03, A04 }\end{array}$ & N32 & 东林外庐 \\
\hline 78) & Yundingju & $\begin{array}{l}\text { В01, B02, } \\
\text { В12 }\end{array}$ & $\begin{array}{l}\text { A01, A02, } \\
\text { A04 }\end{array}$ & N14 & 云顶居 \\
\hline 79) & Shanshuihuating & $\begin{array}{l}\mathrm{B} 02, \mathrm{~B} 03, \\
\mathrm{~B} 12\end{array}$ & A02, (A04) & N07 & 山水华庭 \\
\hline 80$)$ & Weiyucheng & B03 & $\begin{array}{l}\mathrm{A} 02, \mathrm{~A} 03, \\
\text { (A04) }\end{array}$ & N33 & 谓语城 \\
\hline 81$)$ & Jiangnanjiayuan & $\mathrm{B} 03, \mathrm{~B} 12$ & $\begin{array}{l}\text { A01, A02, } \\
\text { A03, A04 }\end{array}$ & N35 & 江南家园 \\
\hline 82) & $\begin{array}{l}\text { Jiazhouxiangshanmeis } \\
\text { hu }\end{array}$ & B03 & $\begin{array}{l}\text { A01, A02, } \\
\text { A03, (A04) }\end{array}$ & N30 & 加州香山美树 \\
\hline 83) & Jinxiulongcheng & B02, B03, & $\begin{array}{l}\text { A01, A02, } \\
\text { A03, A04 }\end{array}$ & N76 & 锦绣龙城 \\
\hline 84) & Zhongguyuan & $\mathrm{B} 02, \mathrm{~B} 12$ & $\begin{array}{l}\mathrm{A} 02, \mathrm{~A} 03, \\
\text { (A04) }\end{array}$ & N18 & 中谷苑 \\
\hline 85$)$ & Baoyeguanggulidu & B02, B03 & $\begin{array}{l}\mathrm{A} 01, \mathrm{~A} 02, \\
(\mathrm{~A} 04)\end{array}$ & N14 & 宝业光谷丽都 \\
\hline 86) & Guanggumeihuawu & B01, B03 & $\begin{array}{l}\text { A01, A02, } \\
\text { (A04) }\end{array}$ & N12 & 光谷梅花坞 \\
\hline 87) & Guangguziyoucheng & $\mathrm{B} 03, \mathrm{~B} 05$ & $\begin{array}{l}\text { A01, A03, } \\
\text { A04 }\end{array}$ & N11 & 光谷自由城 \\
\hline 88) & Nanhushequ & B02, B03 & A02 & N101 & 南湖社区 \\
\hline 89) & Jinfaxiaoqu & B03 & $\mathrm{A} 02, \mathrm{~A} 04$ & N14 & 津发小区 \\
\hline 90) & $\begin{array}{l}\text { Guanggushishangchen } \\
\text { g }\end{array}$ & B03 & $\begin{array}{l}\text { A01, A02, } \\
\text { A03, A04 }\end{array}$ & N07 & 光谷时尚城 \\
\hline 91) & Kadiyagongguan & $\begin{array}{l}\text { B02, B03, } \\
\text { В08 }\end{array}$ & $\mathrm{A} 01, \mathrm{~A} 02$ & N11 & 卡迪亚公馆 \\
\hline
\end{tabular}




\begin{tabular}{|c|c|c|c|c|c|}
\hline 92) & Huanongxiyuanxiaoqu & $\begin{array}{l}\text { B02, B03, } \\
\text { B12 }\end{array}$ & $\begin{array}{l}\mathrm{A} 01, \mathrm{~A} 02, \\
\mathrm{~A} 03,(\mathrm{~A} 04)\end{array}$ & N71 & 华农西苑小区 \\
\hline 93) & Luonanyaju & B03 & A01 & N02 & 珞南雅居 \\
\hline 94) & Nanhushishanmeilu & B02, B11 & $\begin{array}{l}\mathrm{A} 01, \mathrm{~A} 02, \\
(\mathrm{~A} 04)\end{array}$ & N03 & 南湖狮山美庐 \\
\hline & Nanhuayu'an & B02, B03 & $\begin{array}{l}\mathrm{A} 02, \mathrm{~A} 03, \\
\mathrm{~A} 04\end{array}$ & N09 & 澜花语岸 \\
\hline & Ruihutiandi & B03 & $\begin{array}{l}\mathrm{A} 01, \mathrm{~A} 02, \\
(\mathrm{~A} 04)\end{array}$ & N09 & 瑞湖天地 \\
\hline 97) & $\begin{array}{l}\text { Tairannanhumeiguiwa } \\
\mathrm{n}\end{array}$ & $\mathrm{B} 01, \mathrm{~B} 03$ & $\begin{array}{l}\mathrm{A} 01, \mathrm{~A} 02, \\
\mathrm{~A} 03, \mathrm{~A} 04\end{array}$ & N79 & 泰然南湖玫瑰湾 \\
\hline 98) & Nanhushanzhuang & $\begin{array}{l}\text { B02, B03, } \\
\text { B08 }\end{array}$ & $\begin{array}{l}\mathrm{A} 01, \mathrm{~A} 02, \\
\mathrm{~A} 03, \mathrm{~A} 04\end{array}$ & N60 & 南湖山庄 \\
\hline 99) & Mingzeliwan & B03, B08 & $\begin{array}{l}\mathrm{A} 01, \mathrm{~A} 02, \\
\mathrm{~A} 03, \mathrm{~A} 04\end{array}$ & N11 & 明泽丽湾 \\
\hline 100) & Luojiayayuan & B03 & $\begin{array}{l}\mathrm{A} 01, \mathrm{~A} 02, \\
(\mathrm{~A} 04)\end{array}$ & N18 & 珞珈雅苑 \\
\hline 101) & Dahuananhugongyuan & $\begin{array}{l}\text { B02, B03, } \\
\text { B04, B12 }\end{array}$ & $\begin{array}{l}\mathrm{A} 01, \mathrm{~A} 02, \\
\mathrm{~A} 03, \mathrm{~A} 04\end{array}$ & N98 & 大华南湖公园 \\
\hline 102) & )Huadajiayuan & B01, B02 & $\begin{array}{l}\mathrm{A} 01, \mathrm{~A} 02, \\
\mathrm{~A} 04\end{array}$ & N30 & 华大家园 \\
\hline 103) & Jindixi'angushi & B03 & $\begin{array}{l}\mathrm{A} 01, \mathrm{~A} 02, \\
(\mathrm{~A} 04)\end{array}$ & N10 & 金地西岸故事 \\
\hline 104) & Mingzefenghuayuan & B03 & $\begin{array}{l}\mathrm{A} 01, \mathrm{~A} 02, \\
\mathrm{~A} 04\end{array}$ & N20 & 明泽丰华苑 \\
\hline 105$)$ & Hongchengjiayuan & B03 & $\begin{array}{l}\mathrm{A} 01, \mathrm{~A} 02, \\
(\mathrm{~A} 04)\end{array}$ & N09 & 鸿城家园 \\
\hline 106) & Nanhuxinchengjiayuan & B03 & $\begin{array}{l}\mathrm{A} 01, \mathrm{~A} 02, \\
\mathrm{~A} 04\end{array}$ & $\mathrm{~N} 20$ & 南湖新城家园 \\
\hline
\end{tabular}




\begin{tabular}{|c|c|c|c|c|}
\hline 107)Zhianlongting & B02, B03 & $\begin{array}{l}\text { A01, A02, } \\
(\mathrm{A} 04)\end{array}$ & N17 & 芷岸龙庭 \\
\hline 108) Jindigelinxiaocheng & $\begin{array}{l}\text { B02, B03, } \\
\text { B12 }\end{array}$ & $\begin{array}{l}\text { A01, A02, } \\
\text { A03, A04 }\end{array}$ & N93 & 金地格林小城 \\
\hline 109) Nanhuyayuan & $\begin{array}{l}\text { B02, B03, } \\
\text { B04 }\end{array}$ & $\begin{array}{l}\text { A01, A02, } \\
(\mathrm{A} 04)\end{array}$ & N14 & 南湖雅园 \\
\hline 110)Lidaohuayuan & $\mathrm{B} 03, \mathrm{~B} 12$ & $\begin{array}{l}\text { A01, A02, } \\
\text { A03, A04 }\end{array}$ & N26 & 丽岛花园 \\
\hline 111)Lidaoziyuan & $\begin{array}{l}\mathrm{B} 02, \mathrm{~B} 12 \\
(\mathrm{~B} 01)\end{array}$ & $\begin{array}{l}\text { A01, A02, } \\
\text { A03, (A04) }\end{array}$ & N07 & 丽岛紫园 \\
\hline 112)Zhongjiannanhuyihao & B03 & $\begin{array}{l}\text { A01, A02, } \\
\text { A04 }\end{array}$ & N15 & 中建南湖一号 \\
\hline 113)Xingfujiayuan & B03 & $\begin{array}{l}\mathrm{A} 01, \mathrm{~A} 02, \\
(\mathrm{~A} 04)\end{array}$ & N10 & 幸福佳苑 \\
\hline 114) Shichengfenghuaxindu & B02, B03 & $\begin{array}{l}\text { A01, A02, } \\
\text { A04 }\end{array}$ & N13 & 狮城风华新都 \\
\hline 115) Shichenghanyuan & $\begin{array}{l}\text { B02, B03, } \\
\text { B05 }\end{array}$ & $\begin{array}{l}\text { A01, A02, } \\
\text { A04 }\end{array}$ & N06 & 狮城翰园 \\
\hline 116) Shichengmingju & $\begin{array}{l}\text { В01, B02, } \\
\text { B03, B05, } \\
\text { В12 }\end{array}$ & $\begin{array}{l}\text { A01, A02, } \\
(\mathrm{A} 04)\end{array}$ & N13 & 狮城名居 \\
\hline 117)Hongfuhuayuan & $\begin{array}{l}\text { B02, B03, } \\
\text { B04 }\end{array}$ & $\begin{array}{l}\text { A01, A02, } \\
\text { A04 }\end{array}$ & N06 & 鸿福花园 \\
\hline 118) Nanhujingyuan & $\mathrm{B} 02, \mathrm{~B} 03$ & $\begin{array}{l}\text { A01, A02, } \\
\text { A04 }\end{array}$ & N08 & 南湖景苑 \\
\hline 119) Guizihuayuan & $\mathrm{B} 02, \mathrm{~B} 03$ & $\begin{array}{l}\mathrm{A} 01, \mathrm{~A} 02, \\
(\mathrm{~A} 04)\end{array}$ & N09 & 桂子花园 \\
\hline 120)Baoliqianshuiwan & $\begin{array}{l}\text { B02, B04 } \\
(\mathrm{B} 03)\end{array}$ & $\begin{array}{l}\text { A01, A02, } \\
(\mathrm{A} 04)\end{array}$ & N07 & 保利浅水湾 \\
\hline
\end{tabular}




\begin{tabular}{|c|c|c|c|c|}
\hline 121) Wankefeicuitai & B02, B03 & $\begin{array}{l}\text { A01, A02, } \\
(\mathrm{A} 04)\end{array}$ & N19 & 万科翡翠云台 \\
\hline 122) Nanhubandao & B02 & $\begin{array}{l}\text { A01, A02, } \\
\text { A04 }\end{array}$ & $\mathrm{N} 12$ & 南湖半岛 \\
\hline 123)Hanchengchengtou & B02, B03 & $\begin{array}{l}\mathrm{A} 01, \mathrm{~A} 02, \\
(\mathrm{~A} 04)\end{array}$ & N46 & 瀚城城投 \\
\hline 124)Baolilafei & B02, B03 & $\begin{array}{l}\mathrm{A} 01, \mathrm{~A} 02, \\
(\mathrm{~A} 04)\end{array}$ & N11 & 保利拉菲 \\
\hline 125)Baolilanhaijun & B02, B03 & $\begin{array}{l}\mathrm{A} 01, \mathrm{~A} 02, \\
\mathrm{~A} 03,(\mathrm{~A} 04)\end{array}$ & N16 & 保利蓝海郡 \\
\hline 126) Nanhukangtaihuayuan & $\begin{array}{l}\text { B02, B03, } \\
\text { B12 }\end{array}$ & $\mathrm{A} 02,(\mathrm{~A} 04)$ & N09 & 南湖康泰花园 \\
\hline 127)Lvtingyajing & B02, B04 & $\begin{array}{l}\text { A01, A02, } \\
\text { A04 }\end{array}$ & N16 & 绿汀雅境 \\
\hline 128)Xueyafanglin & B02, B03 & $\begin{array}{l}\mathrm{A} 01, \mathrm{~A} 02, \\
(\mathrm{~A} 04)\end{array}$ & N10 & 学雅芳邻 \\
\hline 129)Lumingyuan & B01, B03 & $\begin{array}{l}\mathrm{A} 02, \mathrm{~A} 03, \\
(\mathrm{~A} 04)\end{array}$ & N04 & 鹿鸣苑 \\
\hline 130) Mingduhuayuan & $\mathrm{B} 02, \mathrm{~B} 12$ & $\begin{array}{l}\text { A01, A02, } \\
\text { A04 }\end{array}$ & N43 & 名都花园 \\
\hline 131)Lingxiucheng & $\begin{array}{l}\text { B03, B05, } \\
\text { B08 }\end{array}$ & $\begin{array}{l}\mathrm{A} 01, \mathrm{~A} 02, \\
(\mathrm{~A} 04)\end{array}$ & N08 & 领秀城 \\
\hline 132)Xiongchutiandi & B03 & $\begin{array}{l}\text { A01, A02, } \\
\text { (A04) }\end{array}$ & N04 & 雄楚天地 \\
\hline 133)Baolihuadu & $\mathrm{B} 03, \mathrm{~B} 12$ & $\begin{array}{l}\mathrm{A} 01, \mathrm{~A} 02, \\
\mathrm{~A} 03, \mathrm{~A} 04\end{array}$ & N36 & 保利华都 \\
\hline 134)Donghuyuyuan & B02, B03 & $\begin{array}{l}\mathrm{A} 01, \mathrm{~A} 02, \\
(\mathrm{~A} 04)\end{array}$ & N03 & 东湖御院 \\
\hline 135)Hualeyuan & B02, B05, & $\mathrm{A} 01, \mathrm{~A} 02$, & N05 & 华乐苑 \\
\hline
\end{tabular}




\begin{tabular}{|c|c|c|c|c|}
\hline & B12 & A04 & & \\
\hline 136)Hualeshanzhuang & $\mathrm{B} 05, \mathrm{~B} 12$ & $\begin{array}{l}\text { A01, A03, } \\
\text { A02, A04 }\end{array}$ & N10 & 华乐山庄 \\
\hline 137)Xiejiayuan & B03 & A01 & N02 & 解甲苑 \\
\hline 138) 709suoshequ & $\mathrm{B} 02, \mathrm{~B} 03$ & $\begin{array}{l}\mathrm{A} 02, \mathrm{~A} 04, \\
(\mathrm{~A} 04)\end{array}$ & N36 & 709 所社区 \\
\hline 139)Rongjunjiaoshixiaoqu & B02, B03 & $\mathrm{A} 02$ & N09 & 荣军教师小区 \\
\hline $\begin{array}{l}\text { 140)Zhuodaoquanjiaoshixi } \\
\text { aoqu }\end{array}$ & $\begin{array}{l}\mathrm{B} 01, \mathrm{~B} 02, \\
\mathrm{~B} 03\end{array}$ & $\begin{array}{l}\text { A01, A02, } \\
\text { A04 }\end{array}$ & N23 & 卓刀泉教师小区 \\
\hline 141)Kaileguiyuan & B03 & $\begin{array}{l}\text { A01, A02, } \\
(\mathrm{A} 04)\end{array}$ & N07 & 凯乐桂园 \\
\hline 142)Lantianmingyuan & $\mathrm{B} 02, \mathrm{~B} 03$ & $\begin{array}{l}\text { A01, A02, } \\
(\mathrm{A} 04)\end{array}$ & N05 & 蓝天名苑 \\
\hline 143)Xingguangyuan & $\begin{array}{l}\text { B03, B05, } \\
\text { B12 }\end{array}$ & $\begin{array}{l}\text { A01, A02, } \\
\text { A04 }\end{array}$ & N02 & 星光苑 \\
\hline 144)Fuhushanshequ & B01, B03 & $\mathrm{A} 02$ & N05 & 伏虎山社区 \\
\hline 145)Xinkaishanquanju & B01, B02 & $\begin{array}{l}\text { A01, A03, } \\
\text { A02, A04 }\end{array}$ & N10 & 新凯山泉居 \\
\hline 146)Linkeyuanxiaoqu & B02 & $\mathrm{A} 02$ & N02 & 林科院小区 \\
\hline 147) Jianxinxiaoqu & $\begin{array}{l}\text { B02, B03, } \\
\text { B09 }\end{array}$ & $\begin{array}{l}\text { A01, A02, } \\
\text { A04 }\end{array}$ & N08 & 建信小区 \\
\hline 148)Feicuicheng & $\begin{array}{l}\text { B03 } \\
\text { (reverse) }\end{array}$ & A01 & N01 & 翡翠城 \\
\hline 149)Lantingju & $\mathrm{B} 02, \mathrm{~A} 12$ & $\mathrm{~A} 02, \mathrm{~A} 04$ & N04 & 兰庭居 \\
\hline 150) Nanyuanhuayuan & $\mathrm{B} 02, \mathrm{~B} 03$ & A01 (A04) & N05 & 南苑花园 \\
\hline $\begin{array}{l}\text { 151)Zhuodaoquankejigong } \\
\mathrm{yu}\end{array}$ & $\begin{array}{l}\text { B02, B03, } \\
\text { B12 }\end{array}$ & $\begin{array}{l}\text { A01, A02, } \\
\text { A04 }\end{array}$ & N08 & 卓刀泉科技公寓 \\
\hline 152)Donghumingju & $\mathrm{B} 02, \mathrm{~B} 03$ & $\begin{array}{l}\text { A01, A02, } \\
\text { A03 (A04) }\end{array}$ & N05 & 东湖名居 \\
\hline
\end{tabular}




\begin{tabular}{|c|c|c|c|c|}
\hline 153) Donghuqiaoshequ & B01, B02 & $\mathrm{A} 01, \mathrm{~A} 04$ & N09 & 东湖桥社区 \\
\hline 154)Fengguangyuan & B02, B03 & $\begin{array}{l}\text { A01, A02, } \\
\text { A04 }\end{array}$ & N08 & 风光苑 \\
\hline 155)Donghugongguan & A12 & A 01 & N01 & 东湖公馆 \\
\hline 156)Donghuchutinfu & B02, B03 & A01, A04 & N09 & 东湖楚天府 \\
\hline $\begin{array}{l}\text { 157) Wudaluohanxincunxia } \\
\text { oqu }\end{array}$ & $\begin{array}{l}\text { B02, B03, } \\
\text { B05 } \\
(\mathrm{B} 12)\end{array}$ & $\begin{array}{l}\text { A01, A02, } \\
\text { A04 }\end{array}$ & N07 & 武大珞涵新邨小区 \\
\hline 158)Fengyiyuan & B03 & A 01 & N02 & 丰颐苑 \\
\hline 159) Yinhaiyayuan & B03 & $\begin{array}{l}\text { A01, A02, } \\
\text { (A04) }\end{array}$ & N03 & 银海东湖珞珈 \\
\hline 160) Yinhaiyayuan & $\begin{array}{l}\mathrm{B} 12 \\
\text { (B05) }\end{array}$ & A01 & N04 & 银海雅苑 \\
\hline $\begin{array}{l}\text { 161) Wuhanjingyingxueshe } \\
\text { nggongyu }\end{array}$ & B12 & A 01 & N01 & 武汉精英学生公寓 \\
\hline $\begin{array}{l}\text { 162) Wuhannanzhouqingnia } \\
\text { ngongyu }\end{array}$ & B03 & A 01 & N01 & 武汉南周青年公寓 \\
\hline 163)Rongkeluoyuzhongxin & B03 & A 01 & N02 & 融科珞瑜中心 \\
\hline 164)722yanjiusuoxiaoqu & B03 & $\mathrm{A} 02, \mathrm{~A} 04$ & N02 & 722 研究所小区 \\
\hline 165)Fuhuadasha & B12 & A 01 & N01 & 阜华大厦 \\
\hline 166)Pengchengguoji & $\mathrm{B} 12$ & A01 (A04) & N01 & 鹏程国际 \\
\hline 167)Quanguangshangyuan & $\mathrm{B} 01, \mathrm{~B} 12$ & $\begin{array}{l}\text { A01, A02, } \\
\text { A04 }\end{array}$ & N04 & 群光上苑 \\
\hline 168) Weilaicheng & $\begin{array}{l}\text { B02, B07, } \\
\text { B12 }\end{array}$ & $\begin{array}{l}\text { A01, A02, } \\
\text { A04 }\end{array}$ & N04 & 未来城 \\
\hline 169)Pengchenghuiyuan & B03, B12 & A01 & N03 & 鹏程蕙园 \\
\hline 170)Limingxinju & $\begin{array}{l}\mathrm{B} 12 \\
(\mathrm{~B} 03)\end{array}$ & A01 (A04) & N03 & 黎明新居 \\
\hline 171)Hongdayuan & B12 & A01 & N02 & 鸿达苑 \\
\hline
\end{tabular}




\begin{tabular}{|c|c|c|c|c|}
\hline & (B03) & & & \\
\hline $\begin{array}{l}\text { 172) Wuhanligongmafangsh } \\
\text { andongyuangaocengjia } \\
\text { oshigongyu }\end{array}$ & B04 & A01 & N02 & $\begin{array}{l}\text { 武汉理工马房山东院 } \\
\text { 高层教师公寓 }(14,15 \\
\text { 区) }\end{array}$ \\
\hline 173) Yulanyuan & B02 & $\mathrm{A} 02, \mathrm{~A} 04$ & $\mathrm{~N} 22$ & 玉兰苑 \\
\hline 174)Hongguiyuan & B03 & $\mathrm{A} 02, \mathrm{~A} 04$ & N03 & 鸿桂苑 (东、西、北区) \\
\hline 175) Jianqiaomingdi & $\mathrm{B} 01, \mathrm{~B} 02$ & $\mathrm{~A} 02, \mathrm{~A} 04$ & N05 & 剑桥名邸 \\
\hline 176) Yuanmengmeilijiayuan & B01, B02 & $\begin{array}{l}\text { A02, A03, } \\
\text { A04 }\end{array}$ & N10 & 圆梦美丽家园 \\
\hline 177) Taigeshengtaigongyu & B02, B05 & $\mathrm{A} 02, \mathrm{~A} 04$ & N08 & 泰格生态公寓 \\
\hline $\begin{array}{l}\text { 178)Zhongjianfudixingche } \\
\text { ng }\end{array}$ & B03 & $\mathrm{A} 02,(\mathrm{~A} 04)$ & N08 & 中建福地星城 \\
\hline 179)Bowenhuayuan & B01, B02 & $\begin{array}{l}\text { A01, A02, } \\
\text { (A04) }\end{array}$ & N17 & 博文花园 \\
\hline 180) Yijingyuan & B02 & $\mathrm{A} 02$ & N06 & 逸景苑 \\
\hline 181)Hongyuefu & B03 & $\begin{array}{l}\text { A01, A02, } \\
\text { A04 }\end{array}$ & N09 & 鸿悦府 \\
\hline 182)Honglinghuayuan & B03, B04 & $\begin{array}{l}\text { A01, A02, } \\
\text { A04 }\end{array}$ & $\mathrm{N} 12$ & 鸿岭花园 \\
\hline 183)Lingshangfu & B03 & $\mathrm{A} 02, \mathrm{~A} 04$ & N04 & 岭上府 \\
\hline $\begin{array}{l}\text { 184) Shipailingsanhongling } \\
\text { yu }\end{array}$ & B02, B03 & $\mathrm{A} 02, \mathrm{~A} 04$ & N04 & 石牌岭三鸿领域 \\
\hline 185)Diyahuayuan & B02 & $\begin{array}{l}\text { A01, A02, } \\
\text { A03, (A04) }\end{array}$ & N10 & 迪雅花园 \\
\hline 186) Jingyunhuayuan & B03 & $\mathrm{A} 03$ & N01 & 京韵花园 \\
\hline 187)Zhuyuanxiaoqu & B01, B02 & $\begin{array}{l}\mathrm{A} 01, \mathrm{~A} 02, \\
(\mathrm{~A} 04)\end{array}$ & N07 & 竹苑小区 \\
\hline 188)Zhuyuandongqu & B01, B02 & $\begin{array}{l}\mathrm{A} 01, \mathrm{~A} 02, \\
(\mathrm{~A} 04)\end{array}$ & N16 & 竹苑东区 \\
\hline
\end{tabular}




\begin{tabular}{|c|c|c|c|c|}
\hline 189)Honglinggongyu & B02 & $\mathrm{A} 02, \mathrm{~A} 04$ & N03 & 洪岭公寓 \\
\hline 190)Binghumingdi & B02, B03 & $\begin{array}{l}\mathrm{A} 01, \mathrm{~A} 02, \\
\mathrm{~A} 04\end{array}$ & N16 & 滨湖名邸 \\
\hline 191) Bairuijing (part 1) & B02, B03 & $\begin{array}{l}\mathrm{A} 01, \mathrm{~A} 02, \\
\mathrm{~A} 04\end{array}$ & N08 & 百瑞景（一期） \\
\hline 192) Bairuijing (part 2) & B02, B03 & $\begin{array}{l}\text { A01, A02, } \\
\text { A03 (A04) }\end{array}$ & N14 & 百瑞景（二期） \\
\hline 193)Bairuijing (part 3) & B02, В03 & $\begin{array}{l}\mathrm{A} 01, \mathrm{~A} 02, \\
\mathrm{~A} 04\end{array}$ & N07 & 百瑞景（三期） \\
\hline 194) Bairuijing (part 4) & B02, B03 & $\begin{array}{l}\mathrm{A} 01, \mathrm{~A} 02, \\
\mathrm{~A} 04\end{array}$ & N13 & 百瑞景（四期） \\
\hline 195)Bairuijing (part 5) & B02, B03 & $\begin{array}{l}\mathrm{A} 01, \mathrm{~A} 02 \\
(\mathrm{~A} 04)\end{array}$ & N07 & 百瑞景（五期） \\
\hline 196)Bairuijing (part 6) & B03 & $\begin{array}{l}\mathrm{A} 01, \mathrm{~A} 02, \\
\mathrm{~A} 04\end{array}$ & N09 & 百瑞景（六期） \\
\hline 197)Lidao2046 & B02, B03 & $\begin{array}{l}\mathrm{A} 01, \mathrm{~A} 02, \\
\mathrm{~A} 04\end{array}$ & N08 & 丽岛 2046 \\
\hline $\begin{array}{l}\text { 198) Shenjiawanhuayuanxia } \\
\text { oqu }\end{array}$ & B02, B03 & $\begin{array}{l}\mathrm{A} 01, \mathrm{~A} 02, \\
\mathrm{~A} 03, \mathrm{~A} 04\end{array}$ & N11 & 沈家湾花园小区 \\
\hline 199)Hongyejunyuan & $\mathrm{B} 02, \mathrm{~B} 04$ & $\mathrm{~A} 01, \mathrm{~A} 04$ & N05 & 弘业俊园 \\
\hline 200)Binhuminducheng & B02, В03 & $\begin{array}{l}\mathrm{A} 01, \mathrm{~A} 02, \\
\mathrm{~A} 04\end{array}$ & N15 & 滨湖名都城 \\
\hline 201)Chengshiyinxiang & B02, B03 & $\begin{array}{l}\mathrm{A} 01, \mathrm{~A} 02, \\
(\mathrm{~A} 04)\end{array}$ & N05 & 城市印象 \\
\hline 202) Jinnuoyayuan & B03, B12 & $\begin{array}{l}\mathrm{A} 01, \mathrm{~A} 02, \\
(\mathrm{~A} 04)\end{array}$ & N02 & 金诺雅苑 \\
\hline $\begin{array}{l}\text { 203) Hubeishenggonganting } \\
\text { xiyuan }\end{array}$ & B02 & $\mathrm{A} 02, \mathrm{~A} 04$ & N08 & 湖北省公安厅西院 \\
\hline 204) Yinheyuangongyu & B09 & A01 & N01 & 银河苑公寓 \\
\hline
\end{tabular}




\begin{tabular}{|c|c|c|c|c|}
\hline 205)Shuxiangmendi & B03 & A01 & N01 & 书香门第 \\
\hline 206)Meizhongxiaoqu & B12 & A03 & N01 & 梅中小区 \\
\hline 207)Meiyuanxiaoqu & $\begin{array}{l}\text { B02, B03, } \\
\text { B12 }\end{array}$ & $\begin{array}{l}\text { A01, A02, } \\
\text { A04 }\end{array}$ & N35 & 梅苑小区 \\
\hline 208) Meitanchangsushe & B03, B05 & $\begin{array}{l}\text { A01, A02, } \\
\text { A03, A04 }\end{array}$ & N03 & 煤碳厂宿舍 \\
\hline 209)Meiyuanerqi & $\begin{array}{l}\text { B01, B02, } \\
\text { B03, B12 }\end{array}$ & $\begin{array}{l}\text { A01, A02, } \\
\text { A03, A04 }\end{array}$ & N33 & 梅苑二期 \\
\hline 210)Meiyuansanqi & $\begin{array}{l}\text { B01, B02, } \\
\text { B12 }\end{array}$ & $\begin{array}{l}\text { A01, A02, } \\
(\mathrm{A} 04)\end{array}$ & N03 & 梅苑三期 \\
\hline 211)Boguangyuan & $\begin{array}{l}\text { B01, B02, } \\
\text { B03, B04, } \\
\text { В12 }\end{array}$ & $\begin{array}{l}\text { A01, A02, } \\
\text { A03, A04 }\end{array}$ & $\mathrm{N} 25$ & 波光园 \\
\hline $\begin{array}{l}\text { 212)Zhongnanliushiqihaoy } \\
\text { uan }\end{array}$ & $\mathrm{B} 02, \mathrm{~B} 03$ & A02 & N02 & 中南六十七号院 \\
\hline 213) Jinganshangcheng & B02, B03 & $\begin{array}{l}\text { A02, A03, } \\
(\mathrm{A} 04)\end{array}$ & N08 & 静安上城 \\
\hline 214)Kaiyuanmindi & B03 & $\begin{array}{l}\text { A01, A02, } \\
\text { A04 }\end{array}$ & N13 & 凯旋名邸 \\
\hline $\begin{array}{l}\text { 215) Wuchangchengshiguan } \\
\text { gchang }\end{array}$ & $\mathrm{B} 02, \mathrm{~B} 03$ & $\begin{array}{l}\text { A01, A02, } \\
\text { A04 }\end{array}$ & N10 & 武昌城市公园 \\
\hline 216)Zhanqianyangguang & B03 & A01 & N02 & 站前阳光 \\
\hline 217)Hongxinjiayuan & $\mathrm{B} 02, \mathrm{~B} 03$ & A02, A04 & N05 & 红釒佳园 \\
\hline 218)Qianjiaxinyuan & B03 & A01 & N02 & 千家金视 \\
\hline 219) Yucaijiayuan & $\mathrm{B} 02, \mathrm{~B} 12$ & $\mathrm{~A} 02, \mathrm{~A} 04$ & N02 & 育才嘉苑 \\
\hline 220)Xinlongziyangyuan & $\begin{array}{l}\mathrm{B} 12 \\
(\mathrm{~B} 03)\end{array}$ & A01 & N03 & 欣隆紫阳苑 \\
\hline 221)Donghaiwan & B02 & A02 & N02 & 东海湾 \\
\hline 222)Beihaiwan & $\mathrm{B} 02, \mathrm{~B} 12$ & $\mathrm{~A} 01, \mathrm{~A} 02$, & N09 & 北海湾 \\
\hline
\end{tabular}




\begin{tabular}{|c|c|c|c|c|}
\hline & (B03) & $(\mathrm{A} 04)$ & & \\
\hline 223) Shouyixiaoqu & $\mathrm{B} 03, \mathrm{~B} 12$ & $\begin{array}{l}\text { A01, A02, } \\
\text { A04 }\end{array}$ & $\mathrm{N} 22$ & 首义小区 \\
\hline 224) Shouyixincun & $\begin{array}{l}\mathrm{B} 02, \mathrm{~B} 03, \\
\mathrm{~B} 12\end{array}$ & $\begin{array}{l}\text { A01, A02, } \\
\text { A04 }\end{array}$ & $\mathrm{N} 23$ & 首义新村 \\
\hline 225) Shouyimingju & $\mathrm{B} 02, \mathrm{~B} 03$ & A02, A04 & N05 & 首义名居 \\
\hline 226) Yuandingxiaoqu & B05 & A01 & N03 & 园丁小区 \\
\hline $\begin{array}{l}\text { 227) Wuhantieludadongmen } \\
\text { zhuzhaixiaoqu }\end{array}$ & B01, B02 & $\begin{array}{l}\text { A01, A02, } \\
\text { A04 }\end{array}$ & N39 & $\begin{array}{l}\text { 武汉铁路大东门住宅 } \\
\text { 小区 }\end{array}$ \\
\hline 228) Jintaomingzuo & $\mathrm{B} 12$ & A01 & N01 & 金涛铭座 \\
\hline 229)719suoxiaoqu & B02, B03 & $\begin{array}{l}\text { A01, A02, } \\
\text { (A04) }\end{array}$ & N03 & 719 所小区 \\
\hline 230)Xiaxujiawanxiaoqu & B02 & A02 & N10 & 下徐家湾小区 \\
\hline $\begin{array}{l}\text { 231) Hubeishengjiansheting } \\
\text { xiaoqu }\end{array}$ & $\begin{array}{l}\mathrm{B} 12(\mathrm{~B} 03, \\
\mathrm{B} 09)\end{array}$ & A01 & N01 & 湖北省建设厅小区 \\
\hline 232)Zhongnandagongguan & $\mathrm{B} 12$ & A01 & N01 & 中南大公馆 \\
\hline $\begin{array}{l}\text { 233) Hubeishengjiankeyuan } \\
\text { sushe }\end{array}$ & B07 & A01 & N01 & 湖北省建科院宿舍 \\
\hline 234)Boyazhongnan & B02 & $\mathrm{A} 01, \mathrm{~A} 04$ & N02 & 博雅中南 \\
\hline 235)Qingjianghuayuan & B02 & $\mathrm{A} 02$ & N02 & 清江花园 \\
\hline 236) Yinhaihuating & $\mathrm{B} 03, \mathrm{~B} 12$ & $\begin{array}{l}\text { A01, A02, } \\
\text { A04 }\end{array}$ & N05 & 银海华庭 \\
\hline 237)Xiaohongshanxiaoqu & $\begin{array}{l}\mathrm{B} 02, \mathrm{~B} 03, \\
\mathrm{~B} 12\end{array}$ & $\begin{array}{l}\text { A01, A02, } \\
\text { A04 }\end{array}$ & N56 & 小洪山小区 \\
\hline 238)Nongshanghangxiaoqu & B02 & A02 & N01 & 农商行小区 \\
\hline 239) Yinhaishanzhuang & $\mathrm{B} 12$ & A04 & N02 & 银海山庄 \\
\hline 240)Xingxingxincheng & B03 & A01. A04 & N07 & 星星新城 \\
\hline $\begin{array}{l}\text { 241) Wutiehongshanzhuzha } \\
\text { ixiaoqu }\end{array}$ & $\mathrm{B} 02, \mathrm{~B} 03$ & $\begin{array}{l}\text { A01, A02, } \\
\text { A03, A04 }\end{array}$ & N15 & 武铁洪山住宅小区 \\
\hline
\end{tabular}




\begin{tabular}{|c|c|c|c|c|}
\hline 242) Bayilutieluxiaoqu & B02, B03 & $\begin{array}{l}\mathrm{A} 01, \mathrm{~A} 02, \\
\mathrm{~A} 04\end{array}$ & N30 & 八一路铁路小区 \\
\hline 243)Hongce12 haoyuan & B02, B03 & A02 & N11 & 洪测 12 号院 \\
\hline 244)Chagangdayuan & B02, B12 & $\mathrm{A} 02, \mathrm{~A} 04$ & N36 & 茶港大院 \\
\hline 245)Zhenxingyuanxiaoqu & B02, B03 & A02 & N08 & 振兴苑小区 \\
\hline $\begin{array}{l}\text { 246) Chagangjunzhuanxiao } \\
\mathrm{qu}\end{array}$ & B02 & $\mathrm{A} 02, \mathrm{~A} 04$ & N05 & 茶港军转小区 \\
\hline $\begin{array}{l}\text { 247) Wuhandaxuechagangq } \\
\text { u }\end{array}$ & $\begin{array}{l}\text { B02, B03, } \\
\text { B08 }\end{array}$ & $\begin{array}{l}\mathrm{A} 01, \mathrm{~A} 02, \\
\mathrm{~A} 04\end{array}$ & N27 & 武汉大学茶港区 \\
\hline 248) Shuishengkejiyuan & B03 & A01, A04 & N06 & 水生科技苑 \\
\hline 249) Taoshancun & $\begin{array}{l}\text { B02, B03, } \\
\text { B06 }\end{array}$ & $\begin{array}{l}\mathrm{A} 01, \mathrm{~A} 02, \\
\mathrm{~A} 04\end{array}$ & N08 & 桃山村 \\
\hline 250)Zhangjiawanxiaoqu & B02 & $\mathrm{A} 01, \mathrm{~A} 02$ & N63 & 张家湾小区 \\
\hline 251) Shuxinyuan & B01 & $\mathrm{A} 01, \mathrm{~A} 02$ & N02 & 舒心苑 \\
\hline 252)Hongchengjindu & $\mathrm{B} 02, \mathrm{~B} 03$ & A01 & N02 & 宏城金都 \\
\hline 253) Anshunhuayuan & $\begin{array}{l}\text { B02, B03, } \\
\text { B12 }\end{array}$ & $\begin{array}{l}\mathrm{A} 01, \mathrm{~A} 02, \\
\mathrm{~A} 03, \mathrm{~A} 04\end{array}$ & N15 & 安顺花园 \\
\hline 254)Zhongguotiejian1818 & B03, B12 & A02, A04 & N08 & 中国铁建 1818 \\
\hline 255)Haishanjingutiancheng & $\mathrm{B} 02, \mathrm{~B} 03$ & A02, A04 & N04 & 海山金谷天城 \\
\hline 256)Donghuxiyuan & B02 & A02 & N01 & 东湖熙园 \\
\hline 257)Xianggelijiayuan & $\mathrm{B} 02, \mathrm{~B} 12$ & A04 & N02 & 香格里嘉园 \\
\hline 258)Baizanting & $\mathrm{B} 02, \mathrm{~B} 03$ & A02 & N10 & 拜赞庭 \\
\hline 259)Hongshanhuayuan & $\mathrm{B} 02, \mathrm{~B} 05$ & $\mathrm{~A} 02, \mathrm{~A} 04$ & N07 & 洪山花园 \\
\hline 260)Zhiyinhuayuan & B02 & $\mathrm{A} 02, \mathrm{~A} 04$ & N02 & 知音花园 \\
\hline 261)Huarunzhidigongguan & $\mathrm{B} 12$ & A04 (A01) & N01 & 华润置地公馆 \\
\hline 262)Jinduhuating & B01 & A02 (A01) & N01 & 金都华庭 \\
\hline 263) Kailehuayuan & B02, B03 & $\begin{array}{l}\mathrm{A} 02, \mathrm{~A} 03, \\
\mathrm{~A} 04\end{array}$ & N09 & 凯乐花园 \\
\hline 264)Xinghaihongcheng & B02, B03, & $\mathrm{A} 02, \mathrm{~A} 03$ & N10 & 星海虹城 \\
\hline
\end{tabular}




\begin{tabular}{|c|c|c|c|c|}
\hline & B09 & A04 & & \\
\hline 265)Zishajinyuan & B02, B03 & A02 & N02 & 紫沙金苑 \\
\hline 266)Xingheju & B01, B02 & $\begin{array}{l}\text { A01, A02, } \\
\text { A03, A04 }\end{array}$ & N08 & 星河居 \\
\hline 267) Yaojialing & B02 & A02 & N01 & 姚家岭还建楼 \\
\hline 268)Donglongshijihuayuan & $\begin{array}{l}\mathrm{B} 02, \mathrm{~B} 03, \\
\mathrm{~B} 05, \mathrm{~B} 12\end{array}$ & $\begin{array}{l}\text { A01, A02, } \\
\text { A03, A04 }\end{array}$ & N19 & 东龙世纪花园 \\
\hline 269)Fayuanxiaoqu & B02 & A02 & N04 & 法院小区 \\
\hline 270) Shahumingzhu & $\mathrm{B} 02, \mathrm{~B} 03$ & $\mathrm{~A} 01, \mathrm{~A} 02$ & N06 & 沙湖明珠 \\
\hline 271) Wandayuhushijia & B02, B03 & $\mathrm{A} 02, \mathrm{~A} 04$ & N04 & 万达御湖世家 \\
\hline 272)Hanjieyihaogongguan & B02 & $\mathrm{A} 01, \mathrm{~A} 02$ & N06 & 汉街一号公馆 \\
\hline $\begin{array}{l}\text { 273) Guangxiazhongbeiling } \\
\text { xiu }\end{array}$ & $\mathrm{B} 01, \mathrm{~B} 03$ & $\mathrm{~A} 01, \mathrm{~A} 02$ & N04 & 广厦中北领秀 \\
\hline 274)Chuhanxinju & B03 & A01 & N06 & 楚汉新居 \\
\hline 275)Fudidonghuguoji & B02, B03 & $\begin{array}{l}\text { A01, A02, } \\
\text { A04 }\end{array}$ & N45 & 复地东湖国际 \\
\hline 276) Jinshahaoting & $\mathrm{B} 01, \mathrm{~B} 11$ & A01 & N05 & 金沙豪庭 \\
\hline 277) Jinxiuchuntian & B03 & $\begin{array}{l}\mathrm{A} 01, \mathrm{~A} 02, \\
(\mathrm{~A} 04)\end{array}$ & N07 & 锦绣春天 \\
\hline 278) Jinxiuzhongbei & B02, B03 & $\begin{array}{l}\text { A01, A02, } \\
(\mathrm{A} 04)\end{array}$ & N07 & 锦绣中北 \\
\hline 279) Jinxiujiangnan & B02, B03 & $\begin{array}{l}\text { A01, A02, } \\
\text { A03, (A }\end{array}$ & N31 & 锦绣江南 \\
\hline 280) Weipengyuan & B02 & $\mathrm{A} 02, \mathrm{~A} 03$ & N08 & 伟鹏苑 \\
\hline 281)Donghuxiangxieshuian & $\mathrm{B} 03, \mathrm{~B} 12$ & $\mathrm{~A} 01, \mathrm{~A} 02$ & N05 & 东湖香榭水岸 \\
\hline 282)Shijicaicheng & $\begin{array}{l}\text { B02, B03, } \\
\text { B05 }\end{array}$ & $\begin{array}{l}\text { A01, A02, } \\
\text { A03, A04 }\end{array}$ & N14 & 世纪彩城 \\
\hline 283)Chutiandushiyayuan & $\mathrm{B} 02, \mathrm{~B} 03$ & $\mathrm{~A} 01, \mathrm{~A} 02$ & N10 & 楚天都市雅苑 \\
\hline 284)Donghuchunshuli & В03, В07, & $\mathrm{A} 01, \mathrm{~A} 02$ & N08 & 东湖春树里 \\
\hline
\end{tabular}




\begin{tabular}{|c|c|c|c|c|}
\hline & B08 & & & \\
\hline 285)Donghutianxia & $\mathrm{B} 02, \mathrm{~B} 11$ & $\begin{array}{l}\text { A01, A02, } \\
\text { A04 }\end{array}$ & N11 & 东湖天下 \\
\hline 286)Donghuruiyuan & B02, B03 & $\mathrm{A} 01, \mathrm{~A} 02$ & N03 & 东湖睿园 \\
\hline 287)Donghujingyuan & B03 & $\begin{array}{l}\text { A01, A02, } \\
\text { A03, A04 }\end{array}$ & N37 & 东湖景园 \\
\hline 288) Yuejiazuixiaoqu & $\begin{array}{l}\mathrm{B} 02, \mathrm{~B} 03, \\
\mathrm{~B} 11\end{array}$ & $\begin{array}{l}\text { A01, A02, } \\
\text { A03, A04 }\end{array}$ & N27 & 岳家嘴小区 \\
\hline $\begin{array}{l}\text { 289) Changyuandonghusha } \\
\text { ngjun }\end{array}$ & B02 & $\begin{array}{l}\text { A02, A03, } \\
\text { A04 }\end{array}$ & N07 & 长源东湖尚郡 \\
\hline $\begin{array}{l}\text { 290) Chengkaidongshahuay } \\
\text { uan }\end{array}$ & B03, B05 & $\mathrm{A} 01, \mathrm{~A} 02$ & N16 & 城开东沙花园 \\
\hline 291)Lanjinglvzhou & $\mathrm{B} 03, \mathrm{~B} 11$ & $\mathrm{~A} 01, \mathrm{~A} 02$ & $\mathrm{~N} 20$ & 蓝晶绿洲 \\
\hline 292)Huatengyuan & B03, B04 & $\begin{array}{l}\mathrm{A} 01, \mathrm{~A} 02, \\
\mathrm{~A} 03\end{array}$ & N15 & 华腾园 \\
\hline 293)Dushijingdian & B02, B04 & $\begin{array}{l}\text { A01, A02, } \\
\text { A04 }\end{array}$ & N18 & 都市经典 \\
\hline 294)Xinghuajiatianxia & B03 & $\begin{array}{l}\mathrm{A} 01, \mathrm{~A} 02, \\
\mathrm{~A} 03\end{array}$ & N11 & 兴华嘉天下 \\
\hline 295) Shengshixudong & B03 & A01 & N03 & 盛世徐东 \\
\hline 296)Lianfajiuduguoji & B02 & A01 & N06 & 联发九都国际 \\
\hline $\begin{array}{l}\text { 297)Fuxinghuiyuguojichen } \\
\mathrm{g}\end{array}$ & B02, B03 & $\mathrm{A} 01, \mathrm{~A} 02$ & $\mathrm{~N} 10$ & 福星惠誉国际城 \\
\hline 298)Fuxinghuiyushuian & $\begin{array}{l}\text { B02, B03, } \\
\text { B08 }\end{array}$ & $\mathrm{A} 01, \mathrm{~A} 02$ & N18 & 福星惠誉水岸 \\
\hline $\begin{array}{l}\text { 299) Hubeidaxueqinyuanxia } \\
\text { oqu }\end{array}$ & $\mathrm{B} 02, \mathrm{~B} 03$ & $\mathrm{~A} 01, \mathrm{~A} 02$ & N13 & 湖北大学琴园小区 \\
\hline $\begin{array}{l}\text { 300) Shuianguojishahugong } \\
\text { guan }\end{array}$ & B03 & A01 & N03 & 水岸国际沙湖公馆 \\
\hline
\end{tabular}




\begin{tabular}{|c|c|c|c|c|}
\hline 301)Menghushuian & B02, B03 & $\mathrm{A} 02, \mathrm{~A} 04$ & N14 & 梦湖水岸 \\
\hline 302)Shahujingangyuan & B02, B03 & A02, A04 & N05 & 沙湖金港苑 \\
\hline 303)Meichengqingzhiyuan & B02, B03 & A02 & N09 & 美城清芷园 \\
\hline 304)Hengdashoufu & B02 & $\mathrm{A} 01, \mathrm{~A} 02$ & N10 & 恒大首府 \\
\hline 305) Shuianguoji-1 & B02, B03 & $\mathrm{A} 01, \mathrm{~A} 02$ & N05 & 水岸国际-1 \\
\hline 306) Shuianguoji-2 & B02 & A01 & N07 & 水岸国际-2 \\
\hline 307)Bojintiandi & B03 & $\begin{array}{l}\text { A01, A02, } \\
\text { A04 }\end{array}$ & N06 & 铂金天地 \\
\hline 308)Rongqiaohuafu & B02, B03 & $\begin{array}{l}\text { A01, A02, } \\
\text { A04 }\end{array}$ & N16 & 融侨华府 \\
\hline 309)Zhonghuanguoji & B03 & A01 & N02 & 中环国际 \\
\hline 310) Oulinwan & B03, B05 & $\mathrm{A} 01, \mathrm{~A} 02$ & N03 & 欧林湾 \\
\hline 311) Wandagongguan & B02 & A02 & N05 & 万达公馆 \\
\hline 312) Jiangnanmingzhuyuan & B02, B03 & A03, A04 & N07 & 江南明珠园 \\
\hline 313) Jinjiangguojicheng & B02 & $\mathrm{A} 02, \mathrm{~A} 03$ & N06 & 锦江国际城 \\
\hline 314) Jindiguojihuayuan & B02, B03 & $\begin{array}{l}\text { A01, A02, } \\
\text { A04 }\end{array}$ & N08 & 金地国际花园 \\
\hline 315) Shanglongdiqiucun & B02, B03 & $\begin{array}{l}\text { A01, A02, } \\
\text { A04 }\end{array}$ & $\mathrm{N} 28$ & 尚隆地球村 \\
\hline 316)Chengkaixinqiaoxindu & B02, B03 & $\begin{array}{l}\text { A01, A02, } \\
\text { A04 }\end{array}$ & N09 & 城开玉桥新都 \\
\hline 317)Lanwanjunyuan & B02 & $\mathrm{A} 02, \mathrm{~A} 03$ & N18 & 蓝湾俊园 \\
\hline 318) Jinduhangong & B12 & $\mathrm{A} 02, \mathrm{~A} 04$ & N08 & 金都汉宫 \\
\hline 319)Fenghuangcheng & B03 & $\mathrm{A} 01, \mathrm{~A} 02$ & N08 & 凤凰城 \\
\hline 320) Youyiguoji & $\mathrm{B} 02, \mathrm{~B} 03$ & $\mathrm{~A} 01, \mathrm{~A} 02$ & N19 & 友谊国际 \\
\hline $\begin{array}{l}\text { 321)Fenghuangshijihuayua } \\
\text { n }\end{array}$ & B02 & A02, A04 & N10 & 凤凰世纪花园 \\
\hline 322)Dingxiujiayuan & $\begin{array}{l}\text { B12 } \\
\text { (B03) }\end{array}$ & A01 & N03 & 顶秀嘉园 \\
\hline
\end{tabular}




\begin{tabular}{|c|c|c|c|c|}
\hline 323) Jinbangmingyuan & B01, B02 & $\mathrm{A} 01, \mathrm{~A} 02$ & N05 & 金榜名苑 \\
\hline 324) Shouyishiyuan & B03 & A02 & N02 & 首义师苑 \\
\hline $\begin{array}{l}\text { 325) Wuhandaxuerenminyi } \\
\text { yuansushelou }\end{array}$ & B04 & A01 & N01 & $\begin{array}{l}\text { 武汉大学人民医院宿 } \\
\text { 舍楼 }\end{array}$ \\
\hline 326)Xinyujiayuan & B03 & A02 & N02 & 新宇佳苑 \\
\hline 327)Ziyangjinliwu & $\mathrm{B} 02, \mathrm{~B} 03$ & $\mathrm{~A} 01, \mathrm{~A} 02$ & N03 & 紫阳金利屋 \\
\hline 328)Zidiyuanxiaoqu & B03, B04 & A01 & N10 & 紫笛苑小区 \\
\hline $\begin{array}{l}\text { 329) Huatianmingzhuhuayu } \\
\text { anzhanggongyuan }\end{array}$ & B03 & A02 & N01 & 华天明珠花园张公苑 \\
\hline $\begin{array}{l}\text { 330) Hongyuanguojigonggu } \\
\text { an }\end{array}$ & B02 & $\mathrm{A} 01, \mathrm{~A} 02$ & N04 & 鸿源国际公馆 \\
\hline 331) Aijiaguojihuacheng & $\begin{array}{l}\text { B02, B03, } \\
\text { B08 }\end{array}$ & $\begin{array}{l}\text { A01, A02, } \\
\text { A03, A04 }\end{array}$ & N37 & 爱家国际华城 \\
\hline 332)Lihuayuan & B02, B03 & $\begin{array}{l}\text { A01, A02, } \\
\text { A03 }\end{array}$ & N19 & 丽华苑 \\
\hline 333)Baolicheng & B03, B05 & $\mathrm{A} 01, \mathrm{~A} 02$ & $\mathrm{~N} 23$ & 保利城 \\
\hline 334)Meilinqingcheng & $\mathrm{B} 02, \mathrm{~B} 03$ & $\mathrm{~A} 02, \mathrm{~A} 03$ & N37 & 美林青城 \\
\hline 335) Wutiejiayuan & B03, B04 & $\mathrm{A} 01, \mathrm{~A} 02$ & N29 & 武铁佳苑 \\
\hline 336)Xudongxinyuan & B03 & $\begin{array}{l}\text { A01, A02, } \\
\text { A03 }\end{array}$ & N08 & 徐东馨苑 \\
\hline 337) Junlintianxia & B04 & A01 & N03 & 君临天下 \\
\hline 338) Wankelangyuan & B02 & $\mathrm{A} 02$ & N03 & 万科郎苑 \\
\hline 339)Dongrunshangyu & B02 & $\mathrm{A} 01, \mathrm{~A} 02$ & N03 & 东润上域 \\
\hline 340) Jiangnanhuayuan & $\mathrm{B} 02, \mathrm{~B} 03$ & A02, A04 & N03 & 江南花园 \\
\hline 341) Wankejinyuhuafu & B07 & $\mathrm{A} 02, \mathrm{~A} 04$ & N04 & 万科金域华府 \\
\hline $\begin{array}{l}\text { 342) Wankechengshihuayua } \\
\text { n }\end{array}$ & B02 & $\begin{array}{l}\text { A01, A02, } \\
\text { A04 }\end{array}$ & N82(04) & 万科城市花园 \\
\hline 343)Dangdaiguoji & B02, B03 & $\begin{array}{l}\text { A01, A02, } \\
\text { A04 }\end{array}$ & N97(16) & 当代国际 \\
\hline
\end{tabular}




\begin{tabular}{|c|c|c|c|c|}
\hline 344) Guangguqilinshe & B02 & A01 & N05 & 光谷麒麟社 \\
\hline 345) Tangxunhushequ & B02 & $\mathrm{A} 02$ & N101(00) & 汤逊湖社区 \\
\hline 346)Lidaomeisheng & B03 & $\mathrm{A} 01, \mathrm{~A} 03$ & N08 & 丽岛美生 \\
\hline 347)Maodianqiqixiaoqu & B03 & $\mathrm{A} 01, \mathrm{~A} 02$ & N05 & 茅店七期小区 \\
\hline 348) Wankehongjun & B02 & $\begin{array}{l}\text { A02, A03, } \\
(\mathrm{A} 04)\end{array}$ & $\mathrm{N} 21$ & 万科红郡 \\
\hline 349)Xiandaisenlinxiaozhen & B02, B03 & $\begin{array}{l}\text { A01, A02, } \\
(\mathrm{A} 04)\end{array}$ & N43 & 现代森林小镇 \\
\hline 350)Minghuhaoting & B02, B03 & $\begin{array}{l}\text { A01, A02, } \\
\text { A03 (A04) }\end{array}$ & N31 & 明湖豪庭 \\
\hline $\begin{array}{l}\text { 351)Zhongyenanfangyunhu } \\
\text { shoufu }\end{array}$ & B02 & A01 & N07 & 中冶南方韵湖首府 \\
\hline $\begin{array}{l}\text { 352)Zhongguotiejianwuton } \\
\text { gyuan }\end{array}$ & B02, B03 & $\mathrm{A} 01, \mathrm{~A} 02$ & N13 & 中国铁建梧桐苑 \\
\hline 353) Wanjun & B02, B03 & A01, A02 & N08 & 湾郡 \\
\hline $\begin{array}{l}\text { 354) Dangdaianpudunxiaoz } \\
\text { hen }\end{array}$ & B02 & $\mathrm{A} 01, \mathrm{~A} 02$ & N29 & 当代安普敦小镇 \\
\hline $\begin{array}{l}\text { 355) Baolihaishangwuyueh } \\
\text { ua }\end{array}$ & B03, B05 & $\begin{array}{l}\text { A01, A02, } \\
\text { A03 }\end{array}$ & $\mathrm{N} 23$ & 保利海上五月花 \\
\hline 356)Meilanshanju & B07 & A01 & N02 & 梅兰山居 \\
\hline 357)Huazhoutingan & B03 & $\begin{array}{l}\text { A01, A02, } \\
\text { A03 }\end{array}$ & N06 & 华舟汀岸 \\
\hline 358) Yidunyangguang & $\begin{array}{l}\text { B02, B03, } \\
\text { B07 }\end{array}$ & $\begin{array}{l}\text { A01, A02, } \\
(\mathrm{A} 04)\end{array}$ & N17 & 伊顿阳光 \\
\hline 359) Yijingjiangnan & B03 & $\mathrm{A} 01, \mathrm{~A} 03$ & N14 & 怡景江南 \\
\hline 360)Meijiahubinxincheng & B02, B03 & $\begin{array}{l}\text { A01, A02, } \\
\text { A04 }\end{array}$ & N41 & 美加湖滨新城 \\
\hline $\begin{array}{l}\text { 361) Meijiashidanfogonggu } \\
\text { an }\end{array}$ & B03 & $\mathrm{A} 01, \mathrm{~A} 03$ & N06 & 美加史丹佛公馆 \\
\hline
\end{tabular}




\begin{tabular}{|c|c|c|c|c|}
\hline 362)Ruihehuafu & B03 & A02 & N04 & 瑞和华府 \\
\hline 363) Yangguang100dahudi & $\begin{array}{l}\text { B02, B03, } \\
\text { B04 }\end{array}$ & $\begin{array}{l}\mathrm{A} 01, \mathrm{~A} 02, \\
(\mathrm{~A} 04)\end{array}$ & $\mathrm{N} 27$ & 阳光 100 大湖第 \\
\hline 364) Tianzongshuijinglidu & B03 & $\begin{array}{l}\mathrm{A} 01, \mathrm{~A} 02, \\
(\mathrm{~A} 04)\end{array}$ & N22 & 天纵水晶郦都 \\
\hline $\begin{array}{l}\text { 365)Dangdaikameixiaozhe } \\
\text { n }\end{array}$ & $\mathrm{B} 02, \mathrm{~B} 07$ & $\begin{array}{l}\mathrm{A} 01, \mathrm{~A} 02, \\
\mathrm{~A} 03,(\mathrm{~A} 04)\end{array}$ & N31 & 当代卡梅尔小镇 \\
\hline 366)Zhongyechuangyeyuan & $\mathrm{B} 03, \mathrm{~B} 12$ & $\begin{array}{l}\mathrm{A} 01, \mathrm{~A} 02, \\
\mathrm{~A} 03,(\mathrm{~A} 04)\end{array}$ & N16 & 中冶创业苑 \\
\hline 367)Zhaolinyulongwan & B03 & $\begin{array}{l}\mathrm{A} 01, \mathrm{~A} 02, \\
\mathrm{~A} 03,(\mathrm{~A} 04)\end{array}$ & N05 & 兆麟育龙湾 \\
\hline 368)Canglongxintiandi & $\mathrm{B} 02, \mathrm{~B} 12$ & $\begin{array}{l}\mathrm{A} 01, \mathrm{~A} 02, \\
(\mathrm{~A} 04)\end{array}$ & N15 & 藏龙新天地 \\
\hline 369) Gushangqu & B02 & $\begin{array}{l}\mathrm{A} 01, \mathrm{~A} 02, \\
(\mathrm{~A} 04)\end{array}$ & N33 & 谷尚区 \\
\hline 370)Wankemeilizhicheng & $\mathrm{B} 02, \mathrm{~B} 03$ & $\begin{array}{l}\mathrm{A} 01, \mathrm{~A} 02, \\
\mathrm{~A} 04\end{array}$ & N46 & 万科鬼果力之城 \\
\hline 371)Xindiyijing & B02, B03 & $\begin{array}{l}\mathrm{A} 01, \mathrm{~A} 02, \\
(\mathrm{~A} 04)\end{array}$ & $\mathrm{N} 27$ & 新地艺境 \\
\hline 372)Dangdaiguojicheng & B02, B03 & $\begin{array}{l}\mathrm{A} 01, \mathrm{~A} 02, \\
(\mathrm{~A} 04)\end{array}$ & $\mathrm{N} 21$ & 当代国际城 \\
\hline 373)Fozulingshequ & B02, B03 & $\begin{array}{l}\mathrm{A} 01, \mathrm{~A} 02, \\
\mathrm{~A} 03,(\mathrm{~A} 04)\end{array}$ & N116 & 佛祖岭社区(A-F) \\
\hline 374)Xinxinhuayuan & $\mathrm{B} 02, \mathrm{~B} 12$ & A02 & N07 & 新芯花园 \\
\hline 375)Zaishuiyifang & B02, B03 & $\begin{array}{l}\mathrm{A} 03, \mathrm{~A} 02, \\
(\mathrm{~A} 04)\end{array}$ & N06 & 在水一方 \\
\hline 376) Yijialongchen & $\mathrm{B} 02, \mathrm{~B} 03$ & $\begin{array}{l}\mathrm{A} 01, \mathrm{~A} 02, \\
(\mathrm{~A} 04)\end{array}$ & N11 & 宜家龙臣 \\
\hline 377)Canglongxiaoqu & B02 & $\mathrm{A} 01, \mathrm{~A} 02$, & N06 & 藏龙小区 \\
\hline
\end{tabular}




\begin{tabular}{|c|c|c|c|c|}
\hline & & $(\mathrm{A} 04)$ & & \\
\hline 378) Tinglanyuan & B02 & $\begin{array}{l}\text { A01, A02, } \\
\text { (A04) }\end{array}$ & N43 & 汀兰苑 \\
\hline 379)Binhuyihao & B03 & A02 & N02 & 滨湖一号 \\
\hline 380) Yinhewan & B02, B04 & $\begin{array}{l}\text { A01, A02, } \\
\text { A03, A04 }\end{array}$ & N22 & 银河湾 \\
\hline 381) Wanhaoshuianfenglin & $\begin{array}{l}\text { B02, B03, } \\
\text { B07 }\end{array}$ & $\begin{array}{l}\mathrm{A} 01, \mathrm{~A} 02, \\
\mathrm{~A} 03,(\mathrm{~A} 04)\end{array}$ & N38 & 万豪水岸枫林 \\
\hline 382) Jinghuyuan & B02, B03 & $\begin{array}{l}\text { A01, A02, } \\
\text { A04 }\end{array}$ & N29 & 镜湖园 \\
\hline 383)Foaojunxianyaju & B03 & $\begin{array}{l}\text { A01, A02, } \\
\text { (A04) }\end{array}$ & $\mathrm{N} 20$ & 佛奥俊贤雅居 \\
\hline
\end{tabular}


